\title{
Sigot, Marion
}

\section{Junge Frauen mit Lernschwierigkeiten zwischen Selbst- und Fremdbestimmung. Ergebnisse aus einem partizipativen Forschungsprozess}

Opladen ; Berlin ; Toronto : Verlag Barbara Budrich 2017, 300 S. - (Schriftenreihe der ÖFEB-Sektion Sozialpädagogik; 2)

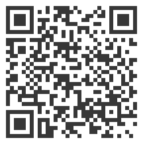

Quellenangabe/ Reference:

Sigot, Marion: Junge Frauen mit Lernschwierigkeiten zwischen Selbst- und Fremdbestimmung.

Ergebnisse aus einem partizipativen Forschungsprozess. Opladen ; Berlin ; Toronto : Verlag Barbara Budrich 2017, 300 S. - (Schriftenreihe der ÖFEB-Sektion Sozialpädagogik; 2) - URN:

urn:nbn:de:0111-pedocs-158335 - DOI: 10.25656/01:15833

https://nbn-resolving.org/urn:nbn:de:0111-pedocs-158335

https://doi.org/10.25656/01:15833

in Kooperation mit / in cooperation with:

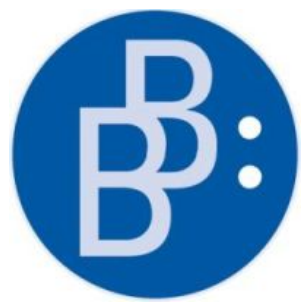

https://www.budrich.de

\section{Nutzungsbedingungen}

Dieses Dokument steht unter folgender Creative Commons-Lizenz: http://creativecommons.org/licenses/by-sa/3.0/de/deed - Sie dürfen das Werk bzw. den Inhalt vervielfältigen, verbreiten und öffentlich zugänglich machen sowie Abwandlungen und Bearbeitungen des Werkes bzw. Inhaltes anfertigen, solange sie den Namen des Autors/Rechteinhabers in der von inm festgelegten Weise nennen und die daraufhin neu entstandenen Werke bzw. Inhalte nur unter Verwendung von Lizenzbedingungen weitergeben, die mit denen dieses Lizenzvertrags identisch, vergleichbar oder kompatibel sind.

Mit der Verwendung dieses Dokuments erkennen Sie die Nutzungsbedingungen an.

\section{Terms of use}

This document is published under following Creative Commons-License: http://creativecommons.org/licenses/by-sa/3.0/de/deed.en - You may copy, distribute and transmit, adapt or exhibit the work or its contents in public and alter, transform, or change this work as long as you attribute the work in the manner specified by the author or licensor. New resulting works or contents must be distributed pursuant to this license or an identical or comparable license.

By using this particular document, you accept the above-stated conditions of use.

\section{(†) (อ)}

\section{Kontakt / Contact:}

peDOcs

DIPF | Leibniz-Institut für Bildungsforschung und Bildungsinformation Informationszentrum (IZ) Bildung

E-Mail:pedocs@dipf.de

Internet: www.pedocs.de

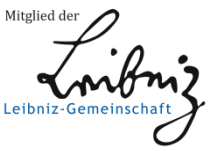




\section{Schriftenreihe der OFEB-Sektion Sozialpädagogik}

\section{Marion Sigot}

\section{Junge Frauen \\ mit Lernschwierigkeiten zwischen Selbst- und Fremdbestimmung}

\section{Ergebnisse aus einem}

partizipativen Forschungsprozess 


\section{Schriftenreihe der ÖFEB-Sektion Sozialpädagogik}




\section{Marion Sigot}

Junge Frauen mit

Lernschwierigkeiten zwischen

Selbst- und Fremdbestimmung

Ergebnisse aus einem partizipativen

Forschungsprozess

Verlag Barbara Budrich

Opladen • Berlin • Toronto 2017 
Bibliografische Information der Deutschen Nationalbibliothek

Die Deutsche Nationalbibliothek verzeichnet diese Publikation in der Deutschen Nationalbibliografie; detaillierte bibliografische Daten sind im Internet über http://dnb.d-nb.de abrufbar.

\section{$\mathbf{K} \cup \mathbf{W}$ \\ @aau.at}

Veröffentlicht mit Unterstützung des Forschungsrates und der Fakultät für Kulturwissenschaften der Alpen-Adria-Universität Klagenfurt.

(C) 2017 Dieses Werk ist im Verlag Barbara Budrich erschienen und steht unter folgender Creative Commons Lizenz:

http://creativecommons.org/licenses/by-nc-nd/3.0/de/

Verbreitung, Speicherung und Vervielfältigung erlaubt, kommerzielle Nutzung und

Veränderung nur mit Genehmigung des Verlags Barbara Budrich

Dieses Buch steht im Open-Access-Bereich der Verlagsseite zum kostenlosen Download bereit (https://doi.org/10.3224/84740127).

Eine kostenpflichtige Druckversion (Printing on Demand) kann über den Verlag bezogen werden. Die Seitenzahlen in der Druck- und Onlineversion sind identisch.

\section{ISBN 978-3-8474-2084-2 \\ DOI $\quad 10.3224 / 84742084$}

Umschlaggestaltung: Bettina Lehfeldt, Kleinmachnow www.lehfeldtgraphic.de Lektorat: Karina Siuda, Berlin Typografisches Lektorat: Anja Borkam, Jena - kontakt@lektorat-borkam.de Verlag Barbara Budrich, http://www.budrich-verlag.de 


\section{Danksagung}

An erster Stelle bedanke ich mich bei all den einzigartigen Frauen, die für mich im Entstehungsprozess der Habilitationsschrift eine wichtige Rolle spielten: Besonders herzlich danke ich den vier Frauen aus der Referenzgruppe, die im Rahmen des partizipativen Forschungsprozesses an der Planung, der Durchführung und der Auswertung der Studie beteiligt waren, über die Orientierungen junger Frauen mit Lernschwierigkeiten erhoben wurden. Ich habe viel von Euch gelernt! An Birgit Grabner ergeht herzlicher Dank für die sorgfältige Protokoll-Erstellung! Alle Frauen, die mir im Rahmen erzählgenerierender Interviews Einblick in ihre Lebenswelt gewährten, danke ich ebenfalls sehr herzlich. Ulrike Loch, Kornelia Tischler und Monika Kastner danke ich für anregende Diskussionen, Anmerkungen und emotionale Unterstützung im Rahmen des Habilitierendenkolloquiums am Institut für Erziehungswissenschaft und Bildungsforschung der Alpen-Adria-Universität Klagenfurt. Gerne denke ich auch an die vielen schönen Stunden, in denen wir es uns nach getaner Arbeit gut gehen ließen.

An Stephan Sting ergeht ganz besonders herzlicher Dank für seine wohlwollende und förderliche Unterstützung, durch die er Raum für Wachstum und Entwicklung zur Verfügung stellt(e). Ernst Kočnik danke ich für die vielen Jahre, in denen er mir als Kollege, Vertreter der SelbstbestimmtLeben-Bewegung und Freund immer ein zentraler und wichtiger Ansprechpartner war und ist. Der Alpen-Adria-Universität Klagenfurt danke ich recht herzlich für die Gewährung einer Freistellung im Wintersemester 2013/14 zum Zweck der Arbeit an der vorliegenden Habilitationsschrift. Danke auch all meinen Kolleginnen und Kollegen am Institut für Erziehungswissenschaft und Bildungsforschung, die mich auf unterschiedliche Art und Weise unterstützten. Nicht zuletzt danke ich meiner Familie und meinem Freundeskreis für den emotionalen Rückhalt, Motivation, gemeinsame Aktivitäten usw. während der Entstehung der Arbeit und natürlich darüber hinaus! Es ist schön, Euch an meiner Seite zu haben! 



\section{Inhalt}

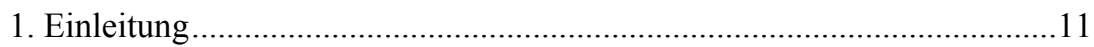

2. Selbstbestimmung von Frauen mit Lernschwierigkeiten:

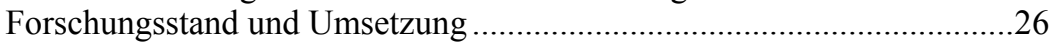

2.1 Der Begriff der Selbstbestimmung....................................................26

2.2 Selbstbestimmung aus der Perspektive der

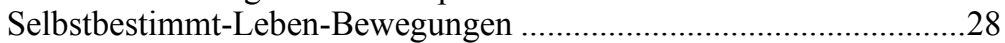

2.3 Selbstbestimmung als Gegenbegriff zu Fremdbestimmung................29

2.4 Abgrenzung von Selbständigkeit und Selbstbestimmung ......................33

2.5 Zur Bezeichnung Menschen mit Lernschwierigkeiten..........................33

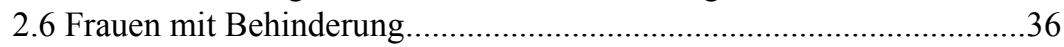

2.7 Die Lebenssituation von Frauen mit Lernschwierigkeiten.....................38

3. Entwicklung, Ansprüche und Umsetzung partizipativer Forschung ..........41

3.1 Von der Forschung über Menschen mit Behinderung zur partizipativen Forschung.

3.2 Grundvoraussetzungen und allgemeine Ansprüche partizipativer Forschung

3.3 Partizipative Forschung mit Menschen mit Lernschwierigkeiten ........45

3.3.1 Verständliche, ,leichte“ Sprache und Anschaulichkeit................45

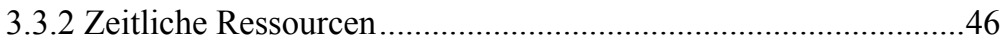

3.4 Die Umsetzung partizipativer Forschung

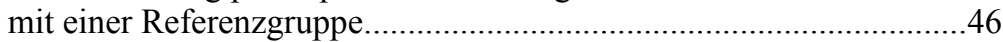

4. Der partizipative Forschungsprozess, Erhebung und Auswertung.............48

4.1 Forschungsleitende Grundfragen .......................................................48

4.2 Der Forschungsprozess mit der Referenzgruppe..................................49

4.2.1 Gruppengröße und -zusammensetzung ....................................50

4.2.2 Der Ort und das Setting der Gruppensitzungen ..........................50

4.2.3 Finanzielle Abgeltung der Arbeit der Expertinnen .......................51

4.2.4 Die Verwendung angemessener Sprache und barrierefreier Materialien .....................................................

4.2.5 Inhalte und Ergebnisse der Referenzgruppensitzungen

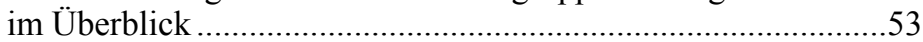

4.2.6 Inhaltlicher Rahmen der Arbeit und Ausgangsbasis aus der Sicht der Frauen aus der Referenzgruppe 


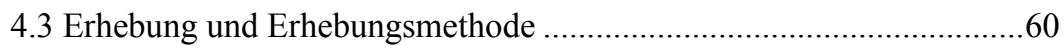

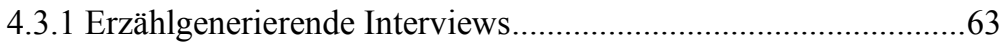

4.3.2 Anregung von Assoziationen und Erzählungen

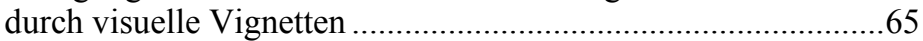

4.3.3 Stichprobe und Zugang zum Feld ............................................68

4.3.4 Das Setting der Interviews ..........................................................

4.4 Die Auswertung der Interviews

mit der dokumentarischen Methode...................................................72

4.4.1 Die Analyseschritte der dokumentarischen Methode und deren Umsetzung im Forschungsprojekt.............................75

5. Die Darstellung der Forschungsergebnisse .............................................88

5.1 Fallrekonstruktionen und sinngenetische Typologie:

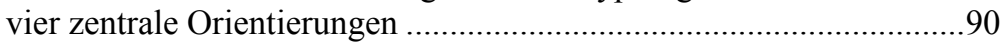

5.1.1 Fallrekonstruktion „Isolde“ (Orientierung A) .............................91

5.1.2 Fallrekonstruktion „Martina“ (Orientierung B) ..........................110

5.1.3 Fallrekonstruktion „Anna“ (Orientierung C) ............................132

5.1.4 Fallrekonstruktion „Eva“ (Orientierung D) ..............................159

5.2 Die vier erhobenen und typisierten Orientierungen in der Einschätzung der Frauen aus der Referenzgruppe ....................183

5.3 Zentrale Themenbereiche der Auseinandersetzung der vier rekonstruierten Orientierungen.

5.4 Soziogenetische Zusammenhänge: Zur Genese der Orientierungen junger Frauen mit Lernschwierigkeiten im Spannungsfeld von Selbst- und Fremdbestimmung

5.4.1 Lebenszusammenhänge als soziogenetische Faktoren

für die Entwicklung von Orientierungen .................................198

5.4.2 Erfahrungsräume als soziogenetische Faktoren

für die Entwicklung von Orientierungen ................................220

5.5 Soziogenetische Zusammenhänge in der Einschätzung

der Frauen aus der Referenzgruppe: ausgewählte Themen.

6. Zusammenfassung, inhaltliche Schlussfolgerungen

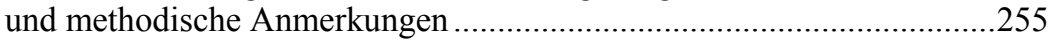

6.1 Die zentralen Forschungsergebnisse im Überblick ............................255

6.1.1 Orientierungen junger Frauen mit Lernschwierigkeiten ...........255

6.1.2 Die Entwicklung der Orientierung und zentrale Wirkungsfaktoren.....

6.2 Schlussfolgerungen zu Selbst- und Fremdbestimmung junger Frauen mit Lernschwierigkeiten 
6.3 Methodische Anmerkungen

6.3.1 Die dokumentarische Methode im Kontext partizipativer Forschung: Erfahrungen aus der vorliegenden Studie und allgemeine Schlüsse

6.3.2 Anmerkungen zur Anregung von Erzählungen im Interview über visuelle Vignetten und zu deren Auswertung mit der dokumentarischen Methode

6.3.3 Methodisch-inhaltliche und forschungsethische Anmerkungen zu partizipativer Forschung...

7. Literatur .283

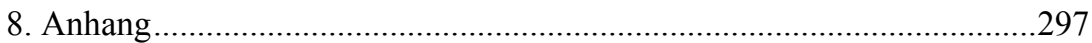

8.1 Regeln für die Belege des Datenmaterials ......................................297

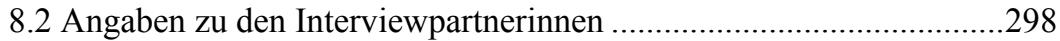

8.3 Verzeichnis der Tabellen und Grafiken ..............................................299

8.4 Quellen der Bilder (visuellen Vignetten) für die Anregung von Erzählungen 300 



\title{
1. Einleitung
}

\begin{abstract}
„Nichts über uns ohne uns! Wir wollen ernst genommen werden! Wir wollen nicht anders behandelt werden als andere! Wir wollen über uns selbst bestimmen! Wir wollen nicht geduzt werden! Wir wollen wie Erwachsene behandelt werden! Nehmen Sie sich für uns Zeit!“" (Wibs 2005: 13).
\end{abstract}

Mit der vorliegenden Habilitationsschrift ${ }^{1}$ ist die Intention verbunden, den hier formulierten, zentralen Ansprüchen von Menschen mit Lernschwierigkeiten $^{2}$ im Rahmen des damit verbundenen Forschungsprozesses gerecht zu werden. Auch mit der durchgängigen Verwendung des Begriffes „Menschen mit Lernschwierigkeiten“ folge ich in der Arbeit den Präferenzen betroffener Menschen, nicht als „Menschen mit geistiger Behinderung“ benannt zu werden, da dieser Begriff von ihnen als stigmatisierend und diskriminierend wahrgenommen wird ${ }^{3}$. Damit möchte ich zum Ausdruck bringen, dass ich sie auch im Kontext der wissenschaftlichen Forschung als Expertinnen und Experten in eigener Sache wahrnehme und anerkenne. Der Begründung für die Ablehnung des Begriffes der ,geistigen Behinderung“, wonach die Verwendung des Begriffes mit negativen Zuschreibungen, Stigmatisierung und Diskriminierung verbunden ist, stimme ich aus wissenschaftlicher Perspektive vorbehaltlos zu.

Forschungsfrage: Die Perspektive von Frauen mit Lernschwierigkeiten auf Fragen im Zusammenhang mit Selbst- und Fremdbestimmung

Ziel der Arbeit ist es, speziell die Perspektive von Frauen mit Lernschwierigkeiten, die bislang in Forschungskontexten wenig berücksichtigt wurde, auf Fragen in Zusammenhang mit Selbst- und Fremdbestimmung in den Fokus

1 Der Originaltitel der Habilitationsschrift lautet: „Junge Frauen mit Lernschwierigkeiten im Spannungsfeld von Selbst- und Fremdbestimmung. Erfahrungen, Ergebnisse und Perspektiven aus einem partizipativen Forschungsprozess mit Teilhabe einer Referenzgruppe“.

2 Wibs [,Wir informieren, beraten und bestimmen selbst“ (Wibs 2005: 3)] ist die Selbstbestimmt-Leben-Initiative von Menschen mit Lernschwierigkeiten in Innsbruck. Mit dieser Forderung knüpft Wibs an die Ausführungen von Andrew Lee, Vertreter der People-FirstBewegung (der Selbstvertretungs-Bewegung von Menschen mit Lernschwierigkeiten in Großbritannien) auf einem Kongress in London im Jahr 2000 an. Dieser sprach sich dabei explizit gegen die Bezeichnung, ,geistig behindert“ aus (vgl. Candussi 2005: 163).

3 Die Verwendung des Begriffes erfolgt in dem Bewusstsein, dass auch der Begriff „Menschen mit Lernschwierigkeiten“ wie jener der „geistigen Behinderung“ ein Konstrukt darstellt, um den gesellschaftlichen und wissenschaftlichen Diskurs zu erleichtern. Auch in der People-First-Bewegung wird der Begriff zwar gegenüber jenem der ,geistigen Behinderung“ präferiert, jedoch zunehmend auch kritisch hinterfragt: „When we talk about people with learning difficulties, we mean 'people labelled as having a learning difficulty'. This is one of the labels that society puts on us to mark us out as not being able to understand things the same as other people" (People First London zit. nach Sigot 2011:156). Auch mit diesem Begriff können demnach defizitorientierte Etikettierungen verbunden sein. 
zu nehmen. Es wird danach gefragt, welche Einstellungen, Erfahrungen und Handlungsstrategien ihr Agieren im Spannungsfeld von Fremd- und Selbstbestimmung prägen. Umgesetzt wird dies in einem partizipativen Forschungsprozess und auf zwei Ebenen: Einerseits indem vier Frauen mit Lernschwierigkeiten in die Planungs-, Erhebungs- und Auswertungsphase eines qualitativen Forschungsprojektes mit einbezogen wurden. Damit kann die Sichtweise von Frauen einer sogenannten Referenzgruppe, die selbst ,in ihrer Sozialisation die Erfahrung des Behindert-Werdens gemacht" (Flieger, 2007: 22) haben, zu Inhalten, Methoden und Ergebnissen der Forschung berücksichtigt werden. Über dieses gemeinsam geplante Projekt wurden auf einer zweiten Ebene Orientierungen junger Frauen mit Lernschwierigkeiten im Spannungsfeld von Selbst- und Fremdbestimmung über erzählgenerierende Interviews mit betroffenen Frauen erhoben und analysiert. Der Einbezug einer Gruppe von Frauen mit Lernschwierigkeiten in die Planungs-, Erhebungs- und Auswertungsphase einerseits und die Adressatinnenorientierung der im Kontext der Studie durchgeführten Forschung andererseits sollten dabei gewährleisten, dass die Perspektive der von der Forschung betroffenen Personen umfassend in den gesamten Forschungsprozess integriert ist.

Der Anspruch, Partizipation im Forschungsprozess umzusetzen, ist auch eine Konsequenz der Berücksichtigung aktueller gesellschaftlicher Entwicklungen, Prämissen und Perspektiven, die die Situation von Menschen mit Behinderungen betreffen. Die UN-Konvention für die Rechte von Menschen mit Behinderungen ${ }^{4}$, verknüpft mit dem Anspruch der Inklusion sowie der Selbstbestimmung von Menschen mit Behinderungen bilden diesen Rahmen und werden in der Folge als Ausgangsbasis partizipativer Forschung, wie sie im vorliegenden Projekt umgesetzt wurde, in den Blick genommen.

\section{Die UN-Konvention für die Rechte von Menschen mit Behinderungen}

Österreich hat sich mit der Ratifizierung der UN-Konvention für Menschen mit Behinderungen im Jahr 2008 verbindlich zur Inklusion und Gleichberechtigung von Menschen mit Behinderungen in allen Lebensbereichen und Lebensphasen bekannt. Damit verpflichtete sich Österreich wie alle anderen Vertragsstaaten dazu, die ,... volle und gleichberechtigte Ausübung aller Menschenrechte und Grundfreiheiten durch alle Menschen mit Behinderungen zu fördern, zu schützen und zu gewährleisten und die Achtung ihrer angeborenen Würde zu fördern“ (Art. 1). Selbstbestimmung ist dabei in einer

4 UN-Konvention. Übereinkommen über die Rechte von Menschen mit Behinderungen. BGB1. III - Ausgegeben am 23. Oktober 2008 - Nr. 155. UN-Konvention. Übereinkommen über die Rechte von Menschen mit Behinderungen. Deutsche Übersetzung des Konventionstextes: http://www.ris.bka.gv.at [Zugriff: 06.04.2017]. 
der acht ,guiding principles ${ }^{\text {c5 }}$ an erster Stelle (a) mit folgender Formulierung angesprochen: „Respect for inherent dignity, individual autonomy including the freedom to make one's own choices, and independence of persons "6. Damit verbunden ist die Idee einer inklusiven Gesellschaft, in der gemeinsames Leben von Menschen mit und ohne Behinderungen in allen Lebensbereichen und Lebensphasen selbstverständlich ist. Mit der Formulierung „full and effective participation and inclusion in society" wird nicht nur ein umfassender und wirksamer Anspruch nach Inklusion, sondern auch nach gesellschaftlicher Teilhabe aller Menschen zum Ausdruck gebracht. Die in der UNKonvention angeführten Rechte sind dabei als „Menschenrechte“ zu begreifen, die konkret hinsichtlich der Personenschutzrechte, umfassender Selbstbestimmungsrechte, Freiheitsrechte, des Rechts auf Familienleben sowie wirtschaftlicher und sozialer Rechte barrierefrei und inklusiv umgesetzt werden müssen (vgl. Schulze 2011: 18f.).

\section{Der Begriff der Inklusion}

Der Begriff der Inklusion, der die gesamte UN-Konvention als Anspruch durchzieht, wurde bereits in den 1990er-Jahren im anglo-amerikanischen Sprachraum geprägt und ging mit der Forderung nach umfassender Aufnahme behinderter Kinder in das Regelschulsystem und der Abschaffung der Sonderpädagogik einher (vgl. Markowetz 2005: 24). Im deutschen Sprachraum wurde der Begriff allerdings weitgehend „schlicht mit Integration“ (ebd.) übersetzt. Der zentrale und tiefgreifende Unterschied der beiden Begriffe wurde damit kaum zum Thema. In vielen Kontexten wurden und werden die beiden Begriffe auch weiterhin ,synonym und nebeneinander“ (ebd.) verwendet. Einen wichtigen Eckpunkt stellte die Salamanca-Erklärung der $\mathrm{UNESCO}^{7}$ von 1994 dar, in der dieser Begriff in den Mittelpunkt rückte und den dazugehörigen Aktionsrahmen bestimmte. In der Erklärung wurde Inklusion mit der Würde des Menschen in Verbindung gebracht und als Menschenrecht formuliert: „Inclusion and participation are essential to human dignity and to the enjoyment and exercise of human rights" (UNESCO 1994). Eine ,präzise Definition” (vgl. Markowetz 2005: 24) des Begriffes der Inklu-

5 In der offiziellen englischen Version der UNO werden die weiteren Prinzipien wie folgt angeführt: „Non-discrimination“, „Full and effective participation and inclusion in society“, „Respect for difference and acceptance of persons with disabilities as part of human diversity and humanity“, „Equality of opportunity“, „Accessibility“, „Equality between men and women“, „Respect for the evolving capacities of children with disabilities and respect for the right of children with disabilities to preserve their identities“. http://www.un.org/ disabilities.html [Zugriff: 06.04.2017].

6 Deutsche Übersetzung: „Die Achtung der dem Menschen innewohnenden Würde, seiner individuellen Autonomie, einschließlich der Freiheit, eigene Entscheidungen zu treffen, sowie seiner Unabhängigkeit“ (Quelle siehe FN 4).

7 Vgl. http://unesdoc.unesco.org/images/0009/000984/098427eo.pdf [Zugriff: 23.05.2017]. 
sion bot die Salamanca-Erklärung allerdings nicht, was dazu führte, dass er etwa in der deutschen Übersetzung der Österreichischen UNESCO-Kommission wieder durchwegs mit „Integration” übersetzt wurde. In der praktischen Umsetzung war damit stets ein Bedeutungsverlust verbunden, der erst mit Blick auf theoretische Grundannahmen deutlich wird: Die Entwicklung des Begriffes der Inklusion ging mit der Kritik am Modell der Integration, das bereits vor beinahe 15 Jahren als „Re-Integration” von Menschen, die zuvor aus gesellschaftlichen Kontexten ausgeschlossen wurden, eingeschätzt wird (vgl. Schumann 2000: 35), einher. Meint Integration ,die Eingliederung von bisher ausgesonderten Personen“ (Krög u.a. 2005: 4), so will Inklusion „die Verschiedenheit im Gemeinsamen anerkennen, d.h., der Individualität und den Bedürfnissen aller Menschen Rechnung tragen“ (ebd.). Mit Inklusion stehen nicht mehr ,besondere Maßnahmen [...], die es Menschen mit Behinderungen ermöglichen sollen, wieder am gesellschaftlichen Leben teilzunehmen, von dem sie vorher ausgeschlossen waren“" (Markowetz 2005: 28), im Fokus. Gesellschaftliche Teilhabe wird im Zusammenhang mit Inklusion demgegenüber als Grundrecht jedes Menschen und somit als Menschenrecht eingeschätzt, das ,selbstverständlich auch und gerade für Menschen mit Behinderungen eigentlich keiner besonderen Begründung bedarf!“” (ebd.). Inklusion beschränkt sich dabei nicht auf Menschen mit Behinderung, sondern nimmt ,alle Dimensionen von Heterogenität“ (Markowetz 2005: 29) in den Blick. Hier wird deutlich, dass Inklusion im Sinne der Umsetzung einer Pädagogik der Vielfalt verstanden werden kann, die ,in der Forschung nach Verschiedenheit fragt und in der Praxis Verschiedenheit $\mathrm{zu}$ ermöglichen versucht“ (Prengel 2009: 104). Inklusive Pädagogik bezieht sich dabei ,auf Prinzipien, Prozesse, Inputs und Ziele im akzeptierenden Umgang mit heterogenen Lernvoraussetzungen“ (Reicher 2011: 162).

Die Integrationspädagogik kann dabei als „Wegbereiterin“ für die Entwicklung einer allgemeinen inklusiven Pädagogik verstanden werden ${ }^{8}$, die nicht als „Regelpädagogik für ,normale’ Menschen“ (Hinz 2005: 78) angelegt sein darf, sondern unterschiedliche Voraussetzungen von Menschen selbstverständlich mit einbeziehen muss.

Der Begriff der Inklusion ist dabei im wissenschaftlichen Diskurs nicht unumstritten. Kritik daran wird vorwiegend im Zusammenhang mit der möglichen Gefahr geübt, dass damit der Anspruch umfassender Teilhabe in allen Lebensbereichen und Lebensphasen zwar erhoben wird, geeignete Rahmenbedingungen aber nicht zur Verfügung stehen würden. Solange dies nicht der Fall sei, könne der Anspruch nach Inklusion dazu führen, dass weniger geeignete Unterstützungsmaßnahmen zur Verfügung gestellt würden als unter dem Postulat der Integration. Der Begriff der Inklusion wird von Kritikerinnen und Kritikern in der Ausgestaltung einer Gesellschaft, ,,in der alle Mit-

8 Damit verbundene Fragen thematisiere ich u.a. in meiner Dissertation „Die Integrationspädagogik als Wegbereiterin einer allgemeinen integrativen Pädagogik“ (Klagenfurt 1998). 
glieder in allen Bereichen selbstverständlich teilnehmen können und die Bedürfnisse aller Mitglieder ebenso selbstverständlich berücksichtigt werden“ (Krög u.a. 2005: 4) auch als utopisch eingeschätzt.

\section{Selbstbestimmung von Menschen mit Behinderung}

Im Kontext der UN-Konvention wird gefordert, dass Menschen mit Behinderung ,selbstbestimmt alle Menschenrechte barrierefrei und - wo notwendig mit Unterstützung - selbst verwirklichen können“ (Schulze 2011: 15). Alle Menschen werden dabei in ihrer Vielfalt wahrgenommen und als gleichberechtigte Mitglieder der Gesellschaft anerkannt. Konsequenterweise bedeutet dies auch, dass ,,jede Person mitgestalten und mitbestimmen darf“ (Krög u.a. 2005: 4). Dieser inklusive Ansatz entspricht dabei den Grundsätzen der Selbstbestimmt-Leben-Bewegung, selbst Verantwortung und Kontrolle über das eigene Leben zu übernehmen. Im Zuge der Entwicklung dieser Bewegung begannen Menschen mit Behinderung, sich für ,die Beseitigung von gesellschaftlichen Benachteiligungen und für eine selbstbestimmte Lebensführung in einer frei gewählten Umgebung“" (Windisch/Miles Paul 1991: 3) $\mathrm{zu}$ engagieren und die Umsetzung entsprechender gesellschaftlicher Rahmenbedingungen einzufordern. Ausgehend von den USA, in denen sich sehr früh solche Bewegungen organisierten, über die skandinavischen Länder und Deutschland in den 1970er- und 80er-Jahren, entwickelte sich die Bewegung gegen Ende des letzten Jahrhunderts auch in Österreich und führte hier zur Gründung entsprechender Initiativen (vgl. Kočnik 2005: 99). Ein zentrales Moment all dieser Initiativen ist die Selbstvertretung, die dadurch gekennzeichnet ist, dass Menschen mit Behinderungen ihre „Probleme selbst definieren, Lösungen selbst ausarbeiten und nie die Initiative an andere abtreten“" (vgl. Kočnik 2005: 102).

Die Entwicklung und Umsetzung notwendiger Unterstützungssysteme knüpft dabei konsequenterweise ,nicht an den traditionellen Konzepten der Behindertenhilfe" (Markowetz 2005: 31) an, die als bevormundend und nicht bedarfsgerecht eingeschätzt werden. Ausgehend vom Befund, dass Menschen mit Behinderungen, die Unterstützung benötigen, von Abhängigkeit, Fremdbestimmung, Freiheitsentzug bis hin zur Körperverletzung bedroht sind (vgl. Brozek 2005: 135), streb(t)en Menschen mit Behinderungen eine bedarfsgerechte und selbstorganisierte Strukturierung der notwendigen Unterstützung an. Die Kritik an nicht bedarfsgerechten Unterstützungsstrukturen, die den Bedürfnissen der betroffenen Menschen häufig nicht entsprechen, führte zum Wunsch, ,sich eigene Organisationsstrukturen anstatt der herkömmlichen Pflegeinstitutionen aufzubauen" (Kaiser 2006: 10). Das im Kontext der Selbstbestimmt-Leben-Bewegung entwickelte Modell der Persönlichen Assistenz kann Menschen mit Behinderungen dazu verhelfen, die ,[...] notwendigen Voraussetzungen $\mathrm{zu}$ gleichen Lebensbedingungen $[\ldots] \mathrm{zu}$ erlangen“ 
(vgl. Ratzka 1988 ${ }^{9}$ ). Damit ist es gleichsam eine logische Konsequenz der Forderung nach Entinstitutionalisierung, die mit den Ansprüchen einer inklusiven Gesellschaft einhergeht.

Die Ausführungen der UN-Konvention verweisen darauf, dass mit dem Anspruch ,der vollen und gleichberechtigten Ausübung aller Menschenrechte und Grundfreiheiten durch alle Menschen mit Behinderungen" (Art. 1) selbstverständlich auch Menschen mit Lernschwierigkeiten gemeint sind. Welche gesellschaftlichen Rahmenbedingungen Menschen mit Lernschwierigkeiten im Kontext von Fragen nach Selbstbestimmung und Inklusion vorfinden, ist Gegenstand der folgenden Ausführungen. Dabei wird insbesondere auf die Zielgruppe im vorliegenden Forschungskontext, Frauen mit Lernschwierigkeiten und deren Lebenssituation, fokussiert.

\section{Selbstbestimmung von Menschen mit Lernschwierigkeiten}

Analysiert man aktuelle Lebensbedingungen aller Menschen mit Behinderungen, so wird deutlich, dass weder unter dem Begriff der Integration noch im Zuge der Hinwendung zum Begriff der Inklusion die gleichberechtigte Teilhabe von Menschen mit Behinderungen in allen Lebensbereichen verwirklicht wurde. Grenzen dafür werden vorrangig an der Art der Behinderung festgemacht: Vor allem für Menschen mit Lernschwierigkeiten gestaltet sich die Umsetzung entsprechender Rechte, die etwa in der UN-Konvention festgeschrieben sind, zögerlich. Dies auch unter der Voraussetzung, dass die UN-Konvention keine diesbezüglichen Einschränkungen vornimmt, also den Anspruch von Rechten nicht von der Art der Behinderung abhängig macht ${ }^{10}$. Die dort angeführten Selbstbestimmungsrechte, verbunden mit der „Gewährleistung von Unterstützungsmaßnahmen - wo erforderlich - auf Basis der individuellen Wünsche von Menschen mit Behinderungen“" (Schulze 2011: 19) werden Menschen mit Lernschwierigkeiten nur eingeschränkt zur Verfügung gestellt ${ }^{11}$. Dies widerspricht der UN-Konvention und den Forderungen von Vertreterinnen und Vertretern der People-First-Initiativen nach Gleich-

9 Vgl. http://www.independentliving.org/docs4/ratzka88a.htm [Zugriff: 06.04.2017].

10 So verweist Marianne Schulze als Vorsitzende des Österreichischen Monitoringausschusses (2008-2015), der die Umsetzung der UN-Konvention überwacht, im Gegenteil darauf, dass durch die Formulierungen alle Menschen mit Behinderung, also auch Personen mit Lernschwierigkeiten, gemeint sind.

11 So haben Menschen mit Lernschwierigkeiten in Österreich kaum Zugang zu Persönlicher Assistenz, sind z.T. sogar explizit vom Anspruch darauf ausgeschlossen. Siehe dazu die IstAnalyse zur Persönlichen Assistenz in Österreich in einer Bundesländer-Übersicht: Stockner, H.: Persönliche Assistenz als Ausweg aus der institutionellen Segregation von Menschen mit Behinderungen. Bericht für Selbstbestimmt Leben Österreich zur Situation der Persönlichen Assistenz in Österreich. http://bidok.uibk.ac.at/library/stockner-assistenz.html [Zugriff: 06.04.2017]. 
stellung und Selbstbestimmung in allen Lebensbereichen (vgl. Wibs 2005: 13).

\section{Frauen mit Lernschwierigkeiten}

Für Frauen und Mädchen mit Behinderungen wird in der Konvention explizit ausgeführt, dass sie „mehrfacher Diskriminierung ausgesetzt sind“ (Art. 6, Abs. 1) und die Vertragsstaaten Maßnahmen zur ,vollen Entfaltung, der Förderung der Autonomie der Frauen“ (Art. 6, Abs. 2) ergreifen. Damit soll laut Konvention eine Garantie der Umsetzung der genannten Menschenrechte und Grundfreiheiten für die Betroffenen verbunden sein. Implizit wird durch diese Ausführungen zum Ausdruck gebracht, dass die angesprochenen Rechte und Freiheiten von Frauen mit Behinderungen im gesellschaftlichen Kontext aktuell nicht als selbstverständlich angesehen, umgesetzt oder ihnen zugestanden werden. So sind sie etwa in der schulischen Förderung und im Bereich von Ausbildung und Beruf benachteiligt (vgl. Bretländer/Schildmann 2011: 41).

Die Wechselwirkungen der „Ungleichheitsindikatoren Geschlecht und Behinderung" (ebd.) wurden lange Zeit in Praxis und Forschung nicht bis kaum beachtet. In der Integrationspädagogik wurde zwar bereits seit Beginn der 1990er-Jahre auf entsprechende Zusammenhänge hingewiesen (ebd.). Allerdings blieb die Situation von Frauen mit Lernschwierigkeiten weiterhin wenig berücksichtigt. Im Zuge der Entwicklung der Selbstbestimmt-LebenBewegung allgemein und damit verbunden der People-First-Bewegung als Selbstvertretungs-Initiative von Menschen mit Lernschwierigkeiten thematisieren betroffene Frauen selbst zunehmend Fragen, die sie für sich selbst als relevant erachten. Erfolgte dies zunächst anfangs eher mit der Forderung, sie überhaupt als Frauen wahrzunehmen, so werden heute fallweise auch inspirierende und vorbildhaft für andere Frauen mit Lernschwierigkeiten wirkende Beispiele selbstbestimmter Lebensgestaltung öffentlich gemacht. So erzählt Elfriede Brauner im „Mutbuch“"12 (2012: 59), wie sie nach schwierigen Erfahrungen in Kindheit und Jugend durch ressourcenorientierte Unterstützung in einer Wohngemeinschaft eine Arbeitsstelle in der freien Wirtschaft gefunden hat und von ihrer persönlichen Assistentin unterstützt wird. Daneben hat sie in einer Selbstvertretungsgruppe Rückhalt. Solche Erfahrungsberichte, die Schritte in Richtung Selbstbestimmung und Autonomie in verschiedenen Lebensbereichen nachzeichnen, vermögen anderen Frauen mit Lernschwierigkeiten Wege aufzuzeigen und sie $\mathrm{zu}$ ermutigen, - wo erforderlich mit Unterstützung - gesellschaftliche Barrieren zu überwinden.

12 Selbstbestimmt Leben Innsbruck - Wibs (Hrsg.) (2012): Das Mut-Buch. Lebensgeschichten von Frauen und Männern mit Lernschwierigkeiten. Neu-Ulm. 


\section{Partizipative Forschung: forschen mit statt über Menschen mit}

Lernschwierigkeiten

Wissenschaftliche Forschung zur Lebenssituation von Menschen mit Behinderungen wurde von Behindertenrechtsaktivistinnen und -aktivisten bislang „eher als Teil des Problems“, denn als Lösungsmöglichkeit hinsichtlich ihrer gesellschaftlichen Benachteiligung gesehen (vgl. König/Buchner 2011: 145). Die Kritik setzt insbesondere an dem Umstand an, dass in den meisten Fällen über Menschen mit Behinderung geforscht wird und sie nicht in die Auseinandersetzung um Fragen, die ihre Lebenssituation thematisieren, mit einbezogen werden. Forschung wird auch als nicht relevant für die Bedürfnisse betroffener Menschen eingeschätzt, weil sie nichts dazu beitragen würde, deren Lebenssituation zu verbessern (vgl. König/Buchner 2011: 145). Nach wie vor sind Menschen mit Behinderung zudem weitgehend ,von den $\mathrm{Zu}-$ gangsbedingungen und Kommunikationsvoraussetzungen der Forschungsproduktion innerhalb der ,sozialen Systeme' Universität und Wissenschaft ausgeschlossen“ (König u.a. 2010: 181). Hier spielt neben vielfältigen Vorurteilen auch das Moment der Sprache als ,[...] der wirksamsten aller Distanzierungstechniken“ (Bourdieu/Passeron 1971: 133) eine nicht zu unterschätzende Rolle. Ausschließungsprozesse erfolgen gerade in der Wissenschaft auch durch Verwendung eines etablierten sprachlichen Codes, der diejenigen, die nicht darüber verfügen, benachteiligt. So gesehen kann die Verwendung „schwerer", akademischer Sprache (nicht nur) für Menschen mit Lernschwierigkeiten auch als Barriere gesehen werden, die Partizipation erschwert.

Dieser ernüchternden Bestandsaufnahme steht die zentrale Forderung aus der People-First-Bewegung „Nichts über uns ohne uns!“ (u.a. Wibs 2005: 13) gegenüber, die für alle Lebensbereiche Geltung hat. Dies kann bezogen auf Forschung als der Anspruch interpretiert werden, eine zentrale Rolle in Forschungsprozessen einnehmen zu wollen. Partizipative Forschung verfolgt die Intention, ,die Beteiligung von gesellschaftlichen Akteuren als Co-Forscher/ innen“ (von Unger 2014: 1) und ,,durch Teilhabe an Forschung mehr gesellschaftliche Teilhabe“ zu ermöglichen (ebd.). Mit der Umsetzung von Forschungsprojekten im Bereich der Disability Studies sowie partizipativer bzw. inklusiver Projekte wird im Bereich der Forschung den Forderungen aus der emanzipatorischen Behindertenbewegung, selbst zu Subjekten der Forschung zu werden, zunehmend Rechnung getragen (vgl. Flieger 2007: 21). Auch wenn es heute ,weitgehend akzeptiert [ist], dass Betroffene selbst am besten wissen, wie es ähnlich Betroffenen geht" (Sierck 2012: 37), hat ein demensprechender, weitreichender Perspektivenwechsel im Bereich der Forschung jedoch noch nicht stattgefunden (vgl. Hermes 2006: 25).

Die Forderung nach der Umsetzung von Forschungsprojekten, die sich an den Interessen von Menschen mit Behinderungen orientieren und sie in die Forschung mit einbeziehen, wird durch die UN-Konvention für Menschen 
mit Behinderungen gestärkt (vgl. Flieger/Schönwiese 2011: 27f.). Mit den in Artikel 3 formulierten allgemeinen Grundsätzen wie der vollen Teilhabe an der Gesellschaft, der Einbeziehung von Menschen mit Behinderungen in die Gesellschaft, der Achtung der Würde, Nichtdiskriminierung, Chancengleichheit und Zugänglichkeit werden auch Standards für die Forschung gesetzt. Forschungsprojekte sollten daher im Interesse von Menschen mit Behinderungen sein, sie in deren Umsetzung mit einbeziehen und in der Durchführung transparent sein. Die barrierefreie Gestaltung von Materialien im Forschungsprozess ist als Grundvoraussetzung für eine gelungene Teilhabe daran unumgänglich. Dieser Anspruch geht auch aus Artikel 9 (1) der UN-Konvention hervor, in dem erforderliche Maßnahmen beschrieben werden, um Menschen mit Behinderung „Zugang zu Information und Kommunikation“ zu gewährleisten. Dies erfordert im Forschungskontext mit Menschen mit Lernschwierigkeiten die Verwendung angemessener, verständlicher, sogenannter ,leichter Sprache“13, im Idealfall verdeutlicht durch Bildmaterial, also mit Anteilen der unterstützten Kommunikation. Neben dem barrierefreien Zugang zu den Inhalten der Forschung müssen darüber hinaus gute Rahmenbedingungen in Abläufen, Methoden und zeitlichen Rahmenbedingungen geschaffen werden (vgl. Flieger 2007: 37ff.).

Der vorliegenden Habilitationsschrift liegt - wie bereits eingangs beschrieben - die Intention zugrunde, partizipative Forschung mit Frauen mit Lernschwierigkeiten umzusetzen. Konkret erfolgte dies dadurch, dass vier Frauen mit Lernschwierigkeiten, organisatorisch erfasst in einer sogenannten Referenzgruppe, in die Konzeption, die Durchführung und die Auswertung einer Studie einbezogen wurden, in der Fragen der Selbstbestimmung von Frauen mit Lernschwierigkeiten selbst thematisiert wurden. Diesem Anspruch entsprechend gestaltet sich der inhaltliche Aufbau der Arbeit.

\section{Zum Aufbau der Arbeit}

Im zweiten, an die Einleitung anschließenden Kapitel der Arbeit wird der Forschungsstand hinsichtlich der zentralen Fragen der Arbeit in den Fokus genommen. Dementsprechend erfolgt eine Ist-Analyse hinsichtlich der Frage der Umsetzung selbstbestimmter Entscheidungs- und Lebensmöglichkeiten von Frauen mit Lernschwierigkeiten. Zunächst werden die Begriffe „Selbstbestimmung“, „Fremdbestimmung“ als zentrale Begriffe der Arbeit in den

13 Die Verwendung „leichter Sprache“ ist ein Anspruch, der ebenfalls aus der People-firstBewegung kommt und erfordert, dass Inhalte in verständlicher Form formuliert werden. Im vorliegenden Forschungskontext präferiere ich selbst die Verwendung des Begriffes der „angemessenen Sprache“, weil er meinen Erfahrungen entspricht, dass Frauen mit Lernschwierigkeiten sprachlich sehr unterschiedliche Voraussetzungen aufweisen. Siehe dazu auch ausführlich Kapitel V, Unterpunkt „Die Verwendung angemessener Sprache und barrierefreier Materialien“. 
Fokus genommen. Die Verwendung der Bezeichnung „Menschen mit Lernschwierigkeiten“" wird mit einem kurzen kritischen Exkurs zum Begriff der „geistigen Behinderung“ reflektiert. Danach wird die Situation von Frauen mit Lernschwierigkeiten auf Grundlage der wenigen vorliegenden Forschungsergebnisse skizziert und so deren mehrfache gesellschaftliche Benachteiligung sichtbar gemacht. Dem partizipativen Anspruch der Arbeit entsprechend, werden in diesem Kapitel dabei bereits neben Forschungsergebnissen Ausführungen und Einschätzungen von Menschen mit Behinderungen mit einbezogen, auch wenn diese nicht (immer) auf Forschungsergebnissen beruhen. Die persönliche Expertise von Personen, die Erfahrungen mit Behinderung in unserer Gesellschaft gemacht haben, wird demnach als Grundlage einer Bestandsaufnahme im Forschungskontext anerkannt.

Das dritte Kapitel der Arbeit thematisiert Entwicklung, Grundannahmen und erforderliche Rahmenbedingungen für die Umsetzung partizipativer Forschung. Diese bilden die Basis für die Umsetzung der entsprechenden Forschungsaktivitäten im Kontext der vorliegenden Arbeit. Ausgehend von der Skizzierung der Entwicklung von Selbstbestimmt-Leben-Bewegungen werden die darin erhobenen Forderungen, dass Menschen mit Behinderungen selbst Verantwortung und Kontrolle über ihr Leben innehaben, ihre „Probleme selbst definieren, Lösungen selbst ausarbeiten und nie die Initiative an andere abtreten" (vgl. Kočnik 2005: 102), thematisiert. Daran anschließend werden zentrale Forderungen daraus auf wissenschaftliche Forschung übertragen reflektiert. Menschen mit Behinderungen wollen demnach als Expertinnen und Experten in eigener Sache gesehen werden, von Objekten zu Subjekten der Forschung werden und eine zentrale Rolle in Forschungsprozessen einnehmen.

Die nächsten beiden Kapitel stehen in engem Zusammenhang miteinander und stellen in ihrer Zusammenschau den Kern der Arbeit dar. In einem partizipativ angelegten Forschungsprozess wurde gemeinsam mit Frauen, die selbst „die Erfahrung des Behindert-Werdens gemacht“ (Flieger 2007: 22) haben, - organisatorisch erfasst in einer sogenannten Referenzgruppe - ein Forschungsprojekt entwickelt. Das gemeinsame Wissen, das Frauen mit Lernschwierigkeiten miteinander teilen, ohne notwendigerweise gemeinsame Erfahrungen gemacht zu haben (vgl. Przyborski 2004: 29), verbindet die Frauen aus der Referenzgruppe dabei miteinander und mit der Zielgruppe des integrierten Forschungsprojektes in einem sogenannten „konjunktiven Erfahrungsraum“"14. Ergebnis dieses gemeinsamen Planungsprozesses war die

14 Die Begriffe „konjunktives Wissen“ und „konjunktive Erfahrung“ spielen eine zentrale Rolle in der theoretischen Fundierung der dokumentarischen Methode als Auswertungsmethode, die für die Studie im Kontext mit der vorliegenden Arbeit gewählt wurde. „Konjunktiv“ verweist dabei auf Wissen oder Erfahrungen, die „milieuspezifisch“ (Bohnsack u.a. 2007: 14) sind. Erfahrungsräume überlagern sich dabei, d.h. dass im vorliegenden Fall der 
Entscheidung, erzählgenerierende Interviews mit Frauen mit Lernschwierigkeiten zu führen und darüber mehr über ihre Erfahrungen und Handlungspraxen im Spannungsfeld von Fremd- und Selbstbestimmung herauszufinden. Nicht nur die Planung, auch die Durchführung und Auswertung dieser Studie erfolgte in einem partizipativen Prozess, in dem die Referenzgruppe durchgängig, aber in unterschiedlicher Funktion und in unterschiedlichem Ausmaß einbezogen war. Damit kommt Fragen im Kontext von Selbstbestimmung und Partizipation inhaltlich wie auch forschungsmethodisch zentraler Stellenwert zu.

Im Fokus des vierten Kapitels der Arbeit stehen der Forschungsprozess und methodische Fragen. Zunächst wird die Entwicklung des Forschungsdesigns und der forschungsleitenden Grundfragen thematisiert. Dabei steht durchgängig parallel dazu der Forschungsprozess im Fokus, in dem insbesondere die Arbeit mit der Referenzgruppe dokumentiert und reflektiert wird. Dafür werden die Inhalte und Ergebnisse der insgesamt sieben stattgefundenen Referenzgruppensitzungen zunächst im Überblick präsentiert. Nach und nach werden auf dieser Grundlage der inhaltliche Rahmen der geplanten Studie sowie die Erhebungs- und Auswertungsmethode entfaltet. Dabei werden jeweils der Einbezug der Frauen aus der Referenzgruppe und die sich daraus ableitenden Ergebnisse für die konkrete Umsetzung im Rahmen der Studie mit beschrieben. Die Besonderheit dieses Kapitels liegt in den Erfordernissen einer Darstellung, die sich von der üblichen wissenschaftlichen Praxis insofern unterscheidet, dass sie kein fertiges Produkt, sondern einen Prozess und dessen Ergebnisse beschreibt. Denn die konkrete Planung einer Studie, die im Rahmen des partizipativ angelegten Forschungsdesigns vorgesehen und letztendlich durchgeführt wurde, konnte folgerichtig ja erst mit Beteiligung der Frauen aus der Referenzgruppe erfolgen. Da die Planung der Studie ein sehr wichtiges Element im partizipatorischen Forschungsprozess darstellt, scheint es mir bedeutsam, diesen Prozess detailliert nachzuzeichnen ${ }^{15}$.

In der Folge wird die gemeinsam geplante Studie selbst methodisch beschrieben. Es werden also die Erhebungs- sowie die Auswertungsmethode differenziert dargestellt. Insbesondere im Zusammenhang mit den erzählgenerierenden Interviews als gewählter Erhebungsmethode wird dabei auch nachgezeichnet, inwiefern sich die Anregungen der Frauen aus der Referenzgruppe hier wiederfinden. Die dokumentarische Methode als Auswertungsmethode wird im Anschluss danach ebenfalls differenziert beschrieben. Diese

Erfahrungsraum, als „behindert“ sozialisiert worden zu sein, sich u.a. mit jenem, weiblichen Geschlechts zu sein, wechselseitig durchdringt (ebd.: 14).

15 Diese differenzierte Dokumentation soll auch der ,,an partizipatorischen Forschungsprojekten häufig geäußerte[n] Kritik [...], dass Prozesse der Beteiligung bzw. konkrete partizipatorische Abläufe im Forschungsprozess nur ungenügend oder nicht ausreichend nachvollziehbar dargestellt werden“ (Flieger 2007: 23), entgegenwirken. 
Methode kommt meiner Einschätzung nach schon von ihrem Ansatz her den Zielsetzungen partizipativer Forschung entgegen, die Perspektive der Zielgruppe auch im Rahmen der Auswertung zu berücksichtigen. Konkret erfolgt dies über ihre speziellen Auswertungsschritte, in denen u.a. im Zuge fallinterner und fallübergreifender Vergleiche die Perspektive der interviewten Frauen zunehmend jene der Forschenden ablöst. Die „Rekonstruktion und Explikation der Vergleichshorizonte“ (Bohnsack 2010: 66) ermöglicht es, dass die Interpretationen nicht auf Grundlage der sogenannten „Standortgebundenheit" oder "Seinsverbundenheit" ${ }^{\text {" }}$ der Forschenden erfolgen, die selbst nicht Erfahrungen gemacht haben, die jenen der Zielgruppe entsprechen. Indem in der dokumentarischen Methode das Wissen der Akteurinnen selbst „die empirische Basis der Analyse“ (Bohnsack u.a. 2007: 11) darstellt, wird der Gefahr, dass es zu Fehlinterpretationen oder Verzerrungen kommen könnte, entgegengewirkt. Im Kontext der vorliegenden Arbeit wird diese Auswertungsmethode um den Einbezug der Referenzgruppe erweitert: Einerseits wurden ausgewählte Interviewpassagen durch die Frauen aus der Referenzgruppe interpretiert und in die Auswertung mit einbezogen, andererseits wurden die von mir als Forscherin herausgearbeiteten Ergebnisse von den Frauen wiederum diskutiert und reflektiert. Besonders im Rahmen der mit der Referenzgruppe durchgeführten Sitzungen, in denen Interviewpassagen von ihnen analysiert wurden, erweist sich die Tatsache, dass „Beschreibungen und Erzählungen [...] unmittelbar verstanden“ (ebd.) werden, als besonders bedeutsam. Durch das Einbringen ihres Wissens, das sie mit den interviewten Frauen mit Lernschwierigkeiten in einem konjunktiven Erfahrungsraum verbindet, sind deren Interpretationen eine meiner Ansicht nach qualitätssichernde Komponente im Auswertungsprozess. Darüber hinaus wurden mit der Rückkoppelung der (Zwischen-)Ergebnisse an die Referenzgruppe diese im Sinne einer kommunikativen Validierung ${ }^{17}$ der „Überprüfung der korrekten Erfassung ihrer Sichtweisen“ (von Kardorff 2009: 247) unterzogen. Mit dem Einbezug der Frauen aus der Referenzgruppe im Rahmen der Auswertung wird auch die sogenannte Investigator-Triangulation (vgl. Flick 2009: 310) umgesetzt, d.h. die Interpretation der erhobenen Daten erfolgt durch verschiedene Personen. Im vorliegenden Projektkontext sind es die Frauen aus der Referenzgruppe und ich selbst mit akademischem Hinter-

16 Unter Bezug auf Mannheim, der die Begriffe „Standortgebundenheit“ und „Seinsverbundenheit" geprägt hat, beschreibt Nohl, dass über die erkenntniskontrollierende Funktion des Einbezugs der Vergleichshorizonte aus den Fällen selbst in die Auswertung ,die Bindung an die Erkenntnis an die alltäglich-existentielle Erfahrungswelt der Forschenden“ (Nohl 2013: 22f.) aufgelöst werden kann.

17 „Kommunikative Validierung“, bei der „Daten oder Ergebnisse der Forschung den Untersuchten mit dem Ziel vorgelegt werden, dass sie von ihnen hinsichtlich ihrer Gültigkeit bewertet werden“ (Steinke 2009: 320), wird auch als Gütekriterium qualitativer Forschung gesehen. 
grund als Forscherin ${ }^{18}$. Planung, Durchführung und Auswertung des integrierten qualitativen Forschungsprojektes erfolgten also in unterschiedlichem Ausmaß, aber durchgängig partizipativ.

Im fünften Kapitel der Arbeit werden die Ergebnisse der im Prozess mit der Referenzgruppe geplanten und durchgeführten Studie präsentiert. Die der Auswertung zugrunde liegenden erzählgenerierenden Einzelinterviews wurden dabei allesamt von mir selbst durchgeführt, wobei ich jene Anmerkungen und Empfehlungen der Referenzgruppe berücksichtigt habe, die im vierten Kapitel der Arbeit beschrieben wurden. Zunächst werden die vier erhobenen zentralen Orientierungen junger Frauen mit Lernschwierigkeiten mittels vier beispielhafter Fallrekonstruktionen differenziert dargestellt. In der dokumentarischen Methode ist dieser Analyseschritt unter dem Begriff der sinngenetischen Typenbildung bekannt. Im Kontext mit Fragen von Fremd- und Selbstbestimmung werden also vier unterschiedliche Formen von Frauen mit Lernschwierigkeiten, mit Rahmenbedingungen und Handlungserfordernissen in verschiedenen Lebensbereichen umzugehen, beschrieben. Die herausgefundenen Orientierungen spannen sich dabei zwischen zwei Polen auf: einerseits einer Herangehensweise, die mit einer weitgehenden Zufriedenheit mit vorgegebenen, mitunter stark strukturierten Rahmenbedingungen verbunden ist, die dem eigenen Bedürfnis nach einem vertrauten Rahmen entgegenkommen. Und andererseits einem umfassenden Anspruch nach Selbstbestimmung und Autonomie, wobei vorgegebene Rahmenbedingungen oftmals als stark einschränkend und bevormundend erlebt werden.

In der darauf folgenden, sogenannten soziogenetischen Analyse steht dann die Frage im Zentrum, wie es zu den unterschiedlichen Orientierungen von Frauen mit Lernschwierigkeiten, die sich zwischen diesen beiden Polen nachzeichnen lassen, kommen kann. Dabei wird auch der Frage nachgegangen, warum es auf Grundlage ähnlicher Erfahrungen zu einer unterschiedlichen Art und Weise kommen kann, aktuellen Herausforderungen zu begegnen. Es wird demnach versucht, ,die sozialen Zusammenhänge“ (Nohl 2012: 110) für die Entstehung unterschiedlicher Orientierungen $\mathrm{zu}$ analysieren. Dabei wird deutlich, dass sich aktuelle Rahmenbedingungen des Lebens und Erfahrungen, die im Verlauf der bisherigen Sozialisation gemacht wurden, auf die aktuelle Orientierung auswirken können, indem sie Handlungsspielräume und Entwicklungsperspektiven der Frauen beeinflussen. Welche Faktoren sich konkret förderlich und welche Faktoren sich hemmend auswirken, ist Gegenstand einer differenzierten Analyse. In diesem Zusammenhang wird auch herausgearbeitet, dass es bei Vorliegen bestimmter Voraussetzungen auch zu einem Wechsel in der Orientierung kommen kann, d.h. dass sich Herangehensweisen und Einstellungen der betroffenen Frauen vor allem durch besondere Erfahrungen auch ändern können. Abgerundet wird dieses

18 Triangulation wird allgemein als qualitätsverbessernde Komponente in der qualitativen Forschung eingeschätzt (vgl. Mayring 2002: 147). 
Kapitel durch die Darstellung der Einschätzung der Ergebnisse durch Frauen aus der Referenzgruppe.

In der Zusammenfassung werden sodann zentrale Ergebnisse der Arbeit im Überblick präsentiert. Diese beziehen sich einerseits auf inhaltliche, andererseits auf methodische Fragen.

Zunächst werden die Ergebnisse der qualitativen Studie zusammengefasst, in der auf Grundlage der Planungen mit der Referenzgruppe die Orientierungen von Frauen mit Lernschwierigkeiten im Rahmen erzählgenerierender Interviews mittels der dokumentarischen Methode analysiert wurden. Die Frage, welche Faktoren im Kontext von Lebensbedingungen und Sozialisationserfahrungen sich als förderlich oder hemmend auf wahrgenommene Handlungsspielräume und Entwicklungsperspektiven erweisen, wird in einer Gesamtzusammenschau in den Blick genommen. Daraus werden als zentrales Ergebnis der Arbeit auch praxisrelevante Schlussfolgerungen abgeleitet, die sich auf Fragen im Zusammenhang von Fremd- und Selbstbestimmung von Frauen mit Lernschwierigkeiten beziehen. Die Überlegungen sind im Sinne einer Ermöglichungsperspektive darauf ausgerichtet, Handlungsperspektiven hinsichtlich der Eröffnung oder Erweiterung selbstbestimmter Entscheidungsräume von Frauen mit Lernschwierigkeiten aufzuzeigen. Im Zuge dessen nehme ich auch Bezug auf vorhandene Forschungsergebnisse und -befunde. Zugleich reflektiere ich die Ergebnisse unter Einbezug der Ausführungen der Frauen aus der Referenzgruppe, die neben dem wissenschaftlichen Wissen jenes von Expertinnen in eigener Sache abbilden, also das konjunktive Wissen von Frauen mit Lernschwierigkeiten selbst. Für mich selbst stellt diese Verwebung der Forschungsergebnisse mit zwei Ebenen von Wissen eine logische Konsequenz dar, in die der gesamte partizipative Forschungsprozess konsequenterweise mündet.

Im durchgeführten Forschungsprojekt kam die dokumentarische Methode als Auswertungsmethode zur Anwendung. Welche Chancen sich meiner Einschätzung nach durch die Anwendung dieser speziellen Methode für die Auswertung von Interviews ergeben, wird in der Gesamtzusammenfassung der Arbeit analysiert. Dort wird auch skizziert, inwiefern der Kontext partizipativer Forschung einen Rahmen darstellt, in dem diese Auswertungsmethode besonders gut zur Anwendung kommen kann. Darüber hinaus wird auch die in den Interviews zum Einsatz gebrachte Vignettenmethode, über die neben erzählgenerierenden Fragen auch visuelle Stimuli zur Anregung von Erzählungen (vgl. Stiehler 2011: 201) gesetzt wurden, hinsichtlich ihrer Bedeutung im Rahmen der Studie reflektiert.

Zuletzt werden die vielfältigen Erfahrungen und Ergebnisse aus dem gemeinsamen Forschungsprozess mit den Frauen aus der Referenzgruppe reflektiert. Daraus resultieren allgemeine Anmerkungen zur Bedeutung partizipativer Forschung im Rahmen wissenschaftlicher Forschung. Kurz gefasst stellen die diesbezüglichen im Rahmen des partizipativen Forschungsprozes- 
ses gewonnenen Ergebnisse durch die Einbeziehung von Frauen mit Lernschwierigkeiten Befunde dar, die es ermöglichen, deren Perspektive in Kontexte zu integrieren, die ihre eigene Lebenssituation betreffen. Dies deckt sich mit der Sicht der Frauen aus der Referenzgruppe, die mit den vorliegenden Ergebnissen der Arbeit ebenfalls diese Möglichkeit verbinden und dies als Chance sehen, die Anliegen von Frauen mit Lernschwierigkeiten öffentlich $\mathrm{zu}$ thematisieren (7RGS-S1-15).

Fragen im Kontext von Selbstbestimmung und Partizipation von Frauen mit Lernschwierigkeiten sind - wie aus der Einleitung hervorgeht - zentrale Themenstellungen der Habilitationsschrift. Vor dem Hintergrund der skizzierten Entwicklungen, durch die Selbstbestimmung und Inklusion von Menschen mit Behinderungen in Gesellschaft und Wissenschaft angesprochen sind, erscheint dabei die Umsetzung partizipativer Forschung nur logisch und konsequent. In allen Phasen des Forschungsprozesses gute Rahmenbedingungen für die Teilhabe von Frauen mit Lernschwierigkeiten zu schaffen, stellte für mich einen zentralen Anspruch dar: angefangen von der freien, gut fundierten, selbstbestimmten Entscheidung, an dem Projekt mitzuwirken, über die Mitgestaltung des Forschungsdesigns bis hin zur Teilhabe im Rahmen der Auswertung. Diese Räume für Teilhabe zu schaffen, ist aus meiner Perspektive eine zentrale Grundvoraussetzung für die Umsetzung qualitätsvoller partizipativer Forschung. 


\section{Selbstbestimmung von Frauen mit Lernschwierigkeiten: Forschungsstand und Umsetzung}

In diesem Kapitel erfolgt eine Ist-Analyse hinsichtlich der Frage der Umsetzung selbstbestimmter Entscheidungs- und Lebensmöglichkeiten von Frauen mit Lernschwierigkeiten. Dafür werden zunächst der Begriff der Selbstbestimmung und dessen Gegenbegriff „Fremdbestimmung“ in den Blick genommen. Danach wird der Begriff der „Lernschwierigkeiten“ als jener, den betroffene Menschen gegenüber dem der geistigen Behinderung präferieren, reflektiert. Nach dieser zunehmenden Konturierung der zentralen Begriffe der Arbeit wird konkret auf Basis vorliegender Forschungsbefunde herausgearbeitet, welche Faktoren die Lebenssituation von Frauen mit Lernschwierigkeiten aktuell prägen. Als Ausgangsbasis für diese Analyse werden Fragestellungen im Kontext von Behinderung und Geschlecht auch aus Perspektive der Geschlechter- und Intersektionalitätsforschung in den Blick genommen.

\subsection{Der Begriff der Selbstbestimmung}

Der Begriff der Selbstbestimmung gilt als Leitidee einer aufgeklärten Gesellschaft. Er ist eng mit der Philosophie der Aufklärung verbunden, in welcher der vernünftige Wille als Voraussetzung für das rationale Handeln des Menschen gilt (vgl. Fornefeld 2009: 184). Im Zuge der Neuzeit löste sich das bislang vorliegende einheitliche christliche Weltbild zunehmend auf (vgl. Gerhardt 1999: 440). „Normen, Werte und Institutionen der eigenen Gesellschaft" (ebd.) werden zunehmend relativiert, die freie Entfaltung des Menschen ist zentrales Anliegen (vgl. Fornefeld 2009: 184). Menschliches Sein wird mit dem Begriff der Freiheit in Verbindung gebracht (vgl. ebd.). In diesem Kontext wird der Begriff der Selbstbestimmung erst relevant. Heute gehört Selbstbestimmung ,zu den weitgehend unbezweifelten Prämissen der gegenwärtigen Ethik“ (Gerhardt 1999: 145) und wird als „ein Schlüsselbegriff der Gegenwart" (Waldschmidt 2012: 11) eingeschätzt. Demnach sollen „alle Menschen selbstbestimmt und autonom ihr Leben gestalten können“ (Waldschmidt 2012: 17).

Mit dem Begriff der Selbstbestimmung ist einerseits die Vorstellung verbunden, ,der Einzelne sei ein autonomes Subjekt, ein Wesen, das losgelöst von Tradition, Erziehung und Sozialstruktur, unabhängig von Zeit, Biografie und Historie persönliche Identität entwickelt und ausgehend von den eigenen Interessen tatkräftig sein Leben gestaltet" (Waldschmidt 2012: 11). Eben jene 
Faktoren werden aber auch als potentiell einschränkend beschrieben. Niemand verfüge ,vollkommen über sich selbst" (Gerhardt 1999: 147), jeder Mensch bleibe „vielmehr an seine psychophysische, sozioökonomische und historische Ausstattung gebunden“ (ebd.). Das ,,jeweilige Niveau dieses Zusammenspiels von Selbst- und Fremdbestimmung“ (Frank 2014: 45) kann dabei u.a. in verschiedenen Lebensphasen variieren. Um ein selbstbestimmtes Leben führen zu können, bedürfe es aus dieser Perspektive auch „Anstrengung“ und „Glück“, dann stünden grundsätzlich „,viele Optionen offen“ (Gerhardt 1992: 146).

Das Leben von Menschen mit Behinderungen war im Zuge der gesellschaftlichen Wandlungsprozesse, die mit der Entwicklung der Leitidee von Selbstbestimmung einhergingen, von Disziplinierung, Ausschluss, Isolation und Exklusion bedroht (Ferdani 2011: 12). Erste Integrationsansätze betreffen die Integration sinnesbeeinträchtigter Kinder in das Schulwesen im 18. Jahrhundert (vgl. Möckel, 2002: 81). Die Integration von Menschen mit zugeschriebener ,geistiger Behinderung“ war vorerst kein Thema. Erst in der Mitte des 19. Jahrhunderts entstanden in mehreren europäischen Staaten „Heil- und Erziehungsanstalten, Heime mit Schulen, später auch Werkstätten für erwachsene Behinderte und mit eigener Landwirtschaft" (ebd.: 84). Die im Zuge damit entstandenen Sonderschulen ermöglichten es erstmals, Schülerinnen und Schüler mit kognitiven Beeinträchtigungen überhaupt erst im Schulwesen zu integrieren (vgl. Möckel 2002: 81), gemeinsamer Unterricht mit nicht beeinträchtigten Kindern war allerdings kein Thema. In der Folge entwickelte sich ein ausdifferenziertes, auf „Behinderungsformen“ fokussiertes Sonderschulsystem (ebd.: 85). In der NS-Zeit wurde Menschen mit Behinderungen der Status des ,unwerten Lebens“ zugeschrieben und sie wurden „unmittelbar von den Maßnahmen faschistischer Gewaltpolitik“ (Ellger-Rüttgardt 1988: 129) erfasst. Nach dem zweiten Weltkrieg wurden Sonderschulen und -einrichtungen ausgebaut, die sich ,vor allem nach den betreuten Behinderungsarten gliedern“ (Jantzen 1974: 65). Im Zuge der Integrationsbewegung und der Entwicklung von Selbstbestimmt-Leben-Bewegungen im letzten Drittel des vergangenen Jahrhunderts wird der Anspruch auf Selbstbestimmung für Menschen mit Behinderung gesellschaftlich erstmals thematisiert.

Gegenwärtig wird Selbstbestimmung nicht nur von Selbstvertretungsbewegungen gefordert, sondern zunehmend auch als Ziel im Bereich der Behindertenarbeit definiert (vgl. Ferdani 2011: 27). Die Umsetzungsmöglichkeiten von Selbstbestimmung im institutionellen Kontext werden jedoch vor allem von Personen aus den Selbstvertretungsbewegungen in Frage gestellt. Institutionelle Strukturen werden dabei an sich als Faktoren eingeschätzt, die fremdbestimmend und diskriminierend wirken.

Der Begriff der Selbstbestimmung wird aus unterschiedlichen Perspektiven auch kritisch reflektiert (vgl. Fornefeld 2009: 186). So wird „,in der neo- 
liberalen Moderne ein Zwang zur Selbstbestimmung“ (ebd.) gesehen. Selbstbestimmung und Autonomie werde damit zur „Pflicht für Menschen mit Behinderung“ (ebd.). Damit sei auch die „Verpflichtung“ zur Eigenverantwortung gegeben, was dazu führen würde, dass der Wohlfahrtsstaat „das Management von Lebenskrisen vermehrt auf das Individuum [...] übertrage“" (ebd.). Selbstbestimmung bleibe aus dieser Perspektive „nur dann eine Errungenschaft der Moderne, wenn sie nicht den ökonomischen Interessen von Sozialpolitik" (ebd.) geopfert werde. Mit der Umsetzung der Forderung nach Selbstbestimmung sei auch die Gefahr verbunden, dass Unterstützung entzogen werde (vgl. Mesdag/Hitzel 2008: 170). Höchst aktuell sind Fragen im Zusammenhang mit Selbstbestimmung auch im Kontext der Diskussionen rund um das Thema Sterbehilfe. Hier wird von Kritikerinnen und Kritikern befürchtet, dass die Forderung nach einem ,individuell beanspruchte[n] Recht auf Selbstbestimmung“ des Lebensendes letztendlich zu einem massiv wahrgenommenen Druck auf schwer kranke, alte und beeinträchtigte Menschen führen könne, Sterbehilfe zu verlangen, um „Kosten im Gesundheitsund Sozialwesen“ einzusparen (vgl. Schumann, 2000: 39f.).

\subsection{Selbstbestimmung aus der Perspektive der Selbstbestimmt- Leben-Bewegungen}

Von den Vertreterinnen und Vertretern aus Selbstbestimmt-LebenBewegungen, also Menschen mit Behinderungen selbst, wird der Begriff der Selbstbestimmung in erster Linie als die Möglichkeit verstanden, Kontrolle über das eigene Leben und benötigte Dienstleistungen zu haben (vgl. Windisch/Miles-Paul 1991: 22) und ,seine Angelegenheiten selber frei regeln zu können“ (Brozek 2005: 136). Menschen mit Behinderung wollen dabei „als Subjekte und nicht in herkömmlicher Weise als Objekte von Hilfe oder Versorgung begriffen“ (Windisch/Miles-Paul 1991: 5) werden.

Besondere Bedeutung kommt auch dem Vorhandensein von Wahlmöglich als Grundvoraussetzung selbstbestimmter Entscheidungen zu (vgl. Brozek 2005: 136). Hier ist eine Anknüpfung an das in den 1950er-Jahren in Skandinavien entwickelte Normalisierungsprinzip feststellbar, in dem ebendiese Wahlmöglichkeiten zwischen akzeptablen Alternativen als Grundvoraussetzung eines normalisierten Lebens beschrieben werden ${ }^{19}$. Mit dem Begriff der Selbstbestimmung ist aus der Perspektive von betroffenen Menschen auch die Kritik am traditionellen Begriff der „Hilfe“ und die Präferenz der Begriffe „Unterstützung“ und „Assistenz“ verbunden. „Hilfeleistungen“ würden zu Abhängigkeit, Ohnmacht und Hilflosigkeit bei Menschen mit Behinderung

19 Zum Normalisierungsprinzip siehe Thimm, Walter (1994): Das Normalisierungsprinzip. Marburg. 
führen (vgl. Frehe 1991: 35). Um dem entgegenzuwirken, wurde in der Selbstbestimmt-Leben-Bewegung das Modell der Persönlichen Assistenz entwickelt, in dem Menschen mit Behinderung nicht mehr „Hilfe “ erhalten, sondern sie selbst entscheiden, welche „Unterstützung“ sie benötigen und wann, wie, wo und von wem diese geleistet wird (vgl. Brozek 2005: 135f.).

Selbstverständlich werden aus dieser Perspektive auch Menschen mit Lernschwierigkeiten umfassende Selbstbestimmungsrechte zuerkannt, da „nicht zwischen Personen unterschieden werden [kann], die die Kompetenz zur Selbstbestimmung haben, diese lernen können oder nicht zur Selbstbestimmung fähig sind“" (Schönwiese 2005: 141). Bei Menschen mit Lernschwierigkeiten müsse es auch darum gehen, ,unterschiedliche Wahlmöglichkeiten aufzuzeigen, um Selbstbestimmung erst konkret zu ermöglichen“ (ebd.: 136).

In People-First-Bewegungen bringen Personen mit Lernschwierigkeiten mit Selbstbestimmung die Möglichkeit, ,alleine zu bestimmen“ (Wibs 2005: 4), wie sie handeln wollen, in Verbindung. Selbstbestimmung sei dann umgesetzt, wenn „Menschen selbst entscheiden, was sie machen“ (Netzwerk People First Deutschland 2004: 7). Eine der wichtigsten Forderungen von Menschen mit Lernschwierigkeiten richtet sich mit dem Slogan „Nichts über uns ohne uns" (Wibs 2005: 13) gegen fremdbestimmte Entscheidungen. Forderungen, die damit verbunden werden, sind u.a. jene nach Selbstbestimmung, Respekt und Gleichbehandlung (ebd.).

Selbstbestimmung wird auch mit dem Begriff der ,Willensfreiheit" in Zusammenhang gebracht (vgl. Dommermuth 2004: 27). „Tätigkeiten“ oder „Formen“, die sich daraus ergeben, werden in diesem Kontext „grundsätzlich akzeptiert" (ebd.). Dies etwa in der institutionellen pädagogischen Praxis umzusetzen, bedeutet, Selbstbestimmung auch in Alltagshandlungen zu ermöglichen bzw. auch zu fördern.

\subsection{Selbstbestimmung als Gegenbegriff zu Fremdbestimmung}

Bei einer differenzierten Perspektive auf den Begriff der Selbstbestimmung wird sichtbar, dass dieser insbesondere auch über seinen Gegenbegriff jenen der Fremdbestimmung - Kontur gewinnt. Als fremdbestimmte Entscheidungen werden solche verstanden, die „über“ oder „für jemanden“ getroffen werden (vgl. Drolshagen/Rothenberg nach Franz 2002: 17). Im Zusammenhang mit der Situation von Menschen mit Behinderungen werden dabei institutionelle Rahmenbedingungen, behindernde Strukturen und Barrieren, einschränkende Rahmenbedingungen und gesellschaftliche Normen als Faktoren möglicher Fremdbestimmung identifiziert (vgl. Drolshagen/Rothenberg nach Franz 2002: 17f.). 
Im Detail wird dies über differenzierte Elemente und Auswirkungen wirksam, die im Überblick in der Tabelle 1 dargestellt und im Anschluss daran differenziert beschrieben werden.

\begin{tabular}{ll}
\hline Faktoren für Fremdbestimmung & Charakteristik und Auswirkung \\
\hline Institutionen & Anpassung an institutionelle Strukturen \\
\hline Sachzwänge & Unterwerfung an fremdbestimmte Tagesabläufe \\
\hline Behindernde Strukturen/Barrieren & $\begin{array}{l}\text { Architektonische Barrieren, } \\
\text { Barrieren im Bereich der Kommunikation }\end{array}$ \\
\hline Fehlende Möglichkeiten der Integration & $\begin{array}{l}\text { Mangelnde Angebote vor Ort legen auf } \\
\text { vorgegebene Möglichkeiten fest }\end{array}$ \\
\hline Normen & Gesellschaftliche Erwartungen können \\
& fremdbestimmend wirken \\
\hline Fachlichkeit und Betreuung & "Entmündigende, fürsorgliche Betreuung durch \\
& Professionelle in Handlungsfeldern wie Pädagogik, \\
& Psychologie, Medizin u.a. \\
\hline
\end{tabular}

Tabelle 1: Faktoren für Fremdbestimmung (eigene Übersicht nach Drolshagen/Rothenberg nach Franz 2002: 17f.)

Institutionelle Strukturen werden demnach als zentrale Faktoren für Fremdbestimmung eingeschätzt ${ }^{20}$. Bereits Foucault und Goffman haben den diskriminierenden Charakter von Institutionen differenziert analysiert und beschrieben. Als „totale Institutionen“ mit „allumfassende[m] Charakter“ (Goffman 1972: 15) gelten u.a. solche, die „zur Fürsorge für Menschen eingerichtet wurden, die als unselbständig oder harmlos gelten“ (ebd.: 16) und „Orte, die der Fürsorge von Personen dienen, von denen angenommen wird, dass sie unfähig sind, für sich selbst zu sorgen [...]" (ebd.). Auch das Gefängnis wird als Beispiel einer totalen Institution beschrieben (ebd.). Dieses wird auch als „erschöpfender Disziplinarapparat" bezeichnet, der zentrale Faktoren des Individuums, ,[...] seine physische Dressur, seine Arbeitseignung, sein alltägliches Verhalten, seine moralische Einstellung, seine Anlagen“" (Foucault 1975: 301), erfasse. Abgeschwächt werden diese Mechanismen auch anderen Institutionen zugeschrieben, die Individuen durch ihre Wirkungsweisen „disziplinieren“. Aus dieser Perspektive erscheinen „sowohl disziplinierende als auch fürsorgliche Interventionen [als] Formen sozialer Kontrolle durch die Professionellen“ (Juhila/Hall/Raitakari 2012: 197).

Für Menschen mit Behinderungen wird der fremdbestimmende Charakter von Institutionen speziell auch darin geortet, dass sie diese zur Anpassung an vorgegebene Rahmenbedingungen führen sollen (vgl. Klee 1980: 90). Unter

20 Zu Fragen der Diskriminierung und Stigmatisierung, insbesondere auch der institutionellen Diskriminierung siehe auch Sigot, Marion (2006): Diskriminierung und Stigmatisierung von Menschen mit Behinderungen. In: Kočnik, Ernst/Kaiser, Herbert/Sigot, Marion (Hrsg.): Stolpersteine auf dem Weg zur Gleichstellung. Klagenfurt, S. 19-48. 
den angeführten Gesichtspunkten wird die Unterbringung von Menschen mit Behinderungen in Heimen aus der Perspektive der Selbstbestimmt-LebenBewegung sogar als ,die größte und schlimmste Verletzung der Menschenrechte gesehen" (Ratzka 2003: 55), der nur durch das Modell der persönlichen Assistenz entgegengewirkt werden kann. Menschen mit Lernschwierigkeiten thematisieren insbesondere den „verwahrenden“ und „bevormundenden" Charakter von Großinstitutionen (vgl. Wibs 2005: 9).

Auch die Begriffe ,strukturelle Gewalt“ (vgl. Jantzen 2004: 157) und ,institutionelle Aussonderung" (Frehe 1991: 35) werden mit fremdbestimmenden, institutionellen Kontexten in Zusammenhang gebracht. Diese würden „Zwangsläufig zu wachsender Entmündigung und Hilflosigkeit“ (ebd.), zu „Sprachlosigkeit und Handlungsunfähigkeit“ (Sierck 2012: 33) führen. Auch ein Zusammenhang von struktureller Gewalt und der Begünstigung von sexuellen Gewalterfahrungen wird festgestellt (vgl. Zemp/Pircher 1996: 120; Mirwald 2009: 39).

Demgegenüber würde eine ,[...] einfache Veränderung der Lebensbedingungen hin zu normalisierten Formen [...]“ (Jantzen 1998: 186) einen wichtigen Beitrag zur „Reorganisation des eigenen Selbst“ (ebd.) darstellen. Noch weitgehender scheint der Befund, dass ,,die sozialen Einrichtungen [...] die Personenkategorien [etablieren], die man dort vermutlich antreffen wird“" (Goffman 1975: 10). Aus dieser konstruktivistischen Perspektive führen institutionelle Strukturen, wenn sie nicht auf die Bedürfnisse der dort lebenden Personen abgestimmten sind, etwa zu Verhaltensweisen, die dann z.B. als „Verhaltensauffälligkeiten“" wahrgenommen und negativ bewertet werden. Erst mit dem „Ende des Verbringens in Sondereinrichtungen und der Verwahrung" wird in der Konsequenz die Entwicklung einer in Richtung Selbstbestimmung und Autonomie weisenden Perspektive (vgl. Feuser 1995: 45) gesehen.

Fremdbestimmung wird auch über vielfältige bauliche oder kommunikative Barrieren erlebt, die Menschen mit Behinderung die Möglichkeit zur Teilhabe erschweren oder nehmen. So sind etwa Menschen im Rollstuhl von Veranstaltungen ausgeschlossen, die keinen barrierefreien Zugang aufweisen. Menschen mit Sinnesbeinträchtigungen werden über Kommunikationsbarrieren Teilhabechancen genommen. Die Verwendung ,schwerer Sprache“ führt zum Ausschluss von Menschen mit Lernschwierigkeiten aus vielfältigen Zusammenhängen. Viele Informationen, die für die selbstbestimmte Gestaltung des eigenen Lebens relevant wären, gehen so an ihnen vorbei.

Auch fehlende Möglichkeiten der Integration wirken fremdbestimmend. Wo Wahlmöglichkeiten fehlen, wird der Begriff der Selbstbestimmung ad absurdum geführt. Gibt es keine akzeptablen Alternativen, so weist der aktuelle Lebenskontext fremdbestimmenden Charakter auf. Wie bereits angeführt, werden in Anknüpfung an das Normalisierungsprinzip Wahlmöglich- 
keiten als Grundvoraussetzung selbstbestimmter Entscheidungen (vgl. Brozek 2005: 136) gesehen.

Gesellschaftliche Normen wirken insofern fremdbestimmend, indem sie Menschen mit Behinderungen als davon abweichend bewerten. Die Gesellschaft wendet sich von einem Individuum, das eigentlich leicht in den ,gewöhnlichen sozialen Verkehr hätte aufgenommen werden können“ (Goffman 1975: 13), aufgrund eines bestimmten Merkmales von ihm ab. Alle anderen Merkmale, Eigenschaften werden in der Folge gar nicht mehr wahrgenommen. Dieser Prozess der Stigmatisierung führt dazu, dass betroffene Menschen auf die Rolle „des/der Behinderten“ festgelegt werden. Demgegenüber müsse es aus einer öko-systemischen Sichtweise in einer idealen Umwelt so vielfältige Rollen geben, dass sie auch von Menschen mit Behinderung ausgefüllt und gesellschaftlich akzeptiert werden könnten (vgl. Sander 2002: 104).

Der fremdbestimmende Charakter von „Fachlichkeit und Betreuung“ wird vor allem in einem Zugang deutlich, in dem von einem Paradigma ausgegangen wird, wonach „Professionelle in ihrer Rolle als Expertinnen und Experten“ (Franz 2002: 30) Entscheidungen über Menschen mit Behinderungen treffen. Unter dieser Perspektive werden Menschen mit Behinderung zu „Patienten“ oder „Klienten“ (ebd.) und werden von ausgebildeten Personen „betreut“. Im Selbstbestimmt-Leben-Paradigma wird die damit verbundene „Abhängigkeit von Fachleuten, Angehörigen etc.“ (ebd.) kritisiert und Menschen mit Behinderung selbst das Expertentum zugeschrieben. Sie werden als „Kundinnen“/,Kunden“ oder „Konsumentinnen“/,Konsumenten“ gesehen, die Unterstützung in einem selbst organisierten Rahmen in Anspruch nehmen (ebd.).

„Fachlichkeit und Betreuung“ kann über defizitorientierte Äußerungen von Fachleuten in unterschiedlichen Kontexten ${ }^{21}$ auch diskriminierenden Faktor aufweisen. Damit wird zur Stigmatisierung von Menschen mit Behinderungen beigetragen (vgl. Klee 1980: 180). Solche Äußerungen können gegenüber Menschen mit Behinderungen selbst, ihren Angehörigen aber auch gegenüber außenstehenden Personen oder Institutionen/Behörden gemacht werden. Nicht selten sind damit auch negative Konsequenzen für die betroffenen Personen verbunden. So etwa, wenn sich defizitorientierte Prognosen in Gutachten über Menschen mit Behinderungen wiederfinden (vgl. Jantzen 2004: 161) und ihnen Teilhabechancen, etwa an der Integration in einem Arbeits- oder Wohnprojekt, nehmen. Mit der "Zurechnungen“" von störendem Verhalten ist auch das Herstellen von „Kontrolle und Herrschaft“ durch professionelle Akteurinnen und Akteure über jene Personen verbunden, die davon betroffen sind (vgl. Juhila/Hall/Raitakari 2012: 197).

Beispiele dafür finden sich in der unter FN 19 angeführten Publikation. 


\subsection{Abgrenzung von Selbständigkeit und Selbstbestimmung}

Der Begriff der Selbstbestimmung wird nicht selten synonym mit jenem der Selbständigkeit gebraucht. Dies ist jedoch insbesondere aus Perspektive von Menschen mit Behinderungen selbst fragwürdig und gefährlich, da damit Personen, die aufgrund von Beeinträchtigungen etwa Alltagsverrichtungen nicht selbständig ausführen können, implizit Selbstbestimmungsrechte abgesprochen werden könnten. Die daraus folgende Notwendigkeit der Abgrenzung der beiden Begriffe wird nicht zuletzt durch ein plakatives praktisches Beispiel von Adolf Ratzka, einem der renommiertesten Vertreter der Selbstbestimmt-Leben-Bewegung, nachvollziehbar: Er verdeutlicht an der wegen körperlicher Einschränkungen nicht vorhandenen Möglichkeit, eine alltägliche Verrichtung selbständig auszuführen, die dennoch gegebene Möglichkeit, selbst die Art und Weise der Verrichtung zu bestimmen, indem die dazu erforderliche Unterstützung zur Verfügung gestellt wird: „lch kann mir selbst einen Pullover anziehen, wozu ich - je nach Tageskondition - mindestens eine halbe Stunde brauche. Nachher bin ich total erschöpft und muß mich mindestens eine weitere halbe Stunde lang ausruhen. Ich brauche also insgesamt mindestens $60 \mathrm{~min}$. Wenn ich einen Assistenten damit beauftrage, mir beim Pulloveranziehen zu helfen, spare ich mindestens 59 Minuten, die ich für wichtigere Tätigkeiten verwenden kann. Genauso wie technische Hilfen müssen wir auch Assistenz einsetzen und zwar so wie wir selbst es in der jeweiligen Situation für angebracht halten“ $\left(\right.$ Ratzka $\left.^{22}\right)$. Auch ein hoher Hilfebedarf begründet demnach kein „Verhältnis fremdbestimmender Abhängigkeit" (Franz 2002: 21).

\subsection{Zur Bezeichnung Menschen mit Lernschwierigkeiten}

In der Einleitung der Habilitationsschrift habe ich erläutert, dass ich in der gesamten Arbeit durchgängig die Bezeichnung „Menschen mit Lernschwierigkeiten“ verwende und damit den Präferenzen betroffener Personen, nicht als „Menschen mit geistiger Behinderung“ benannt zu werden, folge. Insbesondere in People-First-Initiativen, in denen betroffene Menschen für die Umsetzung umfassender Selbstbestimmungsrechte eintreten, wird diese Bezeichnung favorisiert. Der Begriff der ,geistigen Behinderung“ wird abgelehnt, weil er mit negativen Zuschreibungen, Stigmatisierung und Diskriminierung in Verbindung gebracht wird (vgl. Wibs 2005: 2). Der Terminus „Lernschwierigkeiten“ verweist aus Perspektive der betroffenen Menschen auf die Fähigkeit, lebenslang zu lernen und sich weiter entwickeln zu können

22 S. www.independentliving.org/docs5/PersAssistenzinSchweden.html [Zugriff: 07.04.2017]. 
(ebd.: 53), wenn dazu genügend Zeit und geeignete Rahmenbedingungen zur Verfügung stehen.

Die Bezeichnung „Menschen mit Lernschwierigkeiten” wird von einzelnen Vertretern der People-First-Initiativen aber auch kritisch reflektiert, weil auch diese innerhalb der Gesellschaft wieder zur Kategorisierung der betroffenen Menschen verwendet werde ${ }^{23}$. Dennoch erscheint er gegenüber dem Begriff der ,geistigen Behinderung“" als weniger stigmatisierend und diskriminierend. Denn gerade mit der Verwendung dieses Begriffes werden sehr negative, defizitorientierte Merkmale verbunden. Alle anderen Charaktereigenschaften des betroffenen Menschen werden mit der Zuschreibung einer „geistigen Behinderung“ überdeckt, „, sie färbt alle Aussagen, welche über die Person gemacht werden, selbst dort, wo sie von keinerlei Belang wäre“ (Radtke 1994: 110). Auch in fachlichen Kontexten, etwa im Rahmen psychologischer und sonderpädagogischer Diagnostik kann die Klassifizierung als „geistig behindert" zu einem „Leben in Ausgrenzung“ führen, indem dadurch Schullaufbahn, Bildungsangebote, Wohnform und Arbeitsmöglichkeit festgelegt werden (vgl. Feuser 1995: 35). Menschen, die als „geistig behindert“ klassifiziert werden, haben auch geringere Chancen zur Teilhabe am Arbeitsleben (vgl. Niehaus 2006: 181).

Die Frage, ob in einem inklusiven Gesellschaftskonzept überhaupt Bezeichnungen oder Kategorien benötigt werden, um Menschen voneinander $\mathrm{zu}$ unterscheiden, wird an dieser Stelle aktuell ${ }^{24}$. In einem inklusiven Rahmen müsste die „,binäre Unterscheidung ,behindert - nicht behindert" (Finnern/ Thim 2013: 159) grundsätzlich überwunden werden können. Diese und andere Fragen im Zusammenhang mit der Auseinandersetzung mit dem Begriff der Behinderung sind weitgehend ungeklärt bzw. umstritten und werden als die „komplexesten und schwierigsten Probleme der Behindertenpädagogik“ (Dederich 2009: 37) eingeschätzt. So ist selbst eine anerkannte Definition des Begriffes der Behinderung an sich nicht existent, obgleich dieser seit vielen Jahrzehnten in Wissenschaft und Alltag gebraucht wird (ebd.). So unterschiedlich die Positionen mit Fragen im Zusammenhang mit dem Begriff der Behinderung auch sein mögen ${ }^{25}$, ist doch relativ unbestritten, dass die Kategorie der Behinderung wie andere Differenzkategorien ein ,gesellschaftliches Ordnungsformat" (Heite 2010: 187) darstellt und in ein Verhältnis zu ihrem „Gegenpol“, der „Normalität“, gebracht wird (vgl. Schildmann 2004: 38). Allgemein bedeutet „Orientierung an der Normalität [...] Orientierung am gesellschaftlichen Durchschnitt“" (Schildmann 2009: 204). Dies dient den

24 Dieser Frage wird in der Habilitationsschrift nicht weiter nachgegangen, sie begleitet meine wissenschaftliche Tätigkeit aber laufend.

25 Einen sehr differenzierten Überblick dazu gibt Markus Dederich in seinem Beitrag: Behinderung als sozial- und kulturwissenschaftliche Kategorie. In: Dederich, M./Jantzen, W. (Hrsg.) (2009): Behinderung und Anerkennung. Stuttgart, S. 15-40. 
Mitgliedern einer Gesellschaft ,als (soziale) Selbstvergewisserung darüber, dass sie sich in der Mitte der Gesellschaft befinden und auf diese Weise zur Gemeinschaft gehören" (ebd.). Menschen mit Behinderung werden in diesem Kontext als Individuen definiert, die vom Durchschnitt abweichen (vgl. Schildmann 2004: 38). Durch diesen Mechanismus wird auch eine gesellschaftliche Macht- und Diskursstrategie aufrechterhalten (vgl. Schildmann 2004: 38), die jenen, die nicht dem entsprechen, was als „,normal“ definiert wird, einen Platz außerhalb der gesellschaftlichen Mitte zuweist. Der Begriff der „Normalität“ als Maßstab für Abweichung bezeichnet dabei keine objektiven Eigenschaften, ,sondern Perspektiven, mit denen jedes Merkmal über die von außen herangetragene Definition zum Beleg von Normalität und Abnormität werden kann“" (Mesdag/Pforr 2008: 19). Damit verbunden sind der Anspruch und die Erwartung an die Mitglieder einer Gesellschaft, im ,jeweiligen Sozial- und Gesundheitssystem“ uneingeschränkt zu funktionieren (ebd.). Ist dies nicht der Fall, erscheint und verhält sich jemand also anders als der Durchschnitt der Gesellschaft, so führt dies zu Irritation. In der sozialen Interaktion ,verkörpert ein behinderter Mensch das Fremde, das Unvertraute“ (ebd.: 50). Die Wahrnehmung dieses Andersseins kann sich im gesellschaftlichen Kontext in unterschiedlicher Weise äußern. Insbesondere Menschen mit Lernschwierigkeiten sind etwa auch von offener Ablehnung (ebd.: 20) betroffen, die sich u.a. in „Anstarren“, ,diskriminierenden Äußerungen“, „Spott und Hänselein“ bis hin zu „Aggressivität und Vernichtungstendenzen“ (Cloerkes 1997: 79) äußern können.

Mit der Frage nach der Notwendigkeit von Begriffen im Zusammenhang mit Behinderung an sich zeichnet sich gerade mit dem Blick auf die Situation von betroffenen Frauen jedenfalls ab, dass eine eindimensionale, auf „Behinderung" fokussierende Sichtweise zu kurz greift. Denn im Zusammenhang mit Behinderung spielen auch andere Differenzkategorien eine Rolle, wie „Geschlecht, [...] soziale und kulturelle Differenz" (Bruner 2005: 88). Auch die Strukturkategorie „Alter“ ist zu berücksichtigen und stellt mit anderen Strukturkategorien bzw. Ungleichheitsanzeigern ,ein komplexes Wirkungsgefüge“ (Marks 2011: 40) dar. „Soziale Kategorien bzw. soziale Ungleichheiten“ können aus Perspektive der Intersektionalitätsforschung also ,nicht isoliert voneinander konzeptualisiert werden“ (vgl. Walgenbach: 55). Vielmehr müssen damit verbundene Fragen von Wechselwirkung, Überkreuzung, Überlappung oder Verflechtung von Ungleichheitskategorien (vgl. Marks 2011: 45) in den Blick genommen werden. 


\subsection{Frauen mit Behinderung}

In der UN-Konvention wird explizit auf den Zusammenhang von Benachteiligung im Kontext von Behinderung und weiblichem Geschlecht fokussiert. Frauen und Mädchen mit Behinderungen sind demnach ,mehrfacher Diskriminierung ausgesetzt" (UN-Konvention, Art. 6, Abs. 1). Die daraus abgeleitete Notwendigkeit, Maßnahmen zur ,vollen Entfaltung, der Förderung der Autonomie der Frauen“ (UN-Konvention, Art. 6, Abs. 2) zu ergreifen, betrifft alle Lebensbereiche und Lebensphasen und bedeutet in Anlehnung an das Modell des Gender-Mainstreamings, dass alle Maßnahmen daraufhin überprüft und ausgerichtet sein sollen, die Bedürfnisse von Mädchen und Frauen mit Behinderung zu berücksichtigen. Nicht explizit thematisiert werden durch die UN-Konvention Bedürfnisse von Personen, die durch die Zweigeschlechtlichkeit als gesellschaftliche Norm, verbunden mit einer unreflektierten „Klassifizierung der Menschen in Männer und Frauen“ (von Braun/Stephan 2005: 33), nicht erfasst werden bzw. die sich weder der Kategorie ,männlich“ noch der Kategorie „weiblich“ zuordnen können oder wol$\operatorname{len}^{26}$. Hier ist insbesondere die Situation von Transgender-Personen angesprochen, deren Perspektive in vielen gesellschaftlichen Kontexten nicht berücksichtigt wird ${ }^{27}$.

\section{Theoretische Grundannahmen}

Im Kontext der Geschlechterforschung, die sich „,vor dem Hintergrund der Frauenbewegung entwickelt" (Gildemeister 2009: 213) hat, wird durchgängig hervorgehoben, dass Geschlecht nicht nur als körperlich organische, sondern vor allem als soziale Kategorie begriffen werden muss (ebd.: 214). Dabei wird immer weniger auf „substantiell-wesensmäßige“ (ebd.) Differenzen als auf ,die Geschlechterverhältnisse unter Aspekten ihrer Hierarchisierung und sozialen Ungleichheit" (ebd.) verwiesen. Geschlecht wird einerseits ,als Strukturkategorie“" und als ,soziale Konstruktion“ eingestuft (vgl. ebd.: 217). Bei der Frage nach den Geschlechterverhältnissen geht es grundlegend um „die Gesamtheit institutionalisierter Regelungen, durch welche Frauen und Männer [...] zueinander positioniert sind" (ebd.). Hierarchien im Geschlechterverhältnis ergeben sich dabei aus der Trennung von Produktionsarbeit

26 So führt Adi-Ida Landgraf aus, dass es nur sehr wenige Menschen gibt, die seine Situation als Mensch mit Beeinträchtigung, der „körperlich Mann und seelisch Frau“ (Landgraf 2010: 76) ist, verstehen.

27 Dieser Befund trifft auch auf Forschung zu, die die Situation von Menschen mit Behinderungen betrifft. Im vorliegenden Forschungskontext liegt der Fokus auf der Situation von Frauen mit Lernschwierigkeiten. Im Sinne des offen angelegten Forschungsdesigns war aber jedenfalls vorgesehen, sensibel hinsichtlich möglicher Thematisierungen im Zusammenhang damit - etwa im Laufe der Erhebungsphase - zu agieren. 
bzw. beruflicher Arbeit und privater Reproduktion, wobei Frauen darauf festgelegt werden, „Hauptakteurinnen in der privaten Reproduktion“ (vgl. ebd.: 217) zu sein. Berufliche Arbeit von Frauen ,wird nicht in der gleichen Weise honoriert" (ebd.), wie jene im privaten Bereich bzw. wie jene der Männer. In diesem Zusammenhang spielen die beiden Begriffe „sex“ und „gender" eine zentrale Rolle. Meint „sex“ die biologischen Merkmale, so sind mit „gender" soziale und kulturelle Zuschreibungen angesprochen (vgl. ebd). Vorstellungen, die auf der Prämisse biologisch gegebener Geschlechtsunterschiede beruhen, sind demnach „Produkte historischer, gesellschaftlichkultureller Interpretation“ (ebd.). Geschlecht wird aus dieser Perspektive als Konstrukt gesehen, das ,im Alltag, im Austausch mit anderen Menschen und Institutionen ständig hergestellt wird“" (Köbsell 2007: 32). Dieser Prozess wird auch als „doing gender“ bezeichnet. Unterschiede im Sozialverhalten von Männern und Frauen werden mit Blick auf diese Prozesse in der Geschlechterforschung auch ,als Effekt von geschlechtstypischen Entwicklungsverläufen“" (Rendtorff 2011: 51) eingeschätzt.

Für Frauen mit Behinderungen können geschlechtsspezifische Fragestellungen nur auf der Grundlage dieser allgemeinen Erkenntnisse über Geschlechterverhältnisse in Gesellschaftssystemen erörtert werden. Zudem sind „Wechselbeziehungen sozialer Kategorien bzw. Machtverhältnisse“ (Walgenbach 2014: 55) bedeutsam, d.h. dass neben dem Differenzmerkmal Geschlecht auch jenes der Behinderung berücksichtigt werden muss. Mit der Kategorie „Behinderung“ geht es im Wesentlichen darum, „eine bestimmte Art der Abweichung von der männlichen bzw. weiblichen Normalität zu definieren und zu klassifizieren" (Schildmann 2009: 224). Wie bereits erwähnt, bildet der Begriff der „Normalität“ den „Gegenpol“ zu jenem der „Behinderung“ und geht gesellschaftlich mit einer Orientierung am Durchschnitt einher (Schildmann 2004: 37f.). Personen, die nicht der damit festgeschriebenen Norm entsprechen, werden als ,abweichend“ kategorisiert (ebd.: 38). Wie „Geschlecht“ ist auch „Behinderung“ als Strukturkategorie zu verstehen und wird ebenfalls über Alltagshandlungen und soziale Interaktionsprozesse „reproduziert und produziert“ (Reiss 2007: 51). In Anlehnung an den Begriff ,doing gender“ wird hier von „,doing handicap“ gesprochen (vgl. Nagode nach Köbsell 2007: 32). Dies geht vor allem mit stereotypen Zuschreibungen wie „schwach, passiv, abhängig, unselbständig, hilfsbedürftig, kindlich, machtlos, unattraktiv" (vgl. ebd.) an Menschen mit Behinderung in sozialen Interaktionsprozessen einher. Behinderung wir auch als Abweichung „,von der Antizipation einer gesellschaftlichen Normalität [...] an einer Leistungsminderung“ (Reiss 2007: 51) gemessen.

Insbesondere Frauen mit Behinderung sind von der ,Zuschreibung des Unvollständigen im Vergleich zur Norm“ (Reiss 2007: 51) betroffen, indem sie als Frauen und als Menschen mit Behinderung, ,in Personalunion zwei benachteiligten Gruppen angehören“" (Köbsell 2007: 33). Das weibliche Ge- 
schlecht stellt aus dieser Perspektive die „Abweichung von der männlichen Norm“ (ebd.: 52), Behinderung die „Abweichung der Normsetzungen von Gesundheit und Leistung“ (ebd.) dar. Auch Männer mit Behinderung sind gesellschaftlichen Benachteiligungen ausgesetzt, allerdings entsprechen sie einerseits mit ihrer Geschlechtszugehörigkeit der gesellschaftlichen Norm. Zudem ist „die gesellschaftliche Kontrolle über Frauen“ mit Behinderung weitergehend als jene über Männer mit Behinderung (vgl. Schildmann 2004: 42). Vor allem im Zusammenhang mit gesellschaftlichen Schönheitsnormen, Fragen der Ausbildung und Erwerbsarbeit und solchen in Zusammenhang von Beziehungen, Sexualität und Mutterschaft erleben Frauen mit Behinderung ,immer wieder Fremdbestimmung und Diskriminierung“ (Franz 2002: $56)$.

\subsection{Die Lebenssituation von Frauen mit Lernschwierigkeiten}

Frauen mit Lernschwierigkeiten sind von den beschriebenen Mechanismen in besonders starkem Ausmaß betroffen. Im Kontext mit den vorab skizzierten Prozessen wird ihre Situation auch mit dem Status der „Abweichung von der Abweichung" (vgl. Friske 1995: 49) charakterisiert, da sich bei ihnen gleich mehrfach Differenzmerkmale treffen. Dies stellt den Ausgangspunkt für negative Zuschreibungen dar. Neben der weiblichen Geschlechtszugehörigkeit und der Attestierung einer Behinderung bildet vor allem die oft weiterhin entgegen dem Willen der Betroffenen verwendete differenzierte Bezeichnung als ,geistig behindert" ${ }^{\text {‘2 }}$ einen Ausgangspunkt für gesellschaftliche Kontrolle, Fremdbestimmung und Diskriminierung. Werden körperliche Einschränkungen noch „eher als technisch ausgleichbar gesehen“" (Schildmann 2004: 43), so werden kognitive Beeinträchtigungen gesellschaftlich besonders negativ bewertet. Der Zugang zu Erwerbsarbeit und familiärer Reproduktionsarbeit bleibt Frauen mit Lernschwierigkeiten weitgehend verschlossen (vgl. Niehaus 2006: 181; Schildmann 2004: 42). Dabei befinden sie sich in einem „,doppelten Dilemma der Identitätsfindung“ (Reiss 2007: 58), da in der Sozialisation zumeist die häuslichen Fähigkeiten gefördert werden, andererseits berufliche Unabhängigkeit als Ziel formuliert wird (ebd.). Indem letztlich beide Bereiche nicht offenstehen, drohen auf psychischer und ökonomischer Ebene negative Folgen, die sich unter anderem in „Identitätsproblemen“ und „Armut“ manifestieren (vgl. Schildmann 2004: 42).

28 Wie in der Arbeit bereits mehrfach angeführt, präferieren betroffene Menschen den Begriff „Menschen mit Lernschwierigkeiten“, da sie den Begriff der „geistigen Behinderung“ als diskriminierend erleben. Allerdings werden sie in vielfältigen Zusammenhängen weiterhin als ,geistig behindert“ klassifiziert, u.a. darum, wenn es um behördliche Angelegenheiten geht. 
Hinsichtlich der Analyse der Lebenssituation von Frauen mit Lernschwierigkeiten führe ich im Folgenden ergänzend die wichtigsten Ergebnisse meines 2003 durchgeführten Forschungsprojektes ,Zur Lebenssituation von Frauen mit geistiger Behinderung ${ }^{629}$ an (Sigot 2003: 59ff.). Dies erscheint mir im Kontext der vorliegenden Forschung als relevant und aufschlussreich, da damit eine Vergleichsfolie zu den aktuellen Forschungsergebnissen abgebildet werden kann. Die Forschungsergebnisse von 2003 deckten sich weitgehend mit den wenigen damals vorliegenden Befunden, wonach bei Mädchen und Frauen mit Lernschwierigkeiten im Sozialisationsprozess häufig Abhängigkeit und Unselbstständigkeit gefördert werden (vgl. Güttgemann 1997: 11). Dies führt häufig zur Entwicklung passiver Verhaltensstrategien (vgl. Friske 1995: 30). In Bezug auf die gesellschaftlichen Rahmenbedingungen stellte ich 2003 fest, dass für betroffene Frauen in der Regel weder normalisierte Rahmenbedingungen noch alternative Lebensformen mit dem Fokus auf Selbstbestimmung zur Verfügung standen. Für betroffene Frauen schien es demnach ein vorgegebenes Lebensmuster zu geben: Dies war von den Faktoren Absolvierung einer Sonderschule, dem Leben im Heim oder bei Eltern/Angehörigen und der Beschäftigung in einer Werkstätte für Menschen mit Behinderungen bestimmt. Wahlmöglichkeiten standen kaum zur Verfügung. Die Frauen waren in der Regel in einer Werkstätte für Menschen mit Behinderungen mit Haushalts- und Handarbeitstätigkeiten befasst und bekamen dafür lediglich „Taschengeld“ oder gingen keiner Beschäftigung nach. Nur wenige Frauen verrichteten ihre Arbeit in räumlicher Distanz vom Wohnort. Materielle Wünsche der Frauen waren sehr bescheiden und gingen über alltägliche Bedürfnisse kaum hinaus. Altersgemäße, größere Wünsche wie eine eigene Wohnung oder ein selbstständiger Urlaub schienen nicht im Vorstellungsbereich der Frauen zu liegen. Die Frage nach persönlichen Beziehungen nahm im Kontext der Forschung von 2003 einen großen Stellenwert im Leben der Frauen ein. In vielen Fällen beschränkten sich die Kontakte aber auf die Mitbewohnerinnen und Mitbewohner im Heim und/oder auf die Herkunftsfamilie. Freundinnen und Freunde erwiesen sich als besonders wichtige Bezugspersonen, Peer-Beziehungen erschienen mitunter auch als Familienersatz. Die Frage nach der Rolle als Frau verbanden die Frauen vorwiegend mit Familie, Partnerschaft, Haushalt und Schönheit. „Das weibliche Lebensmodell als potentielle Ehefrau und Mutter" wurde als gesellschaftliche „Normalität" wahrgenommen (vgl. Sigot/Wernisch 2005: 193). Gleichzeitig erschienen sie ,von den damit verbundenen Bereichen - wie Paarbindung und Sexualität - ausgegrenzt" (ebd.). Darüber hinaus wurde deutlich, dass sich die Frauen häufig selbst Kompetenzen in diesen Bereichen absprachen.

29 Die weitere inhaltliche Beschäftigung mit der Lebenssituation der betroffenen Frauen und eine kritische Reflexion des Begriffes der ,geistigen Behinderung“ sowie der Rezeption der Ansprüche aus der People-First-Bewegung führte dazu, dass ich diesen Begriff damals zuletzt verwendete. 
Hier wurde auch ein Zusammenhang zwischen der negativen Selbstzuschreibung und der Zuschreibung von Defiziten in der Sozialisation sichtbar. Besonders deutlich wurde in der Forschung von 2003 auch, dass Frauen mit Lernschwierigkeiten vielfältige Erfahrungen mit Gewalt, insbesondere mit sexueller Gewalt hatten. Die Opfer wurden vom vorwiegend männlichen Täter meist mit einem Schweigeverbot belegt. Handlungsstrategien gegen sexuelle Gewalt standen kaum zur Verfügung und wurden in der Regel erst nach erfolgten sexuellen Gewalthandlungen - sofern diese überhaupt im sozialen Umfeld bekannt wurden - vermittelt. Insgesamt fiel auf, dass sich die betroffenen Frauen vordergründig zufrieden mit ihrer Lebenssituation zeigten und keine Veränderungswünsche äußerten. Dies brachte ich 2003 damit in Zusammenhang, dass kaum Wahlmöglichkeiten zur Verfügung standen und „vorgezeichnete Lebensmuster ihnen im Rahmen der Sozialisation als ,Normalität' vermittelt" (Sigot 2003: 63) wurden. Zudem standen zu diesem Zeitpunkt im Lebensraum der interviewten Frauen kaum Wahlmöglichkeiten zur Verfügung.

Die Zusammenschau der theoretischen Grundannahmen und vorliegender Forschungsbefunde deckt sich mit den Ausführungen der UN-Konvention, wonach Frauen mit Behinderung vielfältigen Benachteiligungen ausgesetzt sind. Besonders zutreffend erweist sich dies für die Situation von Frauen mit Lernschwierigkeiten. Es werden aber insbesondere aus der Selbstvertretungsbewegung auch Perspektiven der Eröffnung selbstbestimmter Entscheidungs, Handlungs- und Lebensmöglichkeiten sichtbar. Wie die Frauen selbst sich aktuell innerhalb des Spannungsfeldes von Fremd- und Selbstbestimmung orientieren, ist Gegenstand der vorliegenden Arbeit. 


\section{Entwicklung, Ansprüche und Umsetzung partizipativer Forschung}

Die Perspektive von Menschen mit Behinderung selbst auf Fragen, die ihre konkreten Lebensbedingungen betreffen, wurden lange Zeit in der Forschung weitgehend ausgeblendet. Bis gegen Ende des letzten Jahrhunderts waren Untersuchungen über die Lebensbedingungen von Menschen mit Behinderungen zum überwiegenden Teil von einem unausgewogenen Machtverhältnis zwischen Forschenden und Beforschten gekennzeichnet. Die Forscherinnen und Forscher einerseits ausgestattet ,mit Disziplinar-, Normierungs- und Prüfungsmacht" (Flieger 2007: 21), auf der anderen Seite jene, ,die abweichen, die nicht entsprechen, die anders sind und deren Nützlichkeit sich darin erschließt, Objekt des Handelns anderer zu sein" (ebd.), prägten vorwiegend die Wissensproduktion in den Humanwissenschaften.

In diesem Kapitel erfolgt zunächst eine Skizzierung der Entwicklung partizipativer Forschung. Voraussetzungen und Ansprüche, die sich aus der Teilhabe der von der Forschung betroffenen Personen in Forschungsprozessen ergeben, werden reflektiert. Fragen im Zusammenhang mit der Teilhabe von Menschen mit Lernschwierigkeiten an partizipativen Forschungsprojekten werden auch in Hinblick auf die Möglichkeit der konkreten Umsetzung in den Blick genommen. Dabei liegt der Fokus auf dem Modell der Arbeit mit einer Referenzgruppe, die auch in der vorliegenden Habilitationsschrift praktiziert wurde.

\subsection{Von der Forschung über Menschen mit Behinderung zur partizipativen Forschung}

Eine Abkehr von den eingangs beschriebenen Forschungspraktiken ist in den letzten Jahren parallel zur Entwicklung der Selbstbestimmt-LebenBewegungen, die massiv die Selbstbestimmung und Selbstvertretung von Menschen mit Behinderungen in allen Lebensbereichen einforder(te)n, auszumachen. Ausgehend von den USA, in denen sich sehr früh solche Bewegungen organisierten, über die skandinavischen Länder und Deutschland in den 1970er- und 80er-Jahren, entwickelte sich die Bewegung gegen Ende des letzten Jahrhunderts auch in Österreich und führte hier zur Gründung entsprechender Initiativen (vgl. Kočnik, 2005: 99). Ein zentrales Moment all dieser Initiativen ist die Selbstvertretung, die dadurch gekennzeichnet ist, dass Menschen mit Behinderungen selbst Verantwortung und Kontrolle über ihr Leben innehaben, ihre „Probleme selbst definieren, Lösungen selbst ausarbeiten und nie die Initiative an andere abtreten“ (vgl. Kočnik 2005: 102). 
Diese Forderungen bedeuten übertragen auf wissenschaftliche Forschung, dass die betroffenen Menschen als Expertinnen und Experten in eigener Sache gesehen werden wollen, von „Objekten“ zu „Subjekten“ der Forschung werden und eine zentrale Rolle in Forschungsprozessen einnehmen wollen. Damit wird auch im Bereich der Forschung einer wichtigen Forderung aus der emanzipatorischen Behindertenbewegung Rechnung getragen - „Nichts über uns ohne uns!“ (vgl. Flieger 2007: 21). Mit einiger zeitlicher Verzögerung werden dementsprechende Forschungsansätze, die sich in den USA mit den „Disability studies“ und der „Partizipatorischen Forschung“ etabliert haben (vgl. Flieger 2007: 21), nun auch im deutschsprachigen Raum diskutiert und es wird in einigen wenigen Projekten deren Umsetzung versucht. Im Fokus der Disability studies steht dabei die Frage, auf welche Weise und warum ,historisch, sozial und kulturell - eine Randgruppe wie ,die Behinderten' überhaupt hergestellt" (Waldschmidt 2009: 124) wird. Weitere Themen der Auseinandersetzung sind „Autonomie, Kompetenz, Ganzheit, Normalität, Unabhängigkeit und Abhängigkeit, Gesundheit, körperliche Erscheinung und Ästhetik, Fortschritt und menschliche Perfektibilität" (Dederich 2012: 29). „Die binäre Klassifikation von behindert/nichtbehindert" (Raab 2012: 71) wird dabei in Frage gestellt. Zudem sollen gesellschaftliche „Ausgrenzungsund Diskriminierungsmechanismen“ (Waldschmidt 2009: 126) und Fragen von „Differenz und die Bedeutung von Normen und Normalisierungsprozessen" (Dederich 2010: 171) erforscht werden. Dabei ist das Engagement in Forschung und Theoriebildung mit dem Anspruch verbunden, gesellschaftliche Weiterentwicklung ,hin zu einer Verwirklichung von Bürgerrechten und der Anerkennung behinderter Menschen in einer heterogenitätsfreundlichen Kultur" (ebd.: 173) zu initiieren. Der Fokus partizipativer Forschung liegt auf der Beteiligung der von der Forschung betroffenen Personen in Forschungskontexten. Ausgangspunkt dafür ist die Orientierung am Begriff der Partizipation, mit dem begrifflich „Teilnahme, Teilhabe, Beteiligung oder Mitbestimmung“ (Prosetzky 2009: 89) verbunden werden. „Insbesondere von Marginalisierung bedrohte Zielgruppen" sind dabei Adressatinnen und Adressaten partizipativer Forschung (vgl. Heimgartner/Sting 2012: 15). Auch wenn es heute ,weitgehend akzeptiert [ist], dass Betroffene selbst am besten wissen, wie es ähnlich Betroffenen geht" (Sierck 2012: 37), hat ein demensprechender, weitreichender Perspektivenwechsel in der Forschung allerdings noch nicht stattgefunden (vgl. Hermes 2006: 25). Insbesondere die Lebenserfahrungen von Menschen mit Lernschwierigkeiten, deren "Selbstkonzept" und ihre „subjektiven (Alltags-)Theorien“ wurden „,bis vor kurzem für pädagogisch irrelevant gehalten“ (Lindmeier 2013: 11f.), deren ,persönliche Erfahrungen unterbewertet“ (Johnstone 2000: 265). Die Perspektive der „Forscher und Praktiker" (Falch 2000: 396) wurde als jene der Experten wahrgenommen. 
Massiv gestärkt werden die Forderungen nach der Umsetzung von partizipativen Forschungsprojekten durch die UN-Konvention für Menschen mit Behinderungen $^{30}$ (vgl. Flieger/Schönwiese 2011: 27f.), die 2008 u.a. auch von Österreich und Deutschland ratifiziert wurde. In Artikel 3 werden als allgemeine Grundsätze u.a. die volle Teilhabe an der Gesellschaft, die Einbeziehung von Menschen mit Behinderungen in die Gesellschaft, die Achtung der Würde, Nichtdiskriminierung, Chancengleichheit und Zugänglichkeit postuliert. Hiermit werden auch Standards für die Forschung gesetzt. Forschungsprojekte sollten folgerichtig im Interesse von Menschen mit Behinderungen und in der Durchführung transparent sein sowie darüber hinaus nach Abschluss den Betroffenen zur Verfügung gestellt werden. Trotz des Rückhaltes durch die UN-Konvention kämpfen partizipative Forschungsansätze aber mit Legitimationsproblemen, da sie mit Emanzipation und Teilhabe Zielsetzungen verfolgen, die im Wissenschaftssystem üblicherweise keine (wesentliche) Rolle spielen (vgl. Waldschmidt 2009: 129). Zudem bleiben die Ergebnisse der Forschung in der Regel auch ,in der Verfügungsmacht der Forschenden" (Wrentschur 2012: 138). Als Ursache für die genannten Barrieren wird auch die „Abgrenzungs- und Konkurrenzkultur“ (Flieger/Schönwiese 2011: 31) an Universitäten gesehen.

\subsection{Grundvoraussetzungen und allgemeine Ansprüche partizipativer Forschung}

Die Umsetzung partizipativer Forschung erfordert die Berücksichtigung zentraler Faktoren, die sich aus dem damit verbundenen Verständnis von Forschung und der Teilhabe von der Forschung betroffener Personen ergeben. Diese beziehen sich einerseits auf Beziehungsaspekte, andererseits auf organisatorische Rahmenbedingungen und die konkrete Gestaltung inhaltlicher Abläufe.

Ein ebenso zentrales wie unverzichtbares Element partizipativer Forschung ist ein verändertes Rollenverständnis, das von der Intention getragen ist, einen Machtausgleich zwischen Menschen mit und ohne Behinderungen anzustreben. Dies bedeutet für Forschende, ,die eigene Position, die Beziehungen zum Forschungsfeld und möglicherweise damit verbundene Machtgefüge" (Finnern/Thim 2013: 165) zu erkennen und zu reflektieren. Auf Seiten der Forschenden mit akademischem Hintergrund sind Gegenstand solcher „selbst-reflexive[n] Elemente“ insbesondere „Gegenübertragungs-

30 UN-Konvention. Übereinkommen über die Rechte von Menschen mit Behinderungen. BGB1. III - Ausgegeben am 23.10.2008 - Nr. 155. Deutsche Übersetzung des Konventionstextes: http://www.monitoringausschuss.at [Zugriff: 07.04.2017]. 
phänomene“, die aus deren persönlichen und beruflichen Erfahrungen resultieren (vgl. Flieger/Schönwiese 2011: 32).

Für Forschende ohne akademischen Hintergrund wiederum ist es erforderlich, die eigenen Beiträge als Expertinnen- oder Expertenwissen wahrnehmen und einbringen zu können. Dies kann für Menschen mit Behinderungen, insbesondere für Menschen mit Lernschwierigkeiten vor dem Hintergrund von Sozialisationserfahrungen, in denen ihnen dies oft nicht zugestanden wird, mit vielfältigen Herausforderungen verbunden sein. Raum dafür zu schaffen, in dem „das Eigene zum Sprechen“ (Anhorn/Stehr 2012: 71) gebracht werden kann, ist demnach ein wichtiger Anspruch an partizipative Forschung.

Im Forschungsprozess ist es erforderlich, diese Überlegungen zu integrieren und an geeigneter Stelle, jedenfalls aber zu Beginn und bei Abschluss der gemeinsamen Arbeit (vgl. Flieger 2007: 24f.), zu thematisieren. Dazu ist es unerlässlich, dass die an der Forschung beteiligten Personen tatsächlich ,im Dialog" (Falch 2000: 395) miteinander stehen. Im Verlauf des gemeinsamen Arbeitsprozesses müssen die Rollenverständnisse immer wieder auch reflektiert und ausgehandelt werden. Damit einhergehende Veränderungen traditioneller Rollenzuschreibungen können bei Berücksichtigung der angeführten Aspekte auch emanzipatorisch wirken (vgl. König u.a. 2010: 186ff.).

Als förderlich für einen veränderten und gemeinsame Forschungsarbeit unterstützenden Zugang erweist es sich dabei, wenn sich ,,alle Beteiligten als Lernende verstehen“ (Flieger 2007: 40) und das Forschungsdesign ,prozessorientiert [...] und flexibel" (ebd.) sowie transparent (vgl. Bloemers 2000: 306) angelegt ist und viel Handlungsspielraum ermöglicht.

Auch gegenseitiger Respekt und Anerkennung aller an der Forschung beteiligten Personen wird als Grundvoraussetzung gelungener Teilhabeprozesse eingeschätzt (vgl. Bloemers 2000: 306).

Gemeinsam Ziele zu definieren und darauf abgestimmt das methodische Vorgehen abzuklären, ist wichtiger Teil partizipativer Forschung. Dabei sind relevante Themen solche, ,die für die Akteure in den Lebenswelten und in der Praxis eine Relevanz besitzen“" (von Unger 2014: 53). Die Wahl der Methode soll im Kontext der Fragestellungen und Zielsetzungen sowie entsprechend der jeweiligen Rahmenbedingungen erfolgen (ebd.). Dabei wird davon ausgegangen, dass ,die Akteure in den Lebenswelten über relevante Wissensbestände verfügen [...] und zu kritischer Reflexion in der Lage sind“" (ebd.). 


\subsection{Partizipative Forschung mit Menschen mit Lernschwierigkeiten}

Wenn partizipativen bzw. inklusiven Forschungsansätzen allgemein auch zunehmend Bedeutung beigemessen wird, so sind gerade Menschen mit Lernschwierigkeiten ,nach wie vor von den Zugangsbedingungen und Kommunikationsvoraussetzungen der Forschungsproduktion innerhalb der ,sozialen Systeme' Universität und Wissenschaft ausgeschlossen“" (König u.a. 2010: 181). Sie als Akteurinnen und Akteure zu begreifen, die ,nicht nur in der Lage sind, ihre Sichtweisen und Bedürfnisse zu artikulieren, sondern auch Lösungsansätze zu entwickeln [...]“" (von Unger 2014: 57), scheint im Kontext wissenschaftlicher Forschung besonders schwer vorstellbar.

\subsubsection{Verständliche, ,, leichte“ Sprache und Anschaulichkeit}

Gerade in der Wissenschaft erfolgen Ausschließungsprozesse auch durch die Verwendung eines etablierten sprachlichen Codes, der diejenigen, die nicht darüber verfügen, benachteiligt.

Die Verwendung nicht verständlicher, akademischer, sogenannter ,schwerer" Sprache stellt für Menschen mit Lernschwierigkeiten eine Barriere dar, die deren Partizipation gerade in Forschungskontexten erschwert. Teilhabe von Menschen mit Lernschwierigkeiten zu ermöglichen, erfordert daher in diesem Kontext zunächst ,theoretisch-abstrakte und wissenschaftliche Inhalte in einfacher, allgemein verständlicher Weise zu präsentieren“ (vgl. Flieger 2007: 34). Diese Arbeit ist für Wissenschafterinnen und Wissenschafter ,eine herausfordernde, aber notwendige Aufgabe“ (vgl. Flieger 2007: 34). Im Sinne der in der UN-Konvention geforderten Barrierefreiheit müssen Materialien im Forschungsprozess dementsprechend gestaltet werden, d.h. etwa, Texte in sogenannte ,leichte Sprache ${ }^{\text {(31 }}$, übersetzt werden. Die Verwendung von Bildmaterial kann hier unterstützend wirken.

Auf entsprechende Empfehlungen bzw. Handreichungen von People-FirstInitiativen, wie etwa dem „Wörterbuch für leichte Sprache ${ }^{632}$, sollte dabei zurückgegriffen werden. Denn diese Inhalte sind von betroffenen Personen selbst erstellt und/oder auf ihre Verständlichkeit für Menschen mit Lernschwierigkeiten geprüft.

31 Der Begriff „leichte Sprache“ stammt aus der People-First-Bewegung. So fordert etwa die Selbstvertretungsbewegung WIBS ,Informationen in leichter Sprache“ (Wibs 2005: 41).

32 Netzwerk People First Deutschland e.V. (Hrsg.) (2004): Wörterbuch für leichte Sprache. Kassel. 


\subsubsection{Zeitliche Ressourcen}

In partizipativen Forschungsprozessen muss Menschen mit Lernschwierigkeiten auch ausreichend Zeit zur Verfügung stehen, ,sich in Thema, Inhalt und Sprache zu orientieren“ (vgl. Flieger 2007: 40). Gerade diese Voraussetzung umzusetzen, stellt allerdings im akademischen Forschungsalltag, der von „Zeitdruck“ geprägt ist, eine nicht gering zu schätzende Herausforderung dar (vgl. ebd: 33). In diesem Kontext wird die Frage relevant, ob die „Verlangsamung zeitlicher Abläufe", wie sie als Grundvoraussetzung partizipativer Forschung beschrieben wird (vgl. ebd.), sich nicht grundsätzlich qualitätssteigernd auf Forschung auswirken kann, indem dadurch mehr Spielraum für kreative Prozesse entsteht.

\subsection{Die Umsetzung partizipativer Forschung mit einer Referenzgruppe}

Sehr gut umsetzen lässt sich partizipative Forschung mit Beteiligung einer sogenannten „Referenzgruppe“, die sich aus Personen zusammensetzt, die „von der jeweiligen Forschungsfrage betroffen sind“ (Flieger 2007: 22). Über die Teilhabe im Rahmen einer solchen Konstruktion können „Personengruppen, die in der Forschung traditionell nicht- oder unterrepräsentiert sind, [...] ein Sprachrohr erhalten“ (ebd.: 38). Dieses Kriterium trifft besonders auf Menschen mit Lernschwierigkeiten zu.

Für die Organisation einer Referenzgruppe ${ }^{33}$ ist es zielführend, verschiedene Aspekte zu beachten (vgl. Flieger 2007: 37), die förderlich für die gemeinsamen Forschungstätigkeiten in einem partizipativen Prozess sind. Das zu Beginn erforderliche „Konstituierungsverfahren“ (Flieger 2007: 24) der Gruppe bedingt dabei zunächst die Auseinandersetzung mit konkreten Fragen hinsichtlich der Zusammensetzung der Gruppe. Die institutionelle Verankerung potentieller Mitglieder, Gruppengröße, Organisation der Abläufe und konkrete Arbeitsabläufe und -modalitäten sind dabei Kriterien, die im Kontext mit dem jeweiligen Projekt berücksichtigt werden sollten. Auch die Auswahl eines geeigneten Ortes für die Gruppensitzungen und das spezifische Setting der Gruppentreffen bedürfen auf den jeweiligen Kontext abgestimmter Überlegungen, da sich diese Faktoren unmittelbar auf die inhaltli-

33 Zur Etablierung und Arbeit mit einer Referenzgruppe siehe ausführlich auch Sigot, Marion (2012): Partizipatorische Forschung mit Frauen mit Lernschwierigkeiten - Herausforderung und Bereicherung für die empirische Forschung. In: Heimgartner, A./Loch, U./Sting, S. (Hrsg.): Empirische Forschung in der sozialen Arbeit. Methoden und methodologische Herausforderungen. Wien, S. 153-166. 
che Arbeit auswirken ${ }^{34}$. Die Organisation selbst soll dabei Ansprüchen partizipativer Forschung gerecht werden, indem sie bereits Mitsprachemöglichkeit eröffnen soll (ebd.: 37).

In regelmäßigen Treffen sollen Forschungsaktivitäten gemeinsam geplant und reflektiert werden (ebd.: 24). Die Umsetzung der einzelnen Forschungsschritte kann unterschiedlich starken Einbezug der Referenzgruppe beinhalten, grundsätzlich sollte es jedoch prinzipielle Offenheit hinsichtlich der konkreten Beteiligung an der Umsetzung geben. Ein zu enges Projektdesign ist für partizipatorische Forschungsprojekte nicht zielführend, da dadurch Spielräume eingeschränkt und die Erweiterung von Rollen durch die Mitglieder der Referenzgruppe, aber auch der beteiligten Wissenschafterinnen und Wissenschafter erschwert werden (vgl. ebd.: 39f.).

Angemessene finanzielle Abgeltung stellt im Rahmen partizipativer Forschung mit einer Referenzgruppe einen Faktor dar, der einerseits Anerkennung der geleisteten Arbeit zum Ausdruck bringt, auf der anderen Seite aber auch das Moment der „Verbindlichkeit“ sicherstellt (vgl. Flieger 2007: 38f.).

Aufbereitungs- und Auswertungsverfahren stellen insofern Herausforderung an partizipative Forschung dar, da sie auch dem Anspruch genügen müssen, in angemessener Sprache an die Referenzgruppe rückgekoppelt zu werden. Auf der anderen Seite sind die Ergebnisse dieser Verfahren auch der akademischen Fachwelt in geeigneter Sprache zur Verfügung zu stellen.

34 Eine sehr hilfreiche Orientierungsrichtlinie für die Arbeit mit Referenzgruppen bietet der Aufsatz von Flieger, P. (2007): Der partizipative Ansatz des Forschungsprojektes. Das Bildnis eines behinderten Mannes. Hintergrund - Konzepte - Ergebnisse - Empfehlungen. In: Schönwiese, V./Flieger, P. (Hrsg.): Das Bildnis eines behinderten Mannes. Bildkultur der Behinderung vom 16. bis ins 21. Jahrhundert. Neu-Ulm, S. 19-42. 


\section{Der partizipative Forschungsprozess, Erhebung und Auswertung}

In diesem Kapitel der Arbeit wird der Prozess der im Kontext mit der vorliegenden Habilitationsschrift durchgeführten Forschung mit einer Referenzgruppe auf der Basis der im letzten Kapitel angestellten Überlegungen dokumentiert. Dabei werden methodische Fragen im Zusammenhang mit der Erhebung und der Auswertung differenziert dargestellt. Die Auswertungsschritte der dokumentarischen Methode werden im Detail dargestellt, um auch die konkrete Teilhabe der Frauen aus der Referenzgruppe im Auswertungsprozess nachvollziehbar zu machen.

\subsection{Forschungsleitende Grundfragen}

Wie in der Einleitung der Arbeit ausgeführt, war es für mich im Gesamtkontext der Forschung im Rahmen der vorliegenden Arbeit von zentralem Interesse, Fragen von Fremd- und Selbstbestimmung für Frauen mit Lernschwierigkeiten in den Fokus zu nehmen und dabei über die Teilhabe einer Referenzgruppe dem von der People-First-Bewegung erhobenen Anspruch „Nichts über uns ohne uns!“ zu entsprechen.

Von besonderem Interesse war für mich dabei die Frage, inwieweit sich Frauen mit Lernschwierigkeiten als fremd- und/oder selbstbestimmt in unterschiedlichen Lebenssituationen wahrnehmen, wie sie selbst in diesen Kontexten handeln und was Erfahrungen im Rahmen ihrer Sozialisation damit zu tun haben. Diese und damit von Frauen mit Lernschwierigkeiten selbst verbundene Fragen über ihre im Forschungsverlauf im Zentrum stehende Perspektive selbst zu erhellen, war der durchgehende Anspruch, an dem ich mich in allen Phasen der Forschung zu orientieren versuchte. Die allgemein mit partizipativen Projekten verbundene „Vagheit“ der formulierten forschungsleitenden Grundfragen zu Beginn der Forschung ist nicht selten - vor allem im Zusammenhang mit Projektanträgen (vgl. von Unger 2014: 54) - Anlass für Irritationen, wo dies zunächst oft als nicht üblichen Kriterien entsprechend beurteilt wird und Präzisierungen urgiert werden. Diese Vagheit ist aber dem Umstand geschuldet, dass die eigentliche Projektplanung erst im partizipativen Forschungsprozess unter Mitwirkung der einbezogenen Personen selbst erfolgen kann, um dem Anspruch auf Teilhabe von der Forschung betroffener Personen in allen Projektphasen zu entsprechen. Zudem entspricht dies aber auch einer „konsequenten Umsetzung der Offenheit bei der Erhebung“ (Rosenthal 2014: 15) im Kontext qualitativer und interpretativer Forschung. Auch hier wird der Ausgangspunkt der Forschung „mit einem vagen Interes- 
se an einem bestimmten sozialen Phänomen oder einem bestimmten Milieu“ (ebd.) beschrieben. Damit ist aber bereits dennoch zu Beginn der Forschung „die Blickrichtung auf die Phänomene und damit [der] methodische Zugang“ (ebd.: 16) verbunden. Im Kontext von Forschung zur Frage der Selbstbestimmung von Frauen mit Lernschwierigkeiten ergibt sich daraus, dass deren Perspektive zentrale Berücksichtigung erfahren muss. Methodisch leitet sich daraus - meiner Ansicht nach konsequenterweise - ab, die von der Forschung betroffene Personengruppe auch auf der Ebene der Projektplanung zu berücksichtigen.

\subsection{Der Forschungsprozess mit der Referenzgruppe}

Dem mit partizipativer Forschung einhergehenden Anspruch, Frauen mit Lernschwierigkeiten auch im Kontext wissenschaftlicher Forschung als Expertinnen für alle ihre Lebenssituation betreffenden Fragen anzuerkennen und einzubeziehen, versuchte ich im Kontext der vorliegenden Untersuchung durchgängig und auf verschiedenen Ebenen gerecht zu werden.

$\mathrm{Ob}$ die Frage der Selbstbestimmung von Frauen mit Lernschwierigkeiten grundsätzlich und die von mir damit in Zusammenhang gebrachten Fragen von Frauen mit Lernschwierigkeiten selbst als relevant eingeschätzt werden und wie sie gegebenenfalls erforscht werden könnten, war u.a. Gegenstand dieses Prozesses, für den ich das im letzten Kapitel der Habilitationsschrift beschriebene Modell der Arbeit mit einer Referenzgruppe als zielführend erachtet habe. Im Kontext der vorliegenden Studie besteht die Referenzgruppe aus Frauen, denen in ihrer Sozialisation v.a. in institutionellen Zusammenhängen wie einer Schule oder einer Werkstätte das Vorliegen einer ,geistigen Behinderung“ oder „Lernschwierigkeiten“" zugeschrieben wurden ${ }^{35}$.

Im Folgenden werden die organisatorischen Rahmenbedingungen für die Arbeit mit der Referenzgruppe, deren Bedeutsamkeit und konkrete Ausgestaltung begründet und dargestellt. Im Verlauf der weiteren Arbeit, also im Rahmen der Beschreibung der gewählten Erhebungs- und Auswertungsmethode, der Ergebnisdarstellung und der Zusammenfassung erfolgen dann immer wieder Bezüge auf kontextbezogene Einschätzungen, Reflexionen und Diskussionen der Frauen aus der Referenzgruppe.

35 Zur Definition der Begriffe „geistige Behinderung“ und „Lernschwierigkeiten“ sowie zur Problematisierung im Zusammenhang damit erfolgender gesellschaftlicher Zuschreibungen siehe Kapitel 2, Unterpunkt „Frauen mit Behinderung“. Näheres dazu siehe auch unter „Stichprobe“ im laufenden Kapitel. 


\subsubsection{Gruppengröße und-zusammensetzung}

Im Kontext der vorliegenden Untersuchung konstituiert sich die Referenzgruppe aus vier Frauen mit Lernschwierigkeiten ${ }^{36}$, deren Lebenssituationen von unterschiedlichen aktuellen Rahmenbedingungen des Wohnens und Arbeitens geprägt sind, die aber in ihrer Sozialisation ähnliche Erfahrungen gemacht haben. Vor diesem Hintergrund wird die zentrale Funktion der Referenzgruppe in der Mitgestaltung und Begleitung des Forschungsprozesses aus ihrer eigenen Perspektive deutlich. Über konjunktive Erfahrungsräume, d.h. Erfahrungen, die sie miteinander und mit der Zielgruppe der Forschung teilen, ohne mit diesen real in gemeinsamen Lebenszusammenhängen zu stehen (vgl. Przyborski 2004: 29), kann so die Perspektive von Frauen mit Lernschwierigkeiten auf verschiedenen Ebenen in den Forschungskontext integriert werden.

Bei der Größe der Referenzgruppe wurden zwei zentrale Faktoren berücksichtigt: Einerseits sollten unterschiedliche Lebenshintergründe berücksichtigt werden, andererseits sollte die Größe der Gruppe deren kontinuierliche Arbeit ermöglichen. Im konkreten Fall war auch ausschlaggebend, dass sich die vier Frauen aus einem früheren Projektkontext bereits gut bekannt waren und darüber die Gruppenfindungsphase abgekürzt werden konnte, indem sich alle vier Frauen dafür aussprachen, in dieser Konstellation arbeiten zu wollen.

\subsubsection{Der Ort und das Setting der Gruppensitzungen}

Im Verlauf des Forschungsprojektes wurden sieben je dreistündige Sitzungen mit den Frauen der Referenzgruppe durchgeführt. Alle Sitzungen fanden in dafür organisierten, barrierefreien Büroräumlichkeiten statt ${ }^{37}$. Diese Büroräumlichkeiten konnten über berufliche Kontakte einer der Frauen aus der Referenzgruppe organisiert werden, die von sich aus anbot, damit zusammenhängende Fragen abzuklären. In der Folge wurden von mir selbst nur mehr die Termine mit allen vier Frauen abgestimmt, die Organisation der Räumlichkeiten erfolgte dann über die Frau aus der Referenzgruppe. Hinsichtlich der Raumfrage wurde ganz bewusst darauf geachtet, dass nicht etwa eine Institution, in der eine der Frauen lebt, als Ort für die Treffen gewählt wurde. Getragen war dies von der Intention, mögliche Befangenheiten, Hindernisse oder Konfliktsituationen zu vermeiden, die sich daraus für die Frauen hätten ergeben können. Ein Sitzungsraum in einem Büro entspricht auch

36 Mit den Frauen wurde vereinbart, keine weiteren Angaben zu ihrer Person und zu ihren konkreten Lebensbedingungen zu machen.

37 Mit den Frauen aus der Referenzgruppe wurde vereinbart, keine näheren Angaben zum Ort der Gruppensitzungen zu machen. 
üblichen Bedingungen der Teilnahme von Expertinnen an Forschungsprojekten. Für Frauen, die für das Erreichen dieses Ortes Unterstützung benötigten, wurde diese über einen Fahrtendienst organisiert und finanziert.

\subsubsection{Finanzielle Abgeltung der Arbeit der Expertinnen}

Für die Arbeit als Expertinnen in der Referenzgruppe wurde mit den Frauen ein Honorar für jede der Sitzungen vereinbart und auf die von den Frauen angegebenen Bankverbindungen überwiesen.

Mir selbst war es im Kontext mit der Tatsache, dass die Tätigkeit von Menschen mit Lernschwierigkeiten im überwiegenden Fall über den Bezug eines Taschengeldes in einer Werkstätte nur mit einem äußerst geringfügigen „Taschengeld“ honoriert werden ${ }^{38}$, besonders wichtig, dies im Rahmen des vorliegenden Projektes umzusetzen.

\subsubsection{Die Verwendung angemessener Sprache und barrierefreier Materialien}

Wie im letzten Kapitel der Arbeit ausgeführt, ist für die Teilhabe von Menschen mit Lernschwierigkeiten an einem Forschungsprojekt der Gebrauch verständlicher Sprache unumgänglich. Allen teilnehmenden Personen ohne akademischen Hintergrund muss die von ihnen benötigte Zeit zur inhaltlichen und sprachlichen Orientierung zur Verfügung gestellt werden (vgl. Flieger 2007: 34).

Als hilfreich für die Forschung im vorliegenden Kontext erwiesen sich neben dem Gebrauch von Handreichungen, wie etwa dem „Wörterbuch für leichte Sprache ${ }^{\text {(39 }}$, auch eigene Erfahrungen in der Verwendung sogenannter „leichter Sprache“. Für mich selbst stellten u.a. bereits absolvierte Workshops, Arbeitskreise und Fortbildungen ${ }^{40}$ für und mit Menschen mit Lern-

38 So ergab die Analyse von 30 Interviews von Frauen mit Lernschwierigkeiten im Rahmen meines 2003 durchgeführten Projektes ,Zur Lebenssituation von Frauen mit Lernschwierigkeiten“, dass 23 der Frauen lediglich ein „Taschengeld“ bezogen. Die Höhe bewegte sich damals zwischen 3 und 7 Euro (vgl. Sigot 2003: 41f.). An diesem Umstand hat sich zwischenzeitlich wenig geändert.

39 Vgl. FN 32.

40 So etwa die Leitung eines Arbeitskreises sowie ein eigener Beitrag in leichter Sprache innerhalb dieses Arbeitskreises im Rahmen der 24. Jahrestagung der InklusionsforscherInnen des deutschen Sprachraums „Inklusionsforschung im Lichte der UN-Konvention über die Rechte behinderter Menschen“ (24.-27. Februar 2010) an der Universität Innsbruck. Alle Beiträge der Tagung wurden in einem Sammelband jeweils mit einer Zusammenfassung in „leichter Sprache“ publiziert: Flieger, P./Schönwiese, V. (2011): Menschenrechte - Integration - Inklusion. Aktuelle Perspektiven aus der Forschung. Innsbruck. 
schwierigkeiten sowie die Verfassung eigener Texte in möglichst angemessener Sprache und Rückmeldungen zu deren Verständlichkeit durch die angesprochenen Personen für diese früheren Arbeitszusammenhänge wertvolle Erfahrungen dar, an die ich anknüpfen konnte.

Für die konkrete Arbeit in den einzelnen Sitzungen im aktuellen Projektkontext waren daher auch entsprechende Vorbereitungsarbeiten erforderlich, um die Arbeit an den Inhalten zu ermöglichen bzw. zu erleichtern. So wurden für jede der Sitzungen vorab Inhalte, die thematisiert werden sollten, in meiner Einschätzung nach angemessener Sprache formuliert und auch Arbeitsmaterialien vorbereitet, die die Arbeit unterstützen und strukturieren sollten. Bei der eingangs angeregten Diskussion zu Selbstbestimmung habe ich dafür beispielsweise auf Definitionen ${ }^{41}$ aus Publikation zurückgegriffen, die von Menschen mit Lernschwierigkeiten selbst erarbeitet wurden. Im Zuge der späteren Arbeiten im Zusammenhang mit der Auswertung von Interviewpassagen wurden jeder der Frauen diese schriftlich zur Verfügung gestellt. Dabei wurden allerdings nicht längere, zusammenhängende Passagen, sondern jeweils einzelne Sätze auf handlichen Formaten vorbereitet (Format A5, auf stärkerem Papier ausgedruckt). Dies ermöglichte durch die inhaltliche Eingrenzung auf jeweils einen Satz auf einer Karte die Konzentration auf den jeweiligen eingegrenzten Textausschnitt. Zudem konnte über die Handhabbarkeit durch das handliche Format auch ein ,sinnlicher" Umgang mit dem vorliegenden Material erfolgen, das so auch tatsächlich „begriffen“ werden konnte.

Im gesamten Forschungsprozess habe ich mich auch laufend rückversichert, dass die jeweiligen Inhalte auch verständlich waren und darüber auch eine fundierte Basis für die Diskussionen und Reflexionen innerhalb der Gruppe gegeben war.

Als Ergebnis meiner eigenen Erfahrungen in der Kommunikation mit Menschen mit Lernschwierigkeiten ziehe ich - wie oben ersichtlich - für mich selbst im Kontext der vorliegenden Arbeit die Verwendung des Begriffes der ,angemessenen Sprache“ dem der ,leichten Sprache“ vor. Denn darüber drückt sich für mich der Versuch aus, sie auf die jeweiligen Voraussetzungen der Personen abzustimmen, mit denen ich es im jeweiligen konkreten Kontext der Forschung zu tun habe. Frauen mit Lernschwierigkeiten sind aus meiner Perspektive auch im sprachlichen Bereich keine homogene Gruppe, sondern bringen unterschiedliche Voraussetzungen mit. Dies schließt auch an meine Erfahrungen in einem früheren Forschungskontext an, in dem ich festgestellt habe, dass einige meiner Interviewpartnerinnen mit zugeschriebener ,geistiger Behinderung“ über „sehr hohe sprachliche Kompetenzen“ verfügten, was sich für mich bereits damals als konträr zur ,defizitorientier-

41 „Selbstbestimmung heißt, alleine zu bestimmen, was ich tun möchte“ (Wibs 2005: 4) und „Selbstbestimmung: Wenn Menschen selbst entscheiden, was sie machen“ (Netzwerk People First Deutschland 2004: 7). 
ten These, dass sie sich verbal nicht oder nur unzulänglich ausdrücken können“ (Sigot 2003: 41), darstellte. Dies bestätigte sich auch im Verlauf der aktuellen Forschung, sei es im Kontext der Arbeit mit der Referenzgruppe als auch in einzelnen Interviews ${ }^{42}$ mit Frauen mit Lernschwierigkeiten. Aus diesen Erfahrungen heraus erscheint mir die Verwendung des Begriffes der „angemessenen Sprache“ im vorliegenden Forschungskontext stimmiger ${ }^{43}$.

\subsubsection{Inhalte und Ergebnisse der Referenzgruppensitzungen im Überblick}

Im Kontext des Forschungsprojektes wurden sieben je dreistündige Sitzungen mit den Frauen der Referenzgruppe durchgeführt. In diesen wurde angelehnt an die üblichen Phasen in Forschungsverläufen an Fragen im Zusammenhang mit der Planung, der Umsetzung und der Auswertung gearbeitet, wobei ein unterschiedlich starker Einbezug der Gruppe erfolgte. Grundsätzlich gab es jedoch prinzipielle Offenheit hinsichtlich der konkreten Art der Beteiligung, was als förderlich für partizipative Projekte eingeschätzt wird (vgl. Flieger 2007: 39f.). Keine konkrete Beteiligung der Referenzgruppe erfolgte nach Absprache mit den Frauen im Zusammenhang mit der Datenerhebung. Dies erschien ihnen im Kontext mit ihrer eigenen Lebenssituation aktuell vor allem wegen mangelnder zeitlicher Ressourcen nicht realisierbar ${ }^{44}$. Allerdings wurden erste Erfahrungen aus Interviews mit Frauen mit Lernschwierigkeiten den Frauen der Referenzgruppe reflektiert.

Wie bereits beschrieben, wurden alle Sitzungen der Referenzgruppe inhaltlich strukturiert und methodisch vorbereitet: Für jede der Sitzungen wurden abgestimmt auf übliche Projektabläufe notwendige Arbeitsschritte mit der Referenzgruppe besprochen und geplant. Diese wurden dann vorab von mir selbst in meiner Einschätzung nach angemessener Sprache verschriftlicht. Um Diskussionen und Reflexionen anzuregen sowie die Arbeit im Rahmen der Auswertung zu ermöglichen, wurden ergänzend dazu barrierefreie Arbeitsmaterialien gestaltet. Die Sitzungen wurden auch dokumentiert, indem jeweils ein Verlaufsprotokoll verfasst wurde. Dafür wurde auf die Unterstützung einer Studierenden zurückgegriffen, die den Frauen aus einem

42 So wirkt etwa Evas Sprache im Einzelinterview sehr elaboriert. Siehe dazu insbesondere die Interviewpassagen in der Fallrekonstruktion „Eva“ im Kapitel 6. An Evas Beispiel wird im Vergleich zu anderen Frauen über die Analysen auch deutlich, dass sprachliche Kompetenz auch mit dem Zur-Verfügung-Stellen von Räumen, sich sprachlich weiter zu entwickeln und im Diskurs zu erproben, im Rahmen der Sozialisation zu tun hat.

43 Dies ist keine Kritik der Verwendung des Begriffes der ,leichten Sprache“ durch die PeopleFirst-Initiativen, die dies u.a. in Publikationen umsetzen, die viele Leserinnen und Leser, deren unterschiedliche Voraussetzungen nicht bekannt sind, erreichen sollen.

44 Für die Frauen aus der Referenzgruppe sprachen aktuell u.a. eigene Berufstätigkeit oder Praktika dagegen. 
vergangenen Projektkontext bereits vertraut war. Auf Wunsch der Teilnehmerinnen der Referenzgruppe sind die vollständigen Protokolle nicht im Anhang der Arbeit gesammelt. Es erfolgen aber im Verlauf der Arbeit immer wieder Verweise auf ausgewählte Protokollausschnitte ${ }^{45}$.

Die Arbeit führte auch zu schriftlichen „Produkten“, denen im Verlauf der weiteren Forschungstätigkeit besonderer Stellenwert zukam und auf die in entsprechenden Zusammenhängen auch jeweils verwiesen wird.

Tabelle 2 ermöglicht einen Überblick über Inhalte und Ergebnisse der sieben Referenzgruppensitzungen, deren Dokumentation sowie den Stellenwert der geleisteten Arbeit im weiteren Projektverlauf. Ganz rechts ist dabei ersichtlich, welcher Phase im Projekt - also Planung, Auswertung oder Abschluss - die jeweilige Sitzung vorwiegend zuzuordnen ist. Dabei gibt es auch Überschneidungen, da in jeder der Sitzungen eingangs auch Bezug auf die vorherige Sitzung genommen und deren Inhalte und Ergebnisse zusammengefasst und so als Anknüpfungspunkte für das aktuelle Arbeitsvorhaben präsent gemacht wurden. Die Tabelle dokumentiert und verweist über Fußnoten auch auf Materialien oder Texte, die im Zusammenhang mit der jeweiligen Sitzung entstanden sind und die sich entweder in der Arbeit selbst und/oder in deren Anhang finden.

Wie im Überblick ersichtlich, fungierte die Referenzgruppe einerseits vor allem in der Planungsphase als Beraterin in inhaltlichen und methodischen Fragen. Die Diskussionen und Reflexionen, die in und mit der Gruppe geleistet wurden, bestimmten den Forschungsverlauf von Beginn an entscheidend mit, indem zunächst konkrete Fragen/Bereiche identifiziert wurden, die aus der Perspektive der Frauen aus der Referenzgruppe wesentlich für Fremdund Selbstbestimmung eingeschätzt wurden. Diese Fragen wurden als Orientierungsrichtlinien mit in den Erhebungs- und auch in den Auswertungsprozess genommen. Zudem bot die Einschätzung der Frauen aus der Referenzgruppe zahlreiche Hinweise für die methodische Anlage der Studie. So wurden u.a. Fragen in Zusammenhang mit der Erhebungsmethode, der Zielgruppe und der konkreten Gestaltung der Interviewsituation besprochen. Die Frauen machten auch Anregungen für einen ihrer Ansicht nach idealen Gesprächseinstieg im Interview. Im Rahmen der Auswertung interpretierten die Frauen der Referenzgruppe Interviewpassagen und Vignettenbeschreibungen. Die Auswertungsergebnisse wurden ihnen präsentiert und zur Diskussion gestellt.

Dabei wird jeweils auf die entsprechende Sitzung und die Seite des Protokolls verwiesen. So bedeutet etwa 2RGS-S1, dass diese Anmerkung dem Protokoll der 2. Referenzgruppensitzung (RGS) von Seite 1 entnommen ist. Wörtliche Zitate sind dabei auf Wunsch nicht einzelnen Frauen zugeordnet, sondern es wird jeweils entweder auf eine gemeinsame Einschätzung verwiesen oder auf eine nicht namentlich genannte Frau aus der Referenzgruppe. Siehe dazu auch die Regeln für die Belege des Datenmaterials im Anhang der Arbeit. 


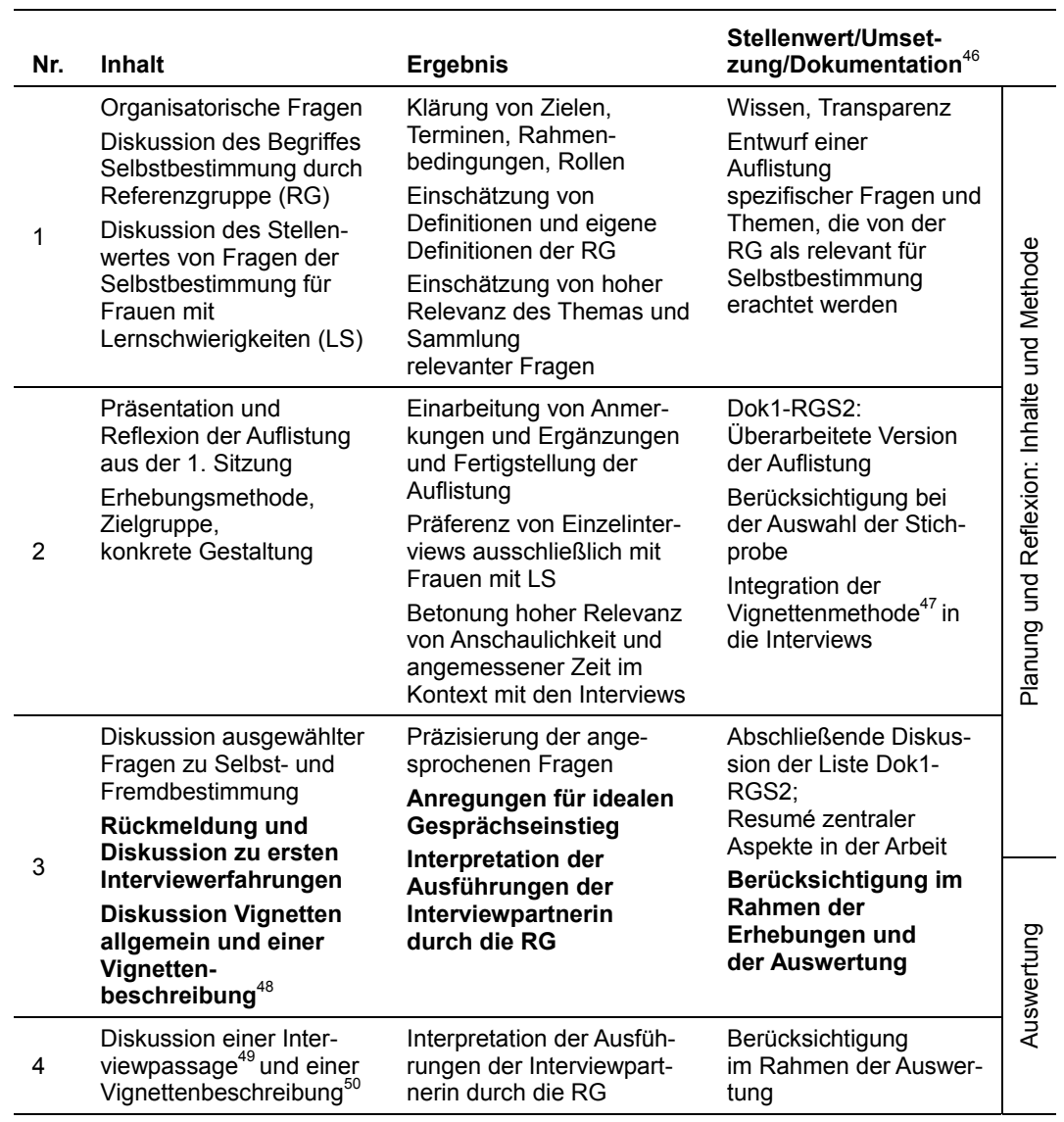

46 Die entsprechenden Dokumente werden im laufenden Kapitel der Habilitationsschrift angeführt. Zudem wird im Zuge der weiteren Arbeit an relevanten Stellen (u.a. in der Ergebnisdarstellung) darauf verwiesen. Entsprechend den Regeln für die Belege des Datenmaterials (siehe Anhang), wird dabei auf das entsprechende Dokument verwiesen, das im Rahmen der entsprechenden Sitzung erarbeitet wurde. So bedeutet etwa Dok1-1RGS, dass es sich um das erste Dokument handelt und dass dieses in der ersten Sitzung der Referenzgruppe erarbeitet wurde.

47 Siehe dazu ausführlich „Erhebungsmethode“ im laufenden Kapitel.

48 ignettenbeschreibung im Interview mit Alina, Passage 952-981.

49 Interview mit Eva, Passage 35-53.

50 Vignettenbeschreibung im Interview mit Alina, Passage 926-949. 


\begin{tabular}{|c|c|c|c|c|}
\hline Nr. & Inhalt & Ergebnis & $\begin{array}{l}\text { Stellenwert/Umset- } \\
\text { zung/Dokumentation }\end{array}$ & \\
\hline 5 & $\begin{array}{l}\text { Diskussion von drei } \\
\text { Interview-passagen } \\
\text { und einer } \\
\text { Vignetten-beschreibung }^{52}\end{array}$ & $\begin{array}{l}\text { Interpretation der } \\
\text { Ausführungen der } \\
\text { Interviewpartnerin } \\
\text { durch die RG }\end{array}$ & $\begin{array}{l}\text { Berücksichtigung } \\
\text { im Rahmen der } \\
\text { Auswertung }\end{array}$ & \multirow{4}{*}{ 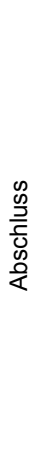 } \\
\hline 6 & $\begin{array}{l}\text { Diskussion von } \\
\text { Zwischenergebnissen } \\
\text { der Auswertung }\end{array}$ & $\begin{array}{l}\text { Interpretation von } \\
\text { Zwischenergebnissen } \\
\text { durch } R G\end{array}$ & $\begin{array}{l}\text { Berücksichtigung } \\
\text { im Rahmen der } \\
\text { Auswertung }\end{array}$ & \\
\hline \multirow[t]{2}{*}{7} & $\begin{array}{l}\text { Diskussion zentraler } \\
\text { Ergebnisse der } \\
\text { Auswertung }\end{array}$ & $\begin{array}{l}\text { Einschätzung der } \\
\text { Typenbildung und } \\
\text { soziogenetischer Faktoren }\end{array}$ & $\begin{array}{l}\text { Zwei Unterkapitel } \\
\text { in der } \\
\text { Habilitationsschrift }^{53}\end{array}$ & \\
\hline & $\begin{array}{l}\text { Klärung von Fragen } \\
\text { im Zusammenhang } \\
\text { mit der Veröffentlichung } \\
\text { Reflexion } \\
\text { Forschungsprozess }\end{array}$ & $\begin{array}{l}\text { Einarbeitung der Beiträge } \\
\text { der Referenzgruppe, } \\
\text { Anonymisierung etc. }\end{array}$ & $\begin{array}{l}\text { Art der Anführung von } \\
\text { Personen, Inhalten; } \\
\text { Belegen in der Arbeit }\end{array}$ & \\
\hline
\end{tabular}

Tabelle 2: Inhalte und Ergebnisse der sieben Referenzgruppensitzungen

Konkrete Ergebnisse der Arbeit (mit) der Referenzgruppe werden jeweils in die entsprechenden weiteren Kapiteln und Unterkapiteln der vorliegenden Habilitationsschrift integriert dargestellt. So werden etwa - wie aus Tabelle 2 ersichtlich - Anmerkungen der Referenzgruppe zur Erhebungsmethode im Punkt „Erhebungsmethode“ und Anmerkungen, die im Zusammenhang mit dem Auswertungsprozess gemacht wurden, im entsprechenden Unterpunkt dokumentiert. Die Evaluation zentraler Auswertungsergebnisse durch die Referenzgruppe ist in zwei eigenen Unterkapiteln der Arbeit erfasst.

\subsubsection{Inhaltlicher Rahmen der Arbeit und Ausgangsbasis aus der Sicht der Frauen aus der Referenzgruppe}

Der inhaltliche Rahmen der Forschung war den Frauen aus der Referenzgruppe durch das Thema meines Habilitationsvorhabens bereits vor ihrer Bekundung zur Bereitschaft der Mitwirkung innerhalb der Referenzgruppe bekannt und erwies sich für sie mit als Grund zur Teilnahme. Die hohe Relevanz der Thematik wurde auch zu Beginn des gemeinsamen Arbeitsprozesses durch die Frauen selbst explizit hervorgehoben (vgl.1RGS-S1f.). Das Thema Selbstbestimmung als Forschungsthema aufzugreifen, erachteten alle Frauen demnach als ganz besonders wichtig, es stellt für sie etwas „ganz Großes“

51 Interview mit Alina, Passage 814-824, und Interview mit Angelika, Passage 369-384 und Passage 642-641.

52 Vignettenbeschreibung im Interview mit Eva, Passage 581-586.

53 Die Rückmeldungen wurden in den Unterkapiteln „Ergebnisse der Evaluation der sinngenetischen Analyse durch die Frauen aus der Referenzgruppe“ und „Soziogenetische Zusammenhänge in der Einschätzung der Frauen aus der Referenzgruppe" zusammengefasst. 
dar, ist „ein großer Bereich“, zu dem es aus ihrer Perspektive viel zu wenig Wissen gibt. Dies wird insbesondere für die Personengruppe, der sie in gesellschaftlichen Kontexten selbst zugeordnet wurden und werden, also der Situation für Menschen mit Lernschwierigkeiten, als besonders bedeutsam eingeschätzt: Es sei weitgehend nicht bekannt, ,was Selbstbestimmung für Menschen mit Lernschwierigkeiten heißt" (vgl. 1RGS-S2). Eigene Erfahrungen von Fremdbestimmung wurden von den Frauen bereits in dieser frühen Projektphase mit sehr negativen Auswirkungen auf das eigene Befinden problematisiert. So wurde übereinstimmend festgehalten, dass es ihnen in fremdbestimmten Kontexten, die von der Aufforderung „du musst...“ geprägt sind, „gar nicht gut“ (vgl. 1RGS-S3) gehe.

\section{Kontexte und Faktoren für Selbstbestimmung}

Zutrauen von Fähigkeiten durch andere und durch sich selbst

Tagesstruktur selbst gestalten, Wunsch nach Freiraum, Vorschriften

Selbstbestimmung im Bereich Arbeit/Beschäftigung

Wunsch nach Privatsphäre, Intimität

Aussehen, Bekleidung, Frisur selbst bestimmen

Gesundheit/Gesunde Ernährung/Bewegung und Selbstbestimmung

Organisation der/Beteiligung bei Verpflegung

Freizeitaktivitäten selbst bestimmen

Selbstbestimmung des Aufenthaltsortes

Religiöse/spirituelle Selbstbestimmung

Partnerschaft/Freundschaft und Selbstbestimmung

Sexualität, Aufklärung, Elternschaft

Zugang zu Informationen

Selbstbestimmung/Fremdbestimmung durch Eltern, Personen aus dem sozialen Umfeld, insbesondere Institutionen

Einengung durch übergroße Fürsorge

Geschlechtsspezifische Fragestellungen

Tabelle 3: Aspekte, die von der Referenzgruppe als relevant für Selbstbestimmung erachtet werden

Ausgehend von diesen Überlegungen wurden von den Frauen der Referenzgruppe spezifische Fragen und Themen, die ihnen als relevant für die Selbstbestimmung von Menschen mit Lernschwierigkeiten erschienen, zur Sprache gebracht. Eigene Erfahrungen, Beobachtungen und Überlegungen dazu wurden diskutiert und reflektiert. Dabei wurde deutlich, dass die Frage, wie betroffene Frauen selbst damit umgehen, wie sie sich also im Spannungsfeld von Fremd- und Selbstbestimmung orientieren und wodurch dies begründet sein kann, für die Frauen aus der Referenzgruppe von besonderem Interesse 
ist, sich also weitgehend mit meinen Interessen als Forscherin deckte ${ }^{54}$. Auf Grundlage dieser von der Referenzgruppe geleisteten Arbeit wurde von mir als Vorbereitung für die folgende Sitzung eine Auflistung von Themen und Fragen, die sie als bedeutsam für Selbstbestimmung von Menschen mit Lernschwierigkeiten einschätzten, erarbeitet. Nach Präsentation, Diskussion und Ergänzung dieser Vorlage in den folgenden beiden Sitzungen wurde diese von den Frauen als geeignete Grundlage für die Orientierung im weiteren Forschungsverlauf eingeschätzt.

Die angeführten Themenstellungen wurden von den Frauen aus der Referenzgruppe als zentral für Selbstbestimmung benannt und anhand von Beispielen erlebter und beobachteter Alltagserfahrungen diskutiert. Die Diskussionen zu den dargestellten Punkten sind jeweils in den entsprechenden Sitzungsprotokollen erfasst. An dieser Stelle fasse ich zentrale Ergebnisse daraus im Überblick zusammen ${ }^{55}$. Sie fungierten für mich als inhaltliche Ausgangsbasis für den weiteren Forschungsprozess, indem sie zentrale Grundüberlegungen von Frauen mit Lernschwierigkeiten als Expertinnen zu Fragen von Selbst- und Fremdbestimmung darstellen:

Die Frage einer selbstbestimmten Lebensführung wird von den Frauen aus der Referenzgruppe eng mit der Frage des Zutrauens von Fähigkeiten durch andere und durch sich selbst in Zusammenhang gebracht (vgl. 1RGS-S2). Dies wird als wechselseitiger Prozess beschrieben, in dem den Frauen häufig von Personen aus ihrem sozialen Umfeld wenig zugetraut wird. Andererseits spielen eigene Ängste und Unsicherheiten eine Rolle, die aus Perspektive der Frauen vermutlich mit eigenen Erfahrungen im Verlauf ihres bisherigen Lebens zu tun haben (vgl. 1RGS-S3). Als Personengruppen, über die Selbstbestimmung gefördert oder Fremdbestimmung erfahren wird, werden Eltern und weitere Personen aus dem sozialen Umfeld, insbesondere Mitarbeiterinnen und Mitarbeiter aus Institutionen, genannt (vgl. 1RGS-S3).

Vorgegebene Rahmenbedingungen, etwa Tagesstrukturen in Institutionen, werden mitunter als massiv fremdbestimmend erlebt. Dies kann sich beispielsweise in den Abläufen im Bereich Arbeit/Werkstätte zeigen, insbesondere aber auch im Freizeitbereich. Gerade hier wird die Einflussnahme aus dem sozialen Umfeld auf dessen Gestaltung sehr negativ wahrgenommen (vgl. 1RGS-S2). Im Zusammenhang mit Fragen im Kontext von Arbeit und Beschäftigung werden Mitsprache- und Gestaltungsmöglichkeiten gewünscht. Sehr starke Kritik wird an dem Umstand geübt, dass in Werkstätten lediglich ein „Taschengeld“ ausbezahlt wird. Dies wird als nicht angemesse-

54 Siehe dazu das Kapitel „Forschungsleitende Grundfragen“.

55 Eine ausführliche Dokumentation dazu ist nachzulesen in Sigot, M. (2012): Partizipative Forschung mit Frauen mit Lernschwierigkeiten - Herausforderung und Bereicherung für die empirische Forschung. In: Heimgartner, A./Loch, U./Sting, S. (Hrsg.): Empirische Forschung in der sozialen Arbeit. Methoden und methodologische Herausforderungen. Wien, S..153-166. 
ne bzw. ungerechte Abgeltung der geleisteten Arbeit erlebt. Die Frauen aus der Referenzgruppe weisen auch auf den Zusammenhang zwischen damit einhergehenden Abhängigkeiten und den damit verbundenen Einschränkungen, das eigene Leben selbstbestimmt zu organisieren, hin (vgl. 1RGS-S3). Auch das Fehlen mit regulärem Lohn verbundener Ansprüche oder Leistungen - etwa eigener Kranken- oder Pensionsversicherung - werden thematisiert (vgl. 2RGS-S3). Im Zusammenhang mit beruflicher Integration, aber auch mit dem Freizeitbereich, wird das Modell der persönlichen Assistenz als sehr positiv hervorgehoben (vgl. 3RGS-S1), weil dadurch die Umsetzung selbstbestimmter Entscheidungen ermöglicht wird.

Fremdbestimmung wird von Frauen mit Lernschwierigkeiten auch hinsichtlich sehr persönlicher und intimer Fragen, die mit der eigenen Körperlichkeit und damit verbundenem Wohlbefinden und weiblicher Identität zu tun haben, wahrgenommen. Häufig werden ihnen bequeme Kleidung, möglichst unauffälliger Haarschnitt und -farbe von Personen aus ihrem Umfeld nahegelegt. Aus der Perspektive der Frauen stehen vor allem zwei Intentionen von Personen aus dem sozialen Umfeld dahinter, die diese aber meist nicht explizit zur Sprache bringen: einerseits sexuelle Gewalthandlungen durch unauffällige Kleidung zu verhindern, aber auch die mögliche Anbahnung von Kontakten zu verhindern. Die damit einhergehenden Versuche, Einfluss auf die Gestaltung des Äußeren zu nehmen, werden als Eingriffe in ganz persönliche Entscheidungen wahrgenommen und vehement abgelehnt (vgl. 1RGS-S1f.).

Auch übergroße Fürsorge (vgl. 1RGS-S2f.) aus dem sozialen Umfeld wird von Frauen mit Lernschwierigkeiten mitunter als Fremdbestimmung wahrgenommen, indem sie sich dadurch in Handlungsspielräumen und in ihrer Privatsphäre eingeschränkt fühlen. Einschränkungen in der Privatsphäre werden auch im Zusammenhang mit alltäglichen Verrichtungen bzw. Vorgängen wahrgenommen. Sie weisen aus der Perspektive der Frauen aus der Referenzgruppe insbesondere dann fremdbestimmte Anteile auf, wenn dritte Personen ungefragt in Kontexten anwesend sind, wo dies nicht erwünscht ist (wie z.B. beim Telefonieren), oder wenn etwa mit Informationen, die die eigene Person betreffen, unsensibel umgegangen wird. Als konkretes Beispiel wird von den Frauen aus der Referenzgruppe etwa der Umgang mit Informationen aus der Krankengeschichte oder über Arzttermine thematisiert (vgl. 1RGS-S3).

Privatsphäre zu haben, bedeutet für Frauen mit Lernschwierigkeiten aus Perspektive der Frauen aus der Referenzgruppe auch, bei der Gestaltung und Dekoration des eigenen Wohnumfeldes eigene Wünsche und Vorlieben umsetzen zu können (vgl. 1RGS-S3).

Fragen in Zusammenhang mit Glauben bzw. Religion werden ebenfalls mit dem Wunsch nach Selbstbestimmung in Zusammenhang gebracht (vgl. 1RGS-S3). De facto wird aber gerade auch in diesem Kontext aus der Per- 
spektive von Frauen mit Lernschwierigkeiten Einfluss zu nehmen versucht. Dies reicht von der Erwartung oder auch der Vorgabe, jeden Sonntag den Gottesdienst zu besuchen bis hin zu massiver Fremdbestimmung hinsichtlich der Konfession.

Aus der Perspektive der Frauen aus der Referenzgruppe weisen auch Fragen in Zusammenhang mit der Verpflegung (vgl. 1RGS-S3f.) Anteile von Fremdbestimmung auf. Dies reicht von der Organisation der Verpflegung bis hin zur Auswahl der Lebensmittel. Hier werden mitunter Beispiele eingebracht, die auf massive Einflussnahme auf ganz persönliche Fragen der Verpflegung abzielen. Als Beispiel wird der darüber oft wahrgenommene Anspruch, sich gesund zu ernähren, erwähnt. Dies insbesondere dann, wenn diese an sie geäußerten Ansprüche im Widerspruch zu den eigenen Ernährungsgewohnheiten der Personen, die diese Ansprüche stellen, stehen. Auch in der Frage der Qualität der gewählten Nahrungsmittel und Getränke aus gesundheitlicher Perspektive sprechen sich die Frauen aus der Referenzgruppe also für selbstbestimmte Entscheidungen aus. Auch bei der Frage der Organisation der Verpflegung wird Mitbestimmung und Mitbeteiligung gewünscht.

Fremdbestimmung für Frauen mit Lernschwierigkeiten wird aus der Perspektive der Frauen aus der Referenzgruppe auch in Zusammenhang mit der Entscheidung des Aufenthaltsortes (vgl. 1RGS-S4) gebracht. Diesbezüglich werden selbstbestimmte Entscheidungsmöglichkeiten gewünscht, real erfolgt hier allerdings ihrer Einschätzung nach häufig Fremdbestimmung. Dies einerseits durch institutionelle oder familiäre Strukturen, die bestimmte Aufenthaltsorte für bestimmte Zeiträume vorsehen oder vorgeben. Zum anderen ist es den Frauen oft nicht möglich, sich auch spontan zu bestimmten Zeiten an Orten einzufinden, an denen sie sich gerne aufhalten würden. Dies wird mit mangelnder Unterstützung, aber auch mit Barrieren in Zusammenhang gebracht, die mit der Mobilität in Zusammenhang stehen. Als wesentlicher Faktor wird auch die „Informationspolitik“ in Einrichtungen eingeschätzt (vgl. 2RGS-S1). Durch verspätete Information werden z.B. Veranstaltungshinweise zu spät wahrgenommen, es besteht daher oft gar keine Möglichkeit mehr, zu partizipieren. Als Beispiel dafür wird angeführt, dass Bewohnerinnen und Bewohnern in Behinderteneinrichtungen häufig veraltete Zeitungen mit nicht mehr aktuellen Veranstaltungshinweisen zugänglich gemacht werden. All diese Prozesse werden von ihnen im Sinne indirekter Fremdbestimmung beschrieben, indem diese nicht explizit, sondern über Prozesse des Vorenthaltens zum Ausdruck kommen.

Fragen, die die Bereiche Partnerschaft/Freundschaft/Sexualität und Selbstbestimmung betreffen, werden von den Frauen aus der Referenzgruppe als Bereiche für Frauen mit Lernschwierigkeiten charakterisiert, in denen Fremdbestimmung in verschiedenen Facetten wahrgenommen, vermutet oder erwartet wird. Beschrieben wird etwa die als Fremdbestimmung erlebte ein- 
geschränkte Möglichkeit, Partnerschaften überhaupt, insbesondere aber außerhalb der eigenen Institution oder des familiären Wohnumfeldes, in Erwägung zu ziehen (vgl. 2RGS-S1). Allgemein werden vor allem Partnerschaft und Sexualität auch als tabuisierte Bereiche wahrgenommen (vgl. 1RGS-S2), $\mathrm{zu}$ denen es auch zu wenig Information gibt. Auch in diesem Zusammenhang werden wie hinsichtlich der Frage der Bekleidung Ängste bei Bezugspersonen vermutet, ganz konkret wird auch deren Sorge vor einer möglichen Schwangerschaft thematisiert (vgl. 2RGS-S1f.). Im Zusammenhang mit der Elternschaft von Frauen mit Lernschwierigkeiten wird als gesellschaftliche Realität das Verhindern einer Schwangerschaft oder das automatische Wegnehmen des Kindes wahrgenommen (vgl. 3RGS-S2). Die Frauen aus der Referenzgruppe sprechen sich demgegenüber für sachliche Information und geeignete Unterstützungssysteme für Fälle, in denen Frauen mit Lernschwierigkeiten Mütter werden, aus (vgl. 3RGS-S2). Eine Wegnahme des Kindes darf aus ihrer Sicht nicht unter anderen Voraussetzungen als bei Frauen ohne Lernschwierigkeiten erfolgen, also nur bei Gefährdung des Kindeswohles (vgl. 2RGS-S1f.)

Auch geschlechtsspezifische Fragestellungen werden von den Frauen aus der Referenzgruppe im Kontext der Diskussionen zu Selbst- und Fremdbestimmung zur Sprache gebracht. Männern mit Lernschwierigkeiten wird demnach aus ihrer Perspektive von ihrem sozialen Umfeld mehr zugetraut und sie entwickeln darüber mehr Mut. Frauen hingegen würden öfter defizitorientierte Zuschreibungen erleben, die sie verunsichern und zur Aufgabe von Vorhaben bringen würden (vgl. 2RGS-S3f.). Dies führt aus Perspektive der Frauen aus der Referenzgruppe auch dazu, dass sie häufig erst gar nicht mehr versuchen würden, sich neuen, herausfordernden Situationen zu stellen. Frauen würden zudem mehr zum Zweifeln an den eigenen Fähigkeiten neigen, Männer hingegen seien eher überzeugt von ihren eigenen Kompetenzen. Männer mit Lernschwierigkeiten würde es auch leichter fallen, an sie geäußerte Ansprüche abzuwehren. Frauen würden sich eher dabei schwer tun, „nein“ zu sagen. Eigene Ansprüche und Wünsche auch gegenüber Widerstand aus dem sozialen Umfeld zu vertreten, wird als notwendiger Entwicklungsschritt von Frauen mit Lernschwierigkeiten im Zusammenhang mit selbstbestimmtem Leben eingeschätzt (vgl. 2RGS-S5).

\subsection{Erhebung und Erhebungsmethode}

Nach der Schaffung einer inhaltlichen Ausgangsbasis durch die Einschätzung der Rahmenbedingungen für die Orientierung von Frauen mit Lernschwierigkeiten im Spannungsfeld von Fremd- und Selbstbestimmung durch die Frauen aus der Referenzgruppe wurde mit ihnen gemeinsam an der Auswahl einer 
geeigneten Erhebungsmethode gearbeitet. Mit der Methode sollte es möglich sein, diese Orientierungen aus der Perspektive von selbst betroffenen Frauen zu erheben.

Im Rahmen dieses Prozesses sah ich es als meine Aufgabe als Wissenschafterin an, zunächst über das Repertoire an möglichen Erhebungsmethoden zu informieren. In partizipativen Forschungsansätzen wird vorrangig auf die Methodologie der qualitativen Forschung zurückgegriffen, da sich solche Forschungsdesigns besonders gut für Forschung eignen, die an persönliche Erfahrungen von Betroffenen anknüpft (vgl. Waldschmidt 2009: 129). Die Frauen aus der Referenzgruppe präferierten von Beginn an eine Methode, in der Frauen mit Lernschwierigkeiten selbst zur Sprache kommen. Auch aus ihrer Perspektive war demnach einer qualitativen Erhebungsmethode gegenüber quantitativen Verfahren der Vorzug zu geben. Nach einer Phase von Rückfragen und Detailinformationen zu verschiedenen qualitativen Erhebungsmethoden wurden Vor- und Nachteile einzelner Verfahren abgewogen und die Präferenzen der Frauen erhoben (vgl. 2RGS-S6-8).

Im Zusammenhang mit der Frage einer geeigneten Erhebungsmethode empfahlen die Frauen aus der Referenzgruppe, „offen“ in die Interviews zu gehen, denn so stehe im Zentrum, ,was sie erzählen“ (vgl. 2RGS-S7). Wenn eine der Frauen von sich aus nicht flüssig erzählen würde, könnten ,gezielte Fragen“" (ebd.) gestellt werden. Dafür spricht aus ihrer Sicht, dass den einzelnen Frauen im Rahmen von Einzelinterviews genügend Raum zur Verfügung steht, sich zu äußern und ihre ganz konkreten Erfahrungen einzubringen. Als am besten geeignete Erhebungsmethode wurden nach eingehender Diskussion und auf Grundlage der Präferenz der Frauen aus der Referenzgruppe Einzelinterviews mit Frauen mit Lernschwierigkeiten als geeignet eingeschätzt und ausgewählt.

Als besonders bedeutsam wurde die Verwendung einer Sprache hervorgehoben, die auf die Voraussetzungen der jeweiligen Frau abgestimmt ist. Damit betonen auch die Frauen aus der Referenzgruppe einen zentralen Anspruch der People-First-Bewegung, nämlich das Verwenden sogenannter „leichter“ Sprache, um barrierefreien Zugang zu Inhalten - hier im Kontext von Interviews - zu gewährleisten. Zudem rieten die Frauen auch dazu, auf Anschaulichkeit zu achten. Dem wurde mit der Integration der Methode der Vignettenbeschreibung ${ }^{56}$ entsprochen, bei der über „visuelle Abbildungen eine stimulierende Aufforderungssituation“ hergestellt wird, „die die befragte Person zu Beurteilungen oder zu weiterführenden Handlungsentwürfen anregt" (Stiehler/Werner 2008: 185). Diese wurde von den Frauen als sinnvolle und zielführende Ergänzung zur Anregung von Erzählungen im Rahmen der Einzelinterviews eingeschätzt. Die konkrete Auswahl der Bilder erfolgte zum

56 Siehe dazu ausführlich Unterpunkt „Anregung von Assoziationen und Erzählungen durch visuelle Vignetten im Rahmen der Interviews“ im laufenden Kapitel. 
Teil mit den Frauen gemeinsam bzw. wurde von diesen als sinnvoll eingeschätzt (vgl. 2RGS-S3).

Auch der Faktor „Zeit“" wurde von den Frauen als sehr bedeutsam im Rahmen der Interviewführung erachtet. Konkret wurde dazu angeführt: „Und wenn es länger dauern sollte, muss man sagen, dass es vielleicht länger braucht. Und sich nicht stressen lässt. Jeder braucht seine Zeit und dass man sich nicht gedrängt fühlt" (2RGS-S8). Damit thematisieren die Frauen aus der Referenzgruppe einen Aspekt, der auch einer zentralen Forderung aus der People-First-Bewegung entspricht ${ }^{57}$, nämlich ausreichend Zeit abgestimmt auf die jeweiligen persönlichen Voraussetzungen zur Verfügung zu stellen.

Die geäußerten Präferenzen der Frauen führten zur Entscheidung, erzählgenerierende Interviews zu führen, in die auch anschauliche Elemente integriert sind. Im Folgenden reflektiere ich Grundüberlegungen zu erzählgenerierenden Interviews als Erhebungsmethode im vorliegenden Forschungskontext. Im Zusammenhang damit wird auch die darin integrierte Vignettenmethode beschrieben.

\subsubsection{Erzählgenerierende Interviews}

Die von der Referenzgruppe als wichtige Komponente im Rahmen der Interviewführung angesprochene Offenheit führte - wie bereits beschrieben - zur Entscheidung, möglichst offen angelegte Interviews mit Frauen mit Lernschwierigkeiten durchzuführen. Dementsprechend wurde eine Variante qualitativer Interviews gewählt, die über eine Eingangsfrage Erzählungen anregen soll. Diese sogenannte „Generierung“ von Erzählungen über eine verbale Aufforderung unterscheidet diese Form des Interviews etwa von fokussierten Interviews, die Ausführungen zu ,vorab definierte[n] Gesprächsgegenstände[n]" (Hopf 2009: 353) anregen sollen. Im Kontext der vorliegenden Studie habe ich mich vom Sprachgebrauch her für den Gebrauch des Begriffes des „erzählgenerierenden Interviews“ entschieden. Dies erscheint mir aus dem Grund naheliegend, da im Zuge der durchgeführten Interviews nicht nur über eine verbale Erzählaufforderung, sondern ergänzend dazu auch über die integrierte Vignettenmethode die Interviewpartnerinnen zu Ausführungen angeregt werden sollten.

Mit der Auswahl dieser Form des Interviews für Frauen mit Lernschwierigkeiten ist eine im wissenschaftlichen Fachdiskurs kontrovers eingeschätzte Frage angesprochen, nämlich, „ob die Fähigkeit, im Interview die Erzählung eines Prozessgeschehens zu entfalten, bei allen Gesellschaftsmitgliedern in gleicher Weise ausgebildet ist (vgl. Küsters 2009: 30). Aufgrund meiner

57 Die Notwendigkeit, angemessene Zeit zur Verfügung zu haben, wird u.a. von der PeopleFirst-Initiative Wibs thematisiert, indem etwa festgehalten wird: „Wir brauchen Zeit, um nachfragen zu können“ (Wibs 2005: 45). 
eigenen Erfahrungen aus einem früheren Forschungskontext ${ }^{58}$ und den aktuellen Interviews war und bin ich der Überzeugung, dass Menschen mit Lernschwierigkeiten wie alle anderen Menschen über unterschiedlich ausgeprägte narrative Kompetenz verfügen. Die theoretischen Ausführungen von Fritz Schütze, der als Begründer dieser Methode gilt, verweisen in ebendiese Richtung. Die Fähigkeit, selbst erlebte Erfahrungen im Rahmen einer „Stegreiferzählung“ zum Ausdruck zu bringen, wird demnach als „Basiskompetenz“ eingeschätzt, die „unabhängig von Schichtungs- und anderen Merkmalen nahezu von allen beherrscht" (Küsters 2009: 31) wird.

Dem Anspruch eines offenen Erhebungsverfahrens folgend, dieses nicht „entsprechend unseren Vorannahmen [zu] strukturieren“ (Rosenthal 2014: 49), wurde dabei zunächst eine Erzählaufforderung gesetzt, die biographisch orientiert war und demnach zu einer Erzählung über den Verlauf des bisherigen Lebens anregen sollte. Je nach Fortgang der Haupterzählung war vorgesehen, erzählgenerierende Nachfragen zu stellen und diese eventuell durch eigene Nachfragen zu ergänzen. Für diese Phase des Nachfragens war im Projektkontext mit den Frauen aus der Referenzgruppe geplant, dass ich mich an der von ihnen erarbeiteten Grundlage ${ }^{59}$ orientiere. Für jeden der angesprochenen Themenkomplexe erarbeitete ich dazu im Vorfeld mit Orientierung am Modell des episodischen Interviews nach Flick spezielle erzählgenerierende Fragen (vgl. Flick 2010: 275), die ich je nach Fortgang und Erfordernis im konkreten Interview zum Einsatz bringen konnte. In der konkreten Konstruktion der auf die einzelnen Themen abgestimmten Fragen orientierte ich mich dabei sehr stark an den innerhalb der Referenzgruppe dazu geführten Diskussionen und Reflexionen.

Diese Vorbereitungsarbeiten, die auf der Grundlage der Arbeit mit der Referenzgruppe basierten, erwiesen sich in der konkreten Erhebungsphase als sehr hilfreich und stellten für mich sicher, dass mit meinen Nachfragen Bereiche thematisiert werden konnten, die von Frauen aus der Zielgruppe selbst als wesentlich für Selbstbestimmung eingeschätzt wurden. Als erzählgenerierende Nachfragen wurden sie abgestimmt auf den speziellen Interviewkontext und die jeweilige Interviewpartnerin, aber lediglich bei Bedarf zum Einsatz gebracht und waren dann meiner Erfahrung nach sehr förderlich für den Fortgang der Erzählung. In einzelnen Fällen wurden spezielle Fragen aus dieser Orientierungsrichtlinie auch bereits zu Beginn des Interviews eingesetzt, um eine Erzählung überhaupt erst in Gang zu setzen. Als besonders brauchbar erwies sich dabei für mich die von der Referenzgruppe als sehr

58 Diese besteht in der Durchführung und Auswertung von Interviews mit Frauen mit Lernschwierigkeiten im Rahmen des Projektes „Zur Lebenssituation von Frauen mit geistiger Behinderung" (Klagenfurt, 2003).

59 Diese Orientierungsgrundlage und ihr Zustandekommen wird im Unterkapitel „Inhaltlicher Rahmen der Arbeit und Ausgangsbasis aus der Sicht der Frauen aus der Referenzgruppe“ beschrieben in Tabelle 2 abgebildet. 
wichtig eingeschätzte Frage nach der Tagesstruktur. Ihre Einschätzung, wonach jede Frau mit Lernschwierigkeiten etwas dazu erzählen können würde (vgl. 3RGS-S1), traf ohne Ausnahme zu. Auch ihre Vermutung, dass über die angeregten Erzählungen oder Beschreibungen im Zusammenhang mit der Tagesstruktur eine gute Basis für Analysen im Zusammenhang mit Selbstund Fremdbestimmung im Alltag der interviewten Frauen gegeben sein würde (vgl. 3RGS-S2), erwies sich als zutreffend.

Grundsätzlich wurde also die Prämisse, das Prinzip der Offenheit im Rahmen der Erhebung ${ }^{60}$ umzusetzen, indem ,zunächst Orientierung am Relevanzsystem der Alltagshandelnden und nicht an den wissenschaftlichen Relevanzsetzungen“ (Rosenthal 2014: 54) erfolgt, berücksichtigt. Dies einerseits durch den prinzipiellen Rahmen von Offenheit im Interviewkontext gegenüber den interviewten Frauen und andererseits an der bedarfsorientierten Orientierung an den Relevanzsetzungen der Frauen aus der Referenzgruppe als Basis für erzählgenerierende Nachfragen, die im o.a. Sinne als „Alltagshandelnde“" gesehen werden können.

Dem Anspruch, Offenheit im Interviewkontext zu gewährleisten, versuchte ich auch insofern gerecht zu werden, dass ich auch auftretende „Störungen als Besonderheit der Befragten“" (Rosenthal 2014: 53) wahrzunehmen versuchte und auch Raum in der Auswertung zukommen ließ. Konkret bedeutet dies, dass ich etwa Fragen, die an mich im Interview gestellt wurden, in die Erhebungssituation und in die Auswertung mit einbezog. Diese Integration aufgetretener „Störungen“ in den Auswertungskontext erweist sich in der Rückschau als aufschlussreich im Zusammenhang mit der Analyse von Orientierungen junger Frauen mit Lernschwierigkeiten.

\subsubsection{Anregung von Assoziationen und Erzählungen durch visuelle Vignetten}

Hinsichtlich der Auswahl der Erhebungsmethode wurde von den Frauen aus der Referenzgruppe mehrmals die - auch aus der People-First-Bewegung bekannte Anregung eingebracht, ,anschaulich“ zu arbeiten. Damit einhergehende Diskussionen und Überlegungen mit der Gruppe (vgl. 2RGS-S6ff.) führten dazu, eine Methode in die Interviews zu integrieren, die über einen nicht verbalen erzählgenerierenden Stimulus zu Ausführungen anregt, die möglicherweise Rückschlüsse auf Orientierungen der befragten Frauen hinsichtlich im Verlauf des Interviews noch nicht thematisierter Fragen ermöglichen. Im Sinne einer Ergänzung der von den Frauen über verbale Stimuli hervorgebrachten Ausführungen wurde diese Methode am Ende des jeweili-

60 Dem Prinzip, Offenheit auch in der interpretativen Textanalyse umzusetzen (vgl. Rosenthal 2014: 54), wird die im vorliegenden Kontext verwendete dokumentarische Methode gerecht. Diese wird ausführlich im Unterkapitel „Auswertungsmethode“ beschrieben. 
gen Interviews zum Einsatz gebracht. Der Einsatz eines solchen Befragungsinstrumentes stellt eine Alternative zu ,erzählexternen Nachfragen“ nach Abschluss des „erzählinternen Frageteils“ in narrativen Interviews dar (vgl. Rosenthal/Loch 2002: 11).

Von den Grundüberlegungen her habe ich mich dabei an den „Dresdner Bewältigungsvignetten“ orientiert, mit denen sehr aufschlussreiche Erfahrungen zur Erfassung von Hilfesuch- und Bewältigungsstrategien von Kindern gemacht wurden (vgl. Stiehler/Werner 2008). Unter Vignetten versteht man in den Sozialwissenschaften ,prägnante Geschichtenanfänge oder visuelle Abbildungen“ (Stiehler/Werner 2008: 185), die dazu dienen ,[...] eine stimulierende Aufforderungssituation herzustellen, die die befragte Person zu Beurteilungen oder zu weiterführenden Handlungsentwürfen anregt. Über Identifikations- und Projektionsprozesse mit den Protagonisten wird eine soziale Wirklichkeit erzeugt, in der die situativen Realitätserzeugungen der befragten Personen rekonstruiert werden“ (ebd). Diese Methode zählt zu den projektiven Verfahren, mit denen „unterdrückte, geleugnete oder schwer ermittelbare Einstellungen“ (ebd.) herausgearbeitet werden können. Mit Reizmaterial werden „Bestrebungen, Gefühle, Gedanken, Eigenschaften in abgebildete Szenen, Figuren und Gestalten oder in Worte, Sätze, Geschichten etc." hineingelegt (ebd.: 185f.). Probandinnen und Probanden reagieren erfahrungsgemäß auf solche Stimuli ,[...] je nach eigenen Gewohnheiten, Interessen, Gefühlen, Erwartungen und Wünschen“ (ebd.: 186). Über die Assoziationen und Entwürfe erfolgt meist eine Identifikation mit der Protagonistin oder dem Protagonisten der Szene, „eigene Motive, Gefühle und Verhaltensweisen“ werden diesem $/ \mathrm{r}$ zugeschrieben (ebd.). Über diese Identifikation können so „persönliche Haltungen, Einstellungen und Verhaltensweisen im sozialinteraktiven Kontext" (ebd.) erhoben werden. Als großer Vorteil projektiver Verfahren gilt, dass durch den speziellen Zugang zu den Befragten ,,auch unterdrückte oder schwer zugängliche Einstellungen“ (ebd.) von diesen zum Thema gemacht werden können. Ein weiterer Vorteil ist der, dass die Befragten „[...] den Erhebungsprozess aktiv mitgestalten und ihre eigenen Grenzen selbst festlegen können“"(ebd.).

Kritisiert werden an projektiven Verfahren die vielfach „noch unzureichend standardisierten Auswertungs- und Interpretationsprozesse“ (ebd.), was auf der anderen Seite aber positiv eingeschätzt wird, da durch die damit einhergehende Offenheit die Auswertung und Interpretation näher an den Antworten der Befragten bleibt (vgl. Stiehler/Werner 2008: 186). Auch für den Anspruch partizipativer Forschung, das Expertentum der Akteurinnen und Akteure anzuerkennen, scheint mir das letztere Argument stimmig. Denn dadurch wird diesem Anspruch im Rahmen der Auswertung auf einer differenzierten Ebene entsprochen, indem deren Ausführungen nicht schematisch in Ergebnisse überführt werden, sondern indem über die Auswertungsschritte der dokumentarischen Methode deren Expertinnenwissen erhalten bleibt. 
In Anlehnung an dieses beschriebene und erprobte Modell habe ich in meiner Untersuchung visuelle Vignetten zum Einsatz gebracht. Dies schien mir gerade bei Fragestellungen in Zusammenhang mit Partnerschaft, Sexualität und Kinderwunsch sehr sinnvoll, für die ich aufgrund eigener Erfahrungen in einem weiter zurückliegenden Projektzusammenhang ${ }^{61}$ die o.a. Einschätzungen der Vorteile eines projektiven Verfahrens durch Stiehler und Werner gut nachvollziehen konnte.

Bei der konkreten Auswahl von visuellen Vignetten war es mir wichtig dem Anspruch auf Ermöglichung von Selbstbestimmung in der Interviewsituation entsprechend - Wahlmöglichkeiten zu eröffnen. Die befragten Frauen sollten also nicht auf ein Bild festgelegt werden, das von mir vorab bestimmt wurde, sondern selbst ein Bild oder zwei Bilder aus einer Reihe von Bildern ${ }^{62}$ auswählen können. Gleichzeitig musste aber aus Gründen der Handhabbarkeit die Auswahlmöglichkeit auf eine überschaubare Anzahl von Vignetten eingeschränkt werden. Die zur Auswahl angebotenen visuellen Vignetten zeigen dabei behinderte und nichtbehinderte Menschen in verschiedenen Lebenssituationen. Die Szenarien auf den Bildern sind dabei eher offen oder thematisieren Fragen, die auf der Grundlage meiner eigenen Erfahrungen in der Forschung und nach Einschätzung der Frauen aus der Referenzgruppe Frauen mit Lernschwierigkeiten in unterschiedlichen Kontexten betreffen ${ }^{63}$. Aus diesem Grund sind auch die dazu entwickelten Fragen offen formuliert und sollen einen erzählgenerierenden Stimulus setzen.

In der Interviewsituation wurde zunächst ausreichend Zeit zur Betrachtung aller Bilder und zur Auswahl zur Verfügung gestellt. Hinsichtlich der Auswahl wurde ersucht, sich am eigenen Interesse $\mathrm{zu}$ orientieren und jene/s Bild/er auszuwählen, durch das/die die betroffene Frau sich angesprochen fühlt. Danach wurde nach einer Beschreibung der dargestellten Situation aus der Perspektive der jeweiligen Interviewpartnerin gefragt. Mit diesen erzählgenerierenden Stimuli im Zusammenhang mit der Entwicklung der jeweiligen Situation war die Intention verbunden, möglicherweise mehr über die Einschätzung soziogenetischer Faktoren auf die Entwicklung von Situationen und Kontexten sowie deren Fortgang aus der Perspektive von Frauen mit Lernschwierigkeiten zu erfahren. Zudem schien es denkbar, über die Frage nach dem Verlauf der jeweiligen Geschichte bei Identifikation mit deren Protagonistin oder Protagonisten mehr über Lebensentwürfe, Vorstellungen und Wünsche der Interviewpartnerinnen zu erfahren. Die konkrete Eingangsfrage lautete (mit situationsangepassten Variationen):

61 Diese besteht in der Durchführung und Auswertung von Interviews mit Frauen mit Lernschwierigkeiten im Rahmen des Projektes „Zur Lebenssituation von Frauen mit geistiger Behinderung" (Klagenfurt, 2003).

62 Die Quellen für die zum Einsatz gebrachten Bilder finden sich im Anhang der Arbeit.

63 Siehe dazu insbesondere Unterpunkt „Inhaltlicher Rahmen der Arbeit und Ausgangsbasis aus der Sicht der Frauen aus der Referenzgruppe“ im laufenden Kapitel der Arbeit. 
Was sehen Sie auf diesem Bild? Beschreiben Sie mir das doch bitte etwas genauer!

Je nach spezifischer Reaktion, Situation sowie Einsetzen und Fortgang einer Beschreibung oder Erzählung wurden gegebenenfalls weitere Fragen angeschlossen, wie etwa:

Was macht die Frau? Was macht der Mann?

Was sagt die Frau? Was sagt der Mann?

Wie fühlt sich die Frau? Wie fühlt sich der Mann?

Abgestimmt auf die jeweilige Interviewpartnerin und den Verlauf des Interviews bzw. der durch die Vignetten angeregten Ausführungen, wurde auch nach der Entwicklung der dargestellten und beschriebenen Situation gefragt:

Was war vorher? Wie ist es dazu gekommen?

Wie geht die Geschichte weiter?

Tabelle 4: Erzählaufforderung und optionale Nachfragen im Rahmen der Vignettenbeschreibungen in den durchgeführten Interviews

\subsubsection{Stichprobe und Zugang zum Feld}

Im Zentrum der vorliegenden Studie stehen Orientierungen junger Frauen mit Lernschwierigkeiten im Spannungsfeld von Selbst- und Fremdbestimmung. Dementsprechend wurden hinsichtlich der Stichprobe Frauen, denen im Verlauf ihrer Sozialisation der Status „Frau mit geistiger Behinderung“ oder „Frau mit Lernschwierigkeit“ zugewiesen wurde, als Interviewpartnerinnen gewählt.

Damit wurde hinsichtlich der Frage, wessen Perspektive bei den Datenerhebungen im Zentrum stehen soll, auch der Präferenz der Frauen aus der Referenzgruppe gefolgt. Deren Ansicht, Frauen mit Lernschwierigkeiten selbst müssten im Fokus der Erhebungen stehen, war sehr eindeutiges Ergebnis der Diskussion in einer der Sitzungen: ,Wichtig ist es, die zu befragen, die es betrifft und das sind wir" (2RGS-S6). Nicht befragt werden sollten explizit Betreuerinnen und Betreuer, weil deren Perspektive ohnehin bekannt sei und immer berücksichtigt würde. Frauen mit Lernschwierigkeiten selbst würden demgegenüber viel zu selten nach ihrer Meinung gefragt (vgl. 2RGSS7). Damit bekräftigen die Frauen aus der Referenzgruppe auch einen zentralen Anspruch der People-First-Bewegung auch im Zusammenhang mit partizipatorischer Forschung, nämlich das Wahrnehmen von Betroffenen als Expertinnen und Experten, deren Perspektive direkt bei ihnen selbst und nicht über andere Personen, über Dritte erhoben werden soll/kann.

Die Zuschreibungen „Frau mit geistiger Behinderung“ oder „Frau mit Lernschwierigkeit" stehen üblicherweise im Kontext mit dem Besuch einer Sonderschule bzw. Integrationsklasse oder einer Institution für Menschen mit Lernschwierigkeiten, die mit entsprechenden Begutachtungs- und Etikettierungsprozessen verbunden sind. Dies dokumentiert sich in der Regel über die Zuschreibung eines Sonderpädagogischen Förderbedarfs oder des Vorliegens der Voraussetzungen für die Aufnahme in eine Werkstätte oder Wohneinrich- 
tung über ein entsprechendes Gutachten. Zumeist wird in diesen Gutachten noch der Begriff der ,geistigen Behinderung“ gebraucht. Auf die Problematik und Relativität entsprechender Zuweisungen wurde im Theorieteil der Arbeit bereits verwiesen ${ }^{64}$.

Im Auswahlprozess wurde eine „geschichtete Stichprobe“ (Merkens 2009: 293) gezogen, bei der die Auswahl ,,absichtsvoll und nicht nach dem Zufallsprinzip“ (ebd.) erfolgt. Neben der Zugehörigkeit zum weiblichen Geschlecht und dem zugeschriebenen Status der Behinderung wurde eine Varianz weiterer Kriterien wie unterschiedliche Wohnformen, der Status hinsichtlich der Einbindung in Arbeit oder Beschäftigung und materielle Ausstattung berücksichtigt $^{65}$. Vom Alter her wurden sehr junge bis junge Frauen im Alter von 18 bis 32 Jahren einbezogen, um Aufschluss über Orientierungen von Frauen zu erhalten, die sich in annähernd vergleichbaren Lebensphasen befinden oder diese vor nicht allzu langer Zeit durchlaufen haben.

Von der Wohnform her wird zwischen dem Wohnen bei den Eltern oder einem Elternteil, dem Wohnen in einer Institution (Wohnheim für Menschen mit Behinderung), dem weitgehend selbständigen Wohnen in einer Wohngemeinschaft mit bedarfsorientierter Unterstützung und dem Wohnen in einer eigenen Wohnung unterschieden. Die Kriterien Arbeit und Beschäftigung gehen mit der materiellen Ausstattung einher. Die Mehrzahl der Frauen ist in Werkstätten in größeren Behinderteneinrichtungen oder bei kleineren Projekten beschäftigt und verfügt über ein geringfügiges Taschengeld, das meist abgestuft nach der eingeschätzten Leistung ausbezahlt wird. Eine der Frauen ist im Rahmen eines Projektes im Status Anlehre, eine weitere Frau ist über eine spezielle Form im Rahmen eines Projektes fix angestellt und erhält realen Lohn. Eine weitere Frau ist am offenen Arbeitsmarkt beschäftigt und erhält ebenfalls realen Lohn. Drei weitere Frauen sind weder in Arbeit noch in Beschäftigung in einer Institution oder am Arbeitsmarkt eingebunden. Diese Frauen beteiligen sich jedoch in unterschiedlichem Ausmaß an Haushaltstätigkeiten in der Herkunftsfamilie. Diese Frauen erhalten weder Lohn noch Taschengeld von einer Arbeitsstelle oder Institution. Alle drei bekommen Taschengeld von den Eltern, bei der Erfüllung ihrer persönlichen materiellen Bedürfnisse sind sie vollständig auf die Unterstützung der Eltern angewiesen.

Bei der Auswahl der Stichprobe wurde der Aspekt der theoretischen Sättigung im Blick gehalten, d.h. sie wurde erst im Verlauf des ,parallele[n] Erhebens und Analysierens der Daten“ (Glaser/Strauss ${ }^{3}$ 2010: 77) abgeschlossen, als absehbar wurde, dass ,keine neuen Phänomene mehr gefunden werden“ (Rosenthal 2014: 85) konnten, die den Einbezug weiterer „Fälle“ erfordern würden. Den Anspruch der theoretischen Sättigung habe ich dabei als

64 Siehe dazu Kapitel 2 ,Zur Bezeichnung ,Menschen mit Lernschwierigkeiten““.

65 Eine Übersichtstabelle mit entsprechenden Angaben $\mathrm{zu}$ den interviewten Frauen befindet sich im Anhang der Arbeit. 
theoretischen Anspruch eigeschätzt, der im eigentlichen Sinne kaum je völlig abgeschlossen sein kann, da keine absolute Sicherheit besteht, „dass keine neuen theoretisch relevanten Einsichten mehr gewonnen werden können“ (ebd.: 85). Im vorliegenden Forschungskontext habe ich aber jedenfalls versucht, mögliche „blinde Flecken“ (ebd.) durch den Einbezug der Einschätzungen der Frauen aus der Referenzgruppe auch im Rahmen des Samplings zu umgehen.

Um ,elementare Verständigungsschwierigkeiten auf verbaler und nonverbaler Ebene“" (Wolff 2009: 341) im Feldzugang möglichst abzufedern und um eine selbstbestimmte Entscheidung über die Teilnahme an den Interviews zu ermöglichen, wurde zunächst über bestehende Vernetzungen mit Personen und Institutionen jeweils Treffen mit einzelnen oder mehreren Frauen vereinbart, die den Teilnahmekriterien entsprachen. Im Rahmen der Bemühungen, Zugang zum Feld zu erlangen, waren die Interaktionen mit den sogenannten „Türstehern“ (Wolff 2009: 342) und „Türsteherinnen“ davon geprägt, das Forschungsvorhaben transparent zu beschreiben und somit der Gefahr entgegenzuwirken, dass sich das Feld als geschlossenes System präsentiert (ebd.: 343) und den Zugang erschwert. Daher wurden zunächst die Personen, über die ein Zugang möglich schien, über das Forschungsvorhaben informiert. Damit einher ging das Ersuchen, im entsprechenden Kontext Gespräche mit Frauen führen zu können und nachzufragen, ob Interesse bzw. Bereitschaft für ein Interview besteht. Als Türöffnerinnen oder Türöffner sind im Kontext des Forschungsprojektes Personen zu verstehen, über deren Arbeits- oder Lebenszusammenhang der Zugang zu Frauen mit Lernschwierigkeiten möglich ist, also Leiterinnen und Leiter von Institutionen sowie Eltern und Angehörige, im Einzelfall Sachwalterinnen oder Sachwalter, aber auch über Mitarbeiterinnen und Mitarbeiter einer Selbstbestimmt-Leben-Initiative. Diese rückwirkend als erfolgreiche einschätzbare Strategie führte $\mathrm{zu}$ mehreren Treffen mit Gruppen von Frauen oder auch mit einzelnen Frauen, in denen sie in sogenannter ,leichter Sprache“ über das Forschungsvorhaben im Allgemeinen, die groben Inhalte, die Mitarbeit einer Referenzgruppe sowie über Rahmenbedingungen der Teilnahme informiert ${ }^{66}$ (Aufnahme, Transkription, Anonymität etc.) wurden. In diesem Kontext war es für die Frauen auch möglich, nachzufragen und Details abzuklären. In diesem Zusammenhang war die besondere Hervorhebung der Bedeutung der Anonymität und Vertraulichkeit auffallend. Auf deren besonderen Stellenwert war ich aber bereits durch die Rückmeldung der Referenzgruppe vorbereitet, die im Zusammenhang mit der Diskussion methodischer Fragen auf deren besonderen Stellenwert für Frauen mit Lernschwierigkeiten hingewiesen hatte (vgl. 2RGS-S6). Nach der Abklärung dieser spezifischen Fragen erfolgte entweder unmittelbar im Anschluss daran oder auf spätere Nachfrage eine $\mathrm{Zu}$ - oder Absage für die Teilnahme als

66 Dafür wurde ein Informationsblatt in „leichter Sprache“ erstellt und übergeben, dessen Inhalte im Gespräch mitgeteilt wurden. 
Interviewpartnerin. Im Einzelfall wurde auch unmittelbar nach der Information über das Projekt bereits auf Wunsch der jeweiligen Interviewpartnerin das entsprechende Interview geführt. Insgesamt war die Bereitschaft, als Interviewpartnerin zur Verfügung zu stehen, sehr hoch. Alle Frauen, die von mir persönlich informiert wurden, entschieden sich zur Teilnahme am Interview. Diese Tatsache war für mich aus einem früheren Projektkontext (vgl. Sigot 2003: 11f.) wenig erstaunlich. Bereits dort hatte ich feststellen können, dass der Großteil der Frauen sehr gerne zu einem Interview bereit war und einige Frauen, die nicht als Interviewpartnerinnen vorgesehen bzw. angefragt waren, sich selbst dafür ins Spiel brachten. Der bereits damals gezogene Schluss, dass Frauen mit Lernschwierigkeiten das Interview als eher seltene Möglichkeit sahen, jemandem etwas über das eigene Leben erzählen zu können, wiederholte sich also im vorliegenden Erhebungskontext. Der Eindruck, dass durch das Ersuchen, etwas über die eigene Lebenssituation und die eigenen Erfahrungen zu erzählen, Interesse und damit verbunden Anerkennung der eigenen Person erlebt werden, verdichtete sich im Zuge der Interviews zunehmend.

\subsubsection{Das Setting der Interviews}

Im Kontext partizipativer Forschung ist es konsequent, auch „die Erzählperson bestimmen zu lassen, wo das Interview durchgeführt wird" (Helfferich 2011: 177). Die Wahl des Ortes kann dabei auch als „Erkenntnismittel“ eingeschätzt werden, indem darüber Aussagen über Kontexte gemacht werden, die als Ort abgelehnt oder präferiert werden.

Im Projektkontext wurden alle Frauen nach ihrer Präferenz für die Vorgespräche und eines Interviewortes gefragt. Alle Vorgespräche und auch die Interviews selbst wurden von mir selbst dann auf Wunsch der Frauen vorwiegend im familiären Umfeld der Frauen oder in Institutionen geführt, in denen sie in Arbeit oder Beschäftigung unter Tags anzutreffen waren. Dass kein anderer Ort von den Frauen selbst aus in Erwägung gezogen wurde, brachte ich mit ihren Lebensbedingungen in Zusammenhang, die offenbar keine Alternativen außerhalb dieser Systeme anboten. In all diesen Fällen wurde vom Setting her großer Wert darauf gelegt, dass im Interviewverlauf Störungen durch dritte Personen möglichst vollständig ausgeschlossen wurden. Dennoch kam es in zwei Fällen zu einer kurzen Unterbrechung des Interviews. In einem Fall verließ die Interviewpartnerin selbst kurz den Raum, weil sie vermeinte, ihre Mutter rufen gehört zu haben. Im zweiten Fall unterbrach eine Mutter das Interview mit der Nachfrage, ob etwas zu trinken gebraucht würde. In beiden Fällen wurde das Interview kurz ausgesetzt und vor dessen Fortsetzung auf das Wieder-Eintreten störungsfreier Rahmenbedingungen gewartet. 
Einige der Interviews fanden im Sommer 2013 in ungestörter Atmosphäre in meinem Büro an der Alpen-Adria-Universität Klagenfurt statt und waren damit verbunden, dass die Frauen auch die Universität und deren Umfeld kennen lernen konnten. Diese Möglichkeit wurde von mir als Variante angeboten, deren Umsetzung über Unterstützung von Personen aus dem sozialen oder beruflichen Umfeld der Frauen ermöglicht.

In fast allen Fällen gab es im Vorfeld oder auch im Anschluss der Interviews auch soziale Kontakte mit den Frauen über ein gemeinsames Essen oder einen gemeinsamen Kaffee. Zu einigen Frauen ergab sich aus dem Interview weiterhin sporadischer Kontakt in verschiedenen Zusammenhängen.

\subsection{Die Auswertung der Interviews mit der dokumentarischen Methode}

Als Auswertungsmethode für die im Rahmen der vorliegenden Studie durchgeführten qualitativen Interviews wurde die dokumentarische Methode gewählt, die von Ralf Bohnsack entwickelt wurde, der sich dabei auf die Wissenssoziologie Karl Mannheims und auf Harold Garfinkels Arbeiten bezog (vgl. van Essen 2013: 164f.). Aus der Palette von bekannten Auswertungstechniken für qualitative Interviews habe ich mich im Kontext der vorliegenden Studie aus diesem Grund für die dokumentarische Methode entschieden, da sie meiner Einschätzung nach sehr stimmig mit der grundsätzlichen Zielsetzung der Arbeit ist, Fragen des Zustandekommens der kommunizierten Einstellungen von Frauen mit Lernschwierigkeiten im Zusammenhang mit Selbst- und Fremdbestimmung zu erhellen. Denn sie bietet schon von ihrem Forschungsansatz her eine theoretische Fundierung, die solche Prozesse sehr differenziert zu berücksichtigen vermag. Zudem korrespondiert diese Methode auch mit der Prämisse partizipativer Forschung, die Personen, die selbst von den Fragestellungen der Forschung betroffen sind, im Forschungsprozess als Expertinnen und Experten zu berücksichtigen: Denn mit den entwickelten Analysetechniken wird es möglich, auch im Rahmen der Auswertung ,nicht ,hinter dem Rücken“ der Akteure“ (Bohnsack/Nentwig-Gesemann/Nohl 2007: 9) nach deren Handlungspraxis zu forschen, sondern deren eigenes Wissen als Ressource zu begreifen und zum Ausdruck zu bringen. Somit kann dieser Anspruch partizipativer Forschung neben dem Einbezug einer aus Frauen mit Lernschwierigkeiten bestehenden Referenzgruppe im gesamten Forschungsprozess auch über die Auswertungsmethode verfolgt werden.

In der dokumentarischen Methode wird insbesondere auch dem Umstand Rechnung getragen, dass den Akteurinnen und Akteuren das Wissen über das Zustandekommen ihrer Einstellungen und Orientierung weitgehend verborgen ist. Sie stellt hier ein sehr ausdifferenziertes Instrumentarium zur Verfü- 
gung, das es ermöglicht, sich nicht vorwiegend mit dem ,[...] kommunikativen, von allen ohnehin geteilten und gewussten Teil des Wissens" (Nohl 2012: 44) zu befassen, sondern zum sogenannten ,,atheoretischen“ oder ,konjunktiven" Wissen der Befragten vorzudringen. Dieses Wissen, das auch als „,handlungspraktisch“, ,handlungsleitend“, ,inkorporiert" und „habitualisiert“ bezeichnet wird, gilt auch als „,implizites Wissen“, da es den Befragten ,[...] reflexiv nicht so ohne weiteres zugänglich ist" (Bohnsack/NentwigGesemann/Nohl 2007: 11). Das unbewusste Wissen der Akteurinnen und Akteure bildet dabei die empirische Basis der dokumentarischen Interpretation, löst sich jedoch von deren Sinnzuschreibungen ab (vgl. Nohl 2012: 45). Die Analyseschritte der dokumentarischen Methode ermöglichen also „,Zugang zur Handlungspraxis und zu der dieser Praxis zugrunde liegenden (Prozess-)Struktur, die sich der Perspektive der Akteure selbst entzieht" (ebd.: 12).

Die zentralen Fragestellungen der vorliegenden Studie, die die Genese des Zustandekommens von Einstellungen und Orientierungen betreffen, decken sich dabei mit der der dokumentarischen Methode zugrunde liegenden Intention, nicht in erster Linie danach zu fragen, ,[...] was die gesellschaftliche Realität in der Perspektive der Akteure ist [sondern] wie diese in der Praxis hergestellt wird“67. Es geht also darum, „einen Zusammenhang von Orientierungen und Erfahrungen zu rekonstruieren“ (Nohl 2012: 2). Da die Handlungspraxis dabei im Vordergrund steht, werden die dokumentarische Methode und die damit vertretene Wissenssoziologie auch als ,praxeologisch“ bezeichnet (vgl. Bohnsack/Nentwig-Gesemann/Nohl 2007: 13). Mit den Fragen nach dem „Wie“, also nach der Herstellung der Realität, sind der sogenannte „modus operandi“, also die Art und Weise, wie diese erfolgt, und damit in weiterer Folge der dieser Praxis zugrunde liegende Habitus angesprochen (vgl. ebd.). Der auf Bourdieu zurückgehende Begriff des Habitus steht dabei ,[...] für die ,Wahrnehmungs-, Denk- und Handlungsschemata' eines Menschen, in dem sämtliche inkorporierten, früheren sozialen Erfahrungen zum Ausdruck kommen" (Lenger u.a. 2013: 14) ${ }^{68}$.

67 Damit einher geht nach Bohnsack (2007: 227) ein „Bruch mit dem common sense“. Dieser auf Pierre Bourdieu zurückgehende Begriff wird definiert durch einen ,grundlegenden Wechsel der Analyseeinstellung“, indem mit den dort „,verankerten Selbstverständlichkeiten des Zugangs zu den subjektiven Motiven [...] konsequent gebrochen wird“ (ebd.).

68 Mit dem Begriff des Habitus verbunden mit der zugrunde liegenden theoretischen Fundierung entwickelte Pierre Bourdieu einen Ansatz zur dialektischen Überwindung von Subjektivismus und Objektivismus, indem er einerseits die ,subjektive Sichtweise der sozialen Akteure" gegenüber dem Objektivismus verteidigt (vgl. Michel 2006: 98). Andererseits betont er , ,...] die Notwendigkeit, die subjektiven Sichtweisen nicht lediglich nachzuzeichnen, sondern sie auf den sozialen Ort ihrer Entstehung zurückzubeziehen“ (ebd.). Der Habitus wird so gesehen auch als „Scharnier“ (ebd.) gesehen, da er einerseits als „System der organischen oder mentalen Dispositionen und der unbewussten Denk-, Wahrnehmungs- und Handlungsschemata“ (ebd.) fungiert. Und andererseits von der ,sozialen Lage des jeweili- 
Aber nicht nur das Handeln selbst, sondern auch die Praxis „des Sprechens, Darstellens und Argumentierens" (ebd.) stehen dabei im Fokus der Forschung. Im Zusammenhang damit ist die Unterscheidung des sogenannten „kommunikativen Wissens“ vom „konjunktiven Wissen“ bedeutsam. Ersteres bezieht sich meist auf Handlungsmotive, die explizit zum Ausdruck gebracht werden. Demgegenüber kann das konjunktive Wissen, das unbewusst wirkt, nicht „kommunikativ expliziert, sondern nur erzählt oder beschrieben werden“ (Nohl 2012: 43). Das konjunktive Wissen, das also „eng mit der spezifischen Praxis von Menschen in Biographien und ihren Milieus verknüpft ist" (Nohl 2012: 5), verbindet Individuen, die bestimmte Erfahrungen teilen können, in einem gemeinsamen, ,konjunktiven Erfahrungsraum“. Mit diesem Begriff wird eine „,von der konkreten Gruppe losgelöste Kollektivität grundlagentheoretisch“ (Przyborski 2004: 29) gefasst. Dabei kommt es in der Praxis allerdings zu Überlagerungen, da jedes Individuum unterschiedliche Erfahrungsräume mit anderen Menschen teilt, die u.a. in der Zugehörigkeit zu einem Geschlecht, zum Bildungsmilieu oder einer Generation bestehen (ebd.: 29f.). Konjunktives Wissen verbindet also auch einander persönlich unbekannte Menschen, ohne dass reale gemeinsame Erfahrungen im Alltag in einem konjunktiven Erfahrungsraum vorliegen müssen.

Für den Kontext der vorliegenden Forschung ist dies aus zwei Gründen von besonderer Bedeutung: Einerseits wurde allen Frauen - also den Frauen aus der Referenzgruppe und den Interviewpartnerinnen - in ihrer Sozialisation in unterschiedlichen Zusammenhängen der Status einer Frau mit ,geistiger Behinderung“ oder „mit Lernschwierigkeiten“ zugeschrieben. Die mit diesen Etikettierungsprozessen einhergehenden Konsequenzen verbinden sie als Frauen in einem konjunktiven Erfahrungsraum. Auf der Ebene des konkreten Forschungsprozesses ermöglicht die Partizipation von Frauen, die diesem Erfahrungsraum angehören, im Rahmen einer Referenzgruppe die Einbindung ihres kommunikativen, aber auch ihres konjunktiven Wissens. Dadurch, dass aufgrund der Teilung eines gemeinsamen Erfahrungsraumes „Beschreibungen und Erzählungen [...] unmittelbar verstanden werden“ (ebd.: 29), liefern ihre Interpretationen und Reflexionen insbesondere auch im Auswertungsprozess wertvolle Impulse und Beiträge. Gehören Erforschte und Forscher üblicherweise unterschiedlichen Milieus an, so ist durch die Mitarbeit von Peers in der Referenzgruppe das Milieu der erforschten Personen im Forschungsverlauf abgebildet.

gen Akteurs“ und seiner damit verbundenen „Kapitalausstattung“ (ebd.) geprägt ist. Der Habitus wird dabei einerseits ,in konkreten Handlungssituationen erworben“ (ebd.), andererseits ,entfaltet er sich auch in der Handlungspraxis“ (ebd.). 


\subsubsection{Die Analyseschritte der dokumentarischen Methode und deren Umsetzung im Forschungsprojekt}

Die Analyseschritte der dokumentarischen Methode im Kontext der Auswertung von Interviews ${ }^{69}$ orientieren sich dabei an den allgemeinen, dieses Kapitel einleitend angeführten theoretischen Fundierungen und sind darauf ausgerichtet, ,einen Zugang zum konjunktiven Wissen“ als dem ,[...] milieuspezifischen Orientierungswissen zu erschließen“ (Bohnsack/Nentwig-Gesemann/ Nohl 2007: 14). Im Überblick werden die unterschiedlichen Stufen und Zwischenstufen der dokumentarischen Methode deutlich:

\begin{tabular}{ll}
\hline Stufen & Zwischenstufen \\
\hline Formulierende Interpretation & $\begin{array}{l}\text { Thematischer Verlauf und Auswahl zu } \\
\text { transkribierender Interviewabschnitte } \\
\text { Formulierende Feininterpretation eines } \\
\text { Interviewabschnittes }\end{array}$ \\
\hline Reflektierende Interpretation & Formale Interpretation mit Textsortentrennung \\
& Semantische Interpretation mit komparativer \\
& Sequenzanalyse \\
\hline Typenbildung & Sinngenetische Typenbildung \\
& Soziogenetische Typenbildung \\
\hline
\end{tabular}

Tabelle 5: Auswertungsschritte der dokumentarischen Methode (vgl. Nohl 2012: 39)

Zentral für die dokumentarische Methode sind zunächst zwei aufeinander aufbauende Analyseschritte: In der formulierenden und daran anschließend in der reflektierenden Interpretation wird zunächst das „Was“, dann das „Wie“ des in den Interviews Gesagten in den Blick genommen. Dabei werden formale und semantische Aspekte berücksichtigt, da die Semantik des Textes "[...] von seiner formalen Konstruktion nicht zu trennen“ (Nohl 2012: 41) ist. Als ganz wesentlicher Schritt in der dokumentarischen Methode als rekonstruktives Verfahren erweist sich gerade im Zuge der reflektierenden Interpretation der Vergleich, „denn die interpretative Fallrekonstruktion vollzieht sich immer vor dem Hintergrund der Vergleichshorizonte, mit denen Forschende an einen Text herantreten" (Nohl in Bohnsack/NentwigGesemann/Nohl 2007: 255). Fallinterne und fallübergreifende Vergleiche münden zunächst in die sogenannte sinngenetische Typenbildung, die zeigt ,$[\ldots]$ in welch unterschiedlichen Orientierungsrahmen die erforschten Personen jene Themenstellungen bearbeiten, die im Zentrum der Forschung ste-

69 Die dokumentarische Methode wurde zunächst von Ralf Bohnsack (1989) für die Auswertung von Gruppendiskussionen entwickelt und umgesetzt. Schon bald wurden zunehmend auch Interviews mit dieser Auswertungsmethode interpretiert. Eine methodologische Begründung des Zusammenhanges von Interview und dokumentarischer Methode wurde aber erst einige Zeit später von Arnd-Michael Nohl (2006) geliefert und weiter entwickelt. 
hen“ (Nohl 2012: 52). Die darauf aufbauende sogenannte soziogenetische Typenbildung macht in einem nächsten Schritt deutlich, ,[...] in welchen sozialen Zusammenhängen und Konstellationen die typisierten Orientierungsrahmen stehen" (ebd.).

Im Folgenden werden die vorab knapp skizzierten Analyseschritte der dokumentarischen Methode differenziert beschrieben und im Kontext der vorliegenden Studie reflektiert: Formulierende und reflektierende Interpretation, fallinterner und fallübergreifender Vergleich sowie sinngenetische und soziogenetische Typenbildung stehen also im Zentrum der Betrachtungen. Dabei wird immer wieder auch das Spezifikum der vorliegenden Studie mit berücksichtigt und diskutiert, das in der begleitenden Arbeit mit einer Referenzgruppe und somit im Einbezug der Akteurinnenperspektive besteht. Dieser Prozess mündet in der Zusammenfassung der Habilitationsschrift auch in die Reflexion eigener Erfahrungen aus der Anwendung der dokumentarischen Methode im Rahmen der vorliegenden Studie und in allgemeine Überlegungen und Schlüsse hinsichtlich der Bedeutung dieser Auswertungsmethode für die partizipative Forschung.

\subsubsection{Erster Schritt: Formulierende Interpretation}

Im Rahmen dieses ersten Analyseschrittes erfolgt eine Zusammenfassung des in den Interviews Gesagten ,in ganzen Sätzen und mit den eigenen Worten der Forschenden“ (Nohl 2012: 41). Diese „Reformulierung des thematischen Gehalts“ dient dazu, ,die Forschenden gegenüber dem Text fremd zu machen" (Nohl 2012: 41). Dies ist insofern von zentraler Bedeutung, da durch diesen zeit- und arbeitsaufwändigen Schritt sichtbar wird, ,[...] dass der thematische Gehalt nicht selbstverständlich, sondern interpretationsbedürftig ist" (Schäffer nach Nohl 2012: 41).

\section{Thematischer Verlauf und Auswahl zu transkribierender Interviewabschnitte}

Konkret sieht die dokumentarische Methode vor, relevante Passagen aus den Interviews für formulierende Interpretationen auszuwählen. Dafür werden zunächst Themen aus den vorliegenden Interviews, die für die Forschung besonders aufschlussreich sein könnten, identifiziert. Um zu diesen Themen $\mathrm{zu}$ gelangen, wird zunächst das tabellarische Verzeichnen „thematischer Verläufe" anhand der Audioaufnahmen und/oder der Durchsicht der Transkriptionen empfohlen (vgl. Nohl 2012: 40). Nach der Skizzierung der thematischen Verläufe wird in der „formulierenden Feininterpretation“ (Nohl 2012: 40) jeder Abschnitt ,sequentiell nach mehr oder weniger markanten Themenwechsel" (ebd.) überprüft, es werden Ober- und Unterthemen herausgearbeitet, die dann - wie bereits beschrieben - in den Worten der Forschenden den thematischen Gehalt des Gesagten festhalten. 
Im Rahmen der Auswertung habe ich mich dafür entschieden, zunächst mehr als die Hälfte aller Interviews zur Gänze formulierend und reflektierend zu interpretieren, um umfassende Rekonstruktionen als Grundlage für die daran anschließende Typenbildung und auch für die soziogenetischen Analysen zur Verfügung zu haben. Dies schien mir im Kontext meiner Forschung auch aus dem Grund relevant, da sich einige der Interviews sprachlich eher karg erwiesen, umfassende Rekonstruktionen über die zugrundeliegenden Vergleiche aber dennoch sehr deutlich vorliegende Orientierungen zum Ausdruck bringen konnten. Bei den weiteren Interviews habe ich zentrale Themen und Passagen ausgewählt und dabei die Kriterien, die in der dokumentarischen Methode dafür herangezogen werden, berücksichtigt. Als relevant für die Auswahl der konkreten Textstellen aus den Interviews werden insbesondere jene Themen eingeschätzt, die vorab von Wissenschaftern und Wissenschafterinnen festgelegt wurden, sowie Themen, die in unterschiedlichen Fällen gleichermaßen vorkommen und sich gut für komparative Analyse eignen (ebd.). Zudem auch Themen, die den interviewten Personen selbst offenbar sehr wichtig sind, was sich an hoher inhaltlicher Dichte und der Verwendung sogenannter Fokussierungsmetaphern zeigt. Der Begriff der „Fokussierungsmetapher“ wird dabei für „Passagen mit hoher narrativer Dichte“ und einem hohen „Detailierungsgrad“ verwendet. Weitere Kriterien von Fokussierungsmetaphern sind besonders prägnante Ausdrucksweise und „metaphorische Dichte“ (vgl. Bohnsack 2007: 225).

In diesem Auswahlprozess erwies sich der Einbezug einer Referenzgruppe, die aus Frauen besteht, die selbst die Erfahrung des Behindert-Seins oder -Werdens gemacht haben, wiederum als sehr unterstützend. Jene Themen, die sie als signifikant für Fragen der Selbstbestimmung identifizierten (vgl. Dok1-RGS2), konnten als zentrale Orientierungsrichtlinie für diesen Prozess herangezogen werden und stellten somit sicher, dass deren Perspektive als zentrales Moment im Forschungsprozess verankert werden kann. So wurde etwa das von der Gruppe als wichtig hervorgehobene Kriterium der Tagesstruktur, anhand derer sich ihrer Ansicht nach sehr deutlich Selbst- und Fremdbestimmung im Alltag von Frauen mit Lernschwierigkeiten zeigen können (vgl. 3RGS-S3), bei der Auswahl berücksichtigt. Diese Anregung der Referenzgruppe erweist sich in der Rückschau als in zweifacher Hinsicht sehr zielführend. Zunächst im Rahmen der Interviews selbst und wie oben angeführt bei der Identifikation zentraler Passagen für die Auswertung.

Passagen, die sogenannte „Fokussierungsmetaphern“"70 beinhalten, wurden in jedem Fall bei der Auswahl berücksichtigt und einer formulierenden und reflektierenden Interpretation unterzogen. Im Auswertungsprozess wurde dabei sichtbar, dass die in die Interviews integrierte Vignettenmethode dabei in einigen Fällen zu Ausführungen anregten, die inhaltlich und sprachlich

70 Zum Begriff der „Fokussierungsmetapher“ siehe auch Unterkapitel „Formale Interpretation und Textsortentrennung“ im laufenden Kapitel. 
von hoher Dichte gekennzeichnet sind und somit „Fokussierungsmetaphern“ darstellen. Offenbar regten die verwendeten visuellen Vignetten auch gedanklich zu bildhaften und verdichteten Ausführungen an und ermöglichten so wichtige Rückschlüsse über vorliegende Orientierungen ${ }^{71}$.

\section{Formulierende Feininterpretation von Interviewabschnitten}

Bei der Feininterpretation von Interviews in ihrer Gesamtheit oder ausgewählter Abschnitte geht es nun - wie dieses Unterkapitel einleitend bereits beschrieben - darum, sich als Forscherin über die „Reformulierung des thematischen Gehalts“ die Ausführungen der interviewten Personen ,fremd zu machen" (Nohl 2012: 41). Der Inhalt der Ausführungen wird somit nicht als selbstverständlich wahrgenommen, sondern vor dem Hintergrund eigener Standortgebundenheit reflektiert. Auch auf die Interpretationsbedürftigkeit des thematischen Gehaltes wurde bereits hingewiesen (vgl. ebd.). Im Zuge der formulierenden Interpretation wird deutlich, was alles in den Texten steckt, wenn man sich das, was gesagt wird, über diesen Schritt „fremd“ macht und nicht nur vor dem eigenen Erfahrungshintergrund reflektiert. Es erweist sich in der Auswertungspraxis, „dass der thematische Gehalt nicht selbstverständlich, sondern interpretationsbedürftig ist" (Schäffer nach Nohl 2012: 41). So gesehen ist dieser arbeitsintensive Schritt unverzichtbar.

Im vorliegenden Forschungskontext konnte ich dafür in mehreren Sitzungen auf die Expertise der Frauen aus der Referenzgruppe zurückgreifen. Konkret wurden mehrere ausgewählte Interviewpassagen ${ }^{72}$ von diesen auf ihren konkreten inhaltlichen Gehalt geprüft. Die Ergebnisse der damit zusammenhängenden Diskussionen und Reflexionen fanden Eingang in den weiteren Forschungsprozess, indem ich sie in meine weiteren Interpretationen mit einbezog.

Aus meiner Perspektive bereichern die Feininterpretationen jener Interviewpassagen, in denen der Prozess des „Fremdmachens“ durch mich als Forscherin als auch von den Frauen aus der Referenzgruppe erfolgte, den Auswertungsprozess. Zum einen, weil dadurch die Perspektive von selbst betroffenen Frauen auch in diesem Auswertungsschritt integriert ist. Zum anderen aber auch dadurch, dass ich durch den Vergleich meiner Interpretati-

71 Sehr deutlich wird dies im Interview mit Eva (IE VA7 582-586), deren starke Orientierung in Richtung Autonomie und Selbständigkeit sich über eine Fokussierungsmetapher dokumentiert, die über eine Vignettenbeschreibung angeregt wurde. Siehe dazu ausführlich Fallrekonstruktion „Eva“, „Ablehnung von Behütung und Kontrolle versus Wunsch nach Freiraum/Freiheit und Privatsphäre“. Anmerkungen zum Einsatz der Vignettenmethode mache ich auch in der Zusammenfassung der Habilitationsschrift.

72 Siehe dazu die Übersichtstabelle (Tabelle 2) im Unterkapitel „Inhalte und Ergebnisse der Referenzgruppensitzungen“. 
onen mit jener der Referenzgruppe Sicherheit für den weiteren Auswertungsprozess bekam.

\subsubsection{Zweiter Schritt: Reflektierende Interpretation}

Nach der Reformulierung des thematischen Gehaltes durch die Forschenden wird bei der reflektierenden Interpretation nun auf die Genese des Gesagten fokussiert: Es wird also das „Wie“ einer differenzierten Analyse unterzogen. Dabei geht es konkret um die Frage, ,wie ein Thema bzw. das in ihm artikulierte Problem bearbeitet [wird] und in welchen (Orientierungs-) Rahmen [es] behandelt“ (Nohl 2012: 41) wird. Die reflektierende Interpretation zielt damit auf die „Rekonstruktion und Explikation des Rahmens, innerhalb dessen das Thema abgehandelt wird, auf die Art und Weise, wie, d.h. mit Bezug auf „[...] welchen Orientierungsrahmen das Thema behandelt wird“ (Bohnsack nach Nohl 2012: 45). Orientierungsrahmen stellen dabei ,sozialisatorisch inkorporierte kollektive Wissensbestände“" (Helsper u.a. 2013: 111) dar und entstehen in „konjunktiven Erfahrungsräumen“ (ebd.).

Bei der reflektierenden Interpretation werden formale und semantische Aspekte des Gesagten berücksichtigt, da davon auszugehen ist, dass „die Semantik des Textes [...] von seiner formalen Konstruktion nicht zu trennen“ (ebd.) ist.

\section{Formale Interpretation und Textsortentrennung}

Als erster Zwischenschritt dient dafür die „formale Interpretation und Textsortentrennung" (Nohl 2012: 41). Unter Bezugnahme auf Schütze werden hier die Ausführungen in den Interviews auf ihre Zugehörigkeit zu den Textsorten „Erzählung“, „Beschreibung“, „Argumentation“ und „Bewertung“ geprüft (ebd.). Dies wird im Interpretationsprozess für jede Passage auch dokumentiert und in die anschließende reflektierende Interpretation mit einbezogen. Den genannten Textsorten wird in der dokumentarischen Methode unterschiedlicher Stellenwert zugemessen bzw. werden Ausführungen in den Interviews auch mit Bezug auf ihre Zugehörigkeit zu einer dieser Textsorten reflektiert und interpretiert.

Der (Stegreif-)Erzählung wird dabei besonderer Stellenwert zugemessen, da sie durch die ihr eigene Dynamik, die sich in verschiedenen Zugzwängen ${ }^{73}$ äußert, die ihr zugrunde liegen, ,besonders nahe an den Erfahrungen des Erzählers“ (Nohl 2012: 42) liegt. Indem die „Darstellung des Geschehens in der Situation des Interviews entwickel[t]“" (Küsters 2009: 13) werden muss,

73 Nohl führt als Zugzwänge, die der Stegreiferzählung zugrunde liegen, konkret den Detaillierungszwang, den Gestaltschließungszwang, den Relevanzfestlegungs- und den Kondensierungszwang an (vgl. ausführlich dazu Nohl 2012: 23ff.). 
lässt die Erzählerin oder der Erzähler tiefen „Einblick in seine Erfahrungsaufschichtung" (Nohl 2012: 42) zu.

\begin{tabular}{ll}
\hline Textsorte & Charakteristik \\
\hline Erzählung & $\begin{array}{l}\text { Darstellung von „Handlungs- und Geschehensabläufe[n], die einen } \\
\text { Anfang, ein Ende und einen zeitlichen Verlauf haben“ (Nohl 2012: 42) }\end{array}$ \\
\hline Beschreibung & $\begin{array}{l}\text { Darstellung „immer wieder kehrende[r] Handlungsabläufe oder } \\
\text { feststehende[r] Sachverhalte“ (ebd.) }\end{array}$ \\
\hline Argumentation & „[...] (alltags-) theoretische Zusammenfassung zu „,den Motiven, \\
& $\begin{array}{l}\text { Günden und Bedingungen für eigenes oder fremdes Handeln“ } \\
\text { (Schütz nach Nohl: 42) }\end{array}$ \\
\hline Bewertung & "[...] evaluative Stellungnahmen zu eigenem oder fremdem Handeln“ \\
& (Nohl: 42) \\
\hline
\end{tabular}

Tabelle 6: Charakteristik verschiedener Textsorten in der dokumentarischen Methode (eigene Übersicht auf Grundlage der angeführten Definitionen)

Hier kann von einem engen Zusammenhang zwischen ,erzählter und erlebter Erfahrung" (ebd.) ausgegangen werden, wobei diese Erfahrung stets in die Haltung der Erzähler eingebunden und somit „konstruiert“ ist. „Es ist also nie die ,Wirklichkeit', sondern eine Erfahrung, die erzählt wird.“ (ebd.). Die Erfahrung unmittelbarer Handlungspraxis, die durch Erzählung und Beschreibung dargelegt wird, ,ist derart an diese Handlungspraxis, an das handlungspraktische Wissen und an die Selbstverständlichkeiten der Informanten gebunden, dass sie von diesen nicht kommunikativ expliziert, sondern nur erzählt oder beschrieben werden kann“ (ebd.: 43). Dieses ,,atheoretische“ und „konjunktive Wissen“ kann entweder beobachtet werden oder man kann „,auf dem Wege von Erzählungen und Beschreibungen zu dieser Handlungspraxis gelangen“ (ebd.). Erzählungen und Beschreibungen dienen daher dazu, dieses atheoretische oder konjunktive Wissen der Interviewten zu erheben. Da es in der qualitativen Sozialforschung nicht um den „kommunikativen, von allen ohnehin geteilten und gewussten Teil des Wissens [...] sondern um das unbekannte konjunktive Wissen“ geht (Nohl 2012: 44), bezieht sich die Interpretation daher vor allem auf die Textsorten Erzählung und Beschreibung, durch die diese Ebene zugänglich wird.

Argumentationen haben demgegenüber einen „starken inhaltlichen Bezug zu Gegenwartsstandpunkt des Erzählers“" (Schütze nach Nohl 2012: 42), denn hier „expliziert und theoretisiert er ja gegenüber der Interviewerin Motive und Gründe seines eigenen Handelns oder nimmt zu diesen argumentativ Stellung" (Nohl 2012: 42). Das hier zum Ausdruck gebrachte kommunikative Wissen bezieht sich vorwiegend auf Motive des Handelns - sogenannte „Um-zu-Motive“ - und „,basiert auf wechselseitigen [...] Motivunterstellungen, die gesellschaftlich institutionalisiert, also ,objektiviert' sind und die explizit oder ,wörtlich' zum Ausdruck gebracht werden“" (Bohnsack nach Nohl 2012: 43). Die interviewte Person nimmt hier auch konkret gegenüber 
dem Interviewer oder der Interviewerin Stellung. Diese Stellungnahmen beziehen sich dabei auf Wissensbestände, die über „Milieugrenzen hinweg gesellschaftlich geteilt" (Nohl 2012: 43) werden. Ein solches kommunikatives, über Argumentationen expliziertes Wissen ist abstrakt und von der Handlungspraxis abgehoben. Argumentation und Bewertungen sind aber ebenfalls interpretierbar, indem man nicht ihrem wörtlichen Sinngehalt folgt, sondern versucht, die ,[...] Herstellungsweise bzw. Konstruktionsweise der Argumentation $[\mathrm{zu}]$ rekonstruieren und auf diesem Wege heraus[zu]arbeiten, wie jemand seine Handlungsweisen rechtfertigt bzw. bewertet." (Nohl 2012: 44). Auch dies kann Aufschluss darüber geben, wie „Themen und Problemstellungen bearbeitet" (Nohl 2012: 44) werden.

Im Kontext der vorliegenden Studie entstand und verdichtete sich bei mir zunehmend der Eindruck, dass die in den Interviews explizierten Argumentationen und Bewertungen tatsächlich Rückschlüsse auf Orientierungsrahmen ermöglichen. Wenn festgehalten wird, es gehe ,in der qualitativen Bildungsforschung nicht um jenen kommunikativen, von allen ohnehin geteilten und gewussten Teil des Wissens" (Nohl 2012: 44), so stellt sich dies für mich im vorliegenden Kontext in spezifischer Weise dar: Menschen mit Lernschwierigkeiten sind von gesellschaftlichen Wissensbeständen aufgrund vielfältiger Barrieren weitgehend ausgeschlossen bzw. werden diese ihnen oft in verzerrter Form nähergebracht. Man kann also meines Erachtens nicht davon ausgehen, dass gesellschaftliche Wissensbestände auch von Menschen mit Lernschwierigkeiten geteilt werden können. Dies wird alleine schon durch die gesellschaftlich übliche Verwendung von Sprache erschwert, die aufgrund der Wortwahl und komplizierter Konstruktionen Distanz schafft. Insofern sind für mich jene Passagen, die sich durch die Textsorte „Argumentation“ erschließen, auch in der Hinsicht relevant, dass sie Rückschlüsse darauf zulassen, welche gesellschaftlichen Werte, Normen und welche Wissensbestände Menschen mit Lernschwierigkeiten in ihrer Sozialisation zugänglich gemacht bzw. nähergebracht wurden.

Bei der Auswertung von Gruppendiskussionen nach der dokumentarischen Methode kommt sogenannten „Fokussierungsmetaphern“ besondere Bedeutung zu. Unter diesem Begriff versteht man Sequenzen, die durch hohe ,interaktive und metaphorische“ Dichte (Bohnsack 2007: 379), „einen hohen Detaillierungsgrad und eine ausgeprägte Bildhaftigkeit der Darstellung“ (Bohnsack 2010: 124) gekennzeichnet sind. Auch in Einzelinterviews kommt spezifischen Passagen der Stellenwert von Fokussierungsmetaphern zu. Sie weisen auf die besondere Bedeutung der Erfahrung hin, die angesprochen ist, oder machen auf prägnante Weise vorliegende Orientierungen sichtbar. Dies erfolgt auch im Einzelinterview durch eine bildhafte Sprache, aber etwa auch 
durch mehrfache Wiederholung von Worten, mehrfache Verneinungen oder Ähnliches ${ }^{74}$.

\section{Semantische Interpretation und komparative Sequenzanalyse}

Auf ,der semantischen Ebene geht es darum, einen Zugang zur Wirklichkeit zu finden, die weder jenseits des Akteurswissens als objektiv definiert wird noch sich im subjektiven Sinn der Akteure (dem ,intentionalen Ausdruckssinn' nach Mannheim) erschöpft" (Nohl 2012: 45). Das Wissen der Akteure bleibt dabei die Basis der Interpretation, „, doch löst sich diese von den Sinnzuschreibungen der Akteure ab" (ebd.).

Die dokumentarische Methode zielt darauf ab, „die implizite Regelhaftigkeit von Erfahrungen und den in dieser Regelhaftigkeit liegenden dokumentarischen Sinngehalt, d.h. den Orientierungsrahmen dieser Erfahrungen zu rekonstruieren“" (Nohl 2012: 45). Das bedeutet, dass über eine Sequenz von erzählten Handlungen hinweg Regelhaftigkeit identifiziert werden muss. Dies kann über die komparative Sequenzanalyse erfolgen. Konkret wird dabei zunächst analysiert, wie die Herangehensweise an ein Thema in einem konkreten Fall ist. Wenn man nun davon ausgeht, „dass in einem Fall ein Thema auf eine (und nur eine) bestimmte Art und Weise (d.h. in einem Rahmen) erfahren wird“ (ebd.), „so kann für eine Textsequenz davon ausgegangen werden, dass auf einen ersten Erzählabschnitt nur ein spezifischer, nämlich ein der jeweiligen Erfahrungsweise, dem jeweiligen Rahmen entsprechender zweiter Abschnitt folgen kann" (ebd.). Wenn man nach Klassen von zweiten Äußerungen sucht, die thematisch sinnvoll erscheinen und homolog zur ersten Äußerung sind, wird die Bestimmung des dokumentarischen Sinngehalts, d.h. die Bearbeitungsweise bzw. der Orientierungsrahmen möglich. Es wird also im internen Vergleich deutlich, welche Herangehensweise auch in Bezug auf unterschiedliche Thematiken überwiegt. Fallübergreifend lassen sich auf dem Weg des Vergleiches im Sinne des minimalen und des maximalen Kontrastes dann unterschiedliche Orientierungen herausarbeiten. Also solche, in denen eine Thematik auf eine strukturgleiche Art und Weise bearbeitet wird, und solche, die zeigen, wie ,eine Thematik auch auf eine ganz andere Art und Weise bearbeitet werden kann“ (Nohl 2012: 47). In der dokumentarischen Methode spricht man dabei von homologen und heterologen Äußerungen (vgl. Nohl 2012: 46f.), also solchen, die sich in ihrer darüber sichtbar gewordenen Orientierung entsprechen oder unterscheiden. Von der konkreten methodischen Vorgangsweise her werden die heterologen zweiten und dritten Äußerungen nicht im selben, sondern in anderen Fällen gesucht.

Als Beispiel verweist Angelikas Ausführung zu ihren schulischen Erfahrungen auf die massiv wahrgenommene Abwertung: „Tag ein Tag aus, behindert=behindert=behindert, jedes Mal das Wort behindert“ (IAng 846) und kann durch die knappe, prägnante Ausführung mit Wiederholungen auch als Fokussierungsmetapher eingestuft werden. 
Erst eine fallübergreifende Gemeinsamkeit, etwa ein gemeinsames Thema, vermag dabei den Vergleich zu strukturieren (vgl. Nohl 2007: 258).

Im Zusammenhang mit der komparativen Sequenzanalyse ist das sogenannte „tertium comparationis“ als das ,gemeinsame Dritte, das den Vergleich [...] strukturiert" (Nohl 2012: 49) von zentraler Bedeutung. Konkret bedeutet dies, dass Themen von Äußerungen in den vorliegenden Interviews verglichen werden. Dabei soll es nicht zufällig zur Identifizierung unterschiedlicher Abfolgen von Textabschnitten in verschiedenen Fällen und zur Rekonstruktion der jeweiligen Orientierungsrahmen kommen, sondern das soll ,in die systematische Variation von Fällen und eine hieran anschließende Typenbildung eingebunden werden" (Nohl 2012: 51). Auf den vorliegenden Forschungskontext übertragen erforderte dies etwa, zu vergleichen, wie verschiedene Frauen mit Lernschwierigkeiten mit unterschiedlichen Herausforderungen umgehen oder wie sie in verschiedenen Situationen agieren. Durch diese spezielle Methode des Vergleiches, in der nicht die eigenen Erfahrungen als Forscherin als Basis des Vergleichs dienen, kann der Gefahr einer „Nostrifizierung“, dass also „das Unbekannte, das zu Erforschende [...] in das Muster der eigenen Selbstverständlichkeiten eingeordnet wird“ (Nohl 2007: 255), entgegengewirkt werden. In der dokumentarischen Methode wird somit mit der „Standortgebundenheit“" der Forschenden bewusst umzugehen versucht, indem diese ,auf dem Wege des empirischen Vergleichs der methodischen Kontrolle zugänglich gemacht" (Nohl in Bohnsack/NentwigGesemann/Nohl 2007: 255f.) wird. Der Vergleich wird dabei nicht im eigentlichen Sinne ,als eine Methode neben anderen“, sondern als ein „die gesamte Forschungspraxis und Einzelmethoden durchwirkende[r] Stil" (ebd.) und unter Bezug auf Durkheim als „Königsweg“ der Sozialforschung (vgl. Nohl 2013: 15) gesehen. Er dient dabei der „Typen- und damit der Theoriebildung" (ebd.) und hat gleichermaßen ,eine erkenntnisgenerierende und eine erkenntniskontrollierende Funktion“ (ebd.). Und zwar, indem schon bei der Rekonstruktion des Einzelfalles der Vergleich eine zentrale Bedeutung einnimmt. Über den Vergleich werden überhaupt erst „Sinnzusammenhänge im untersuchten Fall"“ (ebd.: 16) sichtbar. Darüber hinaus können sich die Forschenden durch das Heranziehen von Vergleichshorizonten aus anderen Fällen ,von der Befangenheit in ihren eigenen Selbstverständlichkeiten und Erwartungen lösen“ (ebd.).

Die komparative Sequenzanalyse stellt über die angesprochenen fallinternen und fallübergreifenden Vergleiche, die unterschiedliche Orientierungsrahmen sichtbar machen, auch die Ausgangsbasis für die darauf folgende Typenbildung dar.

Im vorliegenden Forschungskontext erwies sich im Zuge der Durchführung des Analyseschrittes der semantischen Interpretation und der komparativen Sequenzanalyse der Einbezug der aus Frauen mit Lernschwierigkeiten zusammengesetzten Referenzgruppe aus mehreren Gründen als wertvoll. 
Einerseits konnte bei der Auswahl von Themen, die die Vergleiche im Rahmen der Auswertung des zugrunde liegenden Forschungsprojektes strukturierten, auf die Liste mit Themenstellungen zurückgegriffen werden, die von den Frauen aus der Referenzgruppe als bedeutsam im Kontext mit Fragen von Selbstbestimmung erarbeitet wurden (vgl. Dok1-RGS2). Auf einer zweiten Ebene konnte der Gefahr der „Nostrifizierung“ der eigenen Annahmen nicht alleine durch die Analyseschritte der dokumentarischen Methode selbst, sondern darüber hinaus durch die Verfügbarkeit des konjunktiven Wissens über die Frauen aus der Referenzgruppe begegnet werden. Meine eigenen, durch die fallübergreifenden Vergleiche vor dem Erfahrungshintergrund von betroffenen Frauen erfolgten Interpretationen konnten durch selbst dem erforschten Personenkreis angehörige Frauen überprüft werden. Dadurch konnte meiner Ansicht nach neben der Beachtung der Vergleichshorizonte selbst betroffener Frauen im fallübergreifenden Vergleich auf einer zweiten Ebene der Gefahr entgegengewirkt werden, dass die Interpretationen durch meine „Standortgebundenheit“ als akademische Forscherin geprägt sind.

Im Zusammenhang mit der hohen Bedeutung, die dem Vergleich in der dokumentarischen Methode zukommt, konnte ich im Zuge der vorliegenden Forschungsarbeit Folgendes feststellen: Vergleiche werden auch von Frauen selbst im Interview immer wieder verwendet, um ihre Erzählungen zu strukturieren. So werden etwa im Rahmen der Sozialisation erlebte kontrastierende Erfahrungen thematisiert, Verhalten von Bezugspersonen sowie eigenes Verhalten in unterschiedlichen Kontexten verglichen ${ }^{75}$. Vergleiche in die eigene Erzählung einzubauen, erweist sich offenbar für die Frauen im Interview als Möglichkeit, die jeweiligen Inhalte in Gegenüberstellung zu konträr erlebten Erfahrungen zu schärfen, zu konturieren und somit klarer darzustellen.

\subsubsection{Dritter Schritt: Typenbildung}

Ziel der Typenbildung in der dokumentarischen Methode ist es, „fallübergreifende Orientierungsrahmen, in denen Themen und Probleme bearbeitet werden, zu identifizieren“ (Nohl 2013: 8). Mit dem Begriff des „Orientierungsrahmens“ ist „der zentrale Gegenstand dokumentarischer Interpretation“ (Bohnsack u.a. 2007: 15) angesprochen. Der Begriff des Orientierungsrahmens entspricht dabei jenem des „Habitus“ (ebd.), womit „sozialisatorisch inkorporierte kollektive Wissensbestände“" (Helsper u.a. 2013: 111) gemeint

Dies wird etwa im Interview mit Eva deutlich, die das Wohnen bei den Eltern mit dem Wohnen in der WG vergleicht (vgl. IE 29-35). Anna wiederum vergleicht die Rahmenbedingungen in der Regelschule mit jenen in der Sonderschule (vgl. IA 246-260) und charakterisiert die ,gute“ und die „schlechte“ Lehrerin (vgl. IA 312-323). Sie zieht weiters Vergleiche zwischen den eigenen Anstrengungen und Leistungen in der Schule und der „Faulheit“ anderer Schülerinnen und Schüler (vgl. IA 346-354). 
sind. Orientierungen selbst werden als „Sinnmuster“ gesehen, die sich in spezifischen Handlungen zeigen (vgl. Przyborski 2004: 55). Diejenigen Personen, die über gemeinsame konjunktive Erfahrungsräume verfügen, beziehen sich „unmittelbar und selbstverständlich“ (ebd.) darauf, „sie verstehen einander ohne einander zu interpretieren" (ebd.).

In der dokumentarischen Methode bildet die Ausrichtung an der „Handlungspraxis und Erfahrungswelt der untersuchten Personen“ (Nohl 2013: 41) den Ausgangspunkt der Typisierungen. Weil dabei auf die Orientierungsrahmen fokussiert wird, die sich aus der Handlungspraxis der interviewten Personen beziehen, wird auch von einer ,praxeologischen“ Typenbildung gesprochen (vgl. Nohl 2007: 228).

Die Typenbildung in der dokumentarischen Methode erfolgt in zwei Schritten. Wird in der sinngenetisch angelegten Typologie zunächst vorwiegend auf die Frage des „Was“ unter stellenweisem Einbezug sinngenetischer Überlegungen, also der Frage, wie es zu den Orientierungen gekommen ist, eingegangen, sind diese dann in der soziogenetischen Typologie zentral. Im Folgenden werden beide Schritte gerafft dargestellt.

\section{Sinngenetische Typenbildung}

In der sinngenetischen Typenbildung erhalten die den ersten Fall kontrastierenden und herausgearbeiteten Orientierungsrahmen nun ,eine eigene Signifikanz“ (Nohl 2012: 109), „eine eigene Bedeutung und werden in ihrer eigenen Sinnhaftigkeit gesehen“ (Nohl 2012: 51). Die rekonstruierten Orientierungsrahmen werden abstrahiert, vom Einzelfall abgelöst und zu Typen ausformuliert. Alle herausgefundenen Orientierungsrahmen werden also „erkannt und typisiert" (Nohl 2012: 109).

Dabei darf die Interpretation nicht mehr beim Vergleich zweier Fälle in Bezug auf ein Thema stehen bleiben. Es müssen vielmehr weitere Interviews und Interviewpassagen einbezogen werden, in denen auch andere Themen bearbeitet werden. Je mehr Interviews ,nun auf dem Wege des Vergleichs mit minimal bzw. maximal kontrastierenden [...] Fällen" (Nohl 2013: 6) einbezogen werden, desto deutlicher zeichnen sich bestimmte Orientierungsrahmen nicht nur in einem Fall ab und desto deutlicher werden weitere Orientierungsrahmen rekonstruierbar. „Die Fruchtbarkeit des Vergleichs steigt dabei mit dem Variationsgrad der angewandten tertia comparationis" (Nohl 2012: 53). Das Herausarbeiten maximaler Kontraste ist im Zuge der sinngenetischen Typenbildung von besonderer Bedeutung. Dabei ist der „Kontrast in der Gemeinsamkeit [...] fundamentales Prinzip der Generierung einzelner Typiken und [...] zugleich die Klammer, die eine ganze Typologie zusammenhält“ (Bohnsack 1989: 374 nach Nohl 2012: 55). Eine „komplexe komparative Analyse" mit mehrfacher Variation des tertium comparationis wird 
als Voraussetzung für eine „mehrdimensionale soziogenetische Typenbildung" (Nohl 2012: 51) eingeschätzt.

Im Zusammenhang mit der sinngenetischen Typenbildung müssen zwei Arten von Motiven grundsätzlich voneinander unterschieden werden: „das ,Um-zu-Motiv“, das entwurfs- und damit zukunftsorientiert ist [...] und das ,Weil-Motiv', das erklärt, wie die Handlung zustande gekommen ist" (Nohl 2013: 37). Es wird als sinnvoll erachtet, beide Motive in den Blick zu nehmen (ebd.: 37), also auf das „Was“ und das „Wie“ zu fokussieren.

Die in diesem Analyseschritt erhobenen Orientierungen wurden den Frauen aus der Referenzgruppe präsentiert und zur Diskussion gestellt ${ }^{76}$.

\section{Soziogenetische Typenbildung}

Im Rahmen der soziogenetischen Typenbildung geht es darum, „die sozialen Zusammenhänge aufzuklären, innerhalb derer die sinngenetisch entwickelten Typen [...] stehen“ (Nohl 2012: 110). Dazu sucht man nun nicht mehr ,nach den übergreifenden Gemeinsamkeiten zwischen Fällen [...], sondern nach systematischen Unterschieden zwischen ihnen“ (Nohl 2012: 110). Im Rahmen der soziogenetischen Typenbildung wird also abgeklärt, ,welchem spezifischen Erfahrungsraum, welcher Erfahrungsdimension oder welcher Orientierung eine generelle Orientierung zuzurechnen ist, wofür sie also typisch ist" (Bohnsack 2007: 263).

Mittels der soziogenetischen Typenbildung werden die Soziogenese bzw. „die soziokulturellen Kontexte“ (van Essen 2013: 175) dieser Orientierungen in den Blick genommen. Damit wird die „Ebene der Einzelfallstruktur vollends verlassen“ (ebd.). „Im Vordergrund stehen nun die Strukturen konjunktiver Erfahrungsräume“ (ebd.), die zu den herausgearbeiteten „Orientierungen und habitualisierter Handlungspraxis geführt haben“ (ebd.). Zu berücksichtigen ist dabei, dass „Personen stets in mehrere konjunktive Erfahrungsräume eingebunden sind“ (ebd.).

Die soziogenetische Struktur ist in den Interviews vorwiegend über die Sozialisationsgeschichte und die Biographie erhebbar, wobei „Schlüsselszenen“ dafür in „fokussierten Passagen“ zu Kindheit und Jugend in Interviews (Bohnsack 2007: 250) darstellen.

Auch die soziogenetischen Zusammenhänge wurden den Frauen der Referenzgruppe im Rahmen einer Sitzung präsentiert und zur Diskussion gestellt ${ }^{77}$.

76 Die Ergebnisse dieses Diskussions- und Reflexionsprozesses sind im Kapitel 5, Unterpunkt „Die vier erhobenen und typisierten Orientierungen in der Einschätzung der Frauen aus der Referenzgruppe" dokumentiert.

77 Siehe dazu Kapitel 5, Unterpunkt „Soziogenetische Zusammenhänge in der Einschätzung der Frauen aus der Referenzgruppe: ausgewählte Themen“. 


\subsubsection{Zur Frage der Generalisierung}

In der soziogenetischen Typenbildung werden „Reichweite und Grenzen der Typiken sichtbar gemacht" (Nohl 2012: 115), damit „dient die mehrdimensionale, soziogenetische Typenbildung auch der Generalisierung der empirischen Ergebnisse" (Nohl 2012: 115). Insofern mit der dokumentarischen Methode an einem Fall im systematischen Vergleich mit anderen gleich mehrere Typiken in ihrer Überlappung identifizierbar sind, unterscheidet sie sich von anderen Ansätzen der Typenbildung. „Diese mehrdimensionale Typenbildung ermöglicht dann auch eine Generalisierung von Untersuchungsergebnissen“ (Nohl 2012: 57).

Die Frage der Generalisierungsfähigkeit der vorliegenden Ergebnisse wird von mir selbst nicht beantwortet. Es wurden jedenfalls alle Analyseschritte durchgeführt, die als Grundlage dafür gelten. Wie dafür vorgesehen, wurden die erhobenen Typiken voneinander abgegrenzt, es wurden aber auch die „Verschränkung“ der Typiken vorgenommen (vgl. Bohnsack 2007: 253). Durch diese Schritte wurde auch die „Rekonstruktion der Soziogenese“ (ebd.) der erhobenen Orientierungen möglich.

Im nächsten Kapitel der Arbeit werden die Ergebnisse des gemeinsam mit den Frauen aus der Referenzgruppe geplanten und in diesem Kapitel ausführlich beschriebenen Forschungsprojektes differenziert dargestellt. 


\section{Die Darstellung der Forschungsergebnisse}

Die Darstellung der Ergebnisse der auf Grundlage der im letzten Kapitel angestellten Überlegungen durchgeführten Forschung erfolgt in mehreren Abschnitten. Zunächst werden die vier mittels der dokumentarischen Analyseschritte der formulierenden und reflektierenden Interpretation, der Sequenzanalyse und fallinterner und -über-greifender Vergleiche identifizierten, zentralen Orientierungen im Überblick präsentiert. Es werden also alle herausgefundenen Orientierungsrahmen junger Frauen mit Lernschwierigkeiten, die im Kontext der vorliegenden Untersuchung ,erkannt und typisiert“ (Nohl 2012: 109) wurden, als Zwischenergebnis dargestellt ${ }^{78}$.

Danach werden für jeden Typus markante Dimensionen, die diesen besonders gut charakterisieren, in den Blick genommen. Diese Beispiele werden anhand rekonstruktiv dargestellter Sequenzen aus jeweils einem Fall dokumentiert. Dabei sind die vier differenziert dargestellten Fallrekonstruktionen als Beispiele zu verstehen, an denen sich Dimensionen der abgeleiteten Typik besonders gut nachvollziehen lassen. Nicht der konkret dargestellte Fall in seiner Gesamtheit wird also zum jeweiligen Typus abstrahiert. An einem Fall können im systematischen Vergleich mit anderen Fällen weitere Typiken in ihrer Überlappung sichtbar werden. Dieses Spezifikum, das sich aus den differenzierten Analyseschritten der dokumentarischen Methode ergibt, unterscheidet diese gegenüber anderen typenbildenden Verfahren grundsätzlich (vgl. Nohl 2012: 57). Die im Anschluss an die jeweiligen Rekonstruktionen präsentierten vier Typen sind in der Folge als Kompositionen zu verstehen, die Orientierungen junger Frauen mit Lernschwierigkeiten sichtbar machen. Im Zuge der Beschreibung der vier Typen werden zentrale Dimensionen der jeweiligen Orientierung dann noch durch prägnante Passagen aus anderen Fällen belegt, die deren jeweilige unterschiedliche Ausprägungsvarianten sichtbar machen sollen. Diese zeigen sich auch typenübergreifend, das heißt, dass es auch zu Überlappungen von Orientierungen kommen kann. Sichtbar werden diese v.a. über mehrdimensional angelegte Analysen und Vergleiche erhobenen Überlappungen dann speziell in der soziogenetischen Typenbil-

78 Typologien im Rahmen rekonstruktiver Methoden qualitativer Forschung haben grundsätzlich nicht den Aspekt der Kategorisierung von Personen im Fokus, es werden also nicht wie bei anderem Verfahren ,einzelne Merkmalskriterien summativ“ zusammengefasst (vgl. Rosenthal 2014: 75). Gleichwohl birgt der Prozess der Typisierung grundsätzlich meiner Einschätzung nach gerade im Zusammenhang mit Fragen von Behinderung Gefahren bzw. die Möglichkeit von Missverständnissen, die es zu vermeiden gilt. Im vorliegenden Kontext erfolgt die Auswertung und Darstellung der Ergebnisse, insbesondere bezogen auf die Typenbildung ressourcenorientiert. Die typisierten Orientierungen erfolgten auch unter dem Aspekt der Wertungsfreiheit, d.h. die ausgearbeiteten Orientierungen verweisen auf keine Wertung bzw. Bevorzugung eines speziellen Typus. Sie bilden Lebensformen von Frauen mit Lernschwierigkeiten mit ihren vielfältigen Facetten ab. 
dung. Da in den Rekonstruktionen, die zur sinngenetischen Typenbildung überleiten, soziogenetische Aspekte an verschiedenen Stellen beispielhaft bereits gut angedeutet sind, habe ich mich im Kontext der vorliegenden Studie für diese Art der Darstellung entschieden.

Wird in der sinngenetisch angelegten Typologie zunächst vorwiegend auf die Frage des „Was“ unter stellenweisem Einbezug sinngenetischer Überlegungen, also der Frage, wie es zu den Orientierungen gekommen ist, eingegangen, sind diese dann in der soziogenetischen Typologie zentral. Die Fragen, wie und warum es zu den vorliegenden Orientierungen gekommen ist, werden analysiert und dargestellt und stellen die zentralen Ergebnisse der Studie dar. In dieser Typologie werden soziogenetische Aspekte typenübergreifend in den Blick genommen und zentrale Dimensionen hinsichtlich der Frage analysiert, wie mit einer Problemstellung, die als konjunktive Erfahrung von Frauen mit Lernschwierigkeiten zu begreifen ist, umgegangen wird. Also zum Beispiel, wie Erfahrungen von Abwertung, Ausgrenzung, Diskriminierung oder auch Mobbing als konjunktiven Erfahrungen in unterschiedlichen Orientierungen begegnet wird. Es wird also analysiert, wie die konkrete typenspezifische Sicht- und Herangehensweise zu dieser Problemstellung ist und vor allem auch wie es dazu gekommen ist. Es geht demnach darum, „die sozialen Zusammenhänge aufzuklären, innerhalb derer die sinngenetisch entwickelten Typen [...] stehen" (Nohl 2012: 110). Dazu wird in der soziogenetischen Typenbildung im Unterschied zur Vorgangsweise bei der sinngenetischen Typenbildung nicht mehr nach ,übergreifenden Gemeinsamkeiten [...], sondern nach systematischen Unterschieden“" (ebd.) gesucht. Dabei werden Zusammenhänge zwischen vorliegenden Orientierungen und zugrundeliegenden konjunktiven Erfahrungen deutlich. Ziel dieser soziogenetischen Typenbildung ist es, dies nicht nur im Einzelfall, sondern systematisch zu beleuchten und die Ergebnisse dieser Analyse zu dokumentieren.

\section{Der Einbezug der Referenzgruppe: Rückkoppelung und Reflexion}

Wie im Methodenkapitel ausführlich erläutert, ist ein zentrales Element des gesamten Forschungsprozesses der Einbezug einer Referenzgruppe, die sich aus vier Frauen mit Lernschwierigkeiten zusammensetzt.

Nach deren Einbindung in die inhaltliche und methodische Anlage der Untersuchung sowie in konkrete Auswertungsschritte wurden auch die Ergebnisse der sinngenetischen und soziogenetischen Typenbildung der Referenzgruppe vorgestellt. Konkret wurden die vier erhobenen und typisierten Orientierungsrahmen, die nachfolgend dargestellt werden, der Referenzgruppe präsentiert und mit dieser diskutiert. Auch die Ergebnisse der soziogenetischen Analysen wurden an die Referenzgruppe rückgekoppelt und einer kritischen Diskussion unterzogen. Die Rückmeldungen bzw. Ergänzungen der Frauen aus der Referenzgruppe zur sinngenetischen und soziogenetischen 
Typenbildung werden jeweils im Anschluss an die Präsentation der Auswertungsergebnisse dargestellt. Dieser Einbezug von Frauen mit Lernschwierigkeiten als Expertinnen in eigener Sache stellt meines Erachtens einen sehr wesentlichen Faktor im partizipativ angelegten Forschungsprozess dar, da darüber deren Perspektive darin abgebildet werden kann. Insbesondere auch die Rückkoppelung der (Zwischen-)Ergebnisse an die Gruppe verbunden mit deren Einschätzung der Ergebnisse über kritisches Nachfragen, Bestätigungen, Anregungen und Ergänzungen stellten für mich im Forschungsprozess eine unverzichtbare Orientierungsrichtlinie dar. Durch die Integration der Perspektive der Akteurinnen war es möglich, auch in dieser Phase des Forschungsprozesses die Vergleichshintergründe von Frauen mit Lernschwierigkeiten zu berücksichtigen, die konjunktive Erfahrungen mit den interviewten Frauen teilen.

\subsection{Fallrekonstruktionen und sinngenetische Typologie: vier zentrale Orientierungen}

Mittels der Auswertungsschritte der dokumentarischen Methode wurden vier zentrale Orientierungen identifiziert. Im nächsten Schritt werden diese Orientierungen anhand beispielhafter Fallrekonstruktionen ${ }^{79}$ - „Isolde“, „Martina“, „Anna“ und „Eva“ - dargestellt. Im Anschluss daran werden jeweils weitere ausgewählte Beispiele dafür angeführt, also weitere Belege, die zentrale Dimensionen der jeweiligen Orientierung deutlich machen. Damit dokumentiert sich auch, dass die einzelnen Typen nicht aus einem Fall abgeleitet werden, sondern sich in ihnen Orientierungen widerspiegeln, die mehreren Fällen zugrunde liegen.

In den vier Fallrekonstruktionen werden spezifische Sichtweisen und Orientierungen der jungen Frauen mit Lernschwierigkeiten in den Blick genommen, es steht also die Frage, was die jeweilige Orientierung ausmacht, im Vordergrund. Als Ausgangsbasis für eine im Anschluss daran erfolgende soziogenetische Analyse werden Fragen, wie es zu den Orientierungen gekommen ist, bereits angedeutet.

79 Die wirklichen Namen aller Frauen im Forschungskontext sind anonymisiert, die Pseudonyme wurden z.T. selbst gewählt. 


\begin{tabular}{llc}
\hline Kurzbezeichnung & \multicolumn{1}{c}{ Beschreibung } & Fallrekonstruktion \\
\hline Orientierung A & $\begin{array}{l}\text { Orientierung am vorgegebenen, stark } \\
\text { strukturierten, vertrauten Rahmen, der auf } \\
\text { individuelle, oft phantasievolle, im Detail } \\
\text { selbstbestimmte Weise ausgestaltet wird }\end{array}$ & „Isolde“ \\
\hline Orientierung B & $\begin{array}{l}\text { Ambivalente Orientierung an Selbstbestim- } \\
\text { mung und Autonomie in einzelnen Lebens- } \\
\text { bereichen bei gleichzeitigem Wunsch nach } \\
\text { Bezug von Unterstützungs- und Versor- } \\
\text { gungsleistungen durch das soziale Umfeld }\end{array}$ & „Martina“ \\
& $\begin{array}{l}\text { Starke Orientierung an Selbstbestimmung, } \\
\text { Partizipation und Normalisierung bei } \\
\text { gleichzeitigem Bedürfnis nach Erhalt eines } \\
\text { vertrauten Rahmens, um Konflikte und/oder } \\
\text { negative Erfahrungen innerhalb und } \\
\text { außerhalb dieses Kontextes zu vermeiden }\end{array}$ & \\
& $\begin{array}{l}\text { Starke Orientierung an Selbstbestimmung } \\
\text { und Autonomie, die in allen Lebensbereichen } \\
\text { umfassend aktiv, reflektiert und konsequent } \\
\text { angestrebt werden }\end{array}$ & \\
\hline Orientierung D & "Eva“ \\
& & \\
\hline
\end{tabular}

Tabelle 7: Die vier erhobenen Orientierungen junger Frauen mit Lernschwierigkeiten im Überblick

In den vier Fallrekonstruktionen werden spezifische Sichtweisen und Orientierungen der jungen Frauen mit Lernschwierigkeiten in den Blick genommen, es steht also die Frage, was die jeweilige Orientierung ausmacht, im Vordergrund. Als Ausgangsbasis für eine im Anschluss daran erfolgende soziogenetische Analyse werden Fragen, wie es zu den Orientierungen gekommen ist, bereits angedeutet.

\subsubsection{Fallrekonstruktion „Isolde“ (Orientierung A)}

Isolde repräsentiert die Orientierung am vorgegebenen, stark strukturierten, vertrauten Rahmen, der auf individuelle, oft phantasievolle, im Detail selbstbestimmte Weise ausgestaltet wird.

80 Der Begriff der Normalisierung ist im aktuellen pädagogischen Fachdiskurs umstritten. Er geht auf Nirje und Bank-Mikkelsen (1959) zurück und bedeutet, die Lebensbedingungen von Menschen mit Behinderung zu normalisieren, also solche Rahmenbedingungen zu gestalten und zur Verfügung zu stellen, die auch nicht behinderte Menschen in unserer Gesellschaft vorfinden (vgl. Thimm 2005: 8ff.). Ein in diesem Sinne erkennbares Streben nach solchen Rahmenbedingungen und damit verbundenen Lebensentwürfen ist in den Ausführungen der Frauen mit Lernschwierigkeiten in der vorliegenden Untersuchung deutlich wahrnehmbar und wird zum Teil auch explizit vorwiegend durch den Begriff „,normal“ zum Ausdruck gebracht. Aus diesem Grund wird er in der vorliegenden Arbeit in verschiedenen Zusammenhängen auch verwendet, allerdings unter Anführungszeichen gesetzt, um eine kritische Distanz zu gesellschaftlichen Normalitätskonstrukten zum Ausdruck zu bringen. 
Im Folgenden werden exemplarisch für diese Orientierung markante Eckpunkte aus dem Interview mit Isolde thematisiert, beschrieben und mittels Belegerzählungen, -beschreibungen, -argumentationen und -bewertungen von Isolde dokumentiert. Im Fokus dieser Betrachtungsweise stehen individuelle Orientierungen und Sichtweisen, die zentral für diese Orientierung sind.

\section{Äußerung von genereller Zufriedenheit}

Isoldes Ausführungen über das gesamte Interview dokumentieren die Äußerung von Zufriedenheit mit sämtlichen Lebensbereichen, Lebensphasen, Personen und institutionellen Zusammenhängen.

Für letztere zeichnet Isolde über mehrere Passagen ein Bild, in dem sie beschreibt, dass sie dort vor allem Spaß hatte:

I: „Und wie war es so im Kindergarten?

Isolde: Super, ( ) hat es mir super gefallen, und dann bin ich Kindergarten in M. gegangen, bin ich halt in M. gegangen. [...] War halt eine Gaude ${ }^{81}$ der Kindergarten." (II 8486)

Auch für die Schulzeit führt Isolde an, dass es ihr dort „super“ (II 124) gefallen habe:

I: „Und so die Schulzeit? Wie war die so?

Isolde: Super.

I: Was war da super?

Isolde: Ja, wir haben halt ab und $\mathrm{zu}$ in der Schulklasse gejausnet, also eine Jause und nach der Pause bin ich hinausgegangen zur Jause, ja. War wohl lustig. Ja gejausnet haben wir dann auch, also sind wir nach der Pause hinausgegangen. Vorher ( ) gegessen, haben wir geratscht, dann habe ich ein bisschen aufgeschrieben, meine Dreier als Krähe, dann habe ich noch Vögel gemacht - Krah. Dreier, Einser, Zweier, Dreier - eins, zwei, drei, vier, fünf, sechs, sieben, acht, neun, zehn. ( ) die Oma kann ja alles. Eine alte Frau kocht Rüben, eine alte Frau kocht ( ), schneidet sich den Finger weg. ( ) beigebracht, von der Schulzeit haben sie mir beigebracht wie sie dann - sind wir spazieren gegangen und dann habe ich immer auf den Vogel geschaut. Es war immer was anderes in der Luft, ja sind wir halt herumgegangen mit der Oma. Ja, mit der Oma spazieren gegangen ( ).“( (II 122-134)

Thematisiert werden Aktivitäten mit Mitschülerinnen und Mitschülern, die Isolde über ein „wir“ sprachlich einbringt. Es sei „wohl lustig“ (II 126) gewesen, führt Isolde an. Das ,wohl“ könnte semantisch darauf hinweisen, dass Isolde sich eigentlich gar nicht so gut erinnert, aber offenbar annimmt, es sei „wohl“ lustig gewesen. Jedenfalls scheint Isolde keine schlechten Erinnerungen zu haben bzw. solche auszublenden. Sie stellt die Kindergarten- und Schulzeit der Interviewerin gegenüber durchgängig als „super“ (II 84, 124) und „lustig“ (II 88, 126) dar und weist darauf hin, dass sie sich dort zurecht-

81 Gaude = Spass. In der Ergebnisdarstellung werden schwer verständliche Ausdrücke aus dem lokalen Dialekt erläutert. 
gefunden hat, also orientiert war: „Ja ich kenne mich aus, in der Schule habe ich mich ausgekannt" (II 98-99). Ein vertrauter Rahmen, der hier mit Isoldes Schule beschrieben wird, stellt die Grundlage für Zufriedenheit und Wohlgefühl für Isolde dar. Isolde erfährt über Alltagshandlungen wie Essen und gemeinsame Aktivitäten wie Spazierengehen mit Mitschülerinnen und Mitschülern, Lehrerinnen und Lehrern soziale Integration. Dieses partizipative Element wird sprachlich über die wiederholte (6x vgl. II 122-134) Verwendung des Wortes „wir“ in Zusammenhang mit verschiedenen Aktivitäten deutlich.

Isolde kommt auf Nachfrage in einer weiteren Passage (vgl. II 164-174) nochmals auf die Schule zu sprechen und schreibt hier den Lehrerinnen und Lehrern das Attribut „lustig“ (II 168) zu. Auch die Aktivität ,jausnen“ wird wieder als Fixpunkt für die Schule angeführt (hier 2x - vgl. II 173, 174 und bereits II 126) und verweist auf die große Bedeutung dieser Alltagshandlung als Moment, über das Isolde Partizipation und Integration im sozialen Kontext erfahren kann.

„Ja, also die Lehrer waren lustig, also ich war immer bei der Susanne drüben, bei der Susanne drüben und bei der Frau Huber, da habe ich immer ab und zu gejausnet. Und dann habe ich den Daniel - mit dem ( ) Daniel bin ich dann ein bisschen hinaus gegangen [...] Dann haben wir draußen gefrüh-, haben wir gegessen ( ) gesunde Jause und oben haben wir gekocht, im oberen Raum und meine damaligen Freunde sind auch herunter gekommen. Mit irgendwen Hand gehalten auf den Gang. Ich habe halt voll Liebeskummer gehabt dann immer, ja. ( ) Mädchenklasse und da drinnen war ja - haben wir einen Computer gehabt und da habe ich dann ( ) eingegeben und da haben wir immer so Zahlen gemacht, ja. ( ) sieben Zahlen, ja." (II 170-178)

Eingebettet in diese Passage spricht Isolde von einem Mitschüler, Daniel, mit dem sie ein bisschen ,hinausgegangen“ (II 173) sei. Sie habe auch „mit irgendwen Hand gehalten auf dem Gang“ (II 173-174) und „halt voll immer Liebeskummer gehabt dann immer, ja“ (II 174). Dies scheint neben der allgemeinen Bewertung der Schulzeit etwas zu sein, das für Isolde erinnerbar und erwähnenswert ist. Sie erinnert sich im Detail an den Namen eines Mitschülers, mit dem sie hinausgegangen ist, führt aber nicht den Namen des Burschen an, mit dem sie Händchen gehalten hat, hier ist recht unbestimmt von ,irgendwen“ (II 173) die Rede. Dass ihr Liebeskummer angeführt und mit ,voll“ (II 173) verstärkt wird, deutet ansatzweise auf dessen Intensität hin. Isolde geht aber nicht näher darauf ein, sondern lenkt mit ihrer Erzählung wieder in den für sie in ihrer aktuellen Perspektive offenbar bedeutsameren Orientierungsrahmen, wonach die Schulzeit vor allem „super“ und „lustig“ war.

Auch die Bedingungen des Aufwachsens zu Hause werden von Isolde als „super“ (II 333) eingeschätzt. Das Muster, dass Isolde auf eine Frage, wie ihr etwas gefällt, ,super" sagt, wiederholt sich also hier. Bereits im Zusammenhang mit der Bewertung der Kindergarten- und der Schulzeit ist dies der Fall 
(vgl. II 84, 124). Isolde zeigt sich generell mit ihren Lebensbedingungen zufrieden und nimmt diese rückblickend umfassend positiv wahr.

\section{Große Bedeutung des familiären sozialen Umfeldes für die Alltagsgestaltung}

In Isoldes Orientierung kommt den Personen aus dem familiären Umfeld ein hoher Stellenwert zu, was sich an ihrer bunten Beschreibung eines Ausschnittes daraus dokumentiert. Die Großmutter wird bereits in Isoldes Ausführungen über die Kindergartenzeit als Fixpunkt erwähnt. Sie regt Isolde an, ihr etwas über den Tag im Kindergarten zu erzählen und nimmt auch die Rolle der Vermittlerin zwischen Isolde und Personen aus ihrem sozialen Umfeld ein:

„Ja, ich bin halt in der Kindergruppe gesessen und die Oma hat dann daheim - die hat halt was heut - ( ) immer herumgefragt, was gibt es denn heute zum Essen? Ich habe ihr halt erzählt von den - heute gibt es ( ) und die Anni hat nicht gewusst was es ist, und die Oma hat es dann erklärt, was das ist. Meine Oma ja." (II 78-82)

Über das später im Interview erfolgte Ersuchen, etwas über ihre Großmutter zu erzählen, die Isolde im bisherigen Verlauf des Interviews bereits mehrmals erwähnte, geht sie näher darauf ein:

I: „Erzählen Sie mir noch ein bisschen was über die Oma.

Isolde: Ja, mit der Oma habe ich - die Oma hat ein Schlafzimmer, dann habe ich mit der Oma einmal fern geschaut, weißt eh so ( ) und ( ) immer die Puppe her und gesagt, was schaust denn so ( )? Die Puppen her, so Puppen abgeschmust, die Puppe, ja. Ein bisschen in Oma ihrem Zimmer gesessen. Unten haben wir eine Küche und im Fernsehzimmer haben wir dann auch so einen Fernseher und so einen Computer. Und wenn der Alfi will, gibt er mir auf Youtube was ein, tue ich selber eingeben. Mit der Mama tue ich dann zu die Weihnachten - sind wir immer bei der Mama drüben und tun immer zu die Weihnachten Lieder singen. Und ich singe immer die Drüber-Stimme, wenn ich Duett singe mit der Mama. Mit dem Papa - dann zeigt sie mir Foto von ihrer Hochzeit, aber der Papa hat Kopfweh gehabt, der hat viel Kopfweh ( ) ja, ja. Die Mama, ja -“ (II 151-160)

Für Isolde scheint die Großmutter eine wichtige Bezugsperson zu sein, die im Alltag zu Hause immer vor Ort ist, mit der sie fernsieht und im Zimmer sitzt. Auch weitere Personen aus dem nächsten sozialen Umfeld beschreibt sie als wichtige Fixpunkte für ihr Leben - den Bruder, der ihr etwas zeigt, und die Mutter, mit der sie singt. Der Vater, den Isolde mittels seiner Kopfschmerzen beschreibt, die offenbar für sie bei der Betrachtung seiner Person im Vordergrund stehen, scheint eher nicht erreichbar, er wird jedenfalls nicht in Interaktion mit Isolde sichtbar. Die Beschreibung des Familienlebens vermittelt insgesamt den Eindruck eines harmonischen sozialen Umfeldes, in dem sich Isolde wohl zu fühlen scheint.

Auch die Mutter wird von Isolde öfters erwähnt. Sie spricht diese erstmals im Rahmen von Ausführungen über ihre eigene Geburt an, stellt aber unmit- 
telbar darauf den genauen Zeitpunkt und die Umstände ihrer Geburt in den Fokus ihrer Beschreibung.

„Mama war schwanger und ich bin um elf in der Nacht auf die Welt gekommen, mit Sauerstoffmangel.“ (II 58-59)

Damit und in den weiteren Ausführungen zu ihrer frühen Kindheit überliefert Isolde über ihr im familiären Kontext vermittelte Erzählungen Familiengeschichte.

Die Mutter wird von Isolde vor allem im Zusammenhang mit ihrer eigenen Beeinträchtigung thematisiert und erscheint über den gesamten Verlauf des Interviews als die zentrale Person innerhalb der Familie, die Agenden im Zusammenhang mit Isoldes Beeinträchtigung übernommen hat und wahrnimmt. Sie bringt Isolde etwa das Gehen bei:

„Ja, ich war klein, ja ein, bin ein bisschen mit der - also bin auf Mama ihren Schoß halt gesessen und dann habe ich schon ein bisschen gehen können, hat ( ) mir ein bisschen gehen beigebracht. ( ) geschaut, wie ich gegangen bin, ja gegangen bin ich auch." (II 69-71)

Die Mutter ist innerhalb der Familie offenbar zentral für Isoldes Förderung, aber auch für die Rahmenbedingungen ihrer Alltagsgestaltung verantwortlich. So ist wiederholt davon die Rede, dass die Mutter Isolde abholt (vgl. II 108). Die Mutter holt Isolde vor allem täglich von der Tageswerkstätte ab:

„Genau, und dann wartet schon die Mama, [...] dann steige ich in Mama ihr lilanes Auto ein, zuerst hat sie ein helles Auto gehabt und jetzt ein lilanes, ja. Den Papa kenne ich auch.“( (II 313-315)

Für Isolde ist dieses Abgeholt-Werden und zurück in den Rahmen ihrer Familie Gebracht-Werden von großer Bedeutung. Über den unvermittelt angehängten Satz über ihren Vater verdichtet sich der zuvor (vgl. II 159) gewonnene Eindruck, dass er in Isoldes Leben eine weniger zentrale Rolle spielt als die Mutter.

Isolde thematisiert auch eher beiläufig, dass ihr die Mutter im Zusammenhang mit dem Kauf ihrer Puppe Paula Geld gegeben hat (vgl. II 186) und somit auch an der Ermöglichung des damit verbundenen Bedürfnisses von Isolde nach Ausgestaltung ihres Alltages durch eine individuelle Strategie beteiligt ist ${ }^{82}$.

Die Werkstätte, die Isolde tagsüber besucht, spielt in ihren Ausführungen gegenüber dem familiären Kontext eine eher unwesentliche Rolle.

82 Isolde sieht in ihren Puppen ihre eigenen Kinder und gestaltet mit diesem Konstrukt ihren Alltag aus. Siehe dazu ausführlich Unterpunkt „Konstruktion eines eigenen Rahmens als Bewältigungsstrategie für das Leben im weitgehend vorgegebenen Rahmen " in dieser Fallrekonstruktion. 
„Dann tun wir halt Mittagessen und dann ist es Mittagspause, dann stehen wir auf, dann tun wir ab und zu putzen und um vier ist Heimfahrt und dann Freitag sind die Arbeiten und um zwei ist dann Heimfahren, um zwei.“ (II 291-295)

Im Zusammenhang mit der Frage, was in der Tagesstätte gemacht wird, thematisiert Isolde schnell wieder das Heimfahren, was darauf hindeutet, wie wichtig dieses Daheim für Isolde als vertrauter und bevorzugter Ort ist. Dies verdeutlicht sich an der detailreichen Beschreibung des Heimfahrens an sich. Isolde führt den konkreten Ablauf des Abgeholt-Werdens von der Mutter an und beschreibt auch vertraute Fixpunkte wie das Haus eines Onkels, an dem sie bei der Heimfahrt vorbeikommt (vgl. II 306-331).

\section{Demonstration von Unbehagen und Unzufriedenheit über die Gefühls- und Verhaltensebene führt zu gewünschten Reaktionen im sozialen Umfeld}

Welch große Bedeutung das vertraute, familiäre Umfeld in Isoldes Leben spielt, wird auch über Erfahrungen im Zusammenhang mit einem Klinikaufenthalt zu Therapiezwecken sichtbar. Im Zusammenhang damit dokumentiert sich, dass Isolde durchaus auch mit den ihr verfügbaren Mitteln auf die Erfüllung eigener Bedürfnisse drängt, wenn diese sehr stark sind bzw. wenn die vorgegebenen Rahmenbedingungen ihr nicht entsprechen. Isolde beginnt von sich aus darüber zu berichten, dass sie wegen einer Therapie in einem anderen Bundesland war. Sie thematisiert starkes Heimweh und ihr eigenes Verhalten im Zusammenhang damit, welches offenbar so weit führte, dass diese abgebrochen wurde:

Isolde: ,Ja genau und da wollte ich dann - bei der Tamara bin ich gelegen und ich wollte nur heim, ich wollte nicht mehr ( ) und dann haben sie mich heim geschickt. Da hat es mir nicht gefallen nein.

I: Was hat Ihnen da nicht gefallen?

Isolde: Weiß ich nicht. Ich habe halt geweint, nur mehr geweint und ich will heim, und ich will heim. Dann haben sie mich heim geschickt.“ (II 270-275)

Über gehäufte Wiederholungen im Zusammenhang mit dem Wort „heim“ wird in und um diese Passage (7x vgl. II 270, 271, 2x274, 275, 277) der starke Bezug zu ihrem gewohnten sozialen Umfeld sprachlich sehr deutlich. Auch die Bewertung, dass es ihr außerhalb des vertrauten Kontextes ,nicht gefallen" habe, weist darauf hin und wird durch eine angefügte Verneinung verstärkt. Nochmals intensiviert wird dieser Eindruck über das Weinen als beschriebene, eigene Reaktion, die Isoldes starke Gefühle im Zusammenhang mit dem Heimweh zum Ausdruck bringt. Was genau ihr nicht gefallen hat, kann Isolde auf Nachfrage nicht sagen. Das Heimwollen an sich steht offenbar im Vordergrund und verstärkt den Eindruck, dass Isolde sehr an ihrem gewohnten sozialen Umfeld orientiert ist. Die Passage hat aufgrund der sprachlichen Gestaltung, vor allem der Wiederholungen von Wörtern, den 
Status einer Fokussierungsmetapher und weist auf die Bedeutung hin, die der vertraute familiäre Rahmen für Isolde hat.

Über dieses für Isolde einschneidende Erlebnis wird erstmals im Interview auch ersichtlich, dass Isolde eigenen Willen zeigen kann, der dann auch dazu führt, dass ihrem Bedürfnis nachgegangen wird. Vor allem ihre Gefühlsäußerung über das Weinen wird vom Umfeld auch wahrgenommen und führt somit zum Erfolg. Isolde demonstriert vorwiegend auf der Verhaltensebene Unbehagen und Unzufriedenheit, wenn ihr vorgegebene Rahmenbedingungen nicht entsprechen, und bewirkt eine ihren Bedürfnissen entsprechende Veränderung, hat damit also Erfolg.

\section{Einschneidende Erlebnisse verfestigen die Orientierung am vertrauten Rahmen}

Neben dem Klinikaufenthalt, der für Isolde ein einschneidendes Erlebnis darstellt und ihre Orientierung am Vertrauten verdeutlicht und diese auch verstärkt haben dürfte, wird eine weitere negative Erfahrung thematisiert:

„[...] im Dorfteich, ja da bin ich halt schwimmen ( ) Kopf halt hinter gestreckt und ( ) und ich bin voll hinter ( ) und ich bin durchgedreht. Dann haben sie Gott sei Dank mich herausgebrochen und dann bin ich ins Bett und dann haben sie angerufen wie es der Isolde geht. Und dann habe ich - habe ich glasrige Augen gehabt, liegen gehen, ja. Aber heute traue ich mich schon ins Wasser jetzt." (II 200-205)

Diese für Isolde offenbar traumatische Erfahrung dürfte mit ein Faktor sein, weshalb ihr der vertraute Rahmen so wichtig ist. Sie beschreibt zwar, dass sie sich „schon ins Wasser jetzt“ (II 205) traue. Daraus geht aber implizit hervor, dass Isolde offenbar lange Zeit danach Probleme damit hatte, wieder schwimmen zu gehen. Eine starke Orientierung an der Einbettung in ein Umfeld, das verhindern kann, dass Isolde wieder etwas passieren kann, ist vor dem Hintergrund dieser Erfahrung zu vermuten.

Erfüllung von Grundbedürfnissen und Alltagsgestaltung innerhalb des sozialen Umfeldes als wichtige Elemente der Orientierung

Im Kontext mit der Beschreibung von Isoldes Alltag in der Schule wurde deutlich, dass für sie soziale Integration vorwiegend über gemeinsame Alltags- und Freizeitgestaltung erfolgt. Brachte Isolde über ihre Beschreibung zum schulischen Alltag von sich aus wiederholt das gemeinsame Essen, von ihr vorwiegend als ,jausnen“ bezeichnet, als Fixpunkte ein (vgl. II 122, 123, $171,174,175)$, so dokumentiert sich auch über andere Kontexte sehr deutlich die Bedeutung von Aktivitäten im Zusammenhang mit der Erfüllung von Grundbedürfnissen. Auf die Frage, ob sie noch etwas Wichtiges aus ihrem Leben erzählen will, sagt Isolde , ich tue gern essen, also ab und zu Rindfleisch mit Krensauce und Erdäpfel“" (II 407) und führt weitere Gerichte an, 
die sie gerne isst. Dass auf die Frage, ob sie noch etwas Wichtiges aus ihrem Leben erzählen will, die Beschreibung eines Leibgerichtes und weiterer Lieblingsgerichte erfolgt, ist sehr aussagekräftig. Isolde zeigt sich damit sehr an der Erfüllung ihrer Grundbedürfnisse orientiert. Es gibt keinen Verweis auf Änderungswünsche: Isolde scheint mit dem, was sie hat und wie sie lebt, zufrieden.

Auch an anderer Stelle (vgl. II 347) wird die große Bedeutung von Essen im familiären Kontext deutlich. Isolde führt aus, dass und was sie gerne frühstücken würde und dass sie „daheim im Sommer“ immer grillen würden. Sie beschreibt sehr detailliert, was konkret gegrillt wird und stellt fest: „das liebe ich, da kann ich voll hineinhauen“ (II 348-349). Im Zusammenhang mit der Thematisierung ihres Interesses an Musik zeichnet Isolde als Rahmen dafür ein Szenario, in dem sich die Mutter daneben um Grundbedürfnisse kümmert:

Isolde: „Da kocht dann die Mama und mit der Anni suche ich dann, wenn der Alfi will, mir am Computer was heraus. Ab und zu Filme oder Stars. Die suche ich mir dann immer so heraus.

I: Die Stars, die ihnen gefallen.

Isolde: Genau, genau.

I: Ok, ja also das ist besonders wichtig, die Musik.

Isolde: Genau, genau, genau, genau. ( ) und der hat mir versprochen, wo wir überall auftreten werden. Natürlich bei „Starnacht“ haben wir dann Auftritt. Die Christine wird zuschauen kommen, wie ich mache, wie ich spiele. Ja, das wird lustig werden, ja." (II 413-421)

Über ihren Bruder hat Isolde auch Zugang zum Medium Computer und hat in diesem Rahmen Interesse für Musik entwickelt. Sie bestätigt auf Nachfrage sprachlich viermal, dass ihr Musik besonders wichtig ist. Sie beschäftigt sich damit vorwiegend gemeinsam mit dem Bruder, indem sie mit ihm gemeinsam Videos am Computer anschaut. Über ein von ihr beschriebenes Versprechen des Bruders phantasiert Isolde vom Auftritt mit einer Band, sieht sich also als aktiven Part in einem Rahmen, wo sie partizipiert und im Mittelpunkt steht (vgl. II 365-373).

Über Isoldes Ausführungen wird deutlich, dass Isoldes Freizeit vorwiegend mit Bezugspersonen innerhalb der Familie gestaltet wird. Fernsehen erfolgt vorwiegend mit der Großmutter und anderen weiblichen Bezugspersonen. Aktivitäten, die Isolde alleine ausführt, erfolgen entweder mit phantasierten Bezugspersonen (den Puppen $=$ Kindern) im vertrauten nahen sozialen Umfeld oder in der Phantasie gemeinsam mit dem Bruder (die Auftritte mit der Band). Auch in diesen phantasierten Rahmen verankert Isolde für sie vertraute Faktoren. Einerseits mit einer Frau namens Christine, die zuschauen kommt, eine bekannte Person. Andererseits Inhalte, die ihr bekannt sind, indem sie ausführt: 
„Dann tun wir halt proben, also ( ) und alle Lieder, was ich halt kann, weißt eh, genau. Und ab und $\mathrm{zu}$ - sie kommt ab und zu mit ( ) dann tun wir halt frühstücken.“ (II 397398)

Neben vertrauten Personen und Inhalten wird zudem wieder das für sie wichtige Essen in ihre Vorstellungen integriert. Auch daran dokumentiert sich wieder, dass Isolde offenbar auch in einem phantasierten außerhäuslichen Szenario etwas Vertrautes benötigt, das ihr Sicherheit und Orientierung bietet.

Konstruktion eines eigenen Rahmens als Bewältigungsstrategie für das Leben im weitgehend vorgegebenen Rahmen

Der vertraute familiäre Rahmen scheint Isoldes Bedürfnissen weitgehend gerecht zu werden. Über mehrere Passagen im Interview wird deutlich, dass sie in diesem eine phantasievolle Strategie für die Ausgestaltung ihres Alltages entwickelt hat. Diese scheint darauf ausgerichtet, Anteile auszuleben, die mit der von Isolde wahrgenommenen gesellschaftlichen Frauenrolle einhergehen und möglicherweise eigene Sehnsüchte und Wünsche tangieren. So kommt sie über die Frage nach der Freizeitgestaltung im Schulalter darauf zu sprechen, dass sie daheim mit ihrer "Tochter gespielt“ (II 180) habe. Auf Nachfrage der Interviewerin stellt sich heraus, dass es sich um eine Puppe handelt, der Isolde die Rolle ihrer Tochter zukommen lässt. Sie geht auch darauf ein, dass es eine männliche Puppe, den Bruder der weiblichen Puppe gibt. Die beiden Puppen stellen in Isoldes Beschreibung explizit ihre Kinder dar:

Isolde: „Ja, also daheim habe ich nachher ein bisschen mit meiner Tochter gespielt, mit der Paula.

I: Mit Ihrer Tochter?

Isolde: Ja.

I: Haben Sie eine Tochter?

Isolde: Ja, die Paula, meine Puppe.

I: Die Puppe.

Isolde: Ja, und die haben wir in K. - dann hat die Mama mir Geld gegeben ( ) bin ich voriges Jahr mit der Paula gekommen, die habe ich in F. gelassen. ( ) habe ich den Paul mitgenommen.

I: Wer ist der Paul?

Isolde: Der Bruder von der Paula.

I: Aha.

Isolde: Die zwei Kinder, die habe ich jetzt daheim.“(II 80-192)

Isolde führt auch an anderer Stelle nochmals aus, dass sie die Puppen Paula und Paul als ihre Kinder ansieht:

I: „O.k., können Sie mir noch etwas über die Paula und den Paul erzählen?

Isolde: Ja, mit der Paula spiele ich halt und mit dem Paul. Die Paula liegt im Bett bei mir und der Paul liegt auch im Bett bei mir, die drei Kinder was ich habe." (II 216-218) 
Es deutet sich an, dass Isolde für sich ein Konstrukt geschaffen hat, in dem sie als Mutter von zwei Kindern ${ }^{83}$, einem Bub und einem Mädchen, daheim lebt und mit diesen spielt. Isolde dürfte über diesen selbst konstruierten Rahmen ihr Bedürfnis nach Nähe und Beziehung ausleben und gestaltet damit auch ihre Freizeit. Im Interview entsteht über das mehrmals eingeworfene „weißt eh“ (u.a. II 152, 222) im Redefluss der Eindruck, dass Isolde eine Verbindung zur Interviewerin im Zusammenhang mit der Beschreibung ihrer konstruierten Mutterrolle herstellen will, also mit einer erwachsenen Frau auf Augenhöhe kommuniziert. Auf Ersuchen der Interviewerin führt Isolde aus, dass sie mit den beiden Puppen, die für sie Tochter und Sohn darstellen, spiele und auch mit dem Kinderwagen hinausfahre [,,hinausfahren auf die frische Luft, ein bisschen schaukeln" (II 346)]. Hier verdeutlicht sich die Bedeutung des Konstruktes, in dem sich Isolde als Mutter phantasiert, indem sie auf die Frage nach ihren Freizeitaktivitäten reale Unternehmungen mit der Puppe (,Tochter") anführt. Sie beschreibt auch eine Fahrt an einen anderen Ort, wo sie Paul mitgenommen hat, als Paula keine Zeit hatte. Mit dieser - so führt sie aus - besuchte sie einmal ein Konzert. Isolde vermischt in ihren Ausführungen reale und phantasierte Bezugspersonen. Es scheint so zu sein, dass die beiden Puppen für Isolde tatsächlich den Status von Kindern haben, mit denen sie etwas unternimmt. Im Zusammenhang mit sozialen Aktivitäten inszeniert sich Isolde selbst eher als Person, die mit den Kindern etwas unternimmt, denn als junge Frau, die altersgemäße Interessen hat. In ihren Erzählungen und Beschreibungen thematisiert Isolde auch Aktivitäten mit ihrer Schwester und führt Plüschtiere an, spricht also eher kindliche Aktivitäten und Interessen an:

„Und eine Puppe ist die Schmusekatze, ich weiß nicht, irgendwas Schmusekatze ( ) so ( ) und halt so Bücher, Aschenputtel-Bücher. Tue ich Aschenputtel lesen dann, und bei der Manu tue ich DVD's anschauen, entweder Aschenputtel oder Dornröschen, genau.“ (II 223-226)

Diese real eingenommene, eher kindliche Rolle wird in Ambivalenz zum phantasierten Rollenbild einer Mutter auch deutlich an Isoldes Lebensbedingungen. Sie erzählt über die konkrete Gestaltung ihrer Wohnsituation:

„Ich habe ein eigenes Kinderzimmer. Habe ich Opa sein Bett - wo ich klein war, bin ich immer angestoßen. Ist vom Opa das Bett ( ) mit meiner Tochter der Paula im Bett drinnen und die Mama schläft im Nebenzimmer mit dem Papa, Schlafzimmer.“ (II 375-377)

Aus dieser Beschreibung, in der Isolde sagt, sie habe „ein eigenes Kinderzimmer" (II 375), das neben dem Schlafzimmer der Eltern liege, wird deutlich, dass sie sich in gewisser Weise offenbar selbst den Status des Kindes zuweist. Die Erwähnung der Nähe zu den Eltern, die gleichsam über das

83 Isolde sagt in Zeile 218, sie hätte ,drei Kinder“, geht allerdings hier und im gesamten Verlauf des Interviews mit „Paul“ und „Paula“ nur auf zwei Puppen/Kinder konkret ein. 
Kinderzimmer wachen können, weist auf Isoldes Schutz- und Sicherheitsbedürfnis hin. Isolde gibt in keiner Weise zu erkennen, dass diese Nähe für sie störend ist, es macht eher den Anschein, dass sie diese Rolle als „Kind“, das von den Eltern im Blick gehalten wird, zur eigenen Sicherheit braucht. $\mathrm{Zu}-$ gleich wird in diesem Zusammenhang wieder die eigene phantasierte Mutterrolle angedeutet, indem Isolde erwähnt, dass sie mit ihrer „Tochter Paula“ in ihrem Bett schlafe.

Isoldes Bett wird nochmals, in Zusammenhang mit Postern, die sie darüber aufgehängt hat, erwähnt (vgl. II 231-244). Isolde kommt darauf zu sprechen, dass sie offenbar Fan einer Band ist. Sie beschreibt der Interviewerin, dass sie Poster der Band über ihrem Bett aufgeklebt habe und hängt sprachlich - wie bereits in der Beschreibung im Zusammenhang mit ihren phantasierten Kindern, die bei ihr im Bett schlafen - „weißt eh“ (II 231) an. Auch hier scheint sie wieder eine persönliche Verbindung zur Interviewerin herstellen zu wollen, dieser Eindruck entsteht jedenfalls durch die sprachliche Konstruktion. Interessant ist, dass es sich dabei jeweils um Szenarien im Zusammenhang mit Isoldes Bett handelt: dem Bett, in dem also Isoldes Kinder (Puppen) schlafen und über dem Poster ihrer eigenen Lieblingsband aufgehängt werden. Dieses erscheint dadurch gleichzeitig als Ort einer Jugendlichen, die Fan einer Band ist, und als Ort einer Mutter, deren Kinder hier schlafen. Hier wird der bereits sichtbar gewordene ambivalente Eindruck von Isolde noch deutlicher: selbst Tochter, Kind, Jugendliche zu sein, die im Haus der Eltern unter deren Obhut wohnt und sich Poster übers Bett hängt. Und auf der anderen Seite das Bild, das Isolde auch von sich zeichnet - als Mutter einer Tochter und eines Sohnes, um die sie sich kümmert. Sie hat sich im vorgegebenen, vertrauten Rahmen ihre eigene Welt konstruiert, in der sie sich als Mutter von zwei Kindern, einem Mädchen und einem Buben, wahrnimmt, mit denen sie auch Aktivitäten ausführt. Dass in Isoldes Ausführungen Puppen realen Stellenwert als Kinder zu haben scheinen, könnte darauf hinweisen, dass sich Isolde danach sehnt, selbst Kinder zu haben bzw. Mutter zu sein - im übertragenen Sinn, eine Frau zu verkörpern, die dem von ihr wahrgenommenen gesellschaftlichen Rollenbild entspricht. Isoldes Vorstellung der Rolle einer Mutter scheint von dieser versorgenden Ebene her traditionell. Dass Isolde allerdings keinen Vater in das Szenario phantasiert, ist stimmig mit dem entstandenen Eindruck (vgl. u.a. II 150-160), dass ihr eigener Vater in ihren beschriebenen familiären Interaktionen wenig Raum einnimmt.

Isoldes starke Orientierung an diesem Familienbild, das sie über ein eigenes Konstrukt für sich selbst als Rahmen für das Ausleben eigener Sehnsüchte danach geschaffen hat, dokumentiert sich auch über die Frage nach den Vorstellungen vom eigenen Leben bzw. nach dessen weiterem gewünschten Verlauf. Isolde hält gedanklich am aktuellen Zustand fest und möchte, dass 
alles so bleibt, wie es ist. Isolde baut ihr Konstrukt von sich als Mutter ihrer phantasierten Tochter in diesen Entwurf ein:

„Ja, also ein bisschen schaukeln, mit der Paula, ein bisschen Kinderwagen herum, anschnallen, wir fahren eine Runde. Ja ( ) singe ich dann immer im Duett. Gerne singen mit der Moni, genau. Mit der Sabine ( ). Ja, dann haben wir so einen Fernseher und ein Fernsehzimmer und oben bei der Oma haben wir auch einen Fernseher und da lässt Sie mich ab und zu was anschauen." (II 383-387)

Ihre Wünsche beinhalten also das Ausführen von Unternehmungen mit der phantasierten Tochter im Nahraum der Familie. Isolde sieht sich auch weiter singen und mit der Oma fernsehen, was sie im Verlauf des Interviews schon als zentrale soziale Aktivitäten für ihr aktuelles Leben beschrieben hat (vgl. II 150-160). Als Entwurf für ihr weiteres Leben zeichnet sie also ein Bild, in dem sie mit weiblichen Bezugspersonen für sie angenehme Freizeitbeschäftigungen ausübt (singen, fernsehen) und sich mit ihrer phantasierten Tochter beschäftigt. Der im Konstrukt vorhandene, phantasierte Sohn spielt in diesem Zukunftsentwurf eine ebenso eher nebensächliche Rolle wie Isoldes reale männliche Bezugspersonen. Über diese Vorstellungen zum weiteren Leben zeigt sich Isolde mit dem Status quo zufrieden und vermittelt den Eindruck, dass alles so bleiben soll, wie es derzeit ist. Veränderungen sind nicht im Bereich des Erwünschten oder Vorstellbaren. Das Idealbild einer Frau als Mutter wird in der Phantasie inszeniert und ausgelebt, spielt aber in Isoldes Wünschen und Bedürfnissen real keine Rolle. Dieser Eindruck verdichtet sich auch über eine Vignettenbeschreibung im Rahmen des Interviews:

I: „Also was sehen Sie auf dem Bild?

Isolde: Der Bräutigam und das ist die Braut. Da hat sie so einen Schleier und so einen

( ) und er hat so eine Krawatte an und so eine Jacke und den Ring.

I: Und, ja was machen die beiden da? Was sehen Sie da?

Isolde: Ja, die schauen sich an ( ). Also glücklich, ja glücklich.

I: Die sind glücklich die beiden? Und was machen die gerade?

Isolde: Die schauen sich an, ja. ( ) Die schaut ihn an, er schaut sie an.

I: Was glauben Sie denn, wie geht es den beiden?

Isolde: Gut, gut. Sind verheiratet, ein Ehering ( ) ja genau.

I: Und wie wird denen ihr Leben weitergehen? Was glauben Sie?

Isolde: Ja, ein bisschen spazieren gehen, ja genau ja.“ (II 468-478)

Isolde thematisiert anhand des von ihr ausgewählten Bildes den Bereich Partnerschaft, allerdings ohne einen Konnex zu eigenen Wünschen und Bedürfnissen herzustellen.

\subsubsection{Zusammenfassung der Fallrekonstruktion Orientierung A - „Isolde“}

Die in der Fallrekonstruktion „Isolde“ dargestellten Orientierungen dokumentieren eine als weitgehend zufrieden erscheinende Einrichtung in den vorgegebenen, vertrauten Rahmenbedingungen, die diesen Typus ausmachen. 
Im Detail macht eine selbstbestimmte, sehr individuelle, mitunter auch sehr phantasievolle Ausgestaltung der damit einhergehenden Alltagspraxis vorhandene Ressourcen im Sinne von Bewältigungsstrategien sichtbar. Kompakt dargestellt dokumentiert sich in:

Orientierung A:

Orientierung am vorgegebenen, stark strukturierten, vertrauten Rahmen, der auf individuelle, oft phantasievolle, im Detail selbstbestimmte Weise ausgestaltet wird

Zentral für diese Orientierung ist die hohe Bedeutung eines vertrauten sozialen Umfeldes als Rahmen für die eigene Lebensgestaltung. Dieser kann in familiären, aber auch in institutionellen Strukturen, wie etwa einer Wohneinrichtung, bestehen.

Handlungen im Kontext mit der Alltagsgestaltung stellen dabei wichtige Eckpunkte der Orientierung dar. Die Erfüllung von Grundbedürfnissen nimmt darin einen zentralen Stellenwert ein. Gemeinsame Fixpunkte im Alltag wie die Einnahme von Mahlzeiten oder in den vertrauten Rahmen eingebettete Freizeitaktivitäten sind von wesentlicher Bedeutung, da darüber soziale Interaktion und Partizipation mit dem und am näheren sozialen Umfeld erfolgen kann. Von großer Bedeutung sind in diesem Zusammenhang auch Bezugspersonen, die einerseits mit ihrer eigenen Lebensführung im Allgemeinen und andererseits mit ihrem Verhalten im Rahmen erlebter sozialer Interaktion im Besonderen Orientierungsmodelle darstellen. Vor allem auch über die eigenen Erfahrungen im Rahmen der Herkunftsfamilie und über Bezugspersonen im näheren sozialen Umfeld werden geschlechtsspezifische Rollenbilder und -verteilungen wahrgenommen. Im institutionellen wie im familiären Kontext sind es dabei nicht nur, aber doch vorwiegend Frauen wie die Mutter, die Großmutter, die Lehrerin oder die Mitarbeiterin in einer Institution, die die eigene Orientierung und damit verbunden die Alltagspraxis zentral beeinflussen. Die Mutter, die im Kontext der eigenen Sozialisation als Frau mit Lernschwierigkeiten häufig als zentrale Bezugsperson erlebt wird, die für die Ausgestaltung der familiären Rahmenbedingungen zuständig (gemacht wird und) ist, prägt in besonderer Weise das eigene Frauenbild. Eigene Impulse nach dem Ausleben der über ihre Alltagspraxis vorgelebten, als gesellschaftliche Normalität wahrgenommenen Frauenrolle, die selbst real nicht umgesetzt werden können, werden auf mitunter sehr phantasievolle Weise in der eigenen Alltagsgestaltung ausgelebt. So werden etwa Familienszenarien über die Gestaltung von Alltagshandlungen oder auch über Hilfsmittel konstruiert und spiegeln über deren individuelle Ausgestaltung internalisierte familiäre und gesellschaftliche Bilder wider. Dies wurde etwa am Beispiel der vorweg dargestellten Fallrekonstruktion über die Inszenierung der eigenen Person als Mutter von zwei phantasierten Kindern in Gestalt von Puppen sichtbar, über die die Mutterrolle auch mittels in den Alltag ein- 
gebundener realer Handlungen mit diesen sehr pointiert umgesetzt wird. Die Orientierung am vorgelebten Beispiel zeigt sich hier sehr deutlich, da in der eigenen Inszenierung dem Vater eine noch geringere Rolle zugeschrieben wird als im realen Alltag.

Im familiären oder institutionellen Kontext kann sich die Orientierung an weiblichen Bezugspersonen auch darin äußern, dass Tätigkeiten, die mit „Fürsorge“ in Zusammenhang gebracht und deren Übernahme vom sozialen Umfeld häufig auch erwartet und vorgegeben werden, oftmals zentrale Orientierungspunkte darstellen. So kommt etwa über die Übernahme von Haushaltstätigkeiten nicht nur das Bedürfnis nach Ausleben der dementsprechenden Rolle, sondern auch der Wunsch zum Ausdruck, Erwartungen von Personen aus dem sozialen Umfeld gerecht zu werden.

Dieses Streben ist eingebettet in das generelle Bedürfnis, den Erwartungen und Wünschen von Bezugspersonen zu entsprechen, um den vertrauten Rahmen erhalten zu können. Stimmig mit dieser Orientierung ist die Äußerung von weitgehender Zufriedenheit mit den vorgegebenen Rahmenbedingungen, die sich auf alle Lebensbereiche, Lebensphasen, Lebensbedingungen und Personen erstrecken kann. Dies setzt voraus, dass es innerhalb dieses Rahmens nicht zu massiven Einschränkungen eigenen Wohlbefindens kommt. In diesen Situationen wird Unbehagen, Unzufriedenheit und Kritik daran vorwiegend implizit über die Verhaltensebene oder erst auf Nachfrage zum Ausdruck gebracht. Werden in diesem Rahmen selbst darüber hinaus übergriffige Erfahrungen gemacht, so äußert sich dies häufig über körperliche Ausdrucksformen und in einem Verhalten, das von Rückzug geprägt ist. Negative Erfahrungen im gesellschaftlichen Kontext wie Abbrüche, Abwertung und traumatische Erfahrungen, insbesondere (sexuelle) Gewalt, verstärken diese Orientierung. Der vertraute Rahmen kommt dann dem Bedürfnis nach Sicherheit besonders entgegen und steht im Zentrum dieser Orientierung.

Auch Verhaltensweisen und Interessen von wichtigen Bezugspersonen dienen häufig als Maßstab für die eigene Alltagsgestaltung. Über gemeinsame Aktivitäten kann Beziehung mit diesen hergestellt und erlebt werden. In der dargestellten Fallrekonstruktion dokumentiert sich dies etwa über das gemeinsame Fernsehen mit der Großmutter oder die gemeinsame Beschäftigung mit Musik mit dem Bruder. Im vertrauten Rahmen werden auf der Grundlage gemeinsamer Erfahrungen entwickelte Interessen in der Folge oft individuell und differenziert ausgestaltet. In der damit einhergehenden Handlungspraxis wird gestalterische Tätigkeit und Selbstbestimmung sichtbar.

Im institutionellen Zusammenhang sind Freundschaften mit Peers besonders relevant. Konjunktive Erfahrungen und gemeinsame Lebensräume verbinden und bilden die Basis der damit einhergehenden Interaktionen und Aktivitäten. Geschwister stellen im familiären Rahmen wichtige Bezugspersonen dar, die auch Vorbildfunktion einnehmen können. Am Beispiel der Schwester wird ein weiteres Frauenbild neben dem der Mutter wahrgenom- 
men, das vor allem dazu anregt, das Thema Partnerschaft und Kinderwunsch zu bearbeiten. Peers und Geschwister im gleichen Haushalt nehmen über die gemeinsame Alltags- und Freizeitgestaltung eine zentrale Funktion ein, indem gemeinsam Beziehung erfahren und soziale Interaktion sowie Partizipation im vertrauten, überschaubaren Rahmen gelebt werden kann.

Materielle Rahmenbedingungen werden in dieser Orientierung vorwiegend über Fragen der Finanzierung alltäglicher Bedürfnisse thematisiert. Unzufriedenheit damit wird eher selten geäußert. Wenn dies der Fall ist, dann vorwiegend in Zusammenhang mit der geringfügigen Höhe des Taschengeldes bei Einbindung in eine Werkstätte für Menschen mit Behinderung. Besteht selbst dieser nicht, so ist für die Erfüllung auch geringster materieller Bedürfnisse die finanzielle Zuwendung der Herkunftsfamilie erforderlich. Diese ist im Alltag häufig an gemeinsame Aktivitäten gekoppelt, etwa darüber, dass Einkäufe mit den (finanzierenden) Bezugspersonen erfolgen. Damit haben diese auch Einfluss auf die Art der Aktivität bzw. auch auf die Frage, was konkret gemacht oder angeschafft wird.

\subsubsection{Fallübergreifende Ausprägungen von Orientierung A}

Der in der angeführten Fallrekonstruktion „Isolde“ anhand zentraler Dimensionen herausgearbeitete Orientierungsrahmen wird fallübergreifend in verschiedenen Ausprägungsvarianten sichtbar. Gemeinsamkeiten hinsichtlich der Bearbeitung verschiedener „Themen und Problemstellungen“ (Nohl 2012: 52), die sich in unterschiedlichen Fällen allerdings auch auf unterschiedliche Weise abbilden, werden deutlich. Damit dokumentiert sich auch, dass die einzelnen Typen nicht aus einem Fall abgeleitet werden, sondern sich in ihnen Orientierungen widerspiegeln, die mehreren Fällen zugrunde liegen.

Im Folgenden führe ich einige ausgewählte Beispiele dafür an, also weitere Belege, die zentrale Dimensionen der Orientierung deutlich machen. Die also die „Orientierung am vorgegebenen, stark strukturierten, vertrauten Rahmen, der auf individuelle, oft phantasievolle, im Detail selbstbestimmte Weise ausgestaltet wird" dokumentieren.

Die generelle Artikulation von Zufriedenheit dokumentiert sich beispielsweise auch in Glorias Orientierung. So äußert sie Zufriedenheit mit der Zeit im Kindergarten (vgl. IG 75) und in der Schule (vgl. IG 112). Lebensräume, Lebensumstände und Bezugspersonen werden durchgehend positiv bewertet. Besonders deutlich und umfassend dokumentiert sich Glorias geäußerte $\mathrm{Zu}$ friedenheit im Interview im Zusammenhang mit der Frage nach besonderen Wünschen, indem sie äußert ,ja, ich bin heute glücklich“ (IG 443) und „das habe ich gerne, ja“ (IG 449). Auch wenn Raum geboten wird, Wünsche zu artikulieren, wird also Zufriedenheit, sogar Glück im Zusammenhang mit der aktuellen Lebenssituation zum Ausdruck gebracht. Erst auf die nochmalige 
Frage, diesmal konkret nach einem bisher unerfüllten Wunsch, scheint Gloria zunächst lange überlegen zu müssen und sagt dann , ich weiß was“ (IG 453). So als sei sie froh, auf die Frage der Interviewerin etwas sagen zu können, diese also zufriedenzustellen. Der geäußerte Wunsch besteht dann darin, mit dem Vater in ein Volksmusik-Konzert zu gehen, einkaufen zu gehen und einen bestimmten Schauspieler kennen zu lernen. Eigene Bedürfnisse werden also erst auf Nachfrage geäußert, betreffen eher Freizeittätigkeiten und werden darum ergänzt, dass es um eine gemeinsame Aktivität mit dem Vater geht.

Auch Doris äußert Zufriedenheit mit den Rahmenbedingungen ihres Lebens, konkret mit ihrer Arbeit in einer Werkstätte. Sie beschreibt, dass sie an zwei Tagen im Küchenbereich mitarbeite, den Speisesaal aufräume oder in der Küche das Geschirr sauber mache. An den anderen beiden Tagen arbeite sie in einem anderen Bereich und helfe ,bei der Dekoration und bei die Perlen und bei sonst vieles" (ID 65-66). Auf Nachfrage, ob ihr ein Bereich besser gefalle, sagt Doris ,ich bin mit beiden zufrieden“ (ID 70). Sonja bezieht sich in ihren Ausführungen auf ihr Leben in einer Einrichtung für Menschen mit Behinderungen. Sie bringt über mehrere Passagen (vgl. u.a. IS 53-54) mittels derselben sprachlichen Konstruktion [,,ich bin froh, dass..."] ihre Zufriedenheit zum Teil über Vergleiche zu früheren Erfahrungen, aber auch über die Ausgestaltung der derzeitigen Lebenssituation zum Ausdruck. Karin äußert ebenfalls Zufriedenheit mit dem vorgegebenen Rahmen: „,da gefällt es mir, da komme ich nicht mehr weg, hat der Herr G. gesagt. Das beruhigt mich“ (IK 59-60).

Eine starke Orientierung an Bezugspersonen aus dem sozialen Umfeld wird etwa in der o.a. Passage deutlich, in der Gloria als großen Wunsch eine Aktivität mit dem Vater anführt. Auch bei Doris wird diese Orientierung über die Beschreibung ihrer Freizeitaktivitäten deutlich, die sie vorwiegend mit der Schwester und der Mutter ausführt. Zugleich äußert sie auch Zufriedenheit mit der gemeinsamen Gestaltung der Freizeit (vgl. ID 249-259). Auch die geäußerte Zufriedenheit mit dem gemeinsamen Wohnen mit der Schwester in einem Zimmer (vgl. ID 46) entspricht dieser Orientierung. Brigitte wiederum zeigt sich vollständig am Vater orientiert, mit dem sie alleine zusammenlebt. Auf die Frage nach besonderen Wünschen führt sie an, dass sie gerne schwimme, und ergänzt gleich darauf „mit dem Papa, ja“ (IB 321). Darin dokumentiert sich die starke Orientierung am Vater. Zudem erscheint Brigitte dadurch sehr anspruchslos, sie weiß zunächst gar nichts dazu zu sagen und bringt dann die Erfüllung eines eher kleinen, alltäglichen Wunsches ein. Für sie scheint es allerdings nicht selbstverständlich zu sein, dass dieser Wunsch erfüllt wird. Verdichtet wird ihre Orientierung am Vater über ihre Reaktion auf die Frage, wie sie sich ihr weiteres Leben vorstelle. Dazu sagt Brigitte: „Mein Leben ist der Papa“ (IB 336). Diese Formulierung erscheint im Zusammenhang mit Brigittes Ausführungen in den übrigen Passa- 
gen des Interviews als Fokussierungsmetapher. Hier bringt sie selbst auf den Punkt, was über ihre Beschreibungen, Erzählungen, Argumentierungen und Bewertungen teilweise explizit, zum Teil aber auch implizit zum Ausdruck gekommen ist: Alle ihre Handlungen sind auf den Vater ausgerichtet, sie selbst will seinen Bedürfnissen und Vorstellungen Genüge tun und strebt damit nach dessen Anerkennung. Eigene Bedürfnisse werden bei Brigitte auch auf die ganz konkrete, explizite Nachfrage danach - nicht expliziert bzw. erschöpfen sich im Ausführen dessen, was sie in der Freizeit gerne tue (,schwimmen“). Die Ausführung „mein Leben ist der Papa“ bringt implizit zum Ausdruck, dass Brigitte ihr eigenes Leben in äußerst engem Zusammenhang mit dem des Vaters sieht, und dokumentiert darüber beispielhaft sehr eindrücklich die diesem Typus entsprechende starke, zum Teil auch symbiotische Orientierung an Personen aus dem sozialen Umfeld.

In Brigittes Beispiel ist der Vater die einzige familiäre Bezugsperson, die Mutter ist nach der Trennung der Eltern abwesend. Gibt es im sozialen Umfeld Frauen, so bilden aber eher sie zentrale Bezugs- und Orientierungspunkte, vor allem im Rahmen der Alltagsgestaltung. Dies erfolgt in institutionellen Zusammenhängen über dort vorwiegend tätige Professionistinnen wie die Lehrerin oder die Betreuerin. Dies wurde in der vorab ausführlichen Fallrekonstruktion „Isolde“ deutlich, dokumentiert sich aber auch bei Sonja, die sich „mit der Bezugsbetreuerin so gut“ (ISo 317) versteht und auch in ihren Ausführungen zur Kindergarten- und Schulzeit weibliche Personen als besonders wichtig (vgl. ISo 117-119, 124) bewertet.

Die Bedeutung von Geschwistern für die eigene Alltagsgestaltung wird in vielfältigen Zusammenhängen vor allem in der Freizeitgestaltung deutlich. Doris beschreibt etwa zwei gemeinsame Einkäufe mit der Schwester ,um [...] ihre Pullovers auszusuchen" (ID 142). Sie thematisiert auch gemeinsame Kaffeehausbesuche (vgl. ID 249-259) mit den Eltern und der Schwester, welche dort vor allem Zeitung liest. Sind diese Aktivitäten mit der Schwester quantitativ auch eher gering, so prägen sie Doris Orientierung dennoch sehr stark. Bei Felicitas bekommen gemeinsame Aktivitäten mit ihrer Schwester den Stellenwert besonderer Ereignisse, wie etwa ein gemeinsamer Konzertbesuch in einer weiter entfernten Stadt (IF 440-44).

Sehr deutlich dokumentiert sich die Bedeutung von Geschwistern auch über eine Vignettenbeschreibung im Zuge des Interviews mit Gloria. Diese vergleicht ein Bild eines Mädchens und eines Burschen, die über eine Wiese laufen, mit der Beziehung zu ihrer eigenen Schwester und hält eingangs fest „die sind wie die Minni und ich“ (IG 628). Sie führt weiter aus:

„Ja, sag' ich, die schau'n fröhlich aus und glücklich, wie Geschwister. Die spielen mit einander, sind glücklich, die strecken die Hände aus, sie sind glücklich [...] tun gerne miteinander Rad fahren, Fußball spielen oder so, [...] das finde ich schön." (IG 629631) 
Die Bedeutung von Peers kommt besonders bei Frauen zum Ausdruck, die über die Beschäftigung in einer Werkstätte oder das Leben in einer Institution über entsprechende Kontakte verfügen. So erzählt Sonja, dass sie nach der Mittagspause mit einer Freundin Tischtennis spiele. Danach gehe sie zu einer Kollegin in die Weberei, wo es ihr sehr gut gefalle. Auch im Kontext mit den Beziehungen zu Peers im Rahmen des institutionell organisierten Lebens verwendet Sonja nun die bereits in anderen Kontexten gebrauchte Formulierung, indem sie festhält, ,ich bin froh, dass ich da bin“ (ISo 94). Soziale Eingebundenheit über Beziehungen mit Peers führt nicht nur bei Sonja, sondern auch bei Verena zu Zufriedenheit. Auch diese orientiert sich stark an Peers, sie stimmt ihre Freizeitgestaltung auf deren Interessen ab. Sie ist wie zwei weitere Freundinnen Fan einer Sängerin und sammelt gemeinsam mit diesen Bilder und Zeitungsausschnitte (IV 122-127). Im Zusammenhang damit äußert sie sich sehr positiv über die Beziehungen zu den Freundinnen und hält diese als ,echt wichtig“ (IV 126) für ihr eigenes Leben.

Das dieser Orientierung eigene Streben, den Ansprüchen von Personen aus dem sozialen Umfeld zu genügen, wird über die bereits angeführten Passagen aus dem Interview mit Brigitte deutlich. Sie will den Vorstellungen des Vaters aber nicht nur über ihre Handlungen, sondern auch über ihr Aussehen entsprechen. Brigitte führt aus, dass es ihr wichtig sei, ,fesch“" ${ }^{\prime 84}$ angezogen zu sein [,,immer fesch anziehen, ja“ (IB 280)]. Der Vater habe dazu, so Brigitte auf Nachfrage, einmal gesagt „Uh fesch, Brigitte“ (IB 282). Brigittes Wunsch, ,fesch“ (IB 280) angezogen sein, wird mit der Bestätigung durch den Vater in Zusammenhang gebracht, den Brigitte in ihrer Schilderung Anerkennung für ihr optisches Erscheinungsbild äußern lässt. Darüber dokumentiert sich, dass Brigitte sich sehr stark an der Bewertung durch den Vater orientiert.

Gloria macht bereits über ihre Eingangserzählung, in der sie ihr übermittelte Erzählungen über ihre eigene Geburt berichtet, deutlich, dass sie schon damals ein Verhalten an den Tag gelegt habe, das den Ansprüchen aus dem sozialen Umfeld entspricht. Sie sei bereits als Neugeborenes im Krankenhaus „ruhig“ und „brav“ gewesen, berichtet Gloria und deutet über die sprachliche Formulierung an, dass sie dem auch heute noch entspricht: „Ja, ich war einmal ruhig - ich war - ja brav bin ich" (IG 64). Auch im Kontext einer Beschreibung des schulischen Alltages bringt Gloria eine dementsprechende Selbstzuschreibung ein und äußert wieder, dass sie „ruhig“ (IG 103) war. Eine ähnliche Selbstzuschreibung erfolgt in Zusammenhang mit den Assoziationen zu einem ausgewählten Bild im Rahmen der Vignettenbeschreibung, wo Gloria beschreibt: „Ich war immer ruhig, ich war immer brav, ich war sehr freundlich“ (IG 759). In Glorias wiederholtes Hinweisen auf ihr „,braves" Verhalten im Interview spiegeln sich die von ihr wahrgenommenen

Fesch $=$ hübsch. 
Erwartungen an sie wider. Gloria reproduziert im beschriebenen Kontext das Bild, das Bezugspersonen von ihr zeichnen, und beschreibt somit das von außen an sie herangetragene, erwünschte Verhalten.

Eine selbstbestimmte, individuelle Gestaltung der vorgegebenen Strukturen zeigt sich auch bei Doris in einer starken Fokussierung auf bestimmte Freizeitinteressen, insbesondere bei der Beschäftigung mit seltenen Sprachen. Die Wichtigkeit dieses Bereiches kommt im Interview darüber zum Ausdruck, dass Doris das Thema aktiv einbringt, indem sie die Interviewerin fragt ,ehm, darf ich Ihnen noch etwas erzählen?“ (ID 370). Sie führt aus, dass sie sich „schon lange für drei verschiedene Fremdsprachen“ (ID 373) interessiere. Sie habe sich „schon oft mit Englisch beschäftigt“" (ID 374), habe mit einer Kollegin englische Spiele gespielt. Ihre anderen Fremdsprachen seien Italienisch und Französisch, ,weil diese Sprachen sich einfach hübsch anhören“ (ID 376). Sie führt in einer Passage mit Nachfragen dazu aus, dass sie Sprachen lerne, um einmal zu verreisen. Englisch sei die „zweitwichtigste Sprache, weil viele Leute in verschiedenen Nationen Englisch lernen und es gibt mehrere Gründe, warum ich Englisch lernen möchte“ (ID 394-395). Dazu führt sie aus, dass es sich ,ziemlich schön anhört“ (ID 395) und sie englische Musik interessanter als deutsche finde.

Bei Felicitas dokumentiert sich ihre gestalterische Rolle im familialen Alltag darüber, dass sie etwa versucht, diesen für sich und ihre Eltern am Wochenende durch eine unübliche Aktivität aufzulockern, indem sie den Eltern das Frühstück ans Bett bringt (vgl. IF 99). Diese Tätigkeit bereitet ihr große Freude. Gleichzeitig wird darüber aber auch der Wunsch sichtbar, die Eltern zufrieden zu stellen.

Einen ähnlichen Ausdruck, wie ausführlich an der Fallrekonstruktion „Isolde“ dokumentiert, erfährt die Ausgestaltung des Alltages über ein eigenes Konstrukt bei Ursula. Auch Ursula sieht ihre Puppen als ihre Töchter an [,,aber das ist keine Marionette, sondern meine lei-leibliche To-Tochter" (IU 143)], dies wird auf Nachfrage im Interview bestätigt [,ja, die ist meine Tochter“ (IU 151)] und als „meine leibliche Familie“ (IU 195) bezeichnet. Auf die Frage, was mit der Puppe geschehe, wenn sie ins Theater gehe, sagt Ursula: ,ja dann nehm' ich sie doch mit“ (IU167).

Sonja wiederum führt aus, dass eine ihrer Katzen ,wie ein Baby“ (ISo 421) sei. Aber, so Sonja: „Das braucht man nicht wickeln, das schreit nicht, das macht nur Miau“ (ISo 421-422). Man könne es nehmen, wann man es wolle. ,Ja, das ist mein Baby“ (ISo 423), führt Sonja aus.

Wie bei Isolde wird darüber auch bei Ursula und Sonja implizit eine Orientierung in Richtung des Auslebens als weiblich wahrgenommener Rollenbilder in der eigenen Alltagsgestaltung über individuelle Konstrukte sichtbar. Auch Ursula phantasiert sich demnach als Mutter kleiner Kinder und führt mit ihnen dieselben Tätigkeiten aus, die ihre eigene Mutter mit ihr selbst unternimmt. Über ihre Katzen kommt Sonja implizit auf ihre Einstellung zur 
eigenen möglichen Mutterschaft zu sprechen. Es wird deutlich, dass sie mit der Mutterschaft hohe Anforderungen verbindet, denen sie sich nicht gewachsen zu sein fühlt. Sie argumentiert dies v.a. mit den mangelnden finanziellen Ressourcen für die Ansprüche eines Babys, mit ihrer vielen Arbeit (vgl. So 417-419), aber auch darüber, welche Arbeit ein Baby im Vergleich zu einer Katze mache.

Bei Felicitas dokumentiert sich diese Orientierung an vorgelebten Rollenbildern über die Übernahme von versorgenden Tätigkeiten, wie etwa die Zubereitung des Frühstücks für die Eltern. Auch in ihrem Fall spiegelt dies von ihr real erlebte familiäre Abläufe wider. Sie erlebt die eigenen weiblichen Bezugspersonen wie die Mutter, die Schwester und die Großmutter als diejenigen, die für die Organisation des Haushaltes und der Verpflegung zuständig sind (vgl. IF 103-105, 456, 478-482). Felicitas orientiert sich an diesen vorgelebten traditionellen Rollenbildern und richtet ihre eigene Alltagspraxis im familiären Rahmen stark danach aus.

Brigitte wiederum orientiert sich generell sehr stark am Vater, der von der Mutter getrennt lebt. Sie übernimmt in gewisser Weise die Rolle der abwesenden Mutter und kümmert sich um den Haushalt, legt Wäsche zusammen und räumt die Zimmer auf. Dazu hält sie fest „Papa helfen, ja“ (IB 236) und äußert in diesem Zusammenhang zweimal „muss ich machen“ (IB 241, 243) und ,ich muss das, ja“ (IB 248). Sie orientiert sich damit an den Vorgaben des Vaters und erledigt auf dessen Aufforderung die Hausarbeit. In Kombination mit der Passage über die Trennung der Eltern und die Ausführung von Brigitte, sie würde nun mit dem Vater zusammenleben, scheint Brigitte in gewisser Weise den Platz der Mutter eingenommen zu haben, sie lebt mit dem Vater zusammen [,,ich lebe jetzt mit dem Papa zusammen“" (IB 176)] und führt den Haushalt.

Darüber wird deutlich, dass die Orientierung an einem traditionellen Frauenbild sowohl über vorgelebte Rollen als auch über verbal kommunizierte Erwartungen von Personen aus dem sozialen Umfeld geprägt sein kann. Felicitas identifiziert sich stark mit den vorgelebten Frauenbildern und bringt mit entsprechenden Alltagshandlungen selbstbestimmt Struktur in ihren Tagesablauf. Brigitte orientiert sich an den Vorstellungen ihres Vaters und übernimmt Haushaltstätigkeiten, um dessen Vorstellungen zu entsprechen und ihn damit zufrieden zu stellen.

\subsubsection{Fallrekonstruktion „Martina“ (Orientierung B)}

Martina repräsentiert die ambivalente Orientierung an Selbstbestimmung und Autonomie in einzelnen Lebensbereichen bei gleichzeitigem Wunsch nach Bezug von Unterstützungs- und Versorgungsleistungen durch das soziale Umfeld. 
Im Folgenden werden exemplarisch für diese Orientierung markante Eckpunkte aus dem Interview mit Martina thematisiert, beschrieben und mittels Belegerzählungen, -beschreibungen, -argumentationen und -bewertungen dokumentiert. Im Fokus dieser Betrachtungsweise stehen individuelle Orientierungen und Sichtweisen, die zentral für diese Orientierung sind.

\section{Ablehnung von Regeln und Vorschriften, die nicht den eigene Bedürfnissen und Voraussetzungen entsprechen}

Für Martina ist die Frage von Regeln und Vorschriften zentraler Bezugspunkt vieler ihrer Ausführungen. So streift sie diese Frage bereits in ihrer angeregten Eingangserzählung, wo sie Kindergarten- und Schulzeit thematisiert. Dabei erwähnt sie den Hort, den sie besuchte, und führt an zwei Beispielen an, was man dort machen „musste“ und „durfte“ (vgl. IM 56).

In einer späteren Passage dokumentiert Martina sprachlich und inhaltlich dicht in Form einer Fokussierungsmetapher, dass sie Regeln und Vorschriften in einer institutionellen Wohnform ablehnt. Als Grund dafür macht die deutlich, dass diese vor allem von der Struktur der Abläufe her nicht ihren persönlichen Bedürfnissen entsprechen würde. Diese von ihr vermuteten Bedingungen führt sie als Grund dafür an, warum sie nicht in eine Institution ging, in der sie eine Tätigkeit hätte ausführen können, die ihren Wünschen sehr entsprochen hätte:

M: ,Ja, aber das wollte ich nicht, weil wenn ich müde von der Arbeit nach Hause komme, dann möchte ich mich nicht an irgendwelche Regeln halten müssen. Sowie zum Beispiel, um halb sechs ist Abendessen, dann und dann musst du das machen, um die Zeit musst du das machen, dann und dann ist das. Wenn ich müde von der Arbeit heim komme, möchte ich einmal entspannen, das machen was ich will und nicht mich ständig nach irgendwelchen Vorschriften richten, wo dann vielleicht andere Leute dann auch noch sind, die was mir dann auf den Zeiger auch noch gehen, blöd kommen. Da habe ich lieber meine Ruhe und tue so, wie ich will, wenn ich von der Arbeit heim komme. Ich persönlich jetzt halt, aber anderen taugt das wahrscheinlich, wenn sie unter Leuten sind, ja aber für mich wäre es nichts.

$[\ldots]$

I: Und Sie glauben das wäre dort so gewesen.

M: Ja, ich schätze halt einmal schon, dass man sich da an gewisse Regeln halten muss, also weiß ich nicht. Um fünf kommt man, halb sechs Abendessen, dann und dann das, dann und dann das. Zum Beispiel eine Freundin, die bei mir im [...] gearbeitet hat, die wohnt da in [...] in einer WG und da gibt es auch Tage. Zum Beispiel Donnerstag müssen sie Bettzeug wechseln. An dem Tag müssen sie das machen, an dem Tag das, an dem Tag ist so ein Tag, und an dem Tag ist so ein Tag. Da war es aber egal, wenn sie am Donnerstag heim gekommen ist, müde von der Arbeit. Hat sie am Donnerstag Bettwäsche wechseln müssen, weil an dem Tag war das, obwohl sie müde von der Arbeit Heim gekommen ist, komplett. Also ich weiß nicht.“ (IM 289-314)

Martina will keinem starren Zeitplan folgen, der stark strukturiert ist und für bestimmte Zeiten bestimmte Tätigkeiten vorsieht. Sie orientiert sich in der 
Gestaltung der Tagestruktur lieber an der eigenen Befindlichkeit, wenn sie z.B. müde ist, will sie nicht putzen, nur weil das gerade auf dem Plan steht. Martina hat diesbezüglich keine eigenen Erfahrungen, aber ganz konkrete Vorstellungen dazu. Diese basieren u.a. auf Erfahrungen, die ihr eine Freundin erzählt hat bzw. die eines Bekannten. Es kommt auch zum Ausdruck, dass Martina nicht so sehr an sozialen Kontakten, an Gemeinschaft orientiert ist. Dies deutet sich über die Aussage an, es wäre für sie nicht angenehm, wenn ,dann vielleicht andere Leute dann auch noch sind, die was mir dann auf den Zeiger auch noch gehen, blöd kommen“ (IM 299). Da habe sie lieber ihre Ruhe und, so Martina, ,tue so was ich will“ (IM 300). Sie scheint weniger an Gemeinschaft und sozialem Austausch interessiert als an der Möglichkeit, sich zu entspannen und auszuruhen. Martina verweist damit darauf, dass sie auch gerne einmal alleine ist.

Martina lehnt nicht nur das Leben in einer Institution, sondern auch in einer kleineren Wohngemeinschaft $\mathrm{ab}$ und begründet dies wieder mit zeitlichen Vorgaben, feststehenden Plänen und macht dies an Haushaltstätigkeiten fest:

„Ja, sie müssen halt immer um sechs zurück sein. Jeder muss halt im Haushalt irgendwas tun. Da gibt es einen Plan und ein oder zweimal in der Woche kommt eine Betreuerin vorbei und schaut." (IM 324-326).

Martinas Ablehnung fremdbestimmter Regeln und Vorschriften dokumentiert sich auch über die Beschreibung ihrer eigenen Lebensbedingungen in einer eigenen kleinen Wohnung. Auf die Frage, wie der Haushalt so organisiert werde, sagt Martina:

„Haushalt ist eigentlich nicht so - weil ich nur alleine bin, das ist nicht so. Ich habe jetzt zum Beispiel nicht so einen Putzplan, an dem Tag mache ich das, mache ich das, mache ich das. Ich mache die Sachen halt so, wann Sie zu machen sind und wenn ich gerade Lust dazu habe, zu machen. Weil wenn ich jetzt einen Putzplan habe und ich zum Beispiel jetzt müde von der Arbeit heim komme und ja, schaue ich auf den Kalender, ja neunter ist Putztag, ja, wenn ich es auf dem Tag nicht mache, wenn ich müde bin, nur weil jetzt auf dem Plan der Tag steht, wo das zu machen ist - also ich mache es dann, wenn es notwendig ist und wenn ich gerade Lust dazu habe.“ (IM 478-485)

Martina verdeutlicht und verstärkt hier ihre in Passage IM 292-325 ausgeführte Fokussierungsmetapher zur Ablehnung von Strukturen, die fremdbestimmt sind. Sie macht über den implizit dazu gezogenen Vergleich deutlich, dass sie nicht nach starren Regeln und Plänen lebt, sondern Dinge dann macht, wann sie ihr notwendig erscheinen und wenn sie ,gerade Lust dazu“ (IM 485) hat. Für Martina stellt dabei das eigene Wohlbefinden den zentralen Maßstab für die Gestaltung der Rahmenbedingungen des eigenen Lebens dar. Über diese Orientierung daran und nicht an Vorgaben aus dem sozialen Umfeld oder an allgemeinen Vorstellungen darüber, wie etwas gemacht werden müsste, erscheint Martina in ihrer Orientierung sehr selbstbestimmt. Sie will sich generell keinen Regeln unterwerfen. 
Eine weitere Verstärkung dieser Orientierung wird in der Antwort auf die Frage, was ihr am Wohnen in einer eigenen, kleinen Wohnung gefalle, deutlich. Martina führt an, dass es keine Vorgaben durch andere Personen gibt:

M: „Kommt nicht jemand und sagt, tue einmal ein bisschen aufräumen oder- aber so arg waren meine Eltern gar nicht, dass sie so viel herumgemeckert haben, dass sie so-

I: Aber so im Vergleich jetzt zu dem was Sie meinen, wie es in einem Wohnhaus oder im betreuten Wohnen ist-

M: Nein, das nie freiwillig. Nein, nein, nein. Überhaupt nichts für mich.“ (IM 577-582)

Über die in der Passage sechsmal explizit über eine Verneinung angeführte Ablehnung und die Feststellung, sie würde sich solchen Rahmenbedingungen „nie freiwillig“ aussetzen, wird inhaltlich und sprachlich sehr dicht - wieder über eine Fokussierungsmetapher - Martinas negative Einstellung zu fremdbestimmten Rahmenbedingungen in einer Institution sichtbar.

Martina führt als Grund für die Präferenz einer eigenen Wohnung gleichsam zusammenfassend aus:

„Da kann man machen, was man will, in seiner eigenen Wohnung. Kann man so machen, wie es einem passt.“(IM 586-587)

\section{Orientierung an eigenen Interessen, Bedürfnissen und Präferenzen}

Es wird zunehmend deutlich, dass Martina sich nicht an Pläne, Vorschriften und vorgegebene Regeln halten will. Dies trifft insbesondere auf den Wohnund Freizeitbereich zu. Hier will sie ganz den eigenen Bedürfnissen und Befindlichkeiten gerecht werden und gestaltet diese Bereiche auch weitgehend danach. Sie orientiert sich dabei darüber hinaus an ihrem kleinen Hund, mit dem sie spazieren geht:

„Dann nach dem Essen, wenn ich heim komme, gehe ich so circa schnell eine kleine Runde, so zehn Minuten, dass er einmal hinaus kann. Dann tue ich für mich einmal eine Stunde fernsehen einmal, ein bisschen zum Runterkommen und dann, danach, gehe ich mit ihm circa ein halbe, dreiviertel Stunde, meistens dreiviertel Stunde mit ihm eine große Runde, ja. Das war es dann.“(IM 520-524)

Sie führt weitere Aktivitäten an und deutet dabei an, dass es auch einmal zu Langeweile bzw. zu Phasen komme, wo sie nicht wisse, was sie anfangen solle.

„Viele Sachen mit dem Hund mache ich, Radfahren tue ich viel und die restliche Zeit, wenn ich nicht weiß was anfangen, tue ich irgendwas herum ( ) aufräumen oder was gerade anfällt.“" (IM 527-528)

Dem Bedürfnis nach Entspannung und Ausruhen entspricht Martina, indem sie am Wochenende bis ,halb zwölf fernsehen und erst um halb zehn aufstehen, ja ausschlafen“ (IM 332-332) würde. Haushaltstätigkeiten erledigt Martina dann, wenn sie gerade nichts Besseres zu tun hat oder wenn ihr langweilig ist. 
Martina wird also dann aktiv, wenn sie selbst es möchte und für sinnvoll erachtet. Sie führt Tätigkeiten dann aus, wenn sie den eigenen Antrieb dazu verspürt. Die Vorteile autonomen Lebens liegen für Martina also insbesondere darin, den Alltag nach den eigenen Regeln gestalten zu können und sich nicht nach fremden Vorgaben richten zu müssen. Dies beinhaltet für sie zentral auch, nicht immer aktiv sein zu müssen, sondern auch, sich selbst ausgedehnte Phasen der Entspannung und Erholung zu gewähren.

Im beruflichen Bereich hat Martina ebenfalls deutliche Präferenzen, die sie in verschiedenen Zusammenhängen auch artikuliert. Sie bringt dies v.a. über nicht ihren Vorstellungen entsprechende Aspekte zum Ausdruck. Im Kontext der Thematisierung von Praktikumserfahrungen spricht sie etwa davon, dass der Servicebereich ihr nicht entsprach [„war nichts für mich“ (IM 357), „das war nicht meines“ (IM 359)].

Auch im Gespräch mit ihrer derzeitigen Vorgesetzten macht sie ihre Präferenzen bzw. die Ablehnung bestimmter Bereiche deutlich:

„Sie hat mich auch gefragt, was das Letzte wäre, was ich machen wollen würde, dann habe ich gesagt, Reinigung oder Zimmermädchen, das ist das Letzte was ich machen will, weil -“ (IM 421-422)

Dass Martina ihre Vorstellungen und Wünsche auch Autoritätspersonen gegenüber im beruflichen Kontext zum Ausdruck verleiht, verweist auf die starke Orientierung an den eigenen Wünschen, Vorstellungen und Präferenzen.

Auch hinsichtlich des Themas Urlaub macht Martina eigene Präferenzen deutlich:

„Ach, ich bin nicht so der Typ, der was weiß ich, bis zum anderen Ende der Welt fliegt. Ich bleibe lieber daheim, weil erstens Urlaub kostet viel - Hotel, Flug, das kostet alles viel und ich finde Österreich schön, ist schön. Da braucht man nicht weiß ich wo hin fliegen." (IM 623-625)

Martina gibt sich hinsichtlich der Frage von Urlaub sehr bescheiden. Sie argumentiert dies einerseits mit hohen Kosten, andererseits auch damit, dass es ihr in Österreich ohnehin gut gefalle. Aus ihrer Erzählung geht hervor, dass sie bislang keinen größeren Urlaub hatte, nur mit den Eltern zu Besuch bei ihrer Schwester in Österreich war. Martina verbindet also Urlaub nicht damit, weitere Reisen zu unternehmen, sondern im vertrauten Bereich zu bleiben. Möglicherweise auch, weil sie bislang keine diesbezüglichen Erfahrungen gemacht hat und dies in gewisser Weise außerhalb ihres Vorstellungsbereiches liegt. Da Martina über geringe finanzielle Mittel verfügt und zumindest teilweise auf die Unterstützung der Herkunftsfamilie angewiesen ist $^{85}$, ist das Thema wohl auch in ihrer Perspektive deshalb nicht relevant.

85 Siehe dazu ausführlicher Unterpunkt „Anbindung an die Herkunftsfamilie durch Rückgriff auf deren Versorgungsleistung“" in dieser Fallrekonstruktion. 


\section{Soziale Kontakte innerhalb der Familie und über den beruflichen Kontext}

Martinas soziale Kontakte außerhalb der Familie erfolgen vorwiegend über den beruflichen Kontext. Hier hat sie nach negativen Erfahrungen in der Schule auch positive Aspekte sozialer Gemeinschaft erlebt und „viele nette Leute" (IM 274) kennen gelernt.

Im Wohnort habe sie keine Freunde, stellt Martina fest (vgl. IM 566). Im Haus, in dem sie wohne, gebe es aus folgendem Grund kaum Kontakte:

„Ja, das sind Leute, die haben viele kleine Kinder. Die anderen, die gehen noch Schule, die sind nur 16, 17. Die haben auch ihre Freundeskreise, weil ich bin ja - kann man sagen, auch erst gerade neu dazu gezogen. Ich bin ja noch nicht so lange draußen, aber naja." (IM 570-573)

Freundinnen, die sie ,,ab und zu einmal“ (IM 560) sehen würde, habe sie über einen früheren beruflichen Kontext in einer $30 \mathrm{~km}$ entfernten Stadt. Um diese zu sehen, fahre sie dorthin. Sie würden dann in ein Einkaufszentrum gehen oder, so Martina, machen ,auf was man halt Lust hat“ (IM 564).

Über die spärliche Thematisierung und Beschreibung sozialer Kontakte außerhalb der Familie entsteht der Eindruck, dass Martina eher zurückgezogen lebt und sich ihre Beziehungen vorwiegend über die Familie und das berufliche Umfeld ergeben bzw. zusammensetzen. Außerhalb dieses Rahmens erfolgen Kontakte eher sporadisch: Freundinnen wohnen weit entfernt und werden nur selten besucht. Im Wohnhaus gibt es keine Kontakte, was Martina damit argumentiert, dass sie neu zugezogen ist. Neben diesen ungünstigen Rahmenbedingungen hat es auch den Anschein, dass Martina gar nicht so sehr an Kontakten interessiert ist. Auch die bisherigen Interpretationen lassen darauf schließen. So z.B. auch die Erfahrungen in der Schule und die Schlüsse, die sie daraus zieht. Auch die Überlegungen zum Wohnen in einer Institution lassen dies implizit vermuten. Das Wohnen in einer Institution lehnte Martina ja auch aus dem Grund ab, weil ihr andere ,,auf den Zeiger“ (IM 294) gehen könnten und sie lieber ihre Ruhe hätte (vgl. IM 295).

Diese Orientierung hinsichtlich sozialer Kontakte zu Gleichaltrigen wird aber anhand ihrer Assoziationen im Rahmen der über visuelle Vignetten stimulierten Ausführungen ergänzt und bekommt dadurch ambivalenten Charakter. Martina wählt zwei Bilder aus, über die sie Aspekte von Freundschaft thematisiert. Über ihre Ausführungen deutet sich an, dass Freundschaft sehr wohl ein Thema für Martina darstellt, das aber aufgrund negativer Erfahrungen und ungünstiger Rahmenbedingungen ambivalent besetzt ist.

Zum ersten Bild hält sie fest, dass dieses für sie ,gute Freundschaft, gute Freunde" (IM 733) darstellt, und führt weiter aus:

„Ja, weil meine Freunde halt hauptsächlich in M. sind, sehen wir uns nicht so oft, also eher mehr vom Telefonieren, also dass zu mir einer Heim kommt, das ist ein bisschen kompliziert, weil sie in M. wohnen, aber sonst - wo wir noch in M. unten waren, gleich 
gearbeitet haben, in der Pause haben wir auch ab und zu herumgeblödelt, so wie die zwei jetzt." (IM VA4 733-744)

Martina thematisiert, dass das Zusammenkommen mit Freunden kompliziert sei und deshalb selten zustande komme. Sie erinnert sich an Situationen im Zusammenhang mit der beruflichen Tätigkeit, wo sie mit Freunden Spaß hatte.

Auch über das zweite gewählte Bild kommt Martina schnell auf eine eigene Erfahrung mit Freundschaft zu sprechen, indem sie die Situation wie folgt beschreibt:

M: „Einer ist traurig und der andere gibt ihm was. Ich habe mit einer Freundin zum Beispiel eine Situation gehabt, da hat sie große Probleme gehabt und wollte nicht, dass das irgendwer erfährt und dann habe ich halt keinem was gesagt. Dann war sie mir aber sehr dankbar.

I: Das Bild erinnert Sie ein bisschen an die Situation so ein bisschen? [...]

M: Ja ich habe halt gesagt, ja das wird schon wieder, ich sage keinem was, du brauchst keine Angst haben, ja.“ (IM-VB2 781-789)

Sie beschreibt sich selbst als die Freundin unterstützend, indem sie sich als deren Vertrauen würdig erweist. Dies erscheint konträr zu Martinas Erfahrungen mit Freundschaft im schulischen Kontext und stellt somit wohl Martinas Idealbild von Freundschaft dar.

\section{Ermüdung und Stress durch normalisierte Arbeitsbedingungen}

Martinas Ruhebedürfnis kommt im Interview in verschiedenen Passagen und in unterschiedlichen Kontexten zur Sprache. So kommt wiederholt implizit zum Ausdruck, dass die weitgehend normalisierten Bedingungen, unter denen sie im Arbeitsprojekt tätig ist, sie ermüden. Dies zeichnete sich bereits in Passagen ab, wo Martina das Leben in einer Institution aus dem Grund ablehnte, weil Dinge dann erledigt werden müssen, wenn es vorgegeben ist, auch wenn es der eigenen Befindlichkeit - konkret viermal festgemacht an Müdigkeit (vgl. IM 289, 292, 312, 482), insbesondere nach der Arbeit - widerspricht. Auch der Faktor "Stress" wird von Martina argumentativ als Grund dafür angeführt, dass sie bestimmte Rahmenbedingungen ablehnt, auch wenn sie auf einer anderen Seite ihren Vorstellungen sehr entgegenkommen würden. Martina beschreibt beispielhaft dafür den Ablauf an der derzeitigen Arbeitsstelle und führt aus, dass sie um halb acht komme, die Sachen zusammenpacke, dann entweder zu Fuß oder wenn es weit weg ist mit dem Auto zu den Einsatzbereichen geführt werde, ,,irgendeine Stiegenhäuser putzen, herunter kehren, herunter wischen, solche Sachen" (IM 376377). Sie erzählt, dass sie auch in einem Büro sauber machen würde. Gefragt, ob ihr diese Tätigkeit gefällt, sagt Martina: ,sagen wir so, als Übergangslösung ist es o.k." (IM 390). Sie führt aus, sie würde lieber im Küchenbereich arbeiten. Momentan seien in diesem Bereich aber keine geeigneten Stellen 
frei. Die Stellen, die frei wären, kommen für Martina nicht in Frage, auch wenn sie mehr verdienen könnte:

„Da verdienst in den drei Monaten vielleicht schon ein paar Hunderter mehr, aber für drei Monate und da ist volle Stress die ganze Zeit. Du fängst um sieben Uhr an, bis ich weiß nicht, weit in die Nacht hinein und nach die drei Monate kannst du wieder gehen, weil die Saison vorbei ist." (IM 402-405)

Martina bringt darüber wieder zum Ausdruck, dass ihr das eigene Wohlbefinden wichtiger ist, als mehr zu verdienen oder unter Stress im eigentlich bevorzugten Arbeitsbereich tätig sein zu können. Die Orientierung daran nimmt in Martinas Leben einen zentralen Stellenwert ein. Martina entscheidet sich aus dem Grund für eine an sich weniger passende Variante, wenn sie weniger herausfordernd ist. Sie orientiert sich grundsätzlich an autonomem und selbstbestimmtem Leben und hat bestimmte Vorstellungen und Wünsche dazu. Es ist ihr z.B. klar, dass sie an einer Arbeitsstelle mit schlechteren Bedingungen mehr verdienen könnte. Sie wägt Vor- und Nachteile ab und entscheidet sich eher für eine Situation, die ihr mehr Lebensqualität ermöglicht, als für eine Situation, die mit diesbezüglichen Einbußen oder Herausforderungen verbunden wäre.

Auch über ihre Beschreibung, sie würde nach dem Heimkommen von der Arbeit erst einmal eine Stunde fernsehen, ,ein bisschen zum Runterkommen“ (IM 522), wird deutlich, dass die Arbeit im weitgehend normalisierten $\mathrm{Zu}-$ stand für Martina auch anstrengend und erschöpfend ist.

\section{Der Weg zum autonomen Wohnen auf Initiative der Eltern/Veränderung durch Anbahnung von außen}

Nachdem Martina mehrmals die Vorteile einer eigenen Wohnung in Gegenüberstellung zum Wohnen in einer Institution betont, beschreibt sie auf die Frage der Interviewerin, wie es für sie dazu gekommen sei:
„Derweil ich kleiner war, ist es noch gegangen. Aber dann ist halt mit dem Platz ein bisschen knapp geworden. Dann hat der Papa gesagt: komm, schauen wir um eine eige- ne Wohnung für dich, weil es ist schon der Platz knapp für so drei Personen auf 50 oder 55 Quadratmeter worden - für drei Leute ist ein bisschen knapp. Dann hat der Papa ge- sagt, schauen wir einmal nach einer Wohnung für dich, dann habe ich gesagt, ja können wir machen. Und dann haben wir eh ein Jahr warten müssen bis wir eine gekriegt haben und ja, jetzt..." (IM 447-453)

Es wird deutlich, dass es nicht Martina selbst war, die den Wechsel vom Wohnen bei den Eltern hin zum Wohnen in einer eigenen, kleinen Wohnung initiiert hat. Martina selbst führt zweimal an, dass der Vater diese Veränderung in die Wege geleitet hat (vgl. IM 449, 452). Das Wohnen in einer eigenen Wohnung wird von Martina als Folge des Umstandes beschrieben, dass die elterliche Wohnung für drei Personen zunehmend zu eng wurde. 
Ähnlich verhält es sich mit anderen Veränderungen in Martinas Leben, die auch von außen initiiert bzw. verursacht erscheinen. So beschreibt sie etwa, aus „Zufall“ (IM 256) zur Anlehre in einem Arbeitsprojekt gekommen zu sein:

„Ja, da war ich drei Jahre in der Anlehre, in der Wäscherei und Reinigung. Das war auch nur Zufall, weil ich habe mich mit der Mama damals probiert überall woanders anzumelden, aber sie haben dann überall gesagt, nein das ist ihnen zu gefährlich, wegen der Krankheit und so sind wir halt auf das gekommen. Wollte ich zwar am Anfang auch nicht, Wäscherei und Reinigung, aber weil nichts anderes gegangen ist, habe ich halt da angefangen, für drei Jahre.“ (IM 256-261)

Über die Beschreibung wird sichtbar, dass sie wegen ihrer Beeinträchtigung nicht an anderen Stellen, wo sie sich beworben hatte, aufgenommen wurde.

\section{Anpassung und Einrichtung in veränderten Rahmenbedingungen}

In verschiedenen Zusammenhängen deutet sich an, dass Martina sich an neue, auch nicht selbst gewählte Rahmenbedingungen gut anpassen kann und es sich darin so einrichtet, wie es ihr gefällt, wie es für sie sinnvoll und ihren Bedürfnissen entsprechend erscheint.

So beschreibt Martina, dass sie zunächst nicht in die mangels Alternative gewählte Anlehre wollte:

„Wollte ich zwar am Anfang auch nicht, Wäscherei und Reinigung, aber weil nichts anderes gegangen ist, habe ich halt da angefangen, für drei Jahre." (IM 259-261)

Auf Nachfrage bestätigt Martina, dass sie die Anlehre abgeschlossen habe. Die Zeit dort sei schön gewesen, sagt Martina und verstärkt „echt schön“ (IM 272). Es seien dort viele nette Leute gewesen, sie habe „viele Freunde gefunden, ja“ (IM 274). Die Betreuer seien auch ,alle nett" (IM 276) gewesen. Martina beschreibt verschiedene Haushalts- und Reinigungsarbeiten, die sie dort ausgeübt hat, und führt dazu weiter aus:

„Ja, am Anfang wollte ich überhaupt nicht, am Anfang hat es mir nicht so getaugt, aber mit der Zeit kommt man halt hinein. Mit der Zeit macht das einem nichts mehr aus, hat man sich daran gewöhnt.“ (IM 283-285)

Das Projekt, bei dem sie dann mangels Alternative landet, erscheint zunächst als Notlösung, weil sie wegen ihrer Beeinträchtigung an keiner anderen Stelle aufgenommen wurde. Nach anfänglichem Missfallen findet sich Martina in den neuen Rahmenbedingungen gut zurecht und findet auch Gefallen am sozialen Umfeld. Sie schließt die Anlehre in drei Jahren ab.

Auf den Vorschlag ihrer derzeitigen Chefin, - ,weil ich ordentlich bin“ (IM 413) - einmal eine Tätigkeit im Büro auszuprobieren oder in einem Geschäft etwas zu schlichten, reagiert Martina zweifelnd: „einschlichten - ich glaube zwar nicht, dass das meines ist, aber probieren werde ich es halt einmal"“ (IM 415-416). 
Auch in den veränderten, anfangs ungewohnten Rahmenbedingungen des Wohnens findet sich Martina nach anfänglicher Umgewöhnungs- und Eingewöhnungsphase schnell zurecht. So wohnt sie mittlerweile seit einem Jahr und vier Monaten, wie sie selbst ausführt (vgl. IM 455), in der eigenen Wohnung.

Als wichtigen Faktor hinsichtlich der Rahmenbedingungen, die sie für sich selbst als notwendig erachtet, um mit einer neuen Situation und damit einhergehenden neuen Anforderungen umgehen zu können, nennt Martina im Kontext mit einem abgebrochenen Praktikum ein ihren Bedürfnissen und Voraussetzungen entsprechendes Tempo:

M: ,Ja ein bisschen zu langsam war ich.

I: Aha.

M: Sie haben gesagt, sonst hat alles gut gepasst, nur halt mit der Zeit. Nur ich finde das halt blöd von ihnen, weil ich kann nicht die ersten zwei Tage so arbeiten, wie die arbeiten, mit der gleichen Geschwindigkeit. Das ist nur Zeitsache, finde ich. Geschwindigkeit, da kann man rein kommen, aber - wenn sie meinen...

I: Sie meinen, Sie wären da schon reingekommen mit der Zeit -

M: Ja, ja, schon.

I: Und hätten es dann sicher schneller machen können.

M: Ja, hätte ich mich schon gewöhnt, wäre halt nur eine Zeitfrage gewesen, aber -“ (IM 344-353)

Martinas Argumentation zeigt, dass sie für sich selbst das Etikett „zu langsam“ nicht annimmt. Sie spricht dem gegenüber ihre eigene Fähigkeit an, sich anzupassen und mit neuen Abläufen zurechtzukommen. Durch das Vorenthalten ihren Bedürfnissen entsprechender Rahmenbedingungen wird dies in Martinas Argumentation aber verunmöglicht. Die erforderliche Zeit, sich einzuarbeiten und von ihrem Arbeitstempo her anzupassen, wird nicht zur Verfügung gestellt.

Im Zusammenhang mit Veränderungen greift Martina auf die Unterstützung aus der Familie oder von Personen aus dem beruflichen Umfeld zurück.

\section{Anbindung an die Herkunftsfamilie durch Rückgriff auf deren Versorgungsleistung}

Vor allem über die Alltagsgestaltung im Zusammenhang mit dem selbständigen Wohnen ist Martina stark an die Herkunftsfamilie gebunden. Sie schätzt die Vorzüge des Wohnens in einer eigenen Wohnung, weil sie hier selbstbestimmt nach ihren Bedürfnissen und Befindlichkeiten agieren kann. Zugleich wird aber auch deutlich, dass für sie die Anbindung an die Herkunftsfamilie aus Gründen des Unterstützt- und Versorgt-Werdens gewünschte und gelebte Realität ist. Sie führt an, die Eltern würden sehr nahe bei ihr wohnen - ,wir sind nur eineinhalb Kilometer voneinander entfernt" (IM 461-462). Auf die Frage, wie das tägliche Essen für sie organisiert sei, sagt Martina ,ach, da fahre ich zu Mama“" (IM 489) und führt weiter aus: 
„Ja, weil mich die Mama abholt, hat Sie meistens was - fahre ich zuerst meistens zu meinen Eltern mit ihnen heim mit. Tue ich mit ihnen essen, dann führt Sie mich zu mir in meine Wohnung. Und Wäsche waschen tut auch Sie für mich derweil noch, weil für eine Person..." (IM 492-494)

Auf die Frage, was die Formulierung „,derweil ${ }^{\text {‘8 } 86}$ bedeute, präzisiert Martina: „Ja, derweil ich ja alleine lebe“ (IM 496). Am Wochenende fahre sie immer mit dem Fahrrad zu den Eltern. Frühstücken - so Martina - ,tue ich schon selber. Da gehe ich jeden Samstag einkaufen für mich, für mich alleine, ja, alles was ich brauche" (IM 501-502).

Martina greift in Angelegenheiten der alltäglichen Versorgung stark auf die Mutter zurück. Über verschiedene Ausführungen und sprachliche Formulierungen wird deutlich, dass es Martina bewusst ist, dass völlig autonomes Wohnen letztendlich einen Verzicht darauf bedeuten würde [,derweil noch“" (IM 494), „derweil“ (IM 496), „tue ich schon selber“ (IM 501)]. Bestimmte Tätigkeiten, die mit der Führung eines Haushaltes verbunden sind, wie das wöchentliche Einkaufen, erledigt sie selbst. Derzeit scheint es aber nicht in ihrem Interesse zu liegen, alle mit autonomem Wohnen einhergehenden Anforderungen selbständig zu erledigen. Martina lebt eigenen Vorstellungen entsprechend selbstbestimmt in einer gewissen Komfortzone, die die Versorgung durch die Mutter bietet, und erscheint dadurch ,teil-autonom“.

Martinas Lebensbedingungen haben sich ihrer Einschätzung nach durch den Auszug aus der elterlichen Wohnung und den Übertritt ins selbständige Wohnen „ein bisschen“ (IM 336) verändert. Sie vergleicht diese am Beispiel des Frühstücks, als sie bei den Eltern lebte und der aktuellen Situation:

„Weil damals, wo ich noch bei der Mama gelebt habe, hat sie mich aufgeweckt, ist sie
immer gekommen, mich aufwecken, hat sie für mich Frühstück gerichtet. Wenn ich
aufgestanden bin, war das Frühstück am Tisch, da habe ich mich nur mehr dazusetzen
brauchen. Ja, aber das war dann auch nicht mehr so - ein bisschen eine Umgewöhnung,
aber auch nicht so extrem schlimm. Jetzt muss ich halt ein paar Minuten früher aufste-
hen, weil Frühstück gerichtet ist nicht in zwei Minuten, da brauche ich schon ein biss-
chen länger, aber sonst ist eigentlich nicht so..." (IM 536-542)

Über die Formulierung, die „Umgewöhnung“ sei „nicht so extrem schlimm“ gewesen, dokumentiert sich gleichsam, dass es doch nicht so einfach für Martina gewesen sein dürfte, die Lebensbedingungen mit dem alltäglichen Versorgt-Werden aufzugeben. Teile davon sind über die auch aktuell noch stark ausgeprägte Anbindung an das Elternhaus, durch das tägliche AbgeholtWerden für das Essen bei den Eltern und weitere Unterstützungsleistungen der Herkunftsfamilie, insbesondere der Mutter, erhalten. So ermöglicht ihr dies auch die Haltung eines eigenen Hundes, zu dem Martina zunächst festhält: „Der ist auf mich angemeldet und der ist meiner“ (IM 507). Bei der Versorgung des Hundes spielt die Mutter wieder eine zentrale Rolle:

Derweil $=$ während 
„Ja, ich stehe um viertel sieben auf, dann gehe ich mit ihm eine kleine Runde, eine viertel Stunde, damit er einmal rauskommt. Ja, und um halb acht bin ich dann außer Haus und meine Mama kommt dann so circa um elf, halb zwölf zu mir hinaus, dann geht sie mit ihm eine große Runde, dann geht Sie so um halb eins und dann wartet er halt bis ich nach Hause komme bis halb fünf. Also vier, fünf Stunden alleine daheim bleiben, für ihn ist das gar kein Problem. Das wartet er schon." (IM 513-518)

Gefragt, ob es noch andere Unterschiede gebe, sagt sie ,eigentlich nicht so viel“" (IM 545). Über ihre folgende Ausführung, die Mutter habe sie schon immer lange schlafen lassen, wird sichtbar, dass Martina Abläufe offenbar auch selbst bestimmen konnte, als sie noch zu Hause wohnte.

Martinas Auszug von zu Hause ist mit ihrer Beschreibung der als angenehm beschriebenen Facetten des Versorgt-Werdens und der dort gegebenen Möglichkeit, selbst Abläufe zu bestimmen, auch mit Verlusterfahrungen verbunden. Martina ist sich bewusst, dass autonom zu leben auch bedeutet, gewisse Tätigkeiten in der Alltagsführung selbst wahrnehmen zu müssen. Sie nimmt weiterhin die versorgende Funktion der Mutter dort, wo es ihr angenehm ist, in Anspruch.

Hinsichtlich der finanziellen Ressourcen ist Martina, zumindest teilweise, auf die Unterstützung der Herkunftsfamilie angewiesen. Über eine Passage, in der sie beschreibt, wie die eigene Wohnung eingerichtet wurde, kommt Martina darauf zu sprechen, dass der Vater bislang zur Hälfte für ihre Lebenskosten aufkam:

„Die anderen Sachen habe ich immer mit dem Papa beim Zahlen geteilt. Er die Hälfte, ich die Hälfte. [...] Ja, Hälfte, Hälfte haben wir aufgeteilt.“ (IM 595-599)

Auf die Frage der Interviewerin „das heißt, sie haben ein eigenes Einkommen, oder?" sagt Martina ,ja, von da jetzt ja“ (IM 601). Sie beschreibt, dass sie nunmehr etwa 750 Euro selbst verdiene. Im Rahmen der Anlehre habe sie „wenig“ bekommen, „ein Taschengeld war das“, (IM 606) und führt aus, dass es im ersten Jahr der Anlehre 25, im zweiten 75 und im dritten 125 waren, und sagt „da hat mich der Papa noch unterstützt“ (IM 611).

Bei größeren Anschaffungen ist Martina weiterhin auf die Unterstützung aus der Familie angewiesen, etwa beim Kauf einer Couch für die eigene Wohnung (vgl. IM 593).

Starke Orientierung an weiblichen Bezugspersonen, insbesondere der Mutter

Die Rolle der Mutter in der Unterstützung Martinas hinsichtlich der alltäglichen Lebensgestaltung in einer eigenen kleinen Wohnung wurde im letzten Abschnitt sichtbar.

Die Orientierung an weiblichen Bezugspersonen, insbesondere der Mutter, wird über mehrere Passagen des Interviews deutlich und prägt Martinas Sozialisation. Dies deutet sich bereits in der angeregten Eingangserzählung des Interviews an. In dieser frühen Passage kommt Martina zunächst auf Kinder- 
garten und Schule zu sprechen und beschreibt diese kurz von den Rahmenbedingungen her. Sie hält fest, dass die Mutter sie bereits damals immer abgeholt habe und ihre Versorgung durch die Großmutter organsiert habe, wenn sie selbst verhindert war:

„,...] hat mich die Mama immer abgeholt. Ja und wo kein Kindergarten war, hat Sie mir halt offters zur Oma gegeben, hat die Oma auf mich aufgepasst.“ (IM 57-59)

Im Zusammenhang mit der Abwertung durch Mitschülerinnen und Mitschü$\operatorname{ler}^{87}$ in der Hauptschule vertraut sich Martina der Mutter an und bekommt von dieser emotionale Unterstützung. In diesem Zusammenhang erlebt sie diese aber als handlungsunfähig, indem sie sagt, sie hätten nichts unternehmen können, weil sich die Mitschüler ,nie ändern werden, wenn sie so teppert sind“"(IM 125-126).

Die Mutter unterstützt Martina, als sie wegen einer Krankheit im Zusammenhang mit ihrer Beeinträchtigung längere Zeit im Krankenhaus ist, indem sie sich darum bemüht, dass Martina den Lernstoff nachvollziehen kann:

„Die Mama hat wohl viel bei den Mitschülern gefragt, ob sie es von den Heften abkopieren kann, dass ich das abschreiben kann oder Hefte mitgebracht von den anderen.“ (IM 183-185)

Die Mutter ermöglicht es Martina über ihre Unterstützung auch erst, einen Hund halten zu können, was für sie selbst sehr wichtig ist (vgl. IM 642-644). Indem die Mutter sich um den Hund kümmert und mit ihm spazieren geht, macht sie es möglich, dass Martina trotz Arbeit einen eigenen Hund in der eigenen Wohnung halten kann. Sie schafft also Rahmenbedingungen dafür, dass Martina so leben kann, wie es für sie angenehm ist. Martina greift also nicht nur auf konkrete Versorgungsleistungen durch die Mutter zurück, sondern auch auf deren Unterstützung bei der Gestaltung von Rahmenbedingungen, die ihr entsprechen. Dies erscheint in Martinas Erzählung relativ selbstverständlich.

Negative Erfahrungen im Pflichtschulbereich prägen

Verhältnis zu Gleichaltrigen

Martinas Zeit in der Volksschule ist von umzugsbedingten zweimaligem Wechsel der Schule (vgl. IM 66-69) geprägt, was von ihr selbst auf Nachfrage als ,nicht so arg“ (IM 80) beschrieben wird.

Über Martinas Erzählung ihrer Zeit in der Hauptschule erweckt sie den Eindruck, dass dort lauter „Vollidioten“ (IM 102) und „Pfosten halt“ (IM 90) waren, dass sie geärgert hätten und dass auf sie losgegangen wurde. Sie argumentiert das damit:

Siehe dazu auch die Ausführungen im nächsten Unterpunkt. 
„Ja, weil wenn man da keine Freundin gehabt hat, sind alle auf einen losgegangen.

Wenn man da alleine war, dann hat es schlecht für einen ausgeschaut.“ (IM 97-98)

Sie hätte zwar eine Freundin gehabt, doch wenn diese krank war, hätten die Mitschüler „obercool mit ihrer Clique“ (IM 101) getan. Es sei auch „scheiße erzählt über einen" (IM 107) worden. Es wird deutlich, dass Martina auch Abwertung erfahren hat, zum Beispiel sei sie als ,fette Sau oder solche Sachen" (IM 109) bezeichnet worden. Martina findet selbst keine Begründung für diese Behandlung durch die Mitschülerinnen und Mitschüler:

„Ich weiß es nicht. Ich würde eigentlich keine Punkt finden, was ich da gemacht haben könnten, was ihnen nicht gepasst hätte, dass Sie einen Grund gehabt hätten auf mich loszugehen. Die sind halt so." (IM 113-115)

Sie weist darauf hin, dass sie nicht alleine davon betroffen gewesen sei, es sei auch auf ihre Freundin und ,zwei drei andere in der Klasse“ (IM 117) losgegangen worden. Damit verstärkt sie der Interviewerin gegenüber die Argumentation, die abwertenden Mitschülerinnen und Mitschüler seien ,Vollidioten“ und „Pfosten“ gewesen, es sei nicht an ihr gelegen. Martina spricht einen Zusammenhang mit möglicher Abwertung wegen ihrer Beeinträchtigung nicht an. Sie argumentiert demgegenüber sogar von einer Gegenposition aus, indem sie v.a. mit dem von ihr als Schimpfwort gebrauchten Begriff „Idioten" diese mit einem entsprechenden Status charakterisiert. Gerade über diese Argumentation ist davon auszugehen, dass Martina in der Hauptschule Abwertung wegen ihrer Beeinträchtigung erfahren hat.

Im Zusammenhang damit erhält Martina emotionale Unterstützung von der Mutter. Sie habe, so führt Martina aus, wenn sie heimgekommen sei, „viel der Mama erzählt und das hat dann auch gepasst“" (IM 123-124). Die Mutter bzw. die Eltern wurden diesbezüglich aber nicht tätig, führt Martina aus und findet eine Begründung dafür: Die Eltern hätten, so Martina, aber nichts unternehmen können, denn die Mitschülerinnen und Mitschüler würden sich nicht ändern, ,wenn sie so teppert sind“ (IM 125-126).

Martina geht auf Auswirkungen dieser Abwertung in der Schule nur auf Nachfrage und sehr knapp ein. Sie habe sich „blöd halt“ (IM 130) und „nicht so super" (IM 133) gefühlt, als sie so behandelt wurde.

Über ihre Ausführungen in der Schulzeit erscheint Martina selbst nicht als aktiv, sie wehrt sich nicht, sondern informiert die Mutter, die zwar als emotional unterstützend aber handlungsunfähig wahrgenommen wird.

Gleichaltrige werden in Martinas Erzählung von ihr sprachlich abgewertet, sie verwendet für sie Etiketten wie „Idioten“, „Pfosten“ und „teppert“. Die Verwendung entsprechender Termini als Schimpfworte ${ }^{88}$ ist dabei auffallend.

88 Erving Goffman beschreibt sogenannte „spezifische Stigmatermini“, die in der alltäglichen Kommunikation im abwertenden Sinne verwendet werden, und nennt dabei u.a. „Krüppel, Bastard, Schwachsinniger, Zigeuner als eine Quelle der Metapher und der Bildersprache“ (S. 14). Siehe dazu ausführlich Sigot, Marion (2006): Diskriminierung und Stigmatisierung 
Unbewusst könnte Martina eine Abgrenzung davon zum Ausdruck bringen wollen.

Martina ist aus den beschriebenen Erfahrungen der Abwertung heraus Gleichaltrigen gegenüber negativ eingestellt. Auch über die Ausführungen zur bereits erwähnten Schulfreundin wird dies deutlich. Diese habe sie, Martina, die ganze Zeit ausgenutzt. Sie sei deshalb für sie selbst eine „Notlösung“ (IM 136) gewesen. Die Freundin, so Martina, habe immer ,so teppert herumgetan“ (IM 136). Nach der Hauptschule sei sie froh gewesen, dass sie nichts mehr mit ihr zu tun hatte. Sie habe sie dann mit einer anderen Freundin ,als Retourkutsche“ "einmal so richtig verarscht [...] weil sie mich die ganze Zeit ausgenutzt hat" (IM 138-139). Sie habe auch den Kontakt abgebrochen, indem sie die Nummer geändert habe.

\section{Erfahrungen von Abbrüchen}

Martinas Sozialisation ist geprägt von verschiedenen Abbrüchen, die auf externe Umstände bzw. eigene gesundheitliche Voraussetzungen zurückzuführen sind und für sie massive Veränderungen mit sich brachten.

So kommt es zweimal aufgrund familiärer Veränderungen, nämlich in der Folge von Streit und Trennung der Eltern, zu einem Umzug, verbunden mit zweimaligem Schulwechsel und Wechsel des Hortes, in dem sich Martina wohl fühlte (vgl. IM 54-56).

„[...] dann sind wir umgezogen, weil sich Mama und Papa einmal - weiß nicht, wegen was gestritten haben. Dann haben Sie sich geschieden [...]“ (IM 71-73)

Auf Nachfrage, wie das Umziehen für sie war, sagt sie zunächst ,weiß ich nicht“ (IM 77), dann ,es war nicht so arg“" (IM 80). Martina beschreibt einen familiären Umstand, der für sie weitreichende Konsequenzen hatte, als relativ unbedeutend. In ihrer Erinnerung spielen die eigenen Gefühle dazu keine Rolle. Ihre Beschreibung der einschneidenden Ereignisse, insbesondere auch von Streit und der Trennung der Eltern, wird relativ emotionslos auf einer deskriptiven Ebene abgehandelt.

Eine weitere Abbrucherfahrung Martinas ist durch gesundheitliche Probleme bedingt. Sie erzählt, dass sie nach der Hauptschule eineinhalb Jahre in einer Fachschule in der Landeshauptstadt war, dann hätte sie aus gesundheitlichen Gründen aufhören müssen (vgl. IM 179). Auf das Ersuchen, etwas darüber zu erzählen, berichtet Martina, dass sie Krampfanfälle bekommen habe und ziemlich oft ins Krankenhaus musste, sodass sie mit dem Stoff „fast gar nicht mehr mitgekommen“ (IM 183) sei. Die Mutter habe sich zwar darum bemüht, dass sie Hefte zum Abschreiben bekam, aber als sie wieder in die Schule kam, waren - so Martina - ,alle schon weiß ich wie weit voraus

von Menschen mit Behinderungen. In: Kočnik, Ernst/Kaiser, Herbert/Sigot, Marion (Hrsg.): Stolpersteine auf dem Weg zur Gleichstellung. Klagenfurt, S. 19-48. 
und ich habe nie gewusst, wie man weiterkommt" (IM 188-189). Die Anfälle seien ganz plötzlich aufgetaucht, ,,von der einen Nacht auf die andere einmal“" (IM 194). Es sei ihr damit nicht gut gegangen, sodass sie die Schule, die sie gerne weiter besucht hätte, abbrechen musste. In der Schule habe es ihr deshalb gefallen, weil es eine Klasse gewesen sei, ,wo die Leute so halbwegs normal waren alle. Wo auch wirklich nette Leute dabei waren, ja“ (IM 204205). Über Inhalt und sprachliche Gestaltung wird Martinas Orientierung an „normalen“ Menschen sichtbar, die sie von den im Hauptschulkontext beschriebenen Mitschülerinnen und -schüler, die sie abwerten, ärgern und ausnutzen, implizit abgrenzt.

Die gesundheitlichen Probleme Martinas bewirken einen massiven Einschnitt in einer Phase ihrer Entwicklung, in der sich für sie positive Kontakte in der Fachschule angebahnt hatten. Mit dem Schulabbruch erfolgt auch der Abbruch dieser Kontakte. Anschließende Krankenhausaufenthalte bringen für Martina weitere negative Erfahrungen mit sich.

\section{Negative Erfahrungen durch gesundheitliche Probleme und häufige Krankenhausaufenthalte}

Im Zuge der mit ihren gesundheitlichen Problemen einhergehenden Krankenhausaufenthalte macht Martina massive, negativ behaftete Erfahrungen. Sie berichtet, dass sie nach Beginn der Krankheit immer wieder im Krankenhaus war und viele Medikamente ausprobieren musste, bis eines wirkte. Die Passage hat in ihrer sprachlichen Dichte den Charakter einer Fokussierungsmetapher, was darauf hinweist, wie prägend diese Phase für Martina war:

,[...] ich habe alle durchprobiert und erst bei den letzten zwei hat es dann etwas gebracht. Also habe ich was gehabt, bin ich zwei Wochen ins Krankenhaus, ausprobiert, getestet, wieder heim, dann wieder zwei Wochen später ins Krankenhaus, hinein, hinaus, hinein, hinaus, hinein, hinaus und das ist ein Jahr so gegangen.“ (IM 208-213)

Sie beschreibt diese Zeit allgemein als sehr ,anstrengend“ (IM 215). Zu den gesundheitlichen Problemen und den häufigen Krankenhausaufenthalten kommen weitere negative Erfahrungen in diesem Kontext. So beschreibt Martina auch, dass ihre Glaubwürdigkeit von einer Ärztin im Zusammenhang mit einem Anfall angezweifelt wurde:

„[...] also Untersuchung, nach dem Anfall hat mich die was gefragt und ich habe danach aber nicht reden können, weil alles verkrampft war und die hat mich angesprochen, hat mir Fragen gestellt. Ich habe aber nicht antworten können, und die hat gedacht, ich verarsche sie. Die hat mir das nachher hinterher nicht geglaubt." (IM 208231)

Auch der Tagesablauf im Krankenhaus wird von Martina als sehr negativ erlebt. Sie habe den ganzen Tag nicht gewusst, was sie anfangen solle. Ausschlafen habe sie auch nicht können, weil das Frühstück so früh kam und 
dann die Visite. Sie habe dann „X-tausend Beschäftigungen gesucht“ (IM 238-239), habe Musik gehört und Zeitschriften gelesen, denn ,,man hat irgendwie schauen müssen, dass man den Tag irgendwie herumkriegt" (IM 239-240). Sie habe „schon öfters“ (IM 244) Besuch bekommen, und zwar von „Schwester, Tante, Mama, Papa“ (IM 246).

\section{Zukunftsperspektiven und Wünsche ans Leben:}

Berufliche Perspektive im Fokus

Gefragt nach Wünschen und Vorstellungen für das eigene Leben, führt Martina aus:

„Ja, zuerst einmal in die Küche, Beruf. Beim Privatleben - Hund will ich auf jeden Fall wieder einen haben, weil meiner ist jetzt schon zehn - will ich auf jeden Fall noch einen zweiten haben, wenn der - weil ich finde, wenn man mit Hunden einmal angefangen hat, dann kann man sich ohne nicht mehr vorstellen. Ja, und Freund habe ich derweil noch keinen, aber ist auch nicht so wichtig derweil.“ (IM 641-645)

Momentan steht im Fokus von Martinas Orientierung die Erlangung eines Arbeitsplatzes im von ihr präferierten Küchenbereich. Der Wunsch, nach dem Ableben ihres Hundes wieder einen neuen zu bekommen, verweist auf dessen Bedeutung für Martina. Danach spricht Martina eher beiläufig die Frage einer möglichen Partnerschaft an. Über die Reihung und ihre sprachliche Formulierung entsteht der Eindruck, dass Martina sich dessen bewusst $\mathrm{zu}$ sein scheint, dass auf diese Frage von anderen Frauen der Wunsch nach Partnerschaft oder ein eventueller Kinderwunsch thematisiert werden bzw. möglicherweise im Interview auch erwartet werden könnte. Sie greift dies in ihren Ausführungen auf, verweist aber darauf, dass diese Frage für sie aktuell nicht so wichtig ist.

\section{Ablehnung bis geringe Bedeutung von Partnerschaft und Kinderwunsch}

Die schwach wahrnehmbare Orientierung dokumentiert sich im gesamten Interview darüber, dass Fragen in Zusammenhang mit Partnerschaft und Kinderwunsch kaum thematisiert werden. Bei der Frage nach den Wünschen ans Leben erfolgt der Verweis darauf, dass eine Partnerschaft derzeit nicht aktuell und wichtig erscheint. Auch die Frage eigener Mutterschaft scheint aktuell eher geringe Bedeutung für Martina zu haben. Über eine Erzählung im Zusammenhang mit einem Urlaub bei der Schwester hält sie fest, dass diese ,auch schon eine Kleine“ (IM 630) habe. Auch über die Schwester, die in der Nähe wohne, führt Martina aus, diese habe ,auch einen Kleinen“" (IM 632), der werde bald vier Jahre alt. Offenbar fällt dies Martina sofort zu den Schwestern ein, auch wenn die Frage eigentlich nach ihren Urlaubsaktivitäten ausgerichtet war. Das lässt darauf schließen, dass das Thema eine gewisse 
Bedeutung für Martina haben dürfte, es wird jedoch nicht explizit ausgesprochen und an keiner anderen Stelle thematisiert.

An einer späteren Stelle im Interview deutet sich sogar eine momentan eher negative Haltung zur Frage einer möglichen Partnerschaft an. Im $\mathrm{Zu}-$ sammenhang mit der Auswahl von Bildern, die im Kontext des Interviews als visuelle Vignetten Erzählungen zu Fragestellungen anregen sollen, erscheint im Interview mit Martina die Frage, welche Bilder nicht ausgewählt wurden bzw. dass Bilder, die nicht ausgewählt wurden, in besonderer Art und Weise kommentiert wurden, bedeutsam. Martina betrachtet zwei Bilder, die jeweils einen Mann und eine Frau darstellen, die ein Pärchen sein könnten:

M: „Das muss nicht, ich weiß nicht ob ich das unbedingt will.

I: Irgendein Bild das Sie anspricht. Oder, dass Sie sagen ein Bild, was mir überhaupt nicht gefällt, können wir auch sagen.

M: Die sind alle nicht schön irgendwie. Nur das, das muss nicht unbedingt sein.

I: Das muss nicht sein.

M: Ist nicht das Wichtigste im Leben sage ich halt einmal. Wenn man den Passenden gefunden hat, ist schon schön, aber nicht unbedingt das Wichtigste." (IM 641-645 zu VA3 und VA4)

Martina lehnt an dieser Stelle Partnerschaft sogar eher ab bzw. äußert Zweifel, ob sie „das unbedingt will“ (IM 645). Sehr ablehnend [„Nur das, das muss nicht unbedingt sein" (IM 642)] reagiert sie auf ein Bild, das ein Pärchen zeigt, bei dem Mann und Frau offensichtlich beeinträchtigt sind. Hier spiegelt sich in Martinas Ausführung möglicherweise von ihr wahrgenommene und übernommene gesellschaftliche Ablehnung von Partnerschaft und Sexualität von Menschen mit Lernschwierigkeiten wider. Im Kontext des gesamten Interviews ist aber eher davon auszugehen, dass sich Martina selbst nicht diesem Personenkreis zugehörig sieht.

\subsubsection{Zusammenfassung der Fallrekonstruktion Orientierung B - „Martina“"}

Die in der Fallrekonstruktion „Martina“ dargestellten Herangehens- und Sichtweisen verweisen auf eine Orientierung, die vom Wunsch nach Selbstbestimmung und Autonomie geprägt ist, was sich vorrangig an der Ablehnung fremdbestimmter Strukturen als Basis der eigenen Lebensführung dokumentiert. Daneben wird eine starke Orientierung am vertrauten Rahmen, der über Versorgungsleistungen eigenes Wohlbefinden sichern soll, sichtbar. In dieser Dichotomie erscheint die Orientierung ambivalent. Kompakt dargestellt dokumentiert sich in: 
Orientierung B

Ambivalente Orientierung an Selbstbestimmung und Autonomie in einzelnen Lebensbereichen bei gleichzeitigem Wunsch nach Bezug von Unterstützungs- und Versorgungsleistungen durch das soziale Umfeld

Diese Orientierung ist gekennzeichnet von einem ambivalenten Zugang zu gesellschaftlichen, familiären und institutionellen Strukturen und Rahmenbedingungen. Dies äußert sich darin, dass Selbstbestimmung, Autonomie und Normalisierung einerseits und der Bezug bzw. Erhalt von Versorgungsleistungen durch das soziale Umfeld andererseits nebeneinander im Fokus gehalten werden.

Innerhalb vorgegebener Strukturen werden starre, von anderen Personen aufgestellte Regeln und Vorschriften vehement abgelehnt. Der Rahmen soll selbstbestimmt ausgestaltet sein, sodass er den eigenen Bedürfnissen, Interessen und Präferenzen entspricht. Die Vorteile eines so strukturierten, weitgehend vorgegebenen, den eigenen Bedürfnissen aber entsprechenden Rahmens führen zum Wunsch, diesen zu erhalten. Schritte und Initiativen in Richtung Autonomie sind vor diesem Hintergrund von Ambivalenz geprägt. Sie werden weniger aktiv gesetzt als vom sozialen Umfeld initiiert und mit vollzogen. Die Anpassung und Einrichtung in damit einhergehenden veränderten Rahmenbedingungen erfolgt entsprechend der vorliegenden Orientierung so, dass sie weitgehend an den widersprüchlichen eigenen Bedürfnissen ausgerichtet sind. In der beispielhaft dargestellten Fallrekonstruktion dokumentiert sich dies am Wunsch nach Autonomie und Selbstbestimmung im beruflichen Bereich und im Wohnen bei weiterhin erwünschten und erbrachten Versorgungs- und Unterstützungsleistungen im Alltag durch die Herkunftsfamilie. Diese ermöglicht durch die Bereitstellung von bedürfnisadäquaten Strukturen Lebensbedingungen, die eigenes Wohlbefinden sichern. Im Fallbeispiel dokumentiert sich auch, dass Herausforderungen, die mit normalisierten Lebensbedingungen einhergehen, auch als Überforderung erlebt werden können. Einschränkungen, die sich aus der Behinderung ergeben, und vorliegende Erkrankungen können Gründe dafür darstellen. Im Kontext weitgehend normalisierter Arbeitsbedingungen kann dies auch als Ermüdung oder Stress wahrgenommen werden und zum Bedürfnis führen, in anderen Lebensbereichen über Versorgungsleistungen durch die Herkunftsfamilie entlastet zu werden. Dabei wird eine starke Orientierung an weiblichen Bezugspersonen, insbesondere der Mutter, sichtbar, die im bisherigen Sozialisationsverlauf und auch aktuell in vielfältigen Zusammenhängen diese Grundbedürfnisse und Interessen durch Ausgestaltung entsprechender Rahmenbedingungen im Fokus hat.

Eine ambivalente Ausgestaltung der Rahmenbedingungen innerhalb der Familie oder in Institutionen kann die Ausprägung dieser Orientierung begünstigen. So etwa, wenn im Alltag einerseits selbstbestimmte Entscheidungen gefördert und unterstützt werden. Andererseits aber der Aspekt der For- 
derung weniger stark ausgeprägt ist und dementsprechend Möglichkeiten, die auch Anstrengung erfordern oder die Möglichkeit des Scheiterns beinhalten würden, vermieden werden. So werden z.B. Versuche eines Praktikums schnell abgebrochen, wenn damit Anforderungen verbunden sind, die Entwicklungsschritte voraussetzen oder initiieren würden, die fordernd oder anspruchsvoll erscheinen und vertraute Strukturen und Abläufe beeinflussen oder verändern würden. Die Rolle der Herkunftsfamilie erscheint also ambivalent: Sie unterstützt einerseits Schritte in Richtung Autonomie, verzögert diese anderseits durch die Anbindung an den vertrauten Rahmen über umfassende Versorgungsleistungen. Dementsprechend wird eher bewahrend und betreuend agiert. Entwicklungsmöglichkeiten durch Anforderungen, die sich aus neuen Herausforderungen ergeben könnten, werden über eine solche Struktur eher zögerlich wahrgenommen.

Von der Genese her führen auch negativ erlebte Erfahrungen in vorwiegend außerfamiliären Kontexten zu dieser ambivalenten Orientierung. So können etwa das Erleben von Abwertung und Mobbing in verschiedenen Sozialisationsinstanzen, vorwiegend im Schulbereich wirksam werden. Auch Erfahrungen von Abbrüchen, etwa durch Umzug oder Krankheit bedingter Schulwechsel, sind mögliche soziogenetische Faktoren für die Ausbildung dieser Orientierung. Der dadurch erfolgten fremdbestimmten Veränderung steht dabei das nicht erfüllte Bedürfnis nach Erhalt des vertrauten Rahmens gegenüber und manifestiert sich in einer generellen Orientierung, in welcher auf neuerliche Veränderungen zögerlich reagiert wird. Im aktuellen Alltag kommt diese Erfahrung durch nunmehrige starke Ablehnung von Fremdbestimmung durch Regeln und Vorschriften zum Ausdruck.

Auch Beziehungen und soziale Kontakte außerhalb der Familie sind von Ambivalenz geprägt. Sie entstehen vorwiegend über institutionelle oder berufliche Zusammenhänge. Ausgrenzung und Mobbing durch Gleichaltrige vor allem im schulischen Kontext wirken sich auf spätere Beziehungen in vielfältiger Weise aus. Sie können dazu führen, dass in der aktuellen Lebenssituation Freundschaften ein eher geringer oder ambivalent zum Ausdruck gebrachter Stellenwert zugewiesen wird. In der vorab dargestellten Fallrekonstruktion wurde eine vordergründig sehr geringe Orientierung an Peers dokumentiert. Dahinter verborgen wurde aber der Wunsch nach gelungenen sozialen Beziehungen, insbesondere im Rahmen von Freundschaft deutlich. Im gelebten Alltag scheint jedoch das Bedürfnis nach Autonomie jenes nach Interaktion und Beziehung durch Freundschaften zu überwiegen.

Themen in Zusammenhang mit Beziehung und Partnerschaft nehmen vor diesem Hintergrund einen geringen Stellenwert in der Orientierung ein oder werden abgelehnt. Gesellschaftliche Normkonstrukte und vorgelebte Beispiele werden wahrgenommen, aber als wenig bedeutsam für die aktuelle Lebenssituation eingeschätzt. Hier stehen andere Aspekte wie etwa die berufliche Weiterentwicklung im Vordergrund. 
Die mit der beruflichen Einbindung verbundene Frage materieller Ressourcen macht deutlich, dass auch darüber eine Anbindung an die Herkunftsfamilie zumindest teilweise erhalten bleibt.

\subsubsection{Fallübergreifende Ausprägungen von Orientierung B}

Die in der angeführten Fallrekonstruktion „Martina“ herausgearbeitete Orientierung wird fallübergreifend in verschiedenen Ausprägungsvarianten sichtbar. Gemeinsamkeiten hinsichtlich der Bearbeitung verschiedener „Themen und Problemstellungen" (Nohl 2012: 52), die sich in unterschiedlichen Fällen allerdings auch auf unterschiedliche Weise abbilden, werden deutlich.

Im Folgenden führe ich einige Beispiele aus dem Interview mit Alina an, also weitere Belege, die zentrale Dimensionen der Orientierung B deutlich machen. Die also ebenfalls die „Ambivalente Orientierung an Selbstbestimmung und Autonomie in einzelnen Lebensbereichen bei gleichzeitigem Wunsch nach Bezug von Unterstützungs- und Versorgungsleistungen durch das soziale Umfeld" dokumentieren.

Die beschriebene Ambivalenz der Orientierung wird vor allem in Alinas gedanklicher Auseinandersetzung mit Fragen des autonomen Wohnens deutlich. Dabei dokumentiert sich auch, dass diese Fragestellungen eher durch Anbahnung von außen zum Thema werden. Alina bringt den Wunsch nach autonomem Wohnen einerseits über die geäußerten Präferenzen ihres Freundes ein, indem sie feststellt: ,Also mein Freund würde gerne mit mir zusammen wohnen" (IAl 815-816). Zudem hält sie fest, dass ihre Eltern schon einmal mit ihr über eine eigene Wohnung gesprochen hätten (vgl. IAl 829832). Der Wunsch, bei Verwirklichung des angestrebten Wohnens mit ihrem Freund die Anbindung an den vertrauten Kontext der Herkunftsfamilie zu erhalten, ist deutlich ausgeprägt. Alina nennt als Orte, die sie für das Zusammenleben mit dem Freund in Erwägung zieht, ,irgendwo bei uns daheim“ oder „bei ihm daheim“ (IAl 815). Alina weist also explizit und mehrfach darauf hin, dass sie nicht weit von ihrer Herkunftsfamilie entfernt wohnen möchte (vgl. IAl 821-824). Die große Bedeutung des Wohnens in der Nähe der Familie dokumentiert sich auch an den entsprechenden expliziten Formulierungen in dieser Passage (,,ich möchte“, „das wünsch ich mir so“, ,,ich will einfach"). Als Begründung für das gewünschte Wohnen in der Nähe der Familie äußert Alina zweimal, dass dies „,besser“ für sie sei, ohne dies näher zu begründen.

Die oben angeführten Formulierungen im Zusammenhang mit den Präferenzen im Hinblick auf das Wohnen machen auch eine Orientierung an eigenen Interessen, Bedürfnissen und Präferenzen deutlich. Im Zusammenhang damit dokumentiert sich auch die Ablehnung von Regeln und Vorschriften, die nicht den eigenen Bedürfnissen und Voraussetzungen entsprechen. Bei Alina wird dies eher implizit in verschiedenen Zusammenhängen deutlich. So 
etwa in ihrer Beschreibung, dass sie alle Tätigkeiten im Haushalt „,selbständig“ (IAl 79) plane und selber entscheide, wann sie welche Tätigkeit durchführe (vgl. IAl 81). Alina berichtet auch darüber, dass sie die Freizeit entgegen der elterlichen Vorstellungen gestalte, indem sie etwa viel „Fernseh” schauen“ (IAl 115) und „mit dem Laptop Musik hören“ (IAl 125) würde.

Bei Martina wie Alina prägen vorwiegend negative Erfahrungen im Pflichtschulbereich das Verhältnis zu Gleichaltrigen. Alina führt aus, dass alle anderen in der Schule Freundinnen und Freunde gehabt hätten, nur sie selbst hätten alle „so gehasst, die haben mich einfach gehasst“ (vgl. IAl 395396). Sie sei auch „verarscht“ (IAl. 400) und auch ,gehaut“ (ebd.) worden. Alina führt dies vor allem auf ihr Äußeres zurück. Sie sei als „Brillenschlange" (IAl 405) bezeichnet worden und wegen ihrer Kleidung und ihrer Frisur ausgelacht worden (vgl. IAl 412-413).

Alinas Ausführungen dokumentieren, dass ihre sozialen Kontakte vorwiegend innerhalb der Familie bestehen. Bei Alina ist dieser Kontext um den Freundeskreis der Eltern erweitert, in dem sie auch ihren Freund kennengelernt hat. Wie bei Martina ist dabei eine starke Orientierung an weiblichen Bezugspersonen erkennbar. Sie erzählt davon, dass sie mit Mutter, Schwester und Großmutter „,zusammen essen“ (IAl 102) und „ein bisschen reden“ (IAl 108) würde. Auch die bevorzugten Bezugspersonen aus institutionellen Kontexten sind vorwiegend weiblichen Geschlechts. So beschreibt Alina eine „Chefin“ (IAl 444) und eine Kollegin, mit der sie sich gut verstanden habe. Im selben Arbeitskontext erlebt Alina einen männlichen Vorgesetzten als unangenehm (vgl. IAl 443).

Alina erlebt wie Martina Ermüdung und Stress durch normalisierte Arbeitsbedingungen, die nicht ihrem Arbeitsrhythmus und ihren Vorlieben entsprechen. So erzählt sie, dass der Arbeitsversuch in einer Küche für sie „nur stressig“ (IAl 457) gewesen sei. Sie sei ,zittrig“ geworden und habe "Angst oder so, Kopfschmerzen und so, Schwindelgefühle und so“ (IAl 461462) bekommen. Auch bei einem weiteren Arbeitsversuch, bei dem Alina in einem von ihr gewünschten Bereich eingesetzt war, erlebte sie die Rahmenbedingungen als „nicht so gut“, ,zu stressig, zu viel, zu viel““ (IAl 594-595). Sie habe auch wahrgenommen, dass die Chefin ,nicht so glücklich“ (IAl 608) mit ihr gewesen sei. Im Kontext der Beschäftigung in der Küche einer Behinderteneinrichtung erlebt Alina später Rahmenbedingungen, die ihren Vorlieben und Fähigkeiten entgegenkommen. Zudem wurde dort auf ihre Voraussetzungen Rücksicht genommen, indem die Abläufe in einem angemessenen Tempo erfolgten. Dies führt dazu, dass Alina im Rückblick festhält, es habe ihr dort „sehr gut gefallen“ (IAl 627) und sie habe dabei „Spass gehabt“ (IAl 628).

Aus einem nicht näher ausgeführten Grund erlebt Alina in diesem Kontext allerdings einen Abbruch, indem sie nicht in dem von ihr als angenehm erlebten Arbeitsbereich verbleiben kann, sondern „wo anders eingeteilt“ (IAl 659) 
wird. Auch in einem weiteren Arbeitsversuch, bei dem Alina nach einer Probezeit auf die Verlängerung wartet, erhält sie stattdessen die Nachricht, dass sie nicht weiter beschäftigt werden kann (vgl. IAl 670-672). In weiterer Folge verbleibt Alina auch tagsüber innerhalb der Herkunftsfamilie und geht keiner Beschäftigung mehr nach. Ihre Ausführungen zu ihrem aktuellen Tagesablauf dokumentieren, dass sie sich nach ihren Erfahrungen in einer Einrichtung für Menschen mit Behinderungen und in verschiedenen Arbeitserprobungen und -versuchen an diesen Rahmen anpasst und sich darin entsprechend ihren Vorstellungen einrichtet.

Über Alinas Ausführungen wird deutlich, dass Partnerschaft und Kinderwunsch derzeit geringe Bedeutung zukommt, diese aber über gesellschaftliche Zuschreibungen als für Frauen relevante Themen wahrgenommen werden. So schneidet Alina das Thema Mutterschaft über die Schwangerschaft einer Bekannten an (vgl. IAl 513) und hält in Bezug auf ihre eigenen Vorstellungen dazu fest: ,einfach, nein, naja, wie soll ich das sagen, jetzt, naja, nicht gut, weil, weiß es nicht, also" (IAl 515-516). Zur Familiengründung mit ihrem Freund hält sie ganz konkret fest: „es ist noch Zeit, ist noch Zeit, noch Zeit" (IAl 841).

Trotz Alinas negativer Erfahrungen in verschiedenen Kontexten von $A r$ beit und Beschäftigung sind Zukunftsperspektiven deutlicher in diesem Bereich erkennbar. Bei Alina verbinden sich diese Überlegungen ganz konkret auch mit dem Wunsch nach finanzieller Unabhängigkeit. Sie führt an, etwas machen zu wollen, „wo man auch Geld verdienen kann“ (IAL 791), „dann verdiene ich Geld, das ist gut" (IAl 794). Bei ihren Überlegungen wird aber wieder die Ambivalenz der Orientierung an einem vertrauten Rahmen und Autonomie deutlich. Alina führt an, sie würde gerne ,,von zu Hause aus was arbeiten" und damit Geld verdienen (vgl. IAl 800), wobei sie sich dafür auch Unterstützung durch ihre Mutter wünscht (vgl. IAl 787-789).

\subsubsection{Fallrekonstruktion „Anna“ (Orientierung C)}

Anna repräsentiert die starke Orientierung an Selbstbestimmung, Partizipation und Normalisierung bei gleichzeitigem Bedürfnis nach Erhalt eines vertrauten Rahmens, um Konflikte und/oder negative Erfahrungen innerhalb und außerhalb dieses Kontextes zu vermeiden.

Im Folgenden werden exemplarisch für diese Orientierung markante Eckpunkte aus dem Interview mit Anna thematisiert, beschrieben und mittels Belegerzählungen, -beschreibungen, -argumentationen und -bewertungen dokumentiert. Im Fokus dieser Betrachtungsweise stehen wieder individuelle Orientierungen und Sichtweisen, die zentral für diese Orientierung sind. 
Wunsch, als Person und von den Lebensumständen her dem als gesellschaftliche „Normalität“ wahrgenommenen Konstrukt zu entsprechen

Über Annas Erzählungen und Beschreibungen im Zusammenhang mit ihrem Aufwachsen in einem kleinen Dorf bei ihrer Familie wird ihre Orientierung an Rahmenbedingungen deutlich, die sie als ,normal“ definiert. Dies dokumentiert sich in ihren Erzählungen und Beschreibungen, die sie rund um ihr Aufwachsen in einem kleinen Dorf aufspannt und die sich in unterschiedlichen Zusammenhängen um diesen Begriff drehen. So wird „Normalität“ im Kontext mit dem eigenen Verhalten in der Kindheit zweimal thematisiert (vgl. IA 93, 96). Es scheint so, als wolle Anna gleich eingangs deutlich machen, dass ihre Bedingungen des Aufwachsens so waren, wie die der anderen Kinder, und sie selbst auch nicht von als „Normalität“ definierten Verhalten abwich. Sie erzählt, dass es zu der Zeit, als sie klein war, Kindergärten nur in der Stadt gegeben hat, die teuer und schwer erreichbar waren. Deshalb sei sie bis zum Schuleintritt zu Hause gewesen. Die Zeit zu Hause beschreibt sie als „eigentlich wohl schön so“ (IA 89). Sie habe „die Freiheit auch gehabt“ (IA 90). Sie habe „wohl einmal einen Anpfiff auch dann gekriegt“ (IA 92-93), weil sie mit vier, fünf Jahren ohne sich bei den Eltern abzumelden, zu den Nachbarn gegangen sei. Aber, so Anna, das sei ,[...] eh normal gewesen auch, aber das macht ja jedes Kind einmal, irgendeinen Fehler" (IA 93-94). Der Hinweis auf die eigenen, ,normalen“ Rahmenbedingungen des Aufwachsens erfährt allerdings eine Einschränkung durch den Zusatz ,relativ“:

„Also mein Leben war eigentlich von Kindheit an, also von Baby halt an relativ auch also halt normal aufgewachsen sonst." (IA 96-98)

Die Orientierung an von ihr als „Normalität“ entsprechenden Zusammenhängen dokumentiert sich in vielfältigen Zusammenhängen rund um ihre Erzählungen um ihr Aufwachsen noch an verschiedenen weiteren Sequenzen und durchzieht gleichsam das gesamte Interview als Annas Orientierungsrahmen.

Annas Sichtweise eines ,normalen“ Lebenslaufes spiegelt auch stark eine Orientierung an traditionellen gesellschaftlichen Lebensentwürfen, die Heirat, Familie, Kinder (vgl. IA-VA5), aber auch Erwerbstätigkeit (vgl. IA-VA6) beinhalten, wider. Dies dokumentiert sich an ihren Assoziationen zu zwei von ihr ausgewählten Bildern im Rahmen der Vignettenbeschreibung. Bereits im Rahmen der ersten Bildbeschreibung ist diese gedankliche Orientierung erkennbar:

I: „Könnten Sie mir sagen, was Sie auf dem Bild sehen.

A: Also ein Pärchen, also die dürften verlobt sein oder irgendwas, vermute ich einmal. Also ich weiß, dass sie halt Mongolin halt ist und dass er halt auch ein bisschen eine Behinderung hat. Ich meine, sie sind glücklich, das sehe ich ja. Aber ich meine, wenn sie sich gerne haben, also, dass sie sich verstehen oder so - also ich finde das tadellos, also, dass sie sich - also die passen gut zusammen. 
I: Und wie glaube Sie, geht diese Geschichte weiter von den Zweien? Wenn Sie sich das so vorstellen, wenn Sie die zwei so anschauen. Wie könnte das weitergehen?

A: Ich vermute einmal, dass sie irgendwann einmal vielleicht heiraten werden und, dass sie einmal eine Familie haben würde, also Kinder oder sie halt auch - dass die beide halt nachher arbeiten gehen auch, also so was. Ich meine, weil sie hat ja ein hübsches Gesicht auch und er genauso auch, also ja - also ganz normale Familie gründen und das alles drum und dran." (IA-VA5 890-901)

Die starke Orientierung an einem solchen, als „normal“ wahrgenommenen Entwurf wird auch in der zweiten Bildbeschreibung durch die explizite zweimalige Ausführung des Begriffes deutlich:

I: „Gut, und das Bild, das Sie da ausgesucht haben. Was zeigt das?

A: Also bei - die zwei tun heiraten. Also man sieht, dass sie beide sehr glücklich sind. Also ( ) weinen können auch und das. Also, dass sie halt eine glückliche Zukunft vor sich halt haben und das. Also ja, ganz normal Familie gründen, normal leben, arbeiten gehen." (IA-VA6 902-905)

Partnerschaft, Heirat, Elternschaft und Erwerbstätigkeit von Menschen mit Behinderung stellen sich in Annas Beschreibung als etwas dar, das sie implizit durch ihre Formulierungen zwar gesellschaftlich in Frage gestellt zu sehen scheint. Sie selbst bewertet solche Lebensentwürfe sehr positiv als Quelle des Glücks und entkräftet für sie offenbar latente gesellschaftliche Vorbehalte daran mit ihrer Bewertung ,also ich finde das tadellos“ (IA 894).

\section{Orientierung an einem vertrauten sozialen Rahmen und gewohnten Abläufen}

Neben der Ausrichtung an einem von ihr als gesellschaftliche Normalität wahrgenommenen Maßstab wird bereits in frühen Passagen des Interviews mit Anna auch eine Orientierung an einem vertrauten sozialen Rahmen sichtbar, was wiederum mit dem Begriff der „Normalität“ in Zusammenhang gebracht wird. So erzählt Anna vom Aufwachsen im familiären Umfeld mit den Eltern, der Großmutter, einer Tante und ihrem Bruder in einem Haus in einem kleinen Dorf und bewertet dies in Zusammenhang mit ihrer Orientierung an Lebensumständen, die sich nicht von denen anderer Familien unterscheiden:

I: „Und wie war das so, zusammen, miteinander zu leben, also die ganze Familie?

A: Also relativ- eigentlich ganz normal. Ich meine wir wohnen noch immer auch zusammen - und wir haben - also ich tue auch gerne mit meiner Oma zusammen." (IA 118-120)

Dass Anna explizit anspricht, noch immer und gerne mit der Großmutter zusammen zu wohnen, lässt auf eine Beziehung zu dieser schließen, die für Anna einen hohen Stellenwert zu haben scheint.

In einer späteren Sequenz im Interview dokumentiert sich Annas Orientierung am vertrauten Rahmen im Zusammenhang mit der Frage, was sich für sie ändern würde, wenn sie über ein eigenes Einkommen verfügen würde. 
I: „Würde sich an Ihrer Lebenssituation - würden Sie an Ihrer Lebenssituation was ändern, wenn Sie wirklich ein eigenes Gehalt hätten?

A: Eigentlich nicht, also ich würde viel auf die Seite sparen halt auch, also wenn ich so viel verdienen - also wenn es einmal durchkäme. Also mein Leben würde ganz normal weiterverlaufen wie sonst halt auch immer halt. Also ich würde halt - also ganz normal ganz normal auf die Seite sparen.“(IA 701-706)

Orientierung an Partizipation/Teilhabe und Integration als Ausdrucksformen von ,Normalität“

Anna verweist in einer weiteren Sequenz darauf, dass ihr gesamter Lebenslauf dem in ihrer Orientierung zentralen Fokus auf „Normalität“ entspricht, und bringt dies auch sprachlich zum Ausdruck:

„So bei den Festen, was wir da in unserer Gemeinde haben, tue ich auch immer arbeiten helfen und-also ganz eine normale - also wie halt sonst der ganze Lebensablauf - ganz normal eigentlich." (IA 120-122)

In dieser Passage wird auch eine deutliche Orientierung an Partizipation/Teilhabe in der Gemeinde, also im näheren sozialen Umfeld, sichtbar, indem Anna betont, dass sie bei den Festen in der Gemeinde ,auch immer arbeiten helfe" (IA 121). Sie weist also darauf hin, dass sie eine helfende Funktion einnimmt, etwas zum Gelingen eines Festes beiträgt und somit etwas für die Gemeinde leistet. Es scheint Anna wichtig zu sein, auf ihre soziale Integration in die Gemeinde hinzuweisen. Bei Fokus auf die semantische Ebene schwächen weiterhin Begriffe wie „relativ“ (IA 119) und „eigentlich“ (IA $119,122)$ ihre aktuellen Ausführungen etwas ab. Es erfolgen aber an dieser Stelle keine Verweise auf Aspekte, die zu dieser sprachlichen Einschränkung führen.

Auch über eine Erzählung über soziale Kontakte mit Kindern im Dorf wird eine Orientierung an Partizipation und Integration, verbunden mit der Orientierung an „Normalität“ sichtbar. Anna erzählt, dass sie mit mehreren Kindern in der Nachbarschaft gespielt und gemeinsame Aktivitäten durchgeführt hätte, und führt dazu aus: ,also ich habe genug Spielkameradinnen gehabt, also Spaß bis zum Geht-Nicht-Mehr“ (IA 132-133). Anna resümiert quasi gegenüber der Interviewerin: „Es ist ein ganz normaler Alltag gewesen, zusammen gespielt haben wir" (IA 139-140).

Auch Annas Erzählungen, die sich um den schulischen Kontext aufspannen, lassen eine starke Orientierung an sozialer Einbindung erkennen. Gleichzeitig dokumentieren sich dabei aber - wie im Folgenden sichtbar wird - auch Erfahrungen von Abwertung, Mobbing und Aussonderung. 
Negative Erfahrungen im Regelschulbereich:

unpassende Rahmenbedingungen, mangelnde Anerkennung, Abwertung, Mobbing, Rückstufung, Aussonderung

Die Erfahrung des Scheiterns an den schulischen Erfordernissen verbunden mit einem Schulwechsel stellt für Anna einen sehr wesentlichen Orientierungskontext dar, was sich darüber dokumentiert, dass sie ihn ganz zu Beginn des Interviews über eine generelle Erzählaufforderung von sich aus einbringt:

A: ,Ja, also Volksschule habe ich gemacht. Also in K., das ist gleich ein Stückchen weiter nach N. und nach der zweiten Volksschule bin ich dann hinauf nach M. in die Sonderschule dann gekommen. Weil ich bin da herunten einmal sitzen geblieben.

I: In der Volksschule?

A: Ja, weil ich mir da beim Lernstoff schwer getan habe, bei allen Fächern. Also Sachunterricht, Mathematik, Deutsch oder so Religion. Da habe ich mir am Anfang überhaupt sehr schwer getan auch und nachher bin ich eben von der zweiten Volksschule hinauf nach M. gegangen, also in die Schule, und da habe ich super Lehrer gehabt, die was mich jeden Tag auch unterstützt haben. Mit einer Lehrerin habe ich sogar noch heute Kontakt und -“(IA 20-28)

Anna schreibt sich in der Erzählung über den Schulwechsel zwar zu, sich „schwer getan“ (IA 24) zu haben, macht aber im Vergleich zur neuen Schule, wo sie Unterstützung erfahren hat, gleich eingangs deutlich, dass sie die Bedeutung der Rahmenbedingungen für ihr eigenes Bestehen in der Schule reflektiert. Unterstützung wird dabei von ihr wertgeschätzt und mit Beziehung in Zusammenhang gebracht, was sich in der Formulierung ,super Lehrer" (IA 27) und der Tatsache, dass sie mit einer davon noch heute Kontakt hat, dokumentiert.

Annas weitere Ausführungen zeichnen ein sehr differenziertes Bild zum erlebten Regelschulkontext, den sie über ihre Erfahrungen zunehmend als Raum charakterisiert, der von unpassenden Rahmenbedingungen, mangelnder Anerkennung, Abwertung, Mobbing, Rückstufung und Aussonderung gekennzeichnet ist. In Annas Rückschau dokumentiert sich dabei eine zunächst sehr positive Einstellung zur Schule: ,[...] also ich weiß nur, dass ich mich sehr auf die Schule gefreut habe, weil ich bin gerne auch in die Schule gegangen“ (IA 140-141). Zunehmend berichtet Anna aber von Schwierigkeiten in der Schule. Nach einer Zeit in der Vorschule habe sie sich in der Volksschule ,überhaupt schwer getan [hat], bei allem nachher“ (IA 157). Der Stoff sei schwieriger geworden, sie sei aber in die zweite Klasse gekommen „und dann ist doch immer mehr schwerer geworden“ (IA 158). Letztendlich habe es geheißen:

„Anna wir müssen dich noch einmal zurück stufen, noch einmal in die erste Volksschulklasse.“ (IA 160)

Nachdem sie die erste Klasse wiederholt und die zweite fast fertig hatte, habe es geheißen „das funktioniert nicht“ (IA 163). Anna begründet dies: 
„[...] und da habe ich eine blöde Lehrerin gehabt, die war sehr eingebildet auch und die hat mich wahrscheinlich von Anfang an nie mögen auch" (IA 164)

Sie sagt, sie hätte „es probiert und alles drum und dran“ (IA 165-166). Anna führt dann auch wiederholt (vgl. IA 164, 178) ihre Vermutung an, die Lehrerin hätte ihren Bruder, der in der gleichen Schule war, lieber gehabt. Annas Erzählungen und Beschreibungen rund um Probleme in der Schule, Rückstufung und Schulwechsel sind dabei dadurch geprägt, dass sie eine wahrgenommene Wechselwirkung eigenen „Sich-Schwer-Tuns“ mit unangemessenem Verhalten einer Autoritätsperson, der Lehrerin, dokumentieren. Dass sie Probleme in der Schule hatte, führt Anna dabei nicht in erster Linie auf eigene Unzulänglichkeit zurück, sondern darauf, dass sie eine „blöde“, ,eingebildete" Lehrerin gehabt hätte (vgl. IA 163). Anna macht damit für ihre Probleme in der Schule die Person der Lehrerin zumindest mitverantwortlich. Annas Erzählung gipfelt in dieser Passage in eine Fokussierungsmetapher, die ihre Gefühle in Zusammenhang mit der auf ihr Nicht-Genügen folgenden Aussonderung aus dem Regelschulbereich dokumentiert:

„Und dann haben sie mich eben von dort dann weggetan, ich bin mir vorgekommen wie der letzte Dreck dort.“ (IA 169)

Anna macht eindrucksvoll deutlich, dass sie Erfahrung mit Abwertung und Aussonderung im schulischen Zusammenhang gemacht hat, die bei ihr massive negative Gefühle hervorgerufen haben. Aus der Beschreibung ihrer Gefühle im Zusammenhang damit wird ersichtlich, wie wichtig es für Anna ist, teilzuhaben. Das Verunmöglichen von Partizipation bzw. im Regelbereich eingebunden zu sein, ist für Anna in der Rückschau mit sehr negativen Gefühlen verbunden, die auch ihr Selbstbild massiv beeinträchtigen.

Die Kombination der bereits beschriebenen Passagen zur Schulzeit lassen in der Einstellung von Anna ein gedankliches Konstrukt sichtbar werden, nach dem sie bei passenden Rahmenbedingungen, was sie vor allem in Unterstützung durch „,super Lehrer“ festmacht, wohl keine so massiven schulischen Probleme gehabt hätte. Anna macht durch ihre Zuschreibungen an die Lehrerin auch deutlich, dass sie deren Verhalten in der rückblickenden Bewertung nicht gut heißt und es darauf zurückführt, dass sie von dieser nicht gemocht wurde. Anna präzisiert ihre negativen Erfahrungen in der Volksschule im Zusammenhang mit einem Diktat:

,$[\ldots]$ da habe ich halt die Wörter halt, also keinen richtigen Satz zusammenbauen können und da habe ich halt verdreht und da habe ich halt- wie soll ich sagen, ein Nichtgenügend darauf geschrieben und ich habe mir schwer getan bei gewissen Wörtern, also richtig zusammen zu setzen auch. Es haben aber andere genauso Probleme gehabt, aber ich habe mich dann genauso - ich habe weiterprobiert, bis ich es gekonnt habe und ich hatte gedacht, dass ich wahrscheinlich eine komplette Versagerin bin und sie wollte mich einfach halt- so wie dort einfach nicht mehr haben, also [...]“" (IA 179-185) 
Sie lässt somit die Schwierigkeiten beim Diktat zu etwas werden, das nicht nur sie selbst, sondern auch andere Schüler betraf. Aus der Beschreibung, auch andere hätten in der Schule Probleme gehabt, wird deutlich, dass Anna versucht, sich mit den anderen Kindern als gleichwertig zu präsentieren. Es sei - so geht aus den Ausführungen implizit hervor - unter diesen Rahmenbedingungen „normal“, Probleme zu haben. Anna verstärkt hier auch nochmals ihre früher vorgenommene Argumentation, dass es wohl zum größten Teil an der Lehrerin gelegen habe, dass es in der Regelschule für sie nicht so gut lief. Andererseits dokumentieren sich als Konsequenzen dieser Erfahrungen massive Selbstzweifel, so etwa, ,eine komplette Versagerin“ (IA 184) zu sein. Dieses Gefühl, den Ansprüchen auch bei eigenem Bemühen nicht zu genügen, führt bei Anna zu massiven Selbstzweifeln. Zudem leitet es - verbunden mit dem Gefühl, im Kontext der Regelschule nicht gewollt worden zu sein - zumindest im schulischen Kontext eine Umorientierung in Annas Sichtweise von „Normalität“ ein, wie später im Zusammenhang mit ihrer Einschätzung der Sonderschule noch deutlich wird ${ }^{89}$ (Verweis machen).

Anna führt weiter aus, dass sie nicht direkt ,hinausgeschmissen“ (IA 188) worden sei, es sei alles zwischen Eltern und dem Direktor besprochen worden. Aber die Mutter hätte „selber einen Schleim auf die Lehrerin“ (IA 190) gehabt, hätte die Lehrerin ,selber nicht gemocht, überhaupt nicht" (IA 191). Sie sagt, auch ihr Bruder hätte die Lehrerin nicht mögen, in der Schule hätte die Lehrerin fast gar keiner mögen. Anna sagt, die Lehrerin sei eine unsympathische Frau gewesen, die sie ,auch teilweise mit so gewissen Blicken auch immer so zornig angeschaut" (IA 193-194) habe. Über diese Beschreibung dokumentiert Anna eine aus ihrer Sicht wahrgenommene negative Einstellung der Lehrerin ihr gegenüber und lässt diese mit als Grund, dass sie die Schule verlassen musste, erscheinen. Zu ihr habe die Lehrerin nichts gesagt, aber zu ihren Eltern habe sie gesagt,

„,...] dass es nicht funktioniert mit ihrer Tochter, sie müssen halt leider schauen, sie in eine andere Schule geben, weil es nicht weiter funktioniert mit dem Lernen und das

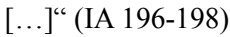

Anna thematisiert hier, dass die Einschätzung der Lehrerin, sie müsse in eine andere Schule, weil es mit ihr nicht „funktioniere“, gegenüber den Eltern offen ausgesprochen wurde. Damit dokumentiert die Lehrerin in Annas Erzählung ihre Unzulänglichkeit im schulischen Rahmen in einem offiziellen Kontext gegenüber den Eltern. Bei Anna selbst resultiert aus diesen Erfahrungen die Entwicklung einer widersprüchlichen Selbsteinschätzung und eine ebensolche Einschätzung der schulischen Rahmenbedingungen.

89 Siehe dazu nächster Unterpunkt ,Wechsel von der Regel- in die Sonderschule: passendere Rahmenbedingungen, Erfolgserlebnisse, Erfahrung von Interesse an der eigenen Person und Anerkennung“" 
Auch die Einschätzung der eigenen sozialen Integration innerhalb der Peergroup im schulischen Kontext erscheint ambivalent. Aus einer Sequenz geht hervor, dass Anna in der Volksschule von einigen Mitschülern schlecht behandelt wurde. Sie erzählt, dass zwei, drei Mitschüler in der Sonderschule „gegen mich halt auch“ (IA 173) waren. Etwas später präzisiert sie auf Nachfrage, dass sie dies „,vom Verhalten her“ (IA 204) gemerkt habe. Sie hätte ,auch normale Schulsachen gehabt, wie jeder andere halt auch“" (IA 206207). Die Mutter hätte darauf geschaut, dass ,wir nicht alles so billige Sachen halt auch nicht gekriegt haben" (IA 207-208), es wären auch teure Sachen darunter gewesen und ,das war der Neid und die Eifersucht auch und da haben wir halt immer dazwischen einen Wickel gehabt auch“" (IA 209-210). Man hätte ihr Sachen weggenommen und kaputt gemacht, ,also waren teilweise schon aggressiv die anderen Kinder" (IA 213-214). Das Verhalten der anderen Kinder führte in Annas Erzählung dazu, dass sie sogar die ungeliebte Lehrerin darüber informiert habe und diese dann gesehen habe, „dass sie mich wirklich nur seckiert ${ }^{90}$ und genervt haben“ (IA 219). Die Lehrerin, der Anna in der vorherigen Passage negative Eigenschaften zugeschrieben hat, wird in dieser Sequenz zur Person, die Anna hilft, indem sie die anderen Schüler ermahnt. Gegen Ende der Passage schreibt Anna der Lehrerin aber wieder zu, sie hätte wegen ihr die Schule verlassen müssen. Als Grund für das Mobbing durch die Mitschüler sieht Anna die Tatsache, dass sie von ihrer Mutter teurere Schulsachen bekommen habe und die anderen Mitschüler „Neid und die Eifersucht“ (IA 209) gehabt hätten, bringt das Mobbing also nicht in Zusammenhang mit der eigenen Beeinträchtigung. Dies entspricht auch dem Streben Annas in einigen vorhergegangenen Passagen, als möglichst „normal“ zu erscheinen, sich der Interviewerin gegenüber so zu präsentieren. Auch in dieser Passage fällt dieser Begriff wieder, diesmal in Zusammenhang mit den „,ganz normalen Schulsachen“ (IA 206).

Wie deutlich wurde, beschreibt sich Anna als von der Lehrerin nicht gewollt und von den Mitschülern als gemobbt. Dennoch habe sie die Schule nicht verlassen wollen, sie habe dort auch viele Freunde gehabt.

A: „Ja, weil ich so viele Freunde auch gehabt habe auch und es war - ein Teil hat auch geweint, weil ich weggehen habe müssen.

I: Wollten Sie lieber dort bleiben?

A: Ich wollte lieber dort bleiben, aber die Lehrerin wollte mich nicht mehr dort haben, also. Also mein Mama war damals auch selber sauer auf die Lehrerin und das - ein paar - ich kann mich eh noch erinnern, wo ich weggegangen bin, dass ein paar dort geweint haben auch, und es auch von meinen Nachbarskindern - die sind auch in meiner Klasse gewesen auch. Also wir sind quasi fast wie eine Familie halt aufgewachsen." (IA 225232)

Dies erscheint in Zusammenhang mit der Erzählung über Mobbing und das Verhalten der Lehrerin als ambivalente Beurteilung und spiegelt auch in

90 Seckieren $=$ ärgern . 
diesem Kontext Annas Orientierung wider, anerkannt zu werden und sozial gut eingebunden zu sein.

\section{Wechsel von der Regel- in die Sonderschule:} passendere Rahmenbedingungen, Erfolgserlebnisse, Erfahrung von Interesse an der eigenen Person und Anerkennung

Beschreibt Anna den erzwungenen Wechsel von der Regel- in die Sonderschule als einschneidend negatives Erlebnis, so beginnt sie ihre Ausführungen über die Sonderschule mit einer Passage, in der dieser Ort als sehr positiv bewertet wird. In der Beschreibung der Akteure und der Rahmenbedingungen spiegelt sich Annas Bedürfnis nach Anerkennung und sozialer Eingebundenheit wider:

I: „Vielleicht erzählen Sie da ein bisschen von Anfang an, wie sich das eben dann in der Sonderschule entwickelt hat für Sie.

A: Also am Anfang, wo ich das erste Mal - also es war nämlich so. Bevor ich da richtig erst oben weiter Schule gegangen bin, da war so eine Art wie ein Vorstellungsgespräch und die Lehrerin, welche mir vorgestellt worden ist, auch die Frau Direktorin und die sind alle ganz anders gewesen, also viel besser als wie herunten, also in $\mathrm{M}$.

I: Inwiefern anders? Also in welcher Art anders?

A: Viel mehr kamoter halt. Also wirklich so lässig, nicht so viel streng oder irgendwas. Ok, nur wenn einer einen Blödsinn gedreht hat, der hat dann genauso zur Lehrerin oder so müssen, ins Büro halt marschieren. Aber es waren meistens fast nur die Burschen hauptsächlich. Ich habe oben - wo ich dann den ersten Schultag gehabt habe, da habe ich auch sofort am ersten Schultag, wo wir Sachen - also wo maximal nur eine Stunde gewesen ist, wo schon das neue Schuljahr angefangen hat, da habe ich sofort, also mindestens ich glaube drei oder vier neue Freundinnen gehabt auch.“ (IA 246-260)

Im Vergleich, den Anna zwischen der Sonderschule und der Volksschule zieht, in der sie sich schwer getan hat, bewertet Anna schon eingangs die Sonderschule als Ort, an dem alles „,kamoter“91 und „lässig, nicht so viel streng oder irgendwas“" (IA 253) war. Der geschütztere Rahmen mit den für sie offenbar besser passenden Rahmenbedingungen wird von Anna in der Rückschau präferiert und verdeutlicht diese Orientierung als Konsequenz der Erfahrungen im Regelschulbereich, die für Anna mit der Wahrnehmung der eigenen Unzulänglichkeit, fehlender Anerkennung, Abwertung und Mobbing verbunden waren. Auch die Orientierung an sozialer Eingebundenheit, die in der Regelschule durch Mobbing massiv beeinträchtigt war, wird bereits in der Eingangssequenz zur Sonderschule deutlich: Anna merkt an, dass sie sehr schnell einige neue Freundinnen gewonnen hat.

Die positive Bewertung des Sonderschulbereiches, der ja Annas zuvor deutlich sichtbar gewordener Orientierung am Begriff der „Normalität“ widerspricht, erscheint als Folge negativer Erfahrungen und ungünstiger Rah-

Kamot $=$ gemütlich, angenehm. 
menbedingungen im Kontext der Regelschule. Anna beschreibt in Passage (vgl. IA 155-169), dass sie sich bei der Aussonderung in die Sonderschule „wie der letzte Dreck“ (IA 169) gefühlt habe. Nun beschreibt sie die Sonderschule sehr positiv und hebt die unterstützenden Rahmenbedingungen hervor. Die Erzählungen und Beschreibungen in den bisherigen Passagen und das darin enthaltene Bemühen Annas, die Interviewerin von der „Normalität“ ihres Lebens zu überzeugen, lassen vermuten, dass es Anna vorwiegend um die Unterstützung geht, die in der Sonderschule viel besser war. Dass es laut Anna auch „lässig“" und „nicht so viel streng“ (IA 253) war, deutet an, dass Anna starre Regeln und Vorschriften ablehnt und ein entspanntes Ambiente bevorzugt.

Die Erfahrungen, die Anna unter diesen Rahmenbedingungen machen konnte, ermöglichen Erfolgserlebnisse und bestärken sie in ihren eigenen Bemühungen, schulische Erfolge zu erzielen:

\begin{abstract}
„Meine Noten sind dann, durch die Sonderschule, sehr viel besser geworden auch. Ich habe nur einmal - in einem Jahr habe ich im Zeugnis nur einen Vierer gehabt, der Rest dann alles - sind dann Einser, Zweier und Dreier gewesen. Nur in Mathematik habe ich mir irrsinnig schwer getan, tue ich mir heute noch immer schwer. Aber wo ich dann später in die Hauptschule übergestiegen bin, ab da sind meine Noten dann immer, dass ich dann Einser und Zweier gehabt habe und nachher das letzte Schuljahr, habe ich dann im Halbjahreszeugnis alles Einser gehabt und das letzte Schuljahreszeugnis, da waren dann ein paar schwere Prüfungen, da sind die Noten leider nur auf Zweier halt herausgekommen, aber ich wollte schauen, dass ich noch einmal beim Abschlusszeugnis noch einmal alles Einser herauskommen." (IA 275-284)
\end{abstract}

Schulischer Erfolg oder Misserfolg hängen in Annas Argumentation von der passenden Unterstützung und von der Person der Lehrerin ab. Zu schnelles Vorgehen wird als unpassender Zugang erlebt, der zu schulischem Misserfolg führt. Dies dokumentiert sich an der Hervorhebung der Bemühungen der Sonderschullehrerin beim Vermitteln der schulischen Inhalte, wobei offenbar Annas persönliches Lerntempo berücksichtigt wurde und so eine Basis für schulische Erfolgserlebnisse geschaffen wurde:
„Also die Lehrerin, die werde ich so nie vergessen. Das ist die Frau K. Das ist eine total super, kamote Lehrerin. Die hat mir jedes - ist egal, was ich für ein Unterrichtsfach ge- habt habe, die hat mir alles ganz langsam beigebracht. Auch wie man richtig dividieren tut, wie man malrechnen tut, oder wie man irgendein Quadrat ausrechnen tut. Ich mei- ne, ich habe zwar wohl auch noch Fehler gehabt und so - ich habe auch normal Schul- arbeiten gehabt, ich habe gelernt, also wie eine Verrückte auf gut Deutsch gesagt, dass ich alles ja gute Noten kriege. Und das habe ich. Also ich habe tadellos, super Noten gekriegt.“(IA 295-301)

Im weiteren Vergleich der beiden Lehrerinnen werden weitere Kriterien sichtbar, die Anna an eine positive Beurteilung schulischer Rahmenbedingungen knüpft. 
A: „Also die andere, die war - die hat sich für mich nicht viel interessiert halt. Ich meine, sie hat wohl so geschaut auch und das alles drum und dran, aber die hat sich nie so richtig für mich irgendwie - nicht so ein Interesse nach mir - sich nicht abgegeben mit mir. Mehr mit den anderen Schülern und auch mehr mit meinem Bruder. Ich bin mir für sie vorgekommen, dass ich für sie so wie Luft gewesen bin - zeitweise dazwischen halt. I: Und das war bei der Sonderschullehrerin dann -

A: - überhaupt nicht der Fall. Da wird kein Schüler vernachlässigt, gar keiner. Also heutzutage finde ich besser- in der heutigen Schulzeit besser, Sonderschule zu gehen, als wie eine normale Volksschule oder Hauptschule oder Gymnasium, weil da werden erstens in einer Sonderschule, hast du auch viel mehr Vorteile auch, du lernst auch viel leichter und du merkst dir auch leichter die Sachen auch. Ich meine, freilich gibt ab und zu auch Differenzen und so, genauso, aber ich habe mir gedacht, das ist meine perfekte Lehrerin, die hilft mir.“ (IA 312-323)

Als zentral für Anna dokumentiert sich über eine mehrmalige explizite Nennung des Begriffes „Interesse“. Wahrgenommen und als Person anerkannt zu werden, also nicht wie „Luft“ (IA 315) behandelt zu werden, stellt sich in Annas Argumentation als Rahmen dar, der eigenes Wohlbefinden, Lernerfolg und Entwicklungsmöglichkeiten fördert. Über ihre diesbezüglichen positiven Erfahrungen mit der Sonderschullehrerin und den Vergleich zu den negativen Erfahrungen mit der Regelschullehrerin bewertet Anna jeweils den gesamten Kontext, in dem diese Erfahrungen gemacht wurden. Dies mündet - wie sich an der o.a. Passage dokumentiert - in eine allgemeine Empfehlung für die Sonderschule als Lernort, an dem man aus Annas Perspektive nicht - wie für die Regelschule implizit hervorgehend - vernachlässigt wird.

In der Einschätzung von Anna ist die Sonderschule der Regelschule ganz allgemein vorzuziehen, als Argument führt sie an: „du lernst auch viel leichter und du merkst dir auch leichter die Sachen auch" (IA 323). Zudem würde dort niemand vernachlässigt. Bei Anna kommt dies sprachlich durch das „du“ so zum Ausdruck, als wäre das für jede/n eine Option und nicht nur für Schülerinnen und Schüler mit einer Beeinträchtigung. In gewisser Weise hebt Anna damit auch den Status der Sonderschule hervor bzw. wertet diese auf.

Dass aber in Annas Sichtweise auch eigenes Bemühen im Sonderschulkontext notwendig ist, um gute Leistungen zu erbringen, wird über einen Vergleich ihrer eigenen Person zu von ihr als ,faul“ eingeschätzten Mitschülerinnen sichtbar.

\footnotetext{
„Aber es war nämlich so, also ich habe eine Schulfreundin oben, die heißt G., die hat sich noch - also sagen wir so, die war faul beim Lernen. Die hat gar nichts getan und dann ist noch eine andere, von der vierten Klasse auch noch in die Sonderschule gekommen und die zwei haben sich dann auf ein Packerl zusammengeschmissen, haben gar nichts mehr gelernt, also sind voll abgesackt. Die haben keine guten Noten gehabt im Zeugnis. Das letzte Schuljahr, wo ich sie noch gesehen habe die ( ), da wollten wir wissen, wie von den anderen auch die Zeugnisse ausschauen und da hat sie sieben Fünfer im Zeugnis gehabt. Also ich hätte mich mit so einem Zeugnis geschämt, weil ich habe da nur noch einen einzigen Zweier gehabt und der Rest alles Einser [...].“ (IA 346-354)
} 
Hier ist zum wiederholten Mal auch Annas Bestreben zu erkennen, sich selbst als gute Schülerin zu beschreiben und hervorzuheben, dass sie keine Probleme hatte und gute Leistungen erbracht hat. Um dies zu verdeutlichen, beschreibt sie die zwei Mitschülerinnen, die „gar nichts mehr gelernt, also [...] voll abgesackt" (IA 350) wären. Schulischer Misserfolg - wie an Annas Ausführungen deutlich wird - ist für sie ein Grund, sich zu schämen. Somit dokumentiert sich über Annas Argumentationskette auch die Erfahrung von Beschämung im Regelschulbereich über für sie in diesem Kontext nicht erbringbar gewesene Leistungen.

\section{Berufliche Orientierung: Unterstützung und Berücksichtigung eigener Interessen als Basis für selbstbestimmte Entscheidungen neben wei- teren Erfahrungen von Misserfolg und Rückstufung}

Über Annas Erzählung, dass sie nach der Sonderschule ,zuerst daheim gewesen halt" (IA 263) sei und in dieser Zeit überlegt habe, „was ich so machen will“" (IA 266), wird ihre Orientierung an eigenen Interessen hinsichtlich des beruflichen Kontextes deutlich. Anna will selbst bestimmen, wie es weitergeht und überlässt Entscheidungen nicht anderen Personen. Aus ihrer Erzählung über diverse Praktika und Kurse, die sie nach der Schule besucht hat, geht hervor, dass sie dabei Unterstützung und Begleitung durch Arbeitsassistenz bekommen hat. In diesem Kontext sei im Rahmen der Beratung gesagt worden, dass die meisten Jugendlichen sich ,schwer tun wegen einer Arbeitsstelle“ (IA 395-396). Das sei auch bei ihr der Fall gewesen, führt Anna aus:

„[...] und nachher habe ich eben auch sehr viele Praktika bei den ganzen Kursen auch gemacht. Ich habe auch Regale eingeräumt, also beim Supermarkt draußen, bei meiner Gemeinde auch. Dann habe ich in S. auch bei verschiedenen - also bei Floristik auch Praktika gemacht. Das war zuerst auch mein Ding und dann habe ich für das auch kein Interesse mehr gehabt und nachher beim Projekt, da habe zuerst auch im Garten gearbeitet und dann bin ich auf Reinigung umgestiegen und dann wollte ich aber Wäscheund Bügeldienst auch noch ausprobieren, weil es dort auch vorhanden ist. Und Wäsche - Bügeldienst habe ich auch gerne gemacht. Nur das Einzige ist, ich vertrage halt sehr viele Putzmittel halt nicht und ich habe auch eine Latexallergie, also muss ich so eigene Handschuhe tragen." (IA 395-405)

Im Rahmen der durch Arbeitsassistenz erfolgten Unterstützung bei der beruflichen Orientierung bekam Anna Gelegenheit, sich auszuprobieren, verschiedene Praktika und Kurse zu machen. Die Erfahrung, dass ihre Wünsche in diesem Prozess ernst genommen und auch berücksichtigt wurden, führt dazu, dass sie zunehmend auch ihre eigenen Interessen, Vorlieben und Voraussetzungen artikulieren kann und als Maßstab für die selbstbestimmte Ausgestaltung des eigenen Lebens sieht.

Dabei ist im Zuge der Beschreibung ihrer derzeitigen Tätigkeiten eine eher auf die Defizite ausgerichtete Einschätzung ihrer eigenen Person erkennbar. 
Sie führt aus: „beim Telefondienst da hapert es noch gewaltig bei mir“ (IA 580-581). Sie tue sich auch schwer beim Vorstellen und würde sich immer wieder versprechen. Sie gebe sich aber Mühe und hält fest: „aber sonst eigentlich, also so taugt mir das wirklich so beim Arbeiten auch" (IA 587-588). Anna betont auch die eigenen Bemühungen in diesem Kontext. Dennoch steht aktuell ein Wechsel im Raum - so führt Anna aus, dass sie in naher Zukunft die Anlehre aufgeben und in die Beschäftigung wechseln werde. Sie könne „nur einen gewissen Druck draufhaben“ (IA 596), sonst würde sie Fehler machen und „,von den Assistenten eines auf den Deckel“ (IA 597-598) bekommen. Sie führt auf Nachfrage nach einem Beispiel dafür eine Situation an, wo sie früher als vorgesehen heimging:

„Und dann habe ich eben eines gewaltig auf den Deckel gekriegt und eine Strafe habe ich halt auch gekriegt, da habe ich am nächsten Tag halt zehn Minuten länger bleiben müssen, aber das ist auch nicht weiß Gott wie schlimm gewesen.“ (IA 606-609)

Sie schließt daraus „Aber man lernt auch genauso aus der Sache“ (IA 609) und legitimiert damit in gewisser Weise die erlebte Sanktion. Aus Annas Beschreibung der Anforderungen in verschiedenen Arbeitsbereichen der Anlehre geht hervor, dass sie diese als sehr hoch einschätzt. Es müsse alles genau gemacht werden, jeder, der die Anlehre mache, müsse „das auch perfekt können“" (IA 616-617). Auf die Frage, wie es nun dazu gekommen sei, dass der Wechsel von der Anlehre in die Beschäftigung im Raum stehe, wechselt Anna in ihrer Beschreibung die Perspektive und beschreibt sich selbst gleichsam aus dem Blickwinkel von Assistenten:

„Es ist nämlich so, ich tue mich - weil die Assistenten tun mich ja beobachten, wie ich mir beim Arbeiten tue und die sehen, dass ich mir wirklich schwer tue. Oft einmal klappt die Arbeit und oft einmal nicht und die Arbeiten müssten eigentlich immer tadellos funktionieren und oft einmal klappt das nachher nicht und dann sehen sie, dass das nicht geeignet für mich ist. Ich wollte das einfach probieren, ich wollte das einfach einmal schaffen, dass ich die Anlehre einmal probiere wie das ist und das." (IA 637642)

Über die Formulierung, die Assistenten würden sehen, „dass das nicht geeignet für mich ist" (IA 640), deutet Anna an, dass nicht sie für den Arbeitsbereich, sondern dieser für sie nicht passend sei. Implizit ist das ein Verweis darauf, dass sie die Rahmenbedingungen als nicht passend für sie einschätzt, sich deshalb „schwer tue“ (IA 638) und deshalb wechseln wolle, sie habe sich das auch gut überlegt. Dennoch deutet Anna das eigene Scheitern an, indem sie sagt, sie hätte das probieren und schaffen wollen. Es wiederholt sich in diesem Zusammenhang eine Erfahrung, die Anna in der Kindheit im schulischen Kontext gemacht hat, dass sie nämlich von einem Bereich in einen wechselt, wo die Anforderungen niedriger sind bzw. ihr eher entsprechen. Druck und erforderliche Genauigkeit scheinen Anna zu überfordern. Wie damals macht sie es nur zum Teil an eigenen fehlenden Voraussetzun- 
gen fest, sondern macht das Umfeld bzw. die nicht für sie passenden Rahmenbedingungen zumindest mit verantwortlich dafür. Interessant für den Aspekt der Selbstbestimmung ist, dass Anna eine Entscheidung, die von Personen aus dem beruflichen Umfeld angedacht wurde, in ihrer Erzählung offenbar zur eigenen Entscheidung werden lässt bzw. Verständnis dafür artikuliert. Dies könnte so interpretiert werden, dass Annas Selbstwert sinkt, sie jedoch vor sich selbst und nach außen hin den Eindruck vermeiden möchte, dass Entscheidungen über sie hinweg getroffen werden und sie somit fremdbestimmt wird. Darin könnte sich eine unbewusste Strategie, Fremdbestimmung für sich und in der Außensicht umzudeuten und sie in gewisser Weise als eigene Entscheidungen erscheinen zu lassen, dokumentieren.

Annas Erfahrungen im Bereich der beruflichen Orientierung erscheinen somit ambivalent. Einerseits erfährt sie Unterstützung und bekommt die Möglichkeit, sich zu erproben. Andererseits erlebt sie dabei wieder Rahmenbedingungen, die es ihr schwer machen, Erfolgserlebnisse zu erzielen. Zudem erfährt Anna bei Nicht-Befolgen von Regeln auch Sanktionen, die sie aber als Möglichkeit zu lernen interpretiert. Letztendlich steht Anna vor einem Wechsel von einer Anlehre in den Bereich der Beschäftigung, was dem bereits in der Schulzeit erlebten erzwungenen Wechsel von der Regel- in die Sonderschule ähnelt. Daneben thematisiert Anna für die Zeit ab Eintritt in die berufliche Orientierungsphase weitere negative Erfahrungen in Form sexueller Übergriffe.

\section{Erfahrungen sexueller Übergriffe beeinträchtigen Streben nach Autonomie und Selbstbestimmung}

Ersucht, über die Zeit ab Eintritt in die berufliche Orientierung noch etwas zu erzählen, das ihr persönlich wichtig sei, erzählt Anna:
A: „Ja, ich habe einmal schlechte Erfahrungen auch gesammelt, also. Also, wie sollte ich sagen. Es war, wo ich das erste Mal beim Projekt gearbeitet habe, da hat es dort - da ist [...] schon ein älterer Mann gewesen, der hat auch unten im Garten gearbeitet, der hat mich - also begrapscht auch und das [...] und der hat mir auf den Hintern geschaut, auf die Brust geschaut, obwohl ich eine normale Arbeitskleidung angehabt habe, mit keinem tiefen Ausschnitt oder irgendwas. Also das ist mir noch nie bei einem Praktikum passiert, oder wo ich Kurse gegangen bin. Das ist mir in meinem ganzen Leben noch nie passiert, dass mich ein Mann angegrapscht hat.
I: Und wie haben Sie sich da gefühlt Frau, wo das passiert ist?
A: Voll scheiße, also wenn so etwas bei den Kursen oder auch beim Projekt - ist egal, wo das auch immer vorkommt, man fühlt sich als Frau total elend, also. Man hat auch zu wenig Beweise auch dafür und ich bin ( ) wie der letzte Dreck, auf gut Deutsch ge- sagt auch." (IA 424-440)

Auf die Frage, wie die Situation weitergegangen sei, führt Anna aus, dass sie dies einer Betreuerin gesagt habe. Sie erzählt weiter: ,ich habe zum Weinen angefangen, ich war nervlich fertig“ (IA 446-447). Die Betreuer seien ,zuerst 
unter Schock“ (IA 446) gestanden, „,waren alle auf einmal auch perplex“ (IA 449). Sie hätten gesehen, dass sie „,nervlich auch fertig gewesen“ (IA 450) sei. Sie sei dann zwei- oder dreimal zu einem Psychologen gegangen, „,betreut worden“ (IA 451). „Mittlerweile“, so Anna, ,kann ich auch mit so was umgehen“" (IA 451-452). Für den Mann, der übergriffig geworden war, habe es keine wesentlichen Sanktionen gegeben, wie Anna ausführt:

A: „Er wollte das abstreiten. Er wollte mich zum Narren machen, also dass ich komplett verrückt wäre. Und dann hat sich aber deswegen herausgestellt, dass ich recht gehabt habe und er hat nachher Pech gehabt. Er hat gesagt, ich soll keine Anzeige machen.

I: Und haben Sie dann eine gemacht oder?

A: Nein, habe ich nicht. Er hat nur damals eine Verwarnung gekriegt und seitdem hat er mich überhaupt in der Ruhe gelassen." (IA 482-487)

Über diese Erzählung wird sichtbar, dass Anna die Erfahrung eines sexuellen Übergriffes durch einen älteren Mann gemacht hat. Ihre gefühlsmäßige Reaktion ähnelt jener, als sie in der Schule gemobbt, von der Lehrerin abgewertet und in der Folge von der Regel- in die Sonderschule wechseln musste. Auf Nachfrage führt sie aus, dass „,man sich als Frau total elend“ (IA 439) fühle, wenn so etwas vorkomme. Auffallend ist die Verwendung derselben sprachlichen Formulierung für ihre Gefühle in beiden erlebten Kontexten. Anna spricht explizit auch hier davon, sie habe sich gefühlt , ,...] wie der letzte Dreck, auf gut Deutsch auch“ (IA 440).

Über Annas Beschreibung, dass sie ja gar keine aufreizende Kleidung getragen hätte [,obwohl ich eine normale Arbeitskleidung angehabt habe, mit keinem tiefen Ausschnitt oder irgendwas" (IA 432-433)], zeigt sich, dass sie ein gesellschaftlich häufig vermitteltes Bild verinnerlicht hat, nachdem Frauen Mitschuld an körperlichen Übergriffen zugewiesen wird, indem sie etwa durch aufreizende Kleidung das männliche Gegenüber dazu auffordern würden. Sie spricht auch häufig im Kontext sexueller Übergriffe Frauen mit Lernschwierigkeiten zugeschriebene mangelnde Glaubwürdigkeit an, indem sie formuliert: ,[...] man hat auch zu wenig Beweise dafür [...]“ (IA 439).

Im Zuge ihrer Erzählung über den Übergriff im Arbeitsprojekt spricht Anna von sich aus weitere negative Erfahrungen im Alter als Jugendliche an:

„Ich habe da noch etwas vergessen zu erzählen. Wo ich 14 Jahre alt gewesen bin, da bin ich auch noch Schule gegangen und da hat es da draußen - gibt es einen, der was mich auch verfolgt hat. Ich habe auch damals etwas Schlimmes durchgemacht. Der hat mich halt auch alles ausgefragt, was ich alles über Sex weiß und das und wenn man das als Jugendliche schon einmal - einmal so etwas in der Art durchgemacht hat- man denkt ja darauf nicht, dass es noch einmal irgendwo auf einen zukommt auch, aber mittlerweile weiß ich, wie ich mich zu wehren habe." (IA 453-458)

Bereits als 14-Jährige war Anna demnach einem sexuellen Übergriff in ihrem ländlichen Wohnumfeld durch einen Mann ausgesetzt, der sie ,alles ausgefragt, was ich über Sex weiß und das“ (IA 455) habe. Sie habe dann nicht geglaubt, dass sie das noch einmal durchmachen müsse. 
Der Eindruck, der sich in der Passage über den sexuellen Übergriff im Projekt ergeben hat, dass Anna keine konkreten Handlungsstrategien parat hatte, verdichtet sich. Anna hatte als Jugendliche in der Situation keine Handhabe gegenüber dem Mann. Sie äußert aber nochmals (wie bereits in IA 452), dass sie nunmehr wisse, wie sie sich ,zu wehren habe“ (IA 458). Eine Nachfrage ergibt, dass Anna nicht durch Unterstützung ihres Umfeldes zu dieser Selbsteinschätzung gelangen konnte, dass ihr also keine Techniken, Strategien in Schule, von den Eltern oder im Projekt vermittelt wurden:

I: „Haben Sie dann was gelernt, wie Sie sich wehren können? Oder woher wissen Sie das jetzt?

A: Ich habe viel von - überhaupt vom Fernsehen auch, habe ich bei so gewissen Filme, wie man sich so verstecken tut auch. Wenn dich - wenn du weißt - du fühlst dich beobachtet auch und das. Ich habe nur so gewisse Kleinigkeiten so gelernt. Ich bin keinen Selbstverteidigungskurs gegangen, sondern - ich habe halt einfach so kleine Sachen halt so - was man in den Filmen halt sieht auch. Also sich so zu verstecken zu lernen halt auch oder irgendwie halt auszuweichen auch. So Kleinigkeiten halt auch.“ (IA 459-465)

Anna hat sich vielmehr über das Fernsehen dort beobachtete Handlungsstrategien abgeschaut und angeeignet. Die in der o.a. Sequenz erwähnten Strategien sind passiv orientiert und auf Rückzug ausgerichtet. Anna denkt im Falle des Falles daran, sich zu verstecken oder auszuweichen. Die Handlungsbzw. Vermeidungsstrategien, die Anna sich angeeignet bzw. für den Fall eines drohenden Übergriffes dadurch parat hat, schränken sie in ihrem persönlichen Freiraum ein, indem sie ihre Bewegung im öffentlichen Raum hemmen. Diese Strategie, Wege und Situationen zu meiden, in deren $\mathrm{Zu}-$ sammenhang sie Gefahr vermutet, wird für Anna zur Gewohnheit. Sie führt an, dass sie dies „einige Jahre, vor dem Typ da draußen“ (IA 499) so praktiziert habe.

Anna spricht in einer weiteren Sequenz nochmals an, dass sie sich ,zu wehren auch“ (IA 541) wisse. Sie wisse, dass die Männer eine „stärkere Körperkraft haben“ (IA 542), dass eine „Frau machtlos dagegen ist, aber gewisse Methoden zum Wehren gibt es immer" (IA 543-544). Anna spricht in dieser späteren Passage auch aktive Formen des Sich-Wehrens an:

A: „Also auch wenn man zwischen die Füße tritt oder sonst irgendwie wegschupft oder irgendwas, wie es halt einem gelingt. Sonst hat man eigentlich Pech gehabt nachher.

I: Und wo haben Sie das gelernt, wie man sich da wehrt? Weil zuerst haben Sie eben gesagt, wie man sich versteckt oder so -

A: Das habe ich nur von den Filmen auch.

I: Und das, wie man sich wehrt, wo haben Sie das gelernt?

A: Also auch so von den Filmen her, weil ich habe ab und zu auch so Karate Filme so geschaut, oder so - wenn so Krimiserien gewesen sind, wo einer auch die Frau verfolgt hat und die hat sich probiert zu wehren, auch so mit Wegschupfen und das. Das sind so Kleinigkeiten halt.“ (IA 545-553)

Anna verdeutlich somit, dass sie sich über das Fernsehen für sich selbst auch aktive Handlungsstrategien angeeignet hat und parat hält, die sie im Falle 
eines drohenden Übergriffes anwenden könnte. Die aktiven Strategien dürfte Anna noch nicht erprobt haben, die passiven, auf Rückzug orientierten Strategien hat sie praktiziert, diese sind ihr vertraut, schränken sie allerdings in ihrem Freiraum ein. Anna selbst schätzt ihre Strategie allerdings als wirksam ein und hält fest, sie könne „mittlerweile [...] auch mit so was umgehen“ (IA 451-452).

In unterschiedlichen Passagen im Interview wird deutlich, dass Anna von Personen aus dem sozialen Umfeld keine nennenswerte konkrete Unterstützung erfahren hat, die ihr dabei helfen, die Situation zu bearbeiten und für sich daraus auch geeignetere Handlungsstrategien zu entwickeln. Die Eltern zeigten sich in Annas Einschätzung zwar in beiden Fällen „total sauer" (IA 476) bzw. „total stinksauer“ (IA 510), wollten ihr das Erlebnis als Jugendliche aber ,zuerst nicht glauben“ (IA 515). Anna erfährt somit vieles von dem, was im Zusammenhang mit sexuellen Übergriffen an Frauen mit Behinderung üblich ist: Ihre Glaubwürdigkeit wird in verschiedenen Kontexten angezweifelt, der Täter stammt aus dem sozialen Umfeld, streitet seine Tat ab [,er wollte das abstreiten, er wollte mich zum Narren machen, also dass ich komplett verrückt wäre" (IA 482)]. Auf das Ersuchen Annas ihrer Mutter gegenüber, den Mann anzuzeigen, reagiert diese mit Ablehnung:

„Nein, ich habe gesagt, bitte Mama eine Anzeige und sie, nein, nein, nein. Die wollten mir das zuerst nicht glauben.“(IA 514-515)

Im Zusammenhang mit ihrem geäußerten Wunsch, weiterhin wie gewohnt am Land leben zu wollen (IA 712), bringt Anna die Erfahrung eines relativ aktuell erlebten weiteren Übergriffes ein. Am Anfang sei es eine „lustige Geschichte“ (IA 719) gewesen. Aber dann sei ,ihr der ein bisschen komisch geworden bei der Sache“ (IA 724-725), er sei „ein bisschen verrückt im Kopf gewesen“ (IA 725). Sie erzählt weiter, dass er sie „knapp vergewaltigt“ (IA 745) habe und führt die Situation und ihre Reaktion aus:

„Ja, ich habe nur gesagt- ich habe ihn zuerst weggeschupft auch, weil er mich so niedergedrückt hat auch und das hat mir weh getan auch und dann habe ich gesagt, aus ich will nicht mehr, habe ich gesagt, geh runter von mir und dann wollte er nicht. Dann habe ich ihn weggeschupft, dann habe ich ihn da vorne beim Brustkorb gekratzt auch. Es war nur ganz leicht, hat er gesagt, spinnst du, das hat ja wehgetan, dann sage ich, du hast mir auch gerade wehgetan, habe ich gesagt, bist selber schuld. Und dann habe ich gesagt, bitte fahre mich jetzt heim, dann ist er normal heim gefahren, hat er mich heim gebracht, ganz normal und nachher, nach ein paar Tagen habe ich das eben meiner Mama auch erzählt. Und dann habe ich gesagt, von so einem Typen will ich nichts mehr wissen, der was Cartoon-Vergewaltigungen anschaut, der ist nicht ganz dicht im Kopf, also da hat man nachher schon ein bisschen ein mulmiges Gefühl bei der Sache." (IA 755-765)

Diese Passage zeigt, dass Anna in der konkreten Situation des Übergriffes auf ihr Repertoire zurückgreifen konnte, dass sie sich aus Fernsehfilmen abgeschaut hat. Anna wendet in der erzählten Szene das ihr aus Filmen bekannte 
„Wegschupfen“ an. Möglicherweise bestärken sie die neuen Erfahrungen der Unterstützung und Anerkennung ihrer Interessen als Entscheidungskriterien in der Phase der beruflichen Orientierung darin, sich zu artikulieren und zur Wehr zu setzen. Diese auch vorliegende Orientierung an Selbstbestimmung dokumentiert sich bereits vorab an der Erzählung von Anna über das vor dem Übergriff geäußerte Ansinnen dieses Mannes, mit ihm zusammen zu ziehen:

„Ich will aber nicht gleich nach drei Monaten, wo ich ihn gerade erst einmal kennen gelernt habe, gleich ausziehen. So etwas macht man nicht so was. Ich würde nichts sagen nach ein, zwei Jahren oder wann, dass ich sage ok, aber nicht nach drei Monaten gleich - der hat das einfach vor mir beschlossen, ja Anna zieh einfach zu mir. Dann habe ich gesagt, ich ziehe gar nirgend wohin.“" (IA 733-737)

Auch für die Zeit nach dem Übergriff beschreibt Anna ein eigenes zunehmend aktiveres Verhalten:

„Ich habe ihn angerufen, habe ich gesagt, du ich will Schluss machen. Dann sagt er: warum? Ja ich habe die Schnauze voll von dir. Sagt er: hast du einen anderen? Sage ich: nein, ich habe einfach nur die Schnauze voll von dir. Und der ist auch ziemlich auch nervig gewesen auch. Richtig, dazwischen zeitweise so aggressiv, aber auf alle Fälle hat er mich um halb eins in der Nacht angerufen, er wird sich was antun und ich habe mir gedacht, auf solche Spielchen - machen sie eh meistens, dabei tun sie ja gar nicht was. Und nachher habe ich ein paar Jahre nicht mehr gesehen und nachher wo ich das zweite Mal beim Projekt gearbeitet habe und nachher ist er durch Zufall auch dort rüber gekommen und hat dort auch gearbeitet, aber er - wo er mich damals gesehen hat, hat er immer noch einen Schleim auf mich gehabt. Habe ich gesagt, du brauchst nicht einen Schleim auf mich haben, das war damals meine Entscheidung." (IA 769-779)

Daneben spricht Anna aber nochmals an, dass sie weiterhin die passiven, auf Rückzug orientierten Strategien praktiziert, diese sind ihr vertraut. Sie greift dies etwas später in einer Beschreibung ihrer ambivalenten Einstellung zum Fortgehen nochmals auf:

„Ja, ich meine Fortgehen möchte ich schon gerne auch wieder einmal und so, aber alleine macht es halt auch keinen Spaß.“ (IA 793-794)

Anna führt an, dass Freundinnen und der Bruder v.a. aus Gründen der Arbeit keine Lust mehr hätten, fortzugehen. Abends alleine wegzugehen, stellt für Anna keine Option dar:

„Nein, das ist ja langweilig nachher. Da fühle ich mich irgendwie auch so unwohl. Also wenn ich jetzt irgendwo alleine - also weggehe gegen Abend oder so. Unter Tags, wenn ich einmal so in die Stadt gehe, macht es mir weniger aus. Ich meine, sonst habe ich meistens auch Freundinnen oder so Arbeitskolleginnen, wo wir meistens nach der Arbeit auch in die Stadt gehen auch und so, aber sonst - also -“ (IA 805-808)

In Verbindung mit Annas Vermeidungsstrategien, die sie in Zusammenhang mit den sexuellen Übergriffen entwickelt hat, ist davon auszugehen, dass sie sich deshalb abends alleine ,unwohl“ fühlt und alleine nicht fortgeht, weil sie auch gefährliche Situationen vermeiden will. Dies spricht Anna explizit auch 
in Zusammenhang mit der Erfahrung des dritten Übergriffes an, bei dem sie sich erstmals zur Wehr gesetzt hatte. An ihrer aktuellen Einstellung zum Ausgehen am Abend haben diese Erfahrungen nichts Wesentliches verändert:

„[...] wie gesagt, ich habe schlechte Erfahrungen gemacht mit Männern und da bin ich heute auch noch immer vorsichtiger. Deswegen gehe ich alleine fast nie fort, also." (IA 750-752)

Es bestätigt sich also, dass Anna durch diese Erfahrungen in ihrem Handlungsspielraum eingeschränkt wird. Sie kann zwar im Notfall auf aktive Handlungsstrategien zurückgreifen, die sie sich selbst über Fernsehfilme angeeignete hat. In ihrer alltäglichen Handlungspraxis zeigt sich aber, dass ihr alte, selbst erarbeitete und über Jahre praktizierte Strategien der Vermeidung vertrauter sind und sie diese auch weitgehend praktiziert.

Alle Übergriffe, die Anna erlebt hat, bleiben - abgesehen von einer Abmahnung an der Projektstelle - ohne tatsächliche Konsequenzen für die Täter. Die Eltern verweigern - möglicherweise wegen eigener und angenommener fremder Anzweiflung von Annas Glaubwürdigkeit - eine von Anna gewünschte Anzeige. Anna erlebt somit in gewisser Weise eine Missachtung oder Abwertung ihrer Person, indem ihre Glaubwürdigkeit angezweifelt und Übergriffe auf sie nicht geahndet werden. In der Kombination mit den von ihr entwickelten, sie selbst einschränkenden Vermeidungsstrategien dürfte dies massive Konsequenzen für Annas Orientierung im Zusammenhang mit ihrem Wunsch nach gesellschaftlicher Partizipation haben. Erste Erfolge im Versuch, sich abzugrenzen und einem Übergriff etwas entgegenzusetzen, vermögen vorerst nichts Wesentliches an ihrer offenbar durch die mehrfachen negativen Erfahrungen entwickelten, verfestigten Vermeidungsstrategie zu ändern.

\section{Wunsch nach Selbstbestimmung wird durch stark fremdbestimmte Kontexte stärker}

Deutlich wird im Kontext der Erzählungen und Beschreibungen im Zusammenhang mit den Übergriffen dennoch eine zunehmende Orientierung am Wunsch, über sich selbst zu bestimmen. Verbal wird dies besonders deutlich an einem Satz, mit dem Anna sich weigert, der Aufforderung des Mannes, zu ihm zu ziehen, nachzukommen:

„Ich kann entscheiden, was ich will““ (IA 738)

Anna ist es offenbar auch wichtig, in der Interviewsituation festzuhalten, dass sie selbstbestimmte Entscheidungen trifft. Im Kontext der erlebten Erfahrungen scheint diese Orientierung Annas einerseits hervorgerufen, andererseits jedoch massiv beeinträchtigt durch die erlebten Übergriffe. Zudem zeichnet sich ab, dass sich das Verhalten von Personen im sozialen Umfeld, die Annas Glaubwürdigkeit in Frage stellten und ihre Wünsche nach Sanktion des Tä- 
ters negierten, negativ auf Annas Selbstbild auswirkt. Die damit verbundenen Gefühle scheinen sich in latenten Selbstzweifeln, Beibehalten von Vermeidungsstrategien und Orientierung am vertrauten sozialen Rahmen zu manifestieren. Dieser Rahmen ist jedoch aus Annas Wahrnehmung von Abhängigkeit charakterisiert, was sie an finanziellen Aspekten deutlich macht.

\section{Wunsch nach finanzieller Unabhängigkeit}

Anna macht auf Nachfrage nach der Höhe der über die Anlehre lukrierten Mittel deutlich, dass sie darüber genau informiert ist:

„Also, die Beschäftigung, die verdient im Monat maximal nur 25 Euro. Also die Bezahlung für die Anlehre ist im ersten Anlehrjahr nur 42 Euro pro Monat, aber wir kriegen Urlaubsgeld und Weihnachtsgeld kriegt jeder, auch die in Beschäftigung auch. Aber das ist überhaupt- ist jetzt egal ob jetzt Anlehre oder Beschäftigung, es ist bei beiden wenig Geld." (IA 622-625)

In einer relativ langen Passage zum Thema Geld dokumentiert sich, dass Anna sehr gut über die Zusammenhänge von fehlenden eigenen finanziellen Mitteln und der Abhängigkeit von den Eltern Bescheid weiß. Diese Passage ist in ihrer inhaltlichen Dichte als Fokussierungsmetapher zu sehen und verweist auf die große Bedeutung dieser Frage für Anna:

„Wie sollte ich sagen, man muss halt wirklich schauen, dass man halt auch ( ) wenn man sich ein Leiberl ${ }^{92}$ kauft, dann muss man halt schauen, dass es im Abverkauf ist. Also maximal fünf oder eventuell vielleicht zehn oder sieben Euro. Also je nachdem, wie es passt und einem gefällt. Ich meine, so wie meine Eltern, die kriegen auch noch Kinderbeihilfe für mich halt auch noch und das ist aber auch nur alle zwei Monate. Ich kriege wohl von meinen Eltern genauso nebenbei auch ein Geld. Also es kriegt jeder, der was drinnen arbeiten tut. Das kriegt jeder von seinen Eltern noch, einen Zuschuss auch. Also mir wäre lieber, dass ich vom Land her - dass ich da den normalen- dass jeder, auch die als Beschäftigung sind und auch die Anlehre, dass jeder den normalen Lohn kriegen würde. Das wäre mein größter Wunsch, also das möchte ich für meine ganze Firma jetzt aussprechen. Also es wäre mein größter Wunsch, dass - [...] als ich möchte halt schon gerne den normalen Lohn kriegen, was die Assistenten genauso auch normal verdienen." (IA 669-682)

Anna macht hier sprachlich durch mehrere Wiederholungen explizit ihren Wunsch nach finanzieller Unabhängigkeit deutlich. Der von Anna schon in vielen Passagen im Interview verwendete Begriff ,normal“ wird hier von ihr wieder mehrmals gebraucht: Anna möchte den „normalen Lohn“, entsprechende Formulierungen erfolgen fünfmal. Zudem möchte sie „das Urlaubsgeld und den normalen Doppelten kriegen, so wie die Assistenten“ (IA 694697). Anna macht also explizit deutlich, dass sie entsprechend den Personen, die als nicht-beeinträchtigte Menschen im Projekt als Assistenten arbeiten, entlohnt werden möchte und fordert also explizit Gleichstellung hinsichtlich

92 Leiberl $=$ T-Shirt. 
Lohn/Gehalt ein. Sie macht sich in dieser Angelegenheit zur Sprecherin aller Personen mit Beeinträchtigung, zumindest an ihrer Arbeitsstelle. Sie beginnt zuerst einen Satz aus der Ich-Perspektive, schwenkt aber unmittelbar um und hält fest ,mir wäre lieber, dass ich [...] den normalen - dass jeder, auch die in Beschäftigung sind und auch die Anlehre, dass jeder den normalen Lohn kriegen würde" (IA 677).

Über die Angabe ihres Alters dokumentiert sie Bewusstsein darüber, dass es aus ihrer Perspektive nicht üblich ist, im Alter von 28 Jahren von den Eltern finanziell abhängig zu sein und nicht selbstbestimmt über eigenes Einkommen verfügen zu können:

„Aber so, dass halt der Unterhalt abgedeckt halt ist. Also so, dass ich halt über ein Monat locker drüber komme. Dass ich nicht alle Ritt ${ }^{93}$ meine Eltern auch fragen brauche, Mama, darf ich ein Geld haben und das, weil ich bin 28 Jahre." (IA 686-688)

Anna führt jedoch auf Nachfrage aus, dass sie mit einem „normalen Lohn“ weiter am Land leben möchte, es wird in dieser Passage nicht deutlich, ob sie damit auch meint, sie wolle bei ihren Eltern bleiben. Zumindest wird angedeutet, dass sie im vertrauten Umfeld verbleiben möchte. Den Verdienst würde sie „ganz normal - ganz normal auf die Seite sparen“ (IA 705-706), was eher darauf hindeutet, dass sie ihn nicht für eine eigene Wohnung brauchen würde. Andererseits sagt sie, sie wolle so viel verdienen ,so, dass halt der Unterhalt abgedeckt halt ist“ (IA 686), dass sie über einen Monat „locker drüber komme“" (IA 687).

Anna erscheint auch in Zusammenhang mit der Frage der finanziellen Unabhängigkeit letztendlich ambivalent. Einerseits weiß sie gut darüber Bescheid, dass finanzielle Abhängigkeit Autonomie und Selbstbestimmung einschränkt und wünscht sich aus diesem Grund eindrücklich finanzielle Eigenständigkeit. Andererseits scheint ihr das vertraute Umfeld als Raum, der ihr Schutz und Unterstützung durch die Eltern bietet und den sie daher nicht verlassen will. In der aktuellen Situation scheint finanzielle Unabhängigkeit real für Anna noch in weiter Ferne zu liegen bzw. in noch weitere Ferne zu rücken, zumal ein Wechsel von der Anlehre in die Beschäftigung bevorsteht, wodurch ihre ohnehin kärglichen Mittel nochmals eingeschränkt werden und Abhängigkeiten verfestigt werden.

\section{Eingeschränkte Lebensperspektiven und -entwürfe durch finanzielle Abhängigkeit}

Wie sehr finanzielle Abhängigkeit sich auf die Wahrnehmung der eigenen Lebensperspektiven und -entwürfe auswirkt, dokumentiert sich bei Anna über ihre Ausführungen zur Frage nach besonderen Wünschen:

Alle Ritt $=$ immer wieder, laufend 
I: „Wie soll Ihr Leben weiter ausschauen, wenn Sie sich das so - ja wenn so eine Fee kommen würde und sagen würde, ich erfülle dir deine Wünsche.

A: Also wünsche hätte ich schon ein paar ja. Ich meine, so wie gerade vorige Woche, [...] ich habe da auch was- ( ) einen gewissen Betrag halt auch gewonnen. Das war ein reiner Zufallstreffer. Es ist auch nicht weiß Gott wie viel gewesen, aber nur halt ein bisschen was. Aber, ja also ich würde trotzdem mein Leben normal weiterleben, wie sonst halt auch immer, vielleicht kommt einmal irgendwann einmal ein großer Lottogewinn, aber ich würde trotzdem ganz normal weiterleben, wie sonst halt auch immer. Also für mich ist halt nur wichtig, dass meine Familie, dass ich gesund bin und, dass es meiner Familie gut geht. Das ist für mich am meisten wichtig auch. Dass ich meine Arbeitsstelle auch behalten kann und - also sonst bin ich eigentlich wunschlos glücklich, also.“ (IA 823-833)

Gefragt nach Wünschen ans Leben spricht Anna zunächst einen realen kleinen Lottogewinn an und überlegt, wie das Leben mit einem größeren Gewinn weitergehen würde. Sie spricht an, dass sie ihr Leben dennoch ,normal weiterleben“ (IA 829) würde, so wie sie es auch für einen „,normalen Lohn“ in Passage geäußert hat. Ansonsten führt sie eher allgemeines wie Gesundheit und Wohlbefinden der Familie an, was auf deren große Bedeutung für Anna schließen lässt.

Eher beiläufig äußert sie dann den bereits einmal ähnlich (vgl. IA 787) formulierten Satz „Irgendwann wird einmal der richtige Mann kommen“ (IA 835). Dies scheint doch eine deutliche Orientierung von Anna zu sein, die sie allerdings eher verhalten thematisiert, so wie etwas, zu dem sie aktiv nicht beitragen kann. Darauf lässt auch die zweimal gebrauchte Formulierung „dann habe ich Glück gehabt“ (IA 838, 845) im Zusammenhang mit den Vorstellungen darüber schließen.

„Also wenn er auch ein Landmensch ist, dann habe ich Glück gehabt. Also, dass wir vielleicht irgendwo da in der Nähe ein Haus bauen oder Haus kaufen würden. Also Kinder möchte ich halt keine haben. Also ich meine, ich kann schon Kinder kriegen und das, aber heutzutage- wenn ich so die anderen Familien anschaue, die gehen auseinander, dann sind die Kinder da. Und da tun mir die Kinder nachher so leid, weil sie nachher hin und hergerissen werden und das. Das ist das ( ) was mich traurig macht. Also deswegen würde ich selber auch keine Kinder haben heute. Also, wenn ich einen Partner haben möchte - also kriege, der vielleicht auch keine Kinder haben will, dann habe ich vielleicht Glück gehabt auch. Ich meine, auch wenn er Kinder haben will und das ich meine, es ist wirklich gut zu überlegen und da habe ich Angst, dass er mich [...] auch irgendwann einmal verlässt und das. Dass ich nachher mit dem Kind dann dastehe." (IA 838-848)

Bei Anna wird das Zusammenleben mit dem Freund auch explizit in die Nähe des derzeitigen Wohnumfeldes verortet, was darauf verweist, dass die räumliche Nähe zu ihrem derzeitigen Wohnumfeld und somit wohl in gewisser Weise ein vertrauter Rahmen gewünscht wird.

In ihrer Argumentationskette kommt Anna auch noch auf den Zusammenhang zu fehlenden finanziellen Ressourcen zu sprechen. Sie führt an, dass man für Kinder Geld brauche, ihnen etwas bieten müsse und wenn sie ,nur 
als Beschäftigung arbeiten tue, ich [...] nachher meinem Kind nicht so viel bieten“ (IA 863) könne. Sie verweist darauf, dass sie es sich mit einem Lottogewinn schon vorstellen könne und macht damit deutlich, dass mangelnde bzw. fehlende finanzielle Ressourcen offenbar ein zentraler Grund für die Ablehnung eigener Kinder sind.

Anna ist hinsichtlich des Zusammenhanges von finanziellen Ressourcen und selbstbestimmtem Leben reflektiert. Sie weiß um die Zusammenhänge mit Abhängigkeiten aufgrund fehlender Ressourcen Bescheid und resümiert für sich auch, dass sie keine Kinder möchte, weil sie ihnen nichts bieten kann. Sie führt auch noch die Angst an, mit einem Kind sitzen gelassen zu werden. Da Anna äußert, dass sie sich bei einem Lottogewinn doch vorstellen könnte, Kinder zu bekommen, dürften mangelnde finanzielle Ressourcen das ausschlaggebende Argument für die hier explizierte Ablehnung eigener Kinder sein. Anna ist es aber grundsätzlich wichtig, darauf hinzuweisen, dass sie auch in der Sichtweise anderer Personen eine geeignete Mutter wäre:

„Es haben auch schon Einige zu mir gesagt, Anna, ich könnte mich dir gut vorstellen mit kleinen Kindern, habe ich gesagt, ja, ja, habe ich gesagt.“ (IA 858-859)

\subsubsection{Zusammenfassung der Fallrekonstruktion Orientierung C - „Anna“}

Die über die Fallrekonstruktion „Anna“ dargestellte Orientierung ist gekennzeichnet vom Wunsch nach Partizipation, Autonomie und Selbstbestimmung. Negative Erfahrungen, Abhängigkeiten und/oder fehlende Unterstützung im Zusammenhang mit der Bewältigung herausfordernder Situationen führen aber zum Bedürfnis nach Erhalt vertrauter Strukturen, auch wenn diese selbst konfliktbehaftet sind bzw. ambivalent erlebt werden.

Kompakt dargestellt dokumentiert sich in:

Orientierung $\mathrm{C}$

Starke Orientierung an Selbstbestimmung, Partizipation und Normalisierung bei gleichzeitigem Bedürfnis nach Erhalt eines vertrauten, geschützten Rahmens

Diese Orientierung kennzeichnet ein deutlich wahrnehmbares Streben nach Selbstbestimmung, Partizipation und Normalisierung, das aber mit verschiedenen Vorbehalten und Ängsten verbunden sein und zögerlich erscheinen kann. Der Aspekt der Vermeidung herausfordernder Situationen innerhalb oder außerhalb des vertrauten Rahmens ist dabei zentral.

Negative Erfahrungen im Zusammenhang mit der eigenen Behinderung, denen wenig entgegengesetzt werden konnte, beeinträchtigen das eigene Selbstbild, die eigene Alltagspraxis und zukünftige Lebensentwürfe. Erlebte und bewusst wahrgenommene Fremdbestimmung im Alltag, Diskriminierung, Stigmatisierung und Abwertung wirken stark einschränkend, indem sie eine eher defizitorientierte Sichtweise der eigenen Person befördern. Eine 
positive Bewertung von Sondereinrichtungen kann Folge solcher Erfahrungen sein, wenn der dort gebotene Rahmen als mehr an den eigenen Bedürfnissen ausgerichtet erscheint. Dies dokumentiert sich am Beispiel der dargestellten Fallrekonstruktion in einer argumentativen Bevorzugung der Sonderschule als Ort, in dem Erfolgserlebnisse möglich sind und über Beziehung zu einer Bezugsperson Interesse an und Anerkennung der eigenen Person erlebt wird.

Erfahrungen von Übergriffen - nicht nur, aber insbesondere in Form sexueller Gewalt - in ihren unterschiedlichen Formen wirken stark hemmend im Zusammenhang mit der Ausprägung einer Orientierung, die in Richtung Selbstbestimmung und Autonomie weist. Können solche Erfahrungen nicht begleitet und mit professioneller Unterstützung aufgearbeitet werden, dann führen sie oft zum Rückzug auf das vertraute Umfeld. Situationen, in denen neuerlich Erfahrungen von Abwertung bis zu Übergriffen als drohend vermutet werden, werden häufig vermieden. Dadurch werden einerseits Handlungsspielräume eingeschränkt. Andererseits werden in diesem Prozess aber auch vielfältige Ressourcen und Potentiale sichtbar, die sich in individuellen Bewältigungsstrategien zeigen. Dies dokumentiert sich am Beispiel der vorab dargestellten Fallrekonstruktion an der Übernahme von Vermeidungs- und Verteidigungsstrategien über das Medium Fernsehen, die es ermöglichen, in einem eingeschränkten Rahmen doch weiterhin am sozialen Umfeld zu partizipieren.

Besonders markant wirken sich negative Erfahrungen innerhalb des nahen sozialen Umfeldes aus. Dies reicht vom Nicht-Beachten und/oder Unterdrücken der persönlichen Bedürfnisse durch Bezugspersonen bis zu Gewalterfahrungen innerhalb dieses Kontextes. Einerseits entwickelt sich so häufig der Impuls, sich daraus zu lösen. Andererseits werden aufgrund bestehender Abhängigkeiten Konflikte mit Personen vermieden, die diesen Rahmen bilden und ausprägen. Im Zentrum steht dann das Bedürfnis, den Rahmen erhalten zu können, weil er zumindest eine vertraute Komponente darstellt und häufig auch ohne Alternative erscheint. Da zu diesen Personen vielfältig ausgeprägte Abhängigkeiten - insbesondere aber nicht nur im materiellen Bereich - vorliegen, über die weitgehend auch Bewusstsein besteht, ist eine Ablösung zudem auch aus damit verbundenen Gründen häufig gar nicht möglich bzw. erschwert Schritte in Richtung eines autonomen Lebens. In diesem Zusammenhang werden auch einengende, konfliktbehaftete und fremdbestimmte Rahmenbedingungen dem unbekannten, fremden Kontext vorgezogen. Allerdings ist das Leben unter diesen Rahmenbedingungen oft auch geprägt von latenter Unzufriedenheit, die sich aus dieser Ambivalenz ergibt. Aktuellen Hindernissen, Barrieren und Herausforderungen wird auf Basis dieser Erfahrungen insgesamt eher passiv und ängstlich begegnet. Sind Erfahrungsräume in der Sozialisation also so ausgestaltet, dass sie stark wahrnehmbar als abwertend, ausgrenzend, stigmatisierend bis übergriffig erlebt 
wurden, kommt es häufig zu einer ambivalent erscheinenden Orientierung: Einerseits wird ein selbstbestimmtes, autonomes Leben angestrebt, das sich von den Bedingungen, die in den Erfahrungsräumen gegeben waren, abgrenzt. Fremdbestimmte Kontexte, Strukturen und Regeln werden vehement kritisiert und abgelehnt. Andererseits ist aber auch eine Orientierung erkennbar, diese aus verschiedenen Gründen und Motiven zu erhalten bzw. darin zu verbleiben. Der dadurch gebildete Rahmen wird einerseits als einengend, andererseits aber auch als vertraut erlebt. In diesem Sinne ist diese Orientierung als ambivalent zu bezeichnen.

Deutlich wahrnehmbar prägen Wünsche nach mehr gesellschaftlicher Teilhabe im Bereich des öffentlichen Lebens und in der Freizeitgestaltung diese Orientierung. Soziale Beziehungen zu Peers erscheinen besonders wichtig und stellen manchmal für das eigene Leben zentrale Orientierungspunkte dar. Entsprechende Kontakte stellen sich unter den gegebenen Rahmenbedingungen, die auch mit eingeschränkter Mobilität verbunden sind, häufig aber als erschwert dar. Beziehungen zu nicht beeinträchtigten Personen, die nicht dem familiären Umfeld angehören, sind eher selten.

Das Bedürfnis nach gesellschaftlicher Partizipation geht auch mit dem Wunsch nach verbesserter finanzieller Ausstattung oder auch finanzieller Unabhängigkeit einher. Materielle Abhängigkeit wird vorwiegend in ihren Auswirkungen auf die eigene Alltagsgestaltung - in erster Linie über eingeschränkte bzw. fremdbestimmte Einkäufe thematisiert - wahrgenommen. Es besteht aber auch Bewusstsein über dadurch eingeschränkte Lebensperspektiven in Hinblick auf autonomes Leben. Fragen, die daran geknüpft werden, wie Partnerschaft und Kinderwunsch, werden vor diesem Hintergrund weitgehend ausgeblendet oder eher unrealistisch betrachtet.

\subsubsection{Fallübergreifende Ausprägungen von Orientierung C}

Die in der angeführten Fallrekonstruktion „Anna“ anhand zentraler Dimensionen herausgearbeitete Orientierung wird fallübergreifend in verschiedenen Ausprägungsvarianten sichtbar. Gemeinsamkeiten hinsichtlich der Bearbeitung verschiedener „Themen und Problemstellungen“ (Nohl 2012: 52), die sich in unterschiedlichen Fällen auf unterschiedliche Weise abbilden, werden deutlich.

Im Folgenden führe ich einige Beispiele aus weiteren Interviews dafür an, also Belegerzählungen, die zentrale Dimensionen von Orientierung $\mathrm{C}$ deutlich machen. Die also die ,starke Orientierung an Selbstbestimmung, Partizipation und Normalisierung bei gleichzeitigem Bedürfnis nach Erhalt eines vertrauten Rahmens " dokumentieren.

Der Wunsch, als Person und von den Lebensumständen her dem als gesellschaftliche „Normalität" wahrgenommenen Konstrukt zu entsprechen, dokumentiert sich auch an Angelikas Ausführungen im Kontext mit der $\mathrm{Zu}$ - 
schreibung von „Behinderung“. Angelika führt aus, von ihrer jüngeren Schwester als ,dumm, behindert“ (IAng 374) bezeichnet worden zu sein, und macht deutlich, dass sie sich von den damit verbundenen Zuschreibungen abgrenzt. Sie greift die Ausführungen der Schwester ein zweites Mal auf und führt an: ,,ja, bin behindert behauptet sie, als ob ich nicht weiß, was behindert heißt" (IAng 378). Gefragt nach ihrer Definition von „behindert-Sein“ führt Angelika aus: „das heißt, das ist ein Mensch, der nicht reden kann, das ist es, ja, sie sogt so was einfach zu mir" (IAng 381). Hier wird sichtbar, dass Angelika selbst den Begriff der Behinderung mit eigenen Zuschreibungen verbindet, zentral ist dabei für sie das Unvermögen, zu reden. Für Angelika besteht Behinderung also in der Unfähigkeit zu kommunizieren, was sie selbst ja im Interview ganz offensichtlich widerlegt und sich damit auch davon abgrenzen kann. Auch die Attestierung einer mit der Behinderung verbundenen „Faulheit“ (IAng 386) will Angelika entkräften, indem sie anführt, welche Tätigkeiten sie im Haushalt ausführt. Als „behindert“ bezeichnet zu werden, kommt für Angelika einer Abwertung gleich. Sie führt Argumente ins Treffen, die belegen sollen, dass sie den damit verbundenen Zuschreibungen nicht entspricht und somit auch nicht als „behindert“ eingestuft werden kann.

Die Orientierung an einem vertrauten sozialen Rahmen und gewohnten Abläufen kommt etwa auch in Marlenes Ausführungen über das Wohnen zum Ausdruck. Sie führt an, dass sie auch bei der Möglichkeit, auszuziehen, weiter bei ihrer Mutter wohnen wolle: „Ich kann ja ausziehen, aber ich mag nicht ausziehen, mag ich nicht“" (IMarl 474). Sie präzisiert, es würde ihr schwerfallen, von ihrer Mutter wegzugehen (vgl. IMarl 479). Trotz der von Marlene im Interview ambivalent beschriebenen Beziehung zu ihrer Mutter (u.a. IMarl 140-145) ist der Wunsch nach Erhalt des vertrauten Rahmens stärker als jener nach Autonomie.

Auch Angelikas Ausführungen dokumentieren eine Orientierung am vertrauten System der Herkunftsfamilie, auch wenn sie dort Abwertung durch die Schwester erlebt und wenig Unterstützung bei der Umsetzung ihrer Wünsche durch ihre Eltern bekommt. Die Beschreibung ,meine Eltern sind ja eh gut, meine Eltern sind ja eh nett, sagen wir einmal so" (IAng 283) dokumentiert dementsprechend eine verhaltene Zufriedenheit mit der Qualität der Beziehung. Der Wunsch nach Ablösung aus diesem System wird über das gesamte Interview allerdings kaum deutlich.

Eine Orientierung am System der Herkunftsfamilie zeigt sich auch bei Melanie, auch wenn sie dieses als grundsätzlich einschränkend und bevormundend erlebt. So beschreibt sie den Wunsch, Probleme mit den Eltern zu vermeiden, als Grund für die Trennung von ihrem Freund: „Ich hab' einfach gesagt zu ihm, sei mir nicht bös', aber es ist besser, wir machen Schluss und alles, weil ich möchte' mit meinen Eltern kein Theater haben“" (IMe 582583). 
Bei Melanie wird dabei auch deutlich, dass der Wunsch nach Selbstbestimmung durch stark fremdbestimmte Kontexte stärker wird. Sie äußert den Wunsch, ausziehen zu wollen, als Konsequenz der strengen Regeln, Vorschriften und Sanktionen der Eltern (vgl. IMe 105-112). Auf Nachfrage nach den konkreten Wünschen und Vorstellungen hinsichtlich des Wohnens macht Melanie deutlich, dass es ihr vorrangig darum geht, selbst bestimmen zu können, was sie macht. Kontrastierend dazu hält sie in Form einer kurzen Beschreibung zur derzeitigen Situation fest, dass sie dann etwa nicht mehr bei der Mutter nachfragen müsse, ob sie aus dem Haus gehen dürfe. Diese Beschreibung macht den von den Eltern bestimmten Handlungsrahmen für Melanie deutlich, der kaum selbstbestimmte Entscheidungen zuzulassen scheint (vgl. IMe 188-200) und Melanies Wunsch nach Selbstbestimmung und Autonomie verstärkt.

Die Orientierung am vertrauten Rahmen geht bei Angelika mit der gleichzeitigen Orientierung an Partizipation und sozialen Kontakten außerhalb der Familie einher, die allerdings aus verschiedenen Gründen (Mobilitätsprobleme, eigene Unsicherheiten, mangelnde Unterstützung) kaum realisiert werden kann. Auch bei Marlene klaffen Wünsche und Realisierungsmöglichkeiten hinsichtlich gesellschaftlicher Partizipation weit auseinander. Im Kontext mit ihren Ausführungen, bei der Mutter wohnen zu wollen, wird deutlich, dass sich Marlene mehr Freiraum und Partizipationsmöglichkeiten bei gleichzeitigem Verbleib im vertrauten System wünscht. Dies wird in Form einer Fokussierungsmetapher deutlich dokumentiert:

„Ja mehr weg von daheim, weil sonst bin ich nur daheim, ich sitz' die ganze Zeit nur daheim, ich kann nichts machen [...] ich kann nirgends hingehen, ich brauch mehr Auszeit, aus, frei, hinaus, ich muss mehr wohin geh'n, irgendwo einkaufen oder ich weiß nicht." (IMarl 1137-1139)

Sie präzisiert auf Nachfrage ,mehr ins Kino gehen, mehr einmal schwimmen gehen, ins Hallenbad, ja, mehr einkaufen" (1148).

Negative Erfahrungen im Regelschulbereich dokumentieren sich bei Angelika mit der erfahrenen Zuschreibung als „Behinderte“ durch die Mitschülerinnen und Mitschüler (vgl. IAng 829). Marlene beschreibt, dass sie in der Schule auch körperlich von Mitschülern angegriffen worden sei und versucht habe, sich durch „Wegschupfen“ zu wehren (vgl. Marl 238-242). Marlene beschreibt auch körperliche Übergriffe durch eine Lehrperson (IMarl 258265). Melanie berichtet davon, von den Mitschülerinnen und Mitschülern ausgelacht und verspottet worden zu sein (IMe 212).

Marlene erlebt körperliche Übergriffe nicht nur in der Schule, sondern auch im familiären Rahmen durch ihren Bruder. Angelika und Melanie erleben Abwertung und Mobbing in der Schule. Situationen, in denen neuerlich Erfahrungen von Abwertung oder Übergriffen vermutet werden, werden in der Folge häufig vermieden. Explizit und besonders deutlich wird dieser Zusammenhang bei Melanie hergestellt, die anführt, abends nicht aus dem 
Haus zu gehen, um nicht ,angepöbelt" zu werden (vgl. IMe 514). Auch bei Angelika dokumentiert sich die Orientierung an einem geschützten Rahmen, in dem ihr keine neuerlichen Abwertungserfahrungen drohen. Sie bevorzugt im öffentlichen Raum die Nähe von vertrauten Personen, wie die des „Busfahrers“ (IAng 911) oder jene der Mutter (vgl. IAng 916-917), von denen sie sich Schutz und Unterstützung erhofft.

Die Erfahrung des Wechsels vom Regel- in das Sondersystem beschreibt, wie ausführlich in der Fallrekonstruktion „Anna“ dargestellt, auch Melanie. Sie erzählt, dass sie aufgrund der eigenen „,späten Entwicklung“ (IMe 259) vom Regel- in den Sonderkindergarten ,geschickt“ wurde (vgl. IMe 264). Nachdem sie danach in die Regelschule eintrat, kam es zu einem neuerlichen Wechsel in einen Sonderkontext. Melanie beschreibt dabei den Wechsel als logische Konsequenz dessen, dass sie sich in der Regelschule „schwer getan“ (IMe 441) habe. Auch bei Angelika erfolgt ein Wechsel von der Regel- in die Sonderschule, den sie als Folge eines Nervenzusammenbruches aufgrund von Abwertung und Mobbing in der Regelschule beschreibt (vgl. IAng 883-885).

Finanzielle Abhängigkeit und damit verbunden eingeschränkte Partizipations-möglichkeiten werden auch über Marlenes Ausführungen im Zusammenhang mit einem Kinobesuch dokumentiert. Sie führt aus, dass sie aufgrund fehlender finanzieller Mittel nur sehr selten ins Kino gehen könne (vgl. IMarl 1058-1059). Diese sind auch der Grund für nur eingeschränkt mögliche Telefonate mit ihrer Freundin: „morgen kann ich noch telefonieren und am Freitag nicht mehr, dann ist aus" (IMarl 1080-1081). Auch Angelika bringt mangelnde finanzielle Ausstattung mit eingeschränkten Partizipationsmöglichkeiten und sozialen Kontakten in Zusammenhang. So erzählt sie, dass sie ihre Freundin aufgrund der teuren Bahnkarte nicht besuchen kann. Der Empfehlung ihrer Mutter, mit ihr zu telefonieren, kann sie nur eingeschränkt nachkommen, denn, so führt sie aus: „das kostet was, jeden Tag telefonieren, das kostet was" IAng 205-206).

\subsubsection{Fallrekonstruktion „Eva“ (Orientierung D)}

Eva repräsentiert die starke Orientierung an Selbstbestimmung und Autonomie, die in allen Lebensbereichen umfassend, aktiv, reflektiert und konsequent angestrebt werden.

Im Folgenden werden exemplarisch für diese Orientierung markante Eckpunkte aus dem Interview mit Eva thematisiert, beschrieben und mittels Belegerzählungen, -beschreibungen, -argumentationen und -bewertungen dokumentiert. Im Fokus dieser Betrachtungsweise stehen individuelle Orientierungen und Sichtweisen, die zentral für diese Orientierung sind. 


\title{
Ablehnung von Behütung und Kontrolle versus Wunsch nach Freiraum/Freiheit und Privatsphäre
}

Die stark ausgeprägte Orientierung in Richtung Selbstbestimmung und Autonomie wird bei Eva sehr explizit und prägnant im Zusammenhang mit der Ablehnung von Behütung und Kontrolle und dem damit verbundenen Wunsch nach mehr Freiraum zum Ausdruck gebracht: Sie schildert bereits in der Eingangspassage ihrer Erzählung sehr dicht und in Form einer Fokussierungsmetapher Unterschiede zwischen dem Wohnen bei den Eltern, das für sie dreißig Jahre lang Rahmenbedingung war, und dem aktuellen, autonomen Wohnen mit Unterstützung nach Bedarf in einer Wohngemeinschaft:

\begin{abstract}
„Aber was mir da sehr=sehr gefehlt hat, war einfach diese Freiheit, diese Unabhängigkeit, du bist immer von irgendjemanden beobachtet worden, behütet worden, wie man das auch immer nennen will ja, ehm: der Freiraum war nicht da, du hast kein Privatleben gehabt du hast kaum Freunde einladen können und wenn du Freunden einladeneingeladen hast, dann ist immer alle halbe Stunde die Tür aufgegangen, so quasi passt alles, ist alles in Ordnung, eh, passiert do so quasi wohl nix und das ist ein gewaltiger Unterschied zu früher auf heute, also." (IE 29-35)
\end{abstract}

Sie macht dabei über diesen Vergleich deutlich, dass das Leben in der Familie für sie von Kontrolle und mangelndem Freiraum gekennzeichnet war. Die Begriffe „Freiraum“ und „Freiheit“ sind in dieser Passage zentral. Sehr deutlich dokumentiert sich diese Orientierung von Eva auch gegen Ende des Interviews wieder über ihre Assoziationen zu einem im Zusammenhang mit der Vignettenbeschreibung im Rahmen des Interviews ausgewählten Bild, das eine Frau im Rollstuhl am Meer zeigt. Für Eva symbolisieren die Weite des Meeres und die weit ausgebreiteten Arme der Frau im Zusammenhang mit Fragen der Selbstbestimmung ein „klassisches Bild [...] für Freiheit“ (IE 581). Sie präzisiert die damit verbundenen Gedanken:

„So quasi ich bin für's Leben bereit, alles, was kommt, wurscht was kommt, ich will's versuchen anzunehmen. Und das Meer spiegelt für mich sehr gut wieder was an=an=an=an Sachen auf dich zukommen können, das heißt manchmal sehr schwungvoll, manchmal sehr schmerzhaft, aber auch manchmal ganz ruhig, und das ist- das ist der Inbegriff von Freiheit für mich.“ (IE VA7 582-586)

Ablehnung von Regeln und Vorschriften versus

Wunsch nach eigener Gestaltungsmöglichkeit

Um diesen Unterschied zu verdeutlichen, thematisiert Eva verschiedene Aspekte, die ihr nun autonomes Leben in der Wohngemeinschaft ausmachen, und hält zunächst fest, dass sie zwar zu viert darin leben würden, aber ,,jeder [...] sein eigenes Leben“ (IE 37) lebe. Man könne es mit der Ordnung halten, wie man wolle [,,wenn i jetzt in meine Wohnung hinein geh', ich kann meine Sachen hinein schmeißen“ (IE 35-36)]. Man könne in der Freizeit tun, was 
man wolle [,„geh'n wir Bier trinken“ (IE 38)], sie könne Freunde mitbringen oder sich mit Freunden treffen. Es gäbe keine Regeln und Vorschriften:

„Du kannst leben, wie du möchtest und keiner schreibt dir vor, nein den darfst du nicht haben oder das ist jetzt ungünstig, unpassend, du kannst einfach deine eigenen Bedürfnisse ausleben jetzt, egal in welcher Form auch immer, du kannst einfach das leben, was du möchtest, und so lernst du am besten, so lernt man am besten seine Grenzen kennen." (IE 39-43)

Die eigenen Bedürfnisse und Wünsche würden demnach im Vordergrund stehen und das eigene Leben bestimmen. Die selbstbestimmte Gestaltung des Alltages, die sich auch in der Entfaltungsmöglichkeit im Rahmen des autonomen Wohnens bietet, wird von Eva als zentral dafür hervorgehoben. Eva hält somit gleichsam ein Plädoyer für Selbstbestimmung und bewertet über den selbst gezogenen Vergleich das Leben im engen Familienverband quasi als Gegenpol, in dem dies alles nicht der Fall ist. In dem die eigenen Bedürfnisse und Wünsche nicht zentral sind, es Regeln und Vorschriften, Kontrolle und Behütung gibt. Im autonomen Wohnen könne man seine konkrete Umwelt ordentlich oder nicht ordentlich halten, ganz wie man selbst es bevorzuge. Auch soziale Kontakte und deren Ausgestaltung seien in dieser Lebensform selbstbestimmt, nicht kontrolliert, niemand würde überwachen, ob wohl nichts passiere. Hier deutet Eva an, dass zu Hause kontrolliert wurde, ob es wohl keine körperlichen Kontakte mit Freunden in ihrem Zimmer gab. Somit sind auch Selbstbestimmung hinsichtlich der Wahl möglicher Partner und sexuelle Selbstbestimmung angesprochen, die Eva nur in einem Wohnumfeld, das von Freiraum gekennzeichnet ist, für möglich hält.

\section{Ablehnung traditioneller Betreuungskonzepte versus \\ Wunsch nach Unterstützung und Begleitung}

Evas starke Orientierung in Richtung Selbstbestimmung und Autonomie wird auch über die Beschreibung der Organisation der Unterstützung in der integrativen Wohngemeinschaft, wo der Bezugsbetreuer, ,je nachdem wie ich das möchte" (IE 245-246), kommen würde, und die differenzierte Beschreibung des WG-Alltags deutlich. Den von ihr idealisierten Jetzt-Zustand beschreibt sie so, dass

„es [...] keine Regeln, keine Vorschriften [gibt] das mich irgendwie am selbständigen, freien Leben hindert, gibt es nicht, also gar nicht." (IE 276-278)

Es gebe ein, zwei Fixpunkte, die man einhalten müsse, könne sonst aber so leben, wie man wolle. Eva führt hier als Beispiel an, dass sie verschlafen habe und bestenfalls damit aufgezogen worden sei. Aber es sage ihr keiner: „du musst um zwölfe daheim sein oder du musst um dreie daheim sein“ (IE 269-270). Auch Evas Vergleich zu den Rahmenbedingungen in einer sonder- 
pädagogischen Einrichtung, in der sie im Alter von 12 Jahren lang lebte, spiegelt ihre Orientierung wider:

\begin{abstract}
„Du hast dich abmelden müssen, du hast dich anmelden müssen, du hast sagen müssen, wohin du willst, du hast sagen müssen, wann du wieder kommst, du hast, eh pf, du bist eigentlich immer am Arsch des Betreuers gehängt, gezwungenermaßen, ja. Und eine Zeit lang macht dir das ja Spaß, eine Zeit lang ist es ganz bequem, wenn du dein Leben Zentimeter für Zentimeter, ahm vorgesetzt kriegst, weil du brauchst nix denken du brauchst nix tun, ist mit- ist, ist bis zu einem gewissen Grad ja ganz angenehm und lasst man sich ja auch gefallen, nur ich hab's dann mit der Zeit hassen angefangen, ich bin dann mit der Zeit wirklich ausgebrochen aus dem Ding. “ (IE 288-295)
\end{abstract}

Eva lehnt hier in Form einer Fokussierungsmetapher über die Beschreibung dieses negativen Gegenpols strenge Regeln und Vorschriften, die sie bereits selbst in ihrer langjährigen Erfahrung in einer sonderpädagogischen Einrichtung erlebt hat, explizit und vehement ab. Thematisiert wird von Eva an dieser Stelle auch, dass das Leben in fremdbestimmten Rahmenbedingungen durchaus auch das eigene Bedürfnis nach Bequemlichkeit bedienen kann. Sie selbst hat zunehmend Aversionen dagegen entwickelt, die sie mit dem stark negativ besetzten Verb „hassen“ beschreibt und so die starke Intensität ihrer ablehnenden Emotion gegenüber Fremdbestimmung deutlich werden lässt.

An einer anderen Stelle führt Eva aus, sie wolle nicht „24 Stunden das Hunderl des Betreuers“ (IE 144) sein, ,das hatte ich zwölf Jahre, das brauche ich nicht mehr" (IE 146-147). Aus der Formulierung wird einerseits erkennbar, dass Eva über langjährige Erfahrungen im betreuten Wohnen verfügt und dass die Erfahrungen, die sie im Zusammenhang mit „Betreuung“ gemacht hat, offenbar so negativ sind, dass sie dies nicht mehr haben möchte. Das von ihr gezeichnete Bild, als Mensch mit Beeinträchtigung „das Hunderl des Betreuers“ zu sein, lässt vermuten, dass sie etwas erlebt hat, das diesem Bild entspricht, also eventuell ,an der Leine zu sein“ und fremdbestimmten Ansprüchen genügen zu müssen. Eva lehnt dieses System aus ihrem heutigen Lebenszusammenhang heraus völlig ab und möchte nur dort Unterstützung und Begleitung, wo es unbedingt erforderlich ist, wo sie herausgefunden hat, „was geht und was geht nicht“ (IE 46).

Eva beschreibt die Rahmenbedingungen in einer Behinderteneinrichtung als so angelegt, dass man „Eigenständigkeit [...], an Selbstbestimmung“ (IE 303) verlieren und verlernen würde, „für das einzustehen, was du eigentlich willst“ (IE 304). Das seien „halt mehr oder weniger die Schattenseiten eines Internats oder in einer Behinderteneinrichtung“" (IE 305-306). Fremdbestimmte Rahmenbedingungen in einer Behinderteneinrichtung gefährden somit laut Eva Eigenständigkeit, Selbstbestimmung und die Fähigkeit, sich selbst zu vertreten. Eva theoretisiert hier also über die Bedingungen von Selbst- und Fremdbestimmung über erfahrungsbasierte Vergleiche. In Verbindung mit den Ausführungen darüber, dass dies für sie heute nicht mehr vorstellbar sei, macht Eva eindeutig eine extrem starke Orientierung in Rich- 
tung Selbstbestimmung deutlich. Sie ist auch bereit, die Bequemlichkeit dafür aufzugeben und an die eigenen Grenzen gehen.

Zugleich hält sie aber an anderer Stelle fest, wie wichtig es sei, die ,richtige Unterstützung“ (IE 491) zu haben, bringt aber im selben Zusammenhang über das Sprichwort „Jeder ist seines Glückes Schmied“ (IE 495) den Anspruch der Eigenverantwortung ein. In Form einer Fokussierungsmetapher argumentiert Eva:

„Du kannst, ich sag immer so schön ,du kannst tausend Leut' um dich haben die dein die $=$ die helfen, aber leben musst du dein Leben selber, es geht nicht anders, das ist - du wirst immer Leut' haben, die bis zu einem gewissen Grad, in gewissen Lebenssituationen unterstützen und begleiten ja, einfach um vielleicht größeren Gefahren oder größere Schmerzgrenzen, vielleicht abzuwenden ja, aber leben musst du im Endeffekt dein Leben selbst, in jeder Minute, in jeder Sekunde, in jeder, das musst du im Endeffekt selbst." (IE 495-501)

Unterstützung greift in der Argumentation von Eva demnach nur bis zu einem bestimmten Grad. Auch in Zusammenhang mit der Annahme von Begleitung und Unterstützung spricht Eva den notwendigen eigenen Anteil an, indem sie formuliert: „aber auch da liegt es an dir, das anzunehmen“ (IE 501). Sie selbst habe damit ,immer noch meine Schwierigkeiten phasenweise“ (IE 502-503), weil sie ein Mensch mit „sehr=sehr großem Stolz“ (IE 504) sei. Sie müsse ,ziemlich schwer schlucken“ (IE 501) und brauche eine Zeit lang, wenn sie bemerke, ,es geht nicht so wie ich möchte jetzt rein vom Körperlichen her" (IE 505-506). Vom Körperlichen her brauche sie ,schon sehr=sehr lange und sehr viel Unterstützung“ (IE 506). Das für sie in den Einklang zu bringen, dass sie mit sich und der Umwelt leben könne, sei sehr schwierig. Denn sie habe „einen sehr=sehr großen Stolz“ (IE 509) und würde wahrscheinlich „so hundert Prozent alles allein machen“ (IE 509), aber das ginge halt nicht, ,,allein von der Körperlichkeit her funktioniert das nicht" (IE 510). Das Stück für Stück zu lernen, sei spannend. Eva bezieht sich dabei auf die Herausforderungen, die es für sie selber mit sich bringt, notwendige Unterstützung auch anzunehmen. Es sei ein notwendiger Lernprozess, diese anzunehmen, auch wenn man gerne alles selbständig erledigen würde, es aber vom Körperlichen her nicht ginge. Eva thematisiert hier implizit das Verhältnis von Selbstbestimmung und Selbständigkeit und macht deutlich, dass in bestimmten Bereichen nicht selbständig $\mathrm{zu}$ sein, nicht heißt, nicht selbstbestimmt mit Unterstützung leben zu können.

Eva betont auch Prozesse sozialer Interaktion im Zusammenhang mit Unterstützung. So komme es manchmal dazu, dass jemand sage „hey, bleib am Teppich, bleib am Boden“ (IE 513), dann sei man vielleicht einmal ,,angefressen", aber wenn man darüber nachdenke, würde man zur Einsicht kommen ,o.k., hat er ja wohl eigentlich eh recht, er tut's nicht, weil er dir nichts zutraut, sondern er tut's weil du dich nicht überschätzen sollst“" (IE 517-518). Es sei also sinnvoll, auch über Empfehlungen aus dem Umfeld nachzudenken 
und zu überlegen, ob sie für das eigene Leben relevant sein könnten, lässt Eva implizit deutlich werden.

\section{Artikulation und Selbstvertretung eigener Wünsche, Ansprüche und Bedürfnisse}

Verbunden ist die starke Orientierung Richtung Selbstbestimmung und Autonomie bei Eva damit, dass sie Wünsche, Ansprüche und Bedürfnisse selbst zum Ausdruck bringen will und dies durch Lernprozesse zunehmend auch umsetzen kann. Sie macht explizit deutlich, dass sie sich selbst und ihre Anliegen vertreten kann und dafür einsteht:

„[...] ich habe gelernt auch - egal, wer vor mir sitzt - meine Meinung zu sagen und zwar so meine Meinung zu sagen, dass ich sie vertreten kann und dass der andere sie auch verstehen kann, was jetzt genau das Problem ist. Und das war bei der Ausbildung, da bin auch immer ich die einzige gewesen die, wenn i gehört hab andere haben genuschelt hinten: ,ja das ist scheiße und das ist scheiße'. Dann sag ich: , warum macht's ihr bitte nicht den Mund auf, warum geht's ihr nicht hin und sagt's: Herr [...], bitte das ist mir zu viel, ich steh an oder das und das versteh' ich jetzt nicht, do gehen Sie mir zu weit oder=oder keine Ahnung, dann sagt's des bitte auch!“ (IE 92-99)

Eva ist es wichtig darauf hinzuweisen, dass sie auch das Bedürfnis hat, dass ihr Gegenüber ihre Meinung auch verstehen kann, die Äußerung ihrer Bedürfnisse im sozialen Kontext also nachvollziehbar ist. Von der semantischen Ebene her ist auffallend, dass Eva selbst ihre Position und auch die eigene Weiterentwicklung über Vergleiche deutlich macht. Eingangs zog Eva etwa einen Vergleich von Wohnen in Abhängigkeit zum selbständigen Wohnen. An dieser Stelle zieht sie einen Vergleich zwischen ihrem eigenen Auftreten und dem von Kolleginnen und Kollegen an einer Ausbildungsstelle, die in Evas Schilderung im Gegensatz zu ihr selbst ihre Meinung nicht selbst vertreten haben. Eva weist auch darauf hin, dass der Chef sie gerade deswegen, weil sie ihre Anliegen vertreten habe, auch besonders geschätzt hätte. Sie selbst hätte im Gegensatz zu ihren Kollegen und Kolleginnen gesagt, was sie wolle, und das sei auch der Grund für Reibereien mit ihrem Chef gewesen. Manchmal sei er zu weit gegangen, manchmal sie selbst, aber sie hätten immer wieder „einen Weg zueinander gefunden“ (IE 102-103) und er hätte sie gerade deswegen ,sehr=sehr geschätzt“ (IE 103). Sie reflektiert also die von ihr wahrgenommene positive Fremdeinschätzung des ihr eigenen Auftretens. Hier deutet sich auch das positive Selbstbild von Eva an. Eva hat also gelernt, auch Autoritätspersonen gegenüber ihre Wünsche und Vorstellungen von Selbstbestimmung zu artikulieren, sie auch bei Widerstand und in Konfrontation zu vertreten. Dies wird später noch an der Haltung gegenüber dem Vater in Zusammenhang mit dessen Einstellung zu Evas Sexualität, aber auch in anderen Zusammenhängen deutlich. So etwa auch im beruflichen Kontext, wo Eva mit ihrem Vorgesetzten Grenzen und Regeln aushandelt. 
Nachdem Eva zunächst festgehalten hat, wie wichtig es sei, seine eigenen Anliegen zu vertreten, bekräftigt sie dies an einer anderen Stelle und geht auf das „wie“ ein. Konkret hält sie fest, dass man jedem seine Meinung sagen könne, ob dies der „Bundespräsident oder dein eigener Chef sei“ (IE 478), dies müsse aber „mit Respekt und Höflichkeit, ja“ (IE 480) erfolgen. Es wird deutlich, dass Eva über die Regeln sozialer Interaktion Bescheid weiß. Indem sie explizit Autoritätspersonen wie den Bundespräsidenten oder den eigenen Chef nennt, macht Eva deutlich, dass sie nichts davon abhalten kann, ihre Meinung zu sagen, dass sie sich auch von solchen Personen nicht davon abbringen bzw. eventuell einschüchtern lasse.

\section{Wahrgenommen- und Akzeptiert-Werden mit den eigenen Wünschen und Bedürfnissen}

Über die Beschreibung, wie sie konkret zum Wohnplatz in einer integrativen WG kam und nun seit einem Jahr dort als ,der glücklichste Mensch, den man sich überhaupt vorstellen kann“ (IE 177), lebt, wird wieder die starke Orientierung von Eva an Selbstbestimmung und Autonomie deutlich. Prägend auf dem Weg dorthin beschreibt Eva eine Situation, wo sie nach ihren Wünschen im Bereich Wohnen gefragt wurde:

\footnotetext{
„Und da sind so Szenen, die ich mein Lebtag niemals vergessen werde, die mich so geprägt haben und auch in meinen Weg immer wieder bestärkt haben, auch wenn es nur Kleinigkeiten waren. Da sind wir zu diesem Herrn [...] gegangen: jetzt geh'n wir zu dritt hinein - Mama, Papa und ich. So, Papa sitzt links, eh rechts von mir, Mama sitzt links von mir und ich sitz halt in der Mitte. Der Herr [...] schaut meine Eltern an, grüßt sie, stellt sich vor. Und dann steht er auf gibt mir die Hand, dann sagt er: ,Und was willst $D u$ ?' [...] Dann sagt der Papa: ,wir suchen einen betreuten Platz für unsere Tochter'. Er schaut meine Eltern an: ,rede ich mit ihnen oder mit ihrer Tochter?' Dann waren sie beide einmal still. ,Ich glaub', Sie reden mit mir', hab' ich i dann gesagt [...] und dann sag ich ,ja, Sie reden mit mir', dann sogt er ,ja was willst Du?' Dann sag' ich: ,ja, ich such' eine Wohnung bzw. so eine Art WG, also im vollbetreuten Wohnen mag ich nicht sein!“ (IE 134-145)
}

Eva erlebte, wie die Eltern für sie sprachen und etwas äußerten, das nicht ihren Wünschen entsprach. Sie erlebte aber auch, wie das Gegenüber darauf nicht einstieg und die Frage nach ihren Wünschen explizit an sie richtete. Diese Szene, in der die Eltern über sie bestimmen wollen, indem sie ungefragt ihre Vorstellungen als Evas Wunsch präsentieren, macht wieder deutlich, dass Eva für sich selbst sprechen, sich selbst vertreten will. Die Erfahrung, dass dieser Wunsch auch vom Gegenüber wahrgenommen und respektiert wird, bestärkt Eva in ihren Vorhaben. Bereits über die Passage, wo sie ehemalige Arbeitskolleginnen aufgefordert hat, ihre Anliegen zu vertreten, wurde diese Orientierung deutlich. Eva hatte damals auch die Möglichkeit, sich in einem entsprechenden Umfeld zu erproben und ihre Grenzen abzustecken. Sie hat Akzeptanz erlebt und wurde als Mensch mit eigenen Wünschen 
und Bedürfnissen wahrgenommen. Dies wiederholt sich jetzt und bestärkt Eva auf ihrem Weg in die Autonomie. Auf die Äußerung ihres Wunsches, nicht im vollbetreuten Wohnen leben zu wollen, wurde mit Erfüllung dieses Wunsches reagiert. Als Eva der Platz in der WG angeboten wurde, begann sie zu träumen, reagierte „euphorisch, weil Du siehst, es geht nach vorne, es geht wirklich dorthin, wo du, für das du jahrelang gearbeitet hast" (IE 172173). Wieder wird die Orientierung an Selbstbestimmung und Autonomie deutlich, aber auch, dass Eva unterstreichen will, dass sie ihren Anteil, dass es dazu gekommen ist, geleistet hat.

Eva theoretisiert an anderer Stelle über Selbstbestimmung und betont dabei den notwendigen eigenen Anteil, indem sie ausführt ,es liegt sehr viel an einem selbst, was man in seinem Leben macht, du kannst dich selbstbestimmen lassen und in die Gesellschaft hineindrücken lassen, aber du wirst nie etwas davon haben, solange du das nicht selber machst" (IE 424-426). Niemand kenne einen besser als man selbst, „nur du kennst deine Bedürfnisse“ (IE 427). Und weiter:

„[...] die Eltern kennen dich nicht wirklich, deine Umgebung kennt dich nicht wirklich, der einzige der dich wirklich kennt, ist man selber.“ (IE 428-439)

Für Eva ist es also wichtig festzuhalten, dass niemand besser über die eigenen Bedürfnisse Bescheid wissen kann, als man selbst. In Verbindung mit ihren Ausführungen über Selbstbestimmung im Allgemeinen erscheint die Orientierung an den eigenen Bedürfnissen als zentral für ein selbstbestimmtes Leben. Es wird deutlich, dass das Äußern eigener Wünsche verbunden mit deren Berücksichtigung Evas weiteren Weg und ihr Selbstbild positiv beeinflusst.

\section{Wahrgenommen werden als eigene Persönlichkeit mit den eigenen Stärken und Schwächen und mit dem eigenen Erscheinungsbild}

Eva verdeutlich an einer erzählten Passage, dass ihr Vater mit der selbstbestimmten, nicht seinen Vorstellungen entsprechenden Gestaltung ihres Äußeren massive Probleme hat. Als er ihre Piercings und Tätowierungen, die sie sich nach Eintritt in die Wohngemeinschaft zugelegt hat, erstmals gesehen habe, habe er die Mutter ,ganz aufgeregt, ganz=ganz=ganz=ganz hektisch, schau=schau“ (IE 349-350) geschupft. Über die gelassene Reaktion der Mutter in dieser Szene wird deutlich, dass diese im Gegensatz zum Vater Evas Weg in die Selbstbestimmung unterstützt. Es wird aber auch deutlich, dass sich Eva hinsichtlich der Gestaltung des eigenen Äußeren nichts sagen lässt, zumal sie es als Ausdrucksmittel für die eigene Befindlichkeit und auch für Entwicklungsprozesse einschätzt:

„Und so verändert man - ich meine man verändert sich auch, weil einen das Leben prägt, ja, und das bringt Veränderungen mit sich und=und das tragt man auch teilweise 
noch außen. Also ich bin so wo- ich bin jemand, der Veränderung sehr stark noch außen trägt, einfach, weil ich damit sagen will: hey Leute passt's auf, mir geht's nicht gut oder $=$ oder $=$ oder es hat sich was verändert in meinem Leben, ich transportier das sehr stark noch außen, auch=auch vom Aussehen, also vom - ja das hängt auch vom - viel vom Aussehen kann man dann viel ableiten und viel überlegen und so, und ich tu' mir damit halt am leichtesten, ja.“ (IE 377-383)

Die Gestaltung des eigenen Aussehens ist also für Eva ein zentrales Mittel, ihre Befindlichkeit auch im sozialen Kontext zum Ausdruck zu bringen und Veränderungsprozesse zu dokumentieren. Im Zusammenhang damit macht Eva deutlich, dass sie so akzeptiert werden möchte, wie sie sei, als Mensch mit Stärken und Schwächen. Sie sage immer,

„der, der in mein Leben will oder in mein Leben möchte, der muss mich so akzeptieren, wie ich bin, mit allen Schwächen, mit allem Negativen, mit allem Positiven, der muss mich halt so nehmen wie ich bin, weil ich tu's anders auch nicht, ja.“ (IE 383-386)

Eva möchte als Person mit all ihren Eigenschaften, mit Schwächen und Stärken wahrgenommen werden. Als eigenständige Person, die sich von niemandem vorschreiben lässt, wie sie ihr Leben und ihr Aussehen gestalten soll. Sie macht deutlich, dass ihr Äußeres und ihre Persönlichkeit stark miteinander verbunden sind, sich letztere über das Aussehen transportiert. So gesehen würde die Orientierung an fremdbestimmten Vorstellungen über ihr Aussehen den Ausdruck von ihr selbst als Persönlichkeit unmöglich machen. Eva arbeitet mit der Gestaltung des Bildes, das sie über ihr Äußeres dokumentiert, auch an ihrem Selbstbild und will wohl auch die Wahrnehmung in der Außensicht über sie beeinflussen.

Der Wunsch Evas, als eigene Person wahrgenommen und akzeptiert zu werden, spiegelt sich auch in ihren Assoziationen zum selbst gewählten Bild von Veronika Hammel im Rahmen der Vignettenbeschreibung wider. Sie begründet die Auswahl gerade dieses Bildes damit, dass

„diese Person auf dem Foto [...] mit sich im Einklang ist, sie weiß vielleicht nicht, was auf sie im Leben zukommt, aber sie ist mit sich im Reinen, sie ist mit sich zufrieden, sie hat, ehm, für mich eine sehr ruhige innere Ausgeglichenheit und diese Hände die - das was, ich gehör' mir selber und alles- was alle anderen sagen, ist mir wurscht. Das sagt für mich das Bild: ich gehör' nur mir selber, und ja [...]“ (IE-VA1 588-592)

Eva beschreibt nach der Begründung für die Auswahl des Bildes sehr detailliert das Äußere von Veronika:

„Sie ist von der Kleidung her, würd’ ich sagen, sehr offen, auch sehr gewagt also Arme und Oberkörper frei - nehmt's mich so wahr, wie i bin, ja und nehmt's mich auch vielleicht in Richtung als sexuelles Wesen wahr, ich bin so wie ich bin und ich suche auch diese Geborgenheit oder auch diese körperliche Nähe vielleicht, ja. Auch mit diesen Ausschnitt, auch mit dieser - schulterfrei, oberkörperfrei, nur da das Wichtigste abgedeckt, sagt für mich aus: ich bin ein sexuelles Wesen nehmt's mich auch wahr ja, nehmt's mich auch so wahr wie ich bin und eben auf der anderen Seite - mit die Hände auf dem Bauch verschränkt oder unterm Bauch verschränkt: ich gehör' aber nur mir, ich 
lass bis zu einem gewissen Grad was zu, aber im Grunde gehör' ich nur mir, und ich bestimme über mich selber - wie weit ich gehen will und was ich nicht möchte, das=das sagt das Bild für mich aus." (IE 595-603)

Damit deutet sich Evas Wunsch an, ganzheitlich als Person - also auch mit sexuellen Bedürfnissen - wahrgenommen und akzeptiert zu werden. Verbunden damit ist, wie Eva auch explizit ausführt, ganz zentral der Wunsch nach selbstbestimmter Absteckung ihrer persönlichen Grenzen. Damit angesprochen erscheint auch der Wunsch nach selbstbestimmter Gestaltung von Sexualität sehr zentral in Evas Orientierung.

\section{Umsetzung selbstbestimmter Gestaltung der Vorstellungen von Partnerschaft und Sexualität}

In Evas Erzählungen und Beschreibungen wird auch in anderen Passagen deutlich, dass ihr Selbstbestimmung in Zusammenhang mit Partnerschaft und Sexualität sehr wichtig ist. Sie beschreibt in diesem Zusammenhang, dass ihr Vater große Probleme damit hat(te), sie als Frau mit selbstbestimmter Sexualität wahrzunehmen und zu akzeptieren, dass Eva jemand nahekommt. Er könne überhaupt nicht damit umgehen - „seine Tochter ist kein sexuelles Wesen, hat auch kein sexuelles Wesen sein zu dürfen“ (IE 193-194), sagt Eva ganz zentral in dieser Passage. Die Ablehnung des Vaters beschreibt Eva sehr eindringlich, man könnte darin auch eine Fokussierungsmetapher sehen, die einerseits auf die Bedeutung selbstbestimmter Sexualität für Eva, andererseits auf die Probleme des Vaters damit hinweist:

\footnotetext{
„Beim Papa war das Problem in Partnerschaft, Sexualität und das ist=ist für ihn nach wie vor ein sehr=sehr großes Thema, also alles andere würd' ich eigentlich als nicht so schlimm empfinden - weil das weiß er mittlerweile, dass ich das kann und dass ich mein Leben auch meistere. Nur womit er sehr große Probleme hat: dass mir jemand zu nahe kommen könnte, und dass da von mir aus Kinder entwickeln könnte, Familie entstehen könnte, und damit kann er überhaupt nicht umgeh'n, also gar nicht. Also das ist für ihn ein Kapitel, seine Tochter ist kein sexuelles Wesen, hat auch kein sexuelles Wesen sein zu dürfen, jetzt in grober Form gesagt ja. Und das ist für ihn ja absolut, ehm, ja schwierig, da fallt ein Schalter im Kopf um, sag' i immer so schön und er sieht rot, also, und das ist immer- sind für ihn so spezielle - also das ist ein spezielles Thema, was gar nicht geht.“ (IE 187-197)
}

Eva spricht im Zuge dieser Beschreibung auch - wenn auch eher beiläufig an, dass es eine große Angst des Vaters sei, dass im Zusammenhang mit der selbstbestimmten Sexualität von Eva auch Kinder entstehen könnten. Deutlich wird in Evas Beschreibung, dass sie mit dem Vater auch in Konfrontation geht, sie hätten schon häufig darüber diskutiert und ,auch schon häufig Auseinandersetzungen gehabt“ (IE 203), er müsse ,es jetzt auf die harte Tour lernen [...] mit dem Vorschlaghammer lernen, weil sonst begreift er das nie“" (IE 203-204). Eva pocht dem Vater gegenüber darauf, dass sie kein - wie er möchte - asexuelles Wesen ist und ihre Sexualität selbstbestimmt gestalten 
will. Auf Nachfrage, was er lernen müsse, erläutert Eva „dass es einfach Männer an meiner Seite gibt und geben wird und auch gibt, ja“" (IE 207). Der Vater wünsche sich immer ,eine normale, eine normale Familie so wie wir sind, Papa, Mama und vielleicht Kinder“ (IE 207-209). Es wird deutlich, dass der Vater bestenfalls ein traditionelles Familienkonzept für Eva akzeptieren könnte, nicht aber einen Zugang von Eva, der ihren eigenen derzeitigen Bedürfnissen und Vorstellungen entspricht. Momentan lebe sie ,sich sexuell ziemlich aus, [...] tobe sich da ziemlich aus“ (IE 213). Eva führt als Gründe dafür an, dass eine fixe Partnerschaft gesicherte Lebensbedingungen für sie und ihren Partner erfordern würde, lehnt also grundsätzlich das Modell einer fixen Partnerschaft nicht ab. Zudem sei ihr auch schon, ,viel zu oft weh getan worden und da schützt man sich halt davor" (IE 220-221), weist Eva auf negative Erfahrung im Bereich Partnerschaft/Sexualität hin und darauf, dass die Abwehr einer fixen Beziehung [,,ich brauche derzeit nichts Fixes, ich brauch zur Zeit nichts Festes" (IE 219)] auch in gewisser Weise eine Vermeidungsstrategie darstellt. Die Mutter wird im Gegensatz zum Vater so dargestellt, dass sie Evas Zugang zur Sexualität und Partnerschaft „,voll akzeptiert" (IE 215), solange es ihr dabei gut gehe.

Es ist Eva auch wichtig, auf die erforderlichen Rahmenbedingungen selbstbestimmter Sexualität hinzuweisen:

„Man kann im Leben alles machen, man kann im Leben so ziemlich alles machen, nur: es muss immer ganz klar geregelt sein, das Gegenüber muss immer ganz genau wissen, was Du möchtest und was nicht und so lang dos offen, egal in welcher Situation, immer angesprochen wird, kannst du das schönste und offenste Leben führ'n, das du dir vorstellen kannst, und du kannst dir selber dabei keinen Vorwurf machen, weil es ist so wie's ist, ja, ganz einfach.“ (IE 223-227)

Eva spricht sich hier für ein Modell selbstbestimmter Sexualität aus, indem jeder selbstbestimmt agieren kann, jedoch unter Aushandlung von individuell zu vereinbarenden Regeln und Rahmenbedingungen im sozialen Kontext.

\section{Einschätzung von Herausforderungen als Chancen persönlicher (Weiter-)Entwicklung}

Auch die dieser Orientierung eigene Reflexion vorhandener Voraussetzungen und gegebener Ansprüche auf dem Weg der Umsetzung eines solchen Lebensentwurfes wird bei Eva explizit thematisiert. Sie beschreibt den Weg zu mehr Selbstbestimmung und Autonomie gekennzeichnet durch große persönliche Herausforderungen, an denen man sich aber weiterentwickeln kann und die so gesehen letztendlich die (Weiter-)Entwicklung der eigenen Persönlichkeit und damit das eigene Selbstbild positiv beeinflussen.

„[Da] lernt man am besten seine eigenen Grenzen kennen, wo muss ich jetzt lernen, stopp zu sagen, wo muss ich jetzt sagen o.k., da geht's jetzt wirklich nimmer weiter, da brauch' ich Hilfe, da=da=da geht's nicht alleine. Das sind alles Sachen wo du sehr viel 
Eigenverantwortung entwickelst und sehr viel an=an Selbstbewusstsein und was geht und was geht nicht. Und vor allem - was das Schönste ist, was ich immer find': du darfst dir erlauben, auf die Schnauze zu fallen, du darfst dir erlauben, Fehler zu machen - ja. Und dann mit Hilfe oder ohne Hilfe, je nachdem, wie schwerwiegend der Fehler ist, da wieder seinen eigenen Weg heraus zu finden. Und genau an solchen Dingen, finde ich, wächst man auch, ob es jetzt positiv ist oder negativ, du=du erlebst dich, vor allem du erfährst sehr viel selber über dich, jeden Tag und immer wieder und das ist mitunter seh $=$ sehr anstrengend und das kann auch manchmal sehr=sehr weh tun, aber es ist sehr=sehr spannend, also es ist immer wieder auf's Neue spannend.“ (IE 43-53)

Man würde seine eigenen Grenzen kennenlernen und darüber viel Eigenverantwortung und Selbstbewusstsein entwickeln. Besonders die Möglichkeit, auch selbst Fehler machen zu dürfen, schätzt Eva in diesem Zusammenhang als äußerst bedeutsam ein. Daran wachse man und erfahre viel über sich selbst. Eva beschreibt diesen Prozess durchaus auch als „sehr=sehr anstrengend“ (IE 51), es würde „manchmal sehr=sehr weh tun“" (IE 52), aber es sei „sehr=sehr spannend“ (IE 53). Diese sprachliche Konstruktion verdeutlicht, dass der Prozess, sich in Richtung umfassender Selbstbestimmung zu entwickeln, von Eva durchaus als sehr mühsam eingeschätzt wird. Im implizit vorgenommenen Vergleich zum Wohnen bei den Eltern erscheint das Leben dort als bequem, weniger herausfordernd und auch weniger schmerzvoll, aber auch nicht so spannend wie ein autonomes Leben, das nach den eigenen Wünschen, Bedürfnissen und Vorstellungen gestaltet werden kann.

Eva grenzt sich in der Beschreibung ihres Weges zur Autonomie ausdrücklich von anderen Frauen mit einer vergleichbaren Ausgangslage ab, die „nicht wirklich wissen und wirklich ahnen, was [...] ein selbständiges Leben wirklich bedeutet“ (IE 106-107), und präzisiert „,von der Psyche und vom Körperlichen“ (IE 109). Man müsse das ,sehr gut überlegen, [...] zehn Mal abwiegen“ (IE 110). Sie führt auch sprachlich sehr deutlich mit verstärkenden Wiederholungen aus, dass sie immer schon gewusst und gesagt habe „irgendwann einmal, aber ich werde irgendwann einmal mein eigenes Leben führen" (IE 112-113). In dieser Kombination der Argumentierung und Beschreibung macht Eva deutlich, dass ihr die Anforderungen, die ein selbständiges Leben mit sich bringen, sehr bewusst sind. Andere Personen, auf die dies nicht zutrifft, so wird in der Argumentation deutlich, müssten sich gründlich überlegen, ob sie dies schaffen würden. Für Eva selbst war dies offenbar keine Frage, ihr war immer klar, dass sie es irgendwann einmal soweit bringen würde. Eva scheint in dieser Argumentation auch sehr selbstbewusst und weist implizit und explizit darauf hin, dass sie über Herausforderungen im Prozess der Ablösung Bescheid weiß und diese auch bewältigt.

Im Zusammenhang mit der Möglichkeit, den Job in einer anderen Stadt anzunehmen und autonom zu leben, beschreibt Eva die Reaktion ihrer Mutter als „voll begeistert" (IE 129). Der Vater wird als bis heute zweifelnd beschrieben, er äußert diese Zweifel auch und fragt Eva, ob sie denn wisse, was selbständiges Leben heiße. Auch in dieser Szene verdeutlicht Eva mit ihrer 
Reaktion [,,ist mir völlig bewusst, mit jedem Zentimeter meines Körpers ist mir das bewusst, ich weiß es eh" (IE 127-128)], dass sie bereit ist, jede damit einhergehende Herausforderung zu meistern. Diese Sequenz verdeutlicht nochmals die starke Orientierung von Eva an Selbstbestimmung und Autonomie. Eva bekräftigt, sich aller Herausforderungen, die damit einhergehen, bewusst zu sein, zeigt sich wieder bereit, diese anzunehmen und scheint sicher, diese auch meistern zu können.

Eva greift auch die bereits in der Eingangspassage erwähnte Thematik wieder auf, wonach der Prozess, sich in Richtung Selbstbestimmung zu entwickeln, als sehr mühsam eingeschätzt wird. Im implizit vorgenommenen Vergleich zum Wohnen bei den Eltern zeichnete sie das Leben dort als bequem, weniger herausfordernd und auch weniger schmerzvoll, aber auch nicht so spannend wie ein autonomes Leben, das nach den eigenen Wünschen, Bedürfnissen und Vorstellungen gestaltet werden kann. Auch in der aktuellen Passage wird das Leben unter fremdbestimmten Rahmenbedingungen als „bequem“ nachgezeichnet. Man müsse ,nichts denken, [...] nichts tun“ (IE 291-293). Es sei bis zu einem ,gewissen Grad ja ganz angenehm“ (IE 294), wiederholt Eva, man „lasst sich das ja auch gefallen“ (IE 294).

Auf die Frage, was Frauen mit Behinderung aus ihrer Perspektive brauchen würden, um selbstbestimmter Leben zu können, ist es laut Eva wichtig,

„,sich selbst ganz stark mit sich selber auseinander zu setzen, wo sind meine Fähigkeiten, was kann ich, was kann ich nicht, was sind Träumereien, was sind Spinnereine, was lässt sich erfüllen." (IE 442-444)

Eva betont also auch hier wieder den eigenen, notwendigen aktiven, selbstreflexiven Beitrag der Auseinandersetzung mit den eigenen Stärken und Schwächen auf dem Weg in Richtung eines selbstbestimmten Lebens. Sie spricht damit auch an, dass es notwendig sei, zwischen realistischen und unrealistischen Überlegungen zu differenzieren und zu überlegen, was sich umsetzen lasse. Es sei auch notwendig, ganz konkret aktiv zu werden, indem man selber auf Menschen im sozialen Umfeld zugehe und sage „hey, ich möchte das und das probieren, bitte stellt's mir einen an die Seite, den ich vertrauen kann“ (IE 451-452). Denn, so Eva, es werde „keiner herkommen und sagen, kann ich Dir helfen?" (IE 450).

Eva spricht hier - wie ihre Ausführungen zum Arbeitsbereich, aber auch zur Umsetzung selbständigen Wohnens zeigen - eigene Erfahrungen an, die sie dahin gebracht haben, wo sie selbst steht. Deutlich wurde in den angesprochenen Passagen, dass der Weg hierher auch ,anstrengend“ war, „weh getan" hat, Eva dafür auch Bequemlichkeit aufgegeben hat, sich auseinandersetzen musste, Konflikte austragen und an sich arbeiten musste. Dies will sie anderen Frauen gleichsam auf den Weg mitgeben, sei erforderlich auf dem Weg zur Selbstbestimmung. Indem Eva implizit darauf hinweist, was sie bislang schon geleistet hat, welche Anteile sie aktiv eingebracht hat, macht 
sie auch deutlich, dass sie ziemlich selbstbewusst ist und ein zunehmend positives Selbstbild entwickeln konnte.

Insgesamt bringt Eva ihre Vorstellungen von einem selbstbestimmten Leben stark in Zusammenhang mit der Chance, Erfahrungen zu machen, Herausforderungen anzunehmen. Eva spricht hier auch von „Prüfungen“ und philosophiert:

\begin{abstract}
„Also das Leben - im Prinzip - ist das Spannendste, was man haben kann, ja, und das ist die größte Prüfung, die du überhaupt hast in deinem Leben, ja. Und da gibt's nix- es gibt nix besseres als Leben, und das Leben ist eine ganze Prüfung und es wird dich zehn Meter noch vorne hauen, aber es kann dich auch 30 Meter wieder zurück hau'n, nur dessen musst du dir bewusst sein, ja. Du kannst nicht immer sagen, das Leben ist schön, das Leben ist perfekt, das ist es im Grunde nicht. Es sind Sequenzen, die wunder=wunderschön sind, und die du auch immer - die dir immer wieder die Kraft geben dann aus der schlechten Situation wieder das Gute zu machen und genau des=des Gesamtparket macht's aus, ja. Und dann auch sich das zu trau'n oder zu sagen ok, gut steh'n wir halt wieder auf, dann geh'n wir halt auch ( ) weiter und schau'n wir, was vorne liegt. Hinter schau'n bringt nix, Vergangenheit ist Vergangenheit, die wird dich eh nie loslassen weil=weil du das im Gesamtpaket eigentlich bist, ja, Vergangenheit lasst dich nie los, das Negative was du erlebt hast, da- weil dich das als Mensch prägt, das bringt ja auch weiter, das macht das Gesamtpaket Mensch ja aus und das muss man lernen, irgendwie $\mathrm{zu}=\mathrm{zu}=\mathrm{zu}$, ehm zu schachteln, das war früher, das ist heute, das ist jetzt und ich bin's eigentlich im Gesamten und es gibt nix was schlimm ist bei mir, und es gibt nix was irgendwie gut ist, es ist die Mischung, die mich als Mensch ausmacht, ja, und wenn man das einmal so halbwegs die Waage halten kann, denk ich funktioniert kann's sehr gut funktionieren also das is ganz klar" (IE 520-534)
\end{abstract}

Eva theoretisiert an dieser Stelle zum wiederholten Mal im Interview und dokumentiert ihre Anschauung, dass positive und negative Erfahrungen im Verlauf der Sozialisation und des weiteren Lebens den Menschen massiv beeinflussen und zu der „Mischung“ führen, die sie ,als Mensch ausmacht“ (IE 535-536).

\title{
Positives Selbstbild mit ressourcen- und umfeldorientiertem Blick auf die eigene Beeinträchtigung
}

Wie bei Eva bereits in Zusammenhang mit der Einschätzung von Herausforderungen als Chancen persönlicher (Weiter-)Entwicklung angedeutet, werden eigene Voraussetzungen damit wahrgenommen, reflektiert und stellen somit eine Grundlage für das aktuelle Selbstbild und den Ausgangspunkt für weitere Schritte dar. Der Blick auf die eigene Beeinträchtigung ist dabei zunehmend ressourcen- und umfeldorientiert. Der Fokus liegt auf dem persönlichen Potential, das im Zusammenhang mit bereits vollzogener Weiterentwicklung abgeschätzt und als Indikator für eigene weitere Entwicklungsschritte gesehen wird. Eva abstrahiert von ihren Ausführungen her Anforderungen und Rahmenbedingungen auch losgelöst von der eigenen Person als allgemein für Menschen mit Behinderung auf dem Weg in die Autonomie zutreffend. Zu- 
gleich macht sie deutlich, dass sie diese Anforderungen und Herausforderungen zu erfüllen vermag und verweist so auf ein positives Selbstbild.

Nicht nur das Fremdbild, auch das Selbstbild von Eva dokumentiert sich bereits in der Eingangserzählung zum Interview mit der Einschätzung der neun Jahre zu Hause, wo sie nie als positiv habe zeigen können, „was du leisten hast können“ (IE 60). Sie weist somit bereits anfangs explizit darauf hin, dass ihr von außen Fähigkeiten - vor allem im sprachlichen und kommunikativen Bereich - zugeschrieben wurden und dass sie sich auch selbst als kompetent und leistungsfähig einschätzt.

Eva verweist in ihren Erzählungen und Beschreibungen im Verlauf des Interviews an mehreren Stellen aus unterschiedlichen Perspektiven auf ihre Kompetenzen und Fähigkeiten. Sie rückt dabei in den Fokus, dass diese auch außenstehenden Personen in verschiedenen Zusammenhängen aufgefallen seien. So führt eine ehemalige Erzieherin in Evas Schilderung aus, sie würde sich diese „supergut" für einen „Traumjob“ vorstellen, weil sie „,verbal und kommunikativ sehr gut drauf" (IE 64) sei. Eva lässt dies in ihrer Erzählung auch den Projektleiter im Rahmen der Bewerbung bei dem Projekt, in dem sie nunmehr tätig ist, ebenso einschätzen. Nachdem er sie erst zwei Minuten gekannt hat, weist er Evas Vater darauf hin: „Ja brauchen Sie ja nur beobachten, wie sie auf jemanden zugeht, wie sie mit jemandem sprachlich umgeht, das passt einfach, ja“ (IE 69-70). Darüber, dass der Vater verwundert war, dass der Projektleiter nach zwei Minuten zu dieser Einschätzung kam, könnte einerseits hervorgehen, dass er selbst keine solche Sichtweise von Eva hatte bzw. zum Ausdruck brachte oder aber, dass Eva in gewisser Weise den Stolz des Vaters auf sie deutlich machen möchte. An der zweimal formulierten Aussage, sie werde diesen „,einen Satz nie vergessen“ (IE 66), „den werde ich mein Lebtag nicht vergessen“ (IE 67), dokumentiert sich, wie wichtig ihr diese positive Einschätzung ihrer Kompetenzen aus dem sozialen Umfeld ist.

Im selbst vorgenommenen Vergleich zwischen zwei Projektstellen, an denen sie gearbeitet hat, dokumentiert sich ein weiterer Aspekt im Zusammenhang mit Evas Selbstbild. Sie beschreibt die Vorzüge des Projektes, bei dem sie jetzt arbeitet, und hebt hervor, dass hier „Eigenständigkeit“ und „Eigenverantwortung ein sehr hohes Prinzip“ (IE 74-75) seien.

„Weil wenn da irgendwas passiert, dann bist du weg vom Fenster, egal ob du im Rollstuhl sitzt oder nicht, also, und das ist schon auch gewesen - ich hab' die Ausbildung in [...] sehr gerne gemacht, aber für mich war sie in vielen Zügen und in vielen Umständen einfach zu weich, es ist immer wieder noch auf die Behinderung Rücksicht genommen worden, was ich ja zum Teil auch nicht schlecht find, ist ja auch richtig, aber es gibt gewisse Grenzen, ja [...]“" (IE 77-81)

Eva führt aus, es habe keine Konsequenzen gegeben, wenn jemand „Mist gemacht" (IE 83) habe. Implizit kommt über diese Beschreibungen und damit verbundene Bewertungen zum Ausdruck, dass Eva selbst im Eigenbild den strengen Kriterien, die bei dem Projekt, in dem sie arbeitet, gelten, entspricht. 
Eva ist es dabei besonders wichtig zu erwähnen, dass es egal sei, ob man im Rollstuhl sitze oder nicht, man müsse funktionieren. Sie als Rollstuhlfahrerin entspricht im Eigenbild diesen Erwartungen. Bei Eva erfolgen im Interview im Vergleich zu den anderen interviewten Frauen Reflexionen über „Behinderung“" explizit und in sehr differenzierter Weise. Dabei ist es Eva wichtig, darauf hinzuweisen, dass ihre Behinderung in der Einschätzung ihrer Fähigkeiten von außen keine Rolle spiele. Sie müsse trotz ihrer Behinderung Leistung erbringen, eigenständig und eigenverantwortlich agieren und auch Konsequenzen von Fehlverhalten tragen. Eva bringt damit zum Ausdruck, dass sie keine Sonderbehandlung aufgrund ihrer Behinderung wünscht und auch nicht erhält. Dies wird auch darin deutlich, dass sie eine andere Stelle, an der sie gearbeitet hat, dafür kritisiert, dass ,,immer wieder noch auf die Behinderung Rücksicht genommen worden“" (IE 79) sei. Eva möchte ihre Fähigkeiten und ihre Ressourcen im Zentrum der Einschätzung von außen sehen. Sie möchte nicht, dass auf ihre Behinderung Rücksicht genommen wird. Dies dokumentiert sich im Vergleich, den Eva zwischen den beiden Projekten zieht, wo sie als positiven Pol eine Einstellung beschreibt, wo es ,egal [ist] ob du im Rollstuhl sitzt oder nicht“ (IE 78), als negativen Pol, dass ,,immer wieder noch auf die Behinderung Rücksicht genommen worden“ (IE 79) sei.

Eva beschreibt weiter, dass sie an der jetzigen Arbeitsstelle immer wieder zu hören bekomme, dass man wohl wisse, dass man sie „oft sehr fordern und zu viel von mir verlangen“ (IE 90) würde. Aber man würde auch wissen, „dass ich das dann doch immer irgendwie hinkriege“ (IE 91). Darüber wird deutlich, dass Eva bewusst machen will, dass die Anforderungen, die an sie gestellt werden sehr hoch seien, manchmal an Überforderung grenzen, weist aber auch darauf hin, dass man wissen würde, dass sie es doch immer irgendwie schaffe. Dadurch, dass Eva in der Erzählung dies als die Perspektive der Arbeitsstelle einbringt, ist wieder die Intention zu erkennen, ihre Fähigkeiten aus der Außenperspektive wahrgenommen darzustellen, somit ein objektives Bild zu zeichnen. Eva vermeidet es hier, selbst zu sagen, was sie alles schafft. Dies scheint die Erzählstrategie von Eva zu kennzeichnen. Die eigenen Fähigkeiten sozusagen aus der Perspektive des sozialen Umfeldes hervorzuheben und somit ein (aus ihrer Perspektive) objektives Bild von ihr zu zeichnen. Auch in der Passage, wo sie die Anforderungen an ihrer Arbeitsstelle beschreibt, wird ein ähnliches Muster sichtbar. Dort beschreibt Eva sachlich die Anforderungen, die an Mitarbeiter gerichtet werden, und zeichnet sich implizit als Person, die diesen Anforderungen genügt.

Aus Evas Äußerungen wird auch deutlich, dass sie gut um die eigene Beeinträchtigung und damit verbundene Einschränkungen Bescheid weiß und sich aktiv damit auseinandersetzt. Sie beschreibt den Prozess, zu erkennen, dass ,es [...] nicht so [geht] wie ich möchte jetzt rein vom körperlichen her“ (IE 505-506), und Unterstützung zu benötigen als sehr schwierig, auch wegen ihres „sehr sehr großen Stolzes“ (IE 509). Wenn sie vom Körperlichen her 
könnte, so Eva, würde sie „,zu hundert Prozent alles alleine machen“ (IE 508), aber „das geht halt nicht, allein von der Körperlichkeit her funktioniert das nicht" (IE 510). Eva beschreibt den Prozess, sich damit auseinanderzusetzen, auch als spannenden Prozess. Eva reflektiert auf einem sehr hohen Niveau die eigene Beeinträchtigung und den daraus resultierenden Unterstützungsbedarf. Die Auseinandersetzung, die nötig ist, um dies zu akzeptieren, beschreibt Eva als spannenden Prozess. Dass Behinderung Unterstützungsbedarf nach sich zieht, liegt für Eva damit auf der Hand und muss nicht begründet werden. Sie sieht die eigene Beeinträchtigung nicht als Unzulänglichkeit, sondern als etwas, das es nun einmal gibt und mit dem sie sich auseinandersetzen muss. Dass sie die Unterstützung, die sie benötigt, auch erhält, scheint für Eva selbstverständlich zu sein.

Aus Evas Erzählung, die ja die Entwicklung von den vielen Jahren zu Hause über eine interessante Erfahrung in einem Projekt bis zur jetzigen Arbeitsstelle, wo sie als vollwertige Mitarbeiterin gesehen und behandelt wird, skizziert, sind soziogenetische Prozesse erkennbar. Dadurch dass Evas Kompetenzen anerkannt und gewürdigt wurden und sie in einem hohen Maße gefördert und auch gefordert wurde, steigen bei Eva zunehmend ein positives Selbstbild und der Selbstwert und tatsächlich auch noch einmal jene Kompetenzen, für die sie Anerkennung bekommen hat. So stellt sich Eva im Interview wirklich als so eloquent dar, wie sie in der eigenen Beschreibung von anderen eingeschätzt wurde. Sie erkennt diese Zusammenhänge auch und reflektiert diese auf einem hohen Niveau. Auch vom sprachlichen her sind Evas Ausführungen sehr differenziert und lassen darauf schließen, dass sie in ihrer beruflichen Sozialisation in Zusammenhängen integriert war, in denen sehr sensibel zu Fragen von Beeinträchtigung umgegangen und auch gesprochen wurde. Eva verwendet z.B. als einzige unter den interviewten Frauen ganz selbstverständlich den Begriff Assistenz neben jenem der Unterstützung.

Perspektivische, realistische Überlegungen im Zusammenhang mit dem Wunsch nach einer eigenen Familie

Für Eva steht im Zentrum ihrer Wünsche für das weitere Leben eine Familie, „also das heißt Mann, Kind, eigenen Haus“ (IE 547):

„Das ist schon so das Ding, was ich mir noch erfüllen möchte, woran ich auch Stück für Stück arbeite, aber ob das jetzt aufgeh'n wird oder nicht sei dahingestellt. Aber das ist einmal der Traum, den ich einmal sicherlich erfüllen wollen würde, weil ich mir denk, dass wir das ganz gut - dass i des ganz gut schaffen kann. Und dass das auch ganz gut funktioniert, wie gesagt, das wär' so das Ultimative eigentlich, das wär so, was sich glaube ich jeder wünscht, ja, einfach, seinen Partner haben, sich aufgehoben zu fühlen, jemanden für sich zu haben.“ (IE 520-537) 
Früher habe sie Familie und Kinder abgelehnt, ,aber jetzt, da du dein eigenes Leben lebst und siehst, was eigentlich schaffbar und machbar ist" (IE 561562), frage man sich, wieso solle das „eines Tages nicht einmal auftauchen“ (IE 562).

Es wird an dieser Stelle deutlich, dass die Lebensbedingungen, die für Eva weitgehende Selbstbestimmung mit der erforderlichen Unterstützung zulassen, auch neue Perspektiven und Möglichkeiten erschließen, die unter anderen Rahmenbedingungen gar nicht vorstellbar gewesen wären. So hat auch Eva früher Kinder und Familie abgelehnt, hat aber im Zuge des Prozesses in Richtung eines selbstbestimmten Lebens und damit auch eigener Entwicklungsprozesse realisiert, dass vieles möglich ist. Sie hat Vertrauen, Selbstbewusstsein und Eigenverantwortung aufgebaut, gelernt, sich selbst zu vertreten, gelernt, Unterstützung anzunehmen. Dies alles hat sie dazu geführt, auch weitere Schritte, die früher für sie nicht vorstellbar waren, als Perspektive zu entwickeln.

In einer früheren Passage sprach Eva davon, derzeit noch keine feste, fixe Beziehung anzustreben, da dies gesicherte Lebensbedingungen für sie und ihren Partner erfordern würde, ging also implizit davon aus, dass dies zum gegebenen Zeitpunkt noch nicht der Fall ist. Im Verlauf des Interviews kommt Eva offenbar zu dem Schluss, dass dies im angesprochenen Zeitraum von drei Jahren der Fall sein könnte. In dieser Passage sprach Eva davon, dass sie sich momentan ,sexuell ziemlich aus[lebe), tobe sich da ziemlich aus“" (IE 213). Letztendlich sehnt sich Eva aber offenbar auch nach dem, was sich ihr Vater für sie wünscht - „eine normale, eine normale Familie so wie wir sind, Papa, Mama und vielleicht Kinder" (IE 207-209).

Eva ist es aber auch wichtig, zu betonen, dass es auch kein „Weltuntergang" (IE 554, 556, 557) sei, wenn sich dieser Wunsch nicht erfüllen würde. Diese sprachliche Verstärkung kann einerseits die Zufriedenheit mit dem bereits Erreichten ausdrücken, andererseits könnte es auch Ausdruck dafür sein, wie sehr Eva sich doch nach Familie und Kindern sehnt. Eva zieht am Ende dieser Passage gleichsam ein Resumé, eine Einschätzung des Status quo ihrer Lebenssituation, die sie als ,so gefestigt“ (IE 563), sozial in einen großen Freundeskreis eingebunden, „stabil im Leben“ (IE 564) stehend beschreibt. Eva beschreibt über die Frage nach Wünschen an das Leben sehr realistisch und reflektiert ihre Vorstellungen von Familie und Kind und lässt deutlich werden, dass sie die Erfüllung dieses Wunsches zwar als „,das Ultimative“ (IE 535) einschätzt, die Nicht-Erfüllung aber auch keinen „Weltuntergang" darstelle.

\section{Einschätzung materieller Autonomie als Grundlage für Selbstbestimmung}

In Evas Einschätzung ist materielle Autonomie eine wichtige Basis für selbstbestimmtes Leben bzw. befördert dieses. 
„Ja, was der Job mit sich bringt, ist ganz einfach: du kannst dein eigenes Leben finanzieren, du kannst dir deine Wünsche erfüllen, du kannst dein - , ja du bist ein Teil der Gesellschaft, wo man eigentlich hingehört, ja, du bist ein ganz ein normaler Teil der Gesellschaft, du erwirtschaftest dein Leben sogar, du gehst sogar einkaufen, du gehst ein Waschmittel kaufen, du gehst - du zahlst deine, deine Arztrechnungen, deine Telefonrechnungen, alles, du=du bist für dein Leben, egal in welcher Hinsicht Job , Arb- , Job, Privatleben, finanzielle Dinge, man ist für sein Leben komplett selber verantwortlich.“( (IE 480-486)

Für Eva sind finanzielle Unabhängigkeit und damit die Möglichkeit der Erfüllung eigener Bedürfnisse somit die Grundlage eines selbstbestimmten Lebens innerhalb der Gesellschaft, also auch für gesellschaftliche Partizipation. Sie gebraucht hier den Begriff „normal“, um darauf hinzuweisen, ein Leben wie alle andere Menschen zu führen, dazuzugehören und ihren eigenen Weg in der Gesellschaft zu gehen. Sie will dazu auch einen eigenen Anteil leisten. Deutlich wird aus der Passage, dass für Eva eindeutig ein $\mathrm{Zu}-$ sammenhang von finanzieller Einbindung und der Möglichkeit, eigene Bedürfnisse selbstbestimmt umzusetzen, hergestellt wird.

Auch an anderer Stelle wird deutlich, dass Eva einen Zusammenhang zwischen materieller Unabhängigkeit und der Möglichkeit, alle Lebensbereiche selbstbestimmt gestalten zu können, sieht. So macht sie eine mögliche Familiengründung bzw. Mutterschaft von guten wirtschaftlichen Rahmenbedingungen abhängig. Sie erachtet es für eine fixe Partnerschaft als notwendig, dass „mein Leben gesichert ist, das Leben meines Partners gesichert ist“ (IE 211-212).

\section{Bewusstsein und Reflexion von Fremd- und Selbstbestimmung und eigene Definitionen dazu}

Gefragt, ob sie die Bedeutung der Begriffe Selbstbestimmung und Fremdbestimmung für sie beschreiben könnte, liefert Eva Definitionen der beiden Begriffe:

„Ist eigentlich mit einem Wort gesagt: wenn du fremdbestimmt wirst, lasst du dir von anderen dein Leben diktieren, und das=das sagt ja das Wort schon, von jemand anders, ja. Und Selbstbestimmung ist eigentlich genau das, was jeder haben sollte, egal - jetzt sag' ich mal jetzt grob g'schlichtet, egal, in welcher Behinderungsart der Mensch sich befindet, jeder, bis zu einem gewissen Grad kann er seinen Lebensweg oder sein Leben selbst bestimmen und des sollte man auch zulassen, egal in welcher Form egal in welcher Weise, weil nur so kann sich ein Mensch weiterentwickeln und nur so kann ein Mensch seine Fähigkeiten, seine Schwächen, seine Stärken entdecken, und dann kann er leben anfangen, aber dazu muss man das erst einmal zulassen.“ (IE 213-321)

In einem fremdbestimmten Rahmen sieht Eva keine Möglichkeit, eigene Entscheidungen zu treffen. Implizit ist in dieser Formulierung aber auch der Aspekt enthalten, dass es dazu komme, weil man das „zulasse“. Insofern sieht Eva auch einen eigenen Anteil am Zustandekommen von Fremd- 
bestimmung. In ihrer Definition von Selbstbestimmung macht Eva deutlich, dass die dafür erforderlichen Rahmenbedingungen in erster Linie in der Gewährung von Freiraum zur Verfügung gestellt werden müssen, betont also den externen Anteil. An der Formulierung, die als Fokussierungsmetapher gesehen werden kann, dokumentiert sich wieder die starke Orientierung von Eva an Selbstbestimmung. Erst, wenn man die Bedingungen für ein Selbstbestimmtes Leben zur Verfügung habe, könne man „leben anfangen“ (IE 321).

\subsubsection{Zusammenfassung der Fallrekonstruktion Orientierung D - „Eva“}

Im Fokus der über die Fallrekonstruktion „Eva“ dargestellten Orientierung stehen umfassende Selbstbestimmung und Autonomie, die in ihrer Wechselwirkung von eigener Person und Umfeldbedingungen reflektiert werden. Kompakt dargestellt dokumentiert sich in Typ D eine

Starke Orientierung an Selbstbestimmung und Autonomie, die umfassend, aktiv, reflektiert und konsequent angestrebt werden

Diese Orientierung ist gekennzeichnet durch eine aktive, zielstrebige und reflektierte Einstellung in Richtung Selbstbestimmung und Autonomie. Sie umfasst alle Lebensbereiche, wird vordergründig jedoch zunächst als Streben nach mehr Freiraum und weniger Kontrolle durch vorgegebene Strukturen sichtbar. Regeln und Vorschriften werden nur dann akzeptiert, wenn sie im gegebenen Kontext nachvollziehbar sind. Der Wunsch, den eigenen Alltag und das persönliche Umfeld selbstbestimmt zu gestalten, ist zentral. Eigene Ansprüche und Bedürfnisse werden artikuliert und auch gegen Vorbehalte und Widerstand aus dem sozialen Umfeld vertreten.

Selbstbestimmtes, möglichst autonomes Wohnen wird als Voraussetzung für die eigene Entfaltungsmöglich eingeschätzt und wird konsequent angestrebt und umzusetzen versucht. Eigene Vorstellungen und Bedürfnisse für die konkrete Gestaltung des eigenen Umfeldes werden im Zuge der Realisierung des autonomen Wohnens zum Ausdruck gebracht. Damit einher gehen mit zunehmendem Umsetzungsgrad auch Wünsche nach Autonomie, Selbstbestimmung, Normalisierung und Partizipation in allen anderen Lebensbereichen. Je weiter diese Orientierung realisiert werden kann, desto umfassender und selbstverständlicher kommen diese Wünsche zum Ausdruck. Häufig münden sie in Vorstellungen und Entwürfen für das eigene Leben, die sich letztendlich stark am als gesellschaftliche „Normalität" wahrgenommenen Bild gleichaltriger Frauen ausrichten. Partnerschaft und Familie verbunden mit zunächst eher verhalten thematisiertem Kinderwunsch stellen perspektivisch wichtige Faktoren für den eigenen Lebensentwurf dar. Zunächst wird aber vor allem der Wunsch sichtbar, Erfahrungen mit Partnerschaft und Se- 
xualität zu erleben, sich darüber selbst besser kennen zu lernen und weiter zu entwickeln.

Im Kontext mit dem eigenen Lebensentwurf erfolgt eine realistische Reflexion vorhandener Voraussetzungen und gegebener Ansprüche für dessen Umsetzung, die auch in Verbindung mit dem Blick auf die eigene Person stehen. Die (Weiter-)Entwicklung der eigenen Persönlichkeit und damit verbunden des eigenen Selbstbildes stehen damit unmittelbar in Zusammenhang. Eigene Stärken und Schwächen werden wahrgenommen, reflektiert und stellen einerseits eine Grundlage für das aktuelle Selbstbild, andererseits den Ausgangspunkt für persönliche Weiterentwicklung dar. Kennzeichnend dafür ist auch der zunehmend stärker werdende Wunsch, als individuelle Persönlichkeit ganzheitlich wahrgenommen und akzeptiert zu werden. Dies beinhaltet auch den Wunsch, sich über das eigene Äußere auszudrücken und vom sozialen Umfeld als erwachsene Frau mit ihrer eigenen Sexualität wahrgenommen und akzeptiert zu werden. Der Blick auf die eigene Beeinträchtigung ist zunehmend ressourcen- und umfeldorientiert. Der Fokus liegt auf dem persönlichen Potential, das im Zusammenhang mit bereits vollzogener Weiterentwicklung abgeschätzt und als Indikator für eigene weitere Entwicklungsschritte gesehen wird. Die Wirkung des sozialen Umfeldes wird als förderlich oder hemmend für die Umsetzung von Wünschen nach Selbstbestimmung, Autonomie und Partizipation wahrgenommen und durchdacht. Barrieren, die sich daraus ergeben, werden thematisiert, deren Überwindung steht im Fokus. Diese Reflexion erfolgt unter Bezugnahme auf eigene Erfahrungen in verschiedenen Phasen und Bereichen der Sozialisation als Beispiele dafür. Dies dokumentiert sich etwa anhand der dargestellten Fallrekonstruktion in der vehementen Ablehnung fremdbestimmter Kontexte, die durch Regeln und Vorschriften gekennzeichnet waren, die über eigene mehrjährige institutionelle Erfahrungen verdeutlicht werden. Demgegenüber wird der Wunsch nach Unterstützung und Begleitung artikuliert, der Selbstbestimmung aus der eigenen Perspektive fördert. Es erfolgt eine realistische Einschätzung des Unterstützungsbedarfes, der sich aus der eigenen Beeinträchtigung ergibt. Diese Überlegungen werden gedanklich in die Umsetzung des eigenen Lebensentwurfes mit einbezogen.

Je mehr Selbstbestimmung und Autonomie in den realen Lebensbedingungen umgesetzt sind, desto mehr befördern diese als Basis die eigene Weiterentwicklung und die reale Umsetzung von weiteren Wünschen und Erfordernissen, die sich daraus ergeben. Abhängigkeit und Kontrolle werden immer weniger als Determinanten für das eigene Leben denkbar, eigene Erfahrungen damit werden als negativer Gegenpol zur gewünschten Lebensform charakterisiert.

Von der soziogenetischen Ebene wirken Erfahrungen von Wahrgenommen-, Akzeptiert-, Anerkannt-, Gefördert- und Gefordert-Werden in verschiedenen Erfahrungsräumen im Verlauf der Sozialisation förderlich auf die 
Entwicklung einer solchen Orientierung und haben damit den Stellenwert biographischer Ressourcen. Dass das Äußern eigener Bedürfnisse in solchen Zusammenhängen als Basis für die Gestaltung des eigenen Lebens berücksichtigt wird, eröffnet real neue Perspektiven und vermittelt das Gefühl von Selbstwirksamkeit, das wiederum weitere Entwicklungsschritte einzuleiten vermag. Wesentlich dafür sind stabile und wohlwollende Beziehungen, die über interaktive Prozesse Möglichkeiten zur eigenen Weiterentwicklung bieten. Dann stellen auch Auseinandersetzungen, Reibungspunkte und Widerstände im sozialen Umfeld wichtige Komponenten dar, indem dadurch Grenzen verhandelt und erweitert werden.

Im Verlauf der Sozialisation durchaus auch als abwertend, diskriminierend und übergriffig erlebte Erfahrungen können durch eine bewusste und durch das Umfeld unterstützte Auseinandersetzung damit auch als Impulse zur Weiterentwicklung wahrgenommen und reflektiert werden. Negative Erfahrungen und Rückschläge werden aus dieser Perspektive mit positiven Konsequenzen in Zusammenhang gebracht und als Initiatoren von Lernprozessen eingeschätzt, die die eigene Persönlichkeitsentwicklung vorangetrieben haben. Sie werden im Sinne dieser Orientierung eher als Herausforderungen gesehen, an denen man sich weiterentwickeln und reifen kann. Auch aktuell wird Herausforderungen und Rückschlägen mit Resilienz begegnet, sie werden zuweilen gar als notwendige Eckpunkte auf dem eigenen Weg in Richtung Selbstbestimmung und Autonomie reflektiert.

Einen wesentlichen Impuls für die Entwicklung dieser Orientierung bildet die Möglichkeit, eigenes (Teil-)Einkommen über eigene Arbeit zu erwirtschaften und zur Verfügung zu haben. Daraus ergeben sich real und gedanklich neue Chancen und Handlungsspielräume, die neue Perspektiven eröffnen. Die Motivation zur Bereitschaft, an sich selbst zu arbeiten und die eigene Entwicklung voranzutreiben, wird dadurch gefördert.

\subsubsection{Fallübergreifende Ausprägungen von Orientierung D}

Die in der angeführten Fallrekonstruktion „Eva“ anhand zentraler Dimensionen herausgearbeitete Orientierung wird fallübergreifend in verschiedenen Ausprägungsvarianten sichtbar. Dabei werden wieder Gemeinsamkeiten hinsichtlich der Bearbeitung verschiedener "Themen und Problemstellungen“ (Nohl 2012: 52), die sich in unterschiedlichen Fällen allerdings auch auf unterschiedliche Weise abbilden, deutlich.

Im Folgenden führe ich einige Beispiele aus einem anderen Fall dafür an, also weitere Belege, die zentrale Dimensionen dieser Orientierung deutlich machen. Die also die „Starke Orientierung an Selbstbestimmung und Autonomie, die umfassend, aktiv, reflektiert und konsequent angestrebt werden “ dokumentieren: 
Im Zusammenhang mit ihren Überlegungen zum autonomen Wohnen dokumentieren sich auch bei Tamara die Ablehnung von Behütung und Kontrolle und damit verbunden der Wunsch nach Freiraum/Freiheit und Privatsphäre. Tamara kritisiert, dass sie in einer Werkstätte immer sagen musste, was sie mache und wohin sie gehen wolle (vgl. IT 553-554). Es sei immer darauf geachtet worden, dass ,wohl nichts passiert“ (IT 556), das habe sie „sehr gestört" (IT 557). Sie führt mehrmals aus, dass sie autonomes Wohnen mit der Möglichkeit, „für sich“ zu sein, in Zusammenhang bringt (vgl. IT 565 2x, IT 567, IT 569). Auch das Bedürfnis, die Freizeit selbstbestimmt zu gestalten und sich nicht an vorgegebenen Strukturen orientieren zu müssen, dokumentiert sich an Tamaras Ausführungen zum autonomen Wohnen. Sie könne dann ihren Hobbys stärker nachgehen und müsse nicht mehr ,immer das machen, was alle gerade machen müssen" (IT 586). Damit dokumentieren sich auch bei Tamara die Ablehnung von Regeln und Vorschriften sowie der Wunsch nach eigenen Gestaltungsmöglichkeiten.

Tamara will ihre Vorstellungen auch umsetzen und setzt sich aktiv dafür ein. Dadurch dokumentiert sich auch das Bestreben nach Artikulation und Selbstvertretung der eigenen Wünsche, Ansprüche und Bedürfnisse. So erzählt Tamara über eine Situation in der Werkstätte (vgl. IT 271-304) einer Behinderteneinrichtung, in der sie sich ungerecht behandelt fühlte. Sie führt aus: ,ja, dann habe ich es halt gesagt, das mich das stört“ (IT 291). Danach habe sich die Situation für sie gebessert. Gefragt, ob es andere solche Situationen gegeben habe, erzählt Tamara, dass sie an der aktuellen Arbeitsstelle gesagt habe, ,welche bestimmte Sachen ich machen möchte" (IT 304), und dem auch gefolgt worden sei. Dies sei für ihr „Selbstbewusstsein sehr gut“ (IT 305) gewesen. Damit wird auch über Tamaras Ausführungen deutlich, dass dem Wahrgenommen- und Akzeptiert-Werden mit den eigenen Wünschen und Bedürfnissen besondere Bedeutung zukommt. Dies führt auch bei Tamara zu mehr Selbstbewusstsein und befördert Weiterentwicklung. Sie lehnt es etwa $a b$, in einer Institution im Küchenbereich zu arbeiten. Das habe sie auch den Betreuerinnen gesagt und dann habe ,sie halt geschnuppert in der Tischlerei und dann haben sie mich dort genommen“" (IT 383). Hier wird stringent beschrieben, dass sie einen Wunsch äußert und dieser dann ohne Widerstände umgesetzt wird. Tamara artikuliert eigene Vorstellungen und Bedürfnisse zunehmend selbstbewusst und selbstverständlich.

Das Bedürfnis, als eigene Persönlichkeit mit den eigenen Stärken und Schwächen und mit dem eigenen Erscheinungsbild wahrgenommen und anerkannt zu werden, dokumentiert sich über Tamaras Erzählung (vgl. IT 587640) über den Einstieg in einen Gymnastik-Kurs. Sie kommt darauf zu sprechen, dass sie sich eingangs „schwer getan“ (IT 608) habe, weil sie nicht gewusst habe, wie die Leute auf sie reagieren würden. Als Gründe für diese Unsicherheit führt sie an, dass sie nicht ganz schlank, „stärker“ (IT 609) sei und auch dass es im Ort „ein bisschen Ablehnung“ bedingt durch ihre leib- 
liche Mutter gebe. Tamara vermutet also Ablehnung aufgrund ihrer äußeren Erscheinung und Stigmatisierung wegen der Person ihrer leiblichen Mutter. Sie führt dann aber aus, dass sie im Gegenteil dafür sogar „bestaunt“ wurde und „Freude“ empfunden habe, dass sie nicht abgelehnt wurde. Dadurch sei sie selbst „stärker" geworden und habe sich weiterentwickelt (vgl. IT 611).

Auch bei Tamara dokumentiert sich ein zunehmend positives Selbstbild mit ressourcen- und umfeldorientiertem Blick auf die eigene Beeinträchtigung. So macht sie deutlich, dass sie über Ressourcen verfügt, anderen Personen Unterstützung zukommen zu lassen. In ihrem aktuellen Arbeitsbereich würde sie Kolleginnen ,ja [...] unterstützen, einfach so unterstützen“ (IT 471). Sie sei „einfach sehr hilfsbereit“ und wolle „halt (...) helfen, ja“ (IT 490). Ihre schulischen Probleme bringt sie in der Rückschau mit ungünstigen Rahmenbedingungen in Zusammenhang, es sei „zu schnell““ (IT 137) vorgegangen worden. Tamara bringt eigene Einschränkungen also zunehmend auch mit Barrieren im Umfeld in Zusammenhang. Sie beschreibt, dass sie im Urlaub mit den Eltern zusammen einkaufen gegangen sei und führt aus: „ich muss immer jemanden zum Fahren haben“" (IT 536). Alleine irgendwo hinzufahren, sei unter anderem wegen weiter Entfernungen und des Umsteigens im Zug schwierig (vgl. IT 536). Tamara stellt somit einen direkten Bezug zwischen mangelhaften Rahmenbedingungen und daraus resultierenden Einschränkungen dar. Wie bereits in den Passagen zu den mangelhaften Rahmenbedingungen in der Schulzeit stellt Tamara auch hier einschränkende Umfeldbedingungen in das Zentrum ihrer Argumentation.

Auch Tamara reflektiert Herausforderungen als Chancen persönlicher (Weiter-)Entwicklung. Diese Herausforderungen liegen bei Tamara vorwiegend in der Frage der Auseinandersetzung mit der eigenen Beeinträchtigung und des Umganges damit im Rahmen sozialer Interaktionen. An einer Passage über eine Begegnung mit zwei jungen Männern wird deutlich, dass Tamara über ihr eigenes „Behindert-Sein“ und den eigenen Umgang damit zunehmend reflektiert. Sie erzählt, dass sie die Kontaktaufnahme durch die beiden jungen Männer von sich aus abgebrochen habe, indem sie gesagt habe, ,dass ich behindert bin" (IT 775). Einer der beiden wollte mehr darüber wissen, aber - so Tamara: ,ich wollte es ihm damals nicht erklären, weil ich ihn noch nicht gekannt habe“" (IT 775-777). Für sich selbst reflektiert Tamara, dass sie sich mit ihrem Verhalten und ihrer Äußerung darüber, eine Behinderung zu haben, selbst die Chance auf das Kennenlernen verbaut hat. Zukünftig sei es besser, sich das zu ,verkneifen“ (IT 784) und gleich zu reden zu beginnen. Man sehe es ihr ja auch nicht gleich an, so Tamara, „dass ich eine Beeinträchtigung habe“ (IT 669). Auf der anderen Seite hätte sie Angst, „,weil ich halt meine Handicaps“ (IT 934) habe. Damit beschäftige sie sich momentan sehr stark (vgl. IT 938).

Auch der Wunsch nach selbstbestimmter Gestaltung von Partnerschaft dokumentiert sich an Tamaras Ausführungen. Dies bezieht sich vorrangig auf 
die Vorstellungen ihres künftigen Partners, der „einfach selbständig und einfach beruflich selbständig und einfach so, ja“ (IT 668) sein soll. Wichtig sei auch, „dass er selber arbeitet und so“ (IT 676). Eine Partnerschaft steht für Tamara aktuell ganz zentral im Fokus ihrer Wünsche. Gefragt, ob sie noch weitere Wünsche ans Leben hat, sagt Tamara ,nein, das wäre für mich jetzt wichtig“, „voll“ (IT 680, IT 682). Tamara stellt auch perspektivische, realistische Überlegungen im Zusammenhang mit dem Wunsch nach einer eigenen Familie an. Sie geht davon aus, dass sich diese Vorstellungen erst im Kontext der Ablösung von ihrem Elternhaus umsetzen lassen. Dabei bringt sie autonomes Wohnen stark mit der Vorstellung, ,einen Lebenspartner [zu] kriegen" (IT 585) in Zusammenhang.

Materielle Autonomie wird dabei von Tamara als Grundlage für selbstbestimmte Entscheidungen eingeschätzt. Sie bringt ihr Gehalt, das sie in einem Arbeitsversuch bekommt, von sich aus sofort mit der Frage nach einer eigenen Wohnung in Zusammenhang. Tamara ist sich dabei ihrer finanziellen Situation und damit verbundener Rahmenbedingungen bewusst. Sie führt aus, dass sie für ihre Arbeit „alle Monat“ „richtiges Gehalt“ (IT 493) beziehe, dass sie versichert sei und Urlaubsgeld bekomme. Zukünftig wolle sie die eigenen finanziellen Mittel dafür einsetzen, eine eigene Wohnung zu finanzieren (vgl. IT 549).

Tamara ist sich dessen bewusst, dass sie aufgrund körperlicher Einschränkungen Unterstützung benötigt. In diesem Zusammenhang wird deutlich, dass sie traditionelle Betreuungskonzepte ablehnt und Unterstützung und Begleitung für die Umsetzung ihrer Vorstellungen möchte. Sie blickt dabei auf positive Erfahrungen mit Unterstützung bei der Arbeitsfindung (vgl. IT 436-437) und mit Jobcoaching (vgl. IT 452) zurück. Tamara verwendet in dieser Passage explizit, ausschließlich und mehrmals (siebenmal) die Begriffe „Unterstützung“ und „Begleitung“. Der Begriff der Betreuung wird in der Rückschau mit vorwiegend negativen Erfahrungen im stark strukturierten, institutionellen Kontext in Zusammenhang gebracht. Sie beschreibt immer wiederkehrende Tätigkeiten und Fixpunkte, an die sich alle halten mussten. Die zugewiesenen Tätigkeiten hätte man schnell erledigen müssen, wenn dies nicht der Fall gewesen sei, sei man ,von den Betreuerinnen ausgeschimpft“ worden (vgl. IT 240-269).

\subsection{Die vier erhobenen und typisierten Orientierungen in der Einschätzung der Frauen aus der Referenzgruppe}

Die vier zuvor anhand von Fallrekonstruktionen typisierten Orientierungen wurden den Frauen aus der Referenzgruppe in einer eigens dafür organsierten Sitzung präsentiert und zur Diskussion gestellt. Dies war verbunden mit der 
Frage, ob damit aus ihrer Perspektive die Lebenswelt von Frauen mit Lernschwierigkeiten in ihrer Vielfalt angemessenen und nachvollziehbar abgebildet wird. Hinsichtlich der sinngenetischen Typen war für mich auch die Frage von Bedeutung, ob die vier rekonstruierten Orientierungen um weitere Orientierungen ergänzt werden müssten.

Zunächst wurden den Frauen aus der Referenzgruppe die vier typisierten Orientierungen in angemessener Sprache und anschaulich anhand von Beispielen aus den Fallbeschreibungen „Isolde“, „Martina“, „Anna“ und „Eva“" vorgestellt. Im Anschluss daran erfolgte eine Diskussion der vier Orientierungen durch die Frauen der Referenzgruppe. Dabei wurden von den Frauen die eingebrachten Beispiele einerseits um eigene Beobachtungen und Erfahrungen ergänzt. Andererseits wurden von ihnen bereits Fragen, wie es zu den spezifischen Orientierungen gekommen sein könnte, ansatzweise reflektiert. Dabei deutete sich für mich bereits an, dass die Frauen vor dem Hintergrund ihrer konjunktiven Erfahrungen als Frauen mit Lernschwierigkeiten, Faktoren thematisierten, die ich bereits über meine eigene soziogenetische Analyse erhoben, ihnen aber zu diesem Zeitpunkt noch nicht präsentiert hatte.

Im Folgenden fasse ich die zu den vier Orientierungen erfolgten Diskussionen und Anmerkungen der Frauen aus der Referenzgruppe kursorisch zusammen, wobei ich mich insgesamt auf das Protokoll ${ }^{94}$ dieser Sitzung beziehe:

Orientierung A - Orientierung am vorgegebenen, stark strukturierten, vertrauten Rahmen, der auf individuelle, oft phantasievolle, im Detail selbstbestimmte Weise ausgestaltet wird

Die zusammenfassende Beschreibung dieser Orientierung wurde von den Frauen als gut nachvollziehbar und stimmig zu ihren eigenen Erfahrungen und Beobachtungen erlebt. Jede der Frauen aus der Referenzgruppe konnte anhand mindestens eines Beispieles aus dem eigenen Erfahrungsbereich diese Orientierung als eine für Frauen mit Lernschwierigkeiten typische Orientierung wiedererkennen. Dabei wurde diese explizit in Zusammenhang mit zwei möglichen Lebensbedingungen, vor allem dem Leben bei den Eltern oder in einer größeren, stark strukturierten Institution gebracht.

Gleich eingangs wurde von einer der Frauen aufgeworfen, dass eine solche Orientierung ihrer Einschätzung nach möglicherweise auch deshalb zustande kommt, weil die betroffene Frau noch keine andere Möglichkeit als das Leben in einem stark strukturierten Rahmen kennengelernt hat. Sie selbst habe aufgrund der Möglichkeit, außerhalb der Einrichtung, in der sie lebt, einer Beschäftigung nachzugehen, ,gesehen, dass es auch anders sein kann“"(S. 3).

94 Siebente Sitzung mit der Referenzgruppe, Protokoll-Seiten 1-15 (7RGS-S1-15). In diesem Kontext beziehe ich mich durchgängig auf das Protokoll dieser Sitzung und führe Seitenzahlen nur mehr bei direkten Zitaten an. Auf Wunsch der Frauen aus der Referenzgruppe werden direkte Zitate nicht explizit genannten Frauen zugeordnet. 
Darüber, dass man aus dem geschützten Rahmen hinauskomme, lerne man Leute kennen, man komme dann mit neuen Erfahrungen nach Hause und sehe ,alles mit anderen Augen“ (ebd.). Wenn man einmal erlebt habe, was außerhalb des geschützten Rahmens möglich sei, dann wolle man das auch wieder haben und sei nicht mehr mit den gegebenen Bedingungen zufrieden. Zwei andere Frauen thematisierten, dass dies auch von der Tagesverfassung abhängig sei. Es gebe Tage, da würde man den vertrauten Rahmen bevorzugen und sich nicht hinausbewegen wollen. Eine weitere Anmerkung betraf die Frage des Alters: Wenn man als Frau älter werde, würde man den vertrauten Rahmen wahrscheinlich wieder bevorzugen, weil man sich darin sicherer fühle. Man hätte dann wahrscheinlich wegen des Alters außerhalb des geschützten Rahmens auch weniger Chancen. Die Orientierung am vertrauten Rahmen wurde von den Frauen aus der Referenzgruppe auch mit verschiedenen Ängsten in Verbindung gebracht, die mit dem Sich-Hinausbewegen aus diesem Rahmen verbunden sein können: zum Beispiel die Angst, nicht in die Arbeitswelt zu passen, weil man etwa als ,zu langsam“ (S. 3) eingeschätzt werde. Oder Ängste in Zusammenhang mit der eigenen Beeinträchtigung, wie "schief angeschaut", ,ausgelacht" (S. 3), ,als Behinderte gesehen werden“ oder ,ausgeschlossen zu werden“. Auch das Gefühl, ,überflüssig“ zu sein und ,nicht dazu zu gehören“, würde dazu führen, dass man lieber im geschützten Bereich bleibe.

In Familien würde zu solchen Orientierungen der Umstand führen, dass „zu viel bemuttert“ (S. 4) werde. Zudem hätten Eltern Angst, dass die Töchter „,ausgegrenzt werden“ (S. 4), „Außenseiter werden“ (S. 4) und „schlecht über sie geredet" (S. 4) werde, wenn sie sich außerhalb des geschützten Rahmens bewegen würden. Manche Eltern, so wurde von einer der Frauen eingeworfen, würden sich auch „schämen für ihre Kinder“ (S. 4) und deshalb auch verhindern, dass sie sich nach außen orientieren. Eltern hätten manchmal auch Angst vor Übergriffen auf ihre Töchter (S. 4). Zuletzt wurde auch noch festgehalten, dass die Orientierung an einem vertrauten, geschützten Rahmen insgesamt dadurch begründet sein kann, dass man ,anders als andere“ (S. 4) und ,nicht perfekt" (S. 4) sei.

Orientierung B - Ambivalente Orientierung an Selbstbestimmung und Autonomie in einzelnen Lebensbereichen bei gleichzeitigem Wunsch nach Bezug von Unterstützungsund Versorgungsleistungen durch das soziale Umfeld

Nach der Vorstellung dieser Orientierung anhand von Beispielen aus der Fallrekonstruktion „Martina“ äußert eine der Frauen als erste Assoziation „Hotel Mama“ (S. 9) und ergänzt: „,das wäre zu viel für mich“ (S. 9). Auch in dieser typisierten Orientierung wurden Verhaltensweisen und Einstellungen von Frauen mit Lernschwierigkeiten aus dem eigenen Erfahrungsbereich wiedererkannt. Anhand von Beispielen wurde darüber diskutiert, dass es immer wieder vorkomme, dass Frauen eigentlich selbst die Möglichkeit hätten, etwas selbst zu machen, sich aber aus Gründen der Bequemlichkeit ver- 
sorgen lassen würden. Man solle aber - so die einhellige Meinung der Frauen aus der Referenzgruppe - ,so weit als möglich alles selber machen“ (S. 9). Erst wenn es nicht mehr gehe, solle man Unterstützung beanspruchen.

Eine der Frauen führt an, dass es ,nicht immer nur Faulheit oder Bequemlichkeit“ (S. 9) sein müsse. Manchmal könne man zwar grundsätzlich etwas alleine machen, aber man habe Angst davor, z.B. beim Hantieren mit heißen Flüssigkeiten. Oder man könne es zwar grundsätzlich alleine machen, aber „es geht so viel Zeit d'rauf“ (S.9). Es könnten auch Ängste vorliegen, etwas falsch zu machen, Fehler zu begehen, die jemanden davon abhalten, etwas zu tun, das er eigentlich kann. Es könne auch sein, dass man es sich nicht zutraue oder dass die Motivation dazu fehle, es selbst zu tun. Fragen im $\mathrm{Zu}-$ sammenhang mit Zutrauen werden im Kontext mit dieser Orientierung eingehend diskutiert. Man müsse vom Umfeld auch dazu motiviert werden, etwas zu tun, und es müsse einem zugetraut werden, das auch zu können. Nur weil z.B. einmal etwas nicht funktioniere oder man etwas falsch gemacht habe, dürfe das auch nicht dazu führen, dass man es nicht mehr tun darf. Wenn das der Fall ist, dann würde man sich immer darauf verlassen, dass man es nicht selber tun müsse, dass einem die Arbeit abgenommen werde. Es dürfe auch nicht so sein, dass einem immer die Arbeit abgenommen werde. Man müsse gefördert, aber auch gefordert werden und auch einmal etwas alleine ausprobieren dürfen. Wenn nötig, soll man zunächst Unterstützung dabei erhalten, dann aber schrittweise lernen, es selbst zu tun.

Orientierung C - Starke Orientierung an Selbstbestimmung, Partizipation und Normalisierung bei gleichzeitigem Bedürfnis nach Erhalt eines vertrauten Rahmens, um Konflikte und/oder negative Erfahrungen innerhalb und außerhalb dieses Kontextes zu vermeiden

Auf die zusammenfassende Beschreibung dieser Orientierung äußerte eine der Frauen unmittelbar daran anschließend: „So bin ich“ (S. 5). Grundsätzlich sei es für sie in Ordnung, mit dem Leben in der Institution einen vertrauten und strukturierten Rahmen zu haben und auch in bestimmten Bereichen Unterstützung in Anspruch nehmen zu können. Innerhalb dieses Rahmens brauche sie aber „Freiraum“ (S. 5) und wolle „selbst gestalten“ (S. 5). Sie brauche auch Rückzugsmöglichkeiten. Sie würde gerne alles, so weit es geht, alleine machen. Wenn es aber einmal nicht so gut gehe, dann sei jemand da, den sie fragen könne und der sie unterstütze. Im Zusammenhang mit dem gewünschten Freiraum wurden spezifische Aspekte eingebracht, die den Frauen dafür bedeutsam erscheinen, wie etwa „das Telefonieren ohne Zuhörer“" (S. 5), das als nicht selbstverständlich beschrieben wurde. Auch die gewünschte Möglichkeit, sich dann zurückzuziehen, wenn man selbst das wolle, wurde als nicht selbstverständlich beschrieben, dem Betreuungspersonal, so wurde wörtlich geäußert, ,passt das oft nicht“ (S. 5). Es sei aber notwendig, sich zurückzuziehen, um Freiraum zu haben. Zur angesprochenen erlebten negativen Haltung des Betreuungspersonals dazu wurde geäußert: „Der Job der 
Betreuer ist es ja eigentlich, uns das Leben zu ermöglichen, das wir haben wollen. Den Job haben sie sich ausgesucht. Aber sie wollen das oft nicht." (S. 5).

Das Betreuungspersonal hätte manchmal Angst, dass es nicht mehr gebraucht werde und dass die Bewohnerinnen und Bewohner zu selbständig werden könnten. Dies sei vergleichbar mit den Ängsten von Eltern, wenn Kinder sich ablösen. Es könne zu der beschriebenen Orientierung kommen, wenn eine Frau in einer Einrichtung eigentlich mehr Freiraum möchte, ihr dieser aber nicht zugestanden wird und sie nicht ermutigt wird, ihren Wünschen nachzugehen. Dann würde sie nicht lernen, alleine zurechtzukommen, und würde immer auch Versorgung und Hilfe im Rahmen der Einrichtung benötigen. Wenn man in einem solchen Kontext lebe, so eine der Frauen, „geht es einem nicht so gut“" (S. 6).

Das diesem Rahmen eigene Moment der Vermeidung von Konflikten und negativen Erfahrungen als Grund, den vertrauten Rahmen auch dann erhalten $\mathrm{zu}$ wollen, wenn er als einengend erlebt werde, wurde ausführlich anhand beobachteter und eigener Beispiele diskutiert. Dabei wurden negative Erfahrungen $^{95}$ außerhalb des geschützten Rahmens - von Abwertung aufgrund der eigenen Behinderung bis zu körperlichen und sexuellen Übergriffen - thematisiert. Auch Abhängigkeit vom sozialen Umfeld, das den Rahmen bildet, wurde als Grund dafür genannt, dass danach gestrebt wird, diesen zu erhalten, auch wenn er als einschränkend oder konfliktreich erlebt wurde.

Orientierung D - Starke Orientierung an Selbstbestimmung und Autonomie, die umfassend, aktiv, reflektiert und konsequent angestrebt werden

Mit der Beschreibung dieser typisierten Orientierung identifizierten sich zwei der Frauen aus der Referenzgruppe explizit unmittelbar nach der Präsentation in der Gruppe mit den Ausführungen „so sehe ich mich“ (S. 7) und ,absolut, das bin ich“"(S. 7). Bei dieser Orientierung mache man alles, was man könne, selbst. Wenn man alleine nicht zurechtkomme, dann ersuche man jemanden aus dem Umfeld um Unterstützung. Eine der Frauen berichtet in diesem $\mathrm{Zu}-$ sammenhang von ihrem Leben mit persönlicher Assistenz im Arbeits- und Freizeitbereich. Sie könne festlegen, wann, wie und wo sie Unterstützung benötige. Das sei für sie Selbstbestimmung. Mit der persönlichen Assistenz könne sie auch soziale Kontakte halten, indem sie etwa selbst Besuche mache oder jemanden zu sich einladen könne.

Im Zusammenhang mit der Frage der persönlichen Assistenz wurde von den Frauen aus der Referenzgruppe auch thematisiert, dass diese für Frauen mit Lernschwierigkeiten nur dann möglich sei, wenn sie auch eine körperli-

95 Die angesprochenen Aspekte wurden von der Gruppe im Zusammenhang mit der Diskussion soziogenetischer Aspekte später noch eingehender diskutiert und werden im laufenden Kapitel im Unterpunkt ,Soziogenetische Zusammenhänge in der Einschätzung der Frauen aus der Referenzgruppe: ausgewählte Themen“ ausführlich dokumentiert. 
che Behinderung hätten. In Institutionen würde es diese Form der Unterstützung nicht geben, da gebe es „,nur Betreuung“ (S. 7). Es hänge also von der Wohnform ab, ob man diese Leistung bekommen könne. Wenn das der Fall ist, sei es „leichter möglich“ (S. 8), selbstbestimmt zu leben. Es sei auch leichter, selbstbestimmt zu agieren, wenn man in einem Umfeld lebe, wo es mehr Möglichkeiten zur Partizipation gibt. Am Beispiel einer den Frauen bekannten Wohneinrichtung, die in einer ländlichen Gegend ohne gute Infrastruktur liegt, wurden entsprechende Nachteile diskutiert. Man könne nicht selbst einkaufen gehen, weil es kein Geschäft gibt, das zu Fuß erreichbar sei. Man könne keine Kontakte außerhalb der Institution vor Ort pflegen, weil die Institution „weit weg von allem“ (S. 8) sei. Es sei also grundsätzlich auch sehr von den Rahmenbedingungen und von der Unterstützung abhängig, ob selbstbestimmtes Leben möglich sei. Man müsse auch gefordert werden, es müsse einem viel zugetraut werden und man müsse zeigen können, dass man etwas leisten kann. Dann würde man diese Orientierung entwickeln können.

\subsection{Zentrale Themenbereiche der Auseinandersetzung der vier rekonstruierten Orientierungen}

Im Folgenden werden die vier vorab dargestellten rekonstruierten Typen als Basis für die nachfolgende soziogenetische Analyse zusammenfassendvergleichend in den Blick genommen. Dies stellt gleichsam einen Zwischenschritt in Richtung der soziogenetischen Analyse dar, womit zentrale Aspekte der vorab dargestellten Fallrekonstruktionen in einer gerafften Zusammenschau abgebildet werden sollen. Es wird dabei auf Gemeinsamkeiten innerhalb der Orientierungen, aber auch schon ansatzweise auf Unterschiede zwischen diesen fokussiert. In der darauffolgenden Analyse werden dann die Unterschiede in den Orientierungen differenziert soziogenetisch nachvollzogen und sollen somit erklärbar machen, wie es zu den vorhandenen Ausprägungen gekommen ist. Dabei werden soziogenetische Faktoren themenübergreifend behandelt. Es wird dann nicht mehr danach gefragt, welche Herangehensweise es zu einem speziellen Bereich - etwa der Familie - gibt, sondern welche soziogenetische Faktoren welche spezifischen Herangehensweisen zu verschiedenen Themen und Problemstellungen hervorbringen.

Als Gemeinsamkeit der vier Typen wird dabei zunächst eine wiederkehrende, sich deutlich abzeichnende Fokussierung auf die Bereiche Familie, damit verbunden das eigene Familienbild und Vorstellungen von eigener Familiengründung sichtbar. Partnerschaft, Sexualität und damit einhergehend Kinderwunsch und Mutterrolle sind weitere relevante Themen. Wohnen in unterschiedlichen Varianten spielt im Zusammenhang mit der damit einhergehenden Alltagsgestaltung eine bedeutende Rolle in den vorliegenden Ori- 
entierungen. Beschäftigung, Arbeit und Beruf bilden einen weiteren Themenkomplex, der einen wesentlichen Orientierungsrahmen darstellt. Ganz zentral für alle vier Typen sind Fragen hinsichtlich der Wahrnehmung geschlechtsspezifischer Rollenbilder über eigene Erfahrungen im Rahmen der Sozialisation. Diese prägen in vielfältiger Weise die aktuelle eigene Orientierung, indem über Identifikation mit vorgelebten Beispielen oder kritische Auseinandersetzung damit die Ausgestaltung des eigenen Alltages sowie Lebensperspektiven beeinflusst werden. Dabei werden unterschiedliche Herangehens- und Bearbeitungsweisen deutlich. Manchmal weist auch gerade die Nicht-Thematisierung oder auch Ablehnung bestimmter Fragen implizit sehr stark darauf hin, dass damit ein Thema angesprochen ist, das tabuisiert, unterdrückt oder sehr herausfordernd ist. In einigen dieser Fälle wurden diese Themen erst über die anschauliche Methode der Vignettenbefragung expliziert. Beispielsweise spricht Martina das Thema Partnerschaft und Familie im Interview nur im Rahmen der Vignettenbeschreibung an (vgl. IM 641-645 zu VA3 und VA4), wo sie Partnerschaft von Menschen mit Lernschwierigkeiten eher ablehnend begegnet bzw. für sich selbst in Frage stellt. Das Thema ist somit nicht irrelevant für sie, es stellt sich eher die Frage, wie es zu der ablehnenden Einstellung gekommen ist und was sich darüber dokumentiert.

Im Folgenden werden die angeführten Themenbereiche gerafft in ihrer unterschiedlichen Ausprägung in den vier Typen beschrieben und verglichen, um in einem Zwischenschritt eine Ausgangsbasis für die soziogenetische Analyse zu bilden, in der dann auf Fragen des ,Wie“ fokussiert wird. In einem ersten Schritt wird also dargestellt, wie sich Orientierungen hinsichtlich der zentralen Themen in den vier Typen unterschiedlich ausdrücken. Wo es notwendig und sinnvoll erscheint, dies über anschauliche Beispiele zu verdeutlichen, werden diese über Belege aus den Interviews eingebracht. Im Anschluss an diese Zusammenfassung stehen in der soziogenetischen Analyse Fragen des Zustandekommens der vorliegenden Orientierungen im Zentrum.

\section{Familie und Familienbild}

Fragen im Zusammenhang mit Familie und Familienbild sind für Frauen aller vier typisierten Orientierungen zentrale Punkte der Auseinandersetzung, die allerdings auf sehr unterschiedliche Art und Weise im Kontext der eigenen Lebenswelt erfolgt.

In der Orientierung von Typ A spielt Familie die zentrale Rolle im eigenen Leben. Sie stellt den vertrauten Rahmen dar, in dem Sicherheit erfahren wird. Die Familie bietet aber auch den Raum, im Rahmen der Partizipation an den Alltagshandlungen ihrer Mitglieder Beziehung zu erleben. Vorgelebte Rollenbilder und Beziehungsmuster führen zudem vorwiegend über Identifikationsprozesse zu einer spezifischen Ausprägung der eigenen Frauenrolle und 
eines damit verbundenen Familienbildes. Auch Erwartungen, die über Bezugspersonen herangetragen werden, können die Ausgestaltung der eigenen Rolle ganz wesentlich beeinflussen. Beispielhaft ist hier eine extrem starke Ausrichtung am Vater durch Brigitte nach dessen Trennung von ihrer Mutter zu erkennen, die von diesem auch gefördert und gefordert wird [,,mein Leben ist der Papa" (IB 336)]. Sie übernimmt die vom Vater auch erwünschte traditionelle Frauenrolle, die die Mutter offenbar nicht (mehr) einnehmen wollte oder konnte. Sie kümmert sich um die Wäsche und räumt die Zimmer auf (vgl. IB 232). In der Folge richtet Brigitte ihr gesamtes Leben am Vater aus, unterstützt diesen und führt Alltagshandlungen wie Einkaufen (vgl. IB 299) und Freizeitaktivitäten wie Schwimmen (vgl. IB 321) mit diesem gemeinsam aus.

Diese Orientierung am vertrauten Umfeld ist auch in Orientierung B zu erkennen. Allerdings kommt sie hier weniger deutlich über den emotionalen Bezug, sondern über erbrachte und erwünschte Versorgungs- und Unterstützungsleistungen durch die Familie zum Ausdruck. Die Familie schafft hier eher den Rahmen für ein Leben nach weitgehend eigenen Vorstellungen. In der vorab dargestellten Fallrekonstruktion „Martina“" wurde dies insbesondere über die Rolle von Martinas Mutter sichtbar, die ihren eigenen Alltag stark an der Erfüllung von Martinas Bedürfnissen ausrichtet, indem sie diese in ihrer beruflichen und privaten Alltagsgestaltung stark unterstützt. Martina wird so ein Leben nach weitgehend eigenen Vorstellungen ermöglicht, in dem sie sehr selbstbestimmt agieren kann und sich auf einen familiären Hintergrund verlassen kann, den sie nicht als einengend, sondern als förderlich erlebt. Ähnlich dokumentiert sich dies bei Alina, bei der die Familie auch den zentralen Orientierungsrahmen bildet, auf den bei Vorstellungen und Phantasien hinsichtlich des eigenen Lebensentwurfes Bezug genommen wird. Sie verbindet ihre Vorstellungen vom Zusammenleben mit dem Freund ausdrücklich mit der Voraussetzung, dass der gemeinsame Wohnort nicht weit von ihrer Familie entfernt sein solle (IAl 814-824).

Auch in Orientierung $\mathrm{C}$ ist ein starker Bezug zur Herkunftsfamilie erkennbar, der insbesondere im Zusammenhang mit entwickelten Vermeidungsstrategien steht, was über die Fallrekonstruktion „Anna“ deutlich sichtbar wurde. Diese Orientierung ist jedoch von ambivalenten Erfahrungen innerhalb dieses Systems charakterisiert. Einerseits wird Familie mit der Möglichkeit des Vermeidens (neuerlicher) negativer Erfahrungen außerhalb dieser, die von Abwertung im Zusammenhang mit der eigenen Behinderung bis zu körperlichen und sexuellen Übergriffen reichen können, in Zusammenhang gebracht. Anders als bei Orientierung B ist die Familie aber auch ein Ort, der negative Erfahrungen begünstigt oder gar (mit-)begründen kann, indem etwa notwendige Unterstützung vorenthalten wird, was sich deutlich bei Anna im Zusammenhang mit den erlebten sexuellen Übergriffen dokumentiert. Bei gleichzeitig vorhandenem Streben nach gesellschaftlicher Partizipation wer- 
den die familiären Strukturen zugleich als Schutz, aber auch als starke Einschränkung erlebt, was zu konfliktbehafteten Beziehungen, Familienkonstellationen und Situationen führen kann. Das Bedürfnis nach einem vertrauten Rahmen steht hier im Widerstreit mit dem ausgeprägten, aber angstbesetzten Bedürfnis nach Partizipation und Autonomie. Sehr deutlich spiegelt sich diese Ambivalenz in der Lebenswelt und der Orientierung Melanies wider. Diese möchte eigentlich gerne mit ihrem Freund zusammenleben und äußert dies auch innerhalb der Familie gegen den starken Widerstand und erlebte Sanktionen des Vaters (vgl. IMe 114-136). Gleichzeitig hat sie u.a. aufgrund abwertender Erfahrungen außerhalb der Familie das Bedürfnis nach einem vertrauten Rahmen. Aus diesem Zwiespalt heraus entwickelt Melanie bei ihrem geäußerten Widerstand an den Vorstellungen des Vaters dennoch ein Modell angepassten Verhaltens, um diesen Rahmen erhalten zu können. Inner- und außerfamiliäres Agieren ist letztendlich bei Melanie von der Intention nach Konfliktvermeidung geprägt (vgl. IMe 142-154). An der Oberfläche scheint sie zwar gegen Regeln und Vorschriften zu rebellieren, im Kern wird jedoch deutlich, dass sie sich sehr stark an den normativen Konzepten der Eltern orientiert. In der Folge schwächt sie eigene Wünsche argumentativ auch sich selbst gegenüber ab, weil sie darum weiß, dass diese ohnehin nicht berücksichtigt werden. Familie wird hier auch als Faktor sichtbar, der zugleich Teil der Orientierung, aber auch soziogenetische Basis der Orientierung bzw. deren Entwicklung sein kann.

In Orientierung D ist die Herkunftsfamilie weniger Teil der aktuellen Orientierung, ihre soziogenetische Bedeutsamkeit bildet sich aber über differenzierte Bezüge im Zusammenhang mit der Reflexion eigener (Weiter-)Entwicklung stark ab. Dies dokumentiert sich an der Fallrekonstruktion „Eva“. Eva zieht im Interview Vergleiche von ihrer aktuellen, autonomen, von der Herkunftsfamilie unabhängigen Lebensform zu ihrem früheren Leben innerhalb dieser. Dabei wird das Leben innerhalb der Familie stark mit Regeln, Vorschriften und Kontrolle in Zusammenhang gebracht. Zugleich wird an den Erzählungen Evas deutlich, dass die Familienmitglieder grundsätzlich ihren Weg in Richtung autonomen Lebens und Selbstbestimmung unterstützen, indem sie entweder direkt förderlich wirken wie die Mutter (vgl. IE 215) oder sich mit ihr wie der Vater diskursiv auseinandersetzen (vgl. IE 203), was dann eher indirekt zu Erweiterung eigenen Handlungsspielraumes führt. Ein ähnlicher Bezug zur Herkunftsfamilie wird bei Tamara sichtbar: Sie möchte sich aus dieser lösen, auch wenn sie einen positiven Pol in Tamaras Leben darstellt. Die Eltern erscheinen in Hinblick auf alltägliche Begleitung förderlich, indem sie sich etwa um deren Mobilität kümmern, unterstützen aber auch Tamaras Intentionen nach autonomem Wohnen (vgl. IT 565). Der förderliche Zugang der Eltern zu Tamara führt dazu, dass diese ausführt, dass sie den Kontakt mit diesen gerne aufrechterhalten, zugleich aber , halt einfach so ein bisschen andere Leute kennenlernen“ (IT 647) möchte. Anders als bei 
Melanie, deren außer- und innerfamiliäres Agieren von Konfliktvermeidung geprägt ist, wird am Beispiel von Eva und Tamara deutlich, wie förderlich gelungene, familiäre Beziehungsstrukturen auf die Entwicklung von Handlungsperspektiven und -spielräumen zu wirken vermögen.

Vor allem die Herkunftsfamilie stellt also für Frauen mit Lernschwierigkeiten aller typisieren Orientierungen einen zentralen Bezugspunkt für eigene Auseinandersetzung dar. Familie kann ein stark die eigenen Lebensbedingungen strukturierender Faktor sein, indem sie einen weitgehend vorgegebenen Rahmen für eigene Alltagsgestaltung im vertrauten, geschützten Kontext bildet. Sie kann auch über konkrete Unterstützungs- und Versorgungsleistungen dazu beitragen, dass ein Leben nach eigenen Vorstellungen erst ermöglicht oder erleichtert wird. Sie kann begleitend und unterstützend wirken und im positiven Sinn Raum für Auseinandersetzungen und Aushandeln von Regeln und Grenzen setzen und somit entwicklungsfördernd wirken. Familie kann aber auch stark einschränkenden Charakter haben, wenn durch vorgegebene Strukturen und Sanktionen bei Aufbegehren dagegen Autonomiebestrebungen und Weiterentwicklung gehemmt werden.

\section{Partnerschaft, Sexualität und Familiengründung}

Die Frage der eigenen Familiengründung und damit im Zusammenhang jene nach Partnerschaft, Sexualität und Kinderwunsch werden bei allen typisierten Orientierungen thematisiert, dies erfolgt jedoch in sehr unterschiedlichem Ausmaß und auf unterschiedliche Art und Weise.

Frauen, deren Orientierung Typ A entspricht, dokumentieren etwa über phantasievoll ausgestaltete Familienszenarien die Orientierung an internalisierten familiären und gesellschaftlichen Bildern. Dies wurde etwa am Beispiel der Fallrekonstruktion „Isolde“ über die Inszenierung der eigenen Person als Mutter von zwei phantasierten Kindern in Gestalt von Puppen sichtbar, über die die Mutterrolle auch mittels in den Alltag eingebundener realer Handlungen umgesetzt wird. Partnerschaft wird in diesem Kontext nur am Rande thematisiert, Fragen im Zusammenhang mit Sexualität bleiben ausgeblendet. Für Frauen, deren Orientierung Typ B oder D entspricht, erscheinen Fragen im Kontext mit Partnerschaft relevant, werden aber je nach eigenen vorgelebten Bildern aus der Herkunftsfamilie oder außerfamiliären Bezügen und eigenen Erfahrungen damit sehr unterschiedlich thematisiert. Dies erfolgt manchmal explizit im Zuge genereller Erzählaufforderungen, häufig aber erst über visuelle Stimuli im Kontext von Vignettenbeschreibungen. Dabei wird in den überwiegenden Fällen eine Orientierung an traditionellen Bildern von Partnerschaft sichtbar, die als gesellschaftliche „Normalität" wahrgenommenen werden. Vorstellungen der Realisierung eigener Intentionen nach Partnerschaft und Familie sind davon geprägt, diesem wahrgenommenen Idealbild zu entsprechen. Damit verbundene Ängste und Unsicherheiten können 
auch dazu führen, dass Partnerschaft einerseits zwar explizit abgelehnt wird, implizit aber eine deutliche Orientierung daran sichtbar wird.

Explizit thematisiert, reflektiert und mit eigenen Vorstellungen verbunden werden Partnerschaft, Sexualität und damit verbunden auch die Frage der eigenen Familiengründung bei Frauen, deren Orientierung Typ D entspricht. Partnerschaft und Familie stellen perspektivisch wichtige Faktoren für den eigenen Lebensentwurf dar. Auch hier erfolgt eine Ausrichtung am als gesellschaftliche „Normalität“ wahrgenommenen Bild gleichaltriger Frauen. Zunächst ist aber vor allem der Wunsch zentral, Erfahrungen mit Partnerschaft und Sexualität zu machen und sich darüber auch selbst besser kennen zu lernen und weiterzuentwickeln.

\section{Wohnen, Alltags- und Freizeitgestaltung}

Neben Fragen im Zusammenhang mit Familie, Familienbild und Familiengründung setzen sich Frauen aller vier rekonstruierten Orientierungen auf sehr unterschiedliche Art und Weise mit Fragen im Zusammenhang mit Wohnen auseinander. In Orientierung A wird Wohnen vor allem mit der Möglichkeit in Zusammenhang gebracht, im vertrauten Rahmen der Herkunftsfamilie oder einer Institution an Alltagshandlungen zu partizipieren. Dies beinhaltet u.a. die Beteiligung an Freizeitaktivitäten, aber auch an Haushaltstätigkeiten. Familienmitglieder wie Mutter, Vater und Geschwister, Mitbewohnerinnen sowie Mitarbeiterinnen und Mitarbeiter in Institutionen stellen dabei primäre Bezugspersonen dar. Die dadurch entstehenden Konstellationen stellen die Rahmenbedingungen des Wohnens dar.

Bei Frauen mit Orientierung B wird Wohnen vorrangig mit dem Wunsch, die eigenen Interessen und Vorlieben umsetzen zu können, in Verbindung gebracht. Selbstbestimmung und Autonomie hinsichtlich der Gestaltung des Alltages und der Freizeit sind zentrale Parameter dieser Orientierung. Das Fehlen vorgegebener Strukturen und Regeln wird als befreiend erlebt. Im Kontext des autonomen Wohnens wird dabei auf Versorgungs- und Unterstützungsleistungen von Bezugspersonen zurückgegriffen. Die damit einhergehende Anbindung an die Systeme, die dies leisten, wird akzeptiert, da sie die eigene Alltagsgestaltung erleichtert und auch als emotionale Unterstützung erlebt wird.

Von Frauen mit Orientierung $\mathrm{C}$ wird das Wohnen in einem vorstrukturierten Rahmen ambivalent erlebt. Einerseits kommt es dem Bedürfnis nach Rückhalt und Unterstützung entgegen. Auf der anderen Seite werden Elemente, die diesen Rahmen bilden, oft auch als einschränkend erlebt. Es besteht einerseits der Wunsch nach Orientierung und Stabilität, die mit den vorgegebenen Strukturen des Wohnens in Zusammenhang gebracht werden. Andererseits besteht der Wunsch nach mehr Autonomie, Selbstbestimmung und sozialen Kontakten außerhalb dieses Systems. Diese Diskrepanz führt 
auch zu Unzufriedenheit mit der aktuellen Wohnsituation. Die Frauen erleben häufig auch, dass ihrem Wunsch nach mehr Autonomie in diesen Systemen nicht entsprochen wird. Zudem nehmen sie immer wieder Einschränkungen hinsichtlich ihrer gesellschaftlichen Partizipationsmöglichkeiten wahr. Die mit dieser Wohnform häufig einhergehende Einbindung in Haushaltstätigkeiten wird mitunter auch als unangenehm und stressig erlebt. Auch hinsichtlich der Freizeitgestaltung werden Anteile von Fremdbestimmung durch Regeln und Vorschriften einerseits sowie mangelnder Mobilität und Unterstützung auf der anderen Seite wahrgenommen.

Frauen mit Orientierung D assoziieren mit autonomem Wohnen persönlichen Freiraum, umfassende Selbstbestimmung und Autonomie. Wohnen stellt aus dieser Perspektive die Basis für die umfassende Berücksichtigung und Umsetzung eigener Bedürfnisse in der Alltagsgestaltung dar. Dabei besteht der Anspruch, aktiv zur Umsetzung der eigenen Ansprüche beizutragen. Einerseits, indem ein eigener finanzieller Beitrag dazu geleistet wird. Andererseits, indem Herausforderungen, die sich mit dem autonomen Wohnen verbinden, aktiv und reflektiert begegnet wird. Wohnen wird auch stark mit der Möglichkeit in Verbindung gebracht, eigene Vorstellungen hinsichtlich der Gestaltung von Partnerschaft und Sexualität umzusetzen und den nötigen Freiraum dafür zu haben.

\section{Arbeit und Beschäftigung}

Fragen im Zusammenhang mit Arbeit und Beschäftigung werden von den Frauen sehr unterschiedlich thematisiert. Dies hängt mit den aktuellen Lebensbedingungen im Bereich des Wohnens und der gegebenen oder nicht vorliegenden Einbindung in Beschäftigungs- und Arbeitsprozesse außerhalb der Herkunftsfamilie zusammen.

Wie bereits angeführt bietet die Herkunftsfamilie den Rahmen, in dem Frauen, deren Orientierung Typ A entspricht, an den Alltagshandlungen der Familienmitglieder, insbesondere der Mutter, partizipieren können. Die Tätigkeiten, in die sie dabei eingebunden werden, umfassen in erster Linie den Haushaltsbereich. Bei Einbindung in institutionelle Beschäftigung werden Kontakte zu Peers und weiblichen Bezugspersonen als Rahmen für gemeinsame Alltags- und Freizeitgestaltung erlebt.

Frauen, deren Orientierung Typ B entspricht, erleben ihre Einbindung in Beruf oder Beschäftigung ambivalent. Einerseits verbinden sie damit die Möglichkeit, im Kontext damit eigene Interessen umsetzen zu können. Dies kann die weitgehende Autonomie und damit verbunden die Unabhängigkeit von vorgegebenen Strukturen sein. Andererseits werden konkrete Arbeitsbedingungen - wie etwa zeitlicher Druck - auch als Stressfaktoren wahrgenommen und führen zu Unzufriedenheit. 
Für Frauen, deren Orientierung Typ C entspricht, stellt die Einbindung in Beschäftigungs- oder Arbeitsprozesse in erster Linie eine Möglichkeit dar, ihrem Bedürfnis nach sozialer Partizipation nachzukommen. Insbesondere die Kontakte zu Peers, die im Kontext damit erfolgen, erscheinen besonders bedeutsam. Dies auch aus dem Grund, dass diese Kontakte vorwiegend ausschließlich darüber entstehen und gehalten werden können. Der Kontext von Arbeit und Beschäftigung erscheint aus dieser Perspektive als zentraler Rahmen für Partizipation und Interaktion außerhalb der Herkunftsfamilie.

Für Frauen, deren Orientierung Typ D entspricht, stellt materielle Autonomie, die durch die berufliche Einbindung möglich ist, eine wichtige Basis für selbstbestimmtes Leben und für gesellschaftliche Partizipation dar. Arbeit stellt einen selbstverständlichen Teil des Alltags und auch die Ausgangsbasis für Erfolgserlebnisse dar, die sich förderlich auf die eigene Entwicklung auswirken. Im Kontext mit autonomem Wohnen führt materielle Autonomie auch dazu, dass dafür erforderliche Alltagshandlungen, wie Einkäufe, als selbstverständlich wahrgenommen und erledigt werden.

Materielle Autonomie, die über eigene Erwerbstätigkeit angestrebt oder erreicht wird, wird auch im Zusammenhang damit reflektiert, einen eigenen gesellschaftlichen Beitrag zu leisten, gesellschaftlich integriert und eigenverantwortlich und somit ,ein Teil der Gesellschaft“ (IE 480) zu sein.

Arbeit und damit verbunden finanzielle Unabhängigkeit wird auch als Basis dafür gesehen, dass Vorstellungen hinsichtlich der Umsetzung von Partnerschaft und Familie umgesetzt werden können.

\section{Geschlecht und Rollenbilder}

Über alle Themen der Auseinandersetzung und in allen Orientierungen wird eine deutliche Orientierung an Fragen im Zusammenhang mit der Geschlechtszugehörigkeit sichtbar. Dies dokumentiert sich insbesondere an Vorstellungen, Handlungen und Lebensentwürfen, die mit dem eigenen Familienbild, Familiengründung, der Alltagsgestaltung, Arbeit und Beschäftigung zu tun haben. Dabei zeigt sich, dass als gesellschaftliche „Normalität“ wahrgenommene und vermittelte Bilder die eigene Auseinandersetzung mit spezifischen Fragen prägen. Im Wunsch, dieser „Normalität“ zu entsprechen, wird auch die Orientierung an Vorstellungen, die die gesellschaftliche Rolle von Frauen betreffen, sichtbar. Diese wird dabei vorwiegend mit Haushaltstätigkeiten und der Versorgung der Familienmitglieder in Verbindung gebracht. Besonders deutlich zeigt sich dies bei Frauen, deren Orientierung Typ A entspricht, etwa über eigene Reinszenierungen der vorgelebten Muster im Rahmen einer phantasievollen Gestaltung des Alltages innerhalb der Herkunftsfamilie ${ }^{96}$.

96 Siehe dazu in diesem Kapitel der Arbeit die detailliert Fallrekonstruktion „Isolde“, die sich als Mutter von zwei phantasierten Kindern in Gestalt von Puppen inszeniert. 
Frauen, deren Orientierung Typ C oder D entspricht, nehmen gesellschaftliche Rollenerwartungen vorwiegend über ihre Einbindung in Haushaltstätigkeiten im Rahmen der Herkunftsfamilie sowie im institutionellen Kontext etwa im Beschäftigungsbereich wahr. In Arbeit und Beschäftigung erfolgen häufig Festlegungen auf Bereiche, die traditionell Frauen zugeschrieben werden, wie etwa Wäscherei oder Küchenbereich.

Manche Frauen erleben in beiden Bereichen Ansprüche, den damit verbundenen Zuschreibungen zu entsprechen und die übertragenen Tätigkeiten zur Zufriedenheit des familiären und institutionellen Umfeldes zu erledigen. Dass dies zuweilen auch als Doppelbelastung erlebt wird, dokumentiert sich an Marlenes Ausführungen. Sie erzählt, dass sie nach der Rückkehr von der Arbeit im Küchenbereich einer Werkstätte in die Herkunftsfamilie auch dort Haushaltstätigkeiten verrichten müsse. Als Beispiel führt sie das Bügeln von Wäsche an, das sie als ihre „Aufgabe“ (IMarl 102) genauer beschreibt: ,alles, komplett die ganze Wäsche machen, das ist so ein Korb“ (IMarl 100-102). Marlene schränkt ein, dass sie an einem Tag nur die Hälfte mache, mehr schaffe sie nicht. Am nächsten Tag nach der Arbeit in der Werkstätte gehe es wieder ,weiter, jeden Tag ist das zu machen“ (IMarl 107).

Bei Frauen, deren Orientierung Typ D entspricht, ist die Orientierung an traditionellen Rollenbildern weniger stark ausgeprägt bzw. deutet sich ein Wechsel von der Orientierung an vorgegebenen Rollenmustern hin zur Orientierung an eigenen Wünschen und Bedürfnissen an. So wird etwa explizit der Anspruch, über berufliche Tätigkeit ein eigenes Einkommen zu erwirtschaften und damit die eigene Existenz zu sichern bzw. zur Absicherung beizutragen, zum Ausdruck gebracht. Zuteilungen zu Arbeitsbereichen, die mit der traditionellen Frauenrolle in Verbindung gebracht werden, werden mitunter auch explizit abgelehnt. So weigert sich Tamara, in einer Institution im Küchenbereich zu arbeiten, und setzt es durch, in die Tischlerei zu wechseln (vgl. IT 383). Leben in einer Partnerschaft wird auch von Frauen dieser Orientierung als Idealbild wahrgenommen und für sich selbst angestrebt. Dies erfolgt aber mit Reflexion von Bedingungen und Voraussetzungen für die Realisierung dieses Wunsches. Diese Bedingungen werden an die gewünschte Person des Partners und an den Rahmen, der für die Umsetzung als notwendig erachtet wird, gestellt. Entsprechen diese Faktoren nicht den eigenen Vorstellungen, so werden gesellschaftliche Rollenerwartungen dem auch untergeordnet. Dies dokumentiert sich am Beispiel Evas, die ihre Vorstellungen von Familie und Kind reflektiert. Sie stellt fest, dass eine Familie zu gründen für sie zwar „das Ultimative“ (IE 535) wäre, die Nicht-Erfüllung aber auch keinen „Weltuntergang“" darstellen würde (vgl. IE 554). 


\subsection{Soziogenetische Zusammenhänge: Zur Genese der Orientierungen junger Frauen mit Lernschwierigkeiten im Spannungsfeld von Selbst- und Fremdbestimmung}

In der sinngenetisch angelegten Typologie wurde über die vier Fallrekonstruktionen vorwiegend auf die Frage des ,Was “ eingegangen, indem verschiedene Dimensionen der jeweiligen Orientierung dargestellt wurden. In diesen wurden aber auch bereits zahlreiche Anknüpfungspunkte für die Erhellung soziogenetischer Fragen sichtbar. Soweit deutlich geworden, wurden diese als Ausgangsbasis für weitere Analysen bereits in der sinngenetischen Typenbildung und der anschließenden vergleichend angelegten Zusammenfassung ansatzweise abgebildet. Die differenzierte soziogenetische Analyse steht nun im Zentrum der folgenden Darstellungen. Es geht nun ganz konkret um die Art und Weise sowie Gründe des Zustandekommens der vorliegenden Orientierungen und Einflussfaktoren darauf. Diese werden auch typenübergreifend in den Blick genommen und hinsichtlich der Überlegungen beleuchtet, wie mit einer Thematik oder einer Problemstellung in den verschiedenen Orientierungen umgegangen wird und wie sich diese spezifischen Herangehensweisen entwickelt haben. Dabei ist insbesondere die Frage des sozialen Kontextes, in dem diese Prozesse stattfinden, interessant. Den Auswertungsschritten der dokumentarischen Methode folgend, wird nun also nach „systematischen Unterschieden“ (Nohl 2012: 110) gesucht und abgeklärt, „welchem spezifischen Erfahrungsraum, welcher Erfahrungsdimension oder welcher Orientierung eine generelle Orientierung zuzurechnen ist, wofür sie also typisch ist“ (Nohl 2012: 115). Dabei werden nun vor allem „Kontraste in der Gemeinsamkeit" in den Blick genommen, da diese als Ausgangspunkt einer mehrdimensionalen Typenbildung (Nohl 2012: 56f.) gelten. Diese Unterschiede bilden darüber hinaus ,die Klammer, die eine ganze Typologie zusammenhält" (Bohnsack 1989: 374 nach Nohl 2012: 55). Im Zuge der Herausarbeitung der spezifischen Erfahrungshintergründe als soziogenetische Pfade für die Entwicklung von Orientierungen wird der Blick auf eine mögliche Systematisierung gelenkt. Es wird also danach gefragt, wie Orientierungen mit spezifischen, konjunktiven Erfahrungshintergründen systematisch zusammenhängen. Wurde in der komparativen Analyse im Rahmen der sinngenetischen Typenbildung jeweils nur ein tertium comparationis als den Vergleich strukturierendes Element herangezogen (z.B. wie die interviewten Frauen über ihre Erfahrungen in der Schule erzählen), so erfolgt nun eine komplexe komparative Analyse, wo das tertium comparationis variiert wird $^{97}$. Diese Vorgangsweise wird als Voraussetzung für eine mehrdimensionale soziogenetische Typenbildung (Nohl 2012: 51) beschrieben. Die

97 Zum „tertium comparationis“ in der Typenbildung nach der dokumentarischen Methode siehe Kapitel 4 ,Semantische Interpretation und komparative Sequenzanalyse“. 
darüber möglich werdende Mehrdimensionalität der Typologie bildet dabei die Basis für eine mögliche Generalisierung (Bohnsack 2007: 263).

Diesen methodischen Annahmen folgend, werden nun Lebenszusammenhänge und Erfahrungsräume von Frauen mit Lernschwierigkeiten in ihren spezifischen Konsequenzen für die Entwicklung der unterschiedlichen Orientierungen beschrieben, mittels Ausführungen aus Interviews belegt und analysiert. Dabei rücken sogenannte „konjunktive Erfahrungen“, also solche, die Individuen verbinden, ohne dass diese in einem konkreten, gemeinsamen Lebenszusammenhang stehen müssen (vgl. Przyborski 2004: 29), in den Blickpunkt.

Für Frauen mit Lernschwierigkeiten zeichnen sich über die sinngenetische Typenbildung und die in diesem Rahmen bereits ansatzweise sichtbar gewordenen soziogenetischen Faktoren konjunktive Lebens- und Erfahrungszusammenhänge $\mathrm{ab}$, die in ihrer unterschiedlichen Erlebens- und Bearbeitungsweise mehrdimensionale Aspekte beinhalten. Von den Lebenszusammenhängen her sind es Faktoren wie die soziale und materielle Verortung bzw. Eingebundenheit, die sich vor allem über Wohnform, Beschäftigung, Einbindung in eine Werkstätte und finanziellen Status ausdrücken. Bei den Erfahrungszusammenhängen werden Prozesse, Interaktionen und Erlebnisse in vielfältigen sozialen Kontexten sichtbar, die soziogenetisch wirksam werden. Dies spannt sich zwischen zwei Polen auf: Negative Erfahrungen stehen positiv erlebten gegenüber und wirken sich sehr unterschiedlich auf die vorliegende Orientierung aus. So wirken sich beispielsweise Erfahrungen von Abwertung, Stigmatisierung und Diskriminierung grundsätzlich einschränkend aus, indem sie Handlungsspielräume und Perspektiven verengen. Akzeptanz und Anerkennung erweitern demgegenüber Handlungsspielräume und befördern auf vielfältige Weise eigene Entwicklungsmöglichkeiten.

Beide Stränge - Lebens- und Erfahrungszusammenhänge - münden ihrerseits in einen für die Ausprägung der Orientierung ganz wesentlichen Faktor, nämlich in die Entwicklung eines dadurch beeinflussten Selbstbildes: Inwieweit dieses davon geprägt ist und inwieweit sich dieses auf die eigene Orientierung auswirkt, wird jeweils mit in den Blick genommen.

Ergänzt wird diese Darstellung der Ergebnisse durch die daran anschließende Präsentation der Einschätzung ausgewählter erhobener soziogenetischer Aspekte durch die Frauen aus der Referenzgruppe.

\subsubsection{Lebenszusammenhänge als soziogenetische Faktoren für die Entwicklung von Orientierungen}

Über die sinngenetische Typenbildung wurden bereits zahlreiche Hinweise darauf sichtbar, dass die Frage der sozialen Eingebundenheit im Sozialisationsverlauf und im aktuellen Kontext soziogenetisch Auswirkung auf die 
Entwicklung der Orientierungen von Frauen mit Lernschwierigkeiten haben dürfte. Unter „sozialer Eingebundenheit" verstehe ich die Art und Weise sowie den Grad der jeweiligen gesellschaftlichen Integration also u.a. die Frage, ob sich diese vorrangig oder ausschließlich auf den familiären Rahmen bezieht oder etwa sich darüber hinaus auf eine alternative Wohnform und/oder Arbeit oder Beschäftigung in einer Werkstätte, aber auch den Freizeitbereich erstreckt. Ganz zentral damit verbunden geht die Frage des materiellen Status einher, der vorwiegend über die Einbindung in Beruf oder Beschäftigung determiniert ist und seinerseits mit Fragen möglicher Abhängigkeiten verbunden ist. Ebenfalls von erheblicher Bedeutsamkeit schätze ich aufgrund meiner Forschungsergebnisse die Frage der wahrgenommenen Art und Weise der Einbindung, also letztendlich die Frage der Qualität bzw. der Passung von Person und Rahmenbedingungen. Dies ist insofern relevant, als etwa vorgegebene, stark strukturierte Lebensbedingungen durchaus auch als angenehm und passend erlebt werden können, wenn sie persönlichen Bedürfnissen entgegenkommen, wie dies vor allem bei der Orientierung Typ A erkennbar ist. Wo diese Passung aber nicht gegeben ist, wo also etwa vorgegebene Rahmenbedingungen vorwiegend als einschränkend erlebt werden, wirkt sich dies in anderer Art auf das eigene Wohlbefinden und die Entwicklung der Orientierung aus. Über diese Kette von Zusammenhängen deutete sich u.a. auch in den Fallrekonstruktionen bereits an, dass das Selbstbild und damit verbunden Prozesse innerhalb der Alltagsgestaltung, aber auch die Entwicklung von Lebensperspektiven von der sozialen Eingebundenheit beeinflusst werden. Darüber hinaus wirken sich durch das soziale Umfeld vermittelte Haltungen und Einstellungen zur gesellschaftlichen Partizipation auf die Orientierung der Frauen aus. Barrieren, die über zwischenmenschliche Interaktionen wirksam werden, existieren neben objektiv wahrnehmbaren hemmenden Beeinträchtigungen etwa im Zusammenhang mit der eigenen Mobilität. Diesbezügliche Faktoren und Zusammenhänge werden in der nachfolgenden soziogenetischen Perspektive mit in den Blick genommen.

\section{Leben in der Familie bietet Struktur und ermöglicht die Teilhabe an deren Alltagshandlungen}

Für zahlreiche Frauen mit Lernschwierigkeiten stellen Strukturen der Herkunftsfamilie den zentralen und mitunter einzigen Rahmen für soziale Kontakte dar und ermöglichen darüber die Teilhabe an Alltagshandlungen. Dies wurde etwa an der Fallrekonstruktion „Isolde“ deutlich, für die dieser Rahmen stimmig mit den eigenen Bedürfnissen zu sein scheint. Die Alltagsgestaltung innerhalb dieses Rahmens ist in der Regel durch die Orientierung an bestimmten Fixpunkten im familiären Tagesablauf vorstrukturiert.

So beschreibt etwa Natalie (vgl. IN 191-193 und IN 229-234) sehr kursorisch und mit Eckpunkten, die durch Haushaltstätigkeiten, Essen und diverse 
Freizeitbeschäftigungen gekennzeichnet sind, ihren sehr an die Familie angepassten Alltag. Aktivitäten außerhalb des engeren Wohnumfeldes werden nicht beschrieben. Natalie hat eher passiv orientierte Interessen, liest und sieht fern. Natalie führt auch an, dass sie gerne im Keller tanze, singe und Gymnastik mache, auf Nachfrage wird aber deutlich, dass sie dies nur „hier und da einmal, eigentlich nicht immer, nur wenn [sie] Zeit habe“" (IN 161) mache. Den Hobbys, so argumentiert Natalie auf Nachfrage, würde sie vor allem nachgehen, um „Abwechslung“ zu haben, was darauf schließen lässt, dass ihr der von Haushaltstätigkeiten und Essen geprägte Alltag doch auch manchmal langweilig und eintönig erscheinen dürfte. Auch Felicitas ist in ihrer Alltagsgestaltung eng in die vorgegebene innerfamiliären Struktur eingepasst. Sie verrichtet ähnlich wie Natalie diverse Haushaltstätigkeiten wie saugen, wischen, Betten machen, zusammenräumen, Geschirr abspülen oder in den Geschirrspüler räumen. Der Nachmittag wird wie bei Natalie mit Freizeittätigkeiten im Bereich des Hauses verbracht. Über Felicitas Beschreibungen wird deutlich, dass soziale Kontakte sich auf die Familienmitglieder beschränken. Genannt werden hier v.a. die Großmutter, der sie beim Kochen hilft und mit der sie sich gegenseitig bei der Wäsche hilft, und die Eltern, mit denen am Abend gemeinsam gegessen wird. Sozialer Austausch erfolgt vorwiegend innerhalb der Familie, so beschreibt Felicitas, dass sie innerhalb der Familie zu den gemeinsamen Mahlzeiten „ein bisschen reden und so“ (IF 108) würden.

Spärliche außerfamiliäre soziale Kontakte bei nicht vorhandener außerfamiliärer sozialer Einbindung

Isoldes soziale Kontakte außerhalb der Herkunftsfamilie sind spärlich. Zwar ist sie tagsüber in einer Werkstätte tätig, orientiert sich aber weitgehend am familiären Rahmen, den sie als vertraut wahrnimmt und in dem sie an den Alltagshandlungen der Familienmitglieder partizipieren kann.

In Felicitas und Natalies Beschreibungen werden vorwiegend soziale Kontakte innerhalb der Familie zur Sprache gebracht. Nur sehr eingeschränkt erfolgen fallweise Verweise auf soziale Bezüge außerhalb der Familie. Bei beiden stellt die Familie den zentralen Rahmen ihrer Handlungs- und Begegnungsmöglichkeiten dar. Soziale Kontexte, die üblicherweise über eine Einbindung in den Bereich Arbeit/Beschäftigung ent- und bestehen, spielen in diesem Rahmen keine Rolle. Freundschaften mit Peers werden nur ansatzweise erwähnt. Wenn, dann werden diese vorwiegend über Aktivitäten oder soziale Kontakte der Eltern angebahnt, wie etwa bei Felicitas und Natalie, deren Freundschaft sich über die Zugehörigkeit ihrer Eltern zum selben Gesangsverein ergeben hat. Dementsprechend sieht Natalie Felicitas nur dann, wenn sich ihre Mütter treffen: „Ja, meistens kommt der Natalie ihre Mutti ein bisserl her oder ratschen und so, dann wir uns sehen“ (IN 546-547). Im Frei- 
zeitbereich dominieren bei beiden Aktivitäten, die im (Nah-)Bereich des Hauses durchgeführt werden können. Gemeinsame Aktivitäten der beiden Freundinnen außer Haus erfolgen eher sporadisch und vorwiegend über Initiative der Bezugspersonen - hier der Mütter - und stellen für beide auch aufgrund ihrer Seltenheit besondere Ereignisse dar.

Werden diese Rahmenbedingungen auch als angenehm und stimmig mit den eigenen Bedürfnissen erlebt, so tragen sie aber auch dazu bei, dass Chancen zur Weiterentwicklung, die sich über neue Möglichkeiten ergeben könnten, nicht wahrgenommen werden können. Indem darüber kaum neue Impulse und Herausforderungen entstehen, bleibt es häufig beim Status quo. Mit der Vorstellung, sich außerhalb des geschützten Rahmens zu bewegen, verbinden bzw. verfestigen sich mitunter auch Vorbehalte und Ängste bei den Frauen selbst oder innerhalb der Familie in Zusammenhang mit möglicher sozialer Partizipation.

\section{Wohnen und Arbeiten in einer Institution als Rahmen für soziale Kontakte und Aktivitäten}

Auch über das kombinierte Wohnen und Arbeiten in einer Einrichtung für Menschen mit Behinderungen werden Möglichkeiten, soziale Kontakte zu führen, wahrgenommen.

Sonja erzählt, dass sie in der Werkstatt arbeite und momentan „Motive für einen Weihnachtskalender mache“ (ISo 89). Danach gehe sie mit Anni Tischtennis spielen und danach immer zu Felix in die Weberei. Im Zusammenhang mit der Beschreibung ihrer sozialen Kontakte innerhalb des Wohn- und Arbeitsverbundes führt Sonja an, sie sei „sehr froh“ (ISo 94) hier zu sein. Sonja ist nicht nur mit der Wohn- und Arbeitssituation in der Institution zufrieden, wie sie mehrmals anführt (vgl. ISo 78, 98, 245), auch die Aktivitäten im Freizeitbereich nutzt sie gerne. Sie scheint auch sozial gut eingebunden zu sein, dies dokumentiert sich explizit an ihrer Einschätzung ihrer sozialen Beziehungen in der Einrichtung mit dem Satz: „Ich verstehe mich gut" (ISo 317). Sonja zeigt sich auch zufrieden mit ihren Beziehungen zu den Betreuern (vgl. ISo 318-319).

Verena orientiert sich im institutionellen Umfeld stark an Peers und stimmt ihre Freizeitgestaltung auf deren Interessen ab. Sie ist wie eine Freundin Fan einer Sängerin und sammelt gemeinsam mit dieser Bilder und Zeitungsausschnitte (vgl. IV 122-127). Ähnlich wie Petra und Sonja zeigt sie sich weitgehend zufrieden damit und hält fest: ,gefallt mir alles da“ (IV 67). Sie ist im vorgegebenen Rahmen auch aktiv und beteiligt sich an einer Tanzgruppe.

Gloria beschreibt gemeinsame Aktivitäten, die im Rahmen der institutionellen Betreuung durchgeführt werden: „Einmal kochen, ( ) einmal einkaufen gehen, oder Kaffeehaus gehen.“ (IG 259-260) 


\section{Leben und Arbeiten in einer Institution: Wunsch nach Erhalt versus Wunsch nach Veränderung}

Über das kombinierte Wohnen und Arbeiten in einer Institution können soziale Kontakte zu Peers entstehen und geführt werden. Strukturierte und gemeinsame Aktivitäten mit diesen und mit Betreuungspersonal innerhalb und außerhalb der Institution bestimmten diesen interaktiven Rahmen. Zufriedenheit damit dokumentiert sich u.a. bei Karin, welche die Beschreibung ihres Lebens in der Institution mit folgendem Resumé abschließt: „da gefällt es mir, da komme ich nicht mehr weg, hat der Herr G. gesagt. Das beruhigt mich“ (IK 59-60). Das Leben im geschützten, vorstrukturierten Rahmen kann also den Wunsch nach dessen Erhalt begründen. Dieser Zusammenhang deutet sich auch über die anderen angeführten Beispiele mit der Äußerung von Zufriedenheit mit der gegebenen Situation ab. In den angeführten Fällen entstehen bei den Frauen selbst kaum Impulse zur Veränderung der Situation.

Das Leben in einem strukturierten Rahmen kann aber auch zu Unzufriedenheit führen, wenn die Rahmenbedingungen als nicht stimmig mit den eigenen Bedürfnissen erlebt werden. Dies dokumentiert sich an Evas Beispiel $^{98}$, für die das Wohnen in der Behinderteneinrichtung über ihre Erfahrungen damit eine Ausgangsbasis für eigene Weiterentwicklung darstellt. In ihrem Fall führen als rigid wahrgenommene Regeln und fehlender Freiraum zur Ablehnung des erlebten Kontextes. Den Zusammenhang von Leben in einer Institution mit Regeln und Vorschriften stellt auch Martina her und lehnt diesen vehement $\mathrm{ab}^{99}$. An Martinas Ausführungen wird sichtbar, dass sie das Leben in einer Institution auch aus dem Grund ablehnt, da sie die darüber bestehende soziale Einbindung nicht als ihrem eigenen Bedürfnis entsprechend einschätzt. Dies deutet sich über die Aussage an, es wäre für sie nicht angenehm, wenn „dann vielleicht andere Leute dann auch noch sind, die was mir dann auf den Zeiger auch noch gehen, blöd kommen“ (IM 299). Da habe sie lieber ihre Ruhe und, so Martina, ,tue so was ich will“ (IM 300). Martina lehnt nicht nur das Leben in einer größeren Institution, sondern auch in einer kleineren Wohngemeinschaft $\mathrm{ab}$ und begründet auch dies mit dort bestehenden zeitlichen Vorgaben und feststehenden Plänen.

98 Siehe dazu ausführlich Fallrekonstruktion „Eva“, insbesondere „Ablehnung von Regeln und Vorschriften versus Wunsch nach eigener Gestaltungsmöglichkeit“ und „Ablehnung traditioneller Betreuungskonzepte versus Wunsch nach Unterstützung und Begleitung“.

99 Dies ist ausführlich abgebildet in der Fallrekonstruktion „Martina“, insbesondere in Punkt „Ablehnung von Regeln und Vorschriften, die nicht den eigene Bedürfnissen und Voraussetzungen entsprechen ". 


\section{Einbindung in Beschäftigung/Arbeit ermöglicht außerfamiliäre soziale Kontakte}

Über die Beschreibung ihres Tagesablaufes (IMarl 65-115) verdeutlicht sich an Marlenes Beispiel, dass die Einbindung in ein Beschäftigungsprojekt untertags bei gleichzeitigem Wohnen bei den Eltern den Tag strukturiert, bestimmte Aktivitäten erfordert und soziale Kontakte außerhalb der Familie mit sich bringt. Marlene beschreibt detailliert ihren täglichen Arbeitsweg: Sie fahre meistens um halb acht mit dem Zug weg, steige um 8 Uhr an ihrer Haltstelle aus und gehe ,her in die Firma“ (IMarl 70). Hier angekommen ziehe sie sich um, richte alles her und ginge dann in eine Besprechung [,tun wir nachher besprechen, was wir arbeiten tun und erklären halt" (IMarl71-72)]. Marlene beschreibt in der Folge detailliert Zeiträume für Pausen und Arbeitsphasen und abschließend wieder den Heimweg in allen Einzelheiten. Ab und zu würde sie auf dem Weg zum Bahnhof mit einer Freundin etwas trinken gehen (vgl. IMarl 1101-1102). Weitere Kontakte mit Freundinnen erfolgen fast ausschließlich über die Beschäftigungseinrichtung. Aktivitäten mit Familienmitgliedern werden als seltene und besondere Ereignisse beschrieben, wie etwa ein Kinobesuch mit dem Bruder:

„Er hat mich zu meinen Geburtstag überrascht, nachher hab' ich gedacht: was will er
jetzt von mir? Überlegen so - eine Überraschung so, ehm, er hat gesagt ich muss meine
Haare machen, daheim bin, daheim sein muss ich, da bleiben. So, die erste Überra-
schung war: ((lacht)) Kino gehen, hab' ich schon g'wusst, das hab' ich eh schon ge-
wusst, aber ich hab's mir nicht, ich hab's aber nimmer gewusst nachher, wie ich in der
Firma war ( ), die ganze Zeit aufgeregt, ja - da hob' ich schon gewusst. O.k., geh'n wir
ins Kino, nein: zuerst sind wir, als erster sind wir noch auf die Messe gegangen. Und
dann sind wir ins Kino gegangen, dann war ich um dreiviertel elf, zwölf daheim
((lacht)).“ (IMarl 1064-1071)

Die Einbindung in die Beschäftigung ermöglicht auch Angelika außerfamiliäre soziale Kontakte und Freundschaften mit Peers, die ihr sehr wichtig sind. Auch für sie stellt die Einbindung in ein Beschäftigungsprojekt offenbar die zentrale Möglichkeit dar, Kontakte zu knüpfen, zu führen und zu halten. So erzählt Angelika von einer Arbeitskollegin, mit der sie „dick befreundet“ (IAng 189) ist, dass sie diese nur an der Arbeitsstelle sehen könne.

\section{Einbindung in Beschäftigung/Arbeit erweckt den Wunsch nach Ausbau/Intensivierung sozialer Kontakte und gesellschaftlicher Partizipation}

Marlene selbst nimmt wahr, dass sie über besondere Ereignisse wie den beschriebenen Kinobesuch mit dem Bruder anlässlich ihres Geburtstages hinaus gesellschaftlich kaum partizipieren kann, was sich über die sprachliche Formulierung in folgender Passage in Form einer Fokussierungsmetapher dokumentiert. Auf die Frage, was sie sich denn wünschen würde, wenn es eine „gute Fee“ erfüllen würde, antwortet Marlene nach längerem Nachdenken: 
„mehr fort gehen“ (IMarl 1133). Sie lacht, bestätigt dies auf Nachfrage zweimal und führt aus:

„Ja, mehr weg von daheim, weil sonst bin ich nur daheim, ich sitz' die ganze Zeit nur daheim, ich kann nix machen, [...] ich kann nirgends hingehen, ich brauch mehr Auszeit, aus, frei, hinaus, ich muss mehr wohin gehen, irgendwo einkaufen oder ich weiß nicht" (IMarl 1137-1139)

Sie präzisiert auf Nachfrage weiter: „mehr ins Kino gehen, mehr einmal schwimmen gehen, ins Hallenbad, ja, mehr einkaufen“ (IMarl 1148). Es wird deutlich, dass sich Marlene nach mehr Freiraum, mehr Partizipation und gesellschaftliche Teilhabe im Alltag sehnt. Dass sich dies in der Interviewsituation sehr deutlich über eine Fokussierungsmetapher dokumentiert, lässt auf die starke Ausprägung dieses Wunsches schließen. Es deutet sich auch an, dass Marlenes Wunsch nach mehr Partizipation über die Möglichkeit in der Einrichtung, soziale Kontakte zu pflegen, wächst. Marlene will soziale Kontakte und Partizipation zunehmend nicht nur im Zusammenhang mit ihrer Arbeit im Beschäftigungsprojekt, sondern auch darüber hinaus.

Angelika hat über die Einbindung in das Beschäftigungsprojekt erstmals die Möglichkeit, soziale Kontakte zu Peers zu führen, nimmt aber schnell wahr, dass ihr die Treffen mit ihrer Freundin an der Arbeitsstelle nicht ausreichen. Ein in seiner sprachlichen Dichte als Fokussierungsmetapher erscheinender Satz dokumentiert die Bedeutung dieser Freundschaft, die Angelika nur im Rahmen der Arbeitsstelle leben kann.

„Sie hat mich gern [...] sie mag die Trennung überhaupt nicht. Das schmerzt, das schmerzt diese Trennung, schmerzt, die Trennung schmerzt" (IAng 182-183)

In dem Satz selbst wird sprachlich ein Wechsel der Person angedeutet. Scheinen es zunächst die Gefühle der Freundin zu sein, so wird darüber deutlich, dass es Angelika selbst ist, die diese Gefühle empfindet. Angelika führt in der Folge explizit die einschränkenden Rahmenbedingungen hinsichtlich der Kontakte zur Freundin aus: Am Wochenende könne sie die Freundin nicht besuchen, weil es schlechte Busverbindungen gebe. Angelika sagt auf Nachfrage, dass dies „ein ziemlich großes“ (IAng 199) Problem sei. Auf die Frage, was ihre Familie dazu sage, führt Angelika den Standpunkt des Vaters aus, der sage, sie sehe die Freundin ohnehin in der Firma. Die Mutter rate ihr, mit der Freundin jeden Tag zu telefonieren (vgl. IAng 205). In diesem $\mathrm{Zu}-$ sammenhang verweist Angelika auch auf dafür erforderliche finanzielle Ressourcen: „das kostet was, jeden Tag telefonieren, das kostet was“ (IAng. 205206). 
Möglichkeit der Fixierung auf konkrete Form der außerfamiliären Einbindung wegen Wunsch nach Erhalt der gegebenen sozialen Kontakte

Am Beispiel von Angelika wird deutlich, dass die Möglichkeit, soziale Kontakte zu ihrer Freundin zu halten, gedanklich bei ihr selbst sehr stark mit dem Beschäftigungsprojekt verbunden ist und dazu führt, dass sie diesen Rahmen erhalten will. Sie beschreibt, sie wäre gerne Köchin geworden, dazu hätte sie aber in eine andere Werkstätte versetzt werden müssen. Dies habe sie abgelehnt, denn dann hätte ihre Freundin „,noch mehr zu leiden darunter“ (IAng 445-456). Sie wolle ,überhaupt nicht" (IAng 452) in die andere Werkstätte, denn sie hätte ,da herinnen“ (IAng 454) ihre Freundinnen. Auch die mangelnde soziale Einbindung am Wohnort bringt Angelika mit der zugeschriebenen Bedeutung des Beschäftigungsprojektes in Verbindung. Am Wohnort habe sie „nicht so“ (IAng 460) Freundinnen und begründet das damit, dass dieser Wohnort sehr abgelegen sei und die Nachbarn schon älter wären. Sie brauche „Freunde die gleich alt sein“ (IAng 475). Sie habe zwar eine Freundin im nächsten größeren Ort, die sie sehen könne, sagt dann aber, sie habe diese zuletzt vor zwei Monaten im Bus getroffen. Für Angelika stellt Freundschaft innerhalb der Peergroup eine starke Orientierung dar. Aus ihren Erzählungen und Beschreibungen wird aber deutlich, dass Wunsch und Realität auseinanderklaffen. Am Wohnort kennt Angelika keine Gleichaltrigen. Über eine Freundin im Nachbarort stellt sich heraus, dass sie diese zuletzt vor zwei Monaten im Bus getroffen hat. Sie kann Freundschaft also nur über ihre Einbindung in das Beschäftigungsprojekt leben und stellt andere Wünsche konkret: in einem präferierten Berufsfeld an einem anderen Ort tätig werden zu können - dafür hintan. Allerdings wird auch die Freundschaft zu ihrer Arbeitskollegin auch als ,schmerzhaft" erlebt, weil sie nicht in dem Maße gelebt werden kann, wie gewünscht und sich ganz auf den beruflichen Rahmen beschränkt. Bei Angelika führen ungünstige Rahmenbedingungen am Wohnort verbunden mit mangelnder Mobilität, eigenen Unsicherheiten und Ängsten dazu, dass ihr Wunsch nach sozialen Kontakten vorwiegend über das Beschäftigungsprojekt, allerdings auch hier nur eingeschränkt, realisiert werden kann. Bei Angelika entstehen dabei auf der Gefühlsebene Schmerzen und Unbehagen. Es deutet sich an, dass durch vielfältige soziale Bezüge eher Offenheit für neue Perspektiven entsteht, da dann nicht der Wunsch nach Erhalt des vorhandenen sozialen Bezugssystems im Vordergrund steht.

\section{Soziale Einbindung erfordert Unterstützungssysteme}

An den Beispielen von Angelika und Marlene zeigt sich, dass die Einbindung in ein Beschäftigungsprojekt für Frauen mit Lernschwierigkeiten häufig die einzige Möglichkeit darstellt, soziale Kontakte zu Peers zu knüpfen und zu pflegen. Ungünstige Bedingungen am Wohnort, eingeschränkte Mobilität bzw. mangelnde Autonomie in der Mobilität und ein negatives Selbstbild 
sind u.a. Barrieren, die verhindern, dass dem Wunsch nach sozialen Kontakten auf anderem Wege nachgekommen werden kann. Wenn diesbezügliche Unterstützung aus dem sozialen Umfeld hinsichtlich der Überwindung dieser Barrieren nicht gegeben ist, wird dies ganz besonders wirksam. Im Fall von Angelika wird deutlich, dass der Wunsch im familiären Umfeld auch wenig ernst genommen bzw. heruntergespielt wird, es werden Beschwichtigungen und Ratschläge geäußert, die wenig dazu beitragen, die Erfüllung von Angelikas Bedürfnis nach sozialen Kontakten zu ermöglichen oder zu erleichtern. Auch Marlene erfährt diesbezüglich wenig Unterstützung. Bei beiden wird deutlich, dass die Integration in das Beschäftigungsprojekt den Wunsch nach sozialen Kontakten mit Peers und zunehmend deren Intensivierung begründet. Dabei wird sichtbar, dass es auch zu Unzufriedenheit führen kann, wenn diese Bedürfnisse nicht umgesetzt werden können. Die Möglichkeiten, die sich durch die Einbindung in ein Beschäftigungsprojekt ergeben, stellen aus dieser Perspektive nur in Kombination mit Strukturen, die deren Realisierung unterstützen, einen Raum dar, über den soziale Kontakte zufriedenstellend realisiert und erlebt werden können. Bei fehlenden Unterstützungssystemen kann aus den über die soziale Einbindung wachsenden, aber nicht realisierbaren Bedürfnissen nach sozialen Kontakten auch Unzufriedenheit entstehen.

\section{Selbstbestimmtes, autonomes Leben als Basis für Weiterentwicklung}

An Evas Beispiel wird deutlich, dass Lebensbedingungen, die weitgehende Selbstbestimmung mit der erforderlichen Unterstützung zulassen, auch neue Perspektiven und Möglichkeiten erschließen, die unter anderen Rahmenbedingungen gar nicht vorstellbar gewesen wären. Bei Eva ergeben sich über erste Erfahrungen nach dem Wohnen bei den Eltern in einer traditionellen Wohneinrichtung zunächst Möglichkeiten, Kontakte zu führen und zu pflegen und eigene Interessen zu entwickeln. Zunehmend nimmt Eva aber den sie einschränkenden Charakter der stark strukturierten, institutionellen Rahmenbedingungen wahr. Über Unterstützung aus dem Elternhaus und durch Anerkennung und Motivation von Schlüsselpersonen im beruflichen Umfeld erhält Eva die Möglichkeit zum Wechsel in eine integrative Wohngemeinschaft und bekommt eine interessante Stelle mit realem Gehalt. In diesem Zusammenhang bekommt Eva genau die Unterstützung, die sie als angemessen erlebt $^{100}$.

Nach dem ersten Jahr in dieser WG und an der Arbeitsstelle reflektiert Eva über zukünftige Lebensperspektiven: Früher habe sie Familie und Kinder abgelehnt, ,aber jetzt, da du dein eigenes Leben lebst und siehst, was eigentlich schaffbar und machbar ist" (IE 561-562), frage sie sich, wieso solle das

100 Siehe dazu auch Punkt ,Soziogenetisch förderlich oder erweiternd auf Handlungsspielräume und Entwicklungsperspektiven wirkende Faktoren“, insbesondere „Nicht bevormundende Unterstützung wirkt entwicklungsfördernd“". 
„eines Tages nicht einmal auftauchen“ (IE 562). Hat Eva zuvor ablehnend auf mögliche Entwicklungsperspektiven reagiert, so entwickelt sie im Zuge des Prozesses in Richtung eines selbstbestimmten Lebens das Gefühl, dass vieles möglich ist, was zuvor unrealistisch erschien. Vertrauen, Selbstbewusstsein, Eigenverantwortung und die Fähigkeit, sich selbst zu vertreten, wachsen zunehmend. Mit der als angemessen erscheinenden Unterstützung, die Eva in einem interaktiven und selbstreflexiven Prozess auch als selbstverständlich anzunehmen lernt, werden zunehmend neue Handlungsspielräume sichtbar. Perspektiven, die früher für sie kaum vorstellbar waren und vordergründig abgelehnt wurden, zeichnen sich zunehmend als realistische Perspektive $a b$.

\section{Soziale Einbindung, materieller Status und dessen Reflexion}

Im Zuge der Analyse der Interviews wurde deutlich, dass Fragen im Zusammenhang mit dem eigenen materiellen Status von Frauen mit Lernschwierigkeiten in den vier typisierten Orientierungen sehr unterschiedlich thematisiert werden. Dies hängt in erster Linie mit den konkreten Lebenssituationen und -bedingungen, aber auch mit spezifischen Erfahrungen zusammen.

Wo vorwiegend oder ausschließlich die Herkunftsfamilie den Rahmen für soziale Einbindung bildet, stellt diese auch den Rahmen für die Ausstattung mit finanziellen Ressourcen dar. Frauen, die sich mit den vorgegebenen Rahmenbedingungen zufrieden zeigen, schließen in diese geäußerte Zufriedenheit implizit in der Regel auch ihren materiellen Status mit ein. Explizit wird die Frage finanzieller Ressourcen kaum thematisiert. Wenn sie doch zur Sprache kommt, dann wird darüber kaum Kritik mit den vorgegebenen Bedingungen und deren Auswirkung auf die eigene Lebensgestaltung sichtbar. Über Ausführungen in Interviewpassagen, die nicht explizit Fragen der finanziellen Ressourcen thematisieren, wird dann manchmal doch eher beiläufig ein Zusammenhang zwischen möglicher Erfüllung dieser Wünsche und fehlender finanzieller Ressourcen hergestellt. In einigen Fällen erfolgt dies im Kontext von Vignettenbeschreibungen, in denen über anschauliche, erzählgenerierende Stimuli Zukunftsperspektiven und Wünsche für das eigene Leben thematisiert werden. Dies verweist darauf, dass Zusammenhänge von finanziellen Ressourcen und eigenen Handlungsmöglichkeiten in der Regel doch unbewusst wahrgenommen und in die eigenen Überlegungen integriert werden. Auch Frauen, die weder über eigenes Einkommen noch über ein in einer Werkstätte ausbezahltes Taschengeld verfügen, erkennen also in der Regel, dass bestimmte Wünsche mangels finanzieller Ressourcen nicht realisierbar sind.

Der Zusammenhang von finanziellen Ressourcen und der darüber möglichen Finanzierung persönlicher Wünsche wird in verschiedenen Kontexten und auf unterschiedliche Art und Weise von jenen Frauen hergestellt, die in 
einer Werkstätte für Menschen mit Behinderung beschäftigt sind. Besteht über das Leben im Rahmen der Herkunftsfamilie diese Einbindung, so werden zu einem sehr geringen Teil selbst finanzielle Mittel über ein sogenanntes „Taschengeld“ erarbeitet, mit dem meistens eher kleine, alltägliche Bedürfnisse finanziert werden. Marlene etwa finanziert sich mit ihrem geringfügigen Taschengeld „meistens Kaugummi“ (IMarl 771) oder „Getränke, Eistee“ (IMarl 777). Angelika geht mit den 25 Euro Taschengeld, die sie monatlich in der Werkstätte bekommt, am liebsten Bekleidung „shoppen“ (IAng 535) und bevorzugt dabei Diskonter (IAng 541). Doris hingegen kann nicht sagen, wofür sie das wenige Geld, das ihr zur Verfügung steht, ausgibt. Sie führt aus: „ich spare, ich habe noch keinen Plan was ich kaufen soll“ (ID 450).

Gibt es über ein geregeltes Dienstverhältnis ein eigenes Einkommen, so kann dieses über die Möglichkeit der (Teil-)Finanzierung die Basis für die eigene Lebensgestaltung darstellen. Dies zeigt sich etwa an Evas Lebenssituation, die ihr Einkommen zur Finanzierung ihrer Lebenshaltungskosten, Grundbedürfnisse und Freizeitinteressen heranzieht. Sie bringt dies auf den Punkt: „du erwirtschaftest dein Leben sogar“ (IE 483). Über ein eigenes Einkommen zu verfügen, bedeutet aus dieser Perspektive auch „ein ganz normaler Teil der Gesellschaft“ (IE 482-483) zu sein. Dass sich ein aus einem regulären Arbeitsverhältnis ergebender Lohn von dem „Taschengeld“ unterscheidet, das in Werkstätten ausbezahlt wird, ist den Frauen, die darüber verfügen, bewusst. So erläutert Tamara, dass sie für ihre Arbeit ,alle Monat“ (IT 493) etwas bekomme, bestätigt, dass dies ,richtiges Gehalt“ sei, und ergänzt, dass sie damit auch versichert sei und Urlaubsgeld bekomme.

\section{Finanzieller Status, geäußerte Zufriedenheit und Bescheidenheit}

Über die Einbindung in eine Werkstätte werden in der Regel sehr geringe finanzielle Mittel lukriert. Angelika beschreibt - wie bereits im Zusammenhang mit der Reflexion des materiellen Status angeführt - dass sie 25 Euro pro Monat in der Werkstätte und zusätzlich 20 Euro vom Vater bekomme. Am liebsten würde sie mit dem Geld „shoppen“ (IAng 519), sagt Angelika lachend, und zwar „Gewand“ (IAng 536), „Gewand ist meine Spezialität“ (IAng 538). Angelika zählt mehrere günstige Geschäfte auf, in denen sie am liebsten einkauft, „weil da haben sie die billigsten Gewänder“ (IAng 542543). Sie führt aus: „es ist ja im Leben nix mehr gratis, oder das Leben gibt nix mehr gratis her, sagen wir einmal so" (IAng 557). Angelika erscheint in ihren Ansprüchen bescheiden, sie sieht die Kombination von 25 Euro Taschengeld, die sie in der Firma bekommt, und 20 Euro vom Vater als ausreichend, das sei für Angelika „viel, sehr viel“ (IAng 511). Angelika scheint insgesamt aber doch wahrzunehmen, dass sie mit den 45 Euro, die ihr monatlich zur Verfügung stehen, sehr sparsam umgehen muss. Die finanzielle Ein- 
schränkung wird bei Angelika in einem vordergründig sehr bescheiden wirkenden Auftreten sichtbar, sie zeigt sich mit den finanziellen Ressourcen im Großen und Ganzen zufrieden.

Doris weiß nur ungefähr, wie viel Geld sie monatlich bekommt [,,manchmal siebzehn Euro und siebenundzwanzig Euro" (ID 446)]. Sie zeigt sich zunächst sehr zufrieden mit dem geringen Taschengeld, das sie in der Werkstätte bekommt.

Angelika wie Doris zeigen sich vordergründig mit den vorhandenen Ressourcen zufrieden. Die Höhe des „Taschengeldes“, das sie in der Werkstatt bekommen, wird erwähnt. Wenn dazu explizit reflektiert wird, dann eher allgemein im Zusammenhang mit den hohen Preisen für materielle Güter.

\section{Implizite Ausdrucksformen von Unzufriedenheit oder Thematisierung derzeit nicht finanzierbarer Wünsche bei geäußerter Zufriedenheit}

Doris zeigt sich mit ihrer gesamten Lebenssituation zufrieden, auch die geringe Höhe des Taschengeldes, das sie über die Beschäftigung bekommt, wird nicht kritisch bewertet. Das Taschengeld wird von Doris offenbar nicht dazu verwendet, aktuelle Bedürfnisse zu finanzieren. „Ich spare, ich habe noch keinen Plan, was ich kaufen soll“ (ID 449), hält Doris fest. Im Zusammenhang mit der Thematisierung spezieller Wünsche für ihre Zukunft hält sie später fest: ,ja, wenn ich eines Tages genug Geld hätte, dann könnte ich irgendwo hin reisen, wo es mir gefällt"“ (ID 441-442). Doris stellt den Zusammenhang von finanziellen Ressourcen und der Finanzierung eigener Wünsche eindeutig her. Dieser erscheint allerdings über die sprachliche Formulierung abgehoben von der eigenen möglichen Beteiligung an der Erwirtschaftung des Geldes in Zusammenhang mit der Finanzierung persönlicher Bedürfnisse und Wünsche.

Dass Angelika sich bei der nach außen dokumentierten Zufriedenheit nach Ablösung aus der bestehenden materiellen Abhängigkeit sehnt, dokumentiert sich an ihren geäußerten Wünschen für ihr weiteres Leben. Gefragt danach, führt sie an, sie hätte gerne ,einen reichen Mann und eine Villa, [...] ohne meine Geschwister“ (IAng 408). Sie beschreibt auf Nachfrage ihre konkreten Vorstellungen dazu und sagt, sie würde jeden Tag in den Pool baden gehen. Der Mann würde arbeiten „als Finanzberater, da verdient er voll viel Kohle“ (IAng 422), wie sie von einem Bekannten wisse. Angelika verbindet mit ihren Vorstellungen der finanziellen Ablösung von der Familie ein Leben, in dem sie unter anderen Rahmenbedingungen, aber wieder in einem geschützten Kontext lebt, der von ihrem zukünftigen Mann finanziert wird. Die Vorstellungen für ihr Leben beinhalten ein eher traditionelles Bild einer Familie mit zwei Kindern und einem sehr gut verdienenden Mann, Haus und Pool. Mit der Anmerkung, sie wünsche sich das Leben in diesem Kontext ohne ihre Geschwister, deutet Angelika eine gewünschte Ablösung von der Herkunfts- 
familie an, die nur über finanzielle Unabhängigkeit von dieser möglich erscheint. Vor dem Hintergrund der Erfahrung Angelikas, von der eigenen Schwester abgewertet und von den Eltern in diesem Zusammenhang nur unzureichend unterstützt zu werden ${ }^{101}$, zeichnet sich ein weiterer Zusammenhang ab: Finanzielle Abhängigkeit verhindert eine mögliche, gewünschte Ablösung aus Lebenszusammenhängen, die soziogenetisch hemmend oder einschränkend auf Handlungsspielräume und Entwicklungsperspektiven wirken.

\section{Wahrgenommenes Missverhältnis von erbrachter Leistung} und Bezug von ,,Taschengeld“ ̈̈ber „,Beschäftigung“ in einer Werkstätte

Thematisieren Angelika und Doris die Frage finanzieller Ressourcen eher unkritisch, so spricht Jessica explizit an, dass sie die Abgeltung ihrer Arbeitsleistung in der Werkstätte als „nicht in Ordnung“ (IJ 329) empfindet. Sie führt an, sie würde bereits seit Jahren in der Werkstätte arbeiten, ,aber fast nichts“ (IJ 306) dafür bekommen. Was sie brauche, bekomme sie ,alles von den Eltern“ (IJ 317). Sie sei auch nicht versichert, „wenn da was passiert, dann haftet die Firma drauf“ (IJ 320). Jessica hält abschließend fest: „Ja, ich finde das nicht normal, dass ich nichts kriege. Das ist nicht in Ordnung" (IJ 329). Damit wird auch Jessicas Orientierung an einem „Normalbereich“ deutlich. Sie zeigt sich darüber bewusst, dass sie über die Einbindung in die Werkstätte und den Bezug eines Taschengeldes nicht über ein gesellschaftlich übliches Arbeitsverhältnis verfügt. Sie nimmt wahr, dass sie für die von ihr geleistete Arbeit keinen regulären Lohn bekommt und auch übliche daran gekoppelte Leistungen wie Urlaub oder Versicherung nicht gegeben sind. Jessica äußert explizit Missfallen darüber, dass sie für ihre Leistung keine gerechte Bezahlung bekommt.

Sehr deutlich wird eine ähnliche Sichtweise auch in der Fallrekonstruktion „Anna“6102, die im Interview quasi als Lobbyistin für gerechten Lohn für Frauen mit Lernschwierigkeiten in Werkstätten auftritt und sich wünscht, dass ,,jeder den normalen Lohn kriegen würde“ (IA 671-672). Sie hält fest, dass sie wie alle anderen Frauen in der Werkstätte auch Geld von den Eltern bekommen würde.

Jessica und Anna orientieren sich in ihren Ausführungen an einem von ihnen als „normal“ eingeschätzten Rahmen. Das Bewusstsein darüber, dass

101 Siehe dazu Punkt ,Soziogenetisch hemmend oder einschränkend auf Handlungsspielräume und Entwicklungsperspektiven wirkende Faktoren“, insbesondere „Erfahrung von Abwertung und Stigmatisierung im Zusammenhang mit zugeschriebener ,Behinderung"“ und „Vorenthalten von bedarfsgerechter Unterstützung im Alltag und in schwierigen Situationen".

102 Siehe dazu ausführlich Fallrekonstruktion Anna, insbesondere ,, Wunsch nach finanzieller Unabhängigkeit“. 
ihre realen Lebensbedingungen davon abweichen, indem ihre Leistungen in der Werkstätte nicht angemessen abgegolten werden und finanzielle Abhängigkeit zu den Eltern besteht, löst bei beiden Unbehagen und Unzufriedenheit aus. Sie verbleiben in einer eher passiven Haltung, in der Unzufriedenheit massiv artikuliert wird, im Endeffekt aber zu keiner Veränderung führt.

\section{Wirtschaftliche und finanzielle Abhängigkeit kann Orientierung am vorgegebenen Rahmen begründen}

Die wirtschaftliche Abhängigkeit zeigt sich in der Lebenssituation von Frauen mit Lernschwierigkeiten in vielfältigen Lebenszusammenhängen und führt oftmals dazu, dass sie sich auch in ihrer konkreten alltäglichen Handlungspraxis stark an den Normkonstrukten und Vorgaben aus dem Umfeld, zu dem diese Abhängigkeit besteht, ausrichten.

Doris orientiert sich ohne sichtbaren inneren oder äußeren Widerstand an den Vorstellungen der Eltern bzw. anderer Autoritätspersonen. So beschreibt sie, dass sie es „nicht so schlimm“ (ID 346) finden würde, wenn ihr die Eltern beim gemeinsamen Einkauf nicht erlauben würden, etwas zu kaufen, das ihr selbst, aber nicht den Eltern gefalle. Auch darüber hinaus findet sich Doris in Regeln, Vorgaben und Strukturen innerhalb der Familie und der Arbeitsstelle ein. Einerseits scheinen diese für Doris Sicherheit und Orientierung zu bieten, andererseits scheint Doris auch wahrzunehmen, dass ihre Abhängigkeit ihr wenig Spielraum lässt.

Besonders deutlich wird der Zusammenhang von Anpassung an vorgegebene Strukturen und Orientierung an vorgegebenen Normkonstrukten und Vorstellungen mit wirtschaftlicher Abhängigkeit am Beispiel von Melanie (vgl. IMe 237-246). Im Zusammenhang mit der Frage, wie der Einkauf von Bekleidung erfolgt, merkt diese an, dass sie das Geld dafür von der Mutter bekomme, dass diese aber auch Einfluss darauf habe, welche Kleidungsstücke gekauft werden. Dass sie in diesen Fällen immer den Vorgaben der Mutter folgt, argumentiert Melanie damit, dass die Mutter ja recht haben könne, weil ein von ihr selbst ausgesuchtes Kleidungsstück sich eventuell nicht für die Arbeitsstelle eigne und von der Größe her nicht passen könnte. Sie führt also aus, dass sie die Entscheidung der Mutter aus verschiedenen Gründen nachvollziehen könne. Im Zusammenhang mit Melanies Bestreben, durch Konfliktvermeidung den geschützten Rahmen zu erhalten, dokumentiert sich darüber aber der eingangs thematisierte Zusammenhang: Melanie orientiert sich auch aus Gründen der wirtschaftlichen Abhängigkeit an den vorgegebenen Strukturen und Regeln. 
Auszahlung von „Taschengeld“ dokumentiert nicht altersgemäße, hierarchische Beziehungsstrukturen

Ihre Forderung nach regulärem Lohn für Frauen mit Lernschwierigkeiten in Werkstätten argumentiert Anna mit dem Wunsch nach finanzieller Unabhängigkeit und der Möglichkeit, den eigenen Unterhalt abzudecken (vgl. IA 686). Sie müsse dann, so Anna: ,nicht alle Ritt ${ }^{103}$ meine Eltern auch fragen brauche, Mama, darf ich ein Geld haben und das, weil ich bin 28 Jahre alt“" (IA 687-688). Über diese Formulierung wird deutlich, dass Anna es auch nicht als altersgemäß empfindet, als junge, erwachsene Frau die Eltern um Geld bitten zu müssen.

Über die mit der Auszahlung von Taschengeld von den Eltern verbundenen Abläufe, etwa konkrete Auszahlungssituationen, dokumentiert sich ein Gefälle in diesen Beziehungen, das darauf verweist, dass finanzielle Abhängigkeit Hierarchien begünstigt. Schon durch die Angabe der Personen aus dem sozialen Umfeld, von denen das Taschengeld übergeben wird, deutet sich sprachlich auch eine darüber wahrgenommene Eltern-,,Kind"-Beziehung erwachsener Frauen mit Lernschwierigkeiten zu ihren Eltern an: Angelika beschreibt, sie bekomme 20 Euro Taschengeld von ihrem „Papa“ (IAng 549), Ursula bezieht dieses ,immer von der Mama“ (IG 476).

Marlene kann offenbar nicht mit einem fix kalkulierbaren Taschengeld von den Eltern rechnen. Sie hält fest, dass sie manchmal von ihrem Vater „einen Zehner oder einen Fünfer oder so“ (IMarl 765) bekommen würde. In Marlenes Beschreibung bekommt dies den Charakter des „Zusteckens“. Die junge erwachsene Frau Marlene erscheint im Verhältnis zu ihrem Vater als Kind. Marlenes Beschreibung, sie sei in der Arbeit „höher g'stellt“ (IMarl 722) worden und würde nun 42 statt 25 Euro monatlich bekommen, lässt sie über die sprachliche Formulierung als passiv und abhängig von den Entscheidungen im beruflichen Umfeld erscheinen.

Sonja beschreibt, dass sie in der Werkstätte der Institution, in der sie auch lebt, sieben Euro Taschengeld in der Woche bekomme. Sie verwendet explizit den Begriff des „Zusteckens“, indem sie festhält, sie bekomme „Gott sei Dank von den Eltern noch was zugesteckt" (ISo 199-201). Sonja nimmt also finanzielle Einschränkung und damit verbundene Abhängigkeiten wahr. Über die sprachliche Formulierung drückt sich ein deutlich wahrnehmbares hierarchisches Gefälle in der Beziehung aus, das sich über die finanzielle Abhängigkeit ergibt.

103 Alle Ritt' = immer wieder. 
Auswirkungen der finanziellen Situation auf Fragen im Zusammenhang mit dem eigenen Äußeren

Finanzielle Abhängigkeit wird insbesondere auch im Zusammenhang mit Fragen des Einkaufs von Bekleidung durch und mit Personen, zu denen ein (finanzielles) Abhängigkeitsverhältnis besteht, sichtbar. Dies dokumentiert sich bei den betroffenen Frauen zunächst vorerst über Ausführungen dazu, dass diese Einkäufe in der Regel mit der finanzierenden Person gemeinsam erfolgen.

Jessica beschreibt, ihre Mutter würde vorschlagen, einkaufen zu gehen und sie sage dann ,ja, gehen wir einkaufen“ (IJ 344). Jessica hält fest, dass die Mutter die Einkäufe finanziere [„Das zahlt alles die Mama bei mir“ (341)], beschreibt aber auch, dass sie selbst aussuchen könne, was ihr gefalle. Die Beziehung zu den Eltern wird von Jessica generell als ,total gut" bewertet.

Brigitte führt aus, dass sie selten Bekleidung kaufe und begründet dies: „Ich habe genug Wäsche zum Anziehen“ (IB 294). Aus ihren Ausführungen geht hervor, dass die eher seltenen Einkäufe dann immer mit ihrem „Papa, zu zweit" (IB 271) erfolgen. Für sie scheint dies selbstverständlich zu sein. Ihre Aktivitäten sind grundsätzlich stark an den Interessen und Bedürfnissen des Vaters ausgerichtet. Es wird auch kein Widerstand oder der Wunsch, alleine einkaufen zu gehen, sichtbar. Brigitte bringt auch zum Ausdruck, dass sie jene Kleidung trägt, die ihrem Vater gefällt. Wenn dies der Fall sei, sage er „Uh, fesch Brigitte“ (IB 286). Es wird sichtbar, dass der Wunsch nach der Anerkennung des Vaters in Kombination mit finanzieller Abhängigkeit bei Brigitte dazu führt, dass sie sich bei der Auswahl von Bekleidung an seinen Vorstellungen orientiert.

Jessicas Freiraum bei der Auswahl der von den Eltern finanzierten Bekleidung stellt im Vergleich zu anderen Frauen eher die Ausnahme dar. An zahlreichen Passagen in unterschiedlichen Interviews kommt auf vielfältige Weise implizit oder explizit die Intention von Personen aus dem sozialen Umfeld zum Ausdruck, Einfluss auf Fragen zu nehmen, die mit dem Ausdruck der eigenen Persönlichkeit über Gestaltung des äußeren Erscheinungsbildes einhergehen. Das sich daraus ergebende hierarchische Eltern- bzw. Erwachsenen-,,Kind"-Gefälle wird auch in Zusammenhang mit dieser Thematik sichtbar. Angelika führt dazu aus:

,ja, ich kauf' aber nur Sachen, die was oben zu sind und hinten, weil manche kaufen sich so eine Miniröcke, [...] so was kauf' ich mir aber nicht, so was [...] Vorne zu und hinten zu, weil die meisten kaufen sich so Leiberln, da ist hinten ein Loch, da sieht man den Rücken voll, bei den meisten." (IAng 705 -711)

Das würde den Eltern ,voll nicht“ (IAng 719) gefallen. Wenn sich Angelika nicht an die Vorstellungen der Eltern hält und etwa einen geschenkten Minirock anzieht, würden die Eltern sofort sagen ,zieh was anderes an, ja“ (IAng 728). Angelika identifiziert sich hier über ihre sprachliche Ausdrucksweise - 
wie auch im Zusammenhang mit einem von den Eltern nicht gewünschten Discobesuch ${ }^{104}$ - mit den Vorstellungen der Eltern. Sie führt deren Argumente als Begründung für ihre Kaufentscheidung an. Zugleich macht sie aber deutlich, dass die Eltern explizit darauf hinweisen, welche Art der Kleidung sie bevorzugen.

Auf eher implizite Weise werden diese Mechanismen bei Marlene sichtbar. Diese beschreibt, dass sie Bekleidung immer mit der Mutter einkaufen würde, die auch bezahlen würde. Die Mutter würde auch meistens entscheiden, was gekauft werde, denn, so argumentiert Marlene, ,wenn ich da was aussuch', dann ist es ein Chaos bei mir" (IMarl 781-782). Die Mutter, so Marlene, „kennt sich da besser aus“ (IMarl 789). Die Mutter sage aber auch manchmal ,such dir schnell was aus, ich hab' keine Zeit“" (IMarl 798). Sie sage ,entscheide dich jetzt, was du willst, sonst kriegst du gar nichts, so in der Art" (IMarl 800-801). Über Marlenes Beschreibungen wird deutlich, dass die finanzielle Abhängigkeit im Zusammenhang mit Einkäufen sich darin äußert, dass entweder die Mutter entscheidet, was gekauft wird, oder Marlene unter Druck gesetzt wird, sich schnell zu entscheiden. Marlene hat offenbar nicht den Raum und wird nicht dabei unterstützt, in Ruhe selbstbestimmte, überlegte Entscheidungen beim Kauf ihrer Kleidung zu treffen. Sie nimmt zeitlichen Druck von der Mutter wahr und kommt damit offenbar auch nicht gut zurecht. Ähnlich wie Melanie überlässt sie die Auswahl beim Einkauf der Mutter und argumentiert dies auch damit, dass diese sich besser auskenne. Beide sind auch finanziell abhängig, beide sehen das familiäre Umfeld als wenig unterstützend, aber doch als Ort, der ihnen in gewisser Weise Schutz bietet und vertraut ist. Bei Melanie ist die familiäre Situation von Konflikten belastet, bei Marlene eher durch Beziehungslosigkeit gekennzeichnet.

Bei Melanie wie bei Angelika wird deutlich, dass die Abhängigkeit im finanziellen Bereich dazu führen kann, dass die finanzierenden Personen Einfluss auf die Auswahl der Kleidung haben und das eigene Äußere somit nicht so gestaltet werden kann, wie es den Frauen selbst entspricht. An Angelikas Ausführungen wird sichtbar, dass deren Eltern bei ihr Kleidung bevorzugen, die alltagstauglich und auf keinen Fall auffallend und aufreizend ist. Dies erscheint im Kontext mit deren implizit zum Ausdruck gebrachten Ängsten im Zusammenhang mit möglichen sexuellen Aktivitäten und einer Schwangerschaft Angelikas im Kontext eines Discobesuches als Verhinderungsstrategie.

Finanzielle Unabhängigkeit hingegen eröffnet Spielraum, sich auch hinsichtlich der Gestaltung des eigenen Äußeren zu erproben. Eva erzählt etwa davon, dass sie im Zuge des autonomen Wohnens und der Erwirtschaftung

104 Dieses Beispiel wird ausführlich beschrieben und analysiert unter Punkt „Erschweren und Verhindern von Partizipation durch das soziale Umfeld“" (,Soziogenetisch hemmend oder einschränkend auf Handlungsspielräume und Entwicklungsperspektiven wirkende Faktoren"). 
eines eigenen Einkommens Piercings und Tatoos machen ließ, die ihren Vater in Aufregung versetzten (IE 349-350). Eva begründet die Gestaltung ihres Äußeren damit, dass sie „Veränderung sehr stark nach außen“ (IE 379) trage. Das eigene Einkommen stellt damit auch die Basis für selbstbestimmte Entscheidungen hinsichtlich der Gestaltung des eigenen Äußeren dar.

\section{Eingeschränkte Möglichkeit zur Partizipation}

durch finanzielle Einschränkungen

Eingeschränkte finanzielle Mittel werden von Frauen mit Lernschwierigkeiten auch im Zusammenhang mit der gesellschaftlichen Partizipation thematisiert. So hält Marlene fest, sie würde gerne mit Freundinnen ins Kino gehen, schränkt aber ein - „wenn ich Geld hätte“ (IMarl 1075). Marlene führt auch die hohen Kosten an, die ein Kinobesuch verursachen würde: ,es kostet ein Geld, Popcorn kostet schon zu viel, dann kostet das Getränk schon einmal so viel“" (IMarl 1058-1059). Ihre früher (vgl. IMarl 720-730) ausgeführte Bewertung, dass ihr das monatliche Geld von 42 Euro ausreichen würde, wird hiermit relativiert. Marlene kann offenbar im Freizeitbereich auch aufgrund fehlender finanzieller Mittel nur eingeschränkt agieren.

Die Möglichkeit, soziale Kontakte außerhalb ihrer Arbeitsstätte zumindest über das Handy zu führen, sind über mangelnde finanzielle Mittel ebenfalls eingeschränkt, wie Marlene im Zusammenhang mit der Frage des Kaufes von Handyguthaben beschreibt: ,also morgen [...] wahrscheinlich kann ich noch telefonieren und dann [...], genau morgen kann ich noch telefonieren und am Freitag nicht mehr dann ist aus“" (IMarl 1081-1082).

Soziale Aktivitäten außerhalb des Familien- oder Arbeitszusammenhanges erfolgen de facto gar nicht. So erläutert Doris auf Nachfrage, ob sie z.B. einmal mit den Freunden in die Stadt oder ins Kino gehe, ,ich habe es nur mit meinen Eltern gemacht" (ID 315).

Angelika thematisiert über die Beschreibung eines Bildes, das eine Frau im Rollstuhl am Meer zeigt, den Zusammenhang mit dem Wunsch nach einem Urlaub am Meer und ihren eingeschränkten finanziellen Ressourcen, indem sie festhält: ,zu wenig, ja, 25 Euro sind zu wenig für Italien für eine Woche, ja“" (IAng 1089). Sie könne daher nicht in den Urlaub fahren, hält Angelika fest, es gehe sich ,zur Zeit mit dem Geld nicht aus“ (IAng VA7 1091).

\section{Eingeschränkte Perspektiven durch finanzielle Abhängigkeit}

Doris äußert vordergründig Zufriedenheit mit ihrer finanziellen Situation und orientiert sich an den Vorgaben aus dem familiären und beruflichen Umfeld. Vor diesem Hintergrund stellt Doris aber gedanklich einen Zusammenhang von finanzieller Abhängigkeit und ihrer derzeitigen Lebenssituation sowie 
möglicher Veränderung derselben her. So hält sie fest: ,ja, also ich hab nicht genug Geld für ein eigenes Haus, also bleibe ich noch ein bisschen hier bei ihnen“ (ID 519-520), gemeint sind hier die Eltern, wie aus dem Interviewzusammenhang hervorgeht.

Anna thematisiert im Interview explizit den Zusammenhang von finanzieller und persönlicher Abhängigkeit (IA 674, 687). Ihre Vorstellungen von gerechtem Lohn für geleistete Arbeit verbinden sich mit möglichen Veränderungsperspektiven - etwa im Wohnen -, die sich erst daraus ergeben könnten. Für Anna scheinen Vorstellungen von einem selbstbestimmten Leben noch in weiter Ferne zu liegen, zumal ein Wechsel von der Anlehre in die Beschäftigung bevorsteht, wodurch ihre finanziellen Mittel noch weiter eingeschränkt werden.

Deutlich dokumentieren sich die von den Frauen mit Lernschwierigkeiten gedanklich hergestellten Zusammenhänge von materieller Abhängigkeit und der eigenen Lebenssituation an Wünschen und Vorstellungen für ihre $\mathrm{Zu}-$ kunft, die aktuell nicht realisierbar scheinen. So wird etwa über Doris Ausführung „Ja, wenn ich eines Tages genug Geld hätte, dann könnte ich irgendwo hinreisen, wo es mir gefällt" (ID 441-442) deutlich, dass sie um die Abhängigkeit der Erfüllung dieses Wunsches von finanziellen Mitteln Bescheid weiß.

\section{Reflexion der Möglichkeit eines selbstbestimmten Lebens durch eigenen Lohn}

Frauen mit Lernschwierigkeiten, die über ein Arbeitsverhältnis eigenen Lohn erwirtschaften können, thematisieren von sich aus Zusammenhänge von finanzieller Unabhängigkeit und einem selbstbestimmten, autonomen Leben. Besonders deutlich dokumentiert sich dies an der Fallrekonstruktion „Eva“. Diese bringt ihre Einschätzung von materieller Autonomie als wichtige Basis für ein selbstbestimmtes Leben explizit zur Sprache:
„Ja, was der Job mit sich bringt, ist ganz einfach: du kannst dein eigenes Leben finan- zieren, du kannst dir deine Wünsche erfüllen, du kannst dein - , ja du bist ein Teil der Gesellschaft, wo man eigentlich hingehört, ja, du bist ein ganz ein normaler Teil der Gesellschaft, du erwirtschaftest dein Leben sogar, du gehst sogar einkaufen, du gehst ein Waschmittel kaufen, du gehst - du zahlst deine, deine Arztrechnungen, deine Tele- fonrechnungen, alles, $\mathrm{du}=\mathrm{du}$ bist für dein Leben, egal in welcher Hinsicht Job , Arb- , Job, Privatleben, finanzielle Dinge, man ist für sein Leben komplett selber verantwort- lich.“( (IE 480-486)

Finanzielle Unabhängigkeit und damit die Möglichkeit der Erfüllung eigener Bedürfnisse stellen somit die Grundlage eines selbstbestimmten Lebens innerhalb der Gesellschaft dar. Gesellschaftliche Partizipation bedeutet aus dieser Perspektive, auch einen eigenen Beitrag innerhalb der Gesellschaft zu leisten. 
Für Tamara sind Vorstellungen von einem selbstbestimmten Leben durch das eigene Gehalt in greifbare, realistische Nähe gerückt. Durch den Lohn könne sie ihr „Handy und in Zukunft vielleicht eine Wohnung, also betreutes Wohnen“ (IT 549) umsetzen, führt Tamara aus und hält fest: „Also, dass ich schon alleine wo wohne [...] das möchte ich für mich, für meine Zukunft (IT 551-552). Das Bewusstsein, selbst etwas zur Umsetzung dieses Wunsches durch eigene finanzielle Mittel beitragen zu können, führt dazu, dass Tamara diesen auch hinsichtlich seiner möglichen Umsetzung zu reflektieren beginnt.

\section{Zusammenfassung: Lebenszusammenhänge als soziogenetische Faktoren für die Entwicklung von Orientierungen}

Die Art und Weise sowie der Grad der sozialen Eingebundenheit stehen in engem Zusammenhang mit den Orientierungen junger Frauen mit Lernschwierigkeiten und bedingen Herangehensweisen und Handlungsstrategien in ihrer Lebenswelt. Hier ist insbesondere die Frage von Bedeutung, welche Rahmenbedingungen zur Verfügung stehen bzw. zugänglich gemacht werden. Die Forschungsergebnisse legen nahe, dass die Eröffnung mehrdimensionaler Erfahrungsräume mit den sich dadurch ergebenden Kontakten und Herausforderungen vielfältige Entwicklungsperspektiven ermöglichen. Die Beschränkung auf ein vorgegebenes System hingegen verengt diese Möglichkeiten.

Hinsichtlich der Lebenszusammenhänge wird deutlich, dass für viele Frauen mit Lernschwierigkeiten der weitgehende Bezug auf den Rahmen der Herkunftsfamilie ihre Lebensrealität darstellt. Dabei bietet das Leben in der Familie Struktur und ermöglicht die Teilhabe an deren Alltagshandlungen. Beschränken sich soziale Kontakte vorwiegend auf diesen Rahmen, was mit nicht vorhandener außerfamiliärer sozialer Einbindung im Bereich Arbeit/ Beschäftigung einhergeht, so wirkt sich dies auf die Wahrnehmung neuer Möglichkeiten oder Erfahrungen aus. Chancen zur Weiterentwicklung, die sich darüber ergeben könnten, können dann oftmals nicht wahrgenommen werden oder werden abgelehnt. Indem darüber kaum neue Impulse und Herausforderungen entstehen, bleibt es häufig beim Status quo. Mit der Vorstellung, sich außerhalb des geschützten Rahmens zu bewegen, verbinden bzw. verfestigen sich mitunter auch Vorbehalte und Ängste bei den Frauen selbst oder innerhalb der Familie in Zusammenhang mit möglicher sozialer Partizipation. Wahlmöglichkeiten, die die Grundlage selbstbestimmter Entscheidungen bilden, bleiben in diesem Prozess verborgen bzw. werden vorenthalten.

Ergänzende bzw. alternative Möglichkeiten, soziale Kontakte außerhalb der Herkunftsfamilie zu knüpfen und zu führen, stellen Wohnen und/oder Arbeiten in einer Institution dar. Interaktionen und gemeinsame Aktivitäten mit Peers und mit Betreuungspersonal innerhalb und außerhalb der Institution 
bieten soziale Begegnungsmöglichkeiten. Beziehungen, die sich in diesem Kontext vor allem zu Peers ergeben, werden vorwiegend als Bereicherung erlebt und ermöglichen u.a. die Freizeitgestaltung mit gleichaltrigen Personen. Tägliche Wege, die im Zusammenhang mit der Einbindung in eine Werkstätte zurückgelegt werden, strukturieren den Tagesablauf und erfordern bestimmte Aktivitäten. Darüber werden auch verschiedene Handlungsabläufe erprobt, was die Bewegung im öffentlichen Raum erleichtert. Soziale Kontakte über den Kontext der Herkunftsfamilie werden in diesem Rahmen ermöglicht. Manchmal stellt die institutionelle Einbindung - etwa in ein Beschäftigungsprojekt - die einzige Möglichkeit dar, Kontakte zu knüpfen, zu führen und zu halten. Dies verdeutlicht sich am Beispiel Angelikas, die davon erzählt, dass sie eine Arbeitskollegin, mit der sie „dick befreundet“ ist, nur an der Arbeitsstelle sehen könne (IAng 189). Die Einbindung in außerfamiliäre, institutionelle Kontexte erweckt dabei häufig erst den Wunsch nach Ausbau bzw. Intensivierung sozialer Kontakte und gesellschaftlicher Partizipation. Dies kann auch zur Fixierung auf eine konkrete Form der außerfamiliären Einbindung führen, wenn damit der Wunsch nach Erhalt der gegebenen sozialen Kontakte, die anders nicht realisierbar erscheinen, verbunden ist. So entscheidet sich Angelika gegen den Wechsel in ein Projekt, in dem sie den von ihr präferierten Tätigkeiten nachgehen könnte, zugunsten des weniger passenden institutionellen Kontextes, um ihre Freundschaft zu einer Kollegin weiter pflegen zu können. Ungünstige Rahmenbedingungen und fehlende Integration am Wohnort verbunden mit Mobilitätsbarrieren, eigenen Unsicherheiten und Ängsten bedingen dabei Angelikas Perspektive. Es deutet sich an, dass durch vielfältige soziale Bezüge eher Offenheit für neue Perspektiven entstehen könnte, da dann nicht der Wunsch nach Erhalt des vorhandenen sozialen Bezugssystems im Vordergrund steht. Bedarfsgerechte Unterstützungsleistungen, die es jungen Frauen mit Lernschwierigkeiten ermöglichen, vielfältige soziale Kontakte zu pflegen, eröffnen neue Perspektiven und Handlungsmöglichkeiten. Dies beinhaltet konkrete Unterstützungsleistung im Zusammenhang mit Mobilitätsbarrieren, aber auch die Förderung und Unterstützung vielfältiger Kontakte auf emotionaler Ebene.

Das Leben in einem strukturierten Rahmen kann aber auch zu Unzufriedenheit führen, wenn die Rahmenbedingungen als nicht stimmig mit den eigenen Bedürfnissen erlebt werden. Dies dokumentiert sich etwa an frühen Erfahrungen von Eva ${ }^{105}$, die das Wohnen in der Behinderteneinrichtung aus diesem Grund kritisiert. Als rigid wahrgenommene Regeln und fehlender Freiraum führen bei ihr zur Ablehnung des institutionellen Kontextes. Unter dieser Perspektive werden mitunter auch die in diesem Rahmen entstehenden sozialen Bezüge als nicht eigenen Bedürfnissen entsprechend eingeschätzt,

105 Siehe dazu ausführlich Fallrekonstruktion „Eva“, insbesondere „Ablehnung von Regeln und Vorschriften versus Wunsch nach eigener Gestaltungsmöglichkeit“ und „Ablehnung traditioneller Betreuungskonzepte versus Wunsch nach Unterstützung und Begleitung“. 
was sich etwa am Beispiel Martinas dokumentiert. Martina lehnt nicht nur das Leben im institutionellen Rahmen ab, was sie mit dort bestehenden zeitlichen Vorgaben und feststehenden Plänen begründet. Sie möchte auch hinsichtlich ihrer sozialen Kontakte nicht auf Personen aus diesem Kontext festgelegt werden.

Die Möglichkeit, in einem selbst bestimmten Rahmen mit bedarfsgerechter Unterstützung leben zu können, eröffnet hingegen neue Perspektiven und Möglichkeiten. Dies wird an Evas Beispiel deutlich, die hier Möglichkeiten wahrnimmt, die ihr zuvor im von ihr kritisierten, stark strukturierten institutionellen Kontext unrealistisch erschienen. Vertrauen, Selbstbewusstsein, Eigenverantwortung und die Fähigkeit, sich selbst zu vertreten, wachsen zunehmend und versetzen Eva in die Lage, neue Herausforderungen aktiv anzunehmen und sich darüber weiterzuentwickeln.

Die Frage des materiellen Status, der vorwiegend über die Einbindung in Beruf oder Beschäftigung determiniert ist, wirkt sich deutlich wahrnehmbar auf die Orientierung junger Frauen mit Lernschwierigkeiten aus. Sie bedingt aber auch Einstellungen und Interaktionen von Personen aus dem sozialen Umfeld. Insbesondere wenn zu diesen Personen materielle Abhängigkeit besteht, ist auch die Beziehungsstruktur selbst von einem Ungleichgewicht geprägt, das sich auch auf das Selbstbild und die Handlungsstrategien der betroffenen Frau auswirken kann.

An die Frage der finanziellen Ausstattung ist auch jene nach der sozialen Einbindung gekoppelt. Wo vorwiegend oder ausschließlich die Herkunftsfamilie den Kontext für soziale Interaktion bildet, stellt diese auch den Rahmen für die Ausstattung mit finanziellen Ressourcen dar. In der Regel äußern Frauen mit Lernschwierigkeiten, die in diesem Rahmen leben, kaum explizit Kritik daran. Auch die Frage der finanziellen Ressourcen bleibt meist ausgespart. Wenn sie thematisiert wird, so eher implizit. Zusammenhänge von finanziellen Ressourcen und eigenen Handlungsmöglichkeiten werden in der Regel aber unbewusst wahrgenommen und in die eigenen Überlegungen integriert. Auch Frauen, die weder über eigenes Einkommen noch über ein in einer Werkstätte ausbezahltes Taschengeld verfügen, erkennen also in der Regel, dass bestimmte Wünsche mangels finanzieller Ressourcen nicht realisierbar sind. Die Perspektive, sich im Kontext von finanzieller Abhängigkeit aus Lebenszusammenhängen zu lösen, die den eigenen Bedürfnissen nicht entsprechen, wird wenn, dann als unrealisierbar wahrgenommen und kaum explizit zur Sprache gebracht. Wirtschaftliche und finanzielle Abhängigkeit kann somit eine Orientierung am vorgegebenen Rahmen und an darin gültigen Regeln und Vorschriften begründen. Dabei dokumentieren sich über die Auszahlung von „Taschengeld“ nicht altersgemäße, hierarchische Beziehungsstrukturen, die die betroffenen Frauen auf eine kindliche Rolle festlegen. Finanzielle Abhängigkeit wirkt sich häufig auch auf Fragen im Zusammenhang mit dem Äußeren aus, indem damit häufig auch Vorgaben verbun- 
den sind, sich etwa in einer bestimmten Art und Weise zu kleiden. Finanzielle Abhängigkeit geht auch mit eingeschränkten Partizipationsmöglichkeiten einher, da etwa Kontakte wegen kostenintensiver Telefonate nicht ausreichend gepflegt werden können. Freizeitaktivitäten, die mit der Notwendigkeit finanzieller Ressourcen einhergehen, wie etwa Kinobesuche, werden vor diesem Hintergrund kaum in Erwägung gezogen. Auch Zukunftsperspektiven werden vor dem Hintergrund finanzieller Abhängigkeit nur verhalten thematisiert.

Auch über das in Werkstätten ausbezahlte, meist sehr geringfügige „Taschengeld" sind nur kleine, eher alltägliche Bedürfnisse finanzierbar. Von betroffenen Frauen wird dieses Missverhältnis von erbrachter Leistung und finanzieller Abgeltung häufig als ,nicht normal“ bis „,nicht in Ordnung“ (IJ 329) wahrgenommen.

An ein über ein geregeltes Dienstverhältnis erwirtschaftetes eigenes Einkommen sind auch sozialrechtliche Ansprüche, wie Urlaub und Versicherungs- und Pensionsansprüche, geknüpft. Finanzielle Unabhängigkeit kann somit die Grundlage eines selbstbestimmten Lebens innerhalb der Gesellschaft darstellen. Finanzielle Unabhängigkeit eröffnet auch neue Perspektiven hinsichtlich selbstbestimmter Entscheidungen über die Gestaltung des eigenen Äußeren.

\subsubsection{Erfahrungsräume als soziogenetische Faktoren für die Entwicklung von Orientierungen}

Waren es mit der Analyse der Auswirkung der ,sozialen Eingebundenheit“ auf Orientierungen vorwiegend bestehende Rahmenbedingungen, die in den Blick genommen wurden, so stehen nun spezifische Ereignisse in unterschiedlichen sozialen Kontexten im Fokus der Betrachtung. Im Zentrum der soziogenetischen Analyse steht somit die Frage, mit welchen spezifischen Erfahrungshintergründen bestimmte Orientierungsrahmen systematisch zusammenhängen. Konkrete Beispiele dafür finden sich typenübergreifend und machen über die darin sichtbaren „Kontraste in der Gemeinsamkeit“ (Nohl 2012: 56) die Mehrdimensionalität in der Typenbildung deutlich. Im Folgenden werden soziogenetisch auf Handlungsspielräume und Entwicklungsperspektiven wirksame Faktoren in den Blick genommen, wobei zwischen diese hemmenden bzw. einschränkenden und förderlichen bzw. erweiternd wirkenden unterschieden wird.

Über diese Analyse wird auch deutlich, dass es bei Vorliegen bestimmter Voraussetzungen auch zu einem Wechsel in der Orientierung kommen kann, sich diese also als wandelbar erweist. Diesem Wechsel oder Wandel in der Orientierung liegen im Kontext der vorliegenden Studie oftmals Momente zugrunde, die von den jeweiligen Frauen auch als nicht alltägliche, sondern 
als besondere Erfahrungen und als Dreh- und Angelpunkte für persönliche (Weiter-)Entwicklung beschrieben werden. Dies wird sprachlich mitunter eindrucksvoll in Form von Fokussierungsmetaphern artikuliert, wobei Frauen unabhängig voneinander dafür zum Teil sehr ähnliche Formulierungen verwenden. Wie etwa Eva, die im Zusammenhang mit der expliziten Wahrnehmung ihrer Interessen durch ihr Gegenüber festhält, das seien ,so Szenen, die ich mein Lebtag niemals vergessen werde, die mich so geprägt haben“ (IE134), oder Anna, die über eine Lehrerin, die sie wahrnahm und förderte sagt: „die Lehrerin, die werde ich so nie vergessen“ (IA 295-296). Dabei sind diese Wechsel in verschiedene Richtungen möglich: Positive Erfahrungen wirken in der Regel entwicklungsfördernd und eröffnen neue Perspektiven, negative hemmen im Gegensatz dazu eigene Handlungsspielräume und Entwicklungspotentiale. In welcher Art dies systematisch deutlich wird, soll ebenfalls über die folgenden Analysen sichtbar gemacht werden.

\subsubsection{Hemmend oder einschränkend auf Handlungsspielräume und Entwicklungsperspektiven wirkende Faktoren}

Im Folgenden wird analysiert und mittels Beispielen aus den vier Fallrekonstruktionen und ergänzenden Ausführungen aus weiteren Interviews belegt, welche Erfahrungen im Zusammenhang mit der Entwicklung der Orientierung junger Frauen mit Lernschwierigkeiten negativ wirksam sind. Solche Erfahrungen werden typübergreifend von allen Frauen berichtet und stellen daher konjunktive Erfahrungsräume von Frauen mit Lernschwierigkeiten dar. In den typisierten Orientierungen werden unterschiedliche Konsequenzen daraus im Sinne soziogenetischer Prozesse deutlich. Diese verweisen darauf, dass die Bearbeitung der erlebten Ereignisse im sozialen Kontext ausschlaggebend für die individuell daraus resultierenden persönlichen Entwicklungsprozesse ist. Dass also die Art und Weise, wie etwa Bezugspersonen auf die erlebten negativen Erfahrungen reagieren bzw. ob sie sogar selbst diese Erfahrungen begründen, sich soziogenetisch maßgeblich auf die Entwicklung von Orientierungen auswirkt. Dies auf Basis der mittels der dokumentarischen Methode vorgenommenen Analyse nachzuzeichnen und darzustellen, steht im Zentrum der folgenden Ausführungen. Soziale Interaktionen, die grundsätzlich sowie im Zusammenhang mit der Bearbeitung negativer Erfahrungen als positiv erlebt werden und auf Handlungsspielräume und Entwicklungsperspektiven fördernd bzw. erweiternd wirken, werden im Anschluss daran beschrieben. Diese können über die damit einhergehenden Prozesse auch zu einem Wandel einer vorliegenden Orientierung führen. 
Erfahrung von Abwertung und Stigmatisierung im Zusammenhang mit zugeschriebener „Behinderung “

Mit Abwertung und Stigmatisierung verbundene Prozesse werden von Frauen mit Lernschwierigkeiten in ihrem Lebensalltag deutlich wahrgenommen, mit unterschiedlichen Gefühlen verbunden erlebt und bearbeitet. Angelika nimmt etwa im Umgang mit den Schwestern deutlich mangelnden Respekt ihr gegenüber wahr: „die respektieren mich überhaupt nicht“ (IAng 370), ,weil die respektiert mich einfach nicht" (IAng 397). Über die Zuschreibungen der jüngeren Schwester, Angelika sei „dumm, behindert“ (IAng 374), und über die damit einhergehende Zuweisung des Etiketts „faul“ (IAng 386) erlebt Angelika Stigmatisierung. Über Angelikas folgende Beschreibung, dass sie nicht nur von der Schwester, sondern auch in der Schule mit dem Begriff „Behinderte“ (u.a. IAng 829) abgewertet wurde, wird deutlich, dass Erfahrungen mit Etikettierung und Stigmatisierung in ihrer Biographie eine zentrale Rolle spielen. Sie erzählt, dass sie ,Tag ein Tag aus, behindert=behindert= behindert, jedes Mal das Wort behindert" (IAng 846) gehört habe. Die Mitschüler (vgl. IAng 833) und Schüler aus der Nebenklasse hätten sie ,alle verarscht von A bis Z, die haben alle gesagt: da kommt die Behinderte daher" (IAng 829-830). Der Begriff „Behinderung“ ist dabei bei Angelika selbst sehr negativ besetzt und wird von ihr als Schimpfwort gesehen, für dessen Verwendung sich ein Schüler bei ihr entschuldigen musste. In derselben Szene bringt Angelika den Begriff der Behinderung auch mit der Zuschreibung ,arm“ in Zusammenhang, indem sie erzählt, dass der Schüler zur Strafe zehnmal schreiben musste: ,sag zu der armen Angelika nicht behindert" (IAng 850). Die Emotionen, die bei ihr das Gefühl des Abgewertet-Werdens hervorbringen, steigern sich gegenüber der Passage, wo es um die Abwertung durch die Schwester geht, zunehmend. War zuvor noch davon die Rede, dass es ihr bei der Abwertung durch die Schwester ,scheiße, buchstäblich nicht gut, buchstäblich nicht gut“ (IAng 384) ging, so führt sie hier an: „das hat weh getan, das war das einzige, das hat weh getan“ (IAng 842). Die abwertenden Erfahrungen in der Schule führen in der Folge dazu, dass es Angelika zunehmend schlechter geht: „Weil die Nerven - die haben = die haben sp- die haben, die haben verrückt gespielt meine Nerven, die haben verrückt gespielt nach dem Thema“ (IAng 882-883). In der Folge dieser Erfahrungen und der Auswirkungen auf ihr Befinden wechselt sie die Schule.

Abwertung und Mobbing durch Gleichaltrige im schulischen Kontext werden u.a. auch bei Alina dokumentiert. Wie Angelika berichtet sie darüber, in der Schule ausgelacht und ,verarscht“ (IAl 400) worden zu sein und sich dadurch „scheiße“ gefühlt zu haben. Anna erzählt, dass einige Mitschüler in der Sonderschule etwas gegen sie gehabt hätten (vgl. IA 173), was sie „vom Verhalten her" (IA 204) gemerkt habe. Sie führt aus, von den Mitschülern nur „seckiert und genervt“ (IA 219) worden zu sein. Wie in der ausführlichen 
Fallrekonstruktion „Anna“ dargestellt, gehen diese Erfahrungen einher mit von Anna als unzureichend wahrgenommener Unterstützung durch ihre Lehrerin.

Erfahrung von mangelndem Interesse, Akzeptanz

und Anerkennung der eigenen Person in sozialen Beziehungen

Neben der Abwertung und dem Mobbing der Mitschüler erfährt Anna über die Lehrerin mangelndes Interesse an ihrer Person, was sie anschaulich damit beschreibt, für diese „wie Luft“ (IA 314) gewesen zu sein. Die Lehrerin habe sich für sie ,halt nicht viel interessiert halt" (IA 312), berichtet Anna und führt aus, sie habe „sich nicht mit [ihr] abgegeben“ (IA 314). Welch zentrale Bedeutung Anna dem „Interesse“ an ihrer Person über das WahrgenommenWerden beimisst, wird durch den Vergleich zwischen der von ihr als „schlecht" eingestuften Lehrerin in der Volksschule und der ,super" Lehrerin in der Sonderschule deutlich. Zu dieser hält Anna fest: ,ich habe mir gedacht, das ist meine perfekte Lehrerin, die hilft mir" (IA 324).

Deutliches Desinteresse an ihrer Person erlebt auch Marlene: Ihre Mutter nimmt die deutlichen Symptome einer sich entwickelnden Magersucht (vgl. IMarl 119-123) nicht wahr. Marlene merkt an, dass die Mutter dazu ,eigentlich fast gar nichts [sage], [ ] nur, dass ich essen soll und das war's" (IMarl 145). Hier schwingt Enttäuschung darüber mit, dass die Mutter deutliche Signale nicht wahrnimmt und sich nicht wirklich mit Marlene auseinanderzusetzen scheint. Marlenes Magersucht könnte als Versuch interpretiert werden, mit ihren Bedürfnissen wahrgenommen zu werden und Beziehung zur Mutter herzustellen, was aber aus Marlenes Sicht nicht funktionieren dürfte.

\section{Erfahrungen von Ausgrenzung und Ausschluss}

In den Fallrekonstruktionen und über die soziogenetische Analyse werden auch Prozesse „sozialer Ausschließung“ in ihrer „Komplexität“ (vgl. Rathgeb 2012: 13) deutlich. Wie über Angelikas Ausführungen im Zusammenhang mit Abwertung und Stigmatisierung in der Schule beschrieben wurde, wechselt sie danach die Schule. Indirekt führen die Erfahrungen von Stigmatisierung und Abwertung zum Ausschluss, indem sie bei Angelika das Gefühl hervorrufen, in diesem Kontext nicht akzeptiert und erwünscht zu sein. Angelika wechselt nur vordergründig ,freiwillig“ die Schule, letztlich sind es die erlebten negativen Erfahrungen, die dazu führen, dass Angelika nicht mehr in diesem Kontext verbleiben will.

Bei Anna führen fehlende Zuwendung, Anerkennung und Förderung zur von ihr sehr schmerzhaft erlebten und explizit beschriebenen Erfahrung der Ausgrenzung: ,und dann haben sie mich eben von dort dann weggetan, ich bin mir vorgekommen wie der letzte Dreck“ (IA 169). Der Prozess beein- 
trächtigt Annas Selbstbild massiv: Sie führt aus, dass sie selbst dachte, „wahrscheinlich eine komplette Versagerin“ (IA 184) zu sein. Das Gefühl, nicht gewollt zu sein, geht damit in Annas Beschreibung einher: ,sie wollte mich halt [...] dort einfach nicht mehr haben" (IA 185). Im Kontext mit der Erfahrung, von der Klassenlehrerin ,wie Luft“ behandelt worden zu sein, verdeutlicht sich die Bedeutung des sozialen Umfeldes auf die Initiierung soziogenetischer Prozesse durch negative Erfahrungen. Anna erlebt in diesem Zusammenhang zwar, dass die Lehrerin die Mitschülerinnen und Mitschüler ermahnt. Das grundsätzlich gegebene Gefühl des Desinteresses der Lehrerin und mangelnder Anerkennung führen allerdings dazu, dass Anna die Situation nicht zufriedenstellend bewältigten kann und zukünftige Erfahrungen dadurch beeinflusst werden. Das Gefühl der Abwertung und des GeringGeschätzt-Werdens im Kontext der Regelschule wirkt sich insofern nachhaltig auf Annas Orientierung aus, dass sie nun für sich den Kontext der Sonderschule, der ihr von ebendieser Lehrerin zuvor zugewiesen wurde, als zukünftig geeigneteren Ort für sie beschreibt. Im Zusammenhang mit dem zuvor beschriebenen erlebten negativen Gefühl (,wie der letzte Dreck“) durch den erzwungenen Schulwechsel dokumentiert sich die Intensität der wahrgenommenen abwertenden Prozesse sehr deutlich. Anna bevorzugt nun die Sonderschule und hebt die unterstützenden Rahmenbedingungen hervor, die es ihr auch möglich machen, über schulische Leistung Anerkennung zu bekommen.

Einen vergleichbaren Ausschluss aus einem Regelzusammenhang erlebt und beschreibt Melanie wiederholt. Zunächst wird sie wegen der eigenen „späten Entwicklung“ (IMe 259) vom Regel- in den Sonderkindergarten verwiesen: „nein, wir brauchen so ein Mädel nicht, wir schicken sie in den [Sonderkindergarten]“ (IMe 264). Melanie reflektiert den „,normalen“ Kindergarten als Ort, an dem sie nicht gewünscht war. Dies wiederholt sich im schulischen Bereich, über den Melanie erzählt, dass sie zunächst in einer „normalen Klass'“ (IMe 349) war. Dann habe man gemerkt, dass „geht bei ihr nimmer, die müssen wir sofort hinüber in die Integrationsklass" " (IMe 351). Melanie führt aus, dass sie dort einen Sprachfehler hatte und in einen Sprachkurs gehen musste, bewertet diese Zeit in der Integrationsklasse aber zusammenfassend mit ,ja, aber es war schön“ (IMe 353). Sie erzählt auch, dass sie in der Sonderschule zur beliebtesten Schülerin des Jahres gewählt wurde: „Melanie, Punkt, Melanie ist jetzt Schüler des Jahres, die beliebteste Schülerin“" (IMe 363-364). Der Wechsel in den Sonderbereich ist in Melanies Argumentation die sofortige und zwingende Konsequenz, wenn im Regelzusammenhang von Kindergarten oder Schule festgestellt wird, dass ,es nimmer geht". Die festgestellten Schwierigkeiten werden aufgrund dieser Erfahrungen von Melanie als individuell begründete Probleme gesehen. Diese Zuschreibungs- bzw. Argumentationskette führt zu einem beeinträchtigten Selbstbild. In Melanies Beschreibung über die „Wahl zur beliebtesten Schü- 
lerin" in der Sonderschulzeit wird implizit auch deutlich, dass sie in der Regelschule keine solche Anerkennung bekam. Den Sonderschulkontext beschreibt Melanie als passend, denn sie hätte eine ,extra Lehrerin gebraucht, für mich ganz alleine“ (IMe 441), was dort der Fall gewesen sei. Die Rahmenbedingungen der Sonderschule, die u.a. über die zeitlichen Ressourcen der Lehrerin, sich Melanie zuzuwenden, positiv erlebt werden, begründen dabei Melanies Sichtweise.

Aus diesen Erfahrungen geht hervor, dass mangelnde Anerkennung im und Ausschluss aus dem Regelkontext dazu führen können, dass eigene Perspektiven eher im Sonderbereich verortet werden, in dem man erwünscht ist, wahrgenommen und anerkannt wird. Solche, vor allem wiederholte Erfahrungen beeinflussen auch die aktuelle Orientierung, indem Herausforderungen und unbekannten Kontexten mit Selbstzweifel, Unsicherheit und Vorbehalten begegnet wird. Bei Angelika und Melanie wird auch deutlich, dass solche Erfahrungen auch dazu führen können, dass die gesellschaftliche Teilhabe beeinträchtigt wird, indem das eigene Bewegen im öffentlichen Raum dadurch vorsichtiger und eingeschränkter wird. So scheint Angelika sich nur in Situationen sicher zu fühlen, wo es jemand bemerken und ihr helfen würde, wenn sie ,verarscht“ (IAng 829) werden könnte. Sie beschreibt in einer weiteren Passage (IAng 910-925), dass es solche Situationen jetzt eigentlich nicht mehr geben würde, und begründet dies damit, dass im Bus der Busfahrer und in der Stadt die Mutter sehen würden, wenn sie jemand schlecht behandeln würde. In der sprachlichen Eindringlichkeit wird die Bedeutung des geschützten Rahmens deutlich: „,as sieht der Busfahrer, das sieht der Busfahrer, wenn einer mich verarscht, das sieht der Busfahrer" (IAng 911) und "das sieht meine Mutter, in der Stadt haben sie das noch nie gemacht, in der Stadt, weil das sieht meine Mutter" (IAng 917). Sie wisse jetzt auch, sich zu wehren, nämlich indem sie eine Freundin anrufen würde, die ihr zur Hilfe kommen würde (IAng 926). Deutlich wird, dass Angelika sich in ihrer Bewegung im sozialen Umfeld offenbar daran orientiert, dass immer jemand in der Nähe ist, der sie beschützen könnte. Melanie wiederum vermeidet es, nach $20 \mathrm{Uhr}$ alleine auswärts unterwegs zu sein, weil sie Angst hat, dass ,so fremde Leute [...] mich anpöbeln oder was" (IMe 514), dass sie also neuerliche abwertende Erfahrungen im öffentlichen Kontext machen könnte. Über erfahrene Prozesse der Ausgrenzung werden so beide in ihrem Handlungsspielraum und in ihrer gesellschaftlichen Teilhabe eingeschränkt.

\section{Erfahrung der Anzweiflung der eigenen Glaubwürdigkeit}

Eine andere Facette von Stigmatisierung erlebt Martina, deren Glaubwürdigkeit im Rahmen eines Krankenhausaufenthaltes angezweifelt wird. Im Zuge eines Krampfanfalles habe sie auf die Frage des medizinischen Personals 
nicht reagieren können. Man habe ihr ,[...] das nachher hinterher nicht geglaubt" (IM 224).

$\mathrm{Zu}$ Melanies zuvor beschriebenen abwertenden Erfahrungen und Ausgrenzung aus Kindergarten und Schule kommt Behandlung ,wie der letzen Dreck“ (IMe 325) im Rahmen eines Krankenhausaufenthaltes. Sie sei von den Ärzten verdächtigt worden, mit einem medizinischen Gerät „herumgespielt" (IMe 329) zu haben. Melanie führt weiter aus, dass sie Schmerzen hatte, dass sich aber darum niemand gekümmert habe, man habe im Gegenteil „nur zugeschaut und sich eines herunter gelacht“ (IMe 331). In ihrer Erinnerung an einen Krankenhausaufenthalt ist ihre Beeinträchtigung also damit verbunden, dass sie nicht gut behandelt wird, dass man ihr nicht hilft und sie sogar auslacht. Melanies bereits zuvor über die eigene Ausgrenzung aus einem Regelzusammenhang entwickelte negative Sichtweise von Behinderung wird durch diese Erfahrung reaktiviert. In der aktuellen Passage steigert sich Melanies Sichtweise von Behinderung als etwas, das in einem Regel-Kontext unerwünscht ist, zum Grund für Verdächtigung, Missachtung und Ausgelacht-Werden.

Die Anzweiflung ihrer Glaubwürdigkeit und damit einhergehende soziale Prozesse im Kontext mit sexuellen Übergriffen erlebt Anna mehrmals. Einerseits über die Reaktion ihrer Eltern (IA 514-515), die ihren Bericht darüber in Frage stellen und Unterstützung vorenthalten. Andererseits über den Umgang mit ähnlichen Erfahrungen im beruflichen Kontext. Auf Annas Bericht reagiert das berufliche Umfeld zunächst „perplex“ (IA 449). Daneben versucht der Täter laut Anna, sie ,zum Narren [zu] machen, also dass ich komplett verrückt wäre" (IA 481-483). Erst als wahrgenommen wird, dass Anna „nervlich auch fertig“ (IA 450) ist, wird reagiert: Anna selbst wird zum Psychologen geschickt. Der Täter allerdings bleibt weitgehend sanktionslos. Anna selbst thematisiert aus diesen Erfahrungen die ihr dadurch zugeschriebene mangelnde Glaubwürdigkeit, indem sie formuliert: ,[...] man hat auch zu wenig Beweise dafür [...]“" (IA 439).

\section{Defizitorientierte Zuschreibungen durch Personen aus dem sozialen Umfeld}

Wiederholt werden von Frauen mit Lernschwierigkeiten auch defizitorientierte Zuschreibungen von Personen aus dem sozialen Umfeld thematisiert. Insbesondere die Zuschreibung des Prädikates ,zu langsam“ - vor allem in institutionellen Zusammenhängen - wird häufig erlebt. Marlene etwa beschreibt im Kontext mit ihrem Praktikum in einer Wäscherei selbst, dass sie zu langsam war und führt aus:

,ich kann nicht schneller, ich kann nicht, das geht nicht bei mir, ich kann nicht so schnell, das geht nicht. Dann hat sie gesagt, wie lang brauchst denn, nachher hat sie mich gefragt, wie lang brauchst Du..."(IMarl 671-673). 
Der in Form einer Fokussierungsmetapher erbrachte Verweis auf die eigenen Defizite über die dargestellte Eigen- und Fremdsicht dokumentiert mögliche soziogenetische Prozesse, die damit einhergehen. Er führt letztendlich dazu, dass diese Sichtweise übernommen und das eigene Selbstbild dadurch nachhaltig beeinflusst und geprägt wird. In Marlenes Beschreibung deuten sich diese Prozesse an: Sie beschreibt weiter, dass ihr dieser Verweis auf die eigenen Defizite „so peinlich“ (IMarl 674) war, und erläutert, was genau dabei schwierig war und dass sie nochmals gefragt wurde und erklärt habe, warum sie so lange brauche. Sie führt weiter aus, dass ihr das dann „zu viel, einfach“ (IMarl 677) war und sie sich ,nachher [...] halt blöd angestellt“ (IMarl 677) habe. Sie fokussiert zunehmend auf eigene Defizite, was sich auch in der aktuellen Orientierung widerspiegelt, indem sie sich vor dem Hintergrund dieser Erfahrungen auch aktuell zuschreibt: ,ich kann nicht schneller, das geht bei mir nicht" (IMarl 671). Die defizitorientierte Zuschreibung scheint also bei Marlene wie eine ,self-fulfilling prophecy“ (Merton 1984; Watzlawick 1988) wirksam zu werden. Marlene nimmt sich selbst letztlich so wahr, wie sie vom Umfeld eingestuft wird. Zu diesen Erfahrungen im institutionellen Kontext kommen ähnliche im familiären Bereich: Marlene erzählt, dass die Mutter zu ersten Schwierigkeiten in einem Praktikum gesagt habe „es schaut nicht gut aus“ (IMarl 653) und die Schuld dafür Marlene zuschrieb, indem sie zu dieser sagte ,wenn dich so komisch anstellst, dann ist nicht gut“" (IMarl 655). Marlene erlebt also auch bei auftretenden Schwierigkeiten im Praktikum negative Zuschreibungen verbunden mit impliziten Schuldzuweisungen durch ihre Mutter.

Auch Tamara beschreibt sich in ihrer Erinnerung an die Regelschule als „einfach zu langsam“ (IT 155). Sie reflektiert allerdings auch Umfeldbedingungen, indem sie demgegenüber die Rahmenbedingungen als nicht passend einstuft - „einfach, weil es ein bisschen zu schnell war für mich, allgemein“ (IT 128-129). Sie geht auf die Rahmenbedingungen und die Auswirkungen auf sich selbst in derselben Passage noch zweimal ein und hält etwa fest, dass sie „einfach nicht mitgekommen“ (IT 141) sei, beim Schreiben und „bei Ansage“ (IT 141). Tamara bringt auch einen geschlechtsspezifischen Aspekt ein, indem sie beschreibt, dass sie das einzige Mädchen in der Schule und „anscheinend einfach zu langsam“ (IT 155) war. Bei Tamara wird aktuell in der Rückschau auf diese Erfahrungen ein Orientierungsrahmen sichtbar, in dem Behinderung in Wechselwirkung mit den Umfeldbedingungen gesehen wird, die sie als nicht stimmig beschreibt. Zum Zeitpunkt der Erfahrungen, also in der Schulzeit, führt die Zuschreibung des Prädikates „zu langsam“ aber zum Wechsel in die Sonderschule. Damit einher geht - ähnlich wie bei Melanie und Anna - auch aktuell noch eine positive Bewertung des Sonderbereiches, den sie als angenehm bezeichnet, weil sie dort „mitgekommen“ (IT 179, 181) sei. Diese erlebten Erfahrungen Tamaras werden später durch 
Erfahrungen von Akzeptanz und Anerkennung ${ }^{106}$ in anderen Bereichen weitgehend ausgeglichen. Bis dahin allerdings prägen sie Tamaras Selbstbild, indem sie ihren Blick auf die eigenen Defizite gerichtet lassen. Durch die später wirksam werdenden positiven Erfahrungen verändert sich auch Tamaras Blick auf die schulischen Erfahrungen. Sie beginnt, den Zusammenhang $\mathrm{zu}$ hemmenden oder förderlichen Umfeldbedingungen herzustellen und kritisch zu reflektieren.

Defizitorientierte Zuschreibungen aus dem sozialen Umfeld erlebt auch Sonja, deren Wunsch, Floristin zu werden, die Mutter mit den Worten begegnet, dass sie „das nicht schaffe“ (ISo 304). Sie führt weiter aus: „das ist dann auch nicht gegangen" (ISo 312). Sie habe kurz in einem Blumengeschäft gearbeitet, es sei schön ,mit Blumen zu arbeiten, aber es ging nicht“ (ISo 51). Aus der sprachlichen Formulierung geht hervor, dass Sonja offenbar die zuvor artikulierte defizitorientierte Zuschreibung der Mutter für sich annahm und bei auftretenden Problemen an der Arbeitsstelle dementsprechend agierte bzw. keine Anstrengungen unternahm, eventuell doch weiter dort tätig sein zu können. Die negativen Zuschreibungen führen dazu, dass gar nicht erst die Idee entwickelt wird, dass eigene Anstrengung und Aktivität etwas an einer Situation ändern könnte.

In den angeführten Beispielen dokumentiert sich, dass defizitorientierte Zuschreibungen der eigenen Person durch das soziale Umfeld internalisiert werden und damit das Selbstbild nachhaltig beeinträchtigen. In der Folge wird Herausforderungen auf der Basis dieser Erfahrungen zögerlich begegnet, geschützte Kontexte werden präferiert. Bei Tamara vermögen spätere Erfahrungen des Anerkannt-Werdens mit den eigenen Kompetenzen einen Wandel in der Orientierung einzuleiten. Dies wird im Rahmen der weiteren Darstellung förderlich oder erweiternd auf Handlungsspielräume und Entwicklungsperspektiven wirkender Faktoren detailliert beschrieben.

\section{Erschweren und Verhindern von Partizipation durch das soziale Umfeld}

Wie im Falle von Angelika sichtbar, wird Partizipation mitunter nicht nur durch ungünstige Rahmenbedingungen, sondern auch durch Handlungen, Aussagen und das Vorenthalten von Unterstützung erschwert bzw. auch verhindert. Besonders drastisch dokumentiert sich dies an Angelikas Ausführungen über einen Discobesuch, wobei sie die ihr gegenüber geäußerten Argumente der Eltern thematisiert. Sie sei einmal „mit der Mama und Papa“ (IAng 604) in der Disco gewesen. Es hätte ihr schon gefallen, so Angelika, ,aber der Mama nicht, die hat so Kopfweh gehabt danach" (IAng 608). Angelika sagt „es war schon schön“ (IAng 612), sie war einmal ,,abgelenkt vom Stress“ (IAng 614). Sie würde gerne wieder hingehen, sagt Angelika, am

106 Siehe dazu Unterkapitel „Akzeptanz, Anerkennung und Interesse als Basis für die Entwicklung von Selbstbewusstsein und für persönliche Weiterentwicklung“ im laufenden Kapitel. 
liebsten mit einer Freundin. Sie müsse aber die Eltern fragen, ob sie das dürfe (vgl. IAng 639). Auf die Frage, was der Vater dazu sagen würde, sagt Angelika „nur mit Begleitschutz“ (IAng 649). Und führt aus: „Ja, da passiert so viel, da passiert ja so viel in einer Disco, ja“ (IAng 651). Auf Nachfrage, was da passiere, sagt Angelika ,da ist schon eine schwanger heimgekommen von der Disco, ja“ (IAng 653). Das würden der Vater und die Mutter befürchten, bestätigt Angelika und äußert Verständnis für deren Ängste, indem sie sagt: „haben irgendwie beide recht“ (IAng 659), denn das „könnte passieren“ (IAng 661). Gefragt, wie es sein könne, dass man schwanger von der Disco heimkommt, sagt Angelika ,wenn man Drogen kriegt zum Beispiel“ (IAng 664), „das gibt's, ja das gibt's“ (IAng 668) und erwähnt, dass sie das schon in der Zeitung gelesen hätte - „hat eine 16jährige Drogen genommen, boah Drogen, witzig, weil Drogen find ich überhaupt nicht witzig, Drogen, Drogen ist Gift, also ich finde, Drogen ist Gift" (IAng 670-671). Am Beispiel eines Discobesuches mit den Eltern wird sichtbar, dass Angelika entsprechende Aktivitäten doch gefallen würden, sie diese zum Teil aber auch deshalb nicht ausführt, weil dies von den Eltern nicht gut bewertet wird und diese Argumente ins Treffen führen, die Angelikas Wunsch abschwächen sollen. Angelika würde also gerne partizipieren, vermeidet Aktivitäten aber auch, um den Vorstellungen der Eltern zu entsprechen. Sie führt scheinbar bei ihr vorhandene gute Gründe an, warum sie die eigentlich gewünschte Aktivität doch nicht interessiere. Implizit wird aber deutlich, dass sie damit den Vorstellungen der Eltern entspricht. Sie äußert deren Vorbehalte im Sinne eigener Argumente, indem sie etwa die Botschaft des Vaters artikuliert, man könne ja „schwanger heimkommen von der Disco, ja“ (IAng 653). Sie bringt sogar noch Drogen ins Spiel und findet dabei ein Argument, die Interviewerin, aber offenbar auch sich selbst davon zu überzeugen, dass ein Discobesuch nicht empfehlenswert ist. Sie gibt damit implizit den Ausführungen des Vaters und der Mutter recht, aber drückt dies auch explizit aus, indem sie sagt: „haben irgendwie beide recht" (IAng 659). Explizit äußert Angelika, dass ihr Disco schon gefallen würde, bei ihrem ersten Besuch (mit den Eltern) hat sie es als schön und stressfrei erlebt und sagt, sie würde gerne mit einer Freundin hingehen. Angelika verbleibt also aus zweierlei Gründen eher passiv hinsichtlich der eigenen Partizipation und rechtfertigt sich dafür: Einerseits will sie den Vorstellungen der Eltern entsprechen, die das nicht für sie vorsehen und „Schreckensszenarien“ aufzeichnen, was alles passieren könne. Andererseits auch aus eigenen Ängsten und Unsicherheiten, die aller Wahrscheinlichkeit nach in diesen Prozessen selbst entwickelt werden. 
Vorenthalten von bedarfsgerechter Unterstützung im Alltag und in schwierigen Situationen

Eine weitere negativ wirksame soziogenetische Facette stellt das Vorenthalten von Unterstützung in vielfältigen Kontexten dar. Im Zusammenhang mit körperlichen und sexuellen Gewalthandlungen ist damit das Nicht-zurVerfügung-Stellen eines geeigneten, präventiv wirkenden Instrumentariums oder zumindest Unterstützung bei der nachträglichen Bearbeitung der aktuellen Situation angesprochen. Ganz deutlich werden diese Prozesse bei Anna dokumentiert, die im beruflichen und familiären Bereich keine adäquate Unterstützung bekommt. In der Folge entwickelt sie eigene Strategien, wird aber in ihrem Handlungsspielraum eingeschränkt. Anna wird auch insofern adäquate Unterstützung vorenthalten, indem ihrem Wunsch, die jeweiligen Täter zur Verantwortung zu ziehen, nicht entsprochen wird. Auf das an ihre Mutter gerichtete Ersuchen, den Täter anzuzeigen, reagiert diese mit Ablehnung und Zweifel an Annas Glaubwürdigkeit: „Nein, ich habe gesagt, bitte Mama eine Anzeige und sie, nein, nein, nein. Die wollten mir das zuerst nicht glauben." (IA 514-515). Auch im beruflichen Kontext erlebt Anna im Zusammenhang mit einem erfolgten sexuellen Übergriff Ähnliches. Weiter zurückliegende Erfahrungen Annas im Regelschulkontext machen deutlich, dass ihr bereits dort keine bedarfsgerechte Unterstützung zukam. Dies führt zum erzwungenen Ausschluss Annas aus dem Regelschulkontext und zu einer Orientierung an einem geschützten Sonder-Bereich durch Anna selbst. Im Zusammenhang mit Annas grundsätzlich vorliegender starker Orientierung an „Normalität" wird deutlich, wie negativ die von ihr erlebten Erfahrungen in Zusammenhang mit vorenthaltener Unterstützung wirksam werden: Anna wünscht sich einen normalisierten Rahmen, ihre Erfahrungen darin führen jedoch dazu, dass sie letztendlich sehr vorsichtig agiert und sich auch zurückzieht. Am Beispiel Annas wird deutlich, dass die Entwicklung der Präferenz eines Sonderkontextes maßgeblich durch eingeschränkte gesellschaftliche Strukturen und Angebote beeinflusst sein kann. Diese bestehen etwa in Rahmenbedingungen, die angemessene Unterstützung im Regelkontext nicht bereitstellen.

Auch Angelikas bereits beschriebene Erfahrungen im Zusammenhang mit der Abwertung durch die Schwester sind durch vorenthaltene Unterstützung geprägt. Die Eltern ermahnen nicht nur die Schwester, sondern auch Angelika selbst. Angelika erhält auch keine Unterstützung in ihrem Wunsch, soziale Kontakte zu Peers zu knüpfen und zu pflegen. In diesem Zusammenhang werden ihre Wünsche von Personen aus dem sozialen Umfeld relativiert bzw. erfolgen Hinweise auf Bearbeitungsmöglichkeiten, die nicht zielführend und nicht praktikable sind ${ }^{107}$.

107 Dieses Beispiel wurde bereits im Kapitel „Lebenszusammenhänge als soziogenetische Faktoren auf die Entwicklung von Orientierungen“, „Einbindung in Beschäftigung/Arbeit 


\section{Angedrohte oder verhängte Sanktionen bei nicht erwünschtem Verhalten}

Neben Verhinderungsstrategien wie dem Erschweren und Verhindern von Partizipation durch das soziale Umfeld wirkt also das Vorenthalten von Unterstützung im Zusammenhang mit dem Wunsch, eigene Bedürfnisse umzusetzen, stark einschränkend. Einen darüber hinaus extrem wirksamen Mechanismus stellen angedrohte oder verhängte Sanktionen im Zusammenhang mit unerwünschtem Verhalten dar, also Verhalten, das nicht Erwartungen, Regeln oder Vorschriften von Personen aus dem sozialen Umfeld entspricht. Dabei wird dies umso wirksamer, je abhängiger die betroffene Person emotional und in ihrer Lebensführung vom sozialen Umfeld ist.

Bei Angelika reicht die bloße Vorstellung von erwartbaren Sanktionen bei nicht den Vorgaben der Eltern entsprechendem Verhalten, was sie am Beispiel des Umgangs mit ihrer Schwester wahrnimmt. Wenn die kleinere Schwester schlimm und frech sei, so Angelika, ,kriegt sie ordentlich einmal eines auf den Arsch“ (IAng 321-322). Sie selber sei „nicht so frech“ (IAng 327), sagt Angelika und führt aus: „Ich war immer die Bravste, auch als Baby, ich war nie frech“ (IAng 331). Und führt weiter aus: ,ich hab' mir das nicht einmal, ich hab' mir das ja nicht einmal erlaubt, frech zu sein, nein, das nicht einmal erlaubt, frech zu sein, ja“ (IAng 333-334). Die Schwester sei einmal frech gewesen, was sie in diesem Zusammenhang auch verstanden hätte. Der Vater habe sie daraufhin ins Zimmer gesperrt und „fest herumgeschrie'n mit ihr“ (IAng 348). Sie rechne mit „ehm Hausarrest und einmal ein Monat Fernsehverbot“" (IAng 354), so Angelikas Vermutung zu möglichen Sanktionen eigenen Fehlverhaltens. Angelika äußert allerdings explizit, sich der Gefahr solcher vermuteten Sanktionen durch eigenes Wohlverhalten erst gar nicht auszusetzen: „darauf setz' ich's nicht an, ganz ehrlich, darauf setz ich's nicht an, auf so was, so was kann ich mir nicht erlauben, ganz ehrlich, nein, ehm“ (IAng 359-361). Die sprachliche Dichte verweist auf die Bedeutung, die Angelika ihrem eigenen Wohlverhalten zuschreibt. Angelika vermeidet im familiären Umfeld Verhalten, das negative Konsequenzen bzw. Sanktionen durch die Eltern nach sich ziehen könnte. Sie sieht am Beispiel ihrer Schwester, welche Konsequenzen drohen könnten, und möchte alles vermeiden, wodurch ihr dies selbst passieren könnte. Schon die Vorstellung vermuteter Sanktionen macht Angelika also Angst und führt dazu, dass sie sich „brav“ und „,nicht frech“ verhält.

Anders als Angelika hat Melanie bereits real Sanktionen der Eltern erlebt und agiert deshalb sehr vorsichtig. Melanie unterdrückt eigene Vorstellungen und Bedürfnisse, um neuerlichen Sanktionen zu entgehen. Im Zusammenhang mit der von ihr angestrebten Erneuerung und vom Vater massiv abgelehnten Beziehung zu ihrem Ex-Freund (vgl. IMe 105-149) erzählt Melanie,

ermöglicht soziale Kontakte und Freundschaften und erweckt den Wunsch nach deren Ausbau bzw. Intensivierung " detailliert beschrieben und analysiert. 
dass die Mutter dem Vater ,wieder recht“" gegeben habe. Diese lasse sie nicht einmal ausreden und habe stattdessen Strafen verhängt, wie Hausarrest im Zimmer oder das Handy-Abgeben-Müssen. Im Verhältnis von Melanie zu ihren Eltern erscheint diese als den Vorstellungen der Eltern ausgeliefert, wobei auch Sanktionen gesetzt werden, wenn Melanie nicht entspricht. Die Eltern erscheinen aus Melanies Perspektive übermächtig und scheinen zu bestimmen, welche Entscheidungen sie trifft. Die Mutter erscheint dabei als Erfüllungsgehilfin des Vaters, die diesem recht gibt und die Sanktionen gegenüber Melanie verhängt. Melanie geht daraufhin noch einmal auf den bereits geäußerten Wunsch, als Konsequenz ausziehen zu wollen, ein. Die Reaktionen der Eltern auf diesen Wunsch beschreibt sie sehr negativ. Indem der Vater u.a. das Einstellen der Beziehung zu Melanie ankündigt und die Mutter angibt, Melanie nicht aufhalten zu wollen, stehen für Melanie zwei Drohungen im Raum, durch die sie unter Druck gesetzt wird. Beugt sie sich dem Wunsch, ist die Beziehung zu ihrem Ex-Freund gefährdet. Auszuziehen würde zum Liebesentzug durch die Eltern führen. Dieser Zwiespalt ist allerdings ein kaum realer, da Melanie, um ausziehen zu können, in ihrer Situation auch der Unterstützung durch die Eltern bedürfen würde. In Melanies Beispiel paaren sich also das Vorenthalten von bedarfsgerechter Unterstützung und die Androhung und Verhängung von Konsequenzen zu einem Rahmen, der ihren Handlungsspielraum und ihr Selbstbild massiv einschränken. In der Folge kann Melanie keine Alternative zum Verbleib in der vorgegebenen, nicht den eigenen Bedürfnissen entsprechenden, aber vertrauten Situation erkennen. Die erlebten Erfahrungen wirken sich dahingehend aus, dass Melanie neuerliche ähnliche Erfahrungen zu vermeiden versucht und sich auch in Alltagshandlungen real stark an den Vorstellungen der Eltern orientiert, auch wenn sie vordergründig dagegen zu opponieren scheint.

\section{Erfahrung unangemessenen oder ablehnenden Verhaltens und Kommunikationsstiles im sozialen Kontext}

Wie etwa bei Angelika eingangs beschrieben, erlebt sie respektloses Verhalten durch ihre jüngere Schwester. Jessica nimmt unangemessenes Verhalten ihrer früheren "Chefin“ in der Werkstätte für Menschen mit Behinderung deutlich wahr. Sie erzählt, dass sie von dieser schlecht behandelt wurde, diese sei ,überhaupt nicht nett“ (IJ 260) zu ihr gewesen, führt Jessica aus und präzisiert, sie sei von ihr immer ,angepfaucht“ (IJ 158, 263) und „so angeflogen“ (IJ 284) worden. Sie sei dann von dort weggekommen, sie „wollte einfach nicht mehr bleiben, weil es nicht gepasst hat" (IJ 291). Sie habe es dann den Eltern gesagt, die hätten gesagt ,ja, das ist o.k." (IJ 297) und dann sei sie in die andere Werkstätte gekommen. Jessica wird hier, wie zuvor schon am Beispiel von Angelika und Anna beschrieben, Unterstützung aus dem sozialen Umfeld im Zusammenhang mit erlebter schlechter Behandlung durch 
andere Personen, hier konkret der Chefin, vorenthalten. Nicht die Chefin muss ihr Verhalten Jessica gegenüber verändern bzw. mit ihr einen beiden entsprechenden Kommunikationsstil aushandeln, sondern Jessica verlässt die Werkstätte. Dieser Mechanismus, dass es eher zum Ausschluss der betroffenen Person als zu einer Anpassung der Rahmenbedingungen an die individuellen Bedürfnisse kommt, wurde bereits in anderen Zusammenhängen sichtbar. So mussten etwa Anna, Melanie und Tamara von der Regel- in die Sonderschule wechseln, weil sie nicht entsprachen, also z.B. als „zu langsam“ eingestuft wurden. Diese Erfahrung kann dazu beitragen, dass nicht den persönlichen Bedürfnissen entsprechende Rahmenbedingungen aus dem Bewusstsein heraus nicht kritisiert werden, dass dies nichts an den Rahmenbedingungen ändert und sogar zu Ausschluss führen kann.

Neben konkretem Verhalten kann auch der Kommunikationsstil im sozialen Kontext als nicht angemessen erlebt werden. Über einen Vergleich zum Verhalten der Chefin zu dem von Freunden wird deutlich, welche Umgangsformen sie als passend erlebt und sich allgemein in der Interaktion mit ihr wünschen würde: „,ich habe immer Zeit für sie und sie haben Zeit für mich und so und das finde ich einfach nett und höflich. Das finde ich so nett und höflich" (IJ 214-216). Sie beschreibt Beziehungen dann als gelungen, wenn man „nett“, „höflich“ und zugänglich ist. Wie sehr das Vorenthalten sozialen Austausches sich auch auf das eigene Befinden und den Selbstwert auswirkt, verdeutlicht Jessica wieder über einen Vergleich. Ihr aktueller Chef in der Werkstätte wolle nicht, wenn man in sein Büro komme. Sie beschreibt, dass man immer warten müsse, bis er herauskomme. Wenn sie anklopfe und sich für die Störung entschuldige, dann frage er ungehalten „Jessica, was ist jetzt?“ (IJ 243-244). Jessica nimmt dies als Ablehnung wahr und bewertet dieses Verhalten: „Das hasse ich voll, das mag ich überhaupt nicht“ (IJ 240241). Mit einer weiteren Chefin habe sie hingegen sehr gute Erfahrungen gemacht, es sei ,einfach nett gewesen [...]. Mit der hast du alles tun dürfen. Da hast du mit ihr ratschen dürfen, du hast alles dürfen. Was wir vor dem Chef nicht dürfen“" (IJ 229-230). Wenn sie sich entschuldigte, weil sie stören musste, sagte diese Chefin anders als der aktuelle Chef ,komm nur, komm nur" (IJ 237). Jessica beschreibt mit ihren Beispielen Verhalten, das sie daran hindert, mit einer Person in Kontakt zu treten, sehr anschaulich über Situationen, die dies real abbilden: Sie darf eine Schwelle zum Raum dieser Person nicht überschreiten und kann daher mit dieser nicht wirklich in Kontakt treten. Offenheit ihr gegenüber wird von Jessica als angenehm erfahren. Erfahrene Ablehnung beeinträchtigt hingegen Jessicas Selbstwert. 


\section{Erfahrung körperlicher Übergriffe im familiären und institutionellen Kontext}

Neben Abwertung, Stigmatisierung und Ausgrenzung stellen konkret übergriffige Erfahrungen durch Personen aus dem sozialen Umfeld Erfahrungsräume von Frauen mit Lernschwierigkeiten dar.

Marlene erfährt im institutionellen schulischen Kontext übergriffiges Verhalten durch den eigenen Lehrer. Sie erzählt von einer Szene, wo sie über den stolpernden Lehrer lachen musste. Daraufhin habe er sie mit Gewalt aus dem Klassenzimmer gezerrt, sie habe sich gewehrt, habe ihn ,getreten alles Mögliche, gehaut, getreten gebissen, gerissen“ (IMarl 282-283). Der Lehrer sei überhaupt „heftig“ (258) gewesen. Er habe „sogar die Eltern angerufen“ (IMarl 265) und diesen gesagt, dass sie länger bleiben müsse. Marlene erlebt also als Konsequenz des eigenen Verhaltens einen körperlichen Übergriff und die Bewertung ihres Verhaltens als unpassend. Das Verhalten des Lehrers wird im Gespräch mit den Eltern offenbar nicht thematisiert, der Vorfall für Marlene nicht zufriedenstellend bearbeitet. Über weitere Passagen zur Schulzeit wird deutlich, dass Marlene selbst die Mittel, sich gegen etwas zu wehren, auf der körperlichen Ebene sieht. Die konkreten Erfahrungen von Marlene prägen bzw. verstärken also das Muster, dass man sich bei einem Angriff wehren muss, indem man auch auf der körperlichen Ebene agiert. Sie nimmt wahr, dass auch eine Autoritätsperson wie der Lehrer auf der körperlichen Ebene reagiert bzw. kommuniziert. Der Lehrer erscheint in Marlenes Erzählung nicht als Begleiter und Unterstützer, sondern als jemand, der übergriffig wird und gegen den man sich körperlich wehren muss. Indem ihre eigene Reaktion auf das Verhalten des Lehrers als ,auffällig“ bewertet wird, erlebt Marlene, dass gleiches Verhalten gesellschaftlich unterschiedlich bewertet wird.

Marlene erlebt neben diesen schulischen Erfahrungen auch körperlich stark übergriffiges Verhalten im familiären Rahmen. Sie erzählt im Detail über einen brutalen Übergriff des Bruders, wobei sie sich an alle Einzelheiten erinnert: „Er hat mich getreten, der hat mich alles geheißen, er hat mich am Kopf, mit'n Fuß von mein Kopf in Kinn voll“" (IMarl 1165-1167). Auf die Frage, wie es dazu gekommen sei, sagt Marlene: ,ich habe ihn geärgert, ich habe ihm nix getan“" (IMarl 1169-1170). Sie führt aus, dass sie den Übergriff vor einem Arzt verschleiert habe: ,ich hab's dem Arzt nicht sagen können, weil das, ich kann nicht, ich muss einfach irgendwie sagen, ich hab' mich ang'haut bei der Ecken, bei der Kanten, hab' ich immer g'sagt" (IMarl 11721174). Marlene thematisiert ihr eigenes Agieren (,Ärgern“) im Zusammenhang mit dem Übergriff des Bruders, wenn sie auch einschränkt, dass sie eigentlich nichts getan hätte. Hier wiederholt sich in extremer Form ein Mechanismus, den sie mit dem Lehrer erlebt hat. Als sie diesen ausgelacht hat, weil er stolperte, wurde er übergriffig. In der Erfahrung von Marlene folgen auf eigentlich recht harmlose Handlungen ihrerseits wie Auslachen oder 
Ärgern Übergriffe durch männliche Personen aus ihrem sozialen Umfeld. Diese Erfahrungen führen bei Marlene zu einem eher als reagierend und passiv, denn selbst als aktiv und gestaltend erscheinendem Verhalten. Die für sie sichtbare Konsequenz, dass auf harmlose Handlungen Übergriffe als Reaktion erfolgen, führen zu massiver Verunsicherung und Passivität, um ähnliche Abläufe zukünftig zu vermeiden. Nachdem für sie eigenes Verhalten und schwierige Situationen mit Übergriffen, Schuldzuweisungen und vorenthaltener Unterstützung verbunden sind, wird Marlene auch nicht ermutigt, jemanden hinsichtlich diesbezüglicher Vorfälle anzusprechen. Der Übergriff des Bruders wird aus Angst vor neuerlichen negativen Konsequenzen verheimlicht. Aktuell entwickelt Marlene - wie sie selbst beschreibt - Magersucht [,,auf Magersucht geh ich jetzt so, ich mag fast nix essen, nein“ (IMarl 119-123)], was sie aber ebenfalls zu verheimlichen versucht. Zugleich sendet Marlene deutliche Signale an die Mutter, mit denen sie den Wunsch nach Unterstützung und Beziehung äußert. Diese werden von der Mutter nicht wahrgenommen bzw. nicht entsprechend berücksichtigt. Die Entwicklung der Magersucht verbindet Marlene selbst mit ihrem negativen Selbst- und Körperbild, indem sie darauf zu sprechen kommt, dass sie sich für zu klein hält: „,ich komm' mir vor als wie ein Kleiner, weiß ich nicht, ich bin viel zu klein“ (IMarl 131). Aus Marlenes Erfahrungen, die von Übergriffen und vorenthaltener Unterstützung durch Bezugspersonen gekennzeichnet ist, deutet sich an, dass diese Formulierung sich weniger auf die äußerliche Erscheinung, sondern auf Marlenes aktuelles Selbstbild bezieht. Auch die von der Mutter im Kontext mit den Schwierigkeiten im Praktikum geäußerten defizitorientierten Zuschreibungen tragen dazu bei, dass Marlenes Selbstbild massiv beeinträchtigt ist.

\section{Erfahrung sexueller Übergriffe in unterschiedlichen Kontexten und Ausprägungen}

Am Beispiel Annas wird besonders deutlich, wie stark Erfahrungen von Übergriffen soziogenetisch in der Entwicklung und Ausprägung von Orientierungen wirksam werden, wie sich in der vorab dargestellten Fallrekonstruktion dokumentiert. Anna erlebt mehrfach sexuelle Übergriffe in unterschiedlichen Kontexten und Ausprägungen: als Jugendliche im Wohnort durch verbale Übergriffe eines Bekannten, im beruflichen Kontext durch konkrete sexuell orientierte Berührungen durch einen Kollegen und durch einen Vergewaltigungsversuch ihres Freundes. Diese Erfahrungen an sich wirken sich schon massiv auf das Befinden und das Selbstbild aus. Sie habe sich dabei „,als Frau total elend“ (IA 435) gefühlt, führt Anna aus und verdeutlicht dies über eine bereits durch sie selbst und eine andere Frau - im Zusammenhang mit Abwertung - eingebrachte Metapher. Sie habe sich gefühlt ,wie der letzte Dreck, auf gut Deutsch gesagt auch“ (IA 440). Im Zu- 
sammenspiel mit den erlebten oder nicht erlebten Reaktionen aus dem sozialen Umfeld liegen zentrale Momente für soziogenetische Prozesse, die sich in der Entwicklung spezifischer Orientierungen ausdrücken. Anna erlebt im Kontext mit den Übergriffen - wie bereits ausgeführt - keine nachhaltig wirksame Unterstützung aus dem sozialen Umfeld. Die Eltern zweifeln ihre Glaubwürdigkeit an und kommen Annas ausdrücklichem Wunsch, den Täter anzuzeigen, nicht nach. Im beruflichen Kontext wird der Täter nicht sanktioniert, Anna selbst wird zum Psychologen geschickt. Anna wird kein Instrumentarium zur Verfügung gestellt, mit dem sie sich vor zukünftigen Übergriffen schützen kann. Demgegenüber erfährt sie in diesem Kontext sogar das Anzweifeln der eigenen Glaubwürdigkeit. All dies wirkt sich massiv auf Annas Selbstbild aus, was sich etwa darin äußert, dass sie die eigene Glaubwürdigkeit anspricht (vgl. IA 439) und auch überlegt, ob sie selbst schuld an den erfolgten Übergriffen sein könnte. Über Annas Beschreibung, dass sie keine aufreizende Kleidung getragen hätte (vgl. IA 432-433), zeigt sich, dass sie durch die erfahrenen Übergriffe und die Reaktionen aus dem sozialen Umfeld ein gesellschaftlich häufig vermitteltes Bild verinnerlicht, nach dem Frauen Mitschuld an körperlichen Übergriffen zugewiesen wird, indem sie etwa durch aufreizende Kleidung das männliche Gegenüber dazu auffordern würden. Im Zuge der übergriffigen Erfahrungen und der vorenthaltenen bzw. mangelnden Unterstützung entwickelt Anna eigene Handlungsstrategien, die sie sich vor allem über das Fernsehen wie etwa „Karate Filme“ (IA 551) und Krimiserien (IA 552) aneignet. Sind diese vorrangig auf Vermeidung neuerlich drohender Übergriffe etwa durch ,sich so zu verstecken“ (IA 464) ausgerichtet, so lassen sie dabei aber auch Kompetenzen und Ressourcen Annas erkennen, indem sie sich damit selbst in die Lage versetzt, überhaupt an gesellschaftlichen Prozessen teilzuhaben. Allerdings werden Annas Potentiale durch diese Bewältigungsarbeit gebunden. Mögliche Entwicklungsschritte werden dadurch gehemmt.

Auch Verena hat Erfahrungen mit sexuellen Übergriffen gemacht. Sie berichtet, dass ihr jemand beim Gang auf die Toilette nachgestiegen sei und ihr „Zwischen die Füße gegriffen“ (IV 419) habe. Verena hat dies anders als Anna vor den Personen aus ihrem sozialen Umfeld verschwiegen [,,dass was passiert ist, habe ich geschwiegen" (IV 423)], aller Wahrscheinlichkeit nach wurde sie vom Täter mit einem Schweigegebot belegt ${ }^{108}$. Sie berichtet darüber, dann krank geworden zu sein. Aktuell habe sie verschiedene Ängste, z.B. vor Hunden, die dazu führen würden, dass sie sich alleine nicht mehr aus dem Haus traue. Auch bei Verena führen Erfahrungen von sexuellen Über-

108 Bereits im Endbericht zum Forschungsprojekt ,Zur Lebenssituation von Frauen mit geistiger Behinderung" (Sigot 2003: 50) dokumentierte sich über Interviews mit betroffenen Frauen, dass sexuelle Übergriffe häufig u.a. deshalb stattfinden, weil der Täter denkt, das Opfer sei wehrlos. Die Opfer werden auch mit einem Schweigeverbot (,Das darf keiner wissen ") belegt, sie verinnerlichen dieses häufig. 
griffen dazu, dass sie sich am vertrauten Bereich orientiert und unbekannte Kontexte eher vermeidet. Ihr selbst ist dieser Zusammenhang aber nicht bewusst. Sie macht ihre Orientierung am vertrauten Bereich an ihrer Angst vor Hunden fest. Ihre eigene Schilderung weist aber darauf hin, dass sich darüber soziogenetisch Folgen des sexuellen Übergriffes zeigen. In Kombination mit nicht erfolgter Unterstützung durch Personen aus dem sozialen Umfeld, vor denen Verena den Übergriff geheim hielt und den diese von sich aus offenbar nicht erkannten, führen diese negativen Erfahrungen zur Einschränkung von Handlungsspielraum und der Orientierung an einem geschützten Rahmen. Diese Orientierung wird von defizitorientierten Zuschreibungen aus dem sozialen Umfeld verfestigt, die Verena internalisiert, in der Folge ihr Selbstbild schwächen und auch dazu führen, dass sie sich mit den vorgegebenen Bedingungen zufrieden zeigt. Das Bestreben, den vertrauten Rahmen zu erhalten, erweist sich als treibende Kraft in Verenas Leben und führt dazu, dass sie auch keine Änderungswünsche artikuliert. Zudem schwächt Verena vor sich selbst vorhandene latente Wünsche ab, indem sie etwa äußert, selbst keine Kinder haben zu wollen. Dies dürfte einerseits Folge des sexuellen Übergriffes, aber auch der explizit geäußerten, defizitorientierten Zuschreibungen der Mutter sein. Von dieser hört Verena, sie könne keine Kinder bekommen, weil ,ihr Körper zu schwach sei“ (IV 90) und sie dabei „d'raufgehen“ (IV 91) würde. Verena hat diese Zuschreibungen offenbar internalisiert und schwächt im Interview eigene Wünsche im Zusammenhang mit einer möglichen Mutterschaft ab. Sie wolle keine Familie, das sei nichts für sie (vgl. IV 115).

Durch Petras Ausführungen dokumentiert sich, dass sie im Erfahrungsraum Regelschule übergriffige Erfahrungen gemacht hat. Sie sei ,gehaut worden“ (IP 67) und es habe sie „,keiner gemocht“ (IP 68), berichtet Petra. Auch zu Hause hat Petra negative Erfahrungen mit körperlichen Übergriffen gemacht, sie erzählt davon, ,im Garten abgewatscht worden“109 (IP 69) zu sein. Mit 13 Jahren - so erzählt Petra - sei sie ,sexuell missbraucht“ (IP 811) worden. Sie führt zur konkreten Situation an, es sei ihr Cousin gewesen, der betrunken gewesen sei. Sie führt differenziert aus, welche Folgen dies für sie hatte. Sie habe ,nur geweint“" (IP 817), hätte Bauchschmerzen gehabt (vgl. IP 818), sie sei „fertig“( (IP 818) gewesen, „habe nicht mehr lernen können“ (IP 818-819) und „,nur geschwitzt“ (IP 837, 838).

Auch Sonja beschreibt körperliche Symptome, die nach einem Übergriff bei ihr zum Ausdruck kamen, ohne jedoch selbst einen Zusammenhang zu den Übergriffen herzustellen. In der Schule habe sie „Angst gehabt“ (ISo 80), habe sich ,zu Tode geschwitzt“ (ISo 85) und „nur geweint“ (ISo 89). Die Lehrerin und ein anderer Lehrer hätten sie nicht gemocht und hätten bei ihren Arbeiten alles durchgestrichen. Ihr schulisches Verhalten und die vorhande-

109 Abgewatscht $=$ geohrfeigt. 
nen körperlichen Symptome wurden vom Lehrpersonal offenbar nicht mit einem möglichen Übergriff in Verbindung gebracht. Sonja erfährt statt Unterstützung zusätzlich Ablehnung und Abwertung. Auch bei ihr kommt es zum Wechsel in die Sonderschule, wo sie sich dann wohler fühlt. Negative Erfahrungen mit Ablehnung, Stigmatisierung, körperlichen und sexuellen Übergriffen führen auch bei Sonja zur Orientierung an einem geschützten Rahmen wie der Sonderschule. Möglichen aktuellen Veränderungen begegnet sie vor dem Hintergrund dieser Erfahrungen mit Selbstzweifeln. So sind Veränderungen wie das Wohnen außerhalb des stark strukturierten, institutionellen Kontextes für sie nicht vorstellbar. „Ich weiß nicht, ob ich das schaffe“ (ISo 623), zweifelt Sonja und führt diesem Satz unmittelbar an: ,ich habe nur Schiaches ${ }^{10}$ erlebt“" (ISo 623-624). Sie stellt also an dieser Stelle selbst den Zusammenhang von Erfahrungen mit Ausgrenzung, Abwertung, Stigmatisierung und der Entwicklung eines negativen Selbstbildes her. Auch bei Sonja wirken sich diese Erfahrungen verbunden mit defizitorientierten $\mathrm{Zu}-$ schreibungen von Personen aus dem sozialen Umfeld auch in der Weise aus, dass eigene, latent vorhandene Wünsche zum Teil umgedeutet und abgeschwächt werden. So thematisiert sie über eine Vignettenbeschreibung zwar eigene vorhandene Wünsche im Zusammenhang mit Partnerschaft (ISo 834842 VA3), führt aber an anderer Stelle aus, sie „brauche keinen Freund“ (IS 804) und denke nicht an eine Partnerschaft.

\section{Erfahrung von Stress und zu hohen Anforderungen}

Unpassende Rahmenbedingungen im Bereich Arbeit und Wohnen können einen Grund für die Präferenz eines vertrauten, familiären Kontextes darstellen und dazu führen, dass neuerliche Herausforderungen außerhalb dieses Rahmens nicht mehr in Angriff genommen werden. Dies dokumentiert sich etwa über die Erfahrungen von Natalie und Felicitas. Beide beschreiben Erfahrungen im Rahmen von Praktika außerhalb des familiären Kontextes im Zusammenhang mit der Präferenz der aktuellen Situation. Natalie etwa beschreibt, dass sie ein Praktikum abgebrochen hat und hält dazu fest, dass es ihr nun zu Hause besser gefalle, denn ,da ist, kann man machen w- was man will und so, hat man mehr Freiheiten ((lacht))“ (IN 788-799). Felicitas bewertet ebenfalls Erfahrungen im Rahmen eines Praktikums: „es war einfach zu viel für mich, also einfach zu viel für mich und zu lang" (IF 560). Auch ein weiteres Praktikum war - so Felicitas - „nicht so gut für mich, gar nicht, weil so viel mir zu stressig war, zu viel Arbeit, zu viel, also zu viel Geschirr zu waschen, wegzuräumen“ (IF 595-595). Felicitas beschreibt auch, dass sie vermittelt bekam, dass man an der Arbeitsstelle mit ihr nicht zufrieden war: „die Chefin, ja [...], ja die war einfach nicht, die war einfach nicht so glücklich mit mir dort und so“"(IJ 607-608).

110 Schiaches $=$ Schlimmes. 
Eine starke Orientierung an einem vertrauten, familiären Rahmen kann also dadurch begründet sein, dass Erfahrungen im Zusammenhang mit außerhäuslichem Wohnen und Arbeiten als einschränkend, unangenehm oder zu stressig erlebt werden. In diesem Fall wird der vertraute Kontext gegenüber den erlebten Rahmenbedingungen präferiert. Dabei dokumentiert sich an den Erzählungen der Frauen, dass es sich vorwiegend um erste, frühe und/oder wiederholte Erfahrungen dazu handelt, die sehr prägend auf die Entwicklung der Orientierung wirken. Entsprechende Zusammenhänge werden häufig abgebrochen, die Inangriffnahme neuerlicher herausfordernder Situationen wird vor diesem Erfahrungshintergrund vermieden.

Nicht immer führen diese Erfahrungen zu Abbruch der entsprechenden Kontexte. Wahrgenommene hohe Anforderungen und Stress im Arbeitsbereich können auch dazu führen, dass diese mit großer Anstrengungen erfüllt werden, dass dann aber im Freizeitbereich Versorgungsleistungen aus dem familiären Umfeld im hohen Ausmaß beansprucht werden (müssen). Dieser Zusammenhang dokumentiert sich etwa an der Fallrekonstruktion „Martina“, die Stress im Arbeitsbereich dadurch ausgleicht, dass sie sich im privaten Bereich sehr auf die Unterstützung der Mutter verlässt und ihr weitgehend die Organisation ihrer Rahmenbedingungen für Wohnen, Essen und Mobilität überlässt ${ }^{111}$.

\section{Zusammenfassung: hemmend oder einschränkend auf Handlungsspielräume und Entwicklungsperspektiven wirkende Faktoren}

Über die angeführten Beispiele wird deutlich, dass Faktoren, die sich negativ auf Handlungsspielräume und Entwicklungsperspektiven auswirken, in unterschiedlichen Kontexten und Dimensionen erfahren werden: direkt und indirekt über Personen aus dem familiären Umfeld wie Geschwister und Eltern, aber auch über Bezugspersonen aus Institutionen wie Kindergarten und Schule. Ebenso über Personen unterschiedlicher Professionen, mit denen im Anlassfall Kontakte erfolgen, wie etwa die Beispiele im Zusammenhang mit medizinischer Behandlung bzw. Krankenhausaufenthalten dokumentieren. Aber auch über Peers und Gleichaltrige, etwa in Form von schulischem Mobbing, können diese Erfahrungen zustande kommen. Dabei sind indirekte Prozesse nicht weniger negativ wirksam als etwa direkt erlebte Abwertung. So etwa, wenn Personen aus dem sozialen Umfeld, von denen eigentlich Unterstützung erwartet wird, diese vorenthalten und eventuell sogar durch eigene Äußerungen oder Handlungen die erfahrene Abwertung noch verstärken. Durch das Anzweifeln von Glaubwürdigkeit und durch das Vorenthalten von Unterstützung werden die negativen Erfahrungen reaktiviert und intensi-

111 Siehe Fallrekonstruktion „Martina“, u.a. Punkte: „Ermüdung und Stress durch normalisierte Arbeitsbedingungen“ und „Anbindung an die Herkunftsfamilie durch Rückgriff auf deren Versorgungsleistung“. 
viert. Im Kontext sexueller Gewalthandlungen kann sich mangelnde oder vorenthaltene Unterstützung auch darin äußern, dass damit ,die Entwicklung einer posttraumatischen Störung“ (Buttolo/Hagl/Krüsmann 2003: 135) begünstigt wird.

Die Art und Weise, wie das soziale Umfeld im Kontext mit der erfolgten Abwertung handelt, prägt so das eigene Selbstbild und wirkt sich auf die damit einhergehende Entwicklung aus. Angelika etwa erlebt die Eltern im Kontext der von diesen miterlebten Abwertung durch die Schwester als nicht unterstützend. Die Eltern würden das ,volle Wäsch”“ (IAng 399) mitbekommen, würden zwar die Schwester ermahnen, aber auch Angelika selbst, „obwohl ich eigentlich gar nix gemacht hab"“ (IAng 401). Zur Abwertung durch die Schwester kommt die Nicht-Anerkennung dieser Situation durch die Eltern, indem diese nicht nur Unterstützung vorenthalten, sondern auch Angelika selbst maßregeln. Durch die ausbleibende Reaktion der Eltern kommt es in der Situation auch zu keiner Korrektur der erlebten Zuschreibungen durch die Schwester. Damit erfolgt implizit eine Bestätigung, eventuell sogar eine Verstärkung der negativen Zuschreibungen durch die Reaktion aus dem Umfeld. Angelikas Selbstbild wird durch diese Prozesse nachhaltig beeinträchtigt.

Die Wahrnehmung von mangelndem Respekt und Abwertung an sich führt grundsätzlich schon zu sehr negativen Gefühlen bei den davon betroffenen Frauen. Angelika führt aus, dass sie sich durch die Abwertung der jüngeren Schwester sehr schlecht fühle (vgl. IAng 384) und wie sehr sie unter der Abwertung durch die Mitschülerinnen und Mitschüler gelitten habe (vgl. u.a. IAng 882-883). In der vorab angeführten Fallrekonstruktion „Anna“ wird deutlich, dass diese sich durch das Verhalten der Lehrerin und die nachfolgende Rückstufung ,wie der letzte Dreck“ fühlte. Im Zusammenhang mit der Beschreibung von Abwertungs- und Aussonderungserfahrungen wird - wie anhand der Beispiele von Anna und Melanie beschrieben - eine auffällige Metapher dafür sichtbar, die in beiden Fällen unabhängig voneinander verwendet wird: Sich-Fühlen oder Behandelt-Werden ,wie der letzte Dreck“ (IA 169, IMe 325) kann so gesehen als Fokussierungsmetapher betrachtet werden, die Gefühle im Zusammenhang mit konjunktiven Erfahrungen von Abwertung, Ausgrenzung, Diskriminierung und Stigmatisierung auf den Punkt bringt. Es wird auch deutlich, wie negativ sich diese Erfahrungen auf die Ausprägung des Selbstbildes und darüber nachhaltig auf das Wahrnehmen von Handlungsspielräumen und Entwicklungsperspektiven auswirken.

\subsubsection{Förderlich oder erweiternd auf Handlungsspielräume und Entwicklungsperspektiven wirkende Faktoren}

Wurde zuvor dokumentiert, wie soziogenetische Faktoren im Zusammenhang mit der Entwicklung der Orientierung junger Frauen mit Lernschwierigkeiten 
negativ wirksam sind, so wird nunmehr auf solche fokussiert, die förderlich oder erweiternd auf Handlungsspielräume und Entwicklungsperspektiven wirken. Dabei stehen wiederum konjunktive Erfahrungsräume von Frauen mit Lernschwierigkeiten im Fokus. Auch in diesem Kontext sind soziale Kontexte, Interaktionen und Bearbeitungsweisen konkreter Situationen ausschlaggebend für individuell daraus resultierende persönliche Entwicklungsprozesse. Diese Erfahrungen werden zum Teil auch als zentrale Dreh- und Angelpunkte für einen Wandel in der Orientierung dokumentiert. Über die dadurch hervorgehobene Besonderheit einer solchen Umgangsweise wird auch dokumentiert, dass der wahrgenommene positive Zugang nicht als selbstverständlich wahrgenommen wird bzw. dass andere Umgangsformen damit in den eigenen Erfahrungen vorliegen. Dies macht wieder die Mehrdimensionalität einer soziogenetischen Typisierung deutlich, indem zum Ausdruck kommt, dass die beschriebenen Erfahrungen über alle Orientierungen gemacht werden, sich aber über unterschiedliche Bearbeitungsweisen im sozialen Kontext soziogenetisch unterschiedlich auswirken.

Werden die zuvor beschriebenen Erfahrungen sehr negativ erlebt, so stellen jene des Respektiert-, Akzeptiert- und Anerkannt-Werdens verbunden mit einem erlebten Interesse an der eigenen Person über soziale Beziehungen sehr positive Erlebnisse dar. Wie bereits angeführt, werden erste diesbezügliche Erfahrungen im Vergleich zu den bisherigen Rahmenbedingungen als zentrale Momente und als Ausgangspunkte eigener Weiterentwicklung beschrieben. Eva beschreibt etwa im Zusammenhang mit dem Wechsel in eine integrative Wohngemeinschaft eine Szene, die sie „nie vergessen“ wird, und Anna beginnt ihre Beschreibung einer sehr förderlichen Lehrerin: „Also die Lehrerin, die werde ich so nie vergessen. [...] Das ist eine total super, kamote Lehrerin“ (IA 295-296). Diese Formulierungen dokumentieren auch, dass die in diesem Kontext gemachte Erfahrung nicht Alltag ist, sondern in ihrer wahrgenommenen Besonderheit eine zentrale soziogenetische Komponente beinhaltet.

In der folgenden Darstellung wird wieder Bezug auf die vier Fallrekonstruktionen genommen, die zu den vier erhobenen und dargestellten sinngenetischen Typen führten. Ergänzend werden auch hier Passagen aus weiteren Interviews analysiert und dargestellt, die zur Verdeutlichung und Anschaulichkeit dieser Zusammenhänge beitragen sollen.

\section{Akzeptanz, Anerkennung und Interesse als Basis für die Entwicklung von Selbstbewusstsein und für persönliche Weiterentwicklung}

Wie sehr Akzeptanz, Anerkennung und Interesse an der eigenen Person das Selbstbild positiv beeinflussen und sich förderlich und erweiternd auf Handlungsspielräume und Entwicklungsperspektiven auszuwirken vermögen, wird 
in vielfältigen Kontexten an der Fallrekonstruktion „Eva“ deutlich ${ }^{112}$. Diese im sozialen Kontext über wichtige Bezugspersonen und Schlüsselszenen wahrgenommenen Erfahrungen führen bei Eva dazu, dass sie selbst zunehmend auf eigene Stärken und Kompetenzen fokussiert und sich in der Folge zunehmend an Selbstbestimmung und Autonomie orientiert. Auch herausfordernde Erfahrungen können in diesem Kontext eine Basis oder auch einen Stimulus für Weiterentwicklung bilden.

Auch bei Tamara wirken sich erste Erfahrungen gesellschaftlicher Akzeptanz sehr positiv auf ihr eigenes Selbstbild aus. Sie reflektiert ihre Erlebnisse in einem Tanzkurs im Wohnort, wo sie sich zunächst ,,schwer getan“ (IT 609) habe, weil sie nicht gewusst hätte, wie die Leute auf sie reagieren würden. Erste positive Erfahrungen des Akzeptiert-Werdens hätten sie jedoch gestärkt, diese Aktivität in einem nicht geschützten, öffentlichen Rahmen weiter zu praktizieren, weil es ,einfach für mich, für mein Selbstbewusstsein“ (IT 608) sei. Die für sie überraschende Erfahrung, im Tanzkurs nicht wie befürchtet auf Ablehnung zu stoßen [,Und ja. Ich werde positiv angenommen, ja“ (IT 650)], ist Ausgangsbasis für einen beginnenden Wandel im Selbstbild. Tamara reflektiert auf Grundlage ihrer Erfahrung auch ganz konkret das bislang negativ wahrgenommene eigene Äußere und plant weitere Schritte der gesellschaftlichen Teilhabe unter der neuen Selbstsicht „weil ich doch nicht so schiach ${ }^{113}$ bin, wie ich gedacht habe, ja“" (IT 648).

Auch an Tamaras Beispiel wir deutlich, dass Anerkannt-Werden zur Wahrnehmung eigener Kompetenzen, Interessen und Bedürfnisse führt. Anerkennung initiiert und befördert Weiterentwicklung, indem sie stärkend und motivierend wirkt. Zudem eröffnen sich daraus neue Perspektiven, indem das eigene Selbstbild positiv beeinflusst wird und neue Denk- und Handlungsräume sichtbar gemacht werden. Das Gefühl, akzeptiert und anerkannt zu werden, stellt also auch insofern die Basis für eine Weiterentwicklung der eigenen Persönlichkeit dar, als es den eigenen Selbstwert hebt und Selbstbewusstsein fördert. Der daraus entstehende Wunsch, wieder Anerkennung im sozialen Kontext zu erfahren, führt dazu, dass auch die Situationen, die dies begründen können, gesucht werden, auch wenn sie zunächst herausfordernd erscheinen.

\section{Berücksichtigung eigener Wünsche und Vorstellungen als Ausgangspunkt von Weiterentwicklung}

Nach den negativen Erfahrungen Tamaras in der Regelschule, als sie als „zu langsam" eingestuft wurde und in die Sonderschule wechseln musste (vgl. IT 119-169), nimmt sie in einem späteren institutionellen Kontext wahr, dass

112 Siehe dazu ausführlich Fallrekonstruktion „Eva“, u.a. Unterpunkt „Wahrgenommen- und Akzeptiert-Werden mit den eigenen Wünschen und Bedürfnissen“.

113 Schiach $=$ hässlich. 
ihren Wünschen nachgekommen wird. Sie habe in der Werkstätte geäußert, „welche bestimmte Sachen [sie] machen möchte“ (IT 304), und sie sei dann dementsprechend eingesetzt worden. Diesen Prozess, dass die eigenen Wünsche überhaupt erst erfragt und als Basis für die Gestaltung ihres Alltages wahrgenommen und anerkannt wurden, schätzt Tamara in der Rückschau als „einfach für mein Selbstbewusstsein sehr gut“ (IT 305) ein. Sie stellt also einen unmittelbaren Zusammenhang zwischen dem Ausdrücken und Wahrgenommen-Werden der eigenen Bedürfnisse mit der Entwicklung des eigenen Selbstbildes her. In der Folge orientiert sich Tamara in ihren Handlungen zunehmend an dem, was ihr für sich selbst gut erscheint, worin ihre eigenen Bedürfnisse liegen.

Auch bei Eva dokumentiert sich die Bedeutung von Prozessen des Wahrgenommen-Werdens mit eigenen Wünschen, Vorstellungen und Bedürfnissen als Ausgangspunkt persönlicher Weiterentwicklung sehr deutlich. Die von ihr beschriebene Szene, in der sie im Kontext der Veränderung der Wohnsituation vom Leiter einer integrativ organisierten Wohngemeinschaft ganz konkret nach ihren Wünschen gefragt wurde, dokumentiert sich in ihrer sprachlichen Ausgestaltung [,,Und da sind so Szenen, die ich mein Lebtag niemals vergessen werde..." (IE 134)] als ein ganz besonders bedeutsamer Ausgangspunkt für persönliche Weiterentwicklung und eine zunehmende Orientierung Richtung Selbstbestimmung und Autonomie.

\section{Wahrgenommen werden mit den eigenen Kompetenzen/Ressourcen}

Evas Erzählung einer Szene, in der ihre Kompetenzen im Zentrum der Betrachtung ihrer Person standen, macht über den Vergleich zu den Bedingungen ihrer Zeit in einer Behindertenwerkstatt die Bedeutung einer solchen Betrachtungsweise durch Personen aus dem sozialen Umfeld deutlich. Die Relevanz dieser ressourcenorientierten Sichtweise für Evas Entwicklung deutet sich bereits dadurch an, dass sie diese Szene bereits in ihrer Eingangserzählung zur Sprache bringt. In der Zeit in der Werkstätte und in den Jahren, die sie zu Hause verbrachte, sei ihr ,,sprichwörtlich die Decke auf den Kopf gefallen“(IE 59) und - so Eva - „du hast nie zeigen können, was du leisten hast können“ (IE 60). Durch eine neue Erzieherin erlebt Eva offenbar erstmals das Gefühl, mit den eigenen Kompetenzen wahrgenommen zu werden. Sie erzählt, wie diese Betreuerin sie schließlich auf einen „Traumjob“ hingewiesen habe und gesagt habe, das würde „supergut“ (IE 62) für sie passen, da könne sie sich Eva ,gut drin vorstellen, weil ich eben verbal und kommunikativ sehr gut drauf bin“ (IE 64). Sie habe sich dann bei diesem Projekt vorgestellt. In diesem Kontext werde sie ,einen Satz, der gekommen ist nie vergessen“ (IE 65-66). Der Projektleiter habe gesagt: ,ja, genau so etwas haben wir gesucht" (IE 66). Sie beschreibt lachend, das sei der erste Satz gewesen, der von dem Projektleiter gekommen sei. Ihr Vater, der beim 
Vorstellungsgespräch dabei war, habe gesagt: „sie kennen meine Tochter zwei Minuten und sagen - ja genau das haben wir gesucht" (IE 68). Darauf habe der Projektleiter gesagt: ,ja brauch'n Sie ja nur beobachten wie sie auf jemanden zugeht, wie sie mit jemandem sprachlich umgeht, das passt einfach, ja“ (IE 69-70). Eva verweist in dieser Passage aus mehreren Perspektiven auf ihre Kompetenzen und Fähigkeiten, rückt in den Fokus, dass diese auch außenstehenden in verschiedenen Zusammenhängen aufgefallen seien. Nicht nur das Fremdbild, auch das Selbstbild von Eva dokumentiert sich über diese Passage als positiv. Sie weist explizit darauf hin, dass ihr von außen Fähigkeiten - vor allem im sprachlichen und kommunikativen Bereich zugeschrieben wurden und sie sich auch selbst als kompetent und leistungsfähig einschätzt, aber lange Zeit nicht die Möglichkeit hatte, dies umzusetzen. Wie sehr die positive Einschätzung der eigenen Kompetenzen und Ressourcen durch Personen aus dem sozialen Umfeld diese eigene Entwicklung initiiert und geprägt hat, wird in dieser Passage sprachlich an der zweimal formulierten Aussage, sie werde „einen Satz nie vergessen“ (IE 66), „,den werde ich mein Lebtag nicht vergessen“ (IE 67), eindrucksvoll dokumentiert. Evas Orientierung wird von dieser Erfahrung nachhaltig positiv geprägt, indem sie ihr neue Perspektiven und auch ganz konkrete Handlungsspielräume eröffnet. Eva nimmt in der Folge eigene Potentiale verstärkt wahr und entwickelt die Intention, diese auch nach außen sichtbar zu machen, ihre eigene Leistungsfähigkeit unter Beweis zu stellen (vgl. IE 60).

Inwiefern eine Herangehensweise im sozialen Umfeld, in dem die Ressourcen und Kompetenzen im Vordergrund der Betrachtung einer Person stehen, förderlich für die Entwicklung eines positiven Selbstbildes ist, dokumentiert sich über Evas Erfahrungen im institutionellen Kontext. Ressourcenorientierung im sozialen Umfeld initiiert also Ressourcenorientierung im Selbstbild und im Zusammenhang mit der Reflexion eigener Lebensbedingungen. Diese Zusammenhänge werden auch bei Tamara deutlich sichtbar. Sie erscheint im Zuge ihrer Erfahrungen, als Person mit Kompetenzen wahrgenommen zu werden, selbst zunehmend ressourcenorientiert. In der Rückschau auf eigene Erfahrungen fokussiert sie dann auch eher auf unpassende Rahmenbedingungen als auf persönliche Unzulänglichkeiten, die zu eigenem Scheitern führten. Die ihr zugeschriebene Langsamkeit (vgl. IT 155) in der Schule relativiert sie beispielsweise mit der Bewertung der damals gegebenen Rahmenbedingungen: Sie schätzt diese als nicht passend ein, indem für sie zu schnell vorgegangen worden sei und sie keine angemessene Unterstützung erhielt (vgl. IT 128, 137, 151). Über den Vergleich zu einer anderen Schule, in der es ihr nach einem Schulwechsel aufgrund passender Rahmenbedingungen besser ging, wird diese Orientierung von Tamara wieder deutlich. Auch für die aktuelle Situation führt Tamara einschränkende, hemmende Rahmenbedingungen für die eigene gesellschaftliche Partizipation ins Treffen. So reflektiert sie die eigene Abhängigkeit von anderen wegen ihrer eige- 
nen eingeschränkten, durch die Behinderung verursachten Mobilität und schlechte Partizipationsmöglichkeiten aufgrund unzureichender Infrastruktur im eigenen Lebensbereich. Auch in diesem Zusammenhang stellt Tamara einen starken Umfeldbezug her: Wären die Umfeldbedingungen besser, so die explizite Argumentation von Tamara, so könnte sie auch leichter am gesellschaftlichen Leben partizipieren. Nicht die eigene Person, sondern das soziale Umfeld wird in seiner Ausgestaltung als defizitär erlebt.

\section{Nicht bevormundende Unterstützung wirkt entwicklungsfördernd}

Konkrete Unterstützung aus dem sozialen Umfeld, die mit Anerkennung und einer ressourcenorientierten Sichtweise einhergeht, vermag initiierte Entwicklungsschritte zu befördern. Die Bedeutung bedarfsgerechter Unterstützung in diesen Prozessen dokumentiert sich differenziert wieder sehr deutlich am Beispiel Evas ${ }^{14}$, die ausführt, wie wichtig es sei, die ,richtige Unterstützung“ (IE 491) zu erhalten. Sie macht deut-lich, dass Unterstützung dann bedarfsgerecht ist, wenn sie ein selbstbestimmtes Leben ermöglicht, also nicht einengt, und zudem mit einer ressourcenorientierten Sichtweise verbunden ist. Unterstützung, die frei vom Element der Bevormundung ist, wird dabei als wichtiges Moment deutlich, über das eigene Weiterentwicklung möglich wird (vgl. IE 517-518).

Wie positiv Unterstützung wahrgenommen wird, dokumentiert sich auch bei Anna, die diesen Aspekt bereits in ihrer Eingangserzählung thematisiert. Nach einem erzwungenen Schulwechsel, weil sie sich „beim Lernstoff schwer getan habe, bei allen Fächern“ (IA 24), verbunden mit mangelnder Unterstützung, berichtet sie im Vergleich dazu von der anschließenden Zeit in der Sonderschule. Hier habe sie ,super Lehrer gehabt, die mich jeden Tag auch unterstützt haben" (IA 27-28). Rahmenbedingungen, in denen sich Anna anerkannt und unterstützt fühlt, ermöglichen ihr in der Folge auch schulische Erfolgserlebnisse. Die erhaltene Unterstützung wird dabei von ihr wertgeschätzt und mit der Beziehung zur Lehrerin in Zusammenhang gebracht, was sich auch in der Tatsache zeigt, dass sie mit ihr noch heute Kontakt hat. An Annas Beispiel wird jedoch auch die Überlappung verschiedener Erfahrungen und deren Konsequenzen auf die Orientierung deutlich: Durch ihre späteren Erfahrungen sexueller Übergriffe, verbunden mit in diesen Kontexten vorenthaltener Unterstützung, werden zuvor gemachte, positiv wahrgenommenen Erfahrungen im Rahmen sozialer Beziehungen überdeckt. Zugleich bewertet Anna aufgrund ihrer negativen Erfahrungen mit der Lehrerin sowie den Mitschülerinnen und Mitschülern in der Regelschule nun die Sonderschule als den Kontext, in dem sie keine Übergriffe, sondern Aner-

114 Siehe dazu detailliert Fallrekonstruktion Eva, Punkt „,Ablehnung traditioneller Betreuungskonzepte versus Wunsch nach Unterstützung und Begleitung “. 
kennung und Unterstützung erfahren hat, als positiv und präferiert diesen in der Folge.

Tamaras zunächst brüchiges Selbstbild hingegen entwickelt sich über bedarfsgerechte, ressourcenorientierte Unterstützung weiter. Nach der Anerkennung ihrer Wünsche und Bedürfnisse in einer Werkstätte erfährt sie damit eine weitere Facette der Anerkennung, die ihre Weiterentwicklung befördert. Über die Arbeitssuche und Tätigkeiten an der Arbeitsstelle (vgl. IT 436-457), im Rahmen derer sie Unterstützung bekommen hat, wird deren Bedeutung für Tamara selbst deutlich. Sprachlich dokumentiert sich dies über die mehrmalige Nennung des Begriffes „Unterstützung“. Tamara erzählt, dass sie mit der Unterstützung von beruflicher Assistenz als Helferin in einer Werkstätte angestellt wurde. Auch im Rahmen der Arbeit dort habe sie ,eine Unterstützung gehabt“ (IT 446). Zuerst habe sie dort „Tisch gedeckt mit Begleitung, halt unterstützt, ja“ (IT 452). Nun sei sie in der Lage, alle Anforderungen zu bewältigen und benötige nur mehr Unterstützung ,in gewissen Bereichen“ (IT 454). Tamara reflektiert - wie bereits beschrieben - den Bedarf nach Unterstützung auch in Zusammenhang mit vorliegenden Barrieren im sozialen Umfeld. Dadurch verbleibt sie in bestimmten Lebensbereichen abhängig von der Unterstützung durch nahestehende Personen oder durch Unterstützung aus Institutionen. Unterstützung, die sich an den eigenen Bedürfnissen orientiert, stellt für Tamara jedenfalls die Basis dar, die ihr gesellschaftliche Partizipation ermöglicht.

\section{Tragfähige Beziehungen als förderliche Rahmenbedingungen für Weiterentwicklung}

Besonders am Beispiel von Eva wird deutlich, welch zentrale Rolle tragfähige Beziehungen in Zusammenhang mit der persönlichen Weiterentwicklung spielen und welchen Einfluss sie auf die Orientierung haben. Eva beschreibt in verschiedenen Passagen im Zusammenhang mit ihrem Äußeren und ihrer Einstellung zur Sexualität vorhandene Vorbehalte und Widerstände ihres Vaters. Dieser bringt seine Zweifel zwar explizit zum Ausdruck, setzt sich mit Eva aber auch intensiv über diese Fragen auseinander. So führt Eva im Zusammenhang mit ihrer offenen Einstellung zur Sexualität, die sich von den Vorstellungen des Vaters abhebt, an, sie hätte ,schon häufig Auseinandersetzungen gehabt“ (IE 203). Er müsse ,es jetzt auf die harte Tour lernen [...] mit dem Vorschlaghammer lernen, weil sonst begreift er das nie" (IE 203204). Eva pocht dem Vater gegenüber auf ihr Recht auf selbstbestimmte Entscheidungen und selbstbestimmte Gestaltung ihres Lebens. Evas Mutter wird von ihr als insgesamt sehr unterstützend beschrieben, was sich auch im Zusammenhang mit der Frage von Evas Einstellung zur Sexualität, aber auch in Zusammenhang mit ihrem äußeren Erscheinungsbild verdeutlicht. Die Mutter wird im Gegensatz zum Vater so dargestellt, dass sie Evas Zugang zur 
Sexualität und Partnerschaft von vornherein „,voll akzeptiert“ (IE 215), solange es ihr dabei gut gehe. Darüber dokumentiert sich neben Akzeptanz auch das Interesse der Mutter an Evas Person. Sie verbindet ihre Offenheit gegenüber Evas Vorstellungen mit der notwendigen Voraussetzung von Evas Wohlergehen und macht damit deutlich, dass ihr Evas Wohl am Herzen liegt.

Auch im Zusammenhang mit der ihr angebotenen Möglichkeit, einen Job in einer anderen Stadt anzunehmen und autonom zu leben, beschreibt Eva die Reaktion ihrer Mutter als ,voll begeistert“ (IE 129). Der Vater äußert auch in diesem Zusammenhang Vorbehalte und Zweifel, ob Eva die damit einhergehenden Herausforderungen auch meistern kann. Die Auseinandersetzung mit dem Vater über diese Fragen wirkt auf Evas Weiterentwicklung förderlich, indem sie zur intensiven Reflexion über Bedingungen und Erfordernisse selbstbestimmten Lebens angeregt wird. Letztendlich führt dies dazu, dass sich Eva sehr bewusst und reflektiert aktuellen Herausforderungen, wie etwa dem autonomen Wohnen, stellt. Sie integriert darin die über die Auseinandersetzung mit dem Vater wahrgenommenen Vorbehalte, indem sie etwa festhält: ,ist mir völlig bewusst, mit jedem Zentimeter meines Körpers ist mir das bewusst, ich weiß es eh" (IE 127-128). Eva bekräftigt also als Ergebnis der Diskussionen mit dem Vater, sich aller Herausforderungen, die damit einhergehen, bewusst zu sein, und zeigt sich bereit, diese anzunehmen.

Die Beziehung zu den Eltern erscheint in Evas Beispiel insofern als tragfähig, als beide sich gedanklich mit Evas Wohlergehen auseinandersetzen. Der Vater thematisiert in der Diskussion mit Eva auch Vorbehalte und Zweifel hinsichtlich ihrer Vorstellungen. Indem er sich mit Eva auseinandersetzt, wird auch deutlich, dass er sie als Person auf Augenhöhe respektiert und wertschätzt. Vater und Mutter respektieren letztendlich Evas Entscheidungen.

Auch am Beispiel Tamaras wird deutlich, wie sich soziale Beziehungen, die grundsätzlich als wohlwollend erlebt werden, auf eigene Handlungsperspektiven und Entwicklungsmöglichkeiten auswirken bzw. dass entsprechende Erfahrungen ausgleichend wirken können. Tamara reflektiert in ihrer Eingangserzählung im Interview als Grund, warum sie zu einer Pflegefamilie gekommen ist, dass die leibliche Mutter sie wegen ihrer Behinderung nicht haben wollte und sie sogar "verräumen wollte“ (IT 67), was Tamara auf Nachfrage explizit mit „töten“ definiert. Tamara beschreibt sich in der Rolle des von der leiblichen Mutter wegen der Behinderung nicht gewollten Kindes, das aber von der Pflegemutter gerade wegen der Behinderung aufgenommen wurde. Hier hält sie explizit fest, dass diese „halt ein beeinträchtigtes" haben wollte. Diesen Kriterien hat Tamara eigener Einschätzung nach entsprochen, weil es bei ihr geheißen habe, ,dass ich nicht gehen und reden werde und es ist ganz gut gegangen“ (IT 90-91). Der positive Zugang der Pflegemutter, die Tamara über die gesamte Zeit des Aufwachsens das Gefühl gab, angenommen zu werden, und sie unterstützte, vermag, zumindest im Ansatz den Erfahrungen der Ablehnung durch die eigene Mutter entgegen- 
zuwirken. An Tamaras Beispiel wird deutlich, dass frühere negative durch positive Erfahrungen kompensiert werden können, wenn dann tragfähige Beziehungen innerhalb stabiler Strukturen zur Verfügung stehen.

\section{Zusammenfassung: förderlich oder erweiternd auf Handlungsspielräume und Entwicklungsperspektiven wirkende Faktoren}

Aus der soziogenetischen Analyse geht hervor, dass vor allem Erfahrungen von Akzeptanz und Anerkennung Handlungsspielräume erweitern und auf vielfältige Weise Entwicklungsmöglichkeiten von jungen Frauen mit Lernschwierigkeiten befördern. Dabei wird insbesondere auch die Bedeutung von „tragfähigen“ Beziehungen deutlich. In solchen Beziehungen erfolgt eine Auseinandersetzung mit den Wünschen und Bedürfnissen der Frau, die ernst genommen werden, auch wenn sie sich nicht mit den Vorstellungen von Personen aus dem Umfeld decken. Damit einhergehende Interaktionsprozesse, die von grundsätzlicher Akzeptanz und Wertschätzung getragen sind, können auch dazu beitragen, dass persönliche Weiterentwicklung initiiert wird. Indem eine intensive Auseinandersetzung über spezifische Themen, die kontrovers eingeschätzt werden, stattfindet, werden Vorstellungen mitunter auch einer gedanklichen Überprüfung zugeführt und modifiziert bzw. konkretisiert. Hier ist auch der Begriff der Resilienz angesprochen, dem in solchen förderlichen Systemen große Bedeutung zukommt. Eine herausfordernde Situation beinhaltet aus dieser Perspektive auch „das Potenzial für eine Transformation der einzelnen Personen und der Beziehungen“ (Retzlaff 2010: 2002). Resilienz bezieht sich in diesem Zusammenhang auch auf „die Fähigkeit von lebenden Systemen, sich nach vorne zu entwickeln [...]“ (ebd.: 112). Diese Wirkungsweise wird besonders deutlich am Beispiel der Auseinandersetzung Evas mit ihrem Vater. Dieser nimmt eine konträre Position zu ihren Vorstellungen ein, setzt sich aber so mit ihr darüber auseinander, dass sie letztendlich zu einer für sie abgesicherten und realistischen Perspektive findet. Am Ende der Auseinandersetzung ist es dem Vater möglich, Evas Position bei eigenen Vorbehalten zu akzeptieren und sie auf ihrem Weg zu unterstützen. Beide entwickeln sich dadurch weiter, die Beziehung erweist sich als tragfähig.

Ein weiterer zentraler, positiv wirkender Faktor ist eine ressourcenorientierte Sichtweise durch Personen aus dem sozialen Umfeld. Verbunden mit Interaktionen, die davon getragen sind, vermag diese Handlungsspielräume zu erweitern und Entwicklungsperspektiven zu fördern. Das Wahrgenommen-Werden mit den eigenen Ressourcen und Kompetenzen wirkt stark förderlich auf die Entwicklung eines positiven Selbstbildes. Ressourcenorientierung im sozialen Umfeld initiiert bzw. verstärkt Ressourcenorientierung im Selbstbild und im Zusammenhang mit der Reflexion eigener Lebensbedingungen. Diese Zusammenhänge werden besonders an Evas Erfahrungen 
deutlich, deren Stärken von unterschiedlichen Schlüsselpersonen im Verlauf ihrer Sozialisation wahrgenommen und ihr in Interaktionsprozessen auch sichtbar gemacht werden. Dadurch gestärkt entwirft sie für sich ein Leben, das von Autonomie und Selbstbestimmung gekennzeichnet ist. Herausforderungen im Zusammenhang damit werden von ihr reflektiert und in Angriff genommen. Tamara erscheint im Zuge ihrer Erfahrungen, als Person mit Kompetenzen wahrgenommen $\mathrm{zu}$ werden, selbst zunehmend ressourcenorientiert. Ihr zunächst brüchiges Selbstbild wird dadurch gestärkt. Dies führt bei ihr sogar zu einer Korrektur eigener, früherer Einschätzungen ihrer eigenen Person. Was sie zuvor als persönliche Unzulänglichkeit wahrnahm, vermag sie nun als Konsequenz unpassender Rahmenbedingungen zu reflektieren. Unter dieser Perspektive werden für Tamara Handlungsmöglichkeiten und Entwicklungsperspektiven im Kontext mit selbstbestimmtem und autonomem Leben sichtbar.

Im Zusammenhang mit Anerkennung und einer ressourcenorientierten Sichtweise erweist sich bedarfsgerechte Unterstützung aus dem sozialen Umfeld als besonders bedeutsam, um initiierte Entwicklungsschritte zu begleiten und zu befördern. Unterstützung wird dann als bedarfsgerecht erlebt, wenn sie nicht einengt und frei vom Element der Bevormundung ist.

\subsection{Soziogenetische Zusammenhänge in der Einschätzung der Frauen aus der Referenzgruppe: ausgewählte Themen}

Im Folgenden fasse ich die Diskussionen und Einschätzungen der Frauen aus der Referenzgruppe zur Frage, wie es zur Ausprägung der unterschiedlichen Orientierungen kommen kann, kursorisch zusammen. Dabei beziehe ich mich insgesamt auf das Protokoll ${ }^{115}$ der Sitzung, in der die Ergebnisse der sinngenetischen Typenbildung und der soziogenetischen Analyse den Frauen präsentiert und zur Diskussion gestellt wurden. Soziogenetische Aspekte wurden von den Frauen auch bereits im Zusammenhang mit der Diskussion der typisierten Orientierungen zur Sprache gebracht ${ }^{116}$, in der Dokumentation jedoch nur ansatzweise abgebildet.

115 Siebente Sitzung mit der Referenzgruppe, Protokoll Seiten 1-15 (7RGS-S1-15). In diesem Kontext beziehe ich mich durchgängig auf das Protokoll dieser Sitzung und führe Seitenzahlen nur mehr bei direkten Zitaten an. Auf Wunsch der Frauen aus der Referenzgruppe werden direkte Zitate nicht konkreten Frauen zugeordnet.

116 Siehe dazu „Die vier erhobenen und typisierten Orientierungen in der Einschätzung der Frauen aus der Referenzgruppe“. 


\section{Bedarfsgerechte Unterstützung}

Auf eine offene Nachfrage zu möglichen Zusammenhängen von Lebenszusammenhängen, Erfahrungen und Orientierungen wurde von der Gruppe selbst zunächst die Frage der als notwendig erachteten Unterstützung eingebracht und eingehend diskutiert. Eine der Frauen erzählte aus der Erfahrung einer Kollegin in der Wohneinrichtung, dass diese gerne einer bestimmten Aktivität außer Haus nachgehen würde, aber keine angemessene Unterstützung oder Begleitung dafür bekomme. Es werde manchmal vom sozialen Umfeld gesagt: ,du kannst das ja gerne machen, aber es gibt keine Unterstützung" (S. 14). Als betroffene Frau erlebe man das als Einschränkung. Unterstützung soll aus der Perspektive aller vier Frauen aus der Referenzgruppe „da sein, wenn sie gebraucht wird“ (S. 5). Und es müsse dann auch wirklich unterstützt werden, es reiche nicht, das immer gesagt werde, dass man unterstützt wird, real aber nicht die Unterstützung geleistet wird, die man benötigt oder gerne hätte. Als Beispiel dafür berichtet eine Frau, dass sie, als sie konkrete Unterstützung benötigt hätte, auf den nächsten Tag vertröstet wurde. Sie hätte aber gerade an diesem Tag gerne eine Aktivität außer Haus durchgeführt, am nächsten Tag war es für sie nicht mehr interessant, da sie bei der gewünschten Aktivität nicht mehr die Freundin hätte treffen können, die am Tag zuvor Zeit gehabt hätte. Eine der Frauen berichtete auch davon, dass ihr Unterstützung für eine Aktivität zugesagt wurde, die Betreuerin zur vereinbarten Zeit aber einfach nicht gekommen sei und auch nicht abgesagt habe. Sie habe dann stundenlang gewartet und sich auch sehr geärgert, weil sie sich aus diesem Grund nicht an einer Aktivität beteiligen konnte, auf die sie sich schon länger gefreut hatte. Unterstützung muss aus der Perspektive der betroffenen Frauen also konkret verfügbar sein, wenn sie gebraucht oder erwünscht wird. Es darf nicht auf später vertröstet werden, denn dann entspricht sie möglicherweise nicht mehr den in jenem Moment vorhandenen Bedürfnissen. Bereits zugesagte Unterstützung muss zum vereinbarten Zeitpunkt geleistet werden. Wenn dies aus bestimmten Gründen nicht erfolgen kann, muss rechtzeitig eine Absage erfolgen. Wenn Unterstützung auf diese Art zur Verfügung steht und geleistet wird, dann begünstigt dies aus der Perspektive der Frauen aus der Referenzgruppe eine Orientierung in Richtung Selbstbestimmung und Autonomie. Nicht bedarfsgerechte Unterstützung wird als unangemessenes Verhalten der Personen aus dem sozialen Umfeld eingeschätzt, von denen diese Unterstützung erwartet wird. Hier wird insbesondere Betreuungspersonal in Institutionen genannt.

Nach dieser - zum Teil sehr emotional geführten - Diskussion über bedarfsgerechte Unterstützung wurden den Frauen die über die soziogenetische Analyse erhobene Art und Weise sowie Gründe des Zustandekommens der vorliegenden Orientierungen und Einflussfaktoren präsentiert und zur Diskussion gestellt. Dabei wurde wie in der vorab dargestellten Analyse zwi- 
schen förderlichen und hemmenden Faktoren unterschieden. Alle beschriebenen Faktoren wurden als Erfahrungen wiedererkannt, die die Frauen selbst oder ihnen bekannte Frauen mit Lernschwierigkeiten bereits gemacht haben bzw. gut kennen. Folgende Aspekte wurden von den Frauen aus der Referenzgruppe als besonders wesentlich identifiziert und eingehend diskutiert ${ }^{117}$ :

Hohe Bedeutung sozialer und beruflicher Einbindung

Die Diskussion der Frauen aus der Referenzgruppe ergab, dass sie es als höchst bedeutsam einschätzen, über die Einbindung in Beschäftigung oder Arbeitsverhältnisse außer Haus soziale Kontakte knüpfen und pflegen zu können. Dies sei für manche Frauen auch mit dem Leben und Arbeiten in einer Einrichtung gegeben, weil sie dort Freundschaften haben und sich an verschiedenen Aktivitäten beteiligen können. Besonders viele Möglichkeiten würden sich aber erst darüber ergeben, dass man zum Beispiel über Beschäftigung außer Haus die Möglichkeit habe, sich auch über den vertrauten Rahmen hinaus gesellschaftlich zu beteiligen. Dies sei zum Beispiel über den täglichen Arbeitsweg möglich, auf dem man neue Leute kennen lernen könne. Man bekomme dadurch auch die Möglichkeit, Angebote außer Haus wahrzunehmen, also auf dem Arbeitsweg etwas einzukaufen, jemanden zu treffen oder auch einen Kaffee trinken zu gehen. Das eröffne neue Perspektiven, man könne und müsse sich in verschiedenen neuen Situationen erproben. Man macht vieles, was man vorher nicht getan hat, weil es sich einfach so ergebe. Wenn man nur zu Hause sei, habe man diese Möglichkeiten nicht. Man sehe nicht, was möglich sein könnte, sondern ,immer nur das, was immer gleich ist" (S. 13).

\section{Abwertung, (Des-)Interesse und Anerkennung}

Alle Frauen berichteten von eigenen Erfahrungen mit Abwertungsprozessen im sozialen Umfeld. Hier wurden insbesondere schulische Erfahrungen, aber auch soziale Interaktionen im Zusammenhang mit gesellschaftlicher Partizipation beschrieben und diskutiert. Man werde häufig aufgrund der eigenen Beeinträchtigung negativ eingeschätzt und dies würde einem auch vermittelt werden. Dies müsse nicht unbedingt ausgesprochen werden, es genüge oft ein Blick von einer Person aus dem sozialen Umfeld, dass ,,man sich klein fühlt“ (S. 13). Abwertung sei schlecht für das eigene Selbstbild, man werde dadurch „,immer kleiner und kleiner“ (S. 13) und traue sich selbst nichts (mehr) zu. Manche Personen würden einen nicht einmal wahrnehmen. Hier wurde eingehend anhand eigener Beispiele die konjunktive Erfahrung der Frauen diskutiert, im Rahmen gesellschaftlicher Aktivitäten nicht wahrgenommen zu

117 Diese Punkte wurden zum Teil auch bereits unter dem Punkt „Die vier erhobenen und typisierten Orientierungen in der Einschätzung der Frauen aus der Referenzgruppe“ andiskutiert. 
werden. Viele Menschen würden sich nicht für sie interessieren oder gar nichts von ihnen wissen wollen. Alle Frauen berichteten hier etwa auch von der Erfahrung, dass über ihre Köpfe hinweg gesprochen und entschieden werde, besonders dann, wenn es schnell gehen müsse. Als Beispiel wurde hier das Aufnehmen von Bestellungen in Gaststätten, wo statt der betroffenen Frauen ihre Begleitpersonen gefragt würden, eingehend beschrieben und diskutiert.

Interesse an der eigenen Person in verschiedenen sozialen Kontexten hingegen wird als eher seltene Erfahrung beschrieben, die als sehr positiv erlebt wird. Verbunden mit Anerkennung „,macht das stark“ (S. 14). Die Erfahrung, dass einem etwas zugetraut wird, führe dazu, dass man sich gut fühle. Man bekomme zunehmend selbst das Gefühl, etwas zu können. Mit Anerkennung - so eine der Frauen - „wächst man“ (S. 14). Eine der Frauen berichtet, dass Anerkennung, die sie im beruflichen Umfeld erhalten habe, dazu geführt habe, dass sie mehr Selbstbewusstsein entwickelt habe und zu ihrer Behinderung stehe: „Ich sage, wer ich bin, dass ich behindert bin, ich schäme mich nicht mehr!" (S. 14). Eine andere Frau erzählt, dass sie erfahren habe, dass sie im Arbeitsumfeld ,so abgegangen“ (S. 14) sei, als sie einmal krank war. Niemand hätte ihre Arbeit ersatzweise tun können, weil sich keiner ausgekannt hätte. Mit dieser Erfahrung sei das Gefühl gewachsen, dass man etwas leisten könne und noch mehr erreichen könne. Sie selbst denke nun immer öfter: „Ich schaffe alles!“.

Selbst bestimmen und entscheiden und die Möglichkeit, Entscheidungen zu revidieren

Die Erfahrung, im Alltag selbst entscheiden und bestimmen zu dürfen, wird als besonders bedeutsam für das eigene Selbstbewusstsein und die eigene Weiterentwicklung eingeschätzt. Diese Möglichkeit stehe sehr oft allerdings nicht zur Verfügung. In Einrichtungen vor allem sei es oft so, dass man sich zwar in verschiedenen Zusammenhängen frei entscheiden könne. Allerdings, so eine der Frauen, dürfe ,man sich nicht mehr um-entscheiden“ (S. 15), das heißt, eine einmal getroffene Entscheidung dürfe meist nicht rückgängig gemacht werden. Wenn man einmal gesagt habe ,das will ich machen, muss ich es machen“ (S. 15). In der Einschätzung der Frauen habe das ,mit Selbstbestimmung nichts zu tun" (S. 15). Man müsse sich immer auch umentscheiden können.

\section{Sexuelle Gewalt und Vermeidung}

Sexuelle Gewalt wurde als Erfahrung thematisiert, die viele Frauen mit Lernschwierigkeiten in unterschiedlichen Kontexten machen. Solche Erfahrungen würden dazu führen, dass man sich nicht mehr ,aus dem Haus traut“" (S. 14), 
man ziehe sich „total zurück“ (S. 14). Eine der Frauen berichtet davon, sich nach einer solchen, weit zurückliegenden Erfahrung lange Zeit nicht „einmal alleine aus Klo getraut" (S. 14) habe. Man fühle sich total eingeschränkt und nicht mehr sicher. In der Gruppe wurden auch psychosomatische Folgen von Gewalterfahrungen beschrieben, die sich mitunter noch viele Jahre später etwa in Form unbestimmter „Angstgefühle“ (S. 14) - immer wieder zeigen würden.

Die der Gruppe im Anschluss an ihre Diskussion über die Fallrekonstruktion „Anna“"118 vermittelte, aus Gewalterfahrungen entwickelte Vermeidungsstrategie wurde anhand der eigenen Diskussionen zuvor wiedererkannt und als ,absolut verständlich“ (S. 14) eingeschätzt. Hervorgehoben wurde auch, dass es „das Schlimmste“ (S. 14) sei, wenn im Kontext mit Gewalterfahrungen den betroffenen Frauen Glaubwürdigkeit abgesprochen werde. Dann fühle man sich „ganz alleine gelassen“ (S. 14), „unverstanden“ (S. 14) und wisse gar nicht mehr, wie man „weitertun“ (S. 14) solle.

\section{Große Bedeutung ,guter" Beziehungen im sozialen Umfeld}

Im Zusammenhang mit der Bewältigung von Gewalterfahrungen wurde die Notwendigkeit ,guter Beziehungen“"(S. 15) hervorgehoben. Eine der Frauen berichtete über die eigene Erfahrung, dass niemand ihr zugehört habe, als sie davon erzählen wollte. Damit sei es ihr ,gar nicht gut gegangen“ (S. 15). Alle Frauen äußerten die Meinung, es sei grundsätzlich wichtig, dass man Beziehungen habe, die unterstützend seien. Man würde oft einfach jemanden zum Reden und mit Verständnis für die eigene Situation brauchen. Man würde das Gefühl benötigen, dass die eigenen Wünsche berücksichtigt werden. Man würde jemanden brauchen, der ,zuhört, verstehen, mitfühlen kann“ (S. 15). Es wäre wichtig, das Angebot zu haben, in jeder Situation zu dieser Person kommen zu können und sich anvertrauen zu können. Im Zusammenhang mit den thematisierten Gewalterfahrungen wurde auch als besonders wichtig eingeschätzt, dass diese Vertrauensperson im Anlassfall aktiv werde und etwas unternehme, ,damit es nicht wieder passiert“ (S. 15). Konkrete Unterstützung in herausfordernden und belastenden Situationen sei also besonders bedeutend und hilfreich, so die Frauen aus der Referenzgruppe. Als besonders wichtig wurde auch die ,gegenseitige Unterstützung von Freundinnen“ (S. 15) hervorgehoben, die bereits ähnliche Erfahrungen gemacht hätten und mit denen man sich austauschen könne.

118 Siehe Fallrekonstruktion „Anna“, insbesondere „Erfahrung von Übergriffen beeinträchtigen Streben nach Autonomie und Selbstbestimmung“ sowie „Soziogenetische Erfahrungen“, insbesondere „Erfahrung sexueller Übergriffe in unterschiedlichen Kontexten und Ausprägungen“. 


\section{Möglichkeiten, Erfahrungen und die Entwicklung des Selbstbildes}

Die Frage des Selbstbildes wurde im Zusammenhang mit den zuvor beschriebenen Aspekten bereits eingehend diskutiert, von den Frauen aber auch als eigener Punkt nochmals in den Blick genommen. Vor allem die im $\mathrm{Zu}-$ sammenhang mit Fragen von Abwertung und Anerkennung thematisierten Wirkungsweisen wurden nochmals aufgegriffen und zusammenfassend diskutiert. Die Einschätzungen der Frauen bestätigen dabei aus ihrem Erfahrungshintergrund, dass sich das Selbstbild über die vorhandenen Lebenszusammenhänge und die dadurch gegebenen Möglichkeiten sowie über eigene Erfahrungen in diesen Kontexten bildet. Dass das eigene Selbstbild die eigene Handlungsweise und Orientierung stark beeinflusst, wurde anhand verschiedener eigener oder von den Frauen beobachteter Beispiele bestätigt.

Die mittels der dokumentarischen Methode erhobenen soziogenetischen Wirkungsweisen wurden also von den Frauen aus der Referenzgruppe weitgehend bestätigt. $\mathrm{Zu}$ ausgewählten, spezifischen Aspekten wurden eigene Erfahrungen berichtet, die diese Wirkungsweisen verdeutlichen. Es wurden auch Ergänzungen zu den analysierten Erfahrungen und Wirkungsweisen vorgenommen, wie etwa die Erfahrung, dass es von Frauen als Fremdbestimmung erlebt wird, wenn man einmal getroffene Entscheidungen nicht mehr revidieren kann.

Die Ergebnisse wurden insgesamt als sehr wichtig für Frauen mit Lernschwierigkeiten eingeschätzt. Denn - so die Argumentation der Frauen aus der Referenzgruppe - wenn diese Zusammenhänge im sozialen Umfeld bewusst seien, sei das für die Frauen selbst wichtig. Sie würden dann selbst darüber nachdenken, warum sie diese oder jene Handlungsweise entwickelt haben und auf der Grundlage dieser Überlegungen eventuell auch etwas verändern können. Als besonders bedeutsam wurde aus ihrer Einschätzung auch die Notwendigkeit hervorgehoben, die Gesellschaft, Eltern, Lehrer, Betreuer usw. darüber zu informieren, welche Folgen die konkreten Formen des Umganges mit den betroffenen Frauen nach sich ziehen könnten. 


\section{Zusammenfassung, inhaltliche Schlussfolgerungen und methodische Anmerkungen}

Im Theorieteil der Arbeit erfolgte zunächst eine auf vorliegenden Forschungsergebnissen beruhende Analyse hinsichtlich der Frage der Umsetzung selbstbestimmter Entscheidungs- und Lebensmöglichkeiten von Frauen mit Lernschwierigkeiten. Dazu wurden die Begriffe „Selbstbestimmung“ und „Fremdbestimmung“ als zentrale Begriffe der Arbeit in den Fokus genommen. Die Verwendung der Bezeichnung „Menschen mit Lernschwierigkeiten“ wurde mit einem kurzen kritischen Exkurs zum Begriff der „geistigen Behinderung" reflektiert. Danach wurde die gesellschaftliche Situation von Frauen mit Lernschwierigkeiten auf der Grundlage vorliegender Forschungsergebnisse und Publikationen skizziert. Im Anschluss daran wurden Entwicklung, Grundannahmen und erforderliche Rahmenbedingungen für die Umsetzung partizipativer Forschung allgemein und mit Fokus auf die Kooperation mit Menschen mit Lernschwierigkeiten thematisiert. Diese bildet auch die Basis für die Umsetzung partizipativer Forschung im Kontext der vorliegenden Arbeit.

\subsection{Die zentralen Forschungsergebnisse im Überblick}

Im Folgenden fasse ich die zentralen Ergebnisse des Forschungsprojektes, das inhaltlich und methodisch mit Frauen mit Lernschwierigkeiten - organisiert in einer sogenannten „Referenzgruppe“ - geplant wurde und in dessen Auswertung diese in verschiedenen Schritten eingebunden waren, zusammen.

\subsubsection{Orientierungen junger Frauen mit Lernschwierigkeiten}

Auf der Grundlage von 20 erzählgenerierenden Interviews, die mit jungen Frauen mit Lernschwierigkeiten durchgeführt wurden, konnten mittels der Analyseschritte der dokumentarischen Methode folgende vier zentrale Orientierungen herausgefunden werden:

Orientierung A: Orientierung am vorgegebenen, stark strukturierten, vertrauten Rahmen, der auf individuelle, oft phantasievolle, im Detail selbstbestimmte Weise ausgestaltet wird

Orientierung B: Ambivalente Orientierung an Selbstbestimmung und Autonomie in einzelnen Lebensbereichen bei gleichzeitigem Wunsch nach Bezug von Unterstützungsund Versorgungsleistungen durch das soziale Umfeld 
Orientierung C: Starke Orientierung an Selbstbestimmung, Partizipation und Normalisierung bei gleichzeitigem Bedürfnis nach Erhalt eines vertrauten Rahmens, um Konflikte und/oder negative Erfahrungen innerhalb und außerhalb dieses Kontextes zu vermeiden

Orientierung D: Starke Orientierung an Selbstbestimmung und Autonomie, die in allen Lebensbereichen umfassend aktiv, reflektiert und konsequent angestrebt werden

Die herausgefundenen vier Orientierungen spannen sich zwischen zwei Polen auf: einerseits einer Herangehensweise, die mit einer weitgehenden Zufriedenheit mit vorgegebenen, stark strukturierten Rahmenbedingungen verbunden ist, die dem eigenen Bedürfnis nach einem vertrauten Rahmen entgegenkommen. Dieser Rahmen wird mitunter auf individuelle, oft phantasievolle, im Detail auch selbstbestimmte Weise ausgestaltet (Typ A). Und andererseits einem umfassenden Anspruch nach Selbstbestimmung und Autonomie, der aktiv, reflektiert und konsequent angestrebt wird. Unter dieser Perspektive werden vorgegebene Rahmenbedingungen oftmals als stark einschränkend und bevormundend erlebt (Typ D). Dazwischen liegen zwei Orientierungen, die jeweils ambivalenten Charakter aufweisen. Die Orientierung an Selbstbestimmung und Autonomie in einzelnen Lebensbereichen kann einerseits mit dem Wunsch nach Bezug von Unterstützungs- und Versorgungsleistungen durch das soziale Umfeld gekoppelt sein (Typ B). Eine starke Orientierung an Selbstbestimmung, Partizipation und Normalisierung kann auch mit dem gleichzeitigen Bedürfnis nach Erhalt eines vertrauten, geschützten Rahmens einhergehen. Dies vor allem, um (neuerliche) Konflikte und/oder negative Erfahrungen innerhalb und außerhalb dieses Kontextes zu vermeiden (Typ C).

Für die vier unterschiedlichen Orientierungen wurden markante Dimensionen anhand rekonstruktiv dargestellter Sequenzen aus jeweils einem Fall differenziert dargestellt. Zentrale Dimensionen dieser Orientierungen wurden ergänzend dazu durch prägnante Passagen aus anderen Fällen belegt. Dadurch konnten unterschiedliche Ausprägungsvarianten der vier Orientierungen sichtbar gemacht werden. Dies soll dokumentieren, dass die Orientierungen nicht jeweils aus einem Fall abgeleitet wurden, sondern mit ihren individuellen Ausprägungsvarianten typische Orientierungen junger Frauen mit Lernschwierigkeiten darstellen. Dieser Prozess der Herausarbeitung von typischen Orientierungen, der in der dokumentarischen Methode „sinngenetische Typenbildung" genannt wird, wurde ebenfalls von den Frauen aus der Referenzgruppe begleitet. Wie im Methodenkapitel der Arbeit differenziert dargestellt, wurden sie einerseits in die Auswertung ausgewählter Interviewpassagen einbezogen, womit ihre Perspektive auch in diesem Prozess integriert ist. Darüber hinaus wurden die herausgefundenen Orientierungen einer kommunikativen Validierung durch die Frauen aus der Referenzgruppe unterzogen. Dieser Prozess ergab, dass die vier erhobenen Orientierungen für 
die Frauen aus der Referenzgruppe sehr gut nachvollziehbar sind und sie ihre Perspektive als korrekt einbezogen wahrnehmen.

\subsubsection{Die Entwicklung der Orientierung und zentrale Wirkungsfaktoren}

In einem weiteren Schritt wurde analysiert, wie und warum es zu den vorliegenden Orientierungen kommen kann und welche Prozesse, Interaktionen und Erfahrungen sich zentral darauf auswirken. Dieser Prozess - in der dokumentarischen Methode „soziogenetische Typenbildung“ genannt - konnte verdeutlichen, welche unterschiedlichen typenspezifischen Sicht- und Herangehensweisen zu verschiedenen Herausforderungen und Problemstellungen, die den konjunktiven Erfahrungsraum von Frauen mit Lernschwierigkeiten kennzeichnen, vorliegen. Besonderer Stellenwert kam dabei auch der Frage $\mathrm{zu}$, wie es auf Grundlage ähnlicher Erfahrungen zu unterschiedlichen Orientierungen kommen kann, also dem Versuch, „die sozialen Zusammenhänge aufzuklären, innerhalb derer die sinngenetisch entwickelten Typen [...] stehen" (Nohl 2012: 110).

Die Frage, wie es zu den unterschiedlichen Orientierungen von Frauen mit Lernschwierigkeiten kommen kann, macht deutlich, dass sich aktuelle Rahmenbedingungen des Lebens und Erfahrungen, die im Verlauf der bisherigen Sozialisation gemacht wurden, auf die aktuelle Orientierung auswirken können. Dabei lassen sich Faktoren identifizieren, die sich als förderlich und solche, die sich hemmend auf Handlungsspielräume und Entwicklungsperspektiven junger Frauen mit Lernschwierigkeiten auswirken.

\section{Lebenszusammenhänge}

Faktoren wie die soziale und materielle Verortung bzw. Eingebundenheit, die sich vor allem über Wohnform, Beschäftigung und finanziellen Status ausdrücken, können sich stark auf die Entwicklung der Orientierung junger Frauen mit Lernschwierigkeiten auswirken. Die Forschungsergebnisse weisen darauf hin, dass die Eröffnung mehrdimensionaler Erfahrungsräume mit den sich dadurch ergebenden Interaktionen und Herausforderungen vielfältige Entwicklungsperspektiven ermöglicht. Die Beschränkung auf ein vorgegebenes System hingegen verengt diese Möglichkeiten. Von besonderer Bedeutung ist auch die Frage materieller bzw. finanzieller Ausstattung, die bei Frauen mit Lernschwierigkeiten mit dem Fehlen eines eigenen Einkommens häufig durch ein Abhängigkeitsverhältnis gekennzeichnet ist. Auf dieser Basis besteht in den sozialen Beziehungen innerhalb der Herkunftsfamilie, aber auch innerhalb institutioneller Kontexte, ein deutlich wahrnehmbares Ungleichgewicht. Dies äußert sich für die betroffenen Frauen nicht nur in der 
Art und Weise, wie mit ihnen kommuniziert wird, sondern hat auch massive Auswirkung auf deren Alltagsgestaltung und höchstpersönliche Lebensbereiche. So sind etwa die Möglichkeiten der Freizeitgestaltung und der Führung sozialer Kontakte darüber eingeschränkt. Zudem wird über die Finanzierung auch oft die Frage der Gestaltung des eigenen Äußeren - etwa über die Auswahl von Kleidung - beeinflusst.

\section{Erfahrungsräume}

Neben diesen - in der Ergebnisdarstellung „Lebenszusammenhänge“ genannten -Faktoren spielen Erfahrungen im Sozialisationsverlauf eine bedeutende Rolle. Diese Ebene wird in der Ergebnisdarstellung mit dem Begriff „Erfahrungsräume" bezeichnetet. Gemeint sind damit Prozesse, Erlebnisse, Interaktionen und deren Verarbeitungsmöglichkeiten in vielfältigen sozialen Kontexten. Die Wirkungsweisen auf dieser Ebene sind mehrdimensional und spannen sich wieder zwischen zwei Polen auf: Negative Erfahrungen stehen positiven gegenüber und wirken sich sehr unterschiedlich auf die Entwicklung bzw. Ausprägung der Orientierung aus. Erfahrungen im gesellschaftlichen Kontext wie Abbrüche, Abwertung, Stigmatisierung, Diskriminierung, Ausgrenzung und traumatische Erlebnisse - insbesondere (sexuelle) Gewalt wirken sich grundsätzlich einschränkend aus, indem sie Handlungsspielräume und Perspektiven verengen. Akzeptanz, Anerkennung und Interesse an der eigenen Person, die in ressourcenorientierten Beziehungen erlebt werden, erweitern demgegenüber Handlungsspielräume und befördern auf vielfältige Weise eigene Entwicklungsmöglichkeiten. Besondere Bedeutung kommt auch der Frage zu, ob, wie und in welchem spezifischen Kontext Erfahrungen be- bzw. verarbeitet werden konnten. Hier wird deutlich sichtbar, dass sozialen Beziehungen als Faktoren, die diesen Rahmen bilden, eine ganz wesentliche Rolle zukommt.

Ein weiteres, zentrales Ergebnis der Forschung ist, dass es bei Vorliegen bestimmter Voraussetzungen auch $\mathrm{zu}$ einem Wandel in der Orientierung kommen kann, d.h. dass sich Herangehensweisen und Einstellungen der betroffenen Frauen vor allem durch spezifische Erfahrungen auch ändern können. Dabei ist dieser Wandel in verschiedene Richtungen möglich: Positive Erfahrungen wirken in der Regel entwicklungsfördernd und eröffnen neue Perspektiven, negative hemmen im Gegensatz dazu eigene Handlungsspielräume und Entwicklungspotentiale. Diese Wirkungsweise untermauert einen ressourcenorientierten pädagogischen Ansatz, in dem von lebenslanger Entwicklungsmöglichkeit von Menschen mit Lernschwierigkeiten ausgegangen wird, indem durch diese Perspektive möglicher Veränderungen auch Handlungsmöglichkeiten sichtbar werden. Dem Wandel der Orientierung auf Grundlage positiver Erfahrungen liegen dabei oftmals Momente zugrunde, die von den jeweiligen Frauen auch als nicht übliche, sondern als besondere 
Erfahrungen und als Dreh- und Angelpunkte für persönliche (Weiter-)Entwicklung beschrieben werden ${ }^{119}$.

Gerade an dieser Stelle wird nochmals deutlich, wie förderlich sich von Respekt, Anerkennung, Interesse und Wohlwollen geprägte soziale Interaktionen und Beziehungen auf die Orientierung junger Frauen mit Lernschwierigkeiten auswirken. In diesem Kontext ist auch zu berücksichtigen, dass Frauen mit Lernschwierigkeiten in der Regel positive und negative Erfahrungen gemacht haben. Wirken positive Erfahrungen generell entwicklungsfördernd, so ist bei negativen Erfahrungen bedeutsam, ob und in welchem Kontext diese verarbeitet werden können. Gelingt eine zufriedenstellende Bearbeitung, was vor allem über Interaktionen in tragfähigen Beziehungen der Fall sein kann, so können negative Erfahrungen gut ausgeglichen werden. Werden negative Erfahrungen allerdings nicht oder nur unzureichend be- und verarbeitet oder gar durch soziale Interaktionen negativ verstärkt ${ }^{120}$, so wirkt sich dies in besonders negativer Weise auf die Orientierung aus. So können etwa durch das Anzweifeln von Glaubwürdigkeit, durch das Vorenthalten von Unterstützung und Äußerungen oder Handlungen im Kontext damit negative Erfahrungen reaktiviert und intensiviert werden. Dies kann sich auch in der Weise auswirken, als durch die damit verbundene Wahrnehmung einer Verengung von Handlungsspielräumen der Rückzug auf einen geschützten Rahmen die Orientierung kennzeichnet oder einen Wandel in diese Richtung begründet ${ }^{121}$.

Mit Blick auf die angeführten Wirkungsweisen werden über spezifische Aspekte im Kontext negativer Erfahrungen aber auch vielfältige Ressourcen und vorhandene Entwicklungspotentiale junger Frauen mit Lernschwierigkeiten deutlich. Dies dokumentiert sich besonders deutlich in der Fallrekonstruktion „Anna“, die mehrfach sexuelle Gewalt erlebt. Anna orientiert sich danach zunehmend stärker am vertrauten familiären Rahmen. Gleichzeitig vermag sie im Zuge dieser Erfahrungen aber eigene Ressourcen zu aktivieren, die sie in die Lage versetzen, doch - wenn aus der Außenperspektive auch eingeschränkt - weiterhin gesellschaftlich zu partizipieren (u.a. IA 453465).

119 So hält Eva im Zusammenhang mit der expliziten Wahrnehmung ihrer Interessen durch ihr Gegenüber fest, das seien ,so Szenen, die ich mein Lebtag niemals vergessen werde, die mich so geprägt haben“ (IE134). Anna hält über eine Lehrerin, die sie als Person wahrnahm und förderte, fest: „die Lehrerin, die werde ich so nie vergessen“ (IA 295-296).

120 Dies dokumentiert sich am Beispiel Angelikas, die von den Eltern gemaßregelt wird, obwohl sie von der Schwester abgewertet wurde (IAng 401).

121 Ein Beispiel dafür ist der Wandel in Annas Orientierung von einer zunächst starken Ausrichtung an „Normalität“ (u.a. IA 120-122) hin zum System Sonderschule über vorenthaltene Unterstützung der Lehrerin und unpassende Rahmenbedingungen in Kombination mit Mobbing durch die Mitschülerinnen und Mitschüler (u.a. IA 20-28). 


\section{Selbstbild}

Lebens- und Erfahrungszusammenhänge beeinflussen auf vielfältige Weise die Entwicklung des Selbstbildes junger Frauen mit Lernschwierigkeiten. Erlebte Erfahrungen und die Rahmenbedingungen der Sozialisation sowie jene des aktuellen Lebens zeichnen deutliche Spuren hinsichtlich der Einschätzung eigener Kompetenzen, Ressourcen und Perspektiven. Die eingangs dargestellten, soziogenetischen Faktoren wirken sich insofern aus, als darüber ein positives, negatives oder auch ambivalentes Selbstbild entstehen kann. Ressourcenorientierung im Umfeld vermag dabei ein Selbstbild zu fördern, das von Selbstvertrauen und der Wahrnehmung eigener Kompetenzen geprägt ist. Negative Zuschreibungen hingegen legen den Grundstein für ein defizitorientiertes Selbstbild. Lebenszusammenhänge, die durch Strukturen gekennzeichnet sind, die nicht den eigenen Bedürfnissen entsprechen, zu denen aber ein Abhängigkeitsverhältnis besteht, untergraben in ihrer Ambivalenz das eigene Selbstbild.

Diese Wirkungsweisen wurden von den Frauen aus der Referenzgruppe als deckungsgleich mit ihren Wahrnehmungen eingeschätzt. Sie bestätigen aus ihrem Erfahrungshintergrund, dass das Selbstbild über die vorhandenen Lebenszusammenhänge und die dadurch gegebenen Möglichkeiten der Teilhabe sowie über eigene Erfahrungen in diesen Kontexten beeinflusst wird. Auch der Zusammenhang von Selbstbild, eigenen Handlungsstrategien und Orientierungen wurde anhand verschiedener eigener oder von den Frauen beobachteter Beispiele bestätigt. Speziell die Erfahrung, im Alltag selbst entscheiden und bestimmen zu dürfen, wird von den Frauen aus der Referenzgruppe als besonders förderlich für das eigene Selbstbewusstsein und die eigene Weiterentwicklung eingeschätzt (7RGS-S15).

\section{Das Wirkungsfeld von Lebenszusammenhängen, Erfahrungsräumen und Selbstbild}

Grafik 1 zeigt die vorab beschriebenen zentralen Einflussfaktoren auf die Orientierung junger Frauen mit Lernschwierigkeiten im Spannungsfeld von Fremd- und Selbstbestimmung im Überblick. Die über das mittige Feld dargestellte Orientierung junger Frauen mit Lernschwierigkeiten wird demnach von den Lebenszusammenhängen, den Erfahrungsräumen sowie dem Selbstbild beeinflusst. Die Lebenszusammenhänge und somit die soziale Einbindung der Frauen wirken auf das Selbstbild und darüber auch auf die Entwicklung der Orientierung, indem dadurch Handlungs- und Entwicklungsperspektiven aufgezeigt oder gehemmt werden. Erlebnisse und Erfahrungen in vielfältigen gesellschaftlichen Zusammenhängen stellen in ihren Auswirkungen weitere förderliche oder hemmende Faktoren dar. Sogenannte Erfahrungsräume, worunter im vorliegenden Kontext spezifische Erfahrungen, Erlebnisse und soziale Interaktionen im Sozialisationsverlauf gemeint sind, wirken als 
dritte Komponente in diesem Feld und verweisen auf die Mehrdimensionalität der Zusammenhänge.

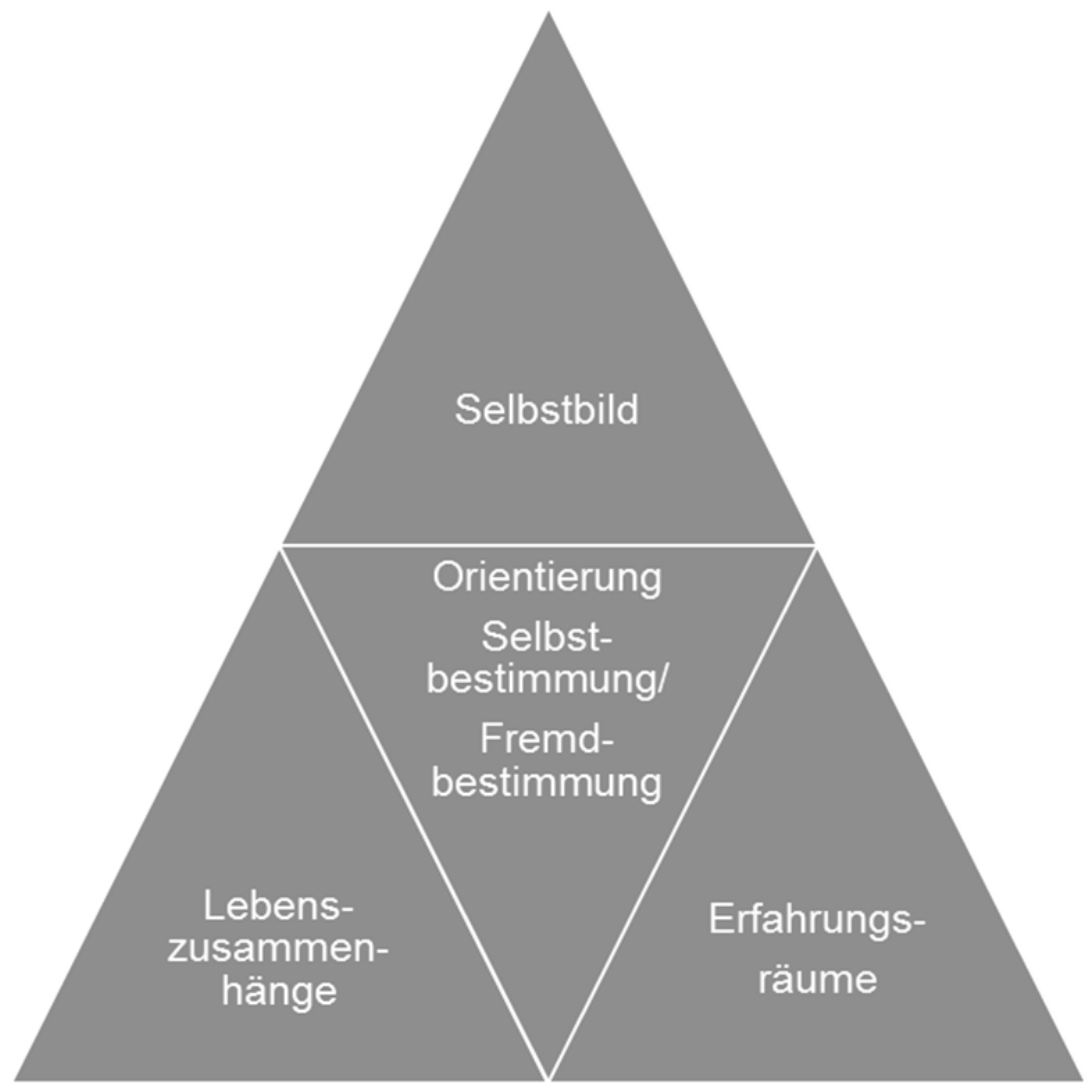

Grafik 1: Einflussfaktoren auf Orientierung im Spannungsfeld von Fremd- oder Selbstbestimmung

Die über das mittige Feld dargestellte Orientierung junger Frauen mit Lernschwierigkeiten wird demnach von den Lebenszusammenhängen, den Erfahrungsräumen sowie dem Selbstbild beeinflusst. Die Lebenszusammenhänge und somit die soziale Einbindung der Frauen wirken auf das Selbstbild und darüber auch auf die Entwicklung der Orientierung, indem dadurch Handlungs- und Entwicklungsperspektiven aufgezeigt oder gehemmt werden. Erlebnisse und Erfahrungen in vielfältigen gesellschaftlichen Zusammenhängen stellen in ihren Auswirkungen weitere förderliche oder hemmende Faktoren dar. Sogenannte Erfahrungsräume, worunter im vorliegenden Kontext spezifische Erfahrungen, Erlebnisse und soziale Interaktionen im Sozialisationsverlauf gemeint sind, wirken als dritte Komponente in diesem Feld und 
verweisen auf die Mehrdimensionalität der Zusammenhänge. Die Orientierungen junger Frauen mit Lernschwierigkeiten stehen also sehr eng in $\mathrm{Zu}-$ sammenhang mit dem Selbstbild, welches sich soziogenetisch neben den Erfahrungsräumen und der sozialen wie materiellen Einbindung auswirkt. Dabei stehen diese soziogenetischen Faktoren in Wechselwirkung zueinander. Erfahrungen in unterschiedlichsten Kontexten stehen in Zusammenhang mit der sozialen und materiellen Einbindung, die Basis dieser Erfahrungen darstellen oder diese beeinflussen können. Diese Erfahrungen wirken sich auch ganz zentral im Selbstbild aus, das darüber positiv oder negativ beeinflusst wird. Das vorhandene Selbstbild wiederum wirkt sich insofern auf die materielle und soziale Einbindung und zukünftige Erfahrungsräume aus, indem es die eigene Herangehensweise dazu prägt. Das bedeutet, dass neuen, herausfordernden Situationen auf Basis des über die individuelle Kombination von Faktoren gebildeten Selbstbildes begegnet wird.

Dabei bestehen in diesem Feld auch Wechselwirkungen bzw. können Wirkungsweisen verstärkt oder abgeschwächt werden. Frauen mit Lernschwierigkeiten, die etwa abhängig von einem Lebenskontext sind, der nicht den eigenen Bedürfnissen entspricht und der zudem von defizitorientierten Zugängen von Personen aus dem sozialen Umfeld gekennzeichnet ist, sehen sich weniger als Akteurinnen im eigenen Leben und orientieren sich eher an fremdbestimmten Lebensentwürfen. Defizitorientierung im Umfeld bedingt dabei einen defizitorientierten Blick auf sich selbst und führt dazu, dass wenig Handlungsspielraum und Entwicklungsmöglichkeiten wahrgenommen werden. Frauen, die einen ressourcenorientierten Zugang zu ihrer eigenen Person erfahren und mit bedarfsgerechter Unterstützung in einem Kontext leben, der ihren eigenen Vorstellungen weitgehend entspricht, entwickeln darüber selbst eine ressourcenorientierte Perspektive, Zufriedenheit und Selbstvertrauen. Chancen, die sich aus neuen Herausforderungen ergeben, können auf dieser Grundlage besser realisiert werden.

\subsection{Schlussfolgerungen zu Selbst- und Fremdbestimmung junger Frauen mit Lernschwierigkeiten}

Aus den zentralen Ergebnissen der Arbeit leite ich nun Schlussfolgerungen ${ }^{122}$ ab, die spezielle Aspekte im Zusammenhang mit Fragen von Selbst- und Fremdbestimmung junger Frauen mit Lernschwierigkeiten thematisieren.

122 Die Schlussfolgerungen werden in den Überschriften als allgemeine Schlussfolgerungen zur Frage der Selbstbestimmung von Menschen mit Lernschwierigkeiten formuliert, wenn sie aus dem vorliegenden Forschungskontext auch als übertragbar erscheinen. Spezifisch auf die Situation von Frauen fokussierte Schlussfolgerungen, die nicht übertragbar erscheinen, werden auch so dargestellt. 
Aus der Perspektive der in der Referenzgruppe am gesamten Forschungsprozess beteiligten Frauen mit Lernschwierigkeiten ergeben sich daraus auch Perspektiven (vgl. 7RGS-S1-15), die auf die Frage der Ermöglichung von Selbstbestimmung in unterschiedlichen Kontexten abzielen. Im Sinne dieser Ermöglichungsperspektive haben die Überlegungen den Fokus auf Grundprämissen, Bedingungen und Umsetzungsmöglichkeiten von Selbstbestimmung für Frauen mit Lernschwierigkeiten. Es werden aber auch zentrale Faktoren analysiert, die die Gefahr des Erlebens von Fremdbestimmung durch Frauen mit Lernschwierigkeiten beinhalten.

\section{Selbstbestimmung im Alltag misst sich an Faktoren, die von Menschen mit Lernschwierigkeiten selbst definiert werden müssen}

Aus den Forschungsergebnissen geht hervor, dass die Außenperspektive auf Frauen mit Lernschwierigkeiten häufig mit Zuschreibungen verbunden ist, die nicht deren Erleben oder deren Wahrnehmung entsprechen. Wenn darüber Handlungsstrategien abgeleitet werden, beinhalten sie die Gefahr der Fremdbestimmung. Dies kann - wie die Ergebnisse verdeutlichen - in unterschiedlicher Weise erfolgen. Einerseits, indem Lebenszusammenhänge aus der Außenperspektive als „fremdbestimmt“ charakterisiert werden, obgleich sie von betroffenen Frauen als ihren Bedürfnissen entsprechend wahrgenommen werden. Sofern die prinzipielle Möglichkeit der Veränderung auch offensteht, indem akzeptable Wahlmöglichkeiten zugänglich gemacht und erprobt werden können, kann eine solche Zuschreibung auch negativ wirken, indem Präferenzen betroffener Personen nicht anerkannt werden. Dies kann Stigmatisierungsprozesse begünstigen, da den davon betroffenen Personen in der Folge das Etikett „fremdbestimmt“ anhaftet. Im Kontext damit gerät der Blick auf eine Mikroebene, die üblicherweise wenig berücksichtigt wird. Damit meine ich Handlungsräume im Kontext der Alltagsgestaltung, die für Frauen mit Lernschwierigkeiten in geschützten, vertrauten Kontexten auch Spielräume für selbstbestimmte Entscheidungen und individuelle Lebensgestaltung ermöglichen. Wenn ein vorgegebener, stark strukturierter Rahmen dem eigenen Bedürfnis entspricht, wird er mitunter auf individuelle, oft phantasievolle Weise selbstbestimmt ausgestaltet ${ }^{123}$. So eröffnen sich in solchen Mikrokontexten durchaus selbstbestimmte Entscheidungs- und Handlungsmöglichkeiten, die von den Frauen auch auf individuelle Weise genutzt werden können.

Auf der anderen Seite können Lebenszusammenhänge, die aus der Außensicht durch weitgehende Selbstbestimmungsmöglichkeiten gekennzeichnet sind, für betroffene Personen selbst im Alltag fremdbestimmende Elemente beinhalten. Dies etwa dann, wenn Unterstützung vorenthalten wird, die erforderlich wäre, um die konkret damit verbundenen Herausforderungen bewälti-

123 Dies dokumentiert sich in der rekonstruktiv dargestellten Fallbeschreibung „Isolde“. 
gen zu können. Auch die wahrgenommene Unmöglichkeit, eine einmal getroffene Entscheidung zu revidieren, wird als massiv fremdbestimmend erlebt. Die grundsätzliche Möglichkeit, sich etwa für eine Freizeitaktivität im institutionellen Kontext entscheiden zu können, bekommt unter dieser Einschränkung aus der Perspektive betroffener Frauen also den Charakter von Fremdbestimmung (vgl. 7RGS-S15).

\section{Selbstbestimmtes Leben von Menschen mit Lernschwierigkeiten bedarf angemessener, nicht bevormundender Unterstützungssysteme}

Wie eingangs angeführt sind selbstbestimmte Handlungsmöglichkeiten an erforderliche Unterstützungsleistungen gekoppelt. Aus der Perspektive betroffener Frauen muss Unterstützung dann konkret verfügbar sein, wenn sie gebraucht und erwünscht wird und kann nicht auf einen Zeitpunkt verschoben werden, der sich etwa aus institutionellen Abläufen oder Ressourcen ergibt. Steht Unterstützung zum konkreten Zeitpunkt des Bedarfs auf die gewünschte Weise zur Verfügung, dann begünstigt dies - auch aus der Perspektive der Frauen aus der Referenzgruppe (vgl. 7RGS-S5) - Selbstbestimmung und Autonomie.

Das Vorenthalten von Unterstützung hingegen bzw. Unterstützung, die nicht den von betroffenen Frauen selbst definierten Bedürfnissen entspricht, kann die Entwicklung eines positiven Selbstbildes untergraben und Entwicklungsperspektiven verengen. Auch die Frage der Anzweiflung der Glaubwürdigkeit von Frauen mit Lernschwierigkeiten ist in diesem Zusammenhang von zentraler Bedeutung. Beides kann zur Einschränkung der Wahrnehmung selbstbestimmter Handlungsmöglichkeiten und Entwicklungsperspektiven führen. Speziell im Zuge solch negativer Erfahrungen von Frauen mit Lernschwierigkeiten selbst entwickelte Handlungsstrategien verweisen aber auch auf vielfältige Ressourcen ${ }^{124}$ und vorliegendes Entwicklungspotential. Mit angemessener Unterstützung würden Energien nicht für die Bewältigung von solchen Herausforderungen bzw. Krisensituationen gebunden, sondern könnten für persönliche Weiterentwicklung genutzt werden. Das Vorenthalten bedarfsgerechter Unterstützung kann von Frauen mit Lernschwierigkeiten auch als unangemessenes Verhalten von Personen aus dem sozialen Umfeld insbesondere Betreuungspersonal in Institutionen - wahrgenommen werden, von denen diese Unterstützung als erwartbar eingeschätzt wird. Dies wird auch von den Frauen aus der Referenzgruppe hervorgehoben (vgl. 7RGS-S5).

Unterstützung und Begleitung wirken dann besonders entwicklungsfördernd, wenn sie im Sinne von Empowerment, also der Stärkung der betroffenen Personen, differenziert in jenen Zusammenhängen und dann geleistet

124 So aktiviert Anna im Kontext vorenthaltener Unterstützung nach sexuellen Gewalthandlungen eigene Ressourcen, die sie in die Lage versetzen, weiterhin handlungsfähig zu bleiben (u.a. IA 453-465). 
werden, wenn sie erforderlich sind. Sie dürfen auch nicht, etwa um Abläufe $\mathrm{zu}$ beschleunigen, ungefragt geleistet bzw. gar aufgezwungen werden. Stehen Bedarf und angebotene Unterstützungsleistung in einem Missverhältnis, kann es auch zu Einbußen an „Eigenständigkeit [...], an Selbstbestimmung “125 (IE 303) kommen.

Erforderliche Unterstützung wahrzunehmen, zu akzeptieren und geleistete Unterstützung auch annehmen zu können, bedarf auch eines Prozesses der persönlichen Auseinandersetzung. Dieser Prozess kann optimal im Kontext förderlicher Beziehungen, deren Wirkungsweisen nachfolgend noch thematisiert werden, erfolgen ${ }^{126}$. Die ,in Unterstützungsbeziehungen verborgene Gefahr von Macht und Abhängigkeit“" (Ferdani 2011: 43) wird in diesem Rahmen erkannt und angesprochen. „Persönliche oder institutionelle Allmachtsphantasien, die sich [...] von einem Konzept der Hilflosigkeit der Betroffenen nähren“ (Schönwiese 2005: 143) spielen in diesem Kontext keine Rolle. Indem entsprechende Zusammenhänge transparent gemacht und reflektiert werden, können auch Handlungsstrategien zur Vermeidung dieser Gefahr reflektiert und umgesetzt werden.

Dieser Austausch wird von jungen Frauen mit Lernschwierigkeiten auch als besonders wirksam im Kontext von Peer-Beziehungen erlebt ${ }^{127}$. Institutionenübergreifende Selbstvertretungsgruppen von Menschen mit Lernschwierigkeiten stellen ideale Räume dafür dar. Aber auch individuelle PeerKontakte von Frauen mit Lernschwierigkeiten innerhalb und außerhalb institutioneller Kontexte bieten Gelegenheit für Freizeitgestaltung, Austausch und Weiterentwicklung. Die Anbahnung und Führung von Peer-Beziehungen auf unterschiedlichen Ebenen wird von jungen Frauen mit Lernschwierigkeiten allerdings häufig als erschwert erlebt ${ }^{128}$. Dementsprechend erfordert die Organisation von Peer-Kontakten selbst systematischer Unterstützung. Dies reicht von der Assistenz hinsichtlich der Überwindung von Mobilitätsbarrieren bis zur Förderung bzw. Gestaltung von Erfahrungsräumen für diese Kontakte. Dadurch können Räume für ,altersgemäße Auseinandersetzung [...] mit eigenen und fremden Werten sowie den eigenen Zukunftsentwürfen“" (Middendorf 2010: 210) geschaffen werden.

125 Diesen Zusammenhang stellt Eva her (IE 303), die Unterstützung nur da haben will, wo es unbedingt erforderlich ist, wo sie herausgefunden hat, ,was geht und was geht nicht“ (IE 46).

126 Diesen Lernprozess und die Bedeutung sozialer Beziehungen im Zusammenhang damit thematisiert u.a. Eva (IE 501-518).

127 Die „gegenseitige Unterstützung von Freundinnen“ (S. 15), die bereits ähnliche Erfahrungen gemacht hätten und mit denen man sich austauschen könne, wird explizit auch von den Frauen aus der Referenzgruppe hervorgehoben (vgl. 7RGS-S15).

128 Middendorf (2010: 209) verweist darauf, dass eine „Freundinnenkultur, wie sie nicht behinderte Mädchen aufbauen [...] für behinderte Mädchen nahezu unerreichbar“" ist. 
Ressourcenorientierung, Selbstbild und Selbstbestimmung stehen in engem Zusammenhang miteinander

Die im Zuge der Ergebnisdarstellung und der Zusammenfassung beleuchteten Zusammenhänge zwischen den Lebens- und Erfahrungszusammenhängen und der Entwicklung des Selbstbildes junger Frauen mit Lernschwierigkeiten legen deren Berücksichtigung im pädagogischen Kontext nahe. Auch bzw. gerade Erfahrungen in alltäglichen Interaktionsprozessen bedingen häufig die Art der Einschätzung eigener Kompetenzen, Ressourcen und Perspektiven. Insbesondere negative Zuschreibungen befördern ein defizitorientiertes Selbstbild. Defizitorientierte Zuschreibungen der eigenen Person durch das soziale Umfeld werden häufig internalisiert und beeinträchtigen damit das Selbstbild nachhaltig. Dies kann in der Folge dazu führen, dass Herausforderungen auf dieser Basis nur zögerlich begegnet wird und Entwicklungschancen somit ungenutzt bleiben.

Die Balance von Selbstbild, vermutetem und ,wie auch immer verifizierte(n) Fremdbild“" (Schönwiese 2011: 143) wirkt sich maßgeblich auf die Identität von Personen aus. Hier lassen sich auch Bezüge zur Anerkennungstheorie erkennen, die davon ausgeht, dass mit der Anerkennung durch andere Personen grundsätzlich die Selbstachtung steigt (vgl. Honneth 1992: 127). Über einen damit verbundenen ressourcenorientierten Zugang wird die Wahrnehmung von Handlungs- und Entwicklungsperspektiven möglich. Ein solcher Zugang wird jedoch von Frauen mit Lernschwierigkeiten häufig nicht erlebt. Sie erleben sich in vielfältigen Kontexten mit negativen Zuschreibungen belegt ${ }^{129}$. Darauf verweist auch die Charakterisierung ressourcenorientierter Zugänge zur eigenen Person im Erfahrungsbereich von Frauen mit Lernschwierigkeiten als besondere, also nicht alltägliche Erfahrungen. Über diese Zusammenhänge werden auch Handlungsperspektiven deutlich, die sich aus einem ressourcenorientierten Zugang eröffnen. Auf Basis der Entwicklung eines positiven Selbstbildes, die mit dieser Erfahrung gefördert wird, ist kontinuierliche Weiterentwicklung möglich. Es werden dann mitunter Perspektiven als realistisch eingeschätzt, die in einem defizitorientierten Kontext kaum in Erwägung gezogen werden. Ressourcenorientierung im Umfeld aktiviert auf diese Weise Ressourcenorientierung im Selbstbild. Dies ermöglicht es Frauen mit Lernschwierigkeiten auch, die eigene Beeinträchtigung vor dem Hintergrund gesellschaftlicher Zusammenhänge und Prozesse $\mathrm{zu}$ reflektieren. Auch defizitorientierte Zuschreibungen aus dem sozialen Umfeld können relativiert werden, wenn der behindernde und einschränkende Teil der Umfeldbedingungen selbst wahrgenommen werden kann $^{130}$.

129 So sehen sich u.a. Marlene im Arbeitsprojekt (vgl. IMarl 671-673) und Tamara in der Regelschule (vgl. IT 155) mit der Zuschreibung, ,zu langsam“ zu sein, konfrontiert.

130 Tamara beginnt auf Basis eines zunehmenden ressourcenorientierten Zuganges von Bezugspersonen auf die eigene Person ihre Beeinträchtigung im Zusammenhang mit hemmenden 
Die Förderung eines positiven Selbstbildes durch einen ressourcenorientierten Zugang von Personen aus dem sozialen Umfeld legt also einen Grundstein für eine aktive Art des Umganges mit herausfordernden Situationen, womit Entwicklungsperspektiven sichtbar werden. Dies wirkt sich insgesamt förderlich auf die Entwicklung einer Orientierung mit dem Fokus auf Selbstbestimmung aus. Wahrgenommenes Interesse an der eigenen Person verbunden mit erlebter Anerkennung führt aus Perspektive von Frauen mit Lernschwierigkeiten zu Selbstbewusstsein, Selbstvertrauen und ,macht [...] stark" (7RGS-S.14). Die dargestellten Zusammenhänge decken sich an dieser Stelle auch mit den Ergebnissen der Resilienzforschung, in der Ressourcenorientierung als Schutzfaktor hinsichtlich der Bewältigung schwieriger Lebensbedingungen eingeschätzt wird (vgl. Witteck 2014: 41).

\section{Die Umsetzung und Wahrnehmung von Selbstbestimmung wird durch die Qualität der Beziehungen zu Personen aus dem sozialen Umfeld beeinflusst}

Im Untersuchungskontext bilden Beziehungen, die von Respekt, Anerkennung, Interesse und einem ressourcenorientierten Zugang gekennzeichnet sind, einen Rahmen, in dem das Selbstbild junger Frauen mit Lernschwierigkeiten und dadurch vielfältige Entwicklungsmöglichkeiten gefördert werden. Somit wirken sich in diesem Sinne qualitätsvolle Beziehungen als allgemein förderlich auf die Entwicklung einer Orientierung in Richtung Selbstbestimmung aus. Herausforderungen können auf der Basis des dadurch gestärkten Selbstvertrauens aktiv angenommen werden. In förderlichen Beziehungen wird auch erforderlicher Bedarf an Unterstützung thematisiert und dazu beigetragen, dass diese real auch zur Verfügung gestellt wird. Förderliche Beziehungen zeichnen sich auch dadurch aus, dass sie über interaktive Prozesse der Auseinandersetzung über spezifische Themen bzw. in Krisensituationen Weiterentwicklung initiieren und befördern. Indem Frauen mit Lernschwierigkeiten im Kontext solcher Beziehungen als Personen mit eigenen Wünschen, Bedürfnissen und Vorstellungen wahrgenommen werden, wird darüber auch Wachstum möglich. Nicht nur die betroffene Frau, auch an diesem Prozess beteiligte Personen können aus konkret damit verbundenen Situationen mit neuen Perspektiven herausgehen. Auch an dieser Stelle erweisen sich die im vorliegenden Forschungskontext erhobenen Befunde als kompatibel zu Befunden, wonach Resilienz in engem Zusammenhang mit der Qualität sozialer Beziehungen steht (vgl. Witteck 2014: 45). Wertschätzung, Respekt und Akzeptanz werden dabei als in Krisensituationen schützende Faktoren eingeschätzt (vgl. Fröhlich-Gildhoff/Ronnau-Böse 2014: 27). Über „Dialog

und förderlichen Umfeldbedingungen kritisch zu reflektieren. Aus dieser Perspektive vermag sie in der Folge auch die Zuschreibung als „zu langsam“ kritisch einzuschätzen und demgegenüber die Umfeldbedingungen, die zu dieser Charakterisierung führten, als nicht passend zu bewerten. 
und Anerkennung“, die solche Beziehungen prägen, werden „erstaunliche Kompetenzen und Entwicklungen" möglich (vgl. Jantzen 2004: 155). Gute Beziehungen werden auch als Grundvoraussetzung persönlicher „Entwicklungs- und Lernprozesse“ eingeschätzt (vgl. Prengel 2013: 123). Für Frauen mit Lernschwierigkeiten können akute Belastungssituationen wie etwa sexuelle Gewalterfahrungen in solchen Beziehungen besser verarbeitet werden. Qualitätsvolle Beziehungen können aber auch präventiv gegen sexuelle Gewalt wirken, worauf ich im Verlauf der weiteren Ausführungen noch differenzierter eingehe.

\section{Hierarchische Strukturen und davon geprägte Interaktionen erschweren Selbstbestimmung}

Die Auswertungsergebnisse verweisen an mehreren Stellen darauf, dass sich hierarchische Strukturen, die von einem unausgewogenen Machtverhältnis charakterisiert sind, hemmend auf das Selbstbild, wahrgenommene Handlungsspielräume und Entwicklungsperspektiven junger Frauen mit Lernschwierigkeiten auswirken. In solchen Kontexten kann auch das Gefühl, ,wie Luft “'131 behandelt zu werden, entstehen, was sich in weiterer Folge sehr negativ auf den eigenen Selbstwert und die Orientierung auswirkt. Die Erfahrung von „Ignoranz, Ausgrenzung und Kränkung“ (Prengel 2005: 28) wird auch allgemein im Kontext der Schule als durch hierarchische Strukturen mitbegründet und als "Schulversagen“ begünstigender Faktor eingeschätzt (ebd.). Rückmeldungen, die sich primär ,,auf nicht Gekonntes, nicht Erbrachtes, über Fehler, Schwächen und Lücken“ (Popp 2011: 135) beziehen, führen bei Schülerinnen und Schülern zum Eindruck ,grundsätzlich defizitär, unvollständig und fehlerhaft zu sein“ (ebd.).

Solche Strukturen sind häufig auch von Interaktionen gekennzeichnet, die von den betroffenen Frauen als respektloses, ablehnendes und zuweilen auch als unangemessenes Verhalten ${ }^{132}$ wahrgenommen werden. Respektloses Verhalten wird auch als „Demütigung und Missachtung [...] der Persönlichkeit““ erlebt (Popp 2011: 135). Im Forschungskontext wird insbesondere ablehnendes Verhalten als Barriere, mit Schlüsselpersonen aus dem sozialen Umfeld in Beziehung zu treten, wahrgenommen und als einschränkend erlebt. Das damit einhergehende Gefühl, ,nicht den Status eines vollwertigen Interaktionspartners zu haben“" (Horster 2009: 154), verweist auch darauf, dass Frauen

131 So beschreibt etwa Anna explizit das mangelnde Interesse der Lehrerin an ihrer Person mit dem Bild, von dieser ,wie Luft“ (IA 314) behandelt worden zu sein. Sie bewertet in der Folge die Regelschule als Kontext, in dem sie diese Erfahrungen gemacht hat, negativ und orientiert sich am geschützten Rahmen der Sonderschule, in dem sie Interesse und Anerkennung erfährt.

132 Jessica etwa bewertet das Verhalten und den Kommunikationsstil ihrer Chefin, die maßregelnd und ablehnend agiert bzw. kommuniziert, als „nicht o.k.“ (IJ 268). 
mit Lernschwierigkeiten in solchen Kontexten häufig nicht soziale Wertschätzung und rechtliche Anerkennung ${ }^{133}$ erhalten.

Hierarchische Beziehungen sind für Frauen mit Lernschwierigkeiten auch mit angedrohten oder real umgesetzten Sanktionen verbunden. Im Kontext emotionaler und materieller Abhängigkeit können Bezugspersonen übermächtig erscheinen, wenn sie unerwünschtes Verhalten, also solches, das nicht deren Erwartungen oder Vorgaben entspricht, sanktionieren oder mit Sanktion bedrohen ${ }^{134}$. Dies führt häufig zu angepasstem Verhalten, um neuerliche Sanktionen oder die Umsetzung angedrohter Sanktionen zu vermeiden. Dieser Mechanismus ist umso wirksamer, je stärker die Abhängigkeit ist und je stärker das hierarchische Gefälle ausgeprägt ist.

\section{Finanzielle Abhängigkeit fördert hierarchische Strukturen und erschwert Selbstbestimmung in zentralen Lebensbereichen}

Finanzielle Abhängigkeit stellt einen zentralen Faktor dar, der das Leben von Frauen mit Lernschwierigkeiten auf vielfältige Weise und auf verschiedenen Ebenen determiniert. Durch finanzielle Abhängigkeit werden Perspektiven der Weiterentwicklung eingeschränkt. Ablösung aus Kontexten, die nicht den eigenen Vorstellungen entsprechen, erscheint vor diesem Hintergrund erschwert oder gar unrealistisch. In der Folge begründet dies häufig eine Orientierung am vorgegebenen Rahmen und an den darin geltenden Regeln.

Hierarchische Strukturen werden dabei nicht nur durch finanzielle Abhängigkeit an sich, sondern auch über speziell damit einhergehende Abläufe bzw. Mechanismen gefördert. So begründet etwa der Auszahlungsmodus für Taschengeld, das von den Eltern bezogen wird, häufig ein Eltern-,,Kind“"Verhältnis, das von einigen Frauen auch so erlebt wird und das zu Unbehagen und Unzufriedenheit führen kann. Dieses hierarchische Gefälle wird sprachlich über die Metapher des „Zusteckens“ geringer finanzieller Mittel dokumentiert (IMarl 765, ISo 199-201). Unzufriedenheit mit Lebensbedingungen, die von finanzieller Abhängigkeit geprägt sind, entsteht vor allem auch bei Frauen, die etwa das in Werkstätten ausbezahlte „Taschengeld“ als nicht angemessen ${ }^{135}$ für die erbrachte Leistung erleben.

Finanzielle Abhängigkeit führt in Kombination mit den sich daraus entwickelten hierarchischen Strukturen bzw. ungleich verteilten Machtverhältnis-

133 Soziale Wertschätzung und rechtliche Anerkennung sind zwei Faktoren, denen im Anerkennungskonzept Axel Honneths zentrale Bedeutung zukommt (vgl. Horster 2009: 154).

134 Für Angelika führen Maßregelungen und die Vorstellung angedrohter Sanktionen durch die Eltern dazu, deren Vorgaben immer entsprechen zu wollen (IAng 333-334). Melanie erfährt reale Sanktionen und passt sich mehr und mehr an, um neuerliche Sanktionen zu vermeiden (IMe 105-114).

135 So bewertet Jessica die Auszahlung eines geringfügigen Taschengeldes für ihre geleistete Arbeit mit ,nicht in Ordnung“ (IJ 329). 
sen dazu, dass die Selbstbestimmung von Frauen mit Lernschwierigkeiten in vielen Lebenszusammenhängen erschwert wird. Häufig bleiben über finanzielle Abhängigkeit auch Perspektiven wie autonomes Wohnen außerhalb des Blickfeldes oder erscheinen nicht realisierbar. Auch Möglichkeiten der Freizeitgestaltung und der Führung sozialer Kontakte werden durch finanzielle Abhängigkeit erschwert. Finanzielle Abhängigkeit kann somit auch einschränkend auf soziale Teilhabe wirken.

Finanzielle Abhängigkeit in Kombination mit hierarchischen Strukturen äußert sich aber auch darin, dass in Angelegenheiten, die die Gestaltung des eigenen Äußeren betreffen, starker Einfluss wahrgenommen wird. Dies dokumentiert sich etwa über den Einkauf von Bekleidung, wenn diese nicht entsprechend den eigenen Vorlieben, sondern auf Grundlage der Präferenzen von Personen aus dem sozialen Umfeld, die diese Einkäufe dann in der Regel auch finanzieren, ausgewählt wird. Die Auswahl der Bezugspersonen entspricht dabei häufig nicht den Vorstellungen der betroffenen Frauen selbst. Sie basiert auch auf Überlegungen, mit denen Personen aus dem sozialen Umfeld sich etwa Schutz vor sexuellen Übergriffen erhoffen, schränken aber die Selbstbestimmung der betroffenen Frauen $\operatorname{ein}^{136}$. Damit verbunden erscheinen auch die Möglichkeiten, über die Gestaltung des Äußeren die eigene Persönlichkeit zum Ausdruck kommen zu lassen, eingeschränkt.

Finanzielle Unabhängigkeit hingegen eröffnet Spielraum, sich auch hinsichtlich der Gestaltung des eigenen Äußeren zu erproben und zu inszenieren. Eigenes Einkommen stellt damit auch die Basis für selbstbestimmte Entscheidungen hinsichtlich der Gestaltung des eigenen Äußeren dar ${ }^{137}$.

\section{Die Selbstbestimmung von Frauen mit Lernschwierigkeiten liegt im Einflussbereich vielfältiger gesellschaftlicher Kontrollmechanismen}

Dem im Theorieteil der Arbeit ausgeführten Befund, dass Frauen mit Behinderung vor allem im Zusammenhang mit gesellschaftlichen Schönheitsnormen, Fragen der Ausbildung und Erwerbsarbeit und solchen in Zusammenhang von Beziehungen, Sexualität und Mutterschaft ,,immer wieder Fremdbestimmung und Diskriminierung“ (Franz 2002: 56) ausgesetzt sind, muss auf der Grundlage der vorliegenden Forschungsergebnisse speziell für die

136 Dieser Zusammenhang wird von den Frauen aus der Referenzgruppe hergestellt, die vor allem zwei Intentionen von Personen aus dem sozialen Umfeld dahinter wahrnehmen, die allerdings nicht explizit zur Sprache gebracht werden: einerseits sexuelle Gewalthandlungen durch unauffällige Kleidung zu verhindern, aber auch die mögliche Anbahnung von Kontakten zu verhindern. Die damit einhergehenden Versuche, Einfluss auf die Gestaltung des Äußeren zu nehmen, werden als Eingriffe in ganz persönliche Entscheidungen wahrgenommen und vehement abgelehnt (vgl. 1RGS-S1f.).

137 So erzählt etwa Eva davon, dass sie im Zuge des autonomen Wohnens und der Erwirtschaftung eines eigenen Einkommens entgegen den Vorstellungen ihres Vaters Piercings und Tatoos machen ließ (IE 349-350). 
Situation von Frauen mit Lernschwierigkeiten bestätigt werden. Wie bereits oben ausgeführt, werden mit dem eingeschränkten Zugang zu Erwerbsarbeit finanzielle Abhängigkeiten begründet. Durch die „Berufswahleinschränkung“ aufgrund der Behinderung, die „Berufswahleinengung“ aufgrund des Geschlechts und die „Berufswahlbegrenzung aufgrund der Verschränkung der beiden Kategorien" (Bretländer/Schildmann 2010: 42) verbleiben die Frauen häufig im Einfluss- und Kontrollbereich von Personen aus ihrem sozialen Umfeld.

Dass familiäre Reproduktionsarbeit Frauen mit Lernschwierigkeiten weitgehend verschlossen bleibt (vgl. Schildmann 2004: 42), dokumentiert sich ebenfalls an den vorliegenden Forschungsergebnissen. Schon früh wird ihnen im Rahmen der Sozialisation vermittelt, „, dass sie nicht schön und begehrenswert sind und die klassische Frauenrolle als Partnerin und Mutter für sie nicht in Frage kommt" (Köbsell 2010: 21). Auch die Frage der Körperlichkeit von Frauen mit Lernschwierigkeiten unterliegt somit stark gesellschaftlichen Kontrollmechanismen. Der Körper wird eher als „Objekt medizinischer und therapeutischer Fürsorge“ (Middendorf 2010: 209), denn als Mittel zur Selbstwahrnehmung und des Ausdrucks der eigenen Persönlichkeit ${ }^{138}$ gesehen. Fragen in Zusammenhang mit Partnerschaft, Sexualität, Familie und Kinderwunsch wurden von den interviewten Frauen wohl auf Basis dieser Erfahrungen vorwiegend implizit zur Sprache gebracht, was auch darauf verweisen dürfte, dass es sich dabei um tabuisierte Bereiche handelt, die im Kontext ihrer sozialen Interaktionen ausgespart bleiben. Explizit wurden Partnerschaft und Kinderwunsch von Frauen mit Lernschwierigkeiten im Interview vorrangig mit Unterstützung des Einsatzes visueller Vignetten als Erzählaufforderung thematisiert. Es ist vor dem Hintergrund vorhandener Wünsche und Vorstellungen im Zusammenhang mit Partnerschaft und Kinderwunsch und der gesellschaftlichen Tabuisierung und Kontrolle dieser Bereiche davon auszugehen, dass dadurch bei den betroffenen Frauen latente Unzufriedenheit und Identitätsprobleme entstehen (vgl. Reiss 2007: 58) können. Eine Auseinandersetzung über Themenstellungen im Rahmen sozialer Interaktionen in Beziehungen, die von Respekt, Anerkennung, Interesse und einem ressourcenorientierten Zugang gekennzeichnet sind, ermöglichen hingegen eine Reflexion eigener Wünsche, Bedürfnisse und Vorstellungen auf einer realistischen Ebene und somit auch persönliche Weiterentwicklung.

Selbstbestimmung von Frauen mit Lernschwierigkeiten wird aus Perspektive betroffener Frauen auch durch eine defizitorientierte Sichtweise durch Personen aus dem sozialen Umfeld untergraben. Männern mit Lernschwierigkeiten wird demnach aus ihrer Perspektive von ihrem sozialen Umfeld mehr zugetraut und sie entwickeln darüber mehr Mut. Frauen hingegen wür-

138 Diese Bedeutung macht Eva im Interview deutlich, indem sie ihre Tatoos und Piercings als zentrales Mittel, ihre Befindlichkeit auch im sozialen Kontext über ihr Äußeres zum Ausdruck zu bringen und Veränderungsprozesse zu dokumentieren, beschreibt (IE 377-383). 
den öfter defizitorientierte Zuschreibungen erleben, die sie verunsichern und zur Aufgabe von Vorhaben bringen würden (vgl. 2RGS-S3f.). Entwicklungsperspektiven in Richtung Autonomie und Selbstbestimmung erscheinen aus dieser Perspektive eingeschränkt (vgl. 2RGS-S5).

\section{Selbstbestimmung von Frauen mit Lernschwierigkeiten erfordert präventive Maßnahmen im Hinblick auf sexuelle Gewalterfahrungen}

Wie die Forschungsergebnisse zeigen, wirken sich Erfahrungen sexueller Übergriffe soziogenetisch auf die Entwicklung und die Ausprägung der Orientierungen junger Frauen mit Lernschwierigkeiten aus. Sie wirken an sich schon massiv negativ auf das Befinden und das Selbstbild ${ }^{139}$ der betroffenen Frau. Sexuelle Übergriffe gehen ,mit einer ausgeprägten Verletzung des Rechts auf Selbstbestimmung“ (vgl. Popp 2002: 158) einher. Wie die Ergebnisse der Arbeit dokumentieren ${ }^{140}$, kann im Zusammenspiel mit der Anzweiflung der Glaubwürdigkeit und dem Vorenthalten von Unterstützung eine Orientierung an einem geschützten, vertrauten Rahmen eingeleitet werden. Mögliche zukunftsgerichtete Entwicklungsschritte werden dadurch jedenfalls erschwert.

In diesem Zusammenhang erweist sich die Förderung einer Orientierung junger Frauen mit Lernschwierigkeiten, die in Richtung der Anpassung an vorgegebene Strukturen weist, als kontraproduktiv. Durch die damit verbundene Orientierung an den Vorstellungen und Wünschen Dritter sind im Anlassfall kaum Strategien des Widerstandes verfügbar. Frauen mit Lernschwierigkeiten werden vorrangig solche Präventionsstrategien nähergebracht, die diese in ihrem Handlungsspielraum einschränken, etwa sich nicht aufreizend $\mathrm{zu}$ kleiden ${ }^{141}$ oder Kontexte $\mathrm{zu}$ vermeiden, in denen Übergriffe vermutet werden. Damit verbunden scheint es dringend erforderlich, möglichen Schuldgefühlen im Kontext mit Gewalterfahrungen, die auch über Aussagen aus dem sozialen Umfeld entstehen können, entgegenzuwirken. Demgegenüber ist die alleinige „Verantwortung beim Täter oder der Täterin“ (Hug 2004: 166) zu verorten. Präventionsarbeit ist auch eng mit dem Ausdrücken eigener Wünsche und Bedürfnisse, mit der Möglichkeit des Verneinens von Ansprüchen aus dem sozialen Umfeld und somit mit Selbstbestimmung verbunden. Eine dementsprechende „Erziehung zum Ungehorsam, zum Nein-

139 So führt Anna aus, sie habe sich dabei ,als Frau total elend“ (IA 435), ,wie der letzte Dreck" (IA 440) gefühlt.

140 Siehe dazu ausführlich die Fallrekonstruktion „Anna“, insbesondere Unterpunkt „Erfahrungen sexueller Übergriffe beeinträchtigen Streben nach Autonomie und Selbstbestimmung“.

141 Dies dokumentiert sich etwa über Annas Beschreibung, dass sie ja gar keine aufreizende Kleidung getragen hätte (IA 432-433), die zur Gewalthandlung angeregt hätte. Angelika entspricht diesen Vorgaben, indem sie ausführt, ,ja, ich kauf' aber nur Sachen, die was oben zu sind und hinten"(IAng 705). 
Sagen“ (Walter 2008: 24) erscheint dabei als besonders wirksame Präventionsmaßnahme (ebd.).

Aussagen über Fremd- und Selbstbestimmung von Menschen

mit Lernschwierigkeiten erfordern die Berücksichtigung

ihrer eigenen Perspektive

Aus den vorab angestellten Überlegungen geht als Schlussfolgerung, die sich auf die Situation von Menschen mit Lernschwierigkeiten allgemein übertragen lässt, hervor, dass Aussagen über Fremd- und Selbstbestimmung von Menschen mit Lernschwierigkeiten immer der Berücksichtigung ihrer eigenen Perspektive bedürfen.

Menschen mit Lernschwierigkeiten selbst vermögen aus dieser Perspektive Faktoren wahrzunehmen, die Selbstbestimmung fördern, hemmen oder gar fremdbestimmend wirken. Daraus werden vielfältige praxisrelevante Schlussfolgerungen möglich, die auf die Ermöglichung von Selbstbestimmung für Menschen mit Lernschwierigkeiten abzielen.

Schlussfolgerungen hinsichtlich der Bedeutung dieses Befundes für den Kontext partizipativer Forschung stelle ich nach den nun folgenden Anmerkungen zur dokumentarischen Methode als Auswertungsmethode, die im Kontext der vorliegenden Arbeit zum Einsatz kam, an.

\subsection{Methodische Anmerkungen}

\subsubsection{Die dokumentarische Methode im Kontext partizipativer Forschung: Erfahrungen aus der vorliegenden Studie und allgemeine Schlüsse}

Im Kontext der vorliegenden Arbeit wurden mit Hilfe der Auswertungsschritte der dokumentarischen Methode Handlungsmuster und Orientierungen von Frauen mit Lernschwierigkeiten erhoben. Die dokumentarische Methode erwies sich dabei als eine Methode der Auswertung, die die Befragten über ihre Analyseschritte „vom Objekt zum Subjekt“ (Kaiser/Kočnik/Sigot: 2004) macht, indem selbst dann Handlungsspielraum sichtbar wird, wenn dieser zunächst aus der Außenperspektive auch als eingeschränkt erscheint. Die Frau mit Lernschwierigkeiten wird im Auswertungskontext als agierendes Subjekt wahrgenommen, das in Interaktion steht und mitgestaltet. Frauen mit Lernschwierigkeiten werden vor diesem Hintergrund auch nicht von vornherein als fremdbestimmte Personen wahrgenommen. Ein solcher Zugang ist bereits von seinem Ansatz her defizitorientiert und geht mit der Gefahr der Stigmatisierung einher. Es wird vielmehr nach dem Anteil der Frauen selbst 
im Spannungsfeld von Fremd- und Selbstbestimmung gefragt. Auf dieser Basis werden auch Räume möglicher selbstbestimmter Entscheidungen sichtbar. Dabei stehen soziogenetische Prozesse im Blickpunkt der Auswertung, d.h. es wird danach gefragt, wie es zu verschiedenen Orientierungen kommen kann. Dieses Wissen kann dazu beitragen, etwas an den Rahmenbedingungen ihres Zustandekommens zu ändern oder wahrzunehmen, wo eine Stärkung der betroffenen Frauen erfolgen könnte.

Mit den Analyseschritten der dokumentarischen Methode wird es möglich, das konjunktive Wissen, das ,eng mit der spezifischen Praxis von Menschen in Biographien und ihren Milieus verknüpft ist" (Nohl 2012: 5) und das Individuen, die diese Erfahrungen in einem sogenannten ,konjunktiven Erfahrungsraum“ (Przyborski 2004: 29) machen, teilen, zum Ausdruck zu bringen. Dieses konjunktive Wissen wird auch als Basis der Analysen ganz zentral berücksichtigt. So erfolgen im Rahmen der reflektierenden Interpretationen fallübergreifende Vergleiche nicht mehr vor dem Erfahrungshintergrund der Forscherin, der ja von ihrem theoretischen Zugang, bisherigen Ergebnissen ihrer Forschung und praktischen Erfahrungen in Beruf und Gesellschaft geprägt ist. Die Forscherin mit akademischem Hintergrund verfügt dabei zudem nicht über das konjunktive Wissen von Frauen mit Lernschwierigkeiten. Die Interpretationen erfolgen demgegenüber vor dem Erfahrungshintergrund von Frauen mit Lernschwierigkeiten selbst. Der Erfahrungshintergrund der akademischen Forscherin wird nach und nach über den Einbezug immer weiterer Interviews in die Vergleiche durch Erfahrungshintergründe anderer Frauen mit Lernschwierigkeiten ersetzt.

Die dokumentarische Methode kann meiner Einschätzung nach auch aus dem Grund als sehr zielführende Auswertungsmethode im Rahmen partizipativer Forschung gesehen werden, da über ihre speziellen Auswertungsschritte die Akteure und Akteurinnen als Expertinnen und Experten für Fragestellungen anerkannt werden, die sie selbst betreffen. Sie verhilft dazu, ,ein den Erforschten bekanntes, von ihnen aber nicht expliziertes handlungsleitendes (Regel-)Wissen (abduktiv) zur Explikation zu bringen“ (Bohnsack/NentwigGesemann 2007: 12). Einem zentraler integrationspädagogischen und inklusiven Ansatz, Akteurinnen und Akteure als erste Expertinnen in eigener Sache anzuerkennen, wird somit im Rahmen dieser Auswertungsmethode Genüge getan. In Kombination mit den Vorteilen partizipativer Forschung erweist sie sich gerade im vorliegenden Forschungszusammenhang als sehr stimmige Methode mit dem Anspruch, keine Aussagen über Menschen mit Lernschwierigkeiten ohne deren Einbezug selbst zu tätigen.

Durch die darüber hinausgehende Teilhabe von Frauen der Referenzgruppe auch am Auswertungsprozess kann gewährleistet werden, dass die Ergebnisse einerseits bereits deren Perspektive beinhalten, indem bei den Analysen auf Interpretationen durch selbst dem erforschten Personenkreis angehörige Frauen zurückgegriffen werden kann. Dadurch kann neben der Beachtung der 
Vergleichshorizonte selbstbetroffener Frauen im fallübergreifenden Vergleich auf einer zweiten Ebene der Gefahr entgegengewirkt werden, dass die Interpretationen durch Orientierung am Erfahrungshintergrund der Forscherin nicht die Orientierungen erfassen, die im Sinne konjunktiven Wissens von Frauen mit Lernschwierigkeiten zum Ausdruck gebracht werden sollen.

\subsubsection{Anmerkungen zur Anregung von Erzählungen im Interview über visuelle Vignetten und zu deren Auswertung mit der dokumentarischen Methode}

Die im Rahmen der Interviews eingesetzten visuellen Vignetten hatten das Ziel, einen nonverbalen Stimulus zu setzen, der die Frauen mit Lernschwierigkeiten zu Erzählungen anregen sollte. Die darüber entstandenen Passagen wurden als Bestandteile der Interviews in die allgemeine Auswertung mit einbezogen, d.h. sie wurden wie alle anderen Interviewpassagen den Analyseschritten der dokumentarischen Methode unterzogen.

Die dokumentarische Methode wird auch zur Analyse von Bildern vor allem hinsichtlich der Frage von Medienrezeption herangezogen. Im Rahmen der vorliegenden Arbeit steht jedoch nicht dieser spezielle Kontext im Fokus, sondern die Frage nach der Eignung dieses Ansatzes zur Generierung von Erzählungen. Hinsichtlich der theoretischen Überlegungen zum Einsatz der Methode wurde im Rahmen der Interviews und deren Auswertung deutlich, dass Bilder auch in diesem Kontext als „Anlass für Sinnkonstruktionen“" fungieren, die „offen für unterschiedliche Sinnzuschreibungen“ (Michel, 2007: 93) sind. Die dokumentarische Methode ermöglicht es über ihre differenzierten Analyseschritte, diese Sinnbildungsprozesse zu rekonstruieren (vgl. ebd.: 94). Dabei erschließt sich der „habitusspezifische Sinn" ${ }^{\text {“142 }}$ nicht so sehr darüber, was geäußert wird, ,sondern wie sich die Personen (szenisch, metaphorisch, praktisch u.a.) zu einem Bild ins Verhältnis setzen“(ebd.: 97). Diese Prozesse laufen in der Regel unbewusst ab, der Sinn der Ausführungen „,...] schleicht sich ,hinter dem Rücken' der Rezipienten in ihre Auseinandersetzungen mit einem Bild ein“" (ebd.: 97).

Die Vignetten-Methode erwies sich im Kontext der Durchführung der erzählgenerierenden Interviews als sehr hilfreich. Erst über den visuellen Stimulus thematisieren manche Frauen Fragen in Zusammenhang mit Themen, die auf erzählgenerierende Fragen nicht angesprochen wurden. Insbesondere Fragen in Zusammenhang mit Partnerschaft konnten mithilfe anschaulicher Vignetten, die als visuelle Erzähl- bzw. Assoziationsaufforderung gedacht und auch so genutzt wurden, thematisiert werden. Auch Frauen, für die verbale Erzählaufforderungen offenbar also nicht den optimalen Stimulus für die

142 Das konjunktive Wissen, das Personen, die über einen gemeinsamen Erfahrungshintergrund verfügen, verkörpert sich im „Habitus“ (vgl. Bohnsack 2013: 179). 
Thematisierung solcher Fragen darstellten, konnten über diese Ebene zu Erzählungen angeregt werden. Dabei wurden über den visuellen Stimulus solche Orientierungen auch bei Frauen, die im Kontext des Gesamtinterviews eher am Alltagsgeschehen orientiert waren, erstaunlich deutlich gemacht. So dokumentieren Glorias Ausführungen auf verbale Erzählaufforderungen Zufriedenheit mit dem Status quo, Fragen in Zusammenhang mit eigenen Lebensperspektiven im Kontext von Partnerschaft oder Familie werden nicht thematisiert. Über den visuellen Erzählstimulus des selbst ausgewählten Bildes, das ein offensichtlich beeinträchtigtes, für Gloria auf den ersten Blick „verlobtes“ Paar zeigt, kommt sie auf eine andere Ebene, wie sich anhand der folgenden Passage dokumentiert:

I: Und was glauben sie denn? Wie geht denn diese Geschichte weiter? Die sind jetzt verlobt und wie geht ihr Leben dann weiter?

M: Bekommen Kinder.

I: Die bekommen Kinder.

M: Ja, und das habe ich gerne, weil ich- ( ) gerne ein kleines Baby haben, ich". (IG 662666)

Somit eröffnet die visuelle Erzählaufforderung Zugang zu Gedanken und Perspektiven, die offenbar unbewusst, aber im Sinne von Phantasien vorhanden sind und darüber artikuliert werden können.

Auch im Interview mit Anna wird anhand von zwei Vignettenbeschreibungen eine zentrale Orientierung deutlich, nämlich jene nach einem Lebensentwurf, der sich an als von ihr wahrgenommener gesellschaftlicher „Normalität" orientiert und Familie und Kinder beinhaltet:

„[...] also ganz normale Familie gründen und das alles drum und dran.“ (IA-VA5 901)

„Also, dass sie halt eine glückliche Zukunft vor sich halt haben und das. Also ja, ganz normal Familie gründen, normal leben, arbeiten gehen." (IA VA6 904-905)

Auch bei Eva fokussiert sich über die Vignettenbeschreibung eine zentrale Orientierung. Für sie stellt die dargestellte Frau im Rollstuhl am Meer ein „klassisches Bild [...] für Freiheit" (IE 581) dar. Eva bringt dies mit ihrer eigenen Orientierung im Zusammenhang mit autonomem Leben und Selbstbestimmung in Verbindung:

„So quasi ich bin für's Leben bereit, alles, was kommt, wurscht was kommt, ich will's versuchen anzunehmen. Und das Meer spiegelt für mich sehr gut wieder was an=an=an=an Sachen auf dich zukommen können, das heißt manchmal sehr schwungvoll, manchmal sehr schmerzhaft, aber auch manchmal ganz ruhig, und das ist - das ist der Inbegriff von Freiheit für mich.“ (IE VA7 582-586)

Die angeführte Passage stellt in ihrer ,,metaphorischen Dichte“, dem „,hohen Detaillierungsgrad" und der prägnanten Ausdrucksweise auch ein Beispiel für eine sogenannte „Fokussierungsmetapher“ dar (vgl. Bohnsack 2007: 225). Diesen Passagen wird in der dokumentarischen Methode generell hoher Stel- 
lenwert zugewiesen, da sie auf eine hohe Bedeutung der darüber angesprochenen Themen für die interviewten Personen schließen lassen.

Konkret dokumentieren sich über die Vignettenbefragung im vorliegenden Forschungskontext besonders deutlich zentrale Orientierungen im Zusammenhang mit Selbstbestimmung, Autonomie, Partizipation und „Normalität“. Dies insbesondere auch im Zusammenhang mit einem als gesellschaftliche „Normalität“ wahrgenommenen Lebensentwurf hinsichtlich Partnerschaft, Ehe, Sexualität und Kinderwunsch. Thematisiert werden über diese visuellen Stimuli auch damit verbundene Sehnsüchte, die zum aktuellen Zeitpunkt für die Frau wenig realisierbar scheinen. Auch Orientierungen im Zusammenhang mit der Frage sozialer Interaktionen kommen zur Sprache. Die visuellen Darstellungen führen auch zu Ausführungen, in denen das aktuelle Selbstbild und angenommene oder gewünschte Fremdbilder thematisiert werden. Diese Methode der Stimulierung von Erzählungen und deren Auswertung mittels der dokumentarischen Methode ist daher aus meiner Perspektive eine sehr zielführende und daher empfehlenswerte Ergänzung im Rahmen von Interviews mit Frauen mit Lernschwierigkeiten.

\subsubsection{Methodisch-inhaltliche und forschungsethische Anmerkungen zu partizipativer Forschung}

Wie in der Einleitung dargestellt, war es das erklärte Ziel der vorliegenden Forschungsarbeit, die Perspektive von Frauen mit Lernschwierigkeiten, die bislang in Forschungskontexten wenig berücksichtigt wurde, auf Fragen in Zusammenhang mit Selbstbestimmung zu erhellen. Umgesetzt wurde dies in einem partizipativen Forschungsprozess und auf zwei Ebenen: Einerseits indem vier Frauen mit Lernschwierigkeiten in die Planungs-, Erhebungs- und Auswertungsphase eines in die Arbeit eingebetteten qualitativen Forschungsprojektes mit einbezogen wurden. Damit konnte die Sichtweise von Frauen einer sogenannten Referenzgruppe, die selbst ,,in ihrer Sozialisation die Erfahrung des Behindert-Werdens gemacht“ (Flieger, 2007: 22) haben, zu Inhalten, Methoden und Ergebnissen der Forschung berücksichtigt werden. Über dieses gemeinsam geplante Projekt wurden auf einer zweiten Ebene Orientierungen junger Frauen mit Lernschwierigkeiten im Spannungsfeld von Selbst- und Fremdbestimmung über erzählgenerierende Interviews mit betroffenen Frauen erhoben und analysiert.

Der Einbezug einer Gruppe von Frauen mit Lernschwierigkeiten in die Planungs-, Erhebungs- und Auswertungsphase einerseits und die Adressatinnenorientierung der im Kontext der Studie durchgeführten Forschung andererseits sollten dabei gewährleisten, dass die Perspektive der von der Forschung betroffenen Personen umfassend in den gesamten Forschungsprozess integriert ist. Wie im Methodenteil der Arbeit und in der Ergebnisdarstellung 
näher ausgeführt, erfolgte die Beteiligung der Referenzgruppe dabei in unterschiedlichem Ausmaß in mehreren Phasen bzw. auf verschiedenen Ebenen der Forschung. Die Frauen aus der Referenzgruppe waren im Rahmen von sieben je dreistündigen Sitzungen in die Planung, Erhebung und Auswertung der Forschung einbezogen. Indem durch die Frauen aus der Referenzgruppe und mich als Forscherin mit akademischem Hintergrund zwei Perspektiven auf Inhalte, Methode und im Auswertungskontext eingenommen wurden, wurde im Forschungsprozess auch das Element der Triangulation umgesetzt. Im Forschungskontext bedeutet Triangulation, ,dass ein Forschungsgegenstand von (mindestens) zwei Punkten aus betrachtet wird" (Flick 2011: 323). Allgemein wird Triangulation in der qualitativen Forschung als Faktor eingeschätzt, der sich qualitätssteigernd auswirken kann (Mayring 2002: 147; Wiezorek/Fritzsche 2010: 140). Neben der Daten- und Theorietriangulation stellt auch die Interpretation durch verschiedene Personen, die sogenannte Investigator-Triangulation (vgl. Flick 2009: 310), einen möglichen Zugang dar. Im vorliegenden Forschungskontext wurde die Triangulation auch auf die Planungs- und Erhebungsphase ausgedehnt, in der neben mir selbst als Forscherin mit akademischem Hintergrund die Frauen aus der Referenzgruppe beteiligt waren. Die angeführten Positionen zu Triangulation legen nahe, dass aus dieser Perspektive partizipatorischer Forschung das Potential zukommt, sich förderlich auf die Qualität der Ergebnisse auszuwirken. Grafik 2 skizziert die Umsetzung von Triangulation im vorliegenden Forschungskontext ${ }^{143}$ :

Der damit umgesetzte mehrperspektivische Ansatz in Planung, Erhebung und Auswertung gewährleistet die Berücksichtigung von Faktoren, die auf andere Weise nicht oder nur unzureichend thematisiert werden könnten. Mit der Umsetzung partizipatorischer Forschung kann es somit „,zu einer Erweiterung der Erkenntnismöglichkeiten durch verschiedene Zugänge“ (Flick 2011: 323) kommen. Partizipativ zu forschen, bedeutet im methodisch-inhaltlichen Sinn also auch, eine mehrdimensionale Perspektive einzunehmen, was sich allgemein auf die Qualität der Ergebnisse förderlich auswirken kann.

Dieser partizipative Zugang bedeutet ganz zentral aber nicht nur aus methodischer Perspektive, dass die Position von Personen, die von der Forschungsfrage betroffen sind, im Forschungskontext berücksichtigt wird. Auch forschungsethische Fragen des (Macht-)Verhältnisses von forschenden Personen und Personen, die im Fokus der Forschung stehen (vgl. Flieger 2007: 19ff.), sind damit angesprochen.

Hier wird insofern ein Ausgleich angestrebt, als die Perspektive der Forschungsadressatinnen und -adressaten in allen Phasen der Forschung berücksichtigt wird. Dies beginnt bei der Überprüfung der Frage, ob der Forschungsgegenstand überhaupt im Interesse der betroffenen Personen ist. In

143 Siehe dazu auch die Übersichtstabelle im Methodenkapitel, die Inhalte und Ergebnisse der Partizipation der Frauen aus der Referenzgruppe im Detail darstellt. 
nteraktiven Prozess können im Rahmen partizipativer Forschung enfalls Neuorientierungen oder Modifizierungen der Forschungsfraten.

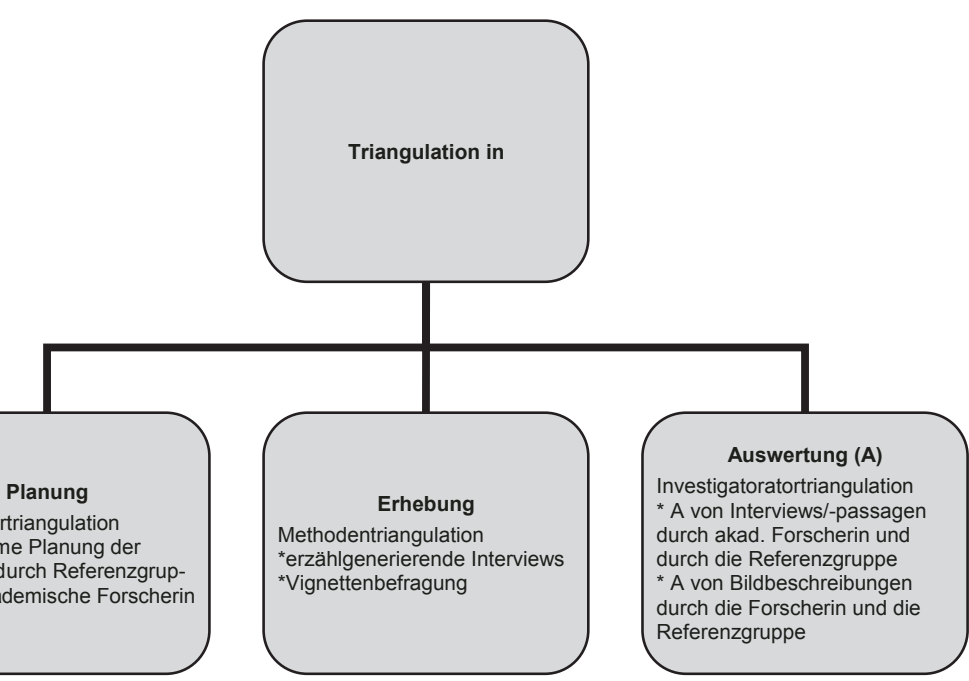

iangulation im vorliegenden Forschungsprojekt

ann gewährleistet werden, dass nicht an den Interessen der betroffeionen vorbei geforscht wird. Im Entwicklungsprozess im vorliegenschungsprojekt war für mich als akademische Forscherin dabei an n zentralen Punkten der Arbeit die Teilhabe der Frauen aus der Refepe von besonderer Bedeutung, indem dadurch das konjunktive WisFrauen mit Lernschwierigkeiten zum Ausdruck gebracht wurde. Nissen wurde mir u.a. in der Planungsphase über eine detaillierte zung zur aktuellen Lage von Frauen mit Lernschwierigkeiten im gsfeld von Fremd- und Selbstbestimmung zur Verfügung gestellt, Diskussionen und Reflexionen im Rahmen von drei Referenzgrupngen (RGS1, RGS2, RGS3) erfolgte. Dieses Wissen wurde von mir n Form eines komprimierten Übersichtspapiers (Dok1-RGS2) gestalder Referenzgruppe auf Korrektheit und Gültigkeit überprüft und von in die Erhebungen genommen. Dort diente es mir im Rahmen der ws mit Frauen mit Lernschwierigkeiten dazu, erzählgenerierende 
Orientierungsrichtlinie für diesen Prozess herangezogen werden und stellten somit sicher, dass ihrer Ansicht nach relevante Aspekte die Auswahl von Interviewpassagen bedingten. So wurde etwa das von der Gruppe als wichtig hervorgehobene Kriterium der Tagesstruktur, anhand derer sich ihrer Ansicht nach sehr deutlich Selbst- und Fremdbestimmung im Alltag von Frauen mit Lernschwierigkeiten zeigen können (vgl. 3RGS-S3), bei der Auswahl berücksichtigt. Diese Anregung der Referenzgruppe erwies sich dann in der Rückschau als sehr zielführend.

Im Zusammenhang mit der Entwicklung des Forschungsdesigns wurden auch methodische Fragen aus der Perspektive der Frauen aus der Referenzgruppe diskutiert und reflektiert. Als Erhebungsmethode präferierten sie nach Diskussion verschiedener anderer Möglichkeiten Einzelinterviews mit Frauen mit Lernschwierigkeiten. Der Einbezug anderer Personen als betroffener Frauen selbst in die Erhebung wurde von der Referenzgruppe eindeutig und vehement abgelehnt. Ihre Begründung dazu, wonach die Meinungen von Frauen mit Lernschwierigkeiten weitgehend unbekannt sei (vgl. 2RGS-S7), machte mir deutlich, wie sehr sich Frauen mit Lernschwierigkeiten in ihrer Lebensrealität als Personen wahrnehmen, die ihre Positionen als gesellschaftlich nicht wahrgenommen erleben. Den von ihnen geäußerten Anspruch umzusetzen, war aus dieser Perspektive für mich selbstverständlich und führte letztendlich zu Ergebnissen, die durch das Expertinnenwissen von Frauen mit Lernschwierigkeiten selbst hervorgebracht wurden.

Ein weiterer wichtiger Aspekt, dessen besondere Bedeutung von den Frauen aus der Referenzgruppe thematisiert wurde, war die Gestaltung des Zuganges zu Frauen mit Lernschwierigkeiten in der Interviewsituation. Hier wurden insbesondere die Notwendigkeit der Verwendung sogenannter „leichter Sprache" und die Bereitstellung ausreichender zeitlicher Ressourcen hervorgehoben. Zudem rieten die Frauen auch dazu, auf Anschaulichkeit zu achten, was letztendlich mit der Integration der Methode der Vignettenbeschreibung auch umgesetzt wurde.

Im Zuge des Auswertungsprozesses, in dem die Frauen aus der Referenzgruppe zunächst ausgewählte Interviewpassagen und Vignettenbeschreibungen der interviewten Frauen mit Lernschwierigkeiten interpretierten, wurde mir darüber Zugang zu Einschätzungen und Erfahrungen aus der Perspektive von der Forschung betroffener Frauen selbst eröffnet. Dies ermöglichte es mir auch, die Auswertung nach der dokumentarischen Methode mit der Abbildung ihres Erfahrungshintergrundes in den Interpretationen auf einer weiteren Ebene zu reflektieren und somit um deren Perspektive zu bereichern. Über einen weiteren Schritt - die Rückkoppelung von (Zwischen-)Ergebnissen an die Referenzgruppe - wurde auch dem ,partizipatorische[n] Anspruch nach kommunikativer Validierung“" (Schönwiese 2005: 56) Genüge getan. Die Ergebnisse wurden also daraufhin überprüft, ob sie als nachvollziehbar und stimmig mit den eigenen Erfahrungen und Einschätzungen ange- 
sehen wurden. In einer abschließenden Sitzung (7RGS-1-15) wurde die Relevanz der Forschungsergebnisse für Frauen mit Lernschwierigkeiten diskutiert. Die Einschätzung, wonach diese zu einer angemesseneren Form des Umganges mit Frauen mit Lernschwierigkeiten führen könnten, machte wiederum deutlich, dass diese Form des Umganges nicht immer als selbstverständlich erlebt wird.

Insgesamt wirkte sich die Arbeit mit den Frauen aus der Referenzgruppe im Forschungsverlauf für mich zunehmend stark wahrnehmbar auf mein eigenes Forschungsverständnis und meinen Zugang zur Forschung im gesamten Forschungsprozess - also in der Konzeptions-, Erhebungs- und Auswertungsphase - aus. Barrierefreiheit und möglichst selbstbestimmtes Agieren für die Probandinnen für möglichst jede Facette des Prozesses zu gewährleisten, war der daraus resultierende von mir an mich selbst erhobene Anspruch. Zudem wirkte sich die Teilnahme der Gruppe insofern auf meine Forschungstätigkeit aus, als ich durch gemeinsame Auswertungssitzungen und die laufende Rückkoppelung und Diskussion von Zwischenergebnissen an und mit der Gruppe, deren Perspektive zunehmend als unverzichtbar und zentral für mein eigenes Verständnis der thematisierten Fragen einzuschätzen begann.

Im Verlauf der Arbeit mit der Referenzgruppe und der Interviews im dargestellten Forschungsprojekt verfestigte sich mein bereits zuvor aus verschiedenen Zusammenhängen ${ }^{144}$ entstandener Zugang, die Kategorie „Behinderung" als gesellschaftliches Konstrukt zu sehen, das sich im Kontext persönlicher Beziehungen und gemeinsamer Arbeit an einer Sache als weitgehend bedeutungslos erweist. Auch der Begriff der Lernschwierigkeiten relativiert sich an dieser Stelle für mich, indem ich diesen im Kontext meiner Erfahrungen eher in einer Umkehr der Bedeutung als „Vermittlungs- oder Unterstützungsschwierigkeit“ bei Personen aus dem sozialen Umfeld betroffener Menschen verorten würde. Im Kontext universitärer Lehre und Forschung verweist dies auf den Anspruch, die eigene Tätigkeit vor allem in Hinblick auf Methoden und sprachliche Gestaltung hinsichtlich vorhandener Barrieren zu überprüfen und entsprechende Modifizierungen vorzuneh$\operatorname{men}^{145}$. Die Teilhabe von Frauen, die im Verlauf ihrer Sozialisation das Etikett einer Behinderung erhalten haben und die mit diesen Zuschreibungen

144 Diese Erfahrungen beruhen u.a. auf der Teilhabe von Menschen mit Lernschwierigkeiten in mehreren meiner Lehrveranstaltungen an der Alpen-Adria-Universität Klagenfurt (u.a. 2009, 2012 und 2014), verschiedenen Fortbildungsaktivitäten für Menschen mit Lernschwierigkeiten sowie der Leitung eines Arbeitskreises in leichter Sprache im Rahmen einer Tagung zur UN-Konvention für die Rechte von Menschen mit Behinderungen an der Universität Innsbruck im Jahr 2010.

145 Im Sinne des Anspruches nach „design-for-all“ würden diese Bedingungen im Übrigen auch anderen Personen den Zugang zu akademischen Inhalten erleichtern. 
leben müssen, stellte durchgängig eine Bereicherung für das Habilitationsprojekt dar, das ohne deren Beteiligung nicht realisierbar gewesen wäre.

Als Resumé möchte ich aufgrund meiner Erfahrungen mit partizipativer Forschung im vorliegenden Kontext festhalten, dass sich Forschung mit statt über Frauen mit Lernschwierigkeiten für mich als unverzichtbarer Zugang darstellt, um deren Perspektive auf Fragen im Kontext von Selbst- und Fremdbestimmung zu erheben. Diesen Ansatz zu integrieren, erachte ich für alle Fragen im Kontext mit der Situation von Menschen mit Behinderung allgemein für unverzichtbar. Denn durch die Teilhabe der von Forschung betroffenen Personen in der Planungs-, Erhebungs- und Auswertungsphase von Forschungsprojekten können empirisch von der Zielgruppe selbst validierte Perspektiven erhoben werden. Dies eröffnet auch neue Handlungsperspektiven in den Praxisfeldern von Sozial- und Integrationspädagogik, indem darüber Anhaltspunkte für eine mögliche Integration dieses Wissens im Sinne des Empowerments der Adressatinnen und Adressaten deutlich werden. Zudem können die Ansprüche auf Inklusion und Selbstbestimmung, die auch Forderungen von Selbstbestimmt-Leben-Bewegungen darstellen und die als zentrale Grundsätze in der UN-Konvention verankert sind, mit der Umsetzung partizipativer Forschung auf wissenschaftlicher Ebene berücksichtigt werden. 


\section{Literatur}

Anhorn, Roland/Stehr, Johannes (2012): Grundmodelle von Gesellschaft und soziale Ausschließung: Zum Gegenstand einer kritischen Forschungsperspektive in der Sozialen Arbeit. In: Schimpf, Elke/Stehr, Johannes (Hrsg.): Kritisches Forschen in der Sozialen Arbeit. Gegenstandsbereiche - Kontextbedingungen - Positionierungen - Perspektiven. Wiesbaden: VS Verlag für Sozialwissenschaften (Perspektiven kritischer Sozialer Arbeit, 11), S. 57-78.

Bloemers, Wolf (2000): Ethische Aspekte der Behindertenforschung. In: Bloemers, Wolf/Wisch, Fritz-Helmut (Hrsg.): Quality of life research and disabled people. Ways to research in different European settings. Frankfurt am Main, New York: Peter Lang (European social inclusion/Sozialgemeinschaft Europa, vol. 2), S. 280 321.

Bohnsack, Ralf (2003): Rekonstruktive Sozialforschung. Einführung in qualitative Methoden. 5. Aufl. Opladen: Leske + Budrich (UTB, 8242: Erziehungswissenschaft, Sozialwissenschaften).

Bohnsack, Ralf (2007): Typenbildung, Generalisierung und komparative Analyse: Grundprinzipien der dokumentarischen Methode. In: Bohnsack, Ralf/NentwigGesemann, Iris/Nohl, Arnd-Michael (Hrsg.): Die dokumentarische Methode und ihre Forschungspraxis. Grundlagen qualitativer Sozialforschung. 2., erw. und aktualisierte Aufl. Wiesbaden: VS Verlag für Sozialwissenschaften, S. 225-253.

Bohnsack, Ralf (2010): Die Mehrdimensionalität der Typenbildung und ihre Aspekthaftigkeit. In: Ecarius, Jutta/Schäffer, Burkhard (Hrsg.): Typenbildung und Theoriegenerierung. Methoden und Methodologien qualitativer Bildungs- und Biographieforschung. Opladen u.a.: Budrich, S. 47-72.

Bohnsack, Ralf (2010): Qualitative Evaluationsforschung und dokumentarische Methode. In: Bohnsack, Ralf/Nentwig-Gesemann, Iris (Hrsg.): Dokumentarische Evaluationsforschung. Theoretische Grundlagen und Beispiele aus der Praxis. Opladen u.a.: Budrich, S. 23-62.

Bohnsack, Ralf (2011): Qualitative Bild- und Videointerpretation. Die dokumentarische Methode. 2., durchges. und aktualisierte Aufl. Opladen [u.a.]: Budrich (UTB, 8407: Erziehungs- und Sozialwissenschaft).

Bohnsack, Ralf (2013): Dokumentarische Methode und die Logik der Praxis. In: Lenger, Alexander/Schneickert, Christian/Schumacher, Florian (Hrsg.): Pierre Bourdieus Konzeption des Habitus. Grundlagen, Zugänge, Forschungsperspektiven. Wiesbaden: Imprint: Springer VS (SpringerLink: Bücher), S. 175-200.

Bohnsack, Ralf/Nentwig-Gesemann, Iris/Nohl, Arnd-Michael (2007): Die dokumentarische Methode und ihre Forschungspraxis. In: Bohnsack, Ralf/Nentwig-Gesemann, Iris/Nohl, Arnd-Michael (Hrsg.): Die dokumentarische Methode und ihre Forschungspraxis. Grundlagen qualitativer Sozialforschung. 2., erw. und aktualisierte Aufl. Wiesbaden: VS Verlag für Sozialwissenschaften, S. 9-27.

Bohnsack, Ralf/Nohl, Arnd-Michael (2007): Exemplarische Textinterpretation: Die Sequenzanalyse der dokumentarischen Methode. In: Bohnsack, Ralf/NentwigGesemann, Iris/Nohl, Arnd-Michael (Hrsg.): Die dokumentarische Methode und ihre Forschungspraxis. Grundlagen qualitativer Sozialforschung. 2., erw. und aktualisierte Aufl. Wiesbaden: VS Verlag für Sozialwissenschaften, S. 303-307. 
Bohnsack, Ralf/Schäffer, Burkhard (2007): Exemplarische Textinterpretation: Diskursorganisation und dokumentarische Methode. In: Bohnsack, Ralf/NentwigGesemann, Iris/Nohl, Arnd-Michael (Hrsg.): Die dokumentarische Methode und ihre Forschungspraxis. Grundlagen qualitativer Sozialforschung. 2., erw. und aktualisierte Aufl. Wiesbaden: VS Verlag für Sozialwissenschaften, S. 309-323.

Bourdieu, Pierre/Passeron, Jean-Claude (1971): Die Illusion der Chancengleichheit. Stuttgart: Klett.

Braun, Christina von/Stephan, Inge (2009): Gender@Wissen. In: Braun, Christina von/Stephan, Inge (Hrsg.): Gender@Wissen. Ein Handbuch der Gender-Theorien. 2., überarb. und erg. Aufl. Köln, Weimar, Wien: Böhlau (UTB, 2584), S. 7-46.

Breger, Claudia (2009): Identität. In: Braun, Christina von/Stephan, Inge (Hrsg.): Gender@Wissen. Ein Handbuch der Gender-Theorien. 2., überarb. und erg. Aufl. Köln, Weimar, Wien: Böhlau (UTB, 2584), S. 47-65.

Bretländer, Bettina (2007): Kraftakte. Lebensalltag und Identitätsarbeit körperbehinderter Mädchen und junger Frauen. Bad Heilbrunn: Klinkhardt (Klinkhardt Forschung).

Bretländer, Bettina/Schildmann, Ulrike (2011): Geschlechtersensible Inklusionsforschung vor dem Hintergrund der neuen UN-Konvention (vor allem Artikel 6, 23, 24, 27, 28). In: Flieger, Petra/Schönwiese, Volker (Hrsg.): Menschenrechte - Integration - Inklusion. Aktuelle Perspektiven aus der Forschung. Bad Heilbrunn: Klinkhardt, S. 39-46.

Brozek, Dorothea (2005): Praxis und Entwicklungsperspektiven von SelbstbestimmtLeben-Bewegungen. In: Kaiser, Herbert/Kočnik, Ernst/Sigot, Marion (Hrsg.): Vom Objekt zum Subjekt. Inklusive Pädagogik und Selbstbestimmung. Klagenfurt [u.a.]: Hermagoras (Beiträge zu Inklusion und Selbstbestimmung, Bd. 1), S. 135144.

Bruner, Claudia Franziska (2005): KörperSpuren. Zur Dekonstruktion von Körper und Behinderung in biografischen Erzählungen von Frauen. Bielefeld: Transcript (Körperkulturen).

Bundeskanzleramt (2014): UN-Konvention über die Rechte von Menschen mit Behinderungen. BGBL. III - ausgegeben am 23. Oktober 2008 - Nr. 155. https:// www.ris.bka.gv.at [Zugriff: 14.10.2014].

Butolli, Willi/Hagl, Maria/Krüsman, Marion (2003): Kreativität und Destruktion posttraumatischer Bewältigung. Forschungsergebnisse und Thesen zum Leben nach dem Trauma. 2., um ein Nachwort erw. Aufl. Stuttgart: Pfeiffer bei KlettCotta (Leben lernen, 132).

Candussi, Klaus (2005): „Wir können alles lernen!“ Was passiert, wenn „geistig Behinderte" mit ihrem Kopf arbeiten? In: Kaiser, Herbert/Kočnik, Ernst/Sigot, Marion (Hrsg.): Vom Objekt zum Subjekt. Inklusive Pädagogik und Selbstbestimmung. Klagenfurt [u.a.]: Hermagoras (Beiträge zu Inklusion und Selbstbestimmung, Bd. 1), S. 163-175.

Cloerkes, Günther (1997): Soziologie der Behinderten. Eine Einführung. Heidelberg: Ed. Schindele.

Dederich, Markus (2009): Behinderung als sozial- und kulturwissenschaftliche Kategorie. In: Dederich, Markus/Jantzen, Wolfgang (Hrsg.): Behinderung und Anerkennung. Stuttgart: W. Kohlhammer (Behinderung, Bildung, Partizipation, 2), S. $15-40$. 
Dederich, Markus (2010): Behinderung, Norm, Differenz - Die Perspektive der Disability Studies. In: Kessl, Fabian (Hrsg.): Differenzierung, Normalisierung, Andersheit. Soziale Arbeit als Arbeit mit den Anderen. 1. Aufl. Wiesbaden: VS Verlag für Sozialwissenschaften, S. 170-184.

Dederich, Markus (2012): Körper, Kultur und Behinderung. Eine Einführung in die Disability Studies (Disability Studies, Bd. 2).

Dederich, Markus/Jantzen, Wolfgang (Hrsg.) (2009): Behinderung und Anerkennung. Stuttgart: W. Kohlhammer (Behinderung, Bildung, Partizipation, 2).

Dommermuth, Ralph (2004): Dürfen was ich möchte. Selbstbestimmungsrechte geistig Behinderter. Freiburg im Breisgau: Lambertus.

Ecarius, Jutta/Schäffer, Burkhard (Hrsg.) (2010): Typenbildung und Theoriegenerierung. Methoden und Methodologien qualitativer Bildungs- und Biographieforschung. Opladen u.a.: Budrich.

Ellger-Rüttgardt, Sieglind (1988): Die Hilfsschule im Nationalsozialismus und ihre Erforschung durch die Behindertenpädagogik. In: Keim, Wolfgang (Hrsg.): Pädagogen und Pädagogik des Nationalsozialismus. Ein unerledigtes Problem der Erziehungswissenschaft. Frankfurt am Main: Suhrkamp, S. 129-146.

Elmer, Corina (Hrsg.) (2004): Stark sein allein genügt nicht. Prävention sexueller Ausbeutung von Mädchen und Jungen. Basel: Lenos-Verlag.

Essen, Fabian (2013): Soziale Ungleichheit, Bildung und Habitus. Möglichkeitsräume ehemaliger Förderschüler. [S.1.]: Springer VS.

Falch, Ase-Britt (2000): Partizipatorische Forschung in der Praxis. In: Bloemers, Wolf/Wisch, Fritz-Helmut (Hrsg.): Quality of life research and disabled people. Ways to research in different European settings. Frankfurt am Main, New York: Peter Lang (European social inclusion/Sozialgemeinschaft Europa, vol. 2), S. 388415.

Ferdani, Sandro (2012): Behinderung als Missachtungserfahrung - Reflexion der Lebenssituation von behinderten Menschen. Hamburg: Diplomica Verlag.

Fetka-Einsiedler, Gerhard/Förster, Gerfried (Hrsg.) (1994): Diskriminiert? Zur Situation der Behinderten in unserer Gesellschaft. Graz: Leykam.

Feuser, Georg (1995): Behinderte Kinder und Jugendliche. Zwischen Integration und Aussonderung. Darmstadt: Wissenschaftliche Buchgesellschaft.

Finnern, Nina-Kathrin/Thim, Anja (2013): Zur Konstruktion und Reifizierung von ,Behinderung' durch Forschung. In: Dorrance, Carmen/Dannenbeck, Clemens (Hrsg.): Doing inclusion. Inklusion in einer nicht inklusiven Gesellschaft. Bad Heilbrunn: Klinkhardt, S. 159-167.

Fischer, Julia (Hrsg.) (2010): Mehr vom Leben. Frauen und Männer mit Behinderung erzählen. 1. Aufl. Bonn: Balance Buch + Medien Verl. (Balance Erfahrungen).

Flick, Uwe (2009): Triangulation in der qualitativen Forschung. In: Flick, Uwe/ Kardorff, Ernst von/Steinke, Ines (Hrsg.): Qualitative Forschung. Ein Handbuch. Orig.-Ausg., 7. Aufl. Reinbek bei Hamburg: Rowohlt-Taschenbuch-Verl. (Rororo Rowohlts Enzyklopädie, 55628), S. 309-318.

Flick, Uwe (2010): Das episodische Interview. In: Oelerich, Gertrud/Otto, Hans-Uwe (Hrsg.): Empirische Forschung und Soziale Arbeit. Ein Studienbuch. Wiesbaden: VS Verlag für Sozialwissenschaften, S. 273-281.

Flieger, Petra (2007): Der partizipatorische Ansatz des Forschungsprojekts Das Bildnis eines behinderten Mannes: Hintergrund - Konzept - Ergebnisse - Empfehlungen. In: Flieger, Petra/Schönwiese, Volker (Hrsg.): Das Bildnis eines behinderten 
Mannes. Bildkultur der Behinderung vom 16. bis ins 21. Jahrhundert: wissenschaftlicher Sammelband. Neu-Ulm: AG SPAK Bücher (AG SPAK Bücher), S. $19-42$.

Flieger, Petra/Schönwiese, Volker (2011): Die UN-Konvention über die Rechte von Menschen mit Behinderungen: Eine Herausforderung für die Integrations- und Inklusionsforschung. In: Flieger, Petra/Schönwiese, Volker (Hrsg.): Menschenrechte - Integration - Inklusion. Aktuelle Perspektiven aus der Forschung. Bad Heilbrunn: Klinkhardt, S. 27-35.

Fornefeld, Barbara (2009): Selbstbestimmung/Autonomie. In: Dederich, Markus/Jantzen, Wolfgang (Hrsg.): Behinderung und Anerkennung. Stuttgart: W. Kohlhammer (Behinderung, Bildung, Partizipation, 2), S. 183-187.

Foucault, Michel (1975): Überwachen und Strafen. Die Geburt des Gefängnisses. 9. Aufl. 1991. Frankfurt am Main: Suhrkamp.

Frank, Klaus D. (2014): Kleine Schule der Dialektik. Hamburg: Tredition.

Franz, Alexandra (2002): Selbstbestimmt Leben mit Persönlicher Assistenz - eine alternative Lebensform behinderter Frauen. Neu-Ulm: AG SPAK Bücher (Materialien der AG SPAK, M 154).

Frehe, Horst (1991): Assistenzgenossenschaft - Eine neue Qualität ambulanter Hilfe. In: Windisch, Matthias/Miles-Paul, Ottmar (Hrsg.): Selbstbestimmung Behinderter. Leitlinien für die Behinderten-Politik und -Arbeit: Vortragsreihe des Fachbereichs Sozialwesen der Universität, Gesamthochschule Kassel, in Kooperation mit dem Verein zur Förderung für Autonomie Behinderter (FAB e.V.). Kassel: GhK, Fachbereich 4, Sozialwesen, S. 35-45.

Friske, Andrea (1995): Als Frau geistig behindert sein: Ansätze zu frauenorientiertem heilpädagogischen Handeln. München: Ernst Reinhardt Verlag.

Fröhlich-Gildhoff, Klaus/Rönnau-Böse, Maike (2014): Resilienz. 3. Aufl. München, München: UTB/Reinhardt, Ernst (UTB Profile, 3290).

Gerhardt, Volker (1999): Selbstbestimmung. Das Prinzip der Individualität. Stuttgart: Reclam (Nr. 9761).

Gildemeister, Regine (2009): Geschlechterforschung (gender studies). In: Flick, Uwe/ Kardorff, Ernst von/Steinke, Ines (Hrsg.): Qualitative Forschung. Ein Handbuch. Orig.-Ausg., 7. Aufl. Reinbek bei Hamburg: Rowohlt-Taschenbuch-Verl. (Rororo Rowohlts Enzyklopädie, 55628), S. 213-223.

Glaser, Barney G./Strauss, Anselm L./Paul, Axel T. (2010): Grounded Theory. Strategien qualitativer Forschung. 3., unveränd. Aufl. Bern: Huber (Programmbereich Gesundheit).

Goffman, Erving (1972): Asyle. Über die soziale Situation psychiatrischer Patienten und anderer Insassen. Frankfurt am Main: Suhrkamp.

Goffman, Erving (1975): Stigma. Über Techniken der Bewältigung beschädigter Identität. 1. Aufl. Frankfurt am Main: Suhrkamp (Suhrkamp Taschenbücher Wissenschaft, 140).

Goffman, Erving/Knoblauch, Hubert (2001): Interaktion und Geschlecht. 2. Aufl. Frankfurt am Main, New York: Campus-Verlag (Campus Studium).

Güttgemann, Petra (1997): Die Adoleszenz bei Mädchen mit geistiger Behinderung und der Einfluß der Mutterbeziehung. In: Behindertenpädagogik (1), S. 10-24.

Heimgartner, Arno/Sting, Stephan (2011): Empirische Forschung zur Sozialen Arbeit in Österreich. In: Heimgartner, Arno/Loch, Ulrike/Sting, Stephan (Hrsg.): Empirische Forschung in der Sozialen Arbeit. Methoden und methodologische Heraus- 
forderungen. 1. Aufl. Münster, Westf: LIT (Soziale Arbeit - Social Issues, 15), S. 9-24.

Heite, Catrin (2010): Anerkennung von Differenz in der Sozialen Arbeit. Zur professionellen Konstruktion des Anderen. In: Kessl, Fabian (Hrsg.): Differenzierung, Normalisierung, Andersheit. Soziale Arbeit als Arbeit mit den Anderen. 1. Aufl. Wiesbaden: VS Verlag für Sozialwissenschaften, S. 187-200.

Helfferich, Cornelia (2011): Die Qualität qualitativer Daten. Manual für die Durchführung qualitativer Interviews. [S.1.]: VS Verlag für Sozialwissenschaften.

Helsper, Werner/Kramer, Rolf-Torsten/Thiersch, Sven (2013): Orientierungsrahmen zwischen Kollektivität und Individualität - ontogenetische und transformationsbezogene Anfragen an die dokumentarische Methode. In: Loos, Peter u.a. (Hrsg.): Dokumentarische Methode. Grundlagen - Entwicklungen - Anwendungen. Opladen: Verlag Barbara Budrich, S. 111-140.

Hermes, Gisela (2006): Der Wissenschaftsansatz Disability Studies - neue Erkenntnisgewinne über Behinderung? In: Hermes, Gisela/Rohrmann, Eckhard (Hrsg.): Nichts über uns - ohne uns! Disability Studies als neuer Ansatz emanzipatorischer und interdisziplinärer Forschung über Behinderung. 1. Aufl. Neu-Ulm: AG-SPAKBücher (Materialien der AG SPAK, 187), S. 15-30.

Hermes, Gisela/Rohrmann, Eckhard (Hrsg.) (2006): Nichts über uns - ohne uns! Disability Studies als neuer Ansatz emanzipatorischer und interdisziplinärer Forschung über Behinderung. 1. Aufl. Neu-Ulm: AG-SPAK-Bücher (Materialien der AG SPAK, 187).

Hildeschmidt, Anne/Schnell, Irmtraud (Hrsg.) (1998): Integrationspädagogik. Auf dem Weg zu einer Schule für alle. Weinheim, München: Juventa.

Hinz, Andreas (2005): Zur disziplinären Verortung der Integrationspädagogik - sieben Thesen. In: Geiling, Ute/Hinz, Andreas (Hrsg.): Integrationspädagogik im Diskurs. Auf dem Weg zu einer inklusiven Pädagogik? Bad Heilbrunn: Klinkhardt, S. 75-78.

Honneth, Axel (1992): Kampf um Anerkennung. Zur moralischen Grammatik sozialer Konflikte. Frankfurt am Main: Suhrkamp.

Hopf, Christel (2009): Qualitative Interviews - ein Überblick. In: Flick, Uwe/ Kardorff, Ernst von/Steinke, Ines (Hrsg.): Qualitative Forschung. Ein Handbuch. Orig.-Ausg., 7. Aufl. Reinbek bei Hamburg: Rowohlt-Taschenbuch-Verl. (Rororo Rowohlts Enzyklopädie, 55628), S. 349-359.

Hug, Sonja (2004): Anders und dennoch gleich. Prävention für Mädchen und Frauen mit geistiger Behinderung. In: Elmer, Corina (Hrsg.): Stark sein allein genügt nicht. Prävention sexueller Ausbeutung von Mädchen und Jungen. Basel: LenosVerlag, S. 155-168.

Jantzen, Wolfgang (1974): Sozialisation und Behinderung. Studien zu sozialwissenschaftlichen Grundfragen der Behindertenpädagogik. Gießen: Focus Verlag.

Jantzen, Wolfgang (1998): Integration heißt Ausschluss vermeiden! Kritische Bemerkungen zum Verhältnis von Integrations- und Enthospitalisierungsdebatte. In: Hildeschmidt, Anne/Schnell, Irmtraud (Hrsg.): Integrationspädagogik. Auf dem Weg zu einer Schule für alle. Weinheim, München: Juventa, S. 179-192.

Jantzen, Wolfgang (2004): Geistige Behinderung und strukturelle Gewalt (2004). In: Wüllenweber, Ernst (Hrsg.): Soziale Probleme von Menschen mit geistiger Behinderung. Fremdbestimmung, Benachteiligung, Ausgrenzung und soziale Abwertung. Stuttgart: W. Kohlhammer, S. 148-169. 
Johnstone, David (2000): Lebensqualitätsforschung und behinderte Menschen. In: Bloemers, Wolf/Wisch, Fritz-Helmut (Hrsg.): Quality of life research and disabled people. Ways to research in different European settings. Frankfurt am Main, New York: Peter Lang (European social inclusion/Sozialgemeinschaft Europa, vol. 2), S. $255-279$.

Juhila, Kirsi/Hall, Christopher/Raitakari, Suvi (2012): Zurechnung von störendem Verhalten: Beschuldigungen, Entschuldigungen und Verantwortlichkeit in den Redeweisen von Professionellen. In: Schimpf, Elke/Stehr, Johannes (Hrsg.): Kritisches Forschen in der Sozialen Arbeit. Gegenstandsbereiche - Kontextbedingungen - Positionierungen - Perspektiven. Wiesbaden: VS Verlag für Sozialwissenschaften (Perspektiven kritischer Sozialer Arbeit, 11), S. 181-200.

Kaiser, Herbert (2006): Die Selbstbestimmt-Leben-Bewegung. Geschichte und Hintergründe. In: Kaiser, Herbert/Kočnik, Ernst/Sigot, Marion (Hrsg.): Stolpersteine auf dem Weg zur Gleichstellung. Das österreichische Bundesbehindertengleichstellungsgesetz. Klagenfurt, Ljubljana, Wien: Mohorjeva (Beiträge zu Inklusion und Selbstbestimmung, Bd. 2), S. 9-18.

Kaiser, Herbert/Kočnik, Ernst/Sigot, Marion (Hrsg.) (2010): Selbst in der Hand! Persönliches Budget und selbstbestimmtes Leben. Klagenfurt, Ljubljana, Wien: Mohorjeva (Beiträge zu Inklusion und Selbstbestimmung, Bd. 3).

Kardorff, Ernst von (2009): Qualitative Evaluationsforschung. In: Flick, Uwe/ Kardorff, Ernst von/Steinke, Ines (Hrsg.): Qualitative Forschung. Ein Handbuch. Orig.-Ausg., 7. Aufl. Reinbek bei Hamburg: Rowohlt-Taschenbuch-Verl. (Rororo Rowohlts Enzyklopädie, 55628), S. 238-250.

Keim, Wolfgang (Hrsg.) (1988): Pädagogen und Pädagogik des Nationalsozialismus. Ein unerledigtes Problem der Erziehungswissenschaft. Frankfurt am Main: Suhrkamp.

Kelle, Udo/Kluge, Susanne (Hrsg.) (2010): Vom Einzelfall zum Typus. Fallvergleich und Fallkontrastierung in der qualitativen Sozialforschung. Wiesbaden: VS Verlag für Sozialwissenschaften.

Klee, Ernst (1980): Behindert. Ein kritisches Handbuch. Frankfurt am Main: Suhrkamp.

Kleemann, Frank/Krähnke, Uwe/Matuschek, Ingo (2013): Interpretative Sozialforschung. Eine Einführung in die Praxis des Interpretierens. 2., korrigierte und aktualisierte Aufl. Wiesbaden: Springer VS (Lehrbuch).

Kniel, Adrian/Windisch, Matthias (2005): People first. Selbsthilfegruppen von und für Menschen mit geistiger Behinderung. München, Basel: Reinhardt.

Köbsell, Swantje (2007): Behinderung und Geschlecht - Versuch einer vorläufigen Bilanz aus Sicht der deutschen Behindertenbewegung. In: Jacob, Jutta/Wollrad, Eske (Hrsg.): Behinderung und Geschlecht. Perspektiven in Theorie und Praxis/Dokumentation einer Tagung. Oldenburg: BIS-Verl. der Carl-von-OssietzkyUniv. (Oldenburger Beiträge zur Geschlechterforschung, Bd. 8), S. 31-50.

Köbsell, Swantje (2010): Gendering Disability: Behinderung, Geschlecht und Körper. In: Jacob, Jutta/Köbsell, Swantje/Wollrad, Eske (Hrsg.): Gendering disability. Intersektionale Aspekte von Behinderung und Geschlecht. Bielefeld: Transcript (Studien interdisziplinäre Geschlechterforschung, 7), S. 17-34.

Kočnik, Ernst (2005): Krüppel aus dem Sack. Das Beratungs-, Mobilitäts- und Kompetenzzentrum an der Universität Klagenfurt. In: Kaiser, Herbert/Kočnik, Ernst/Sigot, Marion (Hrsg.): Vom Objekt zum Subjekt. Inklusive Pädagogik und 
Selbstbestimmung. Klagenfurt [u.a.]: Hermagoras (Beiträge zu Inklusion und Selbstbestimmung, Bd. 1), S. 99-108.

König, Oliver/Buchner, Tobias (2011): Die Bedeutung von Lebensgeschichten für die UN-Konvention. In: Flieger, Petra/Schönwiese, Volker (Hrsg.): Menschenrechte Integration - Inklusion. Aktuelle Perspektiven aus der Forschung. Bad Heilbrunn: Klinkhardt, S. 139-152.

König, Oliver u.a. (2010): Inklusive Forschung und Empowerment. In: Stein, AnneDore/Krach, Stefanie/Niediek, Imke (Hrsg.): Integration und Inklusion auf dem Weg ins Gemeinwesen. Möglichkeitsräume und Perspektiven. Bad Heilbrunn: Klinkhardt, S. 176-190.

Krög, Walter (2005): Herausforderung Unterstützung. Perspektiven auf dem Weg zur Inklusion. Höfen. Hrsg. v. Equal - Entwicklungspartnerschaft MiM. http://bidok. uibk.ac.at/library-mim.broschuere.html [Zugriff: 07.10.2014].

Küsters, Ivonne (2009): Narrative Interviews: Grundlagen und Anwendungen. 2. Aufl. Wiesbaden: VS Verlag für Sozialwissenschaften (Lehrbuch).

Landgraf, Adi-Ida (2010): Wie es ist, anders zu sein. Rankar: Edition WEGE.

Lenger, Alexander/Schneickert, Christian/Schumacher, Florian (2013): Pierre Bourdieus Konzeption des Habitus. In: Lenger, Alexander/Schneickert, Christian/Schumacher, Florian (Hrsg.): Pierre Bourdieus Konzeption des Habitus. Grundlagen, Zugänge, Forschungsperspektiven. Wiesbaden: Imprint: Springer VS (SpringerLink : Bücher), S. 13-41.

Lindmeier, Christian (2013): Biografiearbeit mit geistig behinderten Menschen. Ein Praxisbuch für Einzel- und Gruppenarbeit. 4. Aufl. Weinheim, Basel: Beltz Juventa (Edition Sozial).

Loos, Peter u.a. (Hrsg.) (2013): Dokumentarische Methode. Grundlagen - Entwicklungen - Anwendungen. Opladen: Verlag Barbara Budrich.

Markowetz, Reinhard (2005): Inklusion - Neuer Begriff, neues Konzept, neue Hoffnungen für die Selbstbestimmung und Partizipation von Menschen mit Behinderungen. In: Kaiser, Herbert/Kočnik, Ernst/Sigot, Marion (Hrsg.): Vom Objekt zum Subjekt. Inklusive Pädagogik und Selbstbestimmung. Klagenfurt [u.a.]: Hermagoras (Beiträge zu Inklusion und Selbstbestimmung, Bd. 1), S. 17-66.

Marks, Dana-Kristin (2011): Konstruktionen von Behinderung in den ersten Lebensjahren. Unter besonderer Berücksichtigung der Strukturkategorie Geschlecht. Bochum, Freiburg: Projektverl.

Mayring, Philipp (2002): Einführung in die qualitative Sozialforschung. Eine Anleitung zu qualitativem Denken. 5., neu ausgestattete Aufl. Weinheim: Beltz (Beltz Studium).

Merkens, Hans (2009): Auswahlverfahren, Sampling, Fallrekonstruktion. In: Flick, Uwe/Kardorff, Ernst von/Steinke, Ines (Hrsg.): Qualitative Forschung. Ein Handbuch. Orig.-Ausg., 7. Aufl. Reinbek bei Hamburg: Rowohlt-Taschenbuch-Verl. (Rororo Rowohlts Enzyklopädie, 55628), S. 286-298.

Mesdag, Thomas/Hitzel, Elke (2008): Sage mir, wie Du leben willst (aber bedenke, was ich hören will!). Befragungen von Menschen mit einer geistigen Behinderung als Anerkennung von Selbstbestimmung oder als Paradox ,fremdbestimmter Selbstbestimmung“. In: Mesdag, Thomas/Pforr, Ursula: Phänomen geistige Behinderung. Ein psychodynamischer Verstehensansatz. Orig.-Ausg. Hrsg. v. Thomas Mesdag. Gießen: Psychosozial-Verl. (Psychoanalytische Pädagogik, 28), S. 169188. 
Michel, Burkhard (2006): Bild und Habitus. Sinnbildungsprozesse bei der Rezeption von Fotografien. 1. Aufl. Wiesbaden: VS Verlag für Sozialwissenschaften/GWV Fachverlage, Wiesbaden.

Michel, Burkhard (2007): Fotografien und ihre Lesarten. Dokumentarische Methode von Bildrezeptionsprozessen. In: Bohnsack, Ralf/Nentwig-Gesemann, Iris/Nohl, Arnd-Michael (Hrsg.): Die dokumentarische Methode und ihre Forschungspraxis. Grundlagen qualitativer Sozialforschung. 2., erw. und aktualisierte Aufl. Wiesbaden: VS Verlag für Sozialwissenschaften, S. 93-123.

Michel, Burkhard (2008): Das Gruppendiskussionsverfahren in der (Bild)Rezeptionsforschung. In: Bohnsack, Ralf/Przyborski, Aglaja/Schäffer, Burkhard (Hrsg.): Das Gruppendiskussionsverfahren in der Forschungspraxis. 2., überarb. Aufl. Opladen: Budrich, S. 219-232.

Middendorf, Lena (2010): Körperbilder von Mädchen, Behinderung und Geschlecht. In: Jacob, Jutta/Köbsell, Swantje/Wollrad, Eske (Hrsg.): Gendering disability. Intersektionale Aspekte von Behinderung und Geschlecht. Bielefeld: Transcript (Studien interdisziplinäre Geschlechterforschung, 7), S. 207-222.

Mirwald, Mirjam (2009): Sexualbegleitung für Menschen mit Lernschwierigkeiten. Diskursanalyse und Dokumentarfilm „Die Heide ruft“. 1. Aufl. Aachen: Shaker (Schriften aus dem Institut für Rehabilitationswissenschaften der HumboldtUniversität zu Berlin, 2009, 1).

Möckel, Andreas (2002): Die Funktion der Sonderschulen und die Forderung der Integration. In: Eberwein, Hans/Knauer, Sabine (Hrsg.): Integrationspädagogik. Kinder mit und ohne Beeinträchtigung lernen gemeinsam/ein Handbuch. 6., vollst. überarb. und aktualisierte Aufl. Weinheim, Basel: Beltz (Beltz-Handbuch), S. 8090.

Mürner, Christian/Schönwiese, Volker (2007): Das Bildnis eines behinderten Mannes. Bildkultur der Behinderung vom 16. bis ins 21. Jahrhundert: Ausstellungskatalog und Wörterbuch. Neu-Ulm: AG SPAK Bücher (AG SPAK-Bücher).

Mürner, Christian/Sierck, Udo (Hrsg.) (2011): Behinderte Identität? Neu-Ulm: AGSPAK-Bücher.

Nentwig-Gesemann, Iris (2007): Die Typenbildung der dokumentarischen Methode. In: Bohnsack, Ralf/Nentwig-Gesemann, Iris/Nohl, Arnd-Michael (Hrsg.): Die dokumentarische Methode und ihre Forschungspraxis. Grundlagen qualitativer Sozialforschung. 2., erw. und aktualisierte Aufl. Wiesbaden: VS Verlag für Sozialwissenschaften, S. 277-302.

Netzwerk People First Deutschland e.V. (Hrsg.) (2004): Wörterbuch für leichte Sprache. Halt! Bitte leichte Sprache. 5., überarb. Aufl. Kassel: Netzwerk People First Deutschland.

Niehaus, Mathilde (2006): Exklusion verhindern, Inklusion stärken: Ansätze zur Teilhabe am Erwerbsleben. In: Platte, Andrea/Seitz, Simone/Terfloth, Karin (Hrsg.): Inklusive Bildungsprozesse. Bad Heilbrunn: Klinkhardt, S. 178-185.

Nohl, Arnd-Michael (2006): Bildung und Spontaneität. Phasen biographischer Wandlungsprozesse in drei Lebensaltern. Empirische Rekonstruktionen und pragmatistische Reflexionen. Opladen: Budrich.

Nohl, Arnd-Michael (2007): Komparative Analyse: Forschungspraxis und Methodologie dokumentarischer Interpretation. In: Bohnsack, Ralf/Nentwig-Gesemann, Iris/Nohl, Arnd-Michael (Hrsg.): Die dokumentarische Methode und ihre For- 
schungspraxis. Grundlagen qualitativer Sozialforschung. 2., erw. und aktualisierte Aufl. Wiesbaden: VS Verlag für Sozialwissenschaften, S. 255-276.

Nohl, Arnd-Michael (2012): Interview und dokumentarische Methode. Anleitungen für die Forschungspraxis. 4. Aufl. 2012. Wiesbaden: VS Verlag für Sozialwissenschaften/Imprint: VS Verlag für Sozialwissenschaften (Qualitative Sozialforschung).

Nohl, Arnd-Michael (2013): Relationale Typenbildung und Mehrebenenvergleich. Neue Wege der dokumentarischen Methode. Wiesbaden: Springer VS (Qualitative Sozialforschung).

Nohl, Arnd-Michael u.a. (2013): Zur Entwicklung der dokumentarischen Methode durch Ralf Bohnsack. In: Loos, Peter u.a. (Hrsg.): Dokumentarische Methode. Grundlagen - Entwicklungen - Anwendungen. Opladen: Verlag Barbara Budrich, S. 9-40.

Oelerich, Gertrud/Otto, Hans-Uwe (Hrsg.) (2010): Empirische Forschung und Soziale Arbeit. Ein Studienbuch. Wiesbaden: VS Verlag für Sozialwissenschaften.

Popp, Ulrike (2002): Geschlechtersozialisation und schulische Gewalt. Geschlechtstypische Ausdrucksformen und konflikthafte Interaktionen von Schülerinnen und Schülern. Weinheim [u.a.]: Juventa-Verl. (Jugendforschung).

Popp, Ulrike (2011): Hintergründe und Auswirkungen von Schulversagen. In: Sting, Stephan/Wakounig, Vladimir (Hrsg.): Bildung zwischen Standardisierung, Ausgrenzung und Anerkennung von Diversität. Wien, Berlin, Münster: LIT (Austria: Forschung und Wissenschaft: Erziehungswissenschaft, Bd. 12), S. 131-147.

Prengel, Annedore (2005): Anerkennung von Anfang an - Egalität, Heterogenität und Hierarchie im Anfangsunterricht und darüber hinaus. In: Geiling, Ute/Hinz, Andreas (Hrsg.): Integrationspädagogik im Diskurs. Auf dem Weg zu einer inklusiven Pädagogik? Bad Heilbrunn: Klinkhardt, S. 15-34.

Prengel, Annedore (2009): Vielfalt. In: Dederich, Markus/Jantzen, Wolfgang (Hrsg.): Behinderung und Anerkennung. Stuttgart: W. Kohlhammer (Behinderung, Bildung, Partizipation, 2), S. 105-112.

Prengel, Annedore (2013): Pädagogische Beziehungen zwischen Anerkennung, Verletzung und Ambivalenz. Leverkusen: Verlag Barbara Budrich.

Prosetzky, Ingolf (2009): Isolation und Partizipation. In: Dederich, Markus/Jantzen, Wolfgang (Hrsg.): Behinderung und Anerkennung. Stuttgart: W. Kohlhammer (Behinderung, Bildung, Partizipation, 2), S. 87-95.

Przyborski, Aglaja (2004): Gesprächsanalyse und dokumentarische Methode. Qualitative Auswertung von Gesprächen, Gruppendiskussionen und anderen Diskursen. Wiesbaden: VS Verlag für Sozialwissenschaften (Lehrbuch).

Raab, Heike (2012): Doing Feminism: Zum Bedeutungshorizont von Geschlecht und Heteronormativität in den Disability Studies. In: Rathgeb, Kerstin (Hrsg.): Disability Studies. Kritische Perspektiven für die Arbeit am Sozialen. Wiesbaden: Springer VS (Perspektiven kritischer Sozialer Arbeit, 14), S. 69-90.

Radtke, Peter (1994): Warum die Gesellschaft den ,Behinderten' braucht - Über das Menschenbild des 20. Jahrhunderts. In: Fetka-Einsiedler, Gerhard/Förster, Gerfried (Hrsg.): Diskriminiert? Zur Situation der Behinderten in unserer Gesellschaft. Graz: Leykam, S. 109-119.

Rathgeb, Kerstin (2012): Kritische Perspektiven auf soziale Phänomene und die Arbeit am Sozialen. In: Rathgeb, Kerstin (Hrsg.): Disability Studies. Kritische Per- 
spektiven für die Arbeit am Sozialen. Wiesbaden: Springer VS (Perspektiven kritischer Sozialer Arbeit, 14), S. 9-20.

Ratzka, Adolf (1996): Persönliche Assistenz in Schweden. http://www.independent living.org/node/405 [Zugriff: 07.10.2014].

Ratzka, Adolf (2003): Schweden. Vorbild in Europa? In: Behinderte in Familie, Schule und Gesellschaft 6, S. 54-65.

Reicher, Hannelore (2011): Sozial-emotionales Lernen im Kontext inklusiver Pädagogik. Potenziale und Perspektiven. 1., neue Ausg. Graz: Leykam (Grazer Universitätsverlag: Reihe Habilitationen, Dissertationen und Diplomarbeiten, Bd. 31).

Reiss, Kristina (2007): Behinderung von Geschlecht - Zur Exklusion von Geschlecht durch Behinderung und Produktion des Anderen. In: Jacob, Jutta/Wollrad, Eske (Hrsg.): Behinderung und Geschlecht. Perspektiven in Theorie und Praxis/Dokumentation einer Tagung. Oldenburg: BIS-Verl. der Carl-von-OssietzkyUniv. (Oldenburger Beiträge zur Geschlechterforschung, Bd. 8), S. 51-64.

Rendtorff, Barbara (2010): Bildung, Schule und Geschlecht. Stuttgart: W. Kohlhammer (Praxiswissen Bildung).

Retzlaff, Rüdiger (2010): Familien-Stärken. Behinderung, Resilienz und systemische Therapie. Stuttgart: Klett-Cotta (Fachbuch Klett-Cotta).

Rosenthal, Gabriele (2014): Interpretative Sozialforschung. Eine Einführung. 4. Aufl. Weinheim [u.a.]: Beltz Juventa (Grundlagentexte Soziologie).

Rosenthal, Gabriele/Loch, Ulrike (2002): Das narrative Interview. In: Schaeffer, Doris/Müller-Mundt, Gabriele (Hrsg.): Qualitative Gesundheits- und Pflegeforschung. Bern: Huber, S. 221-232. http://nbn-resolving.de/urn:nbn:de:0168-ssoar57670 [Zugriff: 08.10.2014].

Sander, Alfred (2002): Behinderungsbegriffe und ihre Integrationsrelevanz. In: Eberwein, Hans/Knauer, Sabine (Hrsg.): Integrationspädagogik. Kinder mit und ohne Beeinträchtigung lernen gemeinsam/ein Handbuch. 6., vollst. überarb. und aktualisierte Aufl. Weinheim, Basel: Beltz (Beltz-Handbuch), S. 99-108.

Schildmann, Ulrike (2004): Geschlecht und (geistige) Behinderung. In: Wüllenweber, Ernst (Hrsg.): Soziale Probleme von Menschen mit geistiger Behinderung. Fremdbestimmung, Benachteiligung, Ausgrenzung und soziale Abwertung. Stuttgart: W. Kohlhammer, S. 36-45.

Schildmann, Ulrike (2007): Behinderung und Geschlecht - Datenlage und Perspektiven der Forschung. In: Jacob, Jutta/Wollrad, Eske (Hrsg.): Behinderung und Geschlecht. Perspektiven in Theorie und Praxis/Dokumentation einer Tagung. Oldenburg: BIS-Verl. der Carl-von-Ossietzky-Univ. (Oldenburger Beiträge zur Geschlechterforschung, Bd. 8), S. 11-30.

Schildmann, Ulrike (2009): Geschlecht. In: Dederich, Markus/Jantzen, Wolfgang (Hrsg.): Behinderung und Anerkennung. Stuttgart: W. Kohlhammer (Behinderung, Bildung, Partizipation, 2), S. 222-226.

Schönwiese, Volker (2005): Assistenz, Kontrolle über das eigene Leben und das , begleitende Ich'. In: Geiling, Ute/Hinz, Andreas (Hrsg.): Integrationspädagogik im Diskurs. Auf dem Weg zu einer inklusiven Pädagogik? Bad Heilbrunn: Klinkhardt, S. 141-145.

Schönwiese, Volker (2011): Behinderung und Identität: Inszenierungen des Alltags. In: Mürner, Christian/Sierck, Udo (Hrsg.): Behinderte Identität? Neu-Ulm: AGSPAK-Bücher, S. 143-162. 
Schulze, Marianne (2011): Menschenrechte für alle: Die Konvention über die Rechte von Menschen mit Behinderungen. In: Flieger, Petra/Schönwiese, Volker (Hrsg.): Menschenrechte - Integration - Inklusion. Aktuelle Perspektiven aus der Forschung. Bad Heilbrunn: Klinkhardt, S. 11-26.

Schumann, Monika (2000): Aktuelle Entwicklungen in Biomedizin und Bioethik Neue Herausforderungen an die Integrationspädagogik. In: Hovorka, Hans/Sigot, Marion (Hrsg.): Integration(spädagogik) am Prüfstand. Menschen mit Behinderungen außerhalb von Schule. Innsbruck, Wien, München: Studienverlag, S. 33-60.

Selbstbestimmt Leben Innsbruck - Wibs (2012): Das Mutbuch. Lebensgeschichten von Frauen und Männern mit Lernschwierigkeiten. Neu-Ulm: AG SPAK Bücher.

Sierck, Udo (2011): Goldener Käfig a.D. Identitätsfindung trotz Sonderschule. In: Mürner, Christian/Sierck, Udo (Hrsg.): Behinderte Identität? Neu-Ulm: AGSPAK-Bücher, S. 8-19.

Sierck, Udo (2012): Selbstbestimmung statt Bevormundung. Anmerkungen zur Entstehung der Disability Studies. In: Rathgeb, Kerstin (Hrsg.): Disability Studies. Kritische Perspektiven für die Arbeit am Sozialen. Wiesbaden: Springer VS (Perspektiven kritischer Sozialer Arbeit, 14), S. 31-38.

Sigot, Marion (Hrsg.) (1998): Die Integrationspädagogik als Wegbereiterin einer allgemeinen integrativen Pädagogik. Klagenfurt: Hochschulschrift.

Sigot, Marion (2006): Diskriminierung und Stigmatisierung von Menschen mit Behinderungen. In: Kaiser, Herbert/Kočnik, Ernst/Sigot, Marion (Hrsg.): Stolpersteine auf dem Weg zur Gleichstellung. Das österreichische Bundesbehindertengleichstellungsgesetz. Klagenfurt, Ljubljana, Wien: Mohorjeva (Beiträge zu Inklusion und Selbstbestimmung, Bd. 2), S. 19-48.

Sigot, Marion (2011): Die UN-Konvention für Menschen mit Behinderungen und ihre Auswirkungen auf die Situation von Menschen mit Lernschwierigkeiten. In: Flieger, Petra/Schönwiese, Volker (Hrsg.): Menschenrechte - Integration - Inklusion. Aktuelle Perspektiven aus der Forschung. Bad Heilbrunn: Klinkhardt, S. 47-52.

Sigot, Marion (2011): Zur Gleichstellung von Menschen mit Lernschwierigkeiten. In: Sting, Stephan/Wakounig, Vladimir (Hrsg.): Bildung zwischen Standardisierung, Ausgrenzung und Anerkennung von Diversität. Wien, Berlin, Münster: LIT (Austria: Forschung und Wissenschaft: Erziehungswissenschaft, Bd. 12), S. 151-162.

Sigot, Marion (2012): Partizipatorische Forschung mit Frauen mit Lernschwierigkeiten - Herausforderung und Bereicherung für die empirische Forschung. In: Heimgartner, Arno/Loch, Ulrike/Sting, Stephan (Hrsg.): Empirische Forschung in der Sozialen Arbeit. Methoden und methodologische Herausforderungen. 1. Aufl. Münster, Westf.: LIT (Soziale Arbeit - Social Issues, 15), S. 153-166.

Sigot, Marion (2013): Selbstbestimmung aus der Perspektive von Frauen mit Lernschwierigkeiten. In: Heimgartner, Arno/Lauermann, Karin/Sting, Stephan (Hrsg.): Perspektiven der AkteurInnen in der Sozialen Arbeit. Wien [u.a.]: LIT (Soziale Arbeit, 17), S. 83-90.

Sigot, Marion/Kočnik, Ernst (2010): Persönliches Budget, Persönliche Assistenz und Selbstbestimmung. In: Kaiser, Herbert/Kočnik, Ernst/Sigot, Marion (Hrsg.): Selbst in der Hand! Persönliches Budget und selbstbestimmtes Leben. Klagenfurt, Ljubljana, Wien: Mohorjeva (Beiträge zu Inklusion und Selbstbestimmung, Bd. 3), S. $17-24$.

Sigot, Marion/Wernisch, Barbara (2005): Selbstbestimmung und Inklusion im Leben von Frauen mit einer sogenannten geistigen Behinderung. Folgerungen aus dem 
Forschungsprojekt „Die Lebenssituation von Frauen mit geistiger Behinderung“. In: Kaiser, Herbert/Kočnik, Ernst/Sigot, Marion (Hrsg.): Vom Objekt zum Subjekt. Inklusive Pädagogik und Selbstbestimmung. Klagenfurt [u.a.]: Hermagoras (Beiträge zu Inklusion und Selbstbestimmung, Bd. 1), S. 183-197.

Steinke, Ines (2009): Gütekriterien qualitativer Forschung. In: Flick, Uwe/Kardorff, Ernst von/Steinke, Ines (Hrsg.): Qualitative Forschung. Ein Handbuch. Orig.Ausg., 7. Aufl. Reinbek bei Hamburg: Rowohlt-Taschenbuch-Verl. (Rororo Rowohlts Enzyklopädie, 55628), S. 319-331.

Stiehler, Steve (2011): Der Einsatz von Vignetten in der empirischen Forschung Sozialer Arbeit - Die „Dresdner Bewältigungsvignetten“ für Kinder. In: Heimgartner, Arno/Loch, Ulrike/Sting, Stephan (Hrsg.): Empirische Forschung in der Sozialen Arbeit. Methoden und methodologische Herausforderungen. 1. Aufl. Münster, Westf.: LIT (Soziale Arbeit - Social Issues, 15), S. 201-218.

Stiehler, Steve/Werner, Jillian (2008): „Dresdner Bewältigungsvignetten“ zur Erfassung der Hilfesuch- und Bewältigungsstrategien von Kindern. In: Nestmann, Frank u.a. (Hrsg.): Kindernetzwerke. Soziale Beziehungen und soziale Unterstützung in Familie, Pflegefamilie und Heim. Tübingen: Dgvt-Verl. (Fortschritte der Gemeindepsychologie und Gesundheitsförderung, Bd. 17), S. 185-207.

Stockner, Hubert (2011): Persönliche Assistenz als Ausweg aus der institutionellen Segregation von Menschen mit Behinderungen. Bericht für Selbstbestimmt Leben Österreich zur Situation der persönlichen Assistenz in Österreich. http://bidok. uibk.ac.at/library/stockner-assistenz.html [Zugriff: 08.10.2014].

Thimm, Walter (1995): Das Normalisierungsprinzip. Eine Einführung. 6. Aufl. Marburg: Lebenshilfe-Verl. (Kleine Schriftenreihe/Bundesvereinigung Lebenshilfe für Geistig Behinderte e.V., Bd. 5).

Unesco (1994): The Salamanca statement and framework for action and special needs education. http://unesdoc.unesco.org/images/0009/000984/098427eo.pdf [Zugriff: 23.05.2017].

Unger, Hella (2014): Partizipative Forschung. Einführung in die Forschungspraxis. Wiesbaden: Springer VS (SpringerLink : Bücher).

Waldschmidt, Anne (2009): Disability Studies. In: Dederich, Markus/Jantzen, Wolfgang (Hrsg.): Behinderung und Anerkennung. Stuttgart: W. Kohlhammer (Behinderung, Bildung, Partizipation, 2), S. 125-133.

Waldschmidt, Anne (2010): Warum und wozu brauchen die Disability Studies die Disability History? Programmatische Überlegungen. In: Bösl, Elsbeth/Klein, Anne/Waldschmidt, Anne (Hrsg.): Disability History. Konstruktionen von Behinderung in der Geschichte. Eine Einführung. 1. Aufl. Bielefeld: Transcript (Disability Studies, 6), S. 13-28.

Waldschmidt, Anne (2012): Selbstbestimmung als Konstruktion. Alltagstheorien behinderter Frauen und Männer. 2., korrigierte Auflage. Wiesbaden: VS Verlag für Sozialwissenschaften/Springer Fachmedien Wiesbaden GmbH, Wiesbaden (SpringerLink: Bücher).

Walgenbach, Katharina (2013): Heterogenität - Intersektionalität - Diversity in der Erziehungswissenschaft. Stuttgart: UTB (UTB, 8546: Erziehungswissenschaft).

Walter, Joachim (2004): Sexualbegleitung und Sexualassistenz bei Menschen mit Behinderungen. Heidelberg: Universitätsverlag Winter.

Wibs (Hrsg.) (2005): Das Gleichstellungsbuch. Innsbruck: Alpina. 
Wiezorek, Christine/Fritzsche, Sylke (2010): Methodentriangulation im Forschungsprozess. Zum Beitrag rekonstruktiver Forschungsverfahren im Hinblick auf die Reflexion quantitativer Forschungsverfahren. In: Ecarius, Jutta/Schäffer, Burkhard (Hrsg.): Typenbildung und Theoriegenerierung. Methoden und Methodologien qualitativer Bildungs- und Biographieforschung. Opladen u.a.: Budrich, S. 127142.

Wildwasser Würzburg e.V. (Hrsg.) (2007): Richtig wichtig - stolz und stark. Köln: Eigenverlag.

Windisch, Matthias/Miles-Paul, Ottmar (Hrsg.) (1991): Selbstbestimmung Behinderter. Leitlinien für die Behinderten-Politik und -Arbeit: Vortragsreihe des Fachbereichs Sozialwesen der Universität, Gesamthochschule Kassel, in Kooperation mit dem Verein zur Förderung für Autonomie Behinderter (FAB e.V.). Kassel: GhK, Fachbereich 4, Sozialwesen.

Witteck, Christina (2014): Resilienz in der Sozialpädagogik: Möglichkeiten der Resilienzförderung. 1. Aufl. Hamburg: Diplomica Verlag.

Wolff, Stephan (2009): Wege ins Feld und ihre Varianten. In: Flick, Uwe/Kardorff, Ernst von/Steinke, Ines (Hrsg.): Qualitative Forschung. Ein Handbuch. Orig.Ausg., 7. Aufl. Reinbek bei Hamburg: Rowohlt-Taschenbuch-Verl. (Rororo Rowohlts Enzyklopädie, 55628), S. 334-348.

Wrentschur, Michael (2011): Forumtheater: Szenisch-partizipatives Forschen als Beitrag zur sozialen Teilhabe und politischer Beteiligung. In: Heimgartner, Arno/Loch, Ulrike/Sting, Stephan (Hrsg.): Empirische Forschung in der Sozialen Arbeit. Methoden und methodologische Herausforderungen. 1. Aufl. Münster, Westf.: LIT (Soziale Arbeit - Social Issues, 15), S. 137-152.

Zemp, Aiha/Pircher, Erika/Neubauer, Christine (1996): „Weil es weh tut mit Gewalt““. Sexuelle Ausbeutung von Mädchen und Frauen mit Behinderung. Wien: Institut für Alltagskultur. 



\section{Anhang}

\subsection{Regeln für die Belege des Datenmaterials}

Der Anlage der Arbeit entsprechend, werden nachfolgend Regeln für die Belege aus den Referenzgruppensitzungen und für die durchgeführten Interviews angeführt.

\section{Referenzgruppensitzungen}

Direkte oder indirekte Zitate aus den Referenzgruppensitzungen sind auf Wunsch der Frauen aus der Referenzgruppe nicht einzelnen Frauen zugeordnet, sondern es wird jeweils entweder auf eine gemeinsame Einschätzung verwiesen oder auf eine nicht namentlich genannte Frau aus der Referenzgruppe. Dabei wird jeweils auf die entsprechende Sitzung und die Seite des Protokolls verwiesen. So bedeutet etwa 2RGS-S1, dass diese Anmerkung dem Protokoll der 2. Referenzgruppensitzung (RGS) von Seite 1 entnommen ist.

Schriftliche Dokumente, die auf Basis der Arbeit der Referenzgruppe erstellt wurden, sind zum Teil in der Arbeit selbst, zum Teil im Anhang erfasst. Dabei wird auf das entsprechende Dokument und die Sitzung verwiesen, in der dieses erarbeitet wurde. So bedeutet etwa Dok1-1RGS, dass es sich um das erste Dokument handelt und dass dieses in der ersten Sitzung der Referenzgruppe erarbeitet wurde.

\section{Interviews}

In der Darstellung der Ergebnisse der Interpretationen der Interviews werden wörtliche Zitate der Interviewpartnerinnen angeführt. Aus Gründen der Nachvollziehbarkeit werden in manchen Fällen auch erzählgenerierende Fragen oder auch Nachfragen durch die Interviewerin dargestellt. Für die Belege gelten dabei folgende, auf den Kontext der vorliegenden Studie abgestimmte Regeln:

Grundsätzlich werden wörtliche Zitate dann abgesetzt und in Punktgröße 10 formatiert wiedergegeben, wenn sie mehr als 25 Worte beinhalten. Kürzere Ausführungen sind im Regelfall im Fließtext integriert. Im Einzelfall erfolgt aber auch eine dementsprechende Behandlung wörtlicher Äußerungen, wenn sie als zentrale Aussage für die vorliegende Orientierung oder als Fokussierungsmetapher eingeschätzt werden können. 
Ein Beleg für ein wörtliches Zitat einer interviewten Frau wird wie folgt am Ende dieser Ausführungen in Klammern wie über das folgende Beispiel ersichtlich gekennzeichnet:

(IA 220-231): Der Beleg verweist auf das Interview (I) mit Anna (A) und umfasst die Zeilen 220-231 aus der Transkription.

In den Interviews erfolgen auch Erzählungen, die über die integrierte Vignettenmethode angeregt wurden. Dies wird über einen entsprechenden Zusatz sichtbar gemacht und wie im folgenden Beispiel dargestellt:

(IA-VA5 890-901): Der Beleg verweist darauf, dass die Beschreibung im Interview mit Anna in Zeile 890-901 erfolgt. Der Zusatz VA5 verweist darauf, dass die Aussage im Zusammenhang mit einem selbst ausgewählten Bild im Rahmen der Vignettenmethode (V) erfolgte - hier im Beispiel werden Annas Assoziationen zu Vignette A5 dokumentiert.

Paraphrasierungen von Ausführungen von Interviewteilnehmerinnen werden mit ,,vgl.“ gekennzeichnet - (vgl. IA 20-21).

Die Interviewerin, die in allen Fällen die Autorin selbst war, wird im Interviewverlauf mit „I“ gekennzeichnet.

Die Ausführungen der interviewten Frauen wurden vom Dialekt in eine im deutschen Sprachraum nachvollziehbare Sprache überführt. Spezielle Worte aus dem lokalen Dialekt werden dabei im Original belassen und in Fußnoten erläutert. Veränderungen werden dabei ausschließlich im Hinblick auf eine bessere Gewährleistung des Leseflusses oder im Kontext notwendiger Anonymisierung von Personen, Institutionen etc. vorgenommen. Dort, wo Auslassungen oder Ergänzungen in wörtlichen Zitaten erfolgen, wird dies mit eckigen Klammern gekennzeichnet - [...] bzw. [Veränderung].

\subsection{Angaben zu den Interviewpartnerinnen}

\begin{tabular}{lllll}
\hline $\begin{array}{l}\text { Nummer/ } \\
\text { Pseudonym }\end{array}{ }^{146}$ & Alter & $\begin{array}{l}\text { Wohnform: } \\
\text { Lebt bei/in }\end{array}$ & $\begin{array}{l}\text { Arbeit/ } \\
\text { Beschäftigung }\end{array}$ & $\begin{array}{l}\text { Materieller } \\
\text { Status }\end{array}$ \\
\hline 1 Natalie & 32 & Mutter & $\begin{array}{l}\text { Keine Arbeit, keine } \\
\text { Beschäftigung }\end{array}$ & $\begin{array}{l}\text { Kein Lohn, } \\
\text { Taschengeld } \\
\text { (TG) von Mutter }\end{array}$ \\
\hline 2 Verena & 25 & Institution & Werkstatt & TG \\
\hline 3 Brigitte & 31 & Vater & Werkstatt & TG \\
\hline 4 Doris & 22 & Eltern & Werkstatt & TG \\
\hline 5 Isolde & 21 & Eltern & Werkstatt & TG \\
\hline 6 Marlene & 18 & Mutter & Werkstatt & TG \\
\hline
\end{tabular}

146 Die Vornamen sind anonymisiert, die Pseudonyme wurden zum Teil von den Frauen selbst gewählt, zum Teil (falls kein eigener Name gewählt wurde) von der Autorin vergeben. 


\begin{tabular}{lllll}
\hline 7 Eva & 27 & $\begin{array}{l}\text { WG selbständig } \\
\text { mit Unterstützung }\end{array}$ & Arbeit & Lohn \\
\hline 8 Alina & 21 & Eltern & $\begin{array}{l}\text { Keine Arbeit, } \\
\text { keine Beschäftigung }\end{array}$ & $\begin{array}{l}\text { Kein Lohn, } \\
\text { TG Eltern }\end{array}$ \\
\hline 9 Karin & 20 & Institution & Werkstatt & TG \\
\hline 10 Angelika & 19 & Eltern & Werkstatt & TG \\
\hline 11 Felicitas & 23 & Eltern & $\begin{array}{l}\text { Keine Arbeit, } \\
\text { keine Beschäftigung }\end{array}$ & $\begin{array}{l}\text { Kein Lohn, } \\
\text { TG, Eltern }\end{array}$ \\
\hline 12 Jessica & 24 & Eltern & Werkstatt & TG \\
\hline 13 Martina & 21 & Wohnung allein & Arbeit & Lohn \\
\hline 14 Gloria & 31 & Eltern & Werkstatt & TG \\
\hline 15 Melanie & 19 & Eltern & Werkstatt & TG \\
\hline 16 Anna & 28 & Mutter & Projekt/Anlehre & TG \\
\hline 17 Petra & 26 & Institution & Werkstatt & TG \\
\hline 18 Sonja & 30 & Institution & Werkstatt & TG \\
\hline 19 Tamara & 29 & Eltern & Arbeit & Lohn \\
\hline 20 Ursula & 23 & Mutter & Werkstatt & TG \\
\hline
\end{tabular}

\subsection{Verzeichnis der Tabellen und Grafiken}

Tabelle 1: Faktoren für Fremdbestimmung …..........................................30

Tabelle 2: Inhalte und Ergebnisse der sieben Referenzgruppensitzungen.....55

Tabelle 3: Aspekte, die von der Referenzgruppe als relevant für Selbst bestimmung erachtet werden

Tabelle 4: Erzählaufforderung und optionale Nachfragen im Rahmen der Vignettenbeschreibungen in den durchgeführten Interviews

Tabelle 5: Auswertungsschritte der dokumentarischen Methode ...................75

Tabelle 6: Charakteristik verschiedener Textsorten in der dokumentarischen Methode.

Tabelle 7: Die vier erhobenen Orientierungen junger Frauen mit

Lernschwierigkeiten im Überblick

Grafik 1: Einflussfaktoren auf Orientierung im Spannungsfeld von

Fremd- oder Selbstbestimmung.

Grafik 2: Triangulation im vorliegenden Forschungsprojekt. 


\subsection{Quellen der Bilder (visuellen Vignetten), die für die Anregung von Erzählungen im Rahmen der Interviews verwendet wurden}

Foto von Veronika Hammel (S. 35) von Bothor, Mathias. Aus: Mürner, Christian/Schönwiese, Volker (2007): Das Bildnis eines behinderten Mannes. Bildkultur der Behinderung vom 16. bis ins 21. Jahrhundert: Ausstellungskatalog und Wörterbuch. Neu-Ulm: AG SPAK Bücher (AG SPAK-Bücher).

Coverfoto der Publikation Fischer, Julia (Hrsg.) (2010): Mehr vom Leben. Frauen und Männer mit Behinderung erzählen. 1. Aufl. Bonn: Balance Buch + Medien Verl. (Balance Erfahrungen).

Bilder aus dem Film von Codina, Alexandra: Monica und David (2009/2010).

Frau im Rollstuhl: http://www.barrierefreie-urlaubswelt.de

Bilder/Illustrationen von Breen, Barbara (S. 5, S. 14, S. 15, S. 16, S. 19, S. 21) aus der Publikation: Wildwasser Würzburg e.V. (Hrsg.) (2007): Richtig wichtig - stolz und stark. Ein FrauenBilderLeseBuch. Köln: Eigenverlag. 